\title{
NISTIR 5354
}

(Supersedes NISTIR 5274)

\section{VALIDATED PRODUCTS LIST 1994 No. 1}

\section{Programming Languages Database Language SQL Graphics GOSIP POSIX Computer Security}

Judy B. Kailey Peggy N. Himes Editors

U.S. DEPARTMENT OF COMMERCE Technology Administration National Institute of Standards and Technology

Computer Systems Laboratory Software Standards Validation Group Gaithersburg, MD 20899

January 1994

$Q C$ 

NISTIR 5354

(Supersedes NISTIR 5274)

\section{VALIDATED PRODUCTS LIST 1994 No. 1}

\section{Programming Languages Database Language SQL Graphics GOSIP POSIX Computer Security}

\section{Judy B. Kalley Peggy N. Himes Editors}

U.S. DEPARTMENT OF COMMERCE Technology Administration National Institute of Standards and Technology Computer Systems Laboratory Software Standards Validation Group Gaithersburg, MD 20899

January 1994

(Supersedes October 1993 issue)

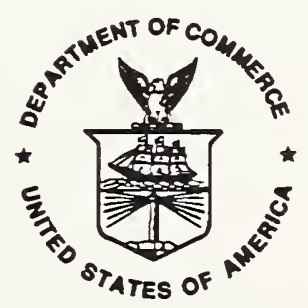

U.S. DEPARTMENT OF COMMERCE Ronald H. Brown, Secretary

TECHNOLOGY ADMINISTRATION Mary L Good, Under Secretary for Technology

NATIONAL INSTITUTE OF STANDARDS AND TECHNOLOGY

AratJ Prabhakar, Director 



\section{FOREWORD}

The Validated Products List is a collection of registers describing implementations of Federal Information Processing Standards (FIPS) that have been validated for conformance to FIPS. The Validated Products List also contains information about the organizations, test methods and procedures that support the validation programs for the FIPS identified in this document.

The Validated Products List is updated quarterly. 


\section{TABLE OF CONTENTS}

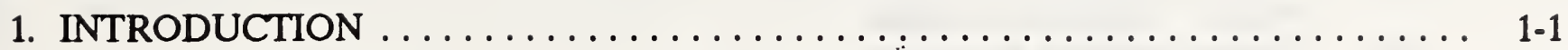

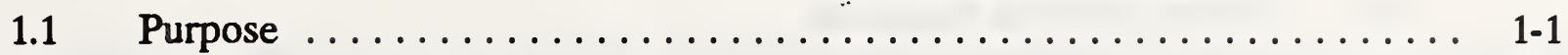

1.2 Document Organization $\ldots \ldots \ldots \ldots \ldots \ldots \ldots \ldots \ldots \ldots \ldots \ldots \ldots \ldots \ldots \ldots \ldots, 1-2$

1.2.1 Programming Languages ......................... $1-2$

1.2.2 Database Language SQL $\ldots \ldots \ldots \ldots \ldots \ldots \ldots \ldots \ldots \ldots \ldots \ldots \ldots \ldots \ldots, 1-2$

1.2 .3 Graphics $\ldots \ldots \ldots \ldots \ldots \ldots \ldots \ldots \ldots \ldots \ldots \ldots \ldots \ldots \ldots \ldots \ldots \ldots, 1-2$

1.2 .4 GOSIP $\ldots \ldots \ldots \ldots \ldots \ldots \ldots \ldots \ldots \ldots \ldots \ldots \ldots \ldots \ldots \ldots \ldots \ldots \ldots, 1-2$

1.2 .5 POSIX $\ldots \ldots \ldots \ldots \ldots \ldots \ldots \ldots \ldots \ldots \ldots \ldots \ldots \ldots \ldots \ldots \ldots \ldots \ldots \ldots \ldots \ldots, 1-2$

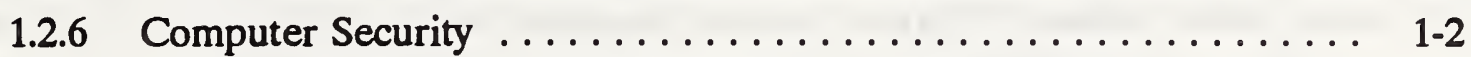

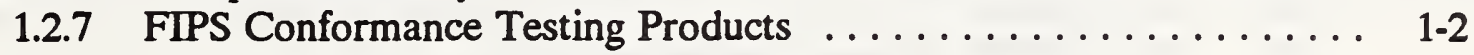

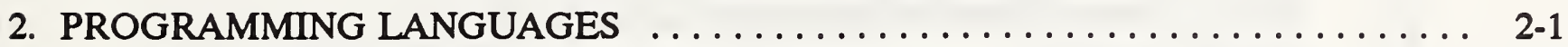

$2.1 \quad$ FIPS Programming Language Standards $\ldots \ldots \ldots \ldots \ldots \ldots \ldots . \ldots \ldots . \ldots .2-1$

2.2 Organization of Programming Language Processor Entries ......... 2-1

$2.3 \quad$ Validation of Processors ....................... 2-2

2.3.1 Validation Requirements $\ldots \ldots \ldots \ldots \ldots \ldots \ldots \ldots \ldots \ldots \ldots \ldots, 2-2$

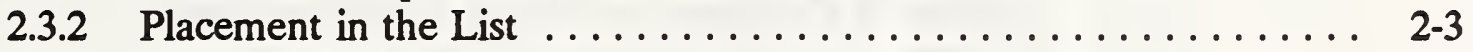

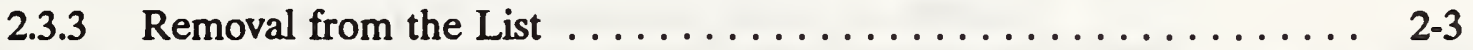

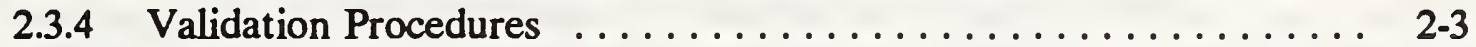

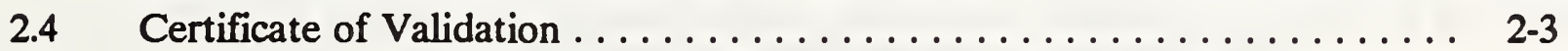

2.5 Language Processor Validation Suites $\ldots \ldots \ldots \ldots \ldots \ldots \ldots \ldots .2-4$

2.6 Testing Laboratories and Supporting Organizations $\ldots \ldots \ldots \ldots \ldots .2-5$

2.7 Language Processors with Certificates - No Nonconformities ... . . . . . 2. 2-7

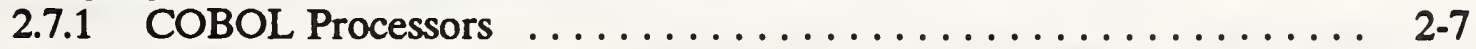

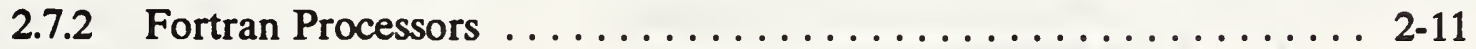

2.7.3 Ada Processors ........................... 2-17

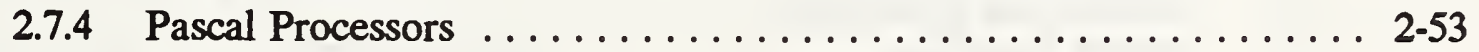

2.7.5 C Processors ........................... 2-55

2.7.6 Mumps Processors ......................... 2-61

2.8 Language Processors with Registered Reports Only .......... 2-62

2.8.1 COBOL Processors with Nonconformities ............. 2-62

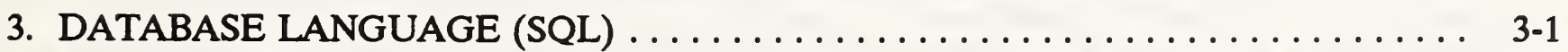

3.1 FIPS Database Language Standards . . . . . . . . . . . .

3.2 Organization of Database Language Processor Entries . . . . . . . . . 3-1

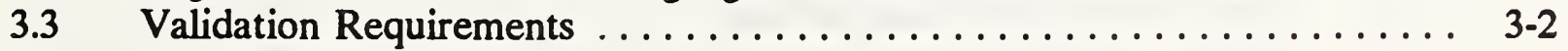

3.4 Certificate of Validation . . . . . . . . . . . . . . . . . . . . . .

$3.5 \quad$ Registered Report ........................... 3-2

3.6 Validation Procedures $\ldots \ldots \ldots \ldots \ldots \ldots \ldots \ldots \ldots \ldots \ldots . \ldots \ldots, 3-2$

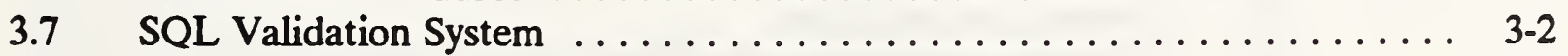

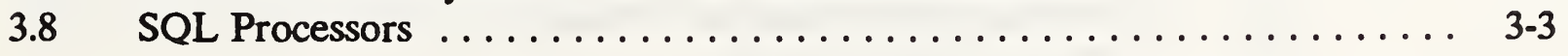

4. GRAPHICS CONFORMANCE TESTING $\ldots \ldots \ldots \ldots \ldots \ldots \ldots \ldots \ldots$ 4 4 .

$4.1 \quad$ FIPS GKS Standard $\ldots \ldots \ldots \ldots \ldots \ldots \ldots \ldots \ldots \ldots \ldots \ldots \ldots \ldots \ldots \ldots, 1$

4.1.1 Organization of GKS Entries $\ldots \ldots \ldots \ldots \ldots \ldots \ldots \ldots \ldots$ 4-1

4.2 FIPS PHIGS Standard $\ldots \ldots \ldots \ldots \ldots \ldots \ldots \ldots \ldots \ldots \ldots \ldots \ldots \ldots \ldots \ldots, 2$ 
4.2.1 Organization of PHIGS Entries $\ldots \ldots \ldots \ldots \ldots \ldots \ldots \ldots \ldots, 4-2$

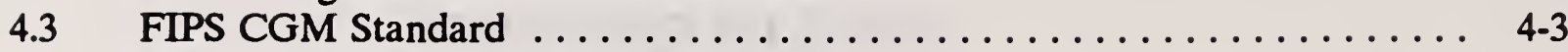

4.3.1 CGM Test Labs and Test Suite $\ldots \ldots \ldots \ldots \ldots \ldots \ldots \ldots . \ldots . \ldots, 4$

4.3.2 Certificate of Validation $\ldots \ldots \ldots \ldots \ldots \ldots \ldots \ldots \ldots, 4,3$

4.3.3 Validated Metafiles ........................ 4-3

$4.4 \quad$ Raster Graphics Standards ........................ 4-4

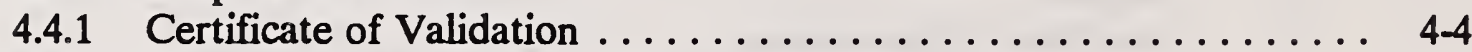

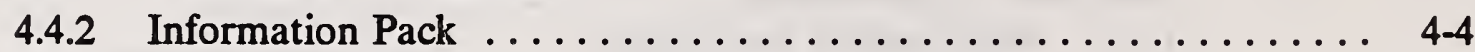

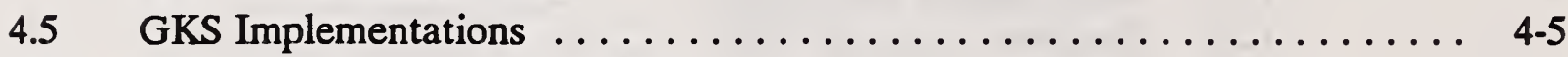

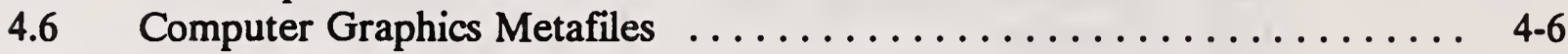

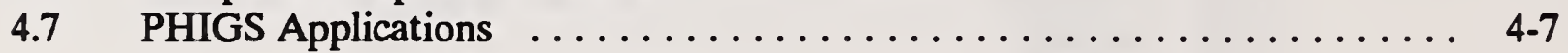

5. U.S. GOSIP Testing Program Register Database System (GRD) $\ldots \ldots \ldots \ldots \ldots$. $5-1$

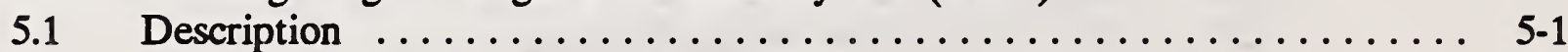

$5.2 \quad$ U.S. GOSIP Register Database (GRD) $\ldots \ldots \ldots \ldots \ldots \ldots \ldots \ldots$. $5-1$

5.3 How To Access The GOSIP Register Database (GRD) ........... 5-1

5.4 GOSIP Registers ........................... 5-3

5.4.1 Register of Conformance Testing Laboratories ........... 5-3

5.4.2 Register of Approved US GOSIP MOT Validation

5.4.3 Register of Conformance Tested GOSIP Products ......... 5-6

5.4.4 Register of GOSIP Interoperability Test Suites .......... 5-57

5.4.5 Register of GOSIP Interoperability Test and Registration

Services ............................ $5-57$

5.4.6 Register of GOSIP Means of Testing $\ldots \ldots \ldots \ldots \ldots \ldots \ldots$ 5-57

5.4 .7 US GOSIP PICS PROFORMA .................. $5-67$

5.4.8 GOSIP Register Database Abstract Test Suites (ATS) $\ldots \ldots \ldots$ 5-69

6. NIST POSIX CONFORMANCE TESTING $\ldots \ldots \ldots \ldots \ldots \ldots \ldots \ldots \ldots \ldots \ldots, 6,1$

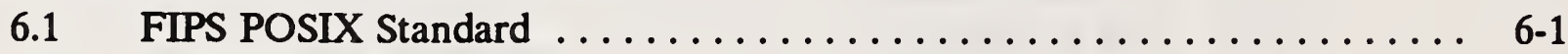

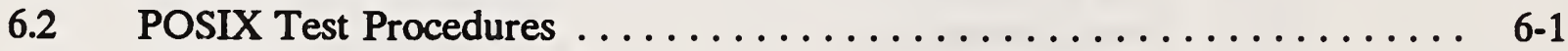

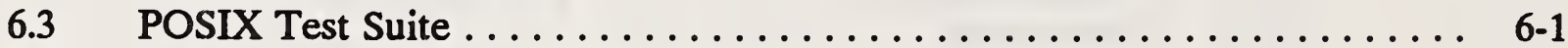

$6.4 \quad$ Validation Requirements ........................ 6-1

6.5 Testing Laboratories for NIST POSIX (FIPS 151-1) . . . . . . . . 6 6-2

6.6 Validated Products for NIST POSIX (FIPS 151-1) .............. 6-3

6.7 Testing Laboratories and Validated Products for NIST POSIX (FIPS 151-2) 6-14

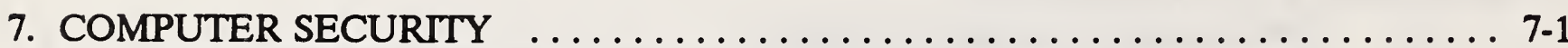

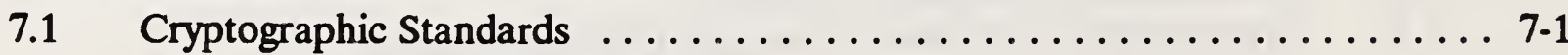

7.2 Data Encryption Validation Tests .................... $7-1$

7.3 Message Authentication Code (MAC) Validation System $\ldots \ldots \ldots \ldots \ldots$ 7-1

7.4 Key Management Validation System (KMVS) ................ 7-1

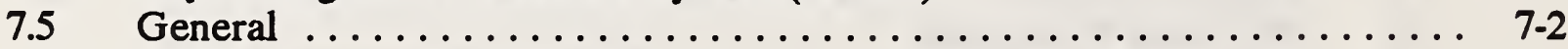

7.5.1 Request for Validation. ................... $7-2$

7.5.2 Information about Validated Products. . . . .

7.5.3 Validation Documentation. ...................... 7.2

7.6 DES Validated Devices ......................... 7-3

7.7 Message Authentication Code (MAC) Implementations $\ldots \ldots \ldots \ldots \ldots$ 7.9

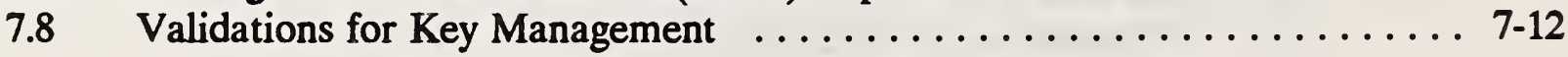




\section{INTRODUCTION}

\subsection{Purpose}

The testing of Information Technology (IT) Products to determine the degree to which they conform to specific Federal Information Processing Standards (FIPS) may be required by Government agencies as specified by the FIPS, Federal Information Resources Management Regulation (FIRMR) Parts 201-20.303, 201-20.304, and 201-39.1002, and the associated Federal ADP and Telecommunications Standards Index. Products having a current validation certificate or test report may be offered or delivered by vendors in response to requirements as set forth in solicitations by Federal agencies. The Validated Products List (VPL) contains conformance testing information for the following IT Standards:

Programming Languages COBOL, Fortran, Ada, Pascal, C, and MUMPS

Database Language SQL

Graphics

GOSIP

POSIX

Computer Security

This List is updated and published quarterly. The information contained herein is supplied by the contributors listed in Section 2.6 and Appendix A, and is current as of the tenth of the month preceding the publication date. Copies of the VPL may be obtained from:

National Technical Information Service

U.S. Department of Commerce

5285 Port Royal Road

Springfield, VA 22151.

Subscriptions: $\quad$ (703) $487-4630$

Individual Copies: (703) $487-4650$

Ordering Number: PB93-937303/AS

The entries in the printed VPL are contained in WordPerfect Version 5.1 files and may be accessed on the Internet using the instructions listed below.

Type: ftp speckle.ncsl.nist.gov (internet address is 129.6.59.2)

Login as user $\mathrm{ftp}$

Type your e-mail address preceded by a dash $(-)$ as the password

Type: cd vpl

Type: binary

Type: get and the name of the file you want; e.g. language

Questions or comments concerning the VPL should be directed to:

National Institute of Standards and Technology (NIST)

Computer Systems Laboratory

Software Standards Validation Group

Building 225, Room A266

Gaithersburg, MD 20899

Telephone (301) 975-3274 


\subsection{Document Organization}

\subsubsection{Programming Languages}

Section 2 identifies those COBOL, Fortran, Pascal, C, Ada, and MUMPS programming language processors that have a current validation certificate or registered test report referencing the applicable FIPS as of the date of this publication.

\subsubsection{Database Language SQL}

Section 3 identifies those SQL language processors that have a validation certificate or a registered test report for FIPS PUB 127-1 as of the date of this publication.

\subsubsection{Graphics}

Section 4 lists the implementations or files for which a validation certificate is currently in place. These entries include:

Graphical Kernel System (GKS) implementations (FIPS PUB 120-1),

Programmer's Hierarchical Interactive Graphics Systems (PHIGS) (FIPS PUB 153),

Computer Graphics Metafiles (CGMs) (FIPS PUB 128),

Raster Graphics data files (FIPS PUB 150).

\subsubsection{GOSIP}

Section 5 contains information regarding FIPS PUB 146-1, GOSIP, conformance testing registers.

\subsubsection{POSIX}

Section 6 identifies POSIX products that have a current validation certificate for FIPS PUB 151-1 and FIPS PUB 151-2.

\subsubsection{Computer Security}

Section 7 contains information regarding validated products for FIPS PUB 46-1, Data Encryption Standard (DES), FIPS PUB 113, Computer Data Authentication (Implements Message Authentication Code, ANSI X9.9), and FIPS PUB 171, Key Management Using ANSI X9.17.

\subsubsection{FIPS Conformance Testing Products}

Appendix A lists FIPS conformance testing products and services available to the public. Information for these products and services may be obtained by contacting the appropriate person listed. 


\section{PROGRAMMING LANGUAGES}

\subsection{FIPS Programming Language Standards}

As specified by the FIPS, FIRMR and the associated Federal ADP and Telecommunications Standards Index, Federal agencies when acquiring language processors, are responsible for assuring that processors are in accordance with the following FIPS for programming languages:

a. COBOL processors must satisfy the provisions of FIPS PUB 21-3, COBOL, and must be identified as implementing all of the language elements of at least one of the subsets of FIPS COBOL as specified in FIPS PUB 21-3.

b. BASIC processors must satisfy the provisions of FIPS PUB 68-2, BASIC.

c. Fortran processors must satisfy the provision of FIPS PUB 69-1, Fortran, and must be identified as implementing all of the language elements of the subset or full levels of FIPS Fortran as specified in FIPS PUB 69-1.

d. Pascal processors must satisfy the provisions of FIPS PUB 109, Pascal.

e. Ada processors must satisfy the provisions of FIPS PUB 119, Ada.

f. MUMPS processors must satisfy the provisions of FIPS PUB 125, MUMPS.

g. C processors must satisfy the provisions of FIPS PUB 160, C.

h. VHDL processors must satisfy the provisions of FIPS PUB 172, VHDL.

Copies of the above publications are for sale by the National Technical Information Service, U.S. Department of Commerce, Springfield, VA 22161.

Conformance testing programs are currently available for all above FIPS except for the programming language BASIC and VHDL. A test suite for BASIC is being developed.

\subsection{Organization of Programming Language Processor Entries}

The entries in the VPL for programming language processors are presented as follows:

- The VENDOR ID column contains the name of the Vendor of the processor.

- The PROCESSOR ID column contains the Processor identification and the Validation Summary Report (VSR) or certificate number. This number refers to the VSR that was produced as a result of the testing. The VSR describes the testing environment and details any processor nonconformity that was detected as a result of the testing. Information for obtaining a VSR is listed in section 2.6.

- Derived processors in the VENDOR \& COMPILER column are Ada processors that have been derived from the processor/hardware/operating system environment used during the testing. In order for derived processors to be listed here, they must be properly registered with the Department of Defense, Ada Joint Program Office (AJPO) by the vendor of the processor. 
- The HARDWARE \& OPERATING SYSTEM column presents the hardware and operating system environment (including pertinent supporting system software) used during the validation. In the case of Ada processors, those environments for derived processors will appear in this column.

- The EXPIRY DATE column lists the expiration date of the Certificate of Validation or Registered Validation Summary Report. A processor may be included in the List after the certificate has expired if the validation is in process. Notification must be received by NIST at least 30 days prior to publication of the List in order for such a processor to be included. In this case the expiration date will be followed by "(pending)".

- For COBOL processors, the SUBSET column cites the applicable Federal Subset. For Fortran processors, the LEVEL column specifies the applicable Federal level. For Pascal processors, the ISO 7185 Pascal Standard Level (ISO 7185 Level 0 is equivalent to FIPS 109). This designation is presented in the PROCESSOR ID column.

- The entries in the OTHER ENVIRONMENTS column are registered hardware and operating system environments for the processor tested. The vendor of the processor has certified that the identified processor, when operating under the environments included in this column, produces the same test results as those obtained from the hardware and operating system environment used during the validation. Test results and other information from these environments may be required as evidence for entries to be included in this column.

Also listed are the programming language processors that have been tested and during the testing were found to have one or more nonconformities.

\subsection{Validation of Processors}

\subsubsection{Validation Requirements}

In accordance with the requirements referenced in Section 1.1, language processors offered to the Government for purchase, lease, or use in connection with ADP services shall be validated for conformance to FIPS for programming languages. To confirm that the specifications of the designated FIPS have been met: .

a. the processor shall be tested with the Compiler Validation System (CVS) approved by NIST,

b. the processor validations shall be conducted in accordance with NIST validation procedures,

c. a Validation Summary Report (VSR) shall be produced summarizing the test results of the CVS on the designated processor for that FIPS,

d. all nonconformities noted in the VSR shall be corrected within twelve months,

e. a Certificate of Validation shall be issued if validation results warrant. In order for a processor to receive a Certificate of Validation the processor must successfully pass all applicable tests of the CVS without exception.

The Federal ADP and Telecommunications Standards Index supplies standard terminology which may allow for delayed validation. When delayed validation is allowed, the offeror may meet this 
requirement by showing evidence of having submitted the processor for validation. Proof of submission is in the form of a letter from NIST scheduling the validation.

Programming language processors offered to the Federal Government must comply with the applicable Government requirements. Failure to comply with these requirements shall be deemed sufficient cause to declare a bidder non-responsive or to declare a vendor in default for failure to deliver required software.

\subsubsection{Placement in the List}

For a processor to be placed in the List it must:
a. have been officially tested within the past twelve calendar months, and
b. have no errors remaining that were identified during a previous test.

\subsubsection{Removal from the List}

A processor is removed from the List when:

a. the processor is not officially tested within twelve calendar months, or

b. testing indicates that the processor still contains errors identified during a previous validation.

\subsubsection{Validation Procedures}

Validation procedures are published in the following documents:

Compiler Validation Procedures, dated January 15, 1993

Ada Compiler Validation Procedures and Guidelines, Version 3.1, August, 1992

Pascal Validation Policy and Procedures, Version 5.5, April 9, 1993

MUMPS Validation Procedures, Version 1.0, dated August 13, 1992

\subsection{Certificate of Validation}

A Certificate of Validation is issued for those programming language processors that have been tested and are considered to be in compliance with the FIPS as specified by the FIPS, FIRMR and the associated Federal ADP and Telecommunications Index.

The requirement for retesting may be waived and the certificate of validation extended at the option of NIST if:

a. no errors were identified during the previous testing of the processor,

b. the vendor certifies, in writing, to NIST that no changes have been made to either the processor or the supporting system software, and

c. no new version of the validation system has been officially released during the interim period. 


\subsection{Language Processor Validation Suites}

Following are the validation suites and ordering information for testing programming language processors for conformance to FIPS.

a. Copies of the COBOL, Fortran, MUMPS, and Ada Compiler Validation Suites may be purchased from:

National Technical Information Service (NTIS)

5285 Port Royal Road

Springfield, VA 22161

Telephone (703) 487-4650 (Voice)

(703) $321-8547$ (FAX)

\section{COMPILER VAUDATION SYSTEM [MEDIUM/FORMAT]}

COBOL 85 (CCVS85)
Fortran (FCVS78)
Ada [Tape/Backup]
Ada [Tape/Tar]
Ada [Tape ANSI Standard]
Ada [Disk (MS/DOS)]
MUMPS [Tape/Backup]
MUMPS [Tape/ANSI]
MUMPS [Tape/Tar]
MUMPS [Disk (MS-DOS)]

VERSION

4.2

2.1

1.11

1.11

1.11

1.11

7.61

7.61

7.61

7.61

\section{NTIS \\ ACCESSION \\ NUMBER}

PB93-504918

PB85-226736

ADA212551

ADA212437

ADA212548

ADA212549

PB91-507699

PB91-507715

PB91-507723

PB91-507707

b. The current version of the Pascal Validation System (PVS) is Version 5.5 and is available from:

British Standards Institution (BSI)

Software Engineering Department

BSI Quality Assurance

P. O. Box 375

Milton Keynes

MK14 6LL

ENGLAND

Telephone (011) +44-908-220908 (Voice)

(011) + 44-908-220671 (FAX)

c. The current version of the ANSI C Validation Suite (ACVS ${ }^{\mathrm{tm}}$ ) is Version 4.0 and is available from:

Perennial, Inc.

4699 Old Ironsides Drive

Suite 210

Santa Clara, CA 95054

Telephone (408) 748-2900 (Voice) 


\subsection{Testing Laboratories and Supporting Organizations}

The organizations listed below have performed validations, supplied information, or are sources for Validation Summary Reports (VSR) for programming languages. These organizations may be contacted for validation information and for copies of VSR(s). COBOL and Fortran VSR(s) may be obtained from NIST. Pascal VSR(s) whose VSR numbers begin with "NIST" or end in "US" may also be obtained from NIST. Pascal VSR(s) whose VSR numbers end in "UK" are available from BSI. Ada VSR(s) may be obtained from the Ada Information Clearinghouse, the National Technical Information Service, or from the Ada Validation Facility (AVF) that produced the VSR. To obtain a copy of a VSR from an AVF, locate the upper case letter in the certificate number (e.g., 870608 W1...). That letter corresponds to the letter in the CODE column to the left of the organizations listed below.

\section{CODE ORGANIZATION}

S National Institute of Standards and Technology Software Standards Validation Group

Building 225, Room A266

Gaithersburg, MD 20899

(301) 975-3274

Telex: 197674 NBS UT

FAX: (301) 948-6213

N National Computing Centre Limited (NCC)

Oxford Road

Manchester

M1 7ED

ENGLAND

$(011)+44(61) 2286333$

$$
+44 \text { (61) } 2364715 \text { (FAX) }
$$

Telex 668962

German National Research Center for Computer Science (GMD)

Department Scientific Visualization

Supercomputer Center (HLRZ)

P. O. 1316, Schloss Birlinghoven

D-W-5205 Sankt Augustin 1

Germany

(011) + 49-2241-14-2706 (voice)

(011) + 49-2241-14-2618 (FAX)

kirsch@gmdzi.gmd.de

Bureau Inter Administration de

Documentation Informatique (BLADI)

21 Rue Bara

92132 Issy

France

Instituto Italiano del Marchio di Qualita (IMQ)

Via Quintiliano, 43

20138 Milano

Italy

$+39-2-5073266$

\section{CONTACTS}

L. Arnold Johnson

Judy Kailey

Carmelo Montanez

William Dashiell

Jane Pink

Jon Leigh

David Bamber

Berthold Kirsch

E. Bialot

Angelo Belloni

$2 \cdot 5$

\section{LANGUAGE}

All

COBOL, Fortran

BASIC

Pascal, C

Ada, MUMPS, SQL, VHDL

COBOL

Fortran

Ada

Fortran

COBOL

Fortran

COBOL

Fortran 
Setagaya-Ku, Tokyo

157 Japan

+81334169600

British Standards Institution (BSI)

John Souter

Pascal

P.O. Box 375

Milton Keynes MK14 6LL

ENGLAND

(011) + 44 0908-220908

Telex: 827682 BSIQAS G

W Ada Validation Facility

Dale Lange

Ada

Language Control Facility

ASD/SCEL

Wright-Patterson AFB, OH 45433-6503

(513) 255-4472

B BNI-AVF

M. Alphonse Philippe

Ada

or AFNOR

A Direction Certification

Tour Europe, Cedex 7

92080 Paris La Defense

FRANCE

(011) 33-142915960

Telefac: (011) 33-142915656

Telex: AFNOR 611974 F

I IABG-AVF

Industrieanlagen-Betriebsgesellschaft

Michael Tonndorf

Ada

Dept. ITE

Einsteinstrasse 20

D-8012 Ottobrunn

Federal Republic of Germany

+49-89-6088-2477

e-mail: tonndorf@ajpo.sei.cmu.edu

Ada Information Clearinghouse

3 D139

1211 S. Fern, C-107

The Pentagon

Washington, D.C. 20301-3081

(703) 685-1477

National Technical Information Service

Ada VSR(s)

U.S. Department of Commerce

5285 Port Royal Road

Springfield, VA 22161

(703) $487-4650$ 


\subsection{LANGUAGE PROCESSORS WITH CERTIFICATES NO NONCONFORMITIES}

2.7.1 COBOL PROCESSORS

\begin{tabular}{cccccc}
\hline VENDOR & $\begin{array}{c}\text { PROCESSOR ID } \\
\& V S R \#\end{array}$ & $\begin{array}{c}\text { HARDWARE \& } \\
\text { OPERATING SYSTEM }\end{array}$ & $\begin{array}{c}\text { EXPIRY } \\
\text { DÄTE }\end{array}$ & $\begin{array}{c}\text { SUBSET } \\
\text { OTHER ENVIRONMENTS } \\
\text { HW/OS }\end{array}$ \\
\hline
\end{tabular}

Bull HN

COBOL 85 Version
$8 \mathrm{c} 83.0$

NIST-92/1681
7/1/94 High

GCOS8 Version 4020

Release 3
DPS 8000 , DPS 9000

GCOS8 Version 4020 Release 3

\section{Computer}

Associates

Control Data

Corporation

Digital

Equipment

Corporation CA-Realia COBOL Version
4.2 Release 9305
NIST-93/1301

CA-Realia COBOL Version 4.2 Release 9305 NIST-93/1302
IBM PS/2 Model 80

OS $/ 2$ Version 2.0

IBM PS/2 Model 80 MS/DOS Version 5.0
$6 / 1 / 94$

Intermediate

IBM PS/2 Model 55SX, 60, 70. 90,95

OS/2 Version 2.0
Micro Focus COBOL/2

Version 3.0 NIST-93/1101
Contol Data 4360

EP/IX Version 2.1.1

$1 / 1 / 94$

$6 / 1 / 94$

Intermediate

IBM PS/2 Model 55sx, 60, 70, 90,95

MS-DOS Version 5.0
High

Control Data 4000 $E P / T X$ Version 2.1.1

\section{VAX COBOL \\ Version 5.1 NIST: $92 / 224 A$}

VAX $8800 \quad 12 / 1 / 94$ High

HewlottPackard Company
COBOL/HP-UX

Version B.07.00 NIST-93/1501
HP9000 Series 720

HP-UX Version 9.0
$5 / 1 / 94$

High

HP9000 Series $635,645,705$, $710,715 / 33,715 / 50,720$, $725 / 50,730,735,750,755$, $807,815,817,822,825,827$. $832,834,835,837,842,845$, $847,850,852,855,857,860$, $865,867,870,870 / 200$, $870 / 300,870 / 400,877,887$, $890 / 1,890 / 2,890 / 3,890 / 4$, 897, F10, F2O, F30, G30, G40, G5O, H2O, H3O, H4O, H5O, 130 , 140,150

HP-UX Version 9.0 


\begin{tabular}{|c|c|c|c|c|c|}
\hline VENDOR & $\begin{array}{c}\text { PROCESSOR ID } \\
\text { \& VSR \# }\end{array}$ & $\begin{array}{c}\text { HARDWARE \& } \\
\text { OPERATING SYSTEM }\end{array}$ & $\begin{array}{r}\text { EXPIRY } \\
\text { DATE } \\
\end{array}$ & SUBSET & OTH \\
\hline & $\begin{array}{l}\text { COBOL/IX } \\
\text { Version A.04.08 } \\
\text { NIST-93/1502 }\end{array}$ & $\begin{array}{l}\text { HP3000 Series } 967 \\
\text { MPE iX Version B.09.66 }\end{array}$ & $5 / 1 / 94$ & High & $\begin{array}{l}\text { HP3O } \\
925, \\
948, \\
960, \\
980 / 2 \\
987, \\
M P E\end{array}$ \\
\hline $\begin{array}{l}\text { IBM Canada, } \\
\text { Ltd. }\end{array}$ & $\begin{array}{l}\text { COBOL/400 } \\
\text { Version } 2 \text { Release } 2 \\
\text { NIST- } 92 / 2071\end{array}$ & $\begin{array}{l}\text { AS } / 400 \\
\text { OS } / 400 \text { Version } 2 \\
\text { Release } 2\end{array}$ & $9 / 1 / 94$ & Intermediate & \\
\hline \multirow[t]{5}{*}{ IBM Corporation } & $\begin{array}{l}\text { IBM SAA AD/CYCLE } \\
\text { COBOL/370 Version } 1 \\
\text { Release } 1 \\
\text { NIST-94/1082 }\end{array}$ & $\begin{array}{l}\text { IBM } 3090 \\
\text { VM/ESA Version ESA } \\
\text { Release } 1.0\end{array}$ & $12 / 1 / 94$ & High & $\begin{array}{l}\text { IBM } 3 \\
\text { VM/E } \\
\text { Relea }\end{array}$ \\
\hline & $\begin{array}{l}\text { IBM SAA AD/CYCLE } \\
\text { COBOL/370 Version } 1 \\
\text { Release } 1 \\
\text { NIST-94/1081 }\end{array}$ & $\begin{array}{l}\text { IBM } 3090 \\
\text { MVS/ESA Version } 4 \\
\text { Release } 3\end{array}$ & $12 / 1 / 94$ & High & $\begin{array}{l}\text { IBM } 3 \\
\text { MVS/ } \\
\text { Relea }\end{array}$ \\
\hline & $\begin{array}{l}\text { VS COBOL. II } \\
\text { Version 1. Release } 4 \\
\text { NIST-93/1801 }\end{array}$ & $\begin{array}{l}\text { IBM } 3090 \\
\text { MVS/ESA Version } 4 \\
\text { Release } 2.2\end{array}$ & $8 / 1 / 94$ & Intermediate & $\begin{array}{l}\text { IBM } 3 \\
\text { MVS/ } \\
\text { MVS/ }\end{array}$ \\
\hline & $\begin{array}{l}\text { VS COBOL II } \\
\text { Version } 1 \text { Release } 4 \\
N S T-93 / 1802\end{array}$ & $\begin{array}{l}\text { IBM } 3090 \\
\text { VSE/ESA Version } 1 \\
\text { Release } 3\end{array}$ & $9 / 1 / 94$ & Intermediate & $\begin{array}{r}\text { IBM } 3 \\
\text { VSE/L }\end{array}$ \\
\hline & $\begin{array}{l}\text { VS COBOL II } \\
\text { Version } 1 \text { Release } 4 \\
\text { NST- } 93 / 1803\end{array}$ & $\begin{array}{l}\text { IBM } 3090 \\
\text { VM/ESA Version ESA } \\
\text { Release } 1.0\end{array}$ & $9 / 1 / 94$ & Intermediate & $\begin{array}{r}\mathrm{IBM} 3 \\
\mathrm{VM} / \mathrm{S}\end{array}$ \\
\hline \multirow{2}{*}{$\begin{array}{l}\text { mbp } \\
\text { Information- } \\
\text { stechnologie } \\
\text { GmbH }\end{array}$} & $\begin{array}{l}\text { Visual COBOL XO } \\
\text { Version } 3.3 \\
\text { NIST/NCC- } 93 / 976\end{array}$ & $\begin{array}{l}\text { Sintronic } 486 \text { DX } 50 \\
M S-D O S \text { Version } 5.0\end{array}$ & $7 / 15 / 94$ & High & \\
\hline & $\begin{array}{l}\text { Visual COBOL XO } \\
\text { Version } 3.3 \\
\text { NIST/NCC-93/977 }\end{array}$ & $\begin{array}{l}\text { Hewlett Packard } \\
\text { HP9000/807S } \\
\text { HP-UX Version } 8.02\end{array}$ & $7 / 15 / 94$ & High & \\
\hline
\end{tabular}

Micro Focus

Micro Focus COBOL for DOS, Windows and OS $/ 2$, Version 3.1 NIST-93/1821
Compaq Deskpro IBM OS/2 Version 2.0 IBM PS/2 Model 90 IBM OS/2 Version 1.3 IBM PS/2 Model 90 Microsofi Windows NT IBM PS/2 Model 80 Microsofi DOS Version 6
8/1/94 High

IBM PS/2 Models 70, 80 IBM OS $/ 2$ Version 2.0 IBM PS/2 Models 60, 65sx, 80 IBM OS $/ 2$ Version 1.3 IBM PS/2 Models 90, 65sx, 60 IBM DOS Version 6.0 IBM PS/2 Models 80, 70-486, 65sx, 60 $M S$ DOS Version 5.0 IBM PS $/ 2$ Models 90, 80, 65sx, 60

MS DOS Version 4.0

IBM PS/2 Models 80, 70-486, $65 \mathrm{sx}, 60$

MS DOS Version 3.3 


\begin{tabular}{|c|c|c|c|c|c|}
\hline VEND & $\begin{array}{l}\text { PROCESSOR II } \\
\text { \& VSR \# }\end{array}$ & $\begin{array}{c}\text { HARDWARE \& } \\
\text { OPERATING SYSTEM }\end{array}$ & $\begin{array}{r}\text { EXPIRY } \\
\text { DATE }\end{array}$ & SUBSET & $\begin{array}{c}\text { OTHER ENVIR } \\
\text { HW/OS }\end{array}$ \\
\hline
\end{tabular}

Micro Focus COBOL V3.1 Sun Sparcstation Model for UNIX (SUn SPARC 2

running Solaris 2) Sun Solaris (SunOS 5.1)

NIST-93/1822

Micro Focus COBOL for

V3.1 for UNIX (IBM

RS/6000)

NIST-93/1823

Micro Focus COBOL V3.1 for UNIX (Intel 80386

running UNIX SVR4.2)

NIST-93/1824

Micro Focus COBOL V3.1

for UNIX (Intel 80386

running UNIX SVR4.2)

NIST-93/1825

Micro Focus COBOL V3.1 for UNIX (Intel 80386

running SCO UNIX)

NIST-93/1826

Micro Focus COBOL

V3.0.50 for UNIX (Sun

SPARC running Solaris 1)

NIST-93/1827

Micro Focus COBOL

V3.0.50 for UNIX

(Pyramid MISserver)

NIST-93/1828

Micro Focus COBOL V3.0

for UNIX (Intel 80386

running UNIX SVR4.0)

NST-93/1829

Microsoft

Corporation

Microsoft COBOL Version

5.0

IBM PS/2 Model 60

IBM DOS Version 5.0

8/1/94 High

80486 Processor)

UNIXWARE SYSTEM $V$,

Release 4.2

HIMS Technology (Intel 80486 processor) SCO UNIX System V,

Release 3.2

Sun Sparcstation Model

$8 / 1 / 94$

High

Sun Solaris (SunOS 4.1.3)

Version 1

Pyramid MISserver-S

UNIX Sysiem $V$ Release

$8 / 1 / 94$

High

4 DC/OSx V1.1

AT\&T Star Server-E UNIX System $V$,

Version 2.1 Release 4

High

High

High

High

8/1/94 High
NIST-92/1962

Compaq Deskpro

Microsofi DOS, Version

4.01

S2000/250

$4 / 1 / 94$

High

$\mathrm{S} 2000 / 450, \mathrm{~S} 2000 / 750$

DYNLX/ptx Version 2

Release 0

Systems Corp. NIST-93/1391

$\$ 2000 / 250$

$2 / 1 / 95$

High

DYNIX/pax Version 2.0

Release 1.0
IBM PS/2 Model 80

DOS Version 3.3
Version 3.0

NIST-93/2001
Micro Focus COBOL 


\begin{tabular}{|c|c|c|c|c|c|}
\hline VENDOR & $\begin{array}{c}\text { PROCESSOR ID } \\
\& V S R \#\end{array}$ & $\begin{array}{c}\text { HARDWARE \& } \\
\text { OPERATING SYSTEM }\end{array}$ & $\begin{array}{r}\text { EXPIRY } \\
\text { DATE }\end{array}$ & SUBSET & OTHER ENVIRONMENTS \\
\hline
\end{tabular}

Siemens Nixdorf COBOL85

Informations-

systeme AG
Version 2.1A

NIST/NCC-93/975

\subsection{1}

BS2000/OSD (BS 2000)

Version 1.0A (11.0A)
5/1/94 High

6/1/94 High

NonStop VLX

Guardian 90 Version $D 10$

Computers, Inc.

COBOL85 Version D20 NIST-93/1541

UCS COBOL (UCOB)

Version 6R1 Release SB5R 1

NIST-93/1841

$2200 / 900$
1100 OS EXEC, Version
$44 R 1$ Release SBSRI

$8 / 1 / 94$

High

2200/600, 2200/400, 1100/90 1100 OS EXEC, Version 44RI Release SBSRI 


\begin{tabular}{|c|c|c|c|c|c|}
\hline VENDOR & $\begin{array}{c}\text { PROCESSOR ID } \\
\& V S R \#\end{array}$ & $\begin{array}{c}\text { HARDWARE \& } \\
\text { OPERATING SYSTEM }\end{array}$ & $\begin{array}{l}\text { EXPIRY } \\
\quad \text { DATE }\end{array}$ & LEVEL & $\begin{array}{c}\text { OTHER ENVIRONMENTS } \\
\text { HW/OS }\end{array}$ \\
\hline
\end{tabular}

$\begin{array}{lll}\text { Bull HN } & \text { FORTRANA } & \text { DPS } 6000 \text { Model } 634 \\ & \text { Release R3.1 } & \text { GCOS6 HVS Version } 2.0 \\ & \text { NIST-93/1202 } & \end{array}$

.

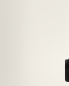

$$
\text { Bu }
$$

$$
\text { NIST-93/1202 }
$$

Fortran T7-ESV Version

Fortran 77 -ESV Versic
8FV4.1
NIST-92/1682

Fortran SXL-3001 Version
01.00
$B L A / 92 / 001$
BOS/X Fortran Compiler
Version 2.2

BLA/92/002

\section{DPS-9000E}

GCOS8 Version $S R 40203$

DPX/2 210

B.O.S.Version 0201

$D P X / 20$

BOS $/ X$ Version 3
$1 / 1 / 94$

DPS6 PLUS

HVS6 PLUS Version 20

DPS 6000

GCOS6 HVS Version 20

DPS-90, DPS-8000

GCOS8 Version SR40203

1/1/94 Full DPX/2 2000 and 300 FAMILES

B.O.S. Version 02.01

1/1/94 Full DPX/20 FAMILIES

BOS $/ X$ Version 3
Concurrent Computer

Corporation

$\begin{array}{ll}\text { SP-2450 (Fortran 77) } & 7100 \\ \text { Version 2 Release 1 } & \text { RTU } 6.1 \\ \text { NIST.92/1501 } & \end{array}$

NIST.92/1501

\begin{tabular}{|c|c|c|c|}
\hline $\begin{array}{l}8500 / 4 \\
R T U 6.0 A\end{array}$ & $6 / 1 / 94$ & Full & $\begin{array}{l}8450,8550,8400 \\
R T U \text { Version } 6.0 A\end{array}$ \\
\hline
\end{tabular}

SP-2450 (Fortran 77 )
Version 2 Release 2
NIST-92/1504
Fortran VI Z
Version RO6
Release 01
NIST-92/1502

Fortran VI O Version RO6
Rolease 01
NIST-92/1503

3280 MPS 6/1/94 Full $3205,3210,3220,3230,3240$, OS $/ 32$ Version $R 09 \quad 3250,3230 X \mathrm{P}, 3250 \mathrm{XP}, 3280 \mathrm{XP}$, Release $01 \quad 3230 M P S, 3260 M P S, 3280 E$ MPS; Micro $3200 \mathrm{CS}^{\star}$, Micro $3200 \mathrm{ES}^{*}$, Micro 3200 MPS*

OS/32 Version R09 Release 01

3280 MPS 6ull $6 / 1 / 94 \quad 3205,3210,3220,3230,3240$, OS/32 Version R09 3250, 3230XP, 3250XP, 3280XP, Release 01 3230MPS, 3260MPS, 3280E MPS;

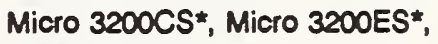
Micro 3200 MPS*

OS/32 Version R09 Release 01

Control Data Corporation
Fortran $/ 77$ Version 3.11 NIST.93/1102
Contol Data 4680

EP/IX Version 2.1.1
$2 / 1 / 94$

Full

Control Data 4000

\begin{tabular}{|c|c|c|c|c|}
\hline $\begin{array}{l}\text { Convex } \\
\text { Computer } \\
\text { Corporation }\end{array}$ & $\begin{array}{l}\text { Convox Fortran } \\
\text { Version } 8 \\
\text { NIST-93/1421 }\end{array}$ & $\begin{array}{l}\text { Convex C3880 } \\
\text { ConverOS Version } 10.2\end{array}$ & $5 / 1 / 94$ & Full \\
\hline \multirow[t]{2}{*}{$\begin{array}{l}\text { Cray Computer } \\
\text { Corporation }\end{array}$} & $\begin{array}{l}\text { f77 Version } 1.0 \\
\text { NIST }-93 / 2201\end{array}$ & $\begin{array}{l}\text { CRAY-3 } \\
\text { CSOS Version } 1.0\end{array}$ & $11 / 1 / 94$ & Full \\
\hline & $\begin{array}{l}f 77 \text { Version } 1.0 \\
\text { NIST-93/2202 }\end{array}$ & $\begin{array}{l}\text { Cray } 3 \\
\text { CsOS Version } 1.0\end{array}$ & $11 / 1 / 94$ & Full \\
\hline
\end{tabular}

EP/LX Version 2.1 .1 


\begin{tabular}{|c|c|c|c|c|c|}
\hline VENDOR & $\begin{array}{c}\text { PROCESSOR ID } \\
\& V S R \text { \# }\end{array}$ & $\begin{array}{c}\text { HARDWARE \& } \\
\text { OPERATING SYSTEM }\end{array}$ & $\begin{array}{l}\text { EXPIRY } \\
\text { M DATE } \\
\end{array}$ & LEVEL & $\begin{array}{c}\text { OTHER ENVIRONMENTS } \\
\text { HW/OS }\end{array}$ \\
\hline
\end{tabular}

Cray Rosearch, inc.

CF77 Compiling System
Rolease 6.0
NIST-93/1221
CFT Compiling System
Release 6.0

NIST-93/1222

Cray CSO

UNICOS Release 7.C.2ck

$8 / 1 / 94$

Cray Y-MP

UNICOS Release 7.C.2cl

$8 / 1 / 94$

Full

Full Cray Coo

UNICOS Release 7.0

DEC Fortran for

RISC/ULTRIX

Version 3.2

NIST-93/2142

Digital

Equipment

Corporation
DECstation 5000, Mod

200

RISC/ULTRX Version 4.3
$12 / 1 / 94$

Full

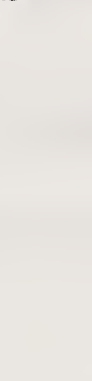
DEC Fortran for OpenVMS VAX, Version 6.1 NIST-93/2143
VAX $6000-420$ OpenVMS VAX Version 60

$12 / 1 / 94$

Full

DEC Fortran for OpenVMS AXP, Version 6.1 NIST-93/2141

DEC 3000-500 OpenVMS AXP Version 1.5
$12 / 1 / 94$

Full
DECstation 2100, 3100, 3100s; Personal DECstation 5000 20/25 MX, HX, TX, PXG +, PXG Turbo+; DECstation 5000 120/125/133 MX, CX, HX, PX, TX, PXG, PXG +, PXG Turbo, PXG Turbo+; $200 M X, C X$, HX, PX, TX, PXG, PXG +, PXG Turbo +; $240 M X, H X, T X, P X G$, PXG Turbo+; DECsystem 3100; 5000 25, 200, 240; 5100; 5400; 5500; 5810; 5820; 5830; 5840; 5900

\section{ULTRIX/RISC Version 4.3}

VAX 4000 Models 100, 100A, 105A, $200,300,400,500,500 A, 600$, 600A, 700A; VAX 6000 Models 200, $300,400,500,600$; VAX 7000 Model 600; VAX 8200, 8250, 8300 , $8350,8500,8550,8600,8650$, $8700,8800,8810,8820,8830$, 8840 , 8842; VAX 9000 Models 110 , 210, 300, 400, 420, 430, 440; VAX 10000 Model 600; VAXft Models 110, 310, 410, 610, 612; VAX $11 / 730,11 / 750,11 / 780,11 / 785$; MicroVAX II, 2000, 3100 10/10E, 20/20E, 30, 40, 80, 90; MicroVAX $3300,3400,3500,3600,3800$, 3900; VAXstation II, 2000; 3100 Models 30, 38, 40, 48, 76; 3200, $3500,3250,3540$; VAXstation 4000 Models 60, 90, VLC; VAXserver 3100 Models 10/10E, 20/20E; $3300,3400,3500,3600,3602$, 3800, 3900; VAXserver 4000 Models $200,300,500$; VAXserver 6000 Models 210, 220, 310, 320, 410, 420, 510, 520, 610, 620, 630; VAXserver 9000 Models 110, 310 , $320,330,340$

OpenVMS VAX Version 6.0, 5.5, 5.4

DEC 2000300 , DEC 3000300 , 300L, 500X, 400 AXP Workstation, 400 AXP Server, 500 AXP Workstation, 500 AXP Server; 600 , 600 Server 800,800 Server, DEC 4000600 AXP, 710; DEC 7000600 AXP; DEC 10000600 AXP Open VMS AXP Version 1.5 


\begin{tabular}{|c|c|c|c|c|c|}
\hline VENDOR & $\begin{array}{c}\text { PROCESSOR ID } \\
\& V S R \#\end{array}$ & $\begin{array}{c}\text { HARDWARE \& } \\
\text { OPERATING SYSTEM }\end{array}$ & $\begin{array}{l}\text { EXPIRY } \\
\quad \text { DATE } \\
\end{array}$ & LEVEL & $\begin{array}{c}\text { OTHER ENVIRONMENTS } \\
\text { HW/OS }\end{array}$ \\
\hline
\end{tabular}

\begin{tabular}{|c|c|c|c|c|c|}
\hline & $\begin{array}{l}\text { DEC Fortran Version } 3.3 \\
\text { for DEC OSF/1 AXP } \\
\text { Systems } \\
\text { NIST-93/1312 }\end{array}$ & $\begin{array}{l}\text { DEC } / 3000-400 \\
D E C \text { OSF } / 1 \text { AXP, Version } \\
1.2\end{array}$ & $3 / 1 / 94$ & Full & $\begin{array}{l}\mathrm{DEC} / 10000, \mathrm{DEC} / 7000, \\
\mathrm{DEC} / 3000, \mathrm{DEC} / 2000, \mathrm{D} \\
D E C \text { OSF } / 1 \text { AXP Version }\end{array}$ \\
\hline & $\begin{array}{l}\text { DEC Fortran for Windows } \\
\text { NT AXP Systems, Version } \\
1.0 \\
\text { NIST- } 93 / 1761\end{array}$ & $\begin{array}{l}\text { DECpc AXP / } 150 \\
\text { Windows NT AXP, } \\
\text { Version } 1.0\end{array}$ & $8 / 1 / 94$ & Full & \\
\hline \multirow[t]{4}{*}{$\begin{array}{l}\text { Encore } \\
\text { Computer } \\
\text { Corporation }\end{array}$} & $\begin{array}{l}\text { Parallel Fortran }+ \\
\text { Version } 1.2 .0 \\
\text { NIST-93/1443 }\end{array}$ & $\begin{array}{l}\text { Encore } 93 \\
\text { UMAX V Version } 3.1 .2\end{array}$ & $5 / 1 / 94$ & Full & \\
\hline & $\begin{array}{l}\text { Parallel Fortran }+ \\
\text { Version } 1.2 .0 \\
\text { NIST-93/1442 }\end{array}$ & $\begin{array}{l}\text { Encore } 91 \\
\text { UMAX V Version 3.0.7 }\end{array}$ & $5 / 1 / 94$ & Full & $\begin{array}{l}\text { Infinity } 90 \\
\text { UMAX V Version 3.0.7 }\end{array}$ \\
\hline & $\begin{array}{l}\text { Fortran } 77+ \\
\text { Version } 5.2 .0 \\
\text { NIST }-93 / 1441\end{array}$ & $\begin{array}{l}\text { Concept } 32 / 97 \\
\text { MPX-32 Version } 3.5402 A\end{array}$ & $5 / 1 / 94$ & Full & $\begin{array}{l}\text { Concept } 32 / 67,32 / 20 x x \text {, } \\
\text { Encore RSX } \\
\text { MPX-32 Version } 3.5402 A\end{array}$ \\
\hline & $\begin{array}{l}\text { GCF Version } 2.0 \\
\text { NIST-92/1542 }\end{array}$ & $\begin{array}{l}\text { Concept } 32 / 97 \\
\text { MPX-32 Version } 3.5402\end{array}$ & $4 / 1 / 94$ & Full & $\begin{array}{l}\text { Concept } 32 / 67,32 / 20 x x \text {, } \\
\text { Encore RSX } \\
\text { MPX-32 Version } 3.5402\end{array}$ \\
\hline
\end{tabular}

Fujitsu America, Inc.
OSI/MSP Fortran77 EX

Version 11 Lovel 10 NIST-91/1383

OSI/MSP Fortran77 EX Version 11 Lovel 10 NIST-91/1384

UXP/M Fortran77 EXNP Version 11 Level 10 NIST-91/1601

UXP/M Fortran77 EXNP Version 11 Lovel 10 NIST.91/1602
Fujitsu VP100E

OSIV/F4 MSP Edition 20

Amdahl 5990

IBM MVS/SP Version 3

Release 1.3

Fujitsu VP2400/10 UXP/M Version 10

Level 10

Fujitsu VP2400/10 UXP $/ M$ Version 10 Level 10

\section{2/1/95}

Full

$2 / 1 / 95$

(1)

2/1/95

$2 / 1 / 95$
Fujitsu M780; M760 OSIV/F4 MSP Edition 20

IBM 3050/200E IBM MVS/SP Version 2 Release 2.3

Fujitsu VP2000 Series UXP/M Version 10 Level 10

Fujitsu VP2000 Series Fujitsu M Series UXP/M Version 10 Level 10
HNSX

Supercomputers Inc.
Fortran77/SX (f77sx)

Release 031

NIST-93/1081
NEC SX-3 Model $22 \quad 1 / 1 / 94$

SUPER-UX Release 2.2
Full
NEC SX-3/11, /12, /14, /24, /42, /44; HNSX SX-3/11, /12, /14, /24, /42, /44 SUPER-UX Release 2.2
Hewlett- Packard Company
HP $\$ 000$ \$800 Fortran 77 Version A.09.00

Rel 9.0

NIST $-93 / 1123$

HP 9000 S700 Fortran 77 Version A.09.00

Rel 9.0

NIST-93/1121
HP9000 Model 835

HP.UX Version 9.0

HP9000 Model 720 HP.UX Version 9.0
$1 / 1 / 95$

Full

$1 / 1 / 95$

Full
HP9000, $\bmod 807,817,825,827$, $834,835,837,840,845,847,850$, $857,860,867,870$ HP-UX Version 9.0

HPS000, $\bmod 705,710,730,750$ HP-UX Version 9.0 


\begin{tabular}{|c|c|c|c|c|c|}
\hline VENDOR & $\begin{array}{c}\text { PROCESSOR ID } \\
\& V S R \text { \# } \\
\end{array}$ & $\begin{array}{c}\text { HARDWARE \& } \\
\text { OPERATING SYSTEM }\end{array}$ & $\begin{array}{l}\text { EXPIRY } \\
\quad \text { DATE } \\
\end{array}$ & LEVEL & $\begin{array}{l}\text { OTHER ENVIRONMENTS } \\
\text { HW } / O S\end{array}$ \\
\hline & $\begin{array}{l}\text { HP } 9000 \text { S300/S400 } \\
\text { Fortran } 77 \text { Version A.09.00 } \\
\text { Rel } 9.0 \\
\text { NIST } 93 / 1122\end{array}$ & $\begin{array}{l}\text { HPSO00 Model } 433 \mathrm{~T} \\
\text { HP-UX Version } 9.0\end{array}$ & $1 / 1 / 95$ & Full & $\begin{array}{l}H P 9000, \bmod 400,425,332,345, \\
350,360,370,375,380,385 \\
\text { HP-UX Version } 9.0\end{array}$ \\
\hline & $\begin{array}{l}\text { HP } 3000 \text { S900 Fortran } 77 \\
\text { Version A.04.31 } \\
\text { Rel } 9.0 \\
\text { NIST-93/1124 }\end{array}$ & $\begin{array}{l}\text { HP3000 Model } 947 L X \\
\text { MPE/LX Version } 4.0\end{array}$ & $1 / 1 / 95$ & Full & $\begin{array}{l}\text { HP3000, mod } 917,922,925,927, \\
930,932,935,937,950,955,957, \\
960,957,980,990 \\
\text { MPE/iX Version } 4.0\end{array}$ \\
\hline \multirow[t]{2}{*}{$\begin{array}{l}\text { IBM Canada, } \\
\text { LTD }\end{array}$} & $\begin{array}{l}\text { IBM ALX XL Fortran } \\
\text { Compiler } / 6000 \text { Version } 2 \\
\text { Release } 3 \\
\text { NIST. } 92 / 2031\end{array}$ & $\begin{array}{l}\text { IBM RISC System/6000 } \\
\text { POWERstation/ } \\
\text { POWERserver } 540 \\
\text { ALX for RISC System/6000 } \\
\text { Version } 3 \text { Release } 2\end{array}$ & $9 / 1 / 94$ & Full & $\begin{array}{l}\text { RISC System/6000 Powerstation } \\
\text { /Powerserver } 220,320 \mathrm{H}, 340,350 \text {, } \\
520 \mathrm{H}, 530,530 \mathrm{5}, 540,550,560 \text {, } \\
560 \mathrm{~F}, 730 \text {, RISC System/6000 } \\
\text { Powerserver } 930,950,970 \\
\text { ALX for RISC System/6000 } \\
\text { Version } 3 \text { Release } 2\end{array}$ \\
\hline & $\begin{array}{l}\text { IBM AIX XL Fortran } \\
\text { Compiler/6000 Version } 3 \\
\text { Release } 1 \\
\text { NIST-93/1921 }\end{array}$ & $\begin{array}{l}\text { IBM RISC System/6000 } \\
\text { POWERstation/ } \\
\text { POWERserver } 550 \\
\text { ADX for RISC System/6000 } \\
\text { Version } 3 \text { Release } 2.5\end{array}$ & 1/1/95 & Full & $\begin{array}{l}\text { RISC System/6000 Powerstation } \\
\text { /Powerserver } 220,250,25 \mathrm{~T}, 25 \mathrm{~W} \text {, } \\
320 \mathrm{H}, 340,350,520 \mathrm{H}, 530,530 \mathrm{E}, \\
540,550,560,560 \mathrm{~F}, 58 \mathrm{H}, 590,730 \text {, } \\
\text { RISC System/6000 Powerserver } \\
25 \mathrm{~S}, 930,950,970,990 \\
\text { AIX for RISC System/6000 } \\
\text { Version } 3 \text { Release } 2.5\end{array}$ \\
\hline \multirow[t]{3}{*}{ IBM Corporation } & $\begin{array}{l}\text { VS Fortran Version } 2 \\
\text { Release } 5 \\
\text { NIST-91/1921 }\end{array}$ & $\begin{array}{l}\text { IBM } 4381 \\
\text { VM/SP Version } 1 \\
\text { Release } 5\end{array}$ & $8 / 1 / 94$ & Full & $\begin{array}{l}\text { S/370 30xx, } 43 \times x, 93 \times x, S / 390, \\
\text { ES/9000 } \\
\text { VM/XA Version 1, Rel 1, } 2 \\
\text { S/370 30xx, 43xx, S/390, ES/9000 } \\
\text { VM/ESA Version 1, Rel 1, } 1.1\end{array}$ \\
\hline & $\begin{array}{l}\text { VS Fortran Version } 2 \\
\text { Release } 5 \\
\text { NIST-91/1922 }\end{array}$ & $\begin{array}{l}\text { IBM S/370 } 3090 \\
\text { MVS/ESA SP Version } 4 \\
\text { Release } 2\end{array}$ & $8 / 1 / 94$ & Full & $\begin{array}{l}\text { S/370 30xx, } 43 \times x, 93 \times x, S / 390, \\
\text { ES/9000 } \\
\text { MVS/SP Version 1, Release } 3 \\
\text { MVS/SP Version 2, Release } 2 \\
\text { MVS/SP Version 3, Release } 1\end{array}$ \\
\hline & $\begin{array}{l}\text { VS Fortran Version } 2 \\
\text { Release } 5 \\
\text { NIST-90/1823 }\end{array}$ & $\begin{array}{l}\text { IBM } 3090 \\
\text { ALX/370 Version } 1 \\
\text { Release } 2\end{array}$ & $8 / 1 / 94$ & Full & $\begin{array}{l}\mathrm{S} / 370,30 \mathrm{xx}, 43 \mathrm{xx}, 93 \times \mathrm{x} \\
A[x / 370 \text { Version } 1, \text { Release } 2\end{array}$ \\
\hline Intel SSD & $\begin{array}{l}\text { f77 Native Compiler } \\
\text { Version R4.5 } \\
\text { NIST-93/1781 }\end{array}$ & $\begin{array}{l}\text { XP/S } \\
\text { OSF/1 AD Release R1.1 }\end{array}$ & $11 / 1 / 94$ & Full & 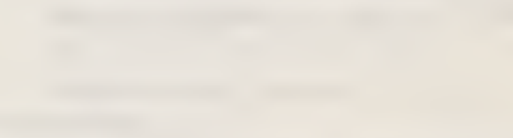 \\
\hline $\begin{array}{l}\text { Intergraph } \\
\text { Corporation }\end{array}$ & $\begin{array}{l}\text { Clipper Advanced } \\
\text { Optimizing Fortran, } \\
\text { Version } 2.0 \\
\text { NIST-94/1104 }\end{array}$ & $\begin{array}{l}\text { Clipper Model } 2730 \\
\text { CLDX, Version } 7.1\end{array}$ & $12 / 1 / 94$ & Full & $\begin{array}{l}\text { Clipper } C_{300} \text { and } \mathrm{C} 400 \\
C L D X, \text { Version } 7.1\end{array}$ \\
\hline $\begin{array}{l}\text { Liant Software } \\
\text { Corporation }\end{array}$ & $\begin{array}{l}\text { Fortran } / 400, \text { Version } 1 \\
\text { Release } 3 \\
\text { NIST-92/1181 }\end{array}$ & $\begin{array}{l}\text { IBM AS } / 400 \text { B4500 } \\
\text { IBM OS } / 400, \text { Version } 1\end{array}$ & $1 / 1 / 94$ & Full & 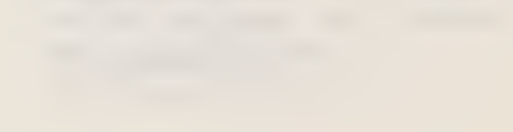 \\
\hline
\end{tabular}




\begin{tabular}{|c|c|c|c|c|c|}
\hline VENDOR & $\begin{array}{c}\text { PROCESSOR ID } \\
\text { \& VSR \# } \\
\end{array}$ & $\begin{array}{c}\text { HARDWARE \& } \\
\text { OPERATING SYSTEM }\end{array}$ & $\begin{array}{l}\text { EXPIRY } \\
\text { DATE } \\
\end{array}$ & LEVEL & $\begin{array}{c}\text { OTHER ENVIRONMENTS } \\
\text { HW } / O S \\
\end{array}$ \\
\hline & $\begin{array}{l}\text { Fortran } / 400 \text {, Version } 2 \\
\text { Release } 1 \\
\text { NIST-92/1182 }\end{array}$ & $\begin{array}{l}\text { IBM AS } / 400 \text { B4500 } \\
\text { IBM OS } / 400 \text {, Version } 2\end{array}$ & $\begin{array}{c}. \cdot \\
1 / 1 / 94\end{array}$ & Full & \\
\hline \multirow[t]{2}{*}{$\begin{array}{l}\text { Microsoft } \\
\text { Corporation }\end{array}$} & $\begin{array}{l}\text { Fortran PowerStation } 32 \\
\text { for Windows NT, } \\
\text { Version } 1.0 \\
\text { NIST } 93 / 2061\end{array}$ & $\begin{array}{l}\text { Dell PC 486D/SO with } \\
\text { Intel } 486 \mathrm{CPU} \\
\text { Microsofi Windows } \\
\text { NT Vension } 3.1\end{array}$ & $10 / 1 / 94$ & Full & \\
\hline & $\begin{array}{l}\text { Microsoft FORTRAN } \\
\text { PowerStation Version } 1.0 \\
\text { NIST-93/1181 }\end{array}$ & $\begin{array}{l}\text { Gateway } 2000486 / 33 C \\
80387 \text { math co- } \\
\text { processor, Microsoft DOS } \\
\text { Version } 5.0 \\
\text { Microsofi Windows Ver } 3.1 \\
\text { Compaq DeskPro } \\
386 / 200 \\
\text { MS-DOS Version } 5.0\end{array}$ & $3 / 1 / 94$ & Full & \\
\hline $\begin{array}{l}\text { Sequent } \\
\text { Computer } \\
\text { Systems, Inc. }\end{array}$ & $\begin{array}{l}\text { ptx/Fortran Version 2.1p } \\
\text { NIST.93/2002 }\end{array}$ & $\begin{array}{l}\text { S2000/250 } \\
D Y N L X / p \times \text { Version } 2 \\
\text { Release } 1.0\end{array}$ & $2 / 1 / 95$ & Full & $\begin{array}{l}52000 / 450,52000 / 750 \\
D Y N L X / p t x \text { Version } 2 \\
\text { Release } 1.0\end{array}$ \\
\hline \multirow[t]{4}{*}{$\begin{array}{l}\text { Siemens Nixdorf } \\
\text { Informations- } \\
\text { systeme AG }\end{array}$} & $\begin{array}{l}\text { Fortran77 V1.3 } \\
\text { NIST/NCC-93/978 }\end{array}$ & $\begin{array}{l}\text { PCE-5S } \\
\text { SINDX-Z V5.41 }\end{array}$ & $11 / 1 / 94$ & Full & $\begin{array}{l}M \times 300 \\
\text { SINLX-L Version } 5.41 \\
M \times 500 \\
\text { SINLX-M Version } 5.41 \\
W \times 200 \\
\text { SINLX-D Version } 5.41\end{array}$ \\
\hline & $\begin{array}{l}\text { Fortran77 V1.3 } \\
\text { NIST/NCC-93/979 }\end{array}$ & $\begin{array}{l}\text { RM400 } \\
\text { SINLX-N V5.41 }\end{array}$ & $11 / 1 / 94$ & Full & $\begin{array}{l}\text { RM } 600 \\
\text { SINLX-P Version } 5.41 \\
\text { RNM600 } \\
\text { SINLX-Y Version } 5.41\end{array}$ \\
\hline & $\begin{array}{l}\text { FOR1 V2.2 } \\
\text { NIST/NCC-93/980 }\end{array}$ & $\begin{array}{l}7.5921 \\
B S 2000 / O S D \text { V1.0A } \\
\text { (BS2000 V11.0A) }\end{array}$ & $11 / 1 / 94$ & Full & \\
\hline & $\begin{array}{l}\text { FORTRANSO V1.0 } \\
\text { NIST/NCC.93/981 }\end{array}$ & $\begin{array}{l}7.5921 \\
B S 2000 / O S D \text { V1.0A } \\
(B S 2000 \text { V11.0A) }\end{array}$ & $11 / 1 / 94$ & Full & \\
\hline \multirow[t]{2}{*}{$\begin{array}{l}\text { Silicon Graphics } \\
\text { Computer } \\
\text { Systems Inc. }\end{array}$} & $\begin{array}{l}\text { Fortran Rolease } 3.6 \\
\text { NIST-93/1164 }\end{array}$ & $\begin{array}{l}\text { IRIS 4D/25 } \\
\text { IRIX } 5.0\end{array}$ & $4 / 1 / 94$ & Full & $\begin{array}{l}\text { Personal IRIS, IRIS, IRIS 4D/50, } \\
4 \mathrm{D} / 70,4 \mathrm{D} / 120,4 \mathrm{D} / 220,4 \mathrm{D} / 280 \\
\text { IRX Release } 5.0\end{array}$ \\
\hline & $\begin{array}{l}\text { Fortran } 77 \text { Release } 3.11 \\
\text { NIST-93/1163 }\end{array}$ & $\begin{array}{l}M / 120 \\
\text { RISC/OS Release } 5.01\end{array}$ & $4 / 1 / 94$ & Full & $\begin{array}{l}\text { M/800, M/1000, RC2030, RC3240, } \\
\text { RC3260, RC4230, RC6280, RS2030, } \\
\text { RS4230 } \\
\text { RISC/OS Release } 5.01\end{array}$ \\
\hline SUNPRO - & $\begin{array}{l}\text { SPARCcompiler Fortran } \\
\text { Version } 4.0 \\
\text { NIST-93/2181 }\end{array}$ & $\begin{array}{l}\text { SPARCstation LX } \\
\text { SPARCserver } 1000 \\
\text { Solaris Version } 2.3\end{array}$ & $2 / 1 / 95$ & Full & \\
\hline
\end{tabular}




\begin{tabular}{|c|c|c|c|c|c|}
\hline VENDOR & $\begin{array}{c}\text { PROCESSOR ID } \\
\text { \& VSR \# } \\
\end{array}$ & $\begin{array}{c}\text { HARDWARE \& } \\
\text { OPERATING SYSTEM } \\
\end{array}$ & $\begin{array}{l}\text { EXPIRY } \\
\quad \text { DATE } \\
\end{array}$ & LEVEL & $\begin{array}{l}\text { OTHER ENVIRONMENTS } \\
\text { HW } / O S \\
\end{array}$ \\
\hline & $\begin{array}{l}\text { SPARCcompiler Fortran } \\
\text { MP (SPARCWORKS } \\
\text { IMPACT } 1.0 \\
\text { NIST- } 93 / 2182\end{array}$ & $\begin{array}{l}\text { SPARCstation } 10 \\
\text { SPARCserver } 1000 \\
\text { SPARCserver } 2000 \\
\text { Solaris Version } 2.3\end{array}$ & $2 / 1 / 95$ & Full & 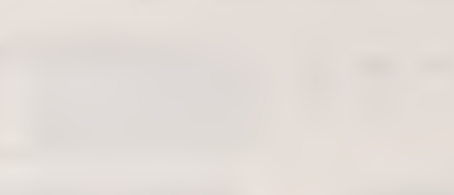 \\
\hline & $\begin{array}{l}\text { SPARCoompiler Fortran } \\
\text { Version } 2.0 .1 \\
\text { NIST-93/1381 }\end{array}$ & $\begin{array}{l}\text { SPARCstation } 2, \\
\text { SPARCstation 10, } \\
\text { SPARCserver } 690 \\
\text { SunOS Version } 4.1 .3\end{array}$ & $7 / 1 / 94$ & Fuil & \\
\hline & & $\begin{array}{l}\text { SPARCstation } 10, \\
\text { SPARCcenter } 2000 \text {, } \\
\text { SPARCserver } 690 \mathrm{MP} \\
\text { SunOS Version } 5.2\end{array}$ & & & \\
\hline & $\begin{array}{l}\text { ProCompiler Fortran } \\
\text { Version } 3.0 .1 \\
\text { NIST-93/1901 }\end{array}$ & $\begin{array}{l}\text { DELL. Model 433DE } \\
\text { Solaris Version } 2.1 \\
\text { Release X86 }\end{array}$ & $8 / 1 / 94$ & Full & \\
\hline $\begin{array}{l}\text { Tandem } \\
\text { Computers, Inc. }\end{array}$ & $\begin{array}{l}\text { Fortran Version D10 } \\
\text { NIST-93/1542 }\end{array}$ & $\begin{array}{l}\text { NonStop VX } \\
\text { Guardian } 90 \text { Version D10 }\end{array}$ & $6 / 1 / 94$ & Full & \\
\hline $\begin{array}{l}\text { Unisys } \\
\text { Corporation }\end{array}$ & $\begin{array}{l}\text { UCS Fortran (UFTN), } \\
\text { Version 5R1 Release } \\
\text { SB5R1 } \\
\text { NIST-93/1842 }\end{array}$ & $\begin{array}{l}2200 / 900 \\
1100 \text { OS EXEC, Version } \\
\text { 44RI Release SBSRI }\end{array}$ & $8 / 1 / 94$ & Full & $\begin{array}{l}2200 / 600,2200 / 400,1100 / 90 \\
1100 \text { OS EXEC, Version } 44 R 1 \\
\text { Release SBSRI }\end{array}$ \\
\hline
\end{tabular}




\subsubsection{Ada PROCESSORS}

The following pages list Ada compilers that have been validated by the Ada Joint Program Office (AJPO). Compilers are listed in order of vendor. The list is updated monthly, and presently includes 330 base compilers and 363 compilers derived from base implementations. For the most current information on validated Ada compilers, please contact the Ada Information Clearinghouse at (703) 685-1477.

(Key: * = Validated through Registration, base system above)

(Key: (\#YYMMDDFX.XXINN): YYMMDD is the date on-site testing was completed;

$F$ is the Ada Validation Facility;

$\mathrm{X} . \mathrm{XX}$ is the ACVC Version;

NNN is a unique sequence number that is assigned by the AVO)

For example, the certificate number \#901120W1.11087 means the compiler completed on-site testing November 20, 1990, at Wright-Patterson AFB under ACVC 1.11.

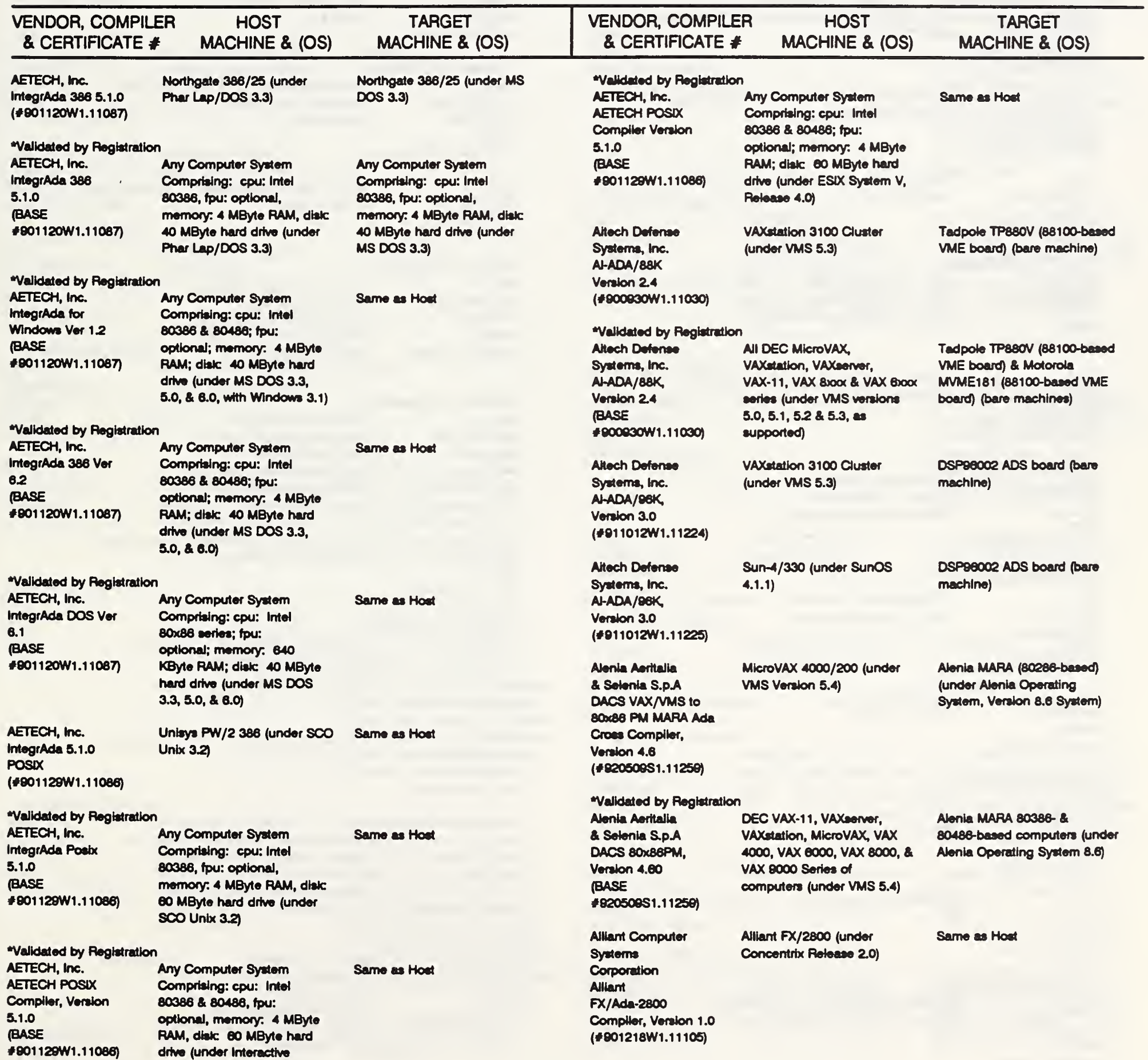


Ada PROCESSORS, Continued

\begin{tabular}{ccc|ccc}
\hline VENDOR, COMPILER & HOST & TARGET & VENDOR, COMPILER & HOST \\
\& CERTIFICATE * & MACHINE \& (OS) & MACHINE \& (OS) & \& CERTIFICATE * & MACHINE \& (OS) & MACHINE \& (OS)
\end{tabular}

\section{Allant Computer}

Sytems Corporation

Allant FX/Ada

Compiler, Version 2.3

(4801218W1.11108)

Aloys

AlycOMP_053,

Verion $1 . \overline{82}$

(100050011.11000)

Anys

Aly COMP O42

Verion 5.3

(1800627N1.11013)

Abye

AtoyCOMP 028,

Vortion $1 . \overline{82}$

(180081411.11040)

\section{Aloye}

Aloycomp_ess,

Voreion $1 . \overline{83}$

(*80081411.11041)

Aloys

AloyCOMP 046,

Verion 5.3

(\$801022A1.11043)

-Valldated by Reglatration

Aly COMP 048,

Sony NEWS series 1250

$15 \times x, 17 \times x, 18 \times x \& 18 x x$

(Under NEWS-OS vereions 3.3

Vortion 5.

(BASE

O01022A1.11043)

Aloys

AloycOMP 004

Apolto DN4000 (under

Veraion $5.3^{-}$

(4001022A1.11044)

-Valdated by Rogiatration

Atrys

Nisycomp con

Apollo DN3000, DN3500,

DN4000 \& DN4500 (under

Vertion 5.3

Domain/OS SR10.2 \& SR10.3)

BASE

C01022A1.11044

Wallidated by Fogistration

Abre

HP Apollo 8000 Series $400 \quad$ Amy Hoet

AlycOMP oO4 (under Domaln/OS SR10.4)

Veraion 5.5 .1

BASE

(801022A1.11044)

Alays

AlyocOMP_OSO,

Bull DPX/2 320 (under

B.0.5. 02.00 .05

Verion $5.3^{-}$

(4801022A1.11045)

-Velldeted by Reglatration

Alays

AlyyCOMP_OSO,

Vereion $5.3^{-}$

(BASE

1801022A1.11045

Aloy

AtyoOMP D

Version $5.3^{-}$

(4801002A1.11048)

-Valdaled by Roglatration

Aloys

AloyCOMP_002

modets funder HPUX 6.5 \&

7.0)

HP 9000.350 (under HP-UX

8.5)

Bull DPX 2/210, /220, /320,

/340 \& /300 funder BOS

$\infty 2.00 .05 \& 2.00 .10)$

(BASE

1801022A1.11046
Same as Hoet

Same as Hoet

Same thot

Same as Hoet

Same as Hoat

Any Hoet

Same as Hoet

Same as Hoet

Any Hoat

Any Hoat
-Valideted by Rogitutation

Anye.

AlyyCOMP_D

Veralon 5.5.1

(BASE

(B01022A1.11040)

Atsy

AlayCOMP_OOS,

Version $5.3^{-}$

(4001002A1.11047)

-Validated by Rogiatration

$\begin{array}{ll}\text { Aloys } & \text { Sun } 3 / 50, / 60, / 75, / 80, \\ \text { Aty COMP_o0, } & / 100, / 260, / 280, / 470 \& \\ \text { Verion } 5.3^{-3} & / 480 \text { (Under SunOS } 3.2,3.5, \\ \text { (BASE } & 4.0 \& 4.1 \text { ) }\end{array}$

(BASE

1001002A1.11047

- Validated by Rogiatration

Sun Microoystems Sum-3

AryCOMP 005 computer family (under

Verion $5.5 .1 \quad$ SunOS 4.1.1)

(BASE

HP 8000 Serles $300 \& 400$

Any Host 8.0

Sum-3/260 (under SunOS 3.2) Same as Ho:

$1001022 A 1.11047$

Alaye

AlyyCOMP_0s5,

CENIA Unigraph 6000 (under Unigraph/X 3.1)

Verion 5.3

(4001002A1.11048)

-Validated by Ragietration

Atoys

Aly COMP_035

Veraion $5.3^{-}$

(BASE

(001002A1.11048)

Unigraph 1000/325, 2000/50 $2000 / 250,2000 / 325$, $3000 / 325-333,6000 / 325-333$ $7000 / 325,8000 / 325 \& 8000$ (under Unigraph/X $3.1 \&$ 3.1.1)

-Valldated by Reglatration

Alese

CETLA Unigraph models

Aly COMP $035 \quad 1000 / 325 ; 2000 / 50, / 250$,

Verion 5.5 .1

1325; $3000 / 325-333$;

(BASE

(801022A1.11046)

$6000 / 325-333 ; 7000 / 325 /$

$8000 / 325 ; 80000$ (under

Unigraph/X 3.2c.1)

Alaye

AlycOMP 016

Compeq Deakpro 386 (under MS-DOS 3.30, Phar Lap 2.0)

Same as Hoet

Version 5.1

(400110ew1.11055)

-Velidated by Ragiatration

\begin{tabular}{|c|c|}
\hline $\begin{array}{l}\text { Nyys } \\
\text { AlyyCOMP_016 } \\
\text { Verion } 5.1 .1 \\
\text { (BASE } \\
\text { P01102W1.11055) }\end{array}$ & $\begin{array}{l}\text { Any Computer Syitem that } \\
\text { executes the Intel } 80388 \text { or } \\
80486 \text { In:truction set } \\
\text { (under MS/DOS } 5.0 \text { \& Pher } \\
\text { Lap 4.0) }\end{array}$ \\
\hline
\end{tabular}

Any Hoat

Aaye

AbycOMP_016

CompuAdd 320 (under MS-DOS Same as Hoet

Veralon 5.1

(*801102W1.11058)

Validated by Reglatration

Alsys

AlycOMP_018,

Version 5.1

(BASE

\$001102W1.11056

Any Hoat

386; and Unioys 388 \&

Deaktop III (under MS-DOS

-Validated by Rogletration

Atoys

3.30, Phar Lap 2.0

Aly COMP 018

Any Computer Sytem

Veraion $5.1^{-}$

Compriaing: cpu: Intel

80386; fpu: optional;

(BASE

$1001102 W 1.11058$

10 MByto (under MS-DOS

3.30, Phar Lep 2.0)
Amy Ho:

Any How

Any How 


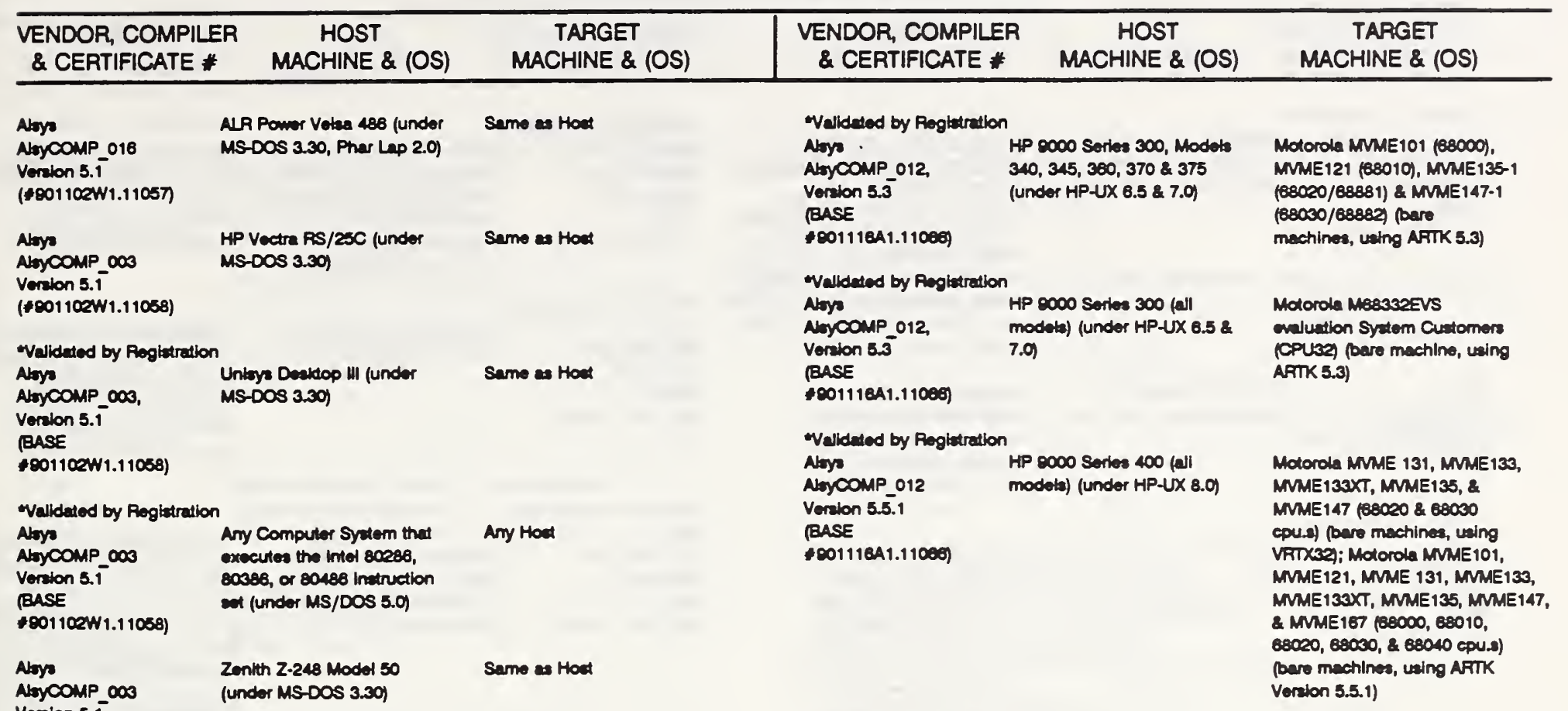

Verion $5.1^{-}$

(under MS-DOS 3.30)

-Velldated by Ringistration

(\$801102W1.11050)

Vallated by Roglatration

Aloys

Asycomp 037

Verion $5 . \overline{4} .2$

(BASE

1901114 N1.11005)
INMOS T800 transputer on a B405 TRAM board (bare), with an WMOS BOOS Communications Hink implemented in an IBM PC/AT (Under MS-DOS 3.1 and INMOS teoner V1.42h)

\section{Aryo} AyycOMP_012 Verion 5.3 (HCO1118A1.11000) 6.5)
- Validated by Registration

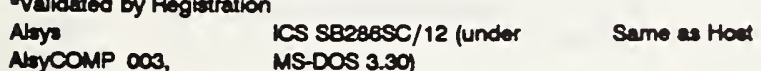

Noys

NyYCOMP 048

Verion $5 . \overline{5} .1$

(BASE

HCO1116A1.11000

Sun SPARCetation \& SPARCeoner computer famlibe; SPARCcenter 2000 (under SunOS 4.1.2); Solboume Series 5/100, /530, / $1800, / 870, / 800$, SE/000; \& \$4000 (under OS/MP 4.1A.1)

BASE

$1001102 W 1.11050)$

Vuldated by Rogletration

Aloy

ALyCOMP 003

Verion 5.1

HP Vectre ES/12; and IBM PC/AT (all modots) (under MS-DOS 3.30)

(BASE

1001 102W1.11050)

\section{Arys}

AnyCOMP 007

Verion 52

(\$001114N1.11005)

INMOS T800 trameputer on a B405 TRAM (bare) with an INMOS B008 Communicationa link implementod in an IBM PC/AT (under MS-DOS 3.1 and WMNOS benver V1.3)

Walidaled by Regiatration

Aloys AtycOMP_037,

BASE 1001114N1.11085)

INMOS T800 traneputer on a B403 TRAM (bare) with an Ink implemented In an IBM PC/AT (under MS-DOS 3.1 and INMOS bermor V1.3)

Walidated by Reglatration
INMOS T800 transputer on a B40S TRAM (baro) uaing an IBM PC/AT under MS-DOS 3.1 running INMOS berver 1.3

for filo-enver support via an INMOS 8008 board link

WHOS T800 trenseputer on a B405 THAM (bere) uaing an EM PC/AT under MS-DOS 3. running INMOS berver 1.3 for file-enver cupport via an INMOS B008 board Hink, INMOS T425 transputer on a B403 TRAM (bare) uaing an IBM PC/AT under MS-DOS 3.1 running INMOS teorver 1.3 for filo-eoner eupport via an WNOS BOOB board Unk

INMOS T800 transputer on a 8405 TRAM (bare), using an IBM PC/AT under MS-DOS 3.1 running INMOS berver V1.42h for filo-eoner eupport vis an INMOS B008 board IInk and INMOS T 425 transputer on a B403 TRAM (baro), waing an IBM PC/AT under MS-DOS 3.1 running INMOS bener V1.42h for file-enver eupport wa an WMOS BO08 board link

Motorola MMME101 (88000) (bare mechine, ueing ARTK Verion 5.3)
Naye HP 8000 Sorios 700 f (ل) NoyCOMP_os3 modeta) (under HP-UX 8.9) Verion $5 . \overline{5.1}$

(BASE

$\$ 0111841.11089$

$\begin{array}{ll}\text { Aloys } & \text { Apollo DN4000 (under } \\ \text { NinycOMP_Q38, } & \text { Domain/OS SR10.2) } \\ \text { Verion 5.3 } & \end{array}$

(\$001116A1.11087)

Walidated by Rogitatration

Aloys AnyCOMP_068, $\quad 24500$ (under Domain/OS Verion $5.3^{-} \quad$ SR10.2 \& SR10.3)

(BASE

N0111841.11087)

-Validated by Regletration Aloye NoyCOMP_os

Verion $5 . \overline{5.1}$

(BASE

\$801116A1.11067

\section{Aloye}

AvyCOMP_015,

Verion $5.3^{-}$

(\$CO1116A1.11088)

Walidaled by Rogistration

Avys $\quad \operatorname{sun} 3 / 50, / 60, / 75, / 80$

Aly,COMP 015, $\quad / 160, / 260, / 280, / 470$ \&

Veraion $5.3^{-} \quad / 480$ (under SunOs 3.2, 3.5,

(BASE

\$001116A1.11088)
1480 (und
4.084 .1 )

Sun 3/280 (under Sunos 3.2)

pare machin uing ARTK

Verion 5.3)

Motorola MMME101 $(68000)$, MNME121 (88010), MMME135-1 (88020/68881) \& MMME147-1 (88050/88882) (Bare machines, uing ARTK 5.3)
MAtorda MVME101, MVME121. MME131, MNME133, MMME133XT, MNME135, MB8332EVS, MMME147, \& MMME167 (88000, 68010, (28000, 68000, \& 68040 cpus) (bare mechines, uaing ARTK Veraion 5.5.1)

Motorola MMME101, MMME121, M68332EVS, MMME131, MMME133, MMME133XT, MMME135, MMME147, 2. MMME 167 108000-, 68010 $68020-, 68030-, 8$ 68040-besed inglo-board ualng ARTK 5.5.1)

Motorola MMME147-1 (88050/68882) (bare machlne, using ARTK Veralon 5.3)

Motorole MMME101 $(88000)$, MMME121 (88010), MMME135-1 (88030/68882) (bare mechines, ueing ARTK 5.3)

Motorole MMME101, MMME121, MMME131, MMME133, MMME133XT, MME135, M68332EVS, MMME147, \& MMME167 (88000, 68010, 68020), 68030 , \& 68040 cpu.\&) (bare mechines, uaing ARTK Verion 5.5.1) 


\begin{tabular}{lcc|ccc}
\hline $\begin{array}{l}\text { VENDOR, COMPILER } \\
\text { \& CERTIFICATE * }\end{array}$ & HOST & TARGET & VENDOR, COMPILER & HOST & TARGET \\
& MACHINE \& (OS) & MACHINE \& (OS) & \& CERTIFICATE * & MACHINE \& (OS) & MACHINE \& (OS) \\
\hline
\end{tabular}

Vallated by Roglatration

Aloys

AloyCOMP 015

Verion 5.5.1

(BASE

(601118A1.11068)
Sun Mierooyntems Sum-3 computer tamily (under Sunos 4.1.1)

\begin{tabular}{|c|c|}
\hline $\begin{array}{l}\text { Abys } \\
\text { AbycoMP 017, } \\
\text { Version } 5.2 \\
\text { (\$CO1118N1.11084) }\end{array}$ & $\begin{array}{l}\text { MicroVAX II (under VMS } \\
\text { V5.3) }\end{array}$ \\
\hline "Valldated by Regiet & \\
\hline $\begin{array}{l}\text { Aby } \\
\text { NoyCOMP_017, } \\
\text { V5.3 } \\
\text { (BASE } \\
\text { \%001118N1.11084) }\end{array}$ & $\begin{array}{l}\text { MicroVAX II (under VMS } \\
\text { V5.3) }\end{array}$ \\
\hline
\end{tabular}

- Vallided by Reglatration

Ator

MicroVAX II (under VMS

Aloycomp 017

V(5.3)

Verion $5 . \overline{4} .3$

(BASE

( 601118N1.11084)
Motorda MMME 131, MMME133, MNME133XT, MVME135, \& MVME147 (68020 \& 68030 cpu.\$) (bare machines, using VRTX32); Motorola MVME101, MMME121, MMME131, MNME133, MNME133XT, MNME135, MB8332EVS, MMME147, 8 MVME167 $(88000,68010,68020,68030, \& 68040$ cpu.s) (bare mechines, uaing ARTK Version 5.5.1)

INMOS T425 traneputer on a B403 TRAM (bare) using the Hot running INMOS berver 1.3 for filo-enver suppont via a CAPUN OTO board Hnk

INMOS T425 transputer on B403 TRAM (bare) ueing the Hout running INMOS borver 1.3 for file-eener support via a CAPUN OTO board link INMOS T800 transputer on B406 TRAM (bare) using the Hot running iNMOS borver via a CAPUN QTO board Hink

INMOS T425 transputer on a B403 TRAM (bare), uing the Hoct running INMOS borver V1.42i for filo-cenver support via a CAPUN OTO board IInk and INMOS T800 traneputer on a B405 TRAM (bare), ueing the Hoet nunning INMOS berver V1.42i for filo-eenver support vie CAPUN OTO board Hnk

\begin{tabular}{|c|}
\hline $\begin{array}{l}\text { Alys } \\
\text { Aleycomp_018 } \\
\text { Verion } 5.2 \\
\text { (\$001120A1.11070) }\end{array}$ \\
\hline $\begin{array}{l}\text { Validated by Regis } \\
\text { Atrys } \\
\text { AtoyCOMP_018, } \\
\text { Vorion } 5.2 \\
\text { (BASE } \\
\text { 1001120A1.11070) }\end{array}$ \\
\hline
\end{tabular}

Alsys

Aly COMP 006 ,

Verion $5.3^{-}$

("001125N1.11071)

Aloys

Aly COMP C23,

IEM 37030840 (under MVS/XA Same as Hot

Vervion $5.3^{-}$

(\$001125N1.11072)

Aloys

Abycomp 011.

Vorion $5 . \overline{3}$

(\$801127A1.11089)

MicroVAX 3100 (under VMS Same as Hoet

5.3)

DEC VAX-11, VAXserver,

VAXtation, MleroVAX, VAX 4000 , VAX 6000 , VAX 8000 \& VAX 8000 Series of

computere (as supportod) (under VMS $5.2 \& 5.4$ )

IBM 2370 Model 80 (under VM/IS CMS reloase 5.1)

Same es Hoat

-Validated by Rogiatration

Atys

AloyCOMP 011,

Verion $5.3^{-}$

(BASE

\%01127A1.11009)
DEC VAX-11, VAXeorver, VAXtation, MleroVAX, VAX 4000 , VAX 6000 , VAX 8000 \& VAX 8000 Series of computer (as supportod) (under VMS 5.2, $5.3 \& 5.4$ )
VAX 6210 (under VMS 5.2)

Motorda MMME135-1 (88020/68881) (bare mechine, uaing AFTK Veraion 5.3) MMME121 (88010), MMME135-1 (88020/68881) \& MMME147-1 (88050/68882) (bere machines, using AFTK 5.3) 1.3 for filo-aner support

-Validated by Rogitutration

Aloye ..

DEC VAX-11, VAXeonver, AloyCOMP_011 VAXitation, MleroVAX, VAX Veraion 5.3.1

(BASE

+001127A1.11089) 4000 , VAX 6000 , VAX 8000,8 VAX 8000 eories of computers (under VMS 5.2, $5.3,25.4$, as eupportod)

"Validated by Roglatration

Aloys

NoyCOMP_Oe8

Compeq Deckpro 386/20

Verion 5.3 (under DOS $3.31 \& 5.0$ )

(BASE

*01127A1.11069)

-Vellated by Regiatration

Aloys

DEC VAX-11, VAXsorver,

ArycOMP 011

Verion 5.5 .1

BASE

(C01127A1.11069)

Xeation, MieroVAX, VAX 4000 , VAX 6000 , VAX 8000 , VAX 8000 computer series (under VMS 5.4)

Aloye

AlsyCOMP_O34,

Multhech 1100 (under SCO

Verion 5.1

(\$001221W1.11103)

-Valldated by Registration

Aloys

AlyyCOMP_O34,

Verion $5.1^{-}$

(BASE

(801221W1.11103)

Everex AGI 30000, Comped Deckpro 388 \& SA Technologies Army Lyhtwoight Computer Unit RCU Va (under Interactive Unbx 3.2)

Validated by Regiatration

Alsys

AbyCOMP_C34,

Prime MBX (under Prime Unix

Vervion 5.

$\$ \infty 01221 W 1.11103$

-Velidated by Regiatration

Aloye

AbyCOMP_OSA,

Verion $5.1^{-}$

(BASE

(001221W1.11103)

Any Computer Syatem compraing: epu: Intel 80386 or 80486 ; fou: optional (under a Unlx

- Validated by Regletration 3.2-baend OS)

Motorola MMME101 (68000),
Alsys

AloyCOMP 02

Verion $5.1^{-}$

(BASE

\%01221W1.11103)

Any Computer Sydtem that 80488 Instruction set (under SCO Open Deaktop 1.1 2. SCO Unix 3.2, sCO Open Deaktop 2.0 \& SCO Unix 3.2.4, Interective Unbx 3.2.2, and AT\&T Unix Syatem $\checkmark$ Roloase 4.0)

-Velidated by Rogletration

Alys Zenith Data Syetems AlyYCOMP_034, Z-Station 433 DEh funder Veraion 5.1 .2 SOO Unix 3.2.4 running BASE SecureWare CMW + Verion \$001221W1.11103) 2.2) executes the intel 80386 or
Motorola MVME101 (68000), MMME121 (88010), MVME133XT \& MMME135-1 (B8020), \& MMME147-1 (88030) (bare machines, ueing ARTK 5.3.1)

Motorola MMME101, MMME121, MMME131, MMME133, MMME133\%T, MVME135-1, MMME147-1, Ms8332EVS (68000, 68010, 68020, \& 68030 cpu.s) (bare machine, using AFTK Version 5.3)

Motorola MMME 131, MMME133, MMME133XT, MMME135, \& MMME147 (88020 \& 68030 cpus) (bare machines, uaing VRTX22); Motorole MMME101, MMME121, MMME131, AMME133, MNME133XT, MMME135, M88332EVS, MMME147, \& MMME167 $(68000,68010,68020,68050,2$ 68040 cpus) (bare machlnes, using AfTK Verion 5.5.1)

Same is Hoet

Each Hoat, eelf-targetted

Any How

Same thet Hot 


\begin{tabular}{lcc|ccc}
\hline $\begin{array}{l}\text { VENDOR, COMPILER } \\
\text { \& CERTIFICATE * }\end{array}$ & $\begin{array}{c}\text { HOST } \\
\text { MACHINE \& (OS) }\end{array}$ & $\begin{array}{c}\text { TARGET } \\
\text { MACHINE \& (OS) }\end{array}$ & $\begin{array}{c}\text { VENDOR, COMPILER } \\
\text { \& CERTIFICATE * }\end{array}$ & $\begin{array}{c}\text { HOST } \\
\text { MACHINE \& (OS) }\end{array}$ & $\begin{array}{c}\text { TARGET } \\
\text { MACHINE \& (OS) }\end{array}$ \\
\hline
\end{tabular}

Valldeded by Regiatration

Aloy

Arycomp_os

Any computer yytem that executes the Intel 80388 or 488 inatruction sel (under SCO Open Deaxtop 2.0 with

Verion 5.5

(BASE

SCO Unix veralon 3.2 .4

Imterective Unix 322, or

AT\&T Unix Syztom V Rolsase 4.0)

Valldatod by Regiatration

Aloys

SAC LCU V2 (under SCO Open Same as Ho:

Aty COMP os

Dealtop 2.0 (SCO Unix

3.2.4))

Verion 5.12

(BASE

$\$ 001221 W 1.11103)$

\section{Aloye}

AnyCOMP O43,

Verion $5.3^{-}$

(\$001221W1.11104)

Aloys

AloycOMP_os

Version $5.1^{-}$

(\$10128W1.11113)

Validated by Roglatration

Noye

AloyCOMP_os4,

Verion $5.1^{-}$

(BASE

010120W1.11113)

- Velidated by Reglatration

Avye

AbyCOMP 070

Verion 5.5 .3

(BASE

010120W1.11113)

Aloye

NiycOMP OS6,

Verion $1 . \overline{82}$

(\$01013111.11127)

Abys

AlycomP_os,

Verion $1 . \overline{82}$

(\%91020111.11128)

Aloye

Alycomp oes.

Verion 5.3

(\$10323W1.11131)

Valldated by Regletration

Aloye

AlycoMP

Verion 5.3.1

(BASE

Apple Macimioen llex (under

Macintoch Syatem Software

6.0.5)

IEM PS/2 Model 80 (under

Lynios Verion $2.0+$

Threects Poloeses 11)

IBM PS/2 Modets 70-xox \&

80-x00 (under Lynxos

Verion 2.0 Rolease 15

Any Hod

Any computer syitem that executes the Intel 80388 or 488 inetruction ent (under Lynxos, Verion 2.1)

Sun $3 / 80$ (under SunOs, Veriton 4.0.3)

KWS EB88020 (under os-8/68020, Verion 2.3)

VAX 8530 (under VMS, Veraion 5.3-1)

KWS EB88020 (under os- $8 / 68020$, Verion 2.3)

Compundd 325 (under DOS 3.31)

Intel ISBC $388 / 116$ (bare mechine, uaing ARTK 5.3)

010323W1.11131)

Aloye

AloycOMP 030

Verion 5.3

(\$810323W1.11132)

Walidated by Rogiatration

Aloys

AloyCOMP_030

Verion 5.3.1

(BASE

010320W1.11132

Aloye

AtycomP 033

Verion $5.3^{-}$

(\$010323W1.11133) executes the Intel 80388 or 80486 instruction ex (under MS-DOS version 5.08

Phar Lap veriolon 4.0)

88 single board computer (bare machino. using ARTK 5.3)

Any 80388 single board computer (bere machino, uing ARTK 5.5) machine, uelng ARTK 5.3)
- Veldated by Reglatration

Anse..

Sun Microoyterms Sun-4,

Intel ISBC $388 / 31$, iSBC

AlyCOMP O62. SPARCanver, \& SPARCatation

Vorion 5.3 .1

computer families (under

$388 / 1 \times x$, ISEC $488 / 1$ xx (bare

BASE

1010323W1.11133)

Atrys

AHOCOMP_040, Sunos 4.1) machines, using AFTK 5.3)

Verion $1 . \overline{8}$

VAX 8530 (under VMS Vercion 5.3-1)

Integrated Dovice Technology IDT7RSs01 Syetem

(\$0104071.11144) (R3000/R3010) (bare machino)

Validated by Rogietration

Alyse

VAX 8530 (under VMS 5.3-1)

Locktheed Sanders STAR MNP

AnYCOMP 040,

Verion $1 . \overline{83} 01$

(R3000/R3010) (bare machine)

(BASE

(0104071.11144

Walideted by Reglatration

Anys

DEC VAX-11, VAXeoner,

NyYCOMP_ $\infty 7$
VAXteation, MicroVAX, VAX

4000 , VAX 6000 , VAX 6000,8

VAX 9000 cories of

Verion 1.8

(BASE

computere (under VMS $5.3 \& 5.4$ )

\$104071.11144)

DECatation 3100 (undor

Alon ULTRIX Verion 4.0)

NoyCOMP_057,

Verion $1 . \overline{83}$

(10100251.11103)

- Velidated by Reglatration

Noye

DEC DECutution \& DECortem

computer tamilies (under

Vericon

ULTRAX $4.0 \& 4.2$ )

$1010025(1.11103)$

Aloys

AtycomP 024,

Veriton $5.3^{-}$

(1010000W1.11105)

- Validated by Roglatration

Noye

AlyCOMP 024 V5.4

IBM RISC Syctem 6000 (all

Amy Hod

(BASE modets) (under $A \times 3.2$ )

IBM RISC Syatem 6000, model Same wo Hod 520 (under $A D \times$ 3.1 )

010000 W1.11185

Anye

NoyCOMP 088,

Untoys B30 (under BTOS II, 10.20

Verion $5.3^{-}$

(4010000W1.11188)

Aloye

AloyCOMP_ 40

Verion $5.3^{-}$

(\$810000W1.11197)

Aloye

AnyCOMP_O62,

Verion $5 . \overline{\bar{s}}$

(1011107W1.11207)

HP Vectre RS/25C (under DOS 3.30)

Unioys 839 (under BTOS II, (3.2.)

Vallated by Roglatration

Noys

ANYCOMP_OS

Veraton $5 . \overline{35}$

(BASE

$1011107 W 1.11227$

HP 8000 Serios 700 , modets (under HP-UX, Veriton A.B8.05 (roloeses 8.05)); HP 8000 Sorles 800 , al modets (Under HP.UX, Vervion A.B8.00 (roleetese $8.001)$

- Validated by Roglatration

Aloys

AlycOMP 00

Vortion 5.5.1

(BASE

HP 0000 Sories 700 ,
Series 800 , all modets

(under HP-UX, Veraion 8.0)
Veraion 0.01); HP 9000

Lhood Sanders STAR MVP board (R3000/R3010) bare machino)

Any Hoat

1011107W1.11227
700 Model 720 (under HP-UX, Verion A.B8.05 (roloce 8.05))
HP 8000 Serios 700 , all modets (under HP.UX, Verion A.B8.05 (roloeses 8.05))

HP 0000 Serios 700, all modets (under HP-UX, Verion 0.01) 


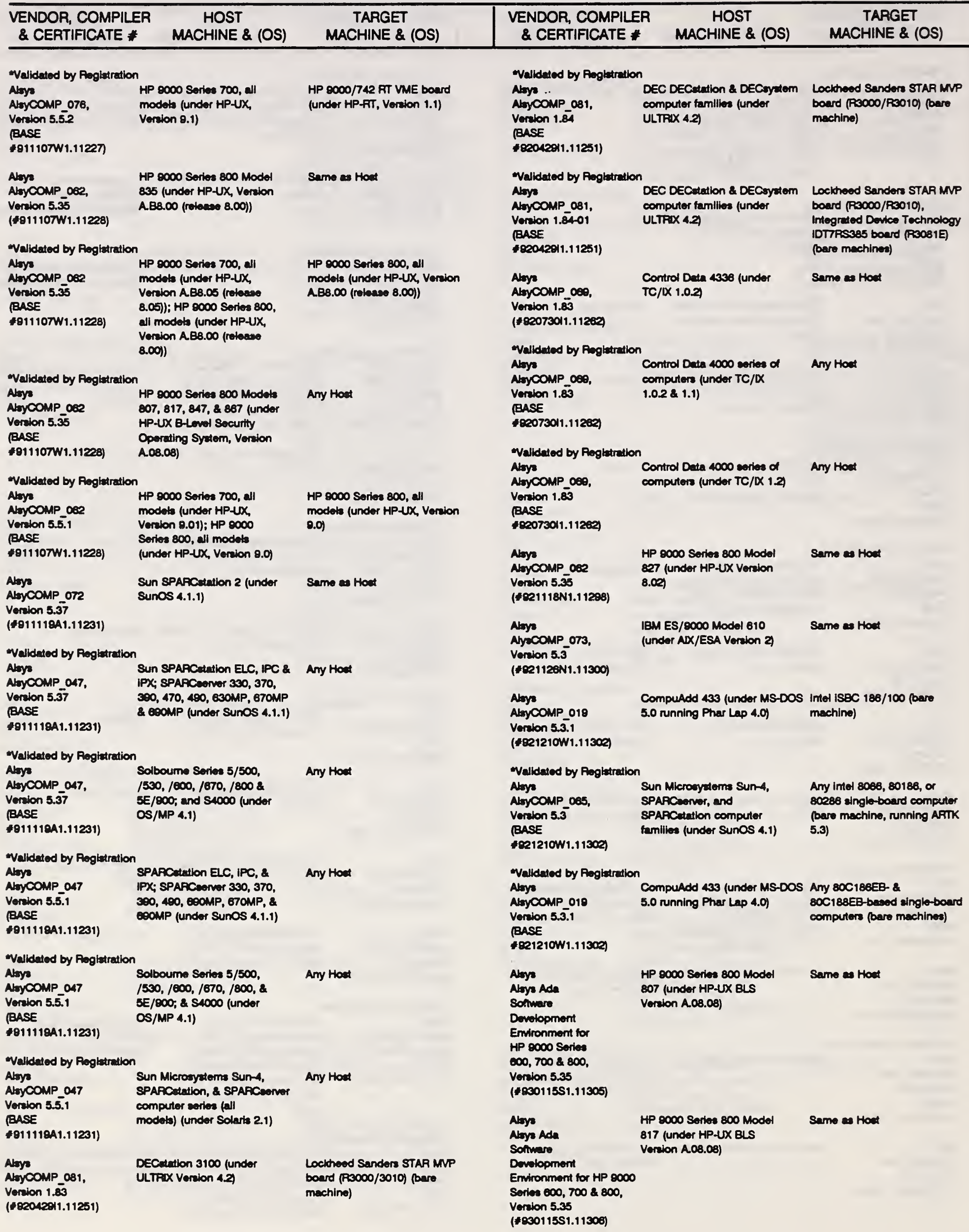


HOST MACHINE \& (OS)
TARGET

MACHINE \& (OS)

VENDOR, COMPILER

\& CERTIFICATE *
HOST

MACHINE \& (OS)

Same at Hoat

HP 8000 Serles 800 Model
847 (Under HP-UX BLS

Veraion A.08.08)

Aloys Ada

Sormare

Devolopment Emvironment

for HP 8000 Serles 600 ,

$700 \& 800$, Verion 5.35

(\$03011551.11307)

\section{Aloys}

Aloys Ade

Solmare

Dovelopment Emvironment

$700 \& 800$, Veralon 5.35

(\$030115S1.11308)

\section{Aloys \\ Aloys Ada \\ Sommere \\ Devilopment \\ Envionment for \\ HP $\$ 000$ Sorles \\ $700 / 800$, Verion \\ 5.35}

(\$03011551.11300)

\section{Aloye}

AlycOMP_088,

Verelon $1 . \overline{83}$

(\$23012511.11310)

\section{Aloys / Germen}

$\mathrm{MOD}$

NATO SWG ON APSE

Compller for

Sunz/SunOS,

Veriton SSC1.82-02

(\$0110161.11233)

Aloys / German

MOD

NATO SWG ON APSE

Compller for VAX/MMS,

Verion VC1.82-02

(\$0111181.11236)

Aloys / Germen

MOD

NATO SWG on APSE

Compller for

VAXNMS to

MC88020, Verion

VCM1.82-02

(\$02030811.11248)

Alys / Gorman

MOD

NATO SWG ON APSE

Compller for

Sunz/Sunos to

MC88020, Verion

S3CM1.82

(\$82072811.11281)

ATLAS EIEKTRONIK

GmbH

ATLAS ELEKTRONIK

Ada Compller WME

1.82

(18103241.11136)

Concurrent

Computer

Corporation

CaAde, Voreion

0.5

(100042711.11008) 887 (under HP-UX BLS Veralon A.08.08)

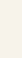

-Valldated by Rogietretion

Concurrent

Compuler

Corporation

CaAde, Vertion 0.5

(BASE

10004271.11008 )

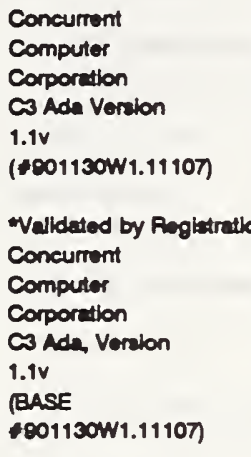

Concurrent Computer Corporation 6850 with Super Lightning Floeting Point (under RTU Verion 5.00 ) Corporation 8500 MIPS R3000/R3010) (Under RTU Voralon 5.1)

Seme es Hoet

Same th Hod

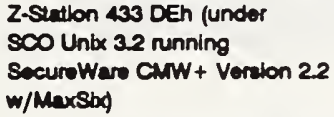

Control Deta 4680 (under

Same as Hoet

$E P /(X 1.4 .3)$

Sur-3/60 (under SunOS

Vervion 4.0.3, with CAS

Sun-3/60 (under SunOS

Veralon 5.50

Veraion 4.0.3)

VAX 8350 (Under VMS Veralon VAX 8350 (Under VMS Veralon 5.4-1, wth CAS Veralon $5.4-1$ ) 5.5e)

VAX 8350 (Under VMS Veralon 5.41 , with CAS Voreion 5.59

Motorola MMME133XT (MC88020) (bare machino)

Valldated by Rogitutration

Concurrent

Computer

Corporation

C3 Ade, Voralon

1.1

(BASE

$1001130 W 1.11107$

Concurrent Computer

Corporation Sories 6000

wth Super Lightning

noating Point, and Series

5000 with Lightning

noating Point (ali modets)

(under RTU Verion 5.04.

$5.08 \& 5.0 \mathrm{C}$

Concurrent Computer

Corporation Series 6000

(MC8s030, with Super

Lohtning Floatling Point) \&

Sorles 5000 (MC88020, wth

Ughtning Floating Point)

(Under RTU Verion 5.04,

$5.08,5.00 \& 8.0)$

$\begin{array}{ll}\text { Concument } & \text { Concurrent Computer } \\ \text { Computer } & \text { Corporation 3280MPS (under } \\ \text { Corporation } & \text { OS/32 Verion R08-03.2) }\end{array}$

Corporation

ROS-OOV

(\$CO1130W1.11108)

Waldated by Reglatration

Concurrent

Computer

Corporation

C. Ade, Vorion

ROB-OOV

(BASE

1901130W1.11108)

Concurrent Computer

Corporation Series 3200:

3200 MPS, 3203, 3205, 3210,

$3220,3230,3250,3230 \times P$,

$3250 \times P, 3230 \mathrm{MPS}, 3260 \mathrm{MPS}$

Miero4, and Mieros (under

OS/32 Verclon: R08-03,

R08-03.1 \& R08-0324

Velldated by Roglatration

\begin{tabular}{|c|c|}
\hline $\begin{array}{l}\text { Concurrent } \\
\text { Computer }\end{array}$ & $\begin{array}{l}\text { Concurrent Computer } \\
\text { Corporation Sytern Bus }\end{array}$ \\
\hline $\begin{array}{l}\text { Corporation } \\
\text { CaAda Vereion }\end{array}$ & $\begin{array}{l}\text { Proceseser family of } \\
\text { computers (under Truate }\end{array}$ \\
\hline $\mathbf{R O 3 - \infty}$ & OS/32 and MTM Version \\
\hline $\begin{array}{l}\text { (BASE } \\
\text { P001130W1.11108) }\end{array}$ & $\begin{array}{l}\text { RDo-03.3S, and OS/32 } \\
\text { Verion ROO-01.1) }\end{array}$ \\
\hline
\end{tabular}

Motorola MMME133XT (MC88020)

Sun-3/60 (under SunOS Verion 4.0.3, with CAS

Vervion 5.5E

(bare machine)

VAX $6000-410$ (under VMS Voralon 5.2

ATLAS ELEKTRONIK GmbH MPR 2300 (under MOS 2300 .

Veraion 2.1)

Valdated by Reglatration

Concurrent

Computer

Corporation

CaAda Verion

ROO-OO

(BASE

+001130W1.11108)

Concurrent

Computer

Corporation

C3 Ada Veraton 1.0

(\$C01130W1.11100)
Concurrent Computer Corporation Sytem Bue Proceseor family of computen (undor OS/32 R08-03.2)

Concurrent Computer Corporation 8400 MIPS R3000/3010) (under RTU Veralon 5.1)
Same as Hot

Any Hoat

Same es Hoet

Any Hod

Any Hoot

Any Hoet
Corporation 8400 (MIPS

TARGET

MACHINE \& (OS) 


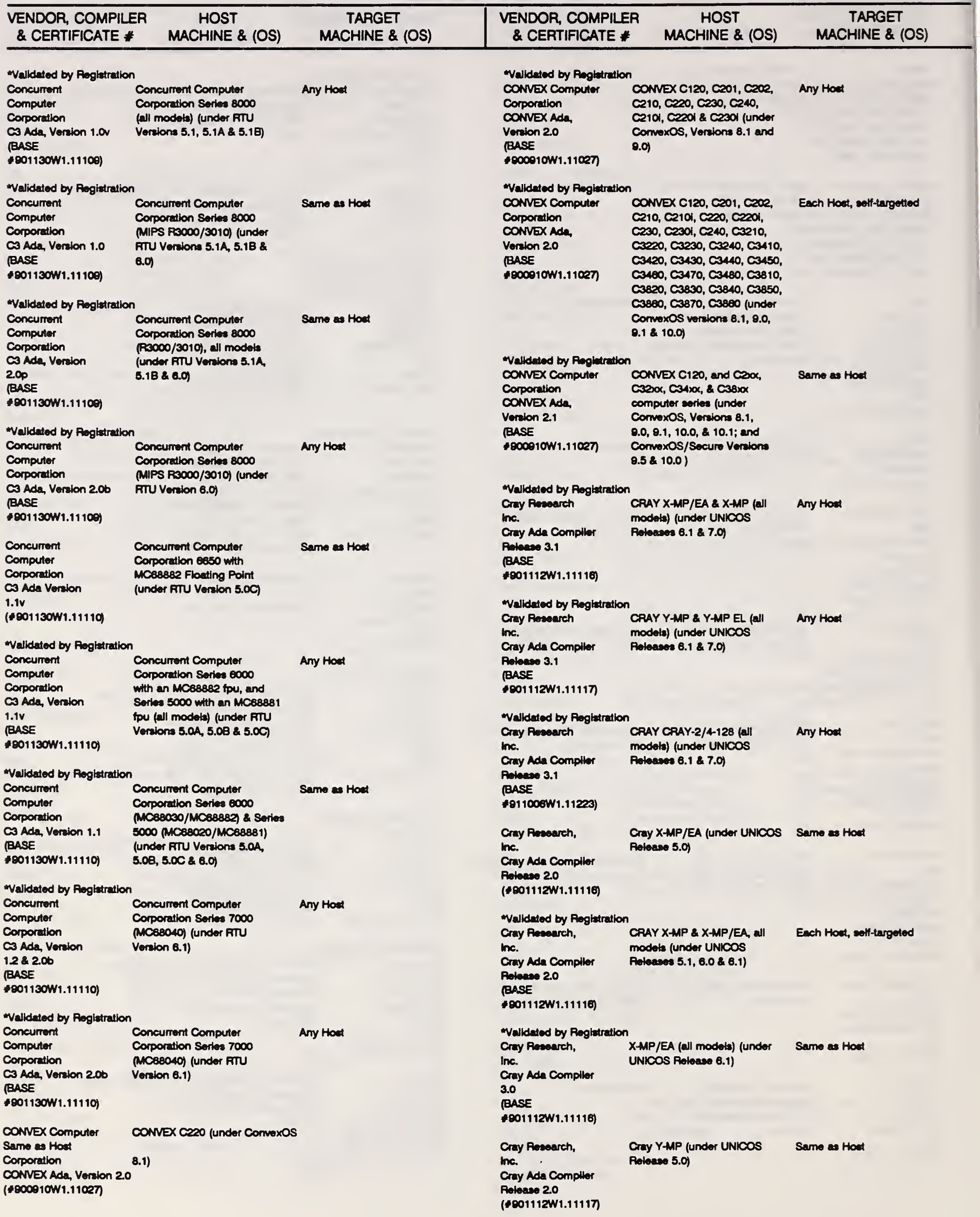




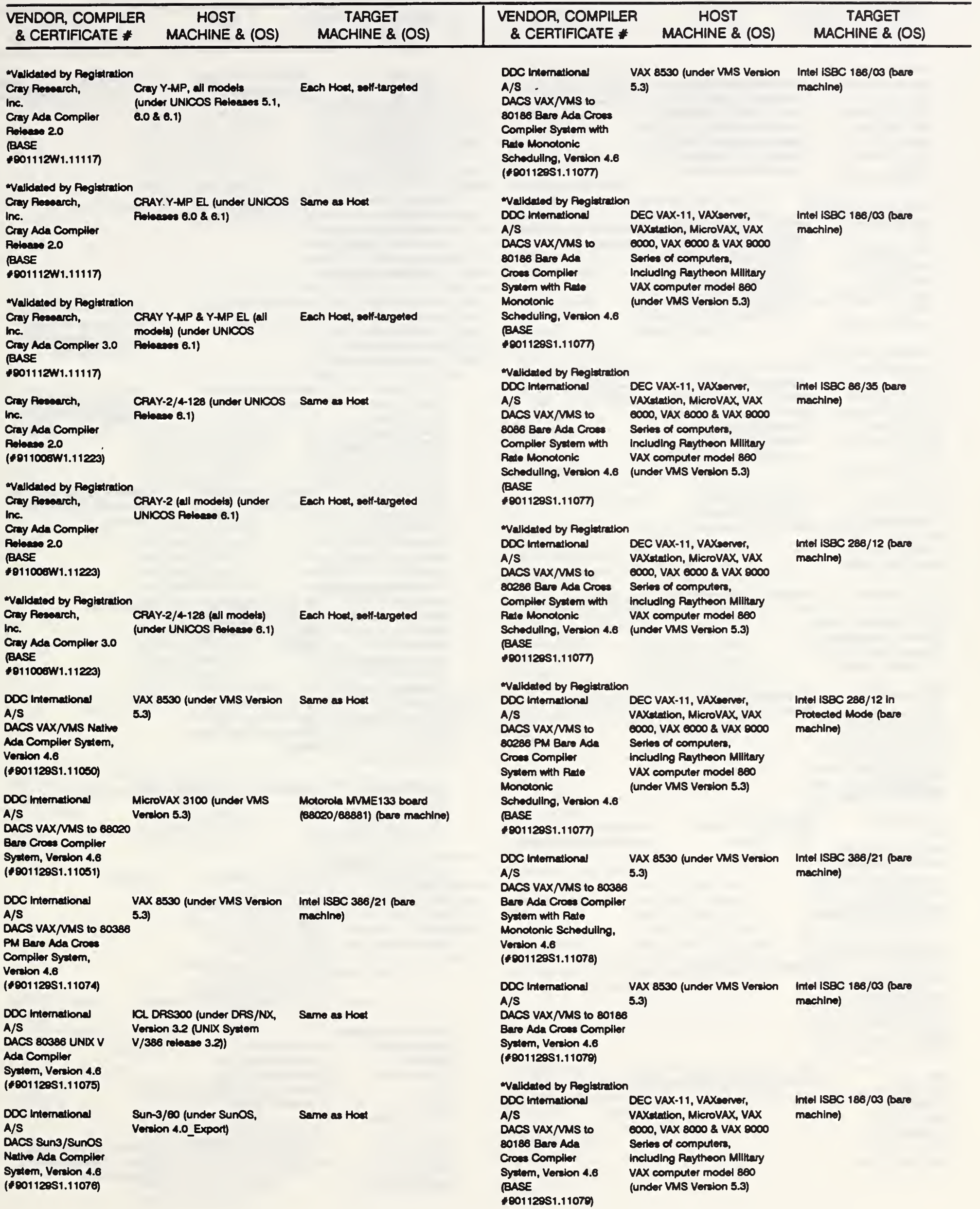




\begin{tabular}{|c|c|c|}
\hline $\begin{array}{l}\text { VENDOR, COMPILER } \\
\text { \& CERTIFICATE * }\end{array}$ & $\begin{array}{c}\text { HOST } \\
\text { MACHINE \& (OS) }\end{array}$ & $\begin{array}{c}\text { TARGET } \\
\text { MACHINE \& } 10\end{array}$ \\
\hline \multicolumn{3}{|l|}{ Validated by Rogiatration } \\
\hline 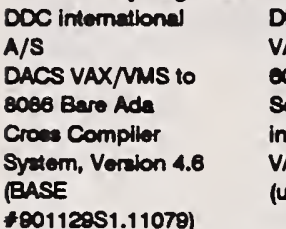 & 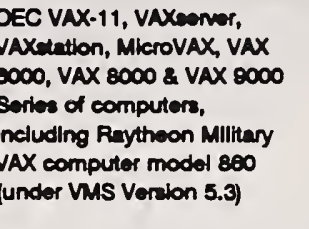 & $\begin{array}{l}\text { Intel ISBC 88/35 (bave } \\
\text { mechine) }\end{array}$ \\
\hline
\end{tabular}

- Validated by Regiatration

DOC imtemational

A/S

DACS VAX/MMS to

80286 Bare Ada

Croes Compller

Syztem, Verion 4.6

(BASE

$\$ 00112951.11070$

DEC VAX-11, VAXconver,

VAXatation, MleroVAX, VAX

8000 , VAX 8000 \& VAX 8000

Sorbes of computers,

Including Raytheon Military

VAX computer model 860

(under VMS Veralon 5.3)

-Validated by Regitutration

DOC Intemational

A/s

DACS VAX/MMS to

80288 PM Bare Ada

Cros Complior

Syctern, Veriolon 4.6

(BASE

+90112051.11070

DOC Internationa

A/S

DACS 80388 DMS/OS

DEC VAX-11, VAXeerver,

VAXetation, MleroVAX, VAX

8000 , VAX 8000 \& VAX 8000

Series of computers.

Including Raythoon Military

VAX computer model 860

(under VMS Verion 5.3)

Ade Compller

Syctem, Verion 4.6

(*001129s1.11112)

DOC International

A/S

DACS VAX/MMS to 80860

Bare Ada Croas Complier

Syztem, Version 4.6.1

(*010502s1.11158)

\section{ODC International \\ A/s \\ DACS Sun-3/Sunos to \\ 68030 Bare Ada Crose \\ Compller Syatem, Verion \\ 4.6.4, MRI IEEE 605 \\ (BASKC MODE \\ (*010502s1.11150)}

$\begin{array}{ll}\text { DDC international } & \text { Sum-3/50 (under SunOS } \\ \text { A/S } & \text { Roleces } 4.0 \text { Export) }\end{array}$

DACS Sum-3/Sunos to

88030 Bare Ada Croes

Compller Sytem, Vercion

4.6.4, MRI IEEE

605 (SECURE MODE)

(\$010502s1.11100)

Velidated by Rogiatration

DOC-1, inc.

IBM PS/2 Model 80-311

(Under LynxOS 388/PS2,

Veraion 2.04)

VAX 8530 (under VMS Voraion 5.3)

Intel ISEC 288/12 (bare machino)

intel isBC 286/12 in

Protected Mode (bare

mechine)

Same as Hod

Sun-3/50 (under SunOS

Roloeses 4.0_Export)

Roleceso 4.0_Export)

Motorda MVME143 board (88030/68882) (bare machine)

Motorola MVME1 43 boand (B8030/68882) (bare machine)

DACS VAXMMS to

80486 PM Bare Ada

Croes Compller

Syatem, Vorsion 4.6

(BASE

+00112851.11074)

DDC-1, ine.

DACS MIPS RaSC/O

to MIPS R3000

Bere Ada Croes

Compllar Syatem,

Roloave 2.1-16

(*020805S1.11263)
MiPS M/120-5 (under RISC/o Loekheod Sanders STAR MMP Vertion 4.501 R3000/R3010 Bourd (bare
VAX 8530 (under VMS Verion 5.3)

mechine) machino)
DDC-1, ine

DACS .

DECatation/ULTRX

to MIPS R3000

Bare Ada Croen

Compliar Syutem

Raleave 2.1-16

(\$02080651.11284)

DDC-1, ine.

DACS Sun

SPAPC/SunOS Nathe

Adu Compllor Syctem,

Verion 4.6.1

$(+020005 s 1.11200)$

\section{DOC-Inter, Inc.}

interACT Ada

17 saA Compller

Syctem, Risteace 3.5

(101070ss1.11101)

DOC-Inter, inc.

InterACT Ada MIPS

Crove-Compller

Syctem, Rolean 2.0

(\$010705S1.11102)

Validated by Regltration

Doc-inter, inc.

InterACT Ada MIPS

MicroVAX 3100 Clueter

Croes-Compller

Syctem, Raberes 2.1

(EASE

\$10706s1.11182

Velldated by Rogiatration

DESC ud

VME Ada Compller

VA3.25

(BASE

$\$ 821008$ N1.11283)

(under VME with VMEB

Environment Option Verion SVese?

- Validated by Rogitration

DESC UId.

VME Ada Compller

VA320

(EASE

+ D21008N1.11293)

Diglal Equipment

Corporation

SPARCatation 2 (under

sunos, Verion 4.1.1)

MicroVAX 3100 Cluater (Under VMS 5.2)

MicroVAX 3100 Ciuster

\section{(under VMS 5.2)}

VAX Ada, Veriolon 22

(*00110051.11053)

- Valldated by Rogiatration

Digital Equipment

Corporation

VAX Ada Verion

22

(BASE

*00110051.11053)

ICL Sories 30 Lovel 80 (under VME with VMEB Environmont Option Verion SV2824$$
5.4
$$

5.4)

Validated by Roglatration

Dighed Equlpment

Corporation

VAX Ada Veriolon

23

(BASE

\$001100S1.11053)

Dightal Equlpment

Corporation

VAX Ada, Verion 22

(*00110051.11054)

DEC VAX-11, VAXconver, VAXtation, VAXt, MicroVAX, VAX 4000, VAX 8000 , VAX 8000 \&AX 8000 Series of computers (as supported); Ratheon Milinary VAX Computer Model 860; and Norden MiNAX Computer Model MINAX II (under VMS Verion 5.4)

Al VAX, MicroVAX, VAXutation, VAXener erites of computers (as supported) (under VMS Versions 5.4 \& 5.5 ) 5.4) (under VMS 5.2)

TARGET

MACHINE \& (OS)

integrated Dovice Technology IDT7R3501 R3000/R3010 Board (bare mechine)

Same en Hoet

ImterACT MIL-STD-1750A In:truction Set Archilecture Simulator Roloase $\mathbf{2 . 3}$ (bare machine simuletion)

Locktheed Sanders STAR MVP R3000/R3010 Board (bere machino)

Locktheed Sanders STAR MVP REO0O/R3010 Board (bare machine)

Same es Hoet

Same as Hod

VAX 8800 (under VMS Vortion

MkerovAX II (under VAXELN Veralon 4.1, uaing VAXELN Ada Verion 2.2 


\begin{tabular}{lcc|ccc}
\hline $\begin{array}{l}\text { VENDOR, COMPILER } \\
\text { \& CERTIFICATE * }\end{array}$ & HOST & TARGET & VENDOR, COMPILER & HOST & TARGET \\
\hline
\end{tabular}

- Validated by Rogiatration

Dight Equipment DEC VAX-11, VAXeoner,

Corporation VAXetation, VAXt,

VAX Ada Verion MicroVAX, VAX 4000 , VAX

$2.2 \quad 6000$, VAX $6000 \&$ VAX 8000

(BASE

100110051.11054
- Validatod by Regiatration Digital Equlpment Corporation

VAX Ada Veraion 2.2

(BASE

100110051.11054 )
- Velidated by Roglatration

Dightel Equlpment Al VAX, MlcroVAX,

Corporation

VAX Ada Version

2.3

(BASE

100110051.11054 )
Digltal Equipment Corporation DEC Ada, Verion 1.0 (\$9110ess1.11220)

- Validatod by Rogiatration Digital Equlpment Corporation DEC Ada, Veralon 1.0 (BASE 10110ess1.11226)
Sories of computers (as supported); Rathoon Military VAX Computer Model 860; and Norden MINAX Computer Model MINAX II (under VMS Veraion 5.4)
VAX 0000 Model 200,300 \& 400 Series; VAX 8200, 8250 $8300,8350,8500,8530$ $8550,8600,8650,8700$, $8800,8810,8820,8830$, 8840, 8842, 8974 \& 8978; I785; MicroVAX II, 2000 , $3100,3300,3400,3500$, 3600,3800 \& 3000 ; VAXstation 11, 2000, 3100 cories, $3200,3500,3520$, 3540 \& 8000; VAXeerver $3100,3300,3400,3500$ $3600,3002,3800,3000$; VAXioner $6000-310$, $0000-410$ \& $0000-420$; Rathoon MIIItary VAX Compuler Model 860 (under VMS Verion 5.4) VAX-11/730, /750, /780,
VAXetation, VAXeenver cories of computers (is upported) (under VMS Veralons 5.4 \& 5.5 )
VAX 4000 models $200 \& 300$; VAX 6000 Sorles $200,300 \& 400$; VAX $8200,8250,8500,8530,8550$, $8700,8800 \& 8810$; VAX-11/730 \& 7750; MleroVAX II, 2000, 3100, 3300, $3400,3500,3600,3800$ \& 3900 ; VAXetation $2000,3100,3150,3200$, 3500 \& II/GPX; VAXeoner 3100, 3300 , $3400,3500,3600,3800,3000$; VAXserver 4000-300; VAXeerver 6000 Models 210, 220, 310, 320, $410 \& 420$; Rethoon Mllitary VAX Computer Modete 810 \& 860; Norden MINAX Computer Model MINAX II, NAX 6208 630; VAX RTA; KA620-8A KA800-M; TIVAX 300, 1000, 3200, 3300, 3305, $3400,3500,3600,3800,4000$ Model $300,8550,8700$, iVAX 6000 Modet $200,300 \& 400$ Serles and rVAXstation 3100 Modets 30 \& 38 (under VACEN Version 42, uaing VNEEN Ada Verion 224

VAX 0000 Model $200,300 \%$ 400 Series; VAX 8200,8250 , $8500,8530,8550,8700,8800$ \&810; VAX.11/730 \& /750; MieroVAX II, 2000, 3100 , $3300,3400,3500,3600,3800$ 23000 ; VACetation 2000 , $3100,3150,3200,35002$

II/GPX; VAXsener 3100, $3300,3400,3500,3000$, 3002,3800 , 3000; VAXeoner 6000 Modets 210220,310 320, 410 \& 420; Ratheon Military VAX Computer Models 810 \& 860; Norden Sydiom: MII VaX II, NAX 620 \& 630; VAX RTA; KAEZO-BA, IVAX $300,1000,3200,3300,3300$, $3400,3500,3600,3800,8550$, 8700 , rVAX 6000 Model 200,300 \& 400 Sorles \& iVAXetation 3100 Modet $30 \& 38$ (under VAXELN Verilon 4.1 using VAXELN Ada Veralon 2.2)

\section{VAX $4000,6000,80000$}

verles of computers; MieroVAX II, 2000, \& 3000 erries of computers;

VAXetation II, 2000, 3000, 8 $3000,4000, \& 0000$ cories of computers; NAX 620 \& 630; KA62O-BA, KABOO-M, KAV3O VME SBC; HVAX $300,1000,3000,4000,6000,28000$ sories of computers; and ivaxetation 3100 sories of computers; (under VAXELN Voralon 4.4, wing VAXELN Ada Verion 22

Same ea Hoet (under ULTRX 4.2)

DECatation 2100, 3100, 3100 s, 5000 Modets 120/125, 120/125CX, 120/125PXG, 120/125PXG TURBO, 200, 200CX 200PX, 200PXG, 200PXG TURBO; and DECyytem 3100, 5000 Model $200,5100,5400,5500,5810,5820$ $5830 \& 5840$ (under ULTRIX Verion 4.0, 4.1 \& 4.2)

Any Hoet

-Velldeted by Roglatration 4000 cories of computers; VAXsonver
Dightel.Equlpment

Corporation

DEC Ada, Veralon

1.0

(BASE

\$9110ess1.11228

validated by Regtatration

Digital Equipment

Corporation

DEC Ade, Verion

1.1

BASE

91102551.11220

Dight Equlpment

Corporation

DEC Ada for

OpenVMS NPP

Syctems, Verion 3.05

(403031051.11315)

- Valldated by Rogletration

Oighal Equlpment

Corporation

DEC Ada for

OpenVMS NXP

Syatem, Verion 3.05

BASE

$\$ 20031051.11315$

Digltel Equlpment

Corporation

DEC Ada for Openvis

VAX Syuterns, Verion

$3.0-7$

(\$03031051.11316)

- Valldated by Regiburation

Dightal Equlpment

VAXT, VAX 4000,6000

DEC Ada for

OpenVuS VAX

Syctems, Version

3.07

(BASE

-03031051.11316

Digltel Equlpment

Corporation

DEC Ada for

OpenVMS VAX

Syctern, Verion 3.0.7

(403031051.11317)

Validated by Rogistration

Digital Equipment

Corporalion

DEC Ada for

Openvis Vax

Syctems, Vercion

3.0-7

BASE

183031951.11317

VAXT, VAX 4000,0000 , $8000,8000,210000$. MicroVAX II, 2000, \& 3000; VAXetation II, 2000, 3000, 4000 ; VAXeerver 3000,4000 , 80000 verive of computere (as eupported) (under VMS Verion 5.4 \& 5.5 )

Digital Equlpment Corporation

DEC Ada for DEC

OSF/1 NXP

Syctems, Verion 3.1

(\$23102951.11330)

$5100,5400,5500,5800$, \&

5000 serles of computers

(under ULTRIX Versions 4.0,

DECetation 2100, 3100, $810,5820,5840, \& 5000$ cories of computers (under Ulurbx Voraion 4.2.

DEC 3000 Model 400 (under OpenVMS AXP Operating

Syctem, Vercion 1.0

DEC 3000 Workatation and computer (under OpenvMS MicroVAX II, $2000, \& 3000$ VAXtation II, 2000,3000 , 4000 ; VAXeener 3000,4000 , 2. 6000 vites of computers (es supported) (under VMS Veraion 5.4 \& 5.5 )

VAxctation 4000 Model 60 (under VMS Veraion 5.5)

DEC 3000 Model 400 (under DEC OSF/1, Veralon 1.3)
DEC DECetition 2100,3100 ,
Any Howt

Any Hot

Same as Hoat

Any Hoet

Any Host

VAXstation 3100 Model 48 (under VAXELN Verclon 4.4, using VAXEUN Ada Veraion 2.4

$\operatorname{VAX} 4000,0000, \& 8000$ MleroVAX II, 2000, 3000; KA620-BA, KAV3O VME SBC KABOO-M; rVAX 300, 1000 , $3000,4000,6000,8000$, \& iVAXetation 3100; NAX 620 2630 ; VAXutation II, 2000 , 3000 , \& 4000; VAXerver $3000,4000,26000$ series of computers (es supported) (under VAXELN Vernion 4.4, ueing VAXELN Ada Veralon 2.2)

Same thos 


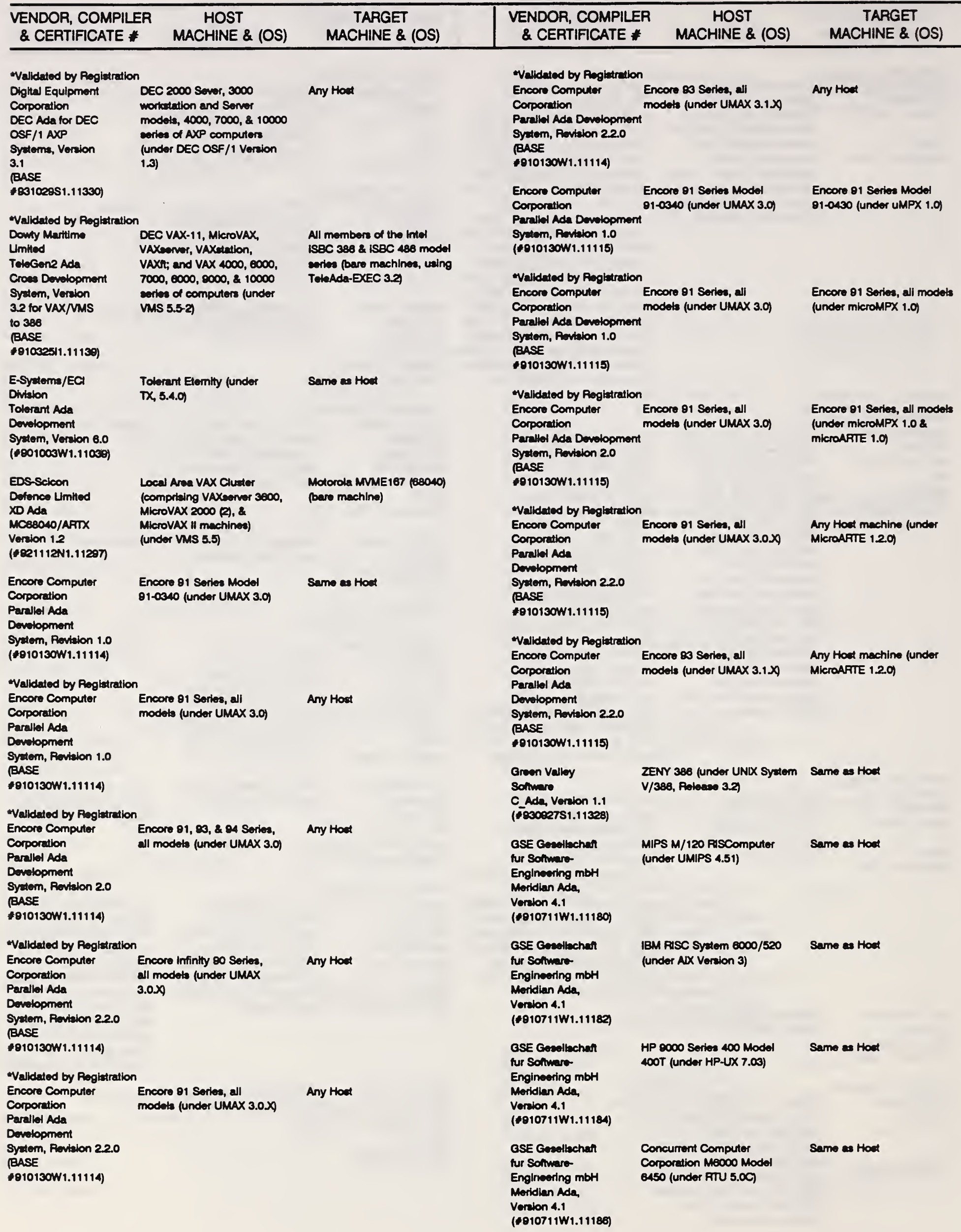


Ada PROCESSORS, Continued

VENDOR, COMPILER
\& CERTIFICATE *

GSE Gesellachen

5.1, CX/RT 5.1, OR CX/SX

TARGET

MACHINE \& (OS) hur Solvware

Englneering mbH

Moridian Ada,

Vorkion 4.1

(\$010711W1.11187)

GSE Gesoltachan

hur Soltwers-

Englineering mbH

Moridian Ade,

Verion 4.1

(\$910711W1.11188)

\section{GSE Geeellachen}

hur Solmwere-

Engineering mbH

Morldian Ade,

Veralon 4.1

(\$910711W1.11190)

\section{Harrb}

Corporation,

Computer Syaterms

Divition

Hartis Ada 5.1

(4000018W1.11028)

Valdated by Registration

Harts

Hamts NH-400 (under CX/UX Any Hoet

Corporation,

5.1)

Data General AVION 400

Same as Hoet

402 (under DG/UX

4.31)

HP 8000 Series 700 Model 720 (under HP-UX 8.01)

Same Hoat

Seme Hoet

Conporation M8000 Mod

8500 (under RTU 5.1A) Harto NH-400 funder CX/UX Same at Hoat
5.1)

Ondaion

Harris Ada 5.1

(BASE

$1000018 W 1.11028)$

Validated by Ragiatration

Hants

Herris NH-400 funder CX/UX Sarne a Hoat

Corporation,

5.2. CX/RT $5.2 \& \mathrm{CX} / \mathrm{s} \times 5.2$

Computer Sytema

Divalon

Hartite Ada

Compller, Version 5.1

(BASE

$1000018 W 1.11028$ )

Valdated by Regiatration

Herits

Corporation,

Hartis NH-400 \& NH-4800

(under CX/UX 5.3, CX/FT 5.3 Marts Ada Run-time Syatem

a. $\mathrm{CX} / \mathrm{SX}$ 5.3)

or ARMS Run-ime Sytem)

Diviaion

Harts Ada 5.1 .1

(BASE

(000918W1.11028)

Validated by Rogitation

Harrts

Corporation,

Computer Syatems

$\mathrm{NH}-400$ \& NH-4800 (under

CX/UX 6.1, CX/RT 6.1, \&

$C \times / 8 \times 6.1)$

Any Hoct (uing elther

Harris Ada Run-time Syctem or ARMS Run-time Syaterm)

Divalon

Harts Ada

Compllor 5.1.1

(BASE

$\$ 00018 W 1.11028)$

Validated by Rogiatration

Harts

Corporation,

Computer Syatems

Divition

Harris Ada

Compliter 5.1 .1

(BASE

$1000918 W 1.11028)$
NH-400, NH-4800, \& NH-5800 Amy Hoot fuaing olther (under CX/UX 6.2, CX/RT Harts Ada Runtime Syatem $6.2,2 \mathrm{CX} / \mathrm{S} \times 6.2)$ or ARMS Run-time Sytem)

VENDOR, COMPILER

\& CERTIFICATE *

HOST
MACHINE \& (OS)

TARGET

MACHINE \& (OS)
Weldeted by Rogiatration

Herite.

Harta NH-400, -4800, \&

Corporation, $\quad \$ 800$ (under CX/UX 62 ,

Computer Syatems $\quad$ CX/RT 6.2, \& CX/SX 6.2)

Divition

Marris Ada veraion 5.2

(BASE

$+000018 W 1.11028$

Valldated by Raghtration

Herrib

Corporation,

Harts NH-400, NH-4800, \&

Compuler Byteme CX/RT 6.2)

Dritaion

Herrts Ade

Compller, werion 62

(BASE

$+000018 W 1.11028)$

Welldaed by Regletration

Hents

Corporation,

Computer Syateme

Hents NH-400, NH-4800, \&

Divalon

Harte Ada

Compliter, veraton 7.1

(BASE

(C00018W1.11028)

Herre

Corporation,

Computer Syctorns

Divalon

Herris Ade 5.1

(1000018W1.11000)

Welidated by Regletration

Herris

Harts NH-1200, NH-3400 \&

Corporation, NH-3800 (under CX/UX 5.1,

Computer Syatems $\quad$ CX/RT 5.1, OR CX/SX 5.1)

Diviaion

Herris Ade 5.1

(BASE

$1000018 W 1.110009$

Walidated by Reglatration

Hants

Corporation,

Hartis NH-3800 (under CX/UX

5.1) Hits 1000 funder CX/UX 7.18 CX/RT 7.1)

Any Hoat (uaing etther Harte Ada Run-time Syctem or ARMS Run-time Syctem)

Computer Sy

$\mathrm{NH}-1200, \mathrm{NH}-3400 \& \mathrm{NH}-3800$ under CX/UX 52, CX/FT 5.2

Diviaion

Harts Ada Compller,

Verion 5.1

(EASE

$+000018 W 1.11020)$

Welladed by Reglatration

Herris

Corporation,

Herts NH-1200, NH-3400 \&

Computer Syutem:

NH 3800 (under CX/UX 5.3

Divtaion

Harrts Ada 5.1.1

(BASE

$\$ 000918 W 1.110009$

Wallated by Rogletration

Harts

Corporation,

Harts NH-1200, NH-3400, 8

Computer Syatem:

NH 3800 (under CX/UX 6.1,

CX/FT 6.1, \& CX/SX 6.1)

Divalon

Harris Ada

Compller 5.1.1

(BASE

-000018W1.11029)

Hownett-Packard

Co./Apollo

$$
\text { DA10.3) }
$$

Any Hoet

Same Hoet

Any Hoat

Any Hoet
Domain Ada V8.Om

(\$910411W1.11137)
Harts NH-440O, NH-4800, \& NH-5800 (Martie Ada runtime Sytem \& ARMS Runtime Sytem)

Any Hoct fusing elther Hants Ada Run-time Syetem or ARMS Run-time Syitem) 
Ada PROCESSORS, Continued

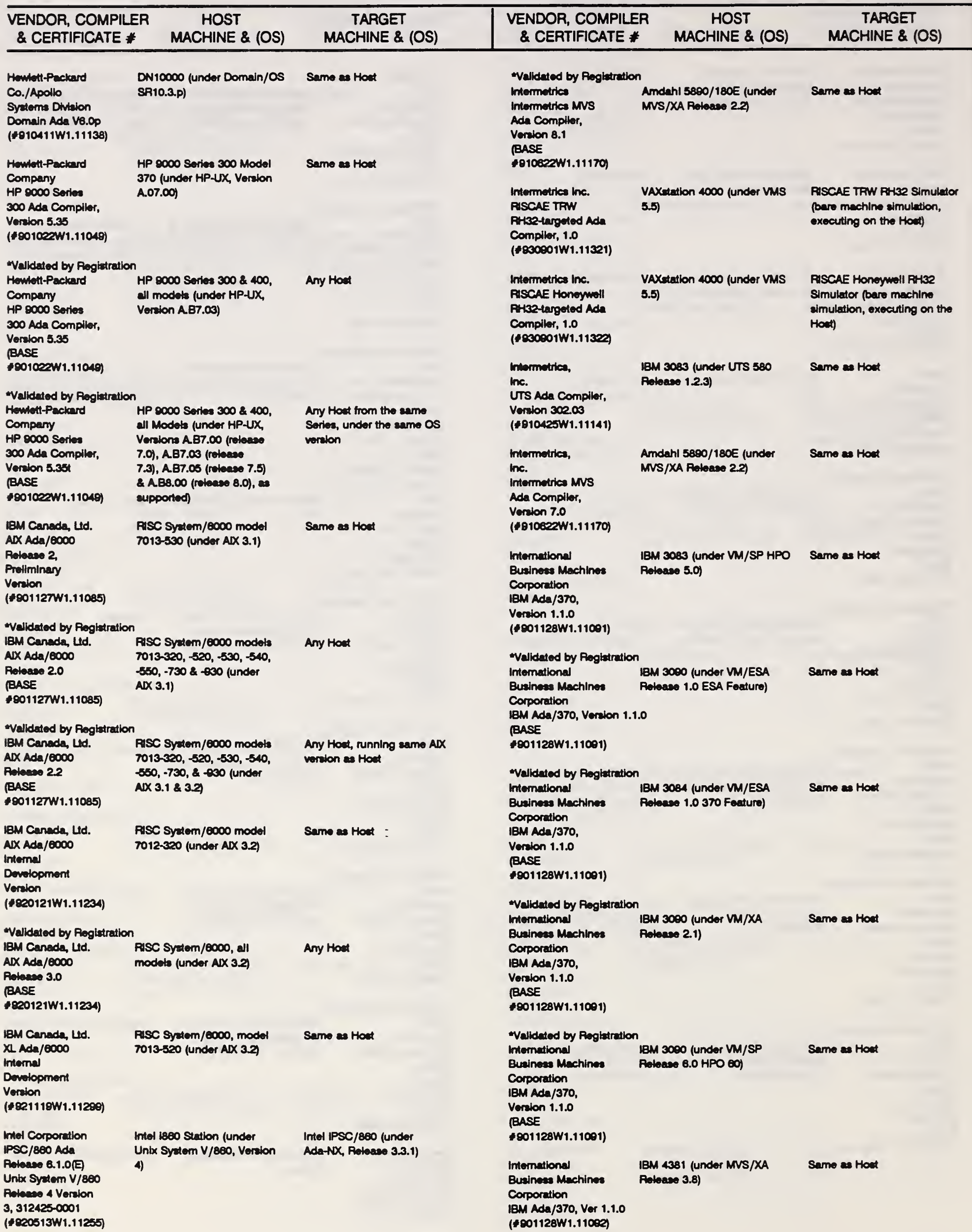




\begin{tabular}{ccc|ccc}
\hline $\begin{array}{c}\text { VENDOR, COMPILER } \\
\text { \& CERTIFICATE * }\end{array}$ & $\begin{array}{c}\text { HOST } \\
\text { MACHINE \& (OS) }\end{array}$ & $\begin{array}{c}\text { TARGET } \\
\text { MACHINE \& (OS) }\end{array}$ & $\begin{array}{c}\text { VENDOR, COMPILER } \\
\text { \& CERTIFICATE * }\end{array}$ & $\begin{array}{c}\text { HOST } \\
\text { MACHINE \& (OS) }\end{array}$ & $\begin{array}{c}\text { TARGET } \\
\text { MACHINE \& (OS) }\end{array}$ \\
\hline
\end{tabular}

Validated by Roglatration

Buaineas Mechines

IBM 3000 (Under MNS/ESA

Corporation

IBM Ada/370,

Verion 1.1.0

(BASE

HOO1128W1.110027

\section{Intemationa}

Busine Machlnes

Corporation

IBM Ada/370,

Verion 12.0

(optimized)

(\$910812W1.11169)

International

Businoses Mechines

Corporation

IBM Ada/370,

Verion 12.0

(optimized)

(\$810812W1.11167)

Intomationa

Busineas Machines

IBM 3083 funder VM/SP HPO Same a Hoet

Potwees 5.09

Corporation

IBM Ada/370

Verion 12.0

(unoptimized)

(1010812W1.11168)

Velidated by Regiatration

Imtemational

IEM 3000 (under VM/SP HPO

Bueineses Mechines

a.0

IBM 3083 (Under VM/SP HPO Same as Hoat

Rolecese 5.0)

IBM 4381 (under MVS/ESA Same Hoet

Roloeves 3.1)

Corporetion

IBM Ada/370,

Veraion 1.20

BASE

\$010812W1.11168)

Waldated by Rogiatration

Intomational

IBM 3000 (under VM/XA 2.1)

Bueineses Mechlnes

Corporation

IBM Ade/370,

Verion 120

BASE

\$010612W1.11168)

- Valldated by Regietration

Intemational

IBM 3084 (under VM/ESA

Busineas Machlnos

1.1.0 (370 Feature))

Corporation

Featuro))

IBM Ada/370,

Veralon 1.20

(BASE

1010812W1.11168)

Waldated by Rogiatration

international

IEM 3000 (under VM/ESA

Busines Machines $\quad 1.1 .0$ (ESA Feature))

Corporation

LBM Ada/370,

Veralon 120

(BASE

\$10812W1.11168)

- Veldated by Reglatration

international

IEN 3000 (under VM/ESA

Bueiness Mechlnos 1.1.1)

Corporation

IBM Ada/370,

Verelon 1.2 .0

(BASE

1010812W1.11168) $\mathrm{VM} / \mathrm{XA}$ 2.1) VM/ESA 1.1.1)
Welldated by Rogitutiotion

$\begin{array}{ll}\text { Internationd } & \text { IBM } 3084 \text { (Under VM/ESA } \\ \text { Bualnees Mechinee } & 1.1 .0(370 \text { Feature)); IBM } \\ \text { Corporation } & 3000 \text { (Under VM/ESA } \\ \text { IBM Ada/370, } & 1.1 .0 \text { (ESA Featuro), VM/ESA } \\ \text { VM/CMS Ada } & 1.1 .1, \text { VM/XA 2.1, \& VM/SP } \\ \text { Compiler, Veraion 1.4.0 } & \text { HPO } 5.0 \& 6.0 \text { ) } \\ \text { (BASE } & \\ \text { to10812W1.11168) } & \end{array}$

$1937 x_{1} 430 x, 308 \times 8000,8$ ES/8000 procesecors (under same OS a Hot?

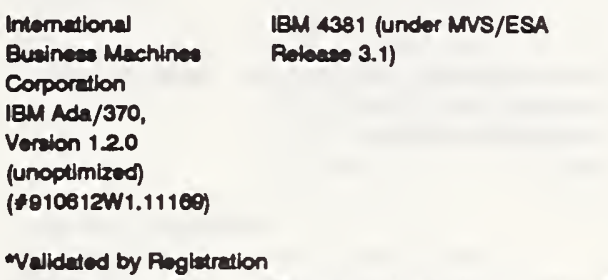

Regituration

Businen Mechines

Corporation

29

IBM Ada/370,

Verion $12.0 \& 1.3 .0$

BASE

(910812W1.11169)

Walleded by Reghatration

IBM 3000 (Under MVS/ESA

Pusines Mechince Rolaese 4.1.0

Corporation

Robases 4.1.9)

IBM Ada/370,

Veralon 120

(BASE

$1010812 W 1.11169$

$3037 x, 4310 x, 308 x, 3000$

ES/0000 proceseon (under VM/SP HPO 6.O)

Validated by Regitutration

IBM 3000 (Under MVS/ESA

Puelnes Mechines 42.0

Corporation

Roloase 4.2.0)

IBM Ada/370,

Verelon 120

(BASE

$\$ 010812 W 1.11169$ )

- Valdated by Reglatration

IEM 3000 (Under MVS/ESA

4.1.0\&420)

Corporation

IBM Ada/370

Vorion 1.3 .0

(BASE

IEM 837x, 4310x, 308x, 30008

ES/9000 procescors (under

$\mathrm{MM} /$ ESA 1.1 .0 \$70

1010612W1.11160

-Velideted by Rogituration

Intemational

Corporation

IBM Ada/370

Vervion 1.3 .0

(BASE

$1010612 W 1.11169$

IBM $937 x, 43 x 0,308 x, 30008$ ES/0000 procescors (under VM/ESA 1.1.0 (ESA Featuro)

IEM $937 x, 43 \times 0,308 x, 30008$ ES/0000 procesicors (under
BM $837 x, 43 \times x, 308 x, 3090$

ES/8000 proceseors (under

IBM $237 x, 43 x 0,308 x, 3000$ \& ES/8000 proceseors (MVS/ESA Rolease 4.1.0

IBM $237 x, 43 x x, 308 x, 3000$ \& ES/8000 procesecors MVS/ESA Rolease 4.2 .0$)$

IBM $937 x, 43 \times x, 308 x, 3000$, 2ES/9000 computers (under camo OS as Host)

IBM $237 x, 430 x$, 308x, 3000, \& ES/8000 computers (under same OS at Hoet)

IBM $037 x, 43 \times 0,308 \times 8000,8$ ES/9000 procesecors (under same OS a Hot)

Same es Hoet MVS/SP XA 2.2
Velldeted by Rogietration International

Bualness Mechines

IBM 3000 (Under MVS/ESA

Corporation

$10,410,820,8$

Compiler, Vereion 1.4 .0

BASE

910812W1.11160

international

Computers Limited

WME Ada Compiler

VA3, 00

(\$011003N1.11222)
1CL Series 39 Low 80

(Under VME with VMEB

Environment Option Verion SV201) 


\begin{tabular}{ll|l|l}
\hline VENDOR, COMPILER HOST TARGET & VENDOR, COMPILER
\end{tabular}

Same es Hoet

Intemational

Computen Umitod

VME Ada Compller

VA3.10

(\$021008N1.11283)

Inine Complier

Corporation

ICC Ada 7.0 .0

(\$10510W1.11145)

*alidated by Roglatration

Inine Compiler

Corporation

ICC Ada for HP

8000 Series

(BASE

(810510W1.11145)

-Vallideted by Rogletration

inine Complier

HP 8000 Sertes $700 \& 800$.

Corporation

ICC Ada for HP

0000 Series

$700 / 8007.4$

all models (under HP.UX

Verions $8.0 \& 8.0$, all

roloacen; and HP.UX BLS

Verion 8.0, all reloesese)

(BASE

\$10510W1.11145)

Inine Compller

Corporation

ICC Ada v7.0.0

(\$910510W1.11140)

-Validated by Rogistration Inine Compler

Corporation

Sun Mieroeydioms Sun-3

computer famlly (under

sunos 4.0 \& 4.1)

Veralon 7.4

BASE

1010510W1.11146)

Inine Compiler

Corporation

ICC Ada v7.0.0

(1010510W1.11147)

- Validated by Rogiatration

Invine Compller

HP 8000 Series $300 \& 400$,

Corporation

ICC Ada for HP

al Modots (under HP.UX

Veralon A.B8.05 (rolacese

8000 Series

8.05)

(BASE

010510W1.11147)

Inine Compller

Corporation

$10 C$ Ada 77.0 .0

VAXtation 3100 Model M38 (under VMS 5.3-1)

(\$10510W1.11148)

- Validated by Regiatration

Inine Compller

Corporation

$1 C C$ Ada for

leeour, Verion

7.4

(BASE

$\$ 810510 W 1.11148$

DEC VAX-11, VAXsonver, VAXctation, MicroVAX, VAX 4000 , VAX 8000 , VAX 8000 VAX 0000 , \& VAX 10000 eries of computers (under VMS 5.4)

- Validated by Rogietration

Inine Compller

Corporation

$10 C$ Ada for

IBoOMC 7.4

(BASE

\$010510W1.11148)

Same as Hoat

Same as Hoet

Any Hoet

Same as Hoet

Amy Hoat

machine)
$700 / 800$, Verion 7.4
HOST

MACHINE \& (OS)

Volideted by Regiatration

inine Compller HP 9000 Series 700, all

Corporation models (under HP.UX Vercion

ICC Ada for $\quad 8.0$, all releases)

ISCONC 7.4

(BASE

\$10510W1.11148)

TARGET

MACHINE \& (OS) 8.0 , all releases)

Intel $1880 \mathrm{MC}$, with/without KCE 800, on an EXV800804C board; any ainglo-board computer using the 1000 chip; and Intel 1000 simuletor, executing on the

How (bare machines)

-Validated by Regiatration

Inine Compllor

Sun Mierosyetems Sun-3

Corporation

$10 C$ Ada for computers, all modets (under SunOS vertion 4.12

be8OMC 7.4

2. Solarts version 1.0.1

(BASE

all reloases)

(010510W1.11148)

-Validated by Rogletration

Inine Compller

Sun Microosyterms Sun-4

Corporation

ICC Ada for

BOONC 7.4 SPARCatalion, \& SPARC computere, all modets

(under SunOS vorion 4.1.2

(BASE

2 Solarte verion 1.0.1

(010510W1.11148)

all roloases)

Validated by Rogiatration

Inino Compller

DEC VAX-11, MieroVAX,

Corporation

ICC Ada tor

locoxA, Verion

VAXsenwer, VAXstation,

VAXt; and VAX 4000, 6000 ,

7.5

BASE

$7000,8000,8000, \& 10000$

(010510W1.11148)

serles of computers (under

VMS 5.4)

Invine Compller

Corporation

VAXtation 3100 Model M38

(under VMS Verion 5.3-1)

ICC Ada v7.4.0

-Validated by Rogiatration

Inine Complier

Corporation

ICC Ada for

beom $X$ and

$1000 \mathrm{MM}$, Version

7.4

(BASE

$\$ 02052011.11260$

DEC VAX-11, VAXsenver, VAX station, MicroVAX, VAX 4000 , VAX 8000 , VAX 8000 VAX $8000, \&$ VAX 10000 Serbes of computers (under VMS 5.4)

-Validated by Regiatration

inine Compiler

HP 8000 Series $300 \& 400$,

Corporation

ICC Ada for

1060MM and beOMX

all modets (under HP-UX

Version 7.4

(EASE

+82052011.11260 )

Intel le60MC with or without

KCESeO on an Intel

EXV80060MC board; any

inglo-board computer the

uses the 1960 chip; Intel

1960 simulator (oxecuting on

the Hoet) (bere machino)

computer uaing the $1960 \mathrm{chlp}$; and

Intel 1080 simulator, executing 8 Solarts vorion 1.0.1,

Intel boomc, wth/without ICE 800, on an EXV8000011C board; any single-board computer uaing the 1960 chip; and Intel 1000 simulator, executing on the How (bare machines)

Imtel leeomc, with/without ICE 880, on an EXV8008014C board; any ainglo-board computer uaing the 1900 chip; and Intel 1000 simulator, executing on the Hot (bare machines)

Intel beaxA with or without ICESSO on an Intel EXV80060XA board; any inglo-board computer that unes the 1se0XA chlp; Intel 19e0XA simulator (executing on the Hoet) (bare machine)

Intel beovx In Hughes DMN running In tagged mode (bere verion 104)

Imtel loc0MM \& beOMX on a TRONIX PLSONXX JXV JAWG Execution Vehicle board; amy anglo-board computer that unes the $1860 \mathrm{MM} / \mathrm{MX}$ euperscalar chlp; Intel 1000 simulator fexecuting on the Hoeff) (bare machino)

Intel loOMM \& lOEOMX with/without ICE $\mathbf{9 0 0}$, on a TRONXX PIBEOMX JXV JIAWG execution Vehicle board; any Hoet (bare machines)

Intel lESOMM \& $1800 \mathrm{MX}$, with/without LEE 800 , on TRONXX PHOEONX JXV JIAWG execution Vehicle board; am Hot (bare machines) with/without ICE 800 , on a TRONIX PISEOMX JXV JIAWG execution Vehicle board; any
(\%02052011.11260)

Intel $180860 M C$ (bare
Intel leconc, with/without

KCE 800, on an EXV80060MC

board; any singlo-board

on the Host (bare machines)

HP 8000 Series $300 \& 400$

Version 8.0, all reloases)
Validated by Regietration

inine Compliter

Corporation

ICC Ada tor

LOOOMM and leoOMX

HP 8000 Series 700 , all

Vortion 7.4

(BASE

$+02052011.11200$ modets (under HP.UX Version 8.0, all roloaces)

Validated by Regiatration

Inine Compller

Corporation

ICC Ada for

locomM and losomx

Version 7.4

(BASE

192052011.1 1280)
Sun Micromytems Sun-3 computers, all modols (under SunOS vertion 4.1.2 all reloseses) machine, ueing CHKSYS komel inglo-board computer uaing the $1960 \mathrm{MM} / \mathrm{MX}$ superscalar chip; and Intel 1080 simulator, executing on the ainglo-board computer uing the $1000 \mathrm{MM} / \mathrm{MX}$ supersealar chip; and Intel 1000 simulator, executing on the

Intel le60MM \& 1860MX, anglo-boerd computer using the $1980 \mathrm{MM} / \mathrm{MX}$ superecalar chip; and Intel loeo imulator, executing on the Hout (bare machines) 


\begin{tabular}{ccc|ccc}
\hline $\begin{array}{c}\text { VENDOR, COMPILER } \\
\text { \& CERTIFICATE * }\end{array}$ & HOST & TARGET & VENDOR, COMPILER & HOST \\
\& CERTINE \& (OS) & MACHINE \& (OS) & TARGET & MACHINE \& (OS) & MACHINE \& (OS) \\
\hline
\end{tabular}

-Velldated by Reglatration

inine Compilat

Corporation

ICC Ada for

1000MM and $1000 \mathrm{Mx}$

Veralon 7.4

(BASE

$182052011.11260)$

Sun Microoystems Sun-4, with/without ICE 860, on a computere, all modets (under SunOS verion 4.12 \& Solarb verion 1.0.1, all relaseas)

Meridian Software

Syctems, Inc.

Meridian Ada,

Verion 4.1

(\$800000W1.11031)

Moridian Sormare

Syutems, Inc.

Morldian Ada, Veriolon 4.1

(\$000000W1.11032)

- Valdated by Rogistration Moridian Softwere

Sun Microsyotems Sun-4,

Syctems, Inc. SPARCEener \& SPARCatation

Moridian Ade

Verion 4.1

computer families (under

SunOS Voralone 4.1 \& 4.1.1)

(BASE

1800000 W 1.11032

Moridlan Softwere

Sytema, Inc.

Meridian Adr, Voralon 4.1

Sun-4/110 (under SunOS, Voralon 4.1)

Sum-3/260 (under SunOS, Verelon 4.1)

TRONIX PIOEOMX JXV JIAWG

execution Vehiclo board; any

angie-board computer ueing

the $1060 \mathrm{MM} / \mathrm{MX}$ euperecalar

chip; and Intel 1060

imulalor, executing on the

Hod (bare machines)

Same as Hod

Moridien Sormere

Syctoma, linc.

Moridian Ade,

Verdion 4.1

IBM PS/2 Model 30 twith

Same as Hoot

flowing-Point

Co-Proceseor) (Under IBM

(1800000W1.11005)

- Velldated by Rogiatration

Meridian Solvere

Any Computer Syetem

Syctems, inc.

Moridlen Ade,

Verelon 4.1

(BASE

1000000W1.11035)

Cortang: cpu: any the

executes the Intel 8088

Inatruction set, tpu: Intel

8087 or equivelent, as

approprlate, memony: 640

KByte RAM minimum, diak 20

MByte hard dive, OS: IBM

PC-DOS 3.30

Same as Hoet

- Valldeted by Rogiatration

Moridien Sormmere

Any Computer Syctem

Sycterns, inc.

Morldien Ade,

Verion 4.1.1

(BASE

$1000000 \mathrm{~W} 1.11035$

Any Hoet

Compriang: Cpu: amy that

execules the Intel 8088

intruction set; Fpu: Intel

8087 or equivalent, as

eppropriate; Memory: 640 or

greater KByte RAM; Disk 20

MByto hard ditwe (under IBM

PC-DOS 3.30)

- Valdated by Reglatration

Velldated by Ruglatration

Meridian Soltware

DECatation 2100,3100 \&

Syatems, lnc. 5000 (under Uitrlx 3.0)

Meridian Ada, Vercion 4.1

BASE

\$800000W1.1 1033)

Moridlan Sormare

Syterms, Inc.

Moridlan Ada,

Version 4.1

nouting-Point

Co-Proceseson) (Under IBM

PC-DOS 3.30)

(\$800000W1.11034)

Validaled by Registration

Meridian Softwere

Any Computer Syctem

Syuteme, inc.

Moridlan Ade,

Veraton 4.1

(BASE

$1000000 W 1.11034$

comprtang: cpu: eny that

executes the Intel 80288

80388 , or 80486 Instruction

cot, fpu: Intel 80287, 80387.

or equivalent, as appropriate,

memory: 640 KByto RAM

minimum, diak 20 MByto hard

ditw, OS: IBM PC-DOS 3.30

Validaled by Registration

Moridian Softumar

Any Computer Syctem

Comprtaing: Cpu: any that

executes the Intel 80288 ,

80386 , or 80486 Intruction

cot; Fpu: Intel 80287, 80387,

or equivalent, as appropriate;

Momory: 640 or greater KByte

RAM; Diak 20 MByte hard drtw

(under IBM PC-DOS 3.30)

-Velidated by Rogiatration

Moridian Sofmare

Any Computer Syatem

Syctems, Inc.

Meridian Ada,

Veralon 4.1.4

(BASE

$\$ 800000$ W1.11034

executes the Intel 80286 .
Meridian Somware Any Computer Sydem

Systems, inc.

Mortdian Ade,

Veraion 4.1.4

(BASE

$\$ 000000$ W1.11005

Compriaing: cpu: any that

executes the Intel 8086

indruction set; fpu:

Intel 8087 or equivalont,

as epropriate; memory:

640 KByte RAM; dlak: 20

MByte hand ditwe (under IBM

PC-DOS 3.30)

Meridien Sotmere

ITT XTRA/286 whth

floating-Point

Co-Procesest) (Under MS-DOS

Seme at Hoet

Mydidian Ade

Verion 4.1

$3.20 / 05286$

(\$800000W1.11036)

- Valldeted by Reglatration

Any Computer System compriaing: cpu: any the

exceutes the Intel 80288

80386, or 80488 Inatruction

cot, fpu: Intel 80287.

80387 , or equivalent,

epproprlate, momory: 1.5

MByte RAM minimum, disk $\mathbf{2 0}$

MByto hard ditwo, OS:

MS-DOS $3.20 / 05288$

-Velidated by Rogiatration

Moridian Solmure Any Computer Syctem

Syctems, Inc. Compriaing: Cpu: any that

Meridian Ada,

Venion 4.1.1

(BASE

executes the Intel 80288 ,

80388 , or 80486 Inatruction

ext; Fpu: intel 80287 .

80387 , or equivalent,

approprlate; Memory: 1.5 or

grouter MByte RAM; Diak 20

MBye hard dive (under

MS-DOS 3.30/OS286)

- Valdeted by Regitration

Moridian Softwere Any Computer Syatem

Syatems, Inc. Compriaing: Cpu: any thet

Moridian Ada,

Verelon 4.1.4

(BASE

executes the Intel 80288 ,

80388 , or 80486 Instruction

eot; tpu: Intel 80287

80387 , or equivalent, as

eppropriate; memony: 1.5

MByte RAM; diak 20 MByto

hard ditve (under MS-DOS

$320 / 05286$
Amy Hoet

Amy Hoot

Any Hoot

Seme at Hod

Amy How

Any Hoat

Any Hod
Compriaing: cpu: any the

80388 , or 80488 Inatruction

ett; fpu: Imtel 80287, 80387,

or equivilent, es appropriate;

momony: 240 KByte RAM; dlak

20 MByto hard ditw

(under IBM PC-DOS 3.30) 


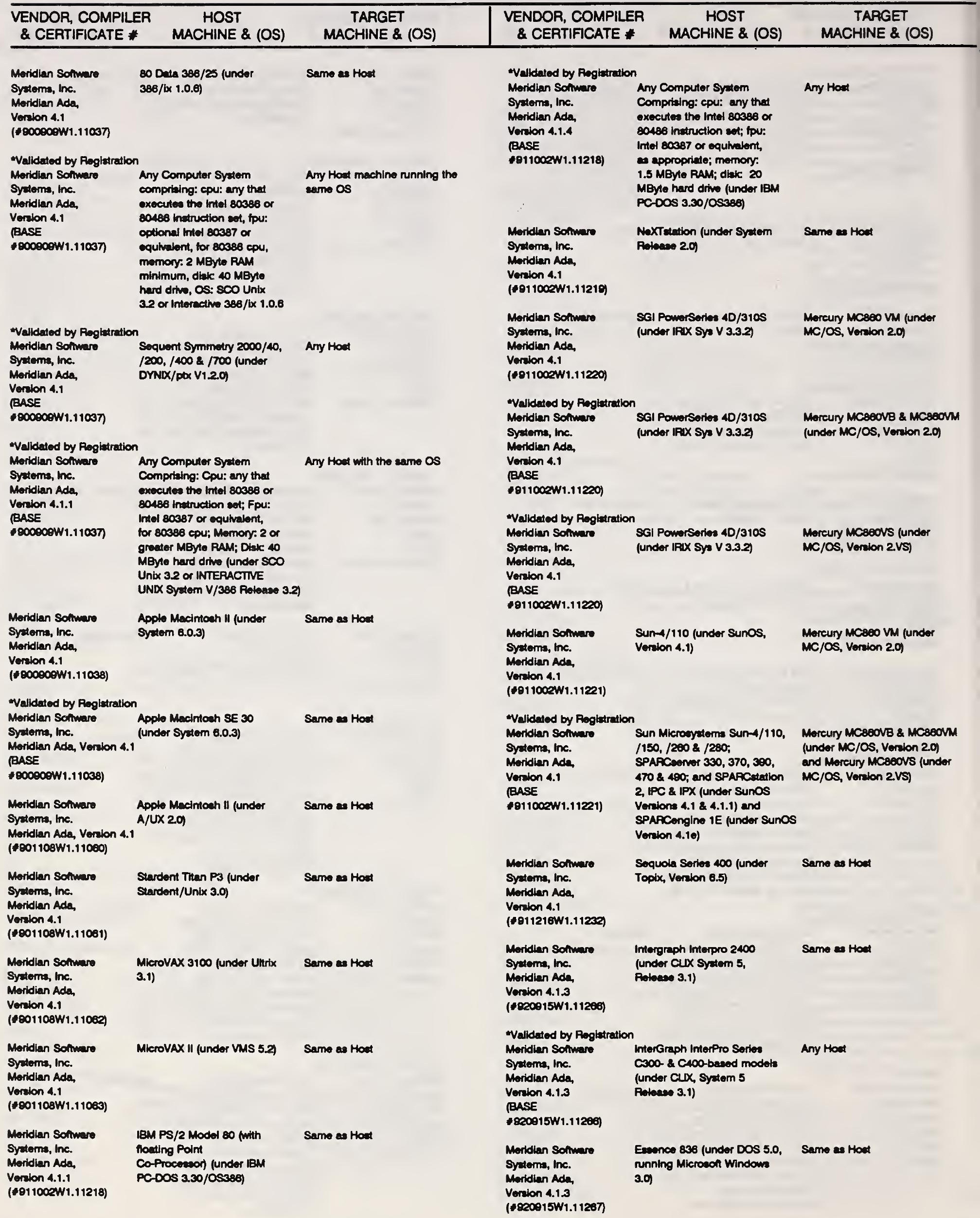




\begin{tabular}{ccc|ccc}
\hline $\begin{array}{c}\text { VENDOR, COMPILER } \\
\text { \& CERTIFICATE * }\end{array}$ & HOST & TARGET & VENDOR, COMPILER & HOST \\
& MACHINE \& (OS) & MACHINE \& (OS) & \& CERTIFICATE * & MACHINE \& (OS) \\
\hline
\end{tabular}

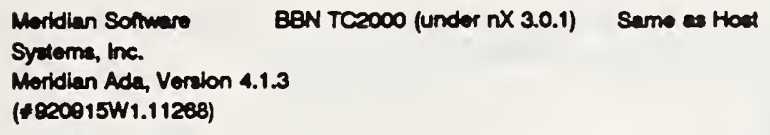

Moridian Soltware

Sycteme, inc.

Motorol VME 167-e8040 Same thot

Mordian Ada, Version 4.1.3

(\$830401W1.11313)

Moridlan Sotmure

Sytems, inc.

Enences 498 (under MS-DOS

Moridian Ada, Verion 4.1.3

(\$830401W1.11314)

MIPS Computer

Syatem

MIPS M/2000 (under PISC/O 4.509

MIPS ASAPP 3.0

(\$000010W1.11010)

MIPS Computer

Sycteme

MIPS M/2000 (under RuSC/os Same as Hoet

MiPS Ada 3.0 4.50

(4000010W1.11011)

Valldeled by Rogiatration

Multiproceseor

Sun Microsyitem

Toolemith inc. SPARCutation worles (under

CASEWorks/RT Ada v1.1 SunOS 4.11, 4.1.2, \& 4.1.3)

for Sun SPARCStation

(BASE

1030722W1.11318

- Validated by Reglitration

Tootemith inc.

Sun Microoyetem

CASEWorto/RT Ada

SPARCetation sorlos (under

Sunos 4.11, 4.1.2, \& 4.1.3)

v1.1 for Sun

SPARCStation

(BASE

1030722W1.11318

Muttiprocecsor

Toolemithe inc.

CASEWorto/RT Ade

for the Sun

SPARCStation, 1.1

(\$830722W1.11318)

Multiprocesesor

Toobemiths Inc.

CASEWorta/RT Ada

MCe80X0, Verion 1.1

(1030722W1.11318)

Multiproceses or

Tookmiths ine.

CASEWorta/RT Ada

1860 , Veraion 1.1

(1830722W1.11320)

Sun SPAFCatation 10 (under SunOS 4.1.3)

Sun SPARCatation 10 (under

sunOs 4.1.3)

Sun SPARCetation 2 (under Sunos 4.1.1)

-Validated by Rogintration

Multiprocesecor

Sun Microsyztem: SPARCetation sories (under SunOS 4.11, 4.1.2, \& 4.1.3)

CASEWorto/PT Ada

v1.1 for 1860

(BASE

$1030722 W 1.11320$
NEC Corporation

NEC Ada Compller

Sytem for

EWS-UXN Rrleace

4.0), Vercion

Paloesen $2.1(4.8)$

(\$1001881.11216)

Valideted by Reglatretion NEC Corporetion

NEC Ada Complter

Syetem, Vercion

RA.1 (4.6.4)

(BASE

101001851.11216

NEC EWS4800/220 (under

EWS-UXN (Rolease 4.0)

R2.1)

UP4800 Serles modets 520 ,

$605,620,825,630, \& 635$

(under UP-UX/N RM.1)

EWS4800 Superetation RISC

Same es Hoet

Any MC88020, MC88030, MC88040-besed single-board computer (bare machines. using Untion 3.1)

R3200-6 CPU board (bere machino)

Same at How

Motorola MMME147 (bere mechino)

Sories (all EWS RISC modets,

only) (under EWS-UX/N(RA.O)

R8.2 \& EWS-UXNVRA.2)

R7.1, es espported)

NEC Corporation

NEC Ada Compllor

NEC EWS4800/80 (under

Syctem for EWS-LX/N to

V70/RX-UX832, Verion 1.0

(\$01001851.11217)

Valleted by Roglatration

NEC Corporation

10 V7O/RX-UX830

CSPI Supercard II fintel 80800 ) with VSB

daughtertoard (bare mechino)

CSPI Supercerd 2 with VSE

daughtertoard, CSPI

Supercand 3 with VSB

daughterboard, CSPI

supercard $3 \times 1$ with VSE

deughterboard, \& CSPI Supercard 4

with VSB daughterboard pare

mechines, uaing Unison/psos + 3.1)
NEC Ada Compller

Syatem for

EWS-UXN Re-1

4.9 to $V 70 / R X-U \times 832$

Verion 1.0

(BASE

101001831.11217

Waldated by Rogitutration

NEC Corporation

NEC Ada Complier

Syatem for

EWS-UXN(Ray 4.0)

wrion R.1 (N4.6.4)

BASE

01001851.11217

North Chine

inathute of

$$
\text { 3.9) }
$$

MieroVAX II (under ULTRIX

Computing Technology

C Ade, Verion 1.0

(\$1010002N1.11188)

Proprictery Sommere Syctema, inc.

PSS VAX/ZRBA3ES

Compller Verion

XB-01.000

(102012311.11250)

R.R sommere

Inc.

Jenue/Ada 220

Phar Lap/DOS

(\$⿻011120W1.11088)

Validated by Rogletration

R.R. Sommere,

Any Computer Syatern

Jenus/Ada 2.2 .0

Phar Lap/DOS

BASE

(D01120W1.11088)

Comprising: cou: Intel

80386, fpu: optional

memory: 4 MByte RAM, dlak

40 MByte hard dive (under

-Valdeted by Roglatration

R.R. Solmere,

Phar Lap/DOS 3.3)

models of the

EWS4800 sories (under

SUportation FISC

(under

VAX 8350 (under VMS Veralon 5.4)

BM PS/2 Model 80 (under Phar Lap/DOS 3.3)

inc.

Janus/Ada 22.

Dos

(BASE

1001120W1.11068)
Any Computer Syatem

Compriaing: cpu: any that

executes Intel 8086/8088

instructions; fpu:

optiona; memory. 640

KByte RAM; diak 20 MByte

hard ditve (Under MS DOS

3.3)
Any Hoet

NEC MN4000 (Under FOK-UX832 V1.6)

NEC MV4000 (under RX-UX832 V1.6)

NEC MN 4000 (under RXX-UX832 V1.63)

Same thot

PSS Zoran ZR34325 Diglta Signal Processor AdaRAID Verion XK-01.000 pere mechine simuletion, executing on the Hod)

IBM PS/2 Model 80 (under MS Dos 3.3)

Any Computer Syctem Comprising: epu: Intel 80386, fpu: optlonal, memory: 4 MByte RAM, diak 40 MByte hard ditwe (under MS DOS 3.3)

Same es Hoat 


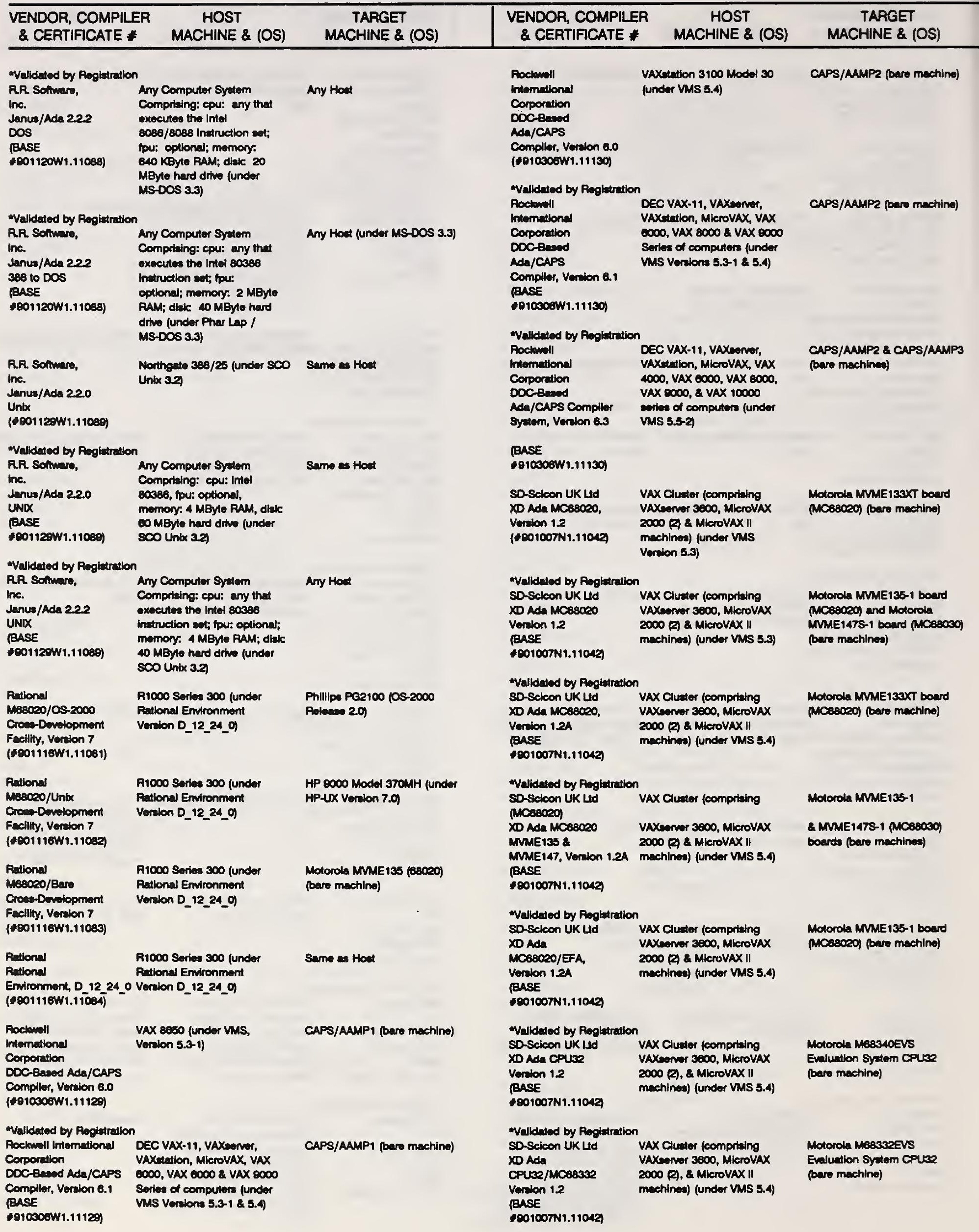




\begin{tabular}{|c|c|c|}
\hline $\begin{array}{l}\text { VENDOR, COMPILER } \\
\& \text { CERTIFICATE * }\end{array}$ & $\begin{array}{c}\text { HOST } \\
\text { MACHINE \& (OS) }\end{array}$ & $\begin{array}{c}\text { TARGET } \\
\text { MACHINE \& (OS) }\end{array}$ \\
\hline $\begin{array}{l}\text { SD-Scicon UK LId } \\
\text { XD Ada } \\
\text { MIL-STD-1750A, } \\
\text { Vorelon } 1.2 \\
\text { (DDO1214N1.11080) }\end{array}$ & $\begin{array}{l}\text { Local Area VAX Clustor } \\
\text { (compraing VAXerver } 3000 \text {, } \\
\text { MicroVAX } 2000 \text { (R) \& } \\
\text { MicroVAX II machinea) } \\
\text { (Under VMS 5.3) }\end{array}$ & $\begin{array}{l}\text { Fairehlld FaAso on a SBC-50 } \\
\text { bowed (MIL-STO-1750A) (Bare } \\
\text { machino) }\end{array}$ \\
\hline $\begin{array}{l}\text { 80-Scicon UK LId } \\
\text { XD Ada MCB8000, } \\
\text { Vorion } 1.2 \\
\text { (1010314N1.11134) }\end{array}$ & $\begin{array}{l}\text { Local Area VAX Cluator } \\
\text { (compriaing VAXsorver } 3000, \\
\text { MieroVAX } 2000 \text { (R) \& } \\
\text { MieroVAX H mechinea) } \\
\text { (under VUS 5.4) }\end{array}$ & $\begin{array}{l}\text { Motorola MC8s000 on an } \\
\text { MYME1 17-3FP board (bare } \\
\text { mechlno) }\end{array}$ \\
\hline
\end{tabular}

validated by Reglatration SD-Scicon UK LId XD Ada

MCB8000/EFA.

Vorelon 1.2

(BASE

010314N1.11134)

80-seteon UK LUd

XD Ada

MCBBCEO/ARTX,

Verelon T1.2

(\$810011N1.11189)

SO-Scicon UK LId

XD Ad MCB8OAO

Veraion 1.2

(4011128N1.11230)

Local Area VAX Cluetor

compriaing VAXeonver 3000 .

MieroVAX 2000 (R) \&

MicroVAX II mechines)

(under VMS 5.4)

Motorole MC88000 on an

MMME1 17-3FP board (bere mechlno)

\section{Local Aven VAX Cuner} loompraing VAXeonor 3000 , MieroVAX 2000 (2) \&

MicroVAX II machines)

(Under VMS 5.4)

Local Aroe VAX Clumer

formprtaing VAXeorwer 3000 , MicrovaX 2000 (a) \&

MicroVAX II mechlines)

(Under VMS 5.4)

\section{Validated by Regitatration}

sa-scicon UK Ud

XD Ade

Local Aroe VAX Cumiter comprialing VAX MicroVAX 2000 (2), \&

MicroVAX II machinea)

MCS8040/FORCE

CPU-40, Veralon

(under Vis 5.5)

(BASE

(011128N1.11230)

Validated by Roglatration

SOScicon UK LId

XD Ada MC8so40,

Vorelon 12

(BASE

1011128N1.11230)

Stomens Nixdort

informetion.

syzeme $A C$

SIEMENS NDXDOFF

QS2000 Ada

Compllor V21

(\$80111911.11111)

- Validated by Regiatration Simoms Nbxdorf

SIEMENS NDDOFF 7.530 , Intormations-

syeteme AG

SIEMENS NDDORF

7.536, 7.541, 7.550, 7.551,

$7.560,7.561,7.570,7.571$,

7.580 \& $7.500 ; 7.500-030$,

$-610,+\infty 0,+100 \&+1120$

(under BS2000 VO.5 \& V10.0)

Compllor V2.1

(BASE

10011191.11111)

Stomens Nixcorf Informations

yydorno $A G$

Ada (SIND) VA.1

(\$010711W1.11181)

valleted by Rogitareation

Stomens Nixdorf

informetion:

Stemens Nbxdort WX200

Siemena Nixdorf WX200

(SNDX-OOT) (under

SINDX-ODT V1.0

syutome $A B$

(BIND-OD) (under

Ade (SIND) V4.1

SINDX-ODT V1.5)

(BASE

(010711W1.11181) (bare machino)

Motorola MMME1478-1

(ACssoso) (bare machino) machine)

Motorola MMME167 (68040)

(bare mechine)

Same as Hoet

Same Hoat

Same as Hoat

VENDOR, COMPILER

\& CERTIFICATE *

HOST
MACHINE \& (OS)

Siomona Nixdort MOX3001

(under SINDX Veralon V5.41)

Simene Nbxdort

gyeme $A$ G

Ade (SIND) VA.1

(†PEO325/1.11249)

Velldeted by Rogitatration

Stomens Nixdorf Stomens Nixdort WX200 \&

informetion- MOXSOOI (Under SINOX Version

oydermo 10 5.41)

Ade (SIND) VA.1

(BASE

- Dec032511.11249)

\begin{tabular}{|c|c|c|}
\hline 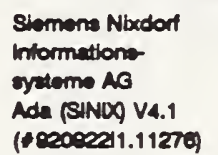 & $\begin{array}{l}\text { Shomen Nixdort RMoco } \\
\text { (Under SINIX Vorrion V5.41) }\end{array}$ & Sam en Hout \\
\hline Wallidelod by Roglext & & \\
\hline 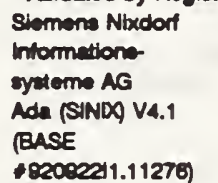 & $\begin{array}{l}\text { Stomone Nbxdorf Fu4400 } \\
\text { (under SINDX Voriton V5.41) }\end{array}$ & Samo as Hod \\
\hline
\end{tabular}

Motorda MVME165 (MC88040)

Motorola MVME165 (MC88040)
(bare machine)

PORCE CPU-40 (MCOs040) (bare

silcon Graphics
Computer Syatems
4D ADA 3.0
$(1000703 W 1.11014)$

Iria-40/380 (under IRIX

Same at Hoet

Rolve: 40-3.3)

Sulcon Graphica
Computer Syctems
4D ADA 3.0
(\$OOOTOSW1.11015)

(100070sW1.1101

Computer Systems

$4 D$ ADA 3.0

(\$000703W1.11016)

Silicon Graphice,

ine.

VADS SGHrbx,

SC4-ADA-4.0,

Veraion 6.1

(\$010020W1.11203)

-Valldeted by Rogitutation

silicon Graphice,

IFis indigo, Personal IRIS

ine.

4D, IFIS 4D seriee of

Irib-4D/25 (under IRAX

Roleace 40-3.3)

Ito-40/220s (under IROX

Rateev 40-3.3)

Same as Hoot

VADS SGHItic,

computere (under $\mathrm{k}$ bx V4.0)

SCA-ADA-4.0,

Veraion 6.1

(BASE

1010020 W1.11203)

Sulcon Graphice,

ine.

SGI Indigo (under Irix

V4.0)

TARGET

MACHINE \& (OS)

Eech Hoet, eelf targeted

Same Hoat

Same as Hoet

\author{
sent
}

Same Host

VADS SCHIIX,

SCA-ADA-4.0,

Verelon 6.1

(\$010020W1.11204)

SKY Computers,

inc.

2.33)

Moridien Ade,

Veralon 4.1

(\$010711W1.11183)

SKY Computers,

Meridien Ada,

Veraion 4.1

(\$010711W1.11185) (under Irtx Syatem V 3.3)

SGI 40/440 (under Irbx v3.3)

Same Hout

Srrbolt 8116-V (under smbolt bernel vertion

SPARCutation 1 (under SunOS releas 4.1)

SKYatation 8117P (under SKYtation lemel verdion 2.33)
SGI Parsonal Iris W-ADes 
Ada PROCESSORS, Continued

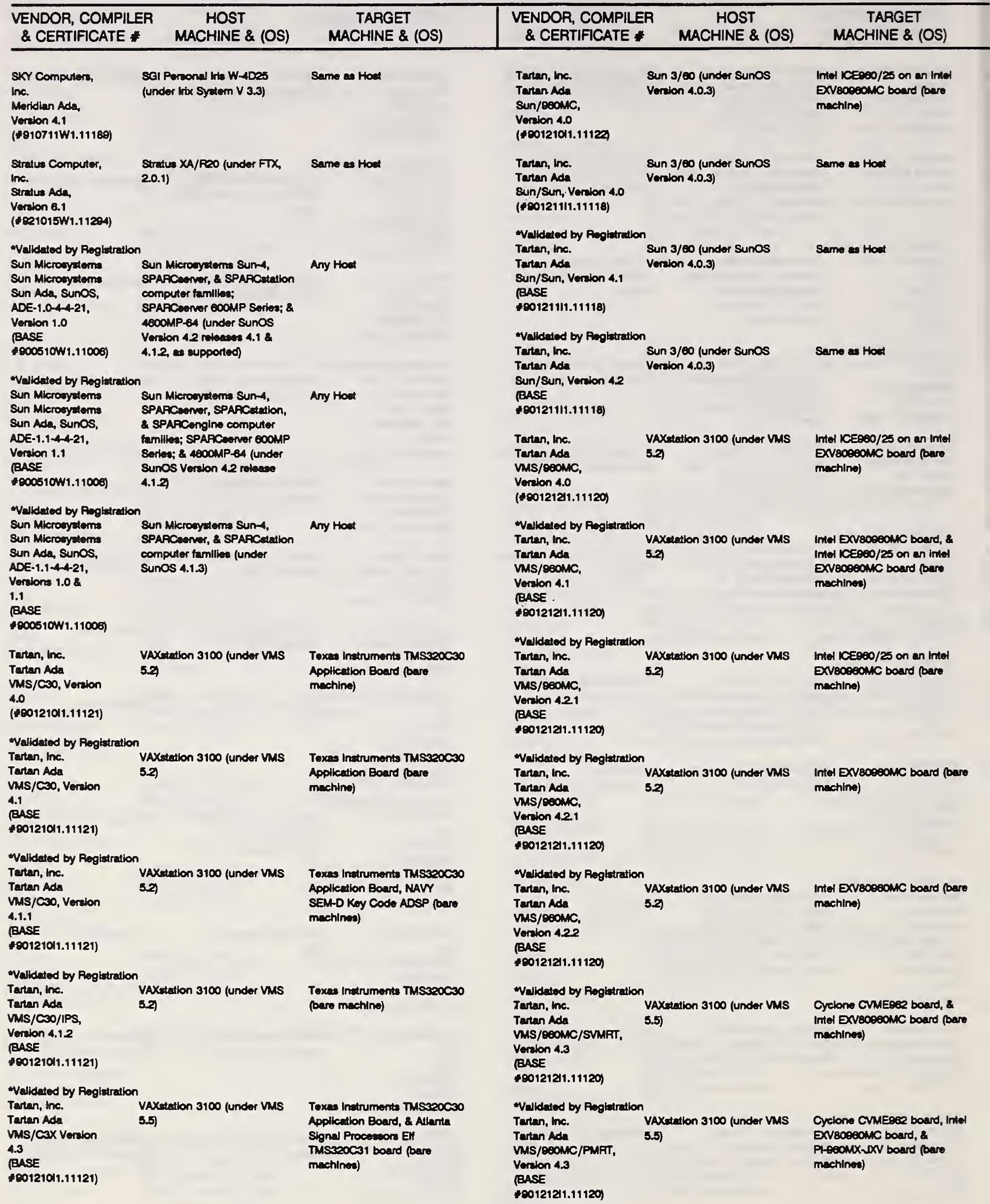




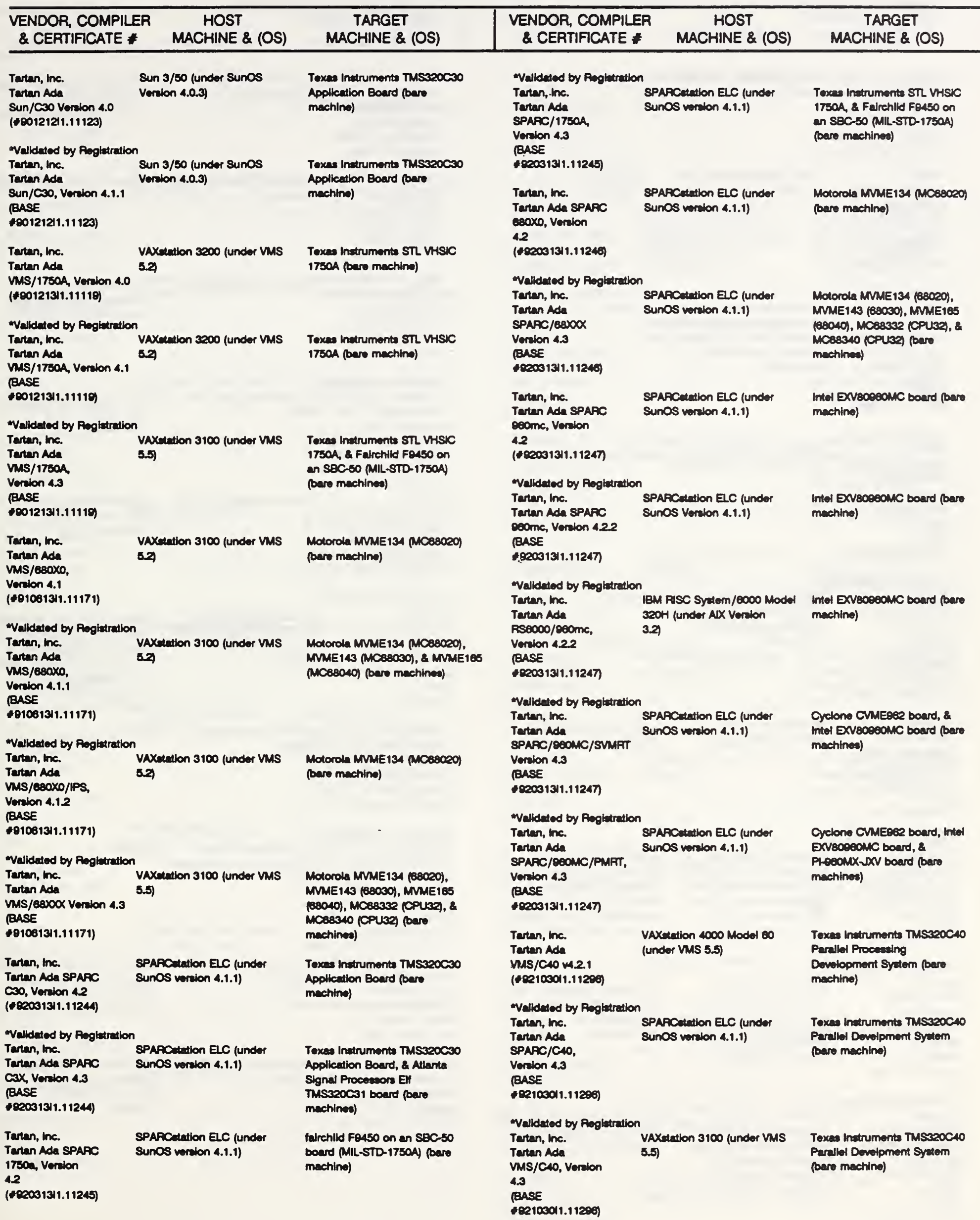




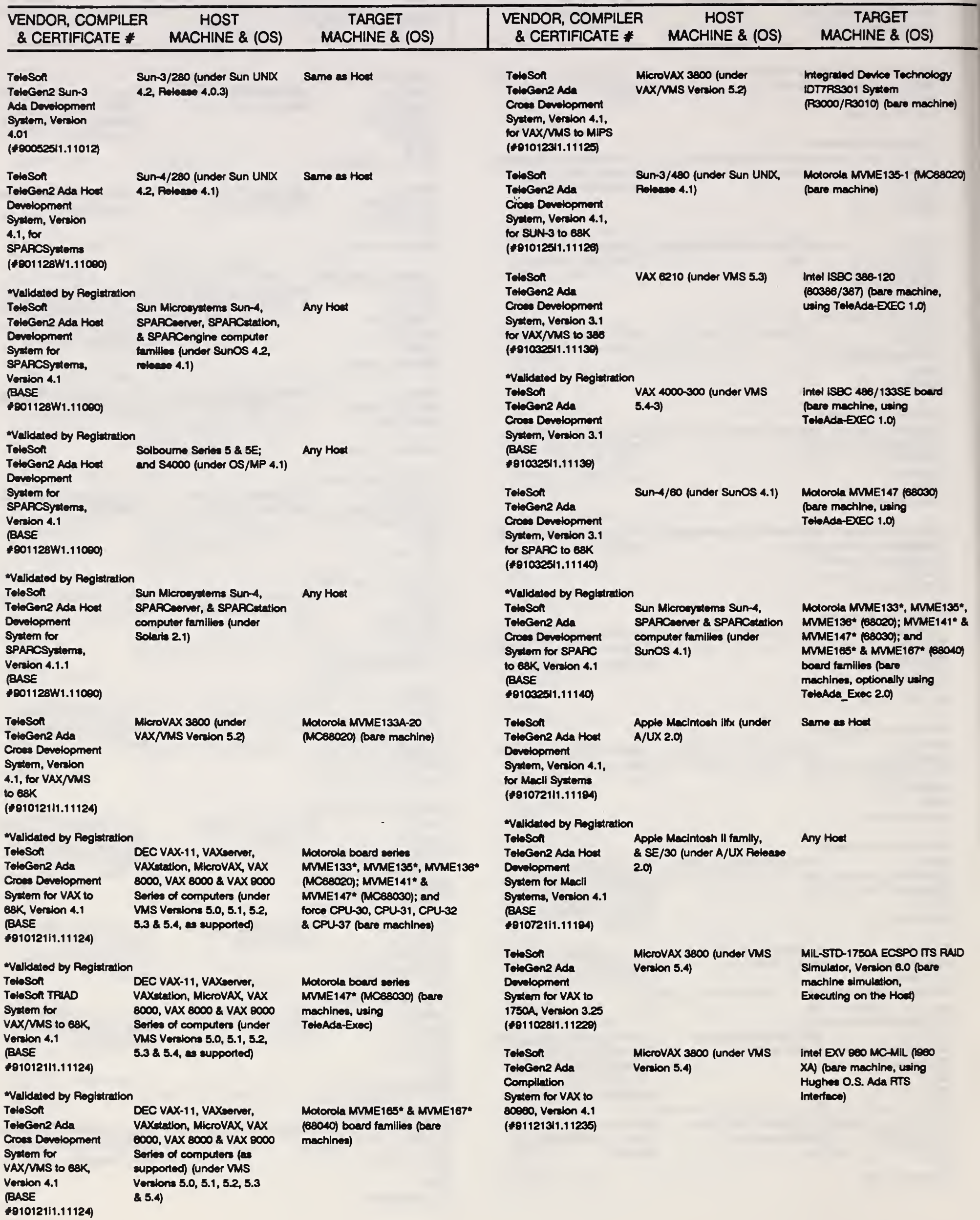




\begin{tabular}{ccc|ccc}
\hline $\begin{array}{c}\text { VENDOR, COMPILER } \\
\text { \& CERTIFICATE * }\end{array}$ & HOST & TARGET & VENDOR, COMPILER & HOST \\
MACHINE \& (OS) & MACHINE \& (OS) & CERTIFICATE * & MACHINE \& (OS) & MACHINE \& (OS)
\end{tabular}

\section{Toleson}

Telecon2 Ada

Croes Development

Syatem Veraton 4.1.1

for SUN-4 to AMIPS

(482102911.11205)

Toloson

TeleGen2(im) Ade

Croes Dowlopment

Syetem for Sun-4

10 $\mathrm{beO}$, Veriton 4.1.1

(\$2212181.11303)

Telesont

TeleGen2(im) Ada

Croes Dowolopment

Sytem for Sun-4

to cesk, Veriton $4.1 \mathrm{C}$

(*8212181.11304)

Toxes indruments

MIPS-Ade, Verion 3.0

(\$001030W1.11052)

Texas Inatruments

7 Ada, Verion

1.0

(*910403W1.11135)

TD Syctems, Ld.

TD

SUn-4/MIL-STD-175

OA Ada Complls

System, Verion

2.8 .0

(4820318W1.11237)

TDD Syeteme, Ltd.

TRD AN/MN Ada

Compller Syatem,

Veraion 2.0.0

(*820310W1.11238)

TrD Syctems, Lid.

\section{TD}

SUn-4/MIL-STD-175

QA Ada Compllor

Syatem, Veriolon

2.8 .0

(\$820318w1.11239)

Th Syetems, Lid.

ח

SUn-4/MIL-STD-175

OA Ada Compller

Syctem, Verdon 2.9 .0

(\$820310W1.11240)

TRD Syatems, Ld.

TRD

PISC6000/MIL-STD

17504 Ade

Compiler Syatem,

Verion $\mathbf{2 . 8 . 0}$

(\$820310W1.11241)

Vallated by Rogiatration

TRD Sydems, Lid.

TDD

RISC6000/MIL-STD

17504 Ada

Compiler Syatem,

Veraion 2.0 .0

BASE

$\$ 020318 W 1.11241$ )
Sun-4/600 (under SunOS

Rolease 4.12)

Integrated Device Technology

IDT7AS301 Syetem

(R3000/R3010) (bare mechine)

Sun-4/690 (under SunOS

Roloes 4.12

CVMEere Syatem (1800XA board with MC Procesecor) (bare mechine)

Sun-4/600 (under SunOS Rolesen 4.12

Motorole MMME1478-1 (38030/88882) (bare mechino)

MIPS M/2000 (under PISC/0 TI DP32 R3000 Procesco $4 . \infty$

MicroVAX 3400 (under VMS 5.3-1)

RTE Vorion 1.0

T1 DP32 R3000 Processor

(bare mechine, uaing TT

Executive and Runtime

Sorvices (EARS) Verion 1.0)

Sun-4/75 (under SunOS,

Verion 4.1.1)

Rockowell international

Ri-1750AB Brasaboard

Devolopment Syctem (bare

mechine, using Tortx Rea

Time Executho, Veraion

1.0.0)

Data Goneral MN/32 20000-2

(under AOS/NS Ii, Verion

2.03)

Sun-4/75 (under SunOS,

Voralon 4.1.1)

tonoymell Program

Dovolopment UnH (PDU) with

Honeywell Generic VHSIC

Spacebome Computer (GVSC)

MIL-STD-1750A pare mechine,

ualng TLPtx Real Time

Executive, Verion 1.0.0

Sun-4/75 (under SunOs,

Veraion 4.1.1)

TL MIL-STD-1750A Muiliple

Procesecor Simuletor pare machine imulation, ueing

TrDitx Roal Time Exocutive,

Venton 1.0.0, and executing on the Hot

IBM RISC Sytem 6000, Model TLDmpe MIL-STD-1750A 630 (under ADX, Venton 3.1)

Multiplo Proceseco Simulato (bere mechine simulation, using TLDibx Rod Time

Executive, Veraton 1.0.0, and executing on the Hodt

IBM RISC Syetem 6000 sories (under AIX, Veriton 3.1)
IBM Uner Consolo with IBM Generic VHSIC Speceborne Computer (bare mechine, using Trotx Rod Time Execution, Veraton 1.0.0)
TR Syptems, Ld.

TD .

VAX/MIL-STD-17SOA

MileroVAX 3500 (under VMS, Version 5.1)

Ada Compllor

Syctem, Veraion

2.0 .0

(\$820310W1.11242)

-Velideded by Reglatration

TRD Sytems, Lid.

TD

VAX/MUL-STD-1750A

Ada Complier

Sytem, Verion 2.0 .0

(BASE

$1020310 W 1.11244$

TD Syctems, Ld.

TRD HP

$\infty 000 /$ MIL-STL-1750

A Ada Complier

System, Verion

2.0.0

(1020310W1.11243)

TRD Sytems, LId.

TRD Comanch

VAX/MIL-STD-1750A

Ada Compller

Syctem, Vereton

3.4

(\$031012W1.11329)

U.S. Nr Force

AFCAS 17504 Ada

Compiler, Veriton

1.0

(\$010425W1.11142)

-Valdated by Rogiatration

U.S. Ar Force

AFCAS 1750A Ade

Complier, Veralon

1.1

(BASE

010425W1.11142

U.S. Air Force

AFCAS 1750A/XMEM

Ada Compller,

Verelon 1.0

(\%010125W1.11143)

VAxutation 4000 Model 60 (under VMS 5.5)

VAXetation 3100 (Under VMS Veraton 5.3)

Valideted by Rogitatration

U.S. Ar Force AFCAS 1750A/XMEM

Ada Compller,

Veraion 1.1

(BASE

$1010425 W 1.11143$

U.S. NAWY

AdaVAX, Veraion

5.0 VOPTIMIZE)

(\$010517S1.11162)

U.S. NAVY

AdeVAX, Vercion

5.0 (/NO OPTIMIZE)

(\$81051751.11163)

$\because$

U.S. NAVY

AdeVAX, Veralon

5.0 VOPTIMIZE)

(101051751.11164)

U.S. NAYY

AdaVAX, Voralon

5.0 (NNO_OPTMIIEE)

(\$81051751.11165)

DEC VAX-11, VAXeonver,

DAxtation, MieroVAX, VAX

(6000, VAX 6000,8

VNX 0000 Series of

computere (under VMS 5.4)

HP $0000 / 350$ (under MP-UX, Veraion 7.9

VMS Voraton 5.4)

Verion 5.3 )

VMS Verton 5.4)

VAX 8600 (under VMS Verilon 5.3)

VAX 8000 (under VMS Veraton 5.3)

VAX-11/785 (under VMS Veraion 5.3)

VAX-11/785 (under VMS

Veraton 5.3)
TL MiL-STD-1750A Multiple

Proceseor Simulator pare

machine imulation, using

TrDrx Roal Thmo Executhe,

Verion 1.0.0, and executing

on the Hoed

IBM Uner Console with IBM Generic VHSIC Spaceborne

Computer peare mechino.

uaing Thortx Real Time

Execution, Vorsion 1.0.0)

TLDmpe MIL-STD-1750A

Multiple Procesen Si Simulator pere machino simulation, uving TLDitx Raal Time Executhe, Version 1.0.0 and executing on the Hoet!

TD MIL-STD-1750A Muitiple Procescer Simulator (TLmpe), executing on the Hod foare mechine Imulation, using TRD Roal Time Executive (TDrta). 3.4.C)

Ar Force RAD MIL-STD-1750A inulator (bare mechine ilmulation, executing on the Hoet)

Nir Force RAD MIL-STD-1750A Amuletor Bare machine imulation, executing on the Hoat)

Ni Force RAD MiL-STD-1750A imulator (bare mechine imulation, executing on the Hoet:

Ai Force RAD MiL-STD-1750A inulator pare mechine imulation, executing on the Hod)

Same as Hoet

Same es Hod 
Ada PROCESSORS, Continued

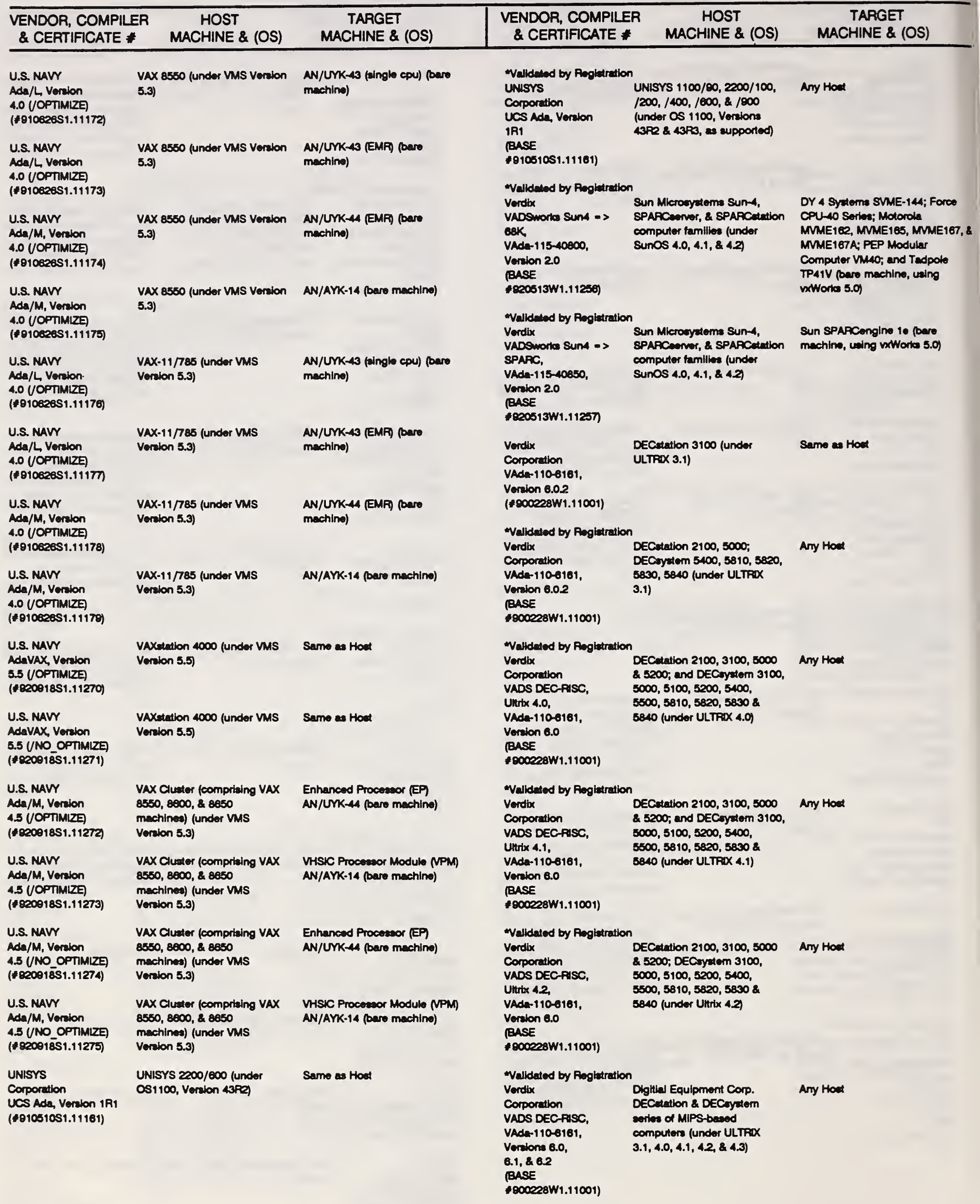


Ada PROCESSORS, Continued

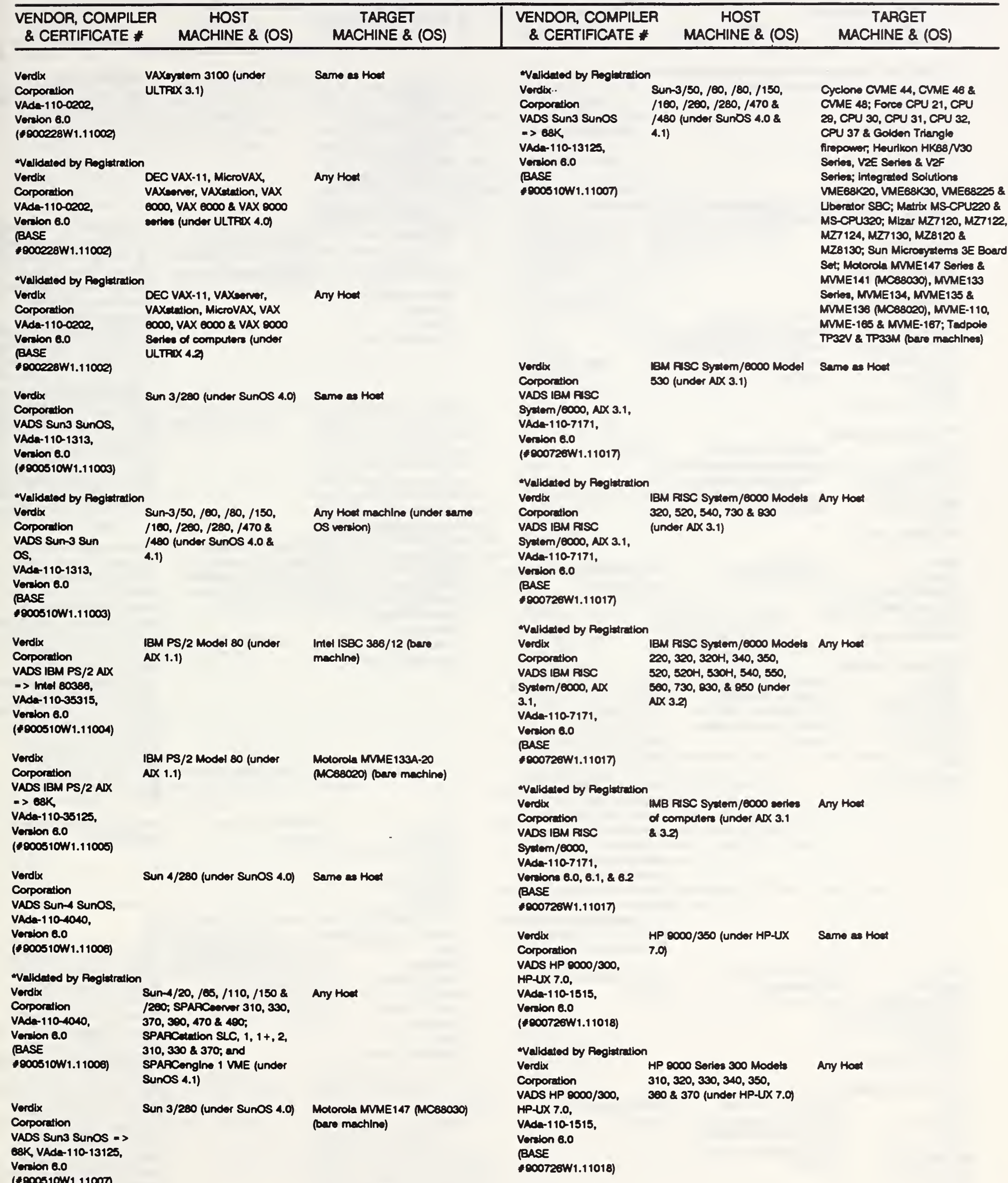

(\$500s10w1.11007) 
Ada PROCESSORS, Continued

\begin{tabular}{|c|c|c|c|c|c|}
\hline $\begin{array}{l}\text { VENDOR, COMPILE } \\
\text { \& CERTIFICATE * }\end{array}$ & $\begin{array}{c}\text { HOST } \\
\text { MACHINE \& (OS) }\end{array}$ & $\begin{array}{c}\text { TARGET } \\
\text { MACHINE \& (OS) }\end{array}$ & $\begin{array}{l}\text { VENDOR, COMPILER } \\
\text { \& CERTIFICATE * }\end{array}$ & $\begin{array}{c}\text { HOST } \\
\text { MACHINE \& (OS) }\end{array}$ & $\begin{array}{c}\text { TARGET } \\
\text { MACHINE \& (OS) }\end{array}$ \\
\hline $\begin{array}{l}\text { Vordix } \\
\text { Corporation } \\
\text { VADS Prime } \\
\text { EXL/320, UNIX } \\
\text { Syetom V/386 3.2, } \\
\text { VAda-1 10-3232, } \\
\text { Verlon 6.0 } \\
\text { (1000728W1.11018) }\end{array}$ & $\begin{array}{l}\text { Prime EXL/320 (under UNIX } \\
\text { Syatem V/386 3.2) }\end{array}$ & Same as Hoot & $\begin{array}{l}\text { Verdlix } \\
\text { Corporation } \\
\text { VADS } \\
\text { VAX/Ultrix = > 68k, } \\
\text { Ultrix 3.1, } \\
\text { VAda-110.02125, } \\
\text { Vortion 6.0 } \\
\text { (H000720W1.11023) }\end{array}$ & $\begin{array}{l}\text { MikroVAX } 3100 \text { (under Ultrix } \\
\text { 3.1) }\end{array}$ & 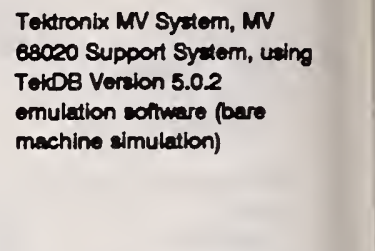 \\
\hline $\begin{array}{l}\text { Vordix } \\
\text { Corporation } \\
\text { VADS VAX/MMS 5.2, } \\
\text { VAda-110-0303, } \\
\text { Vortion } 6.0 \\
\text { (180072061.11002) } \\
\text { "Valldeted by Rogitration }\end{array}$ & $\begin{array}{l}\text { MicroVAX } 3100 \text { (under } \\
\text { VAX/NMS V5.2) }\end{array}$ & Same is Hoat & 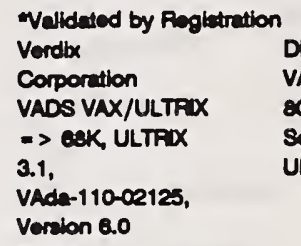 & $\begin{array}{l}\text { DEC VAX-11, VAXeonver, } \\
\text { VAXtation, MieroVAX, VAX } \\
8000, \text { VAX } 8000 \text { \& VAX } 8000 \\
\text { Sories of computers (under } \\
\text { Utirix 3.1) }\end{array}$ & $\begin{array}{l}\text { Cyclone CVME 44, CVME } 48 \& \\
\text { CVME 48; Force CPU 21, CPU } \\
29, \text { CPU 30, CPU 31, CPU } 32 \text {, } \\
\text { CPU } 37 \text { \& Golden Trlangle } \\
\text { firepower, Hourikon HK88/V30 } \\
\text { Sorles, VZE Sorles \& VZF } \\
\text { Serbes; Intograted Solutions }\end{array}$ \\
\hline $\begin{array}{l}\text { Vordlix } \\
\text { Corporation } \\
\text { VADS VAX/MMS 5.3, } \\
\text { VAda-110-0303, } \\
\text { Vorion } 6.0 \\
\text { (BASE } \\
\text { I800726W1.11020) }\end{array}$ & 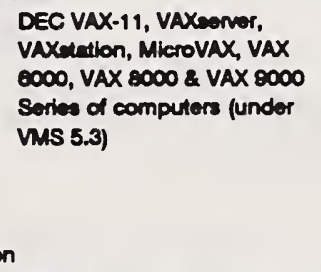 & Any Hoot & $\begin{array}{l}\text { (BASE } \\
\text { PCOOT2EW1.11023) }\end{array}$ & & 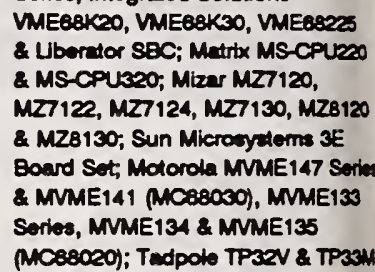 \\
\hline $\begin{array}{l}\text { Vordix } \\
\text { Corporation } \\
\text { VADS VAX/MMS 5.2, } \\
\text { VAda-110.0303, } \\
\text { Vordons } 8.0 \& 8.2\end{array}$ & 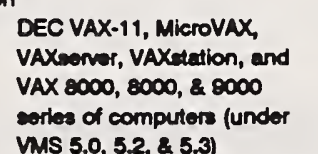 & Any thoed & & & 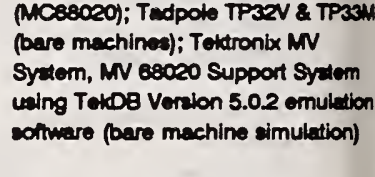 \\
\hline
\end{tabular}

Verilons $6.0 \& 8.2$ VMS 5.0, 5.2, \& 5.3)

(BASE

1000720W1.11020)

Verdbx

Corporation

MicroVAX 3100 (under Motorola MMME147 (MC88030)

VADS VAX/MMS $=>68 k$

(bare mechine)

Velldated by Regletration

Verdbx

Corporation

VADS

DEC VAX-11, VAXsenver, $\begin{array}{ll}\text { VADS } & 6000, \text { VAX } 8000,2 \text { VAX } 8000 \\ \text { VNX/ULTRXX }=>68 K, & \text { Sories of computers (under }\end{array}$

ULTRAX 3.1,

Vereion 6.0

(\$000726W1.11021)

Valdated by Rogitation

VAde-110-02125,

Uitrib 4.0, 4.1, \& 4.2)

Vercion 6.0

(BASE

1000720W1.11020)

Verdix

Corporation

VADS VAXMMS $=>$

eaK VMS 5.2 ,

VAda-110-03125,

DEC VAX-11, VAXserver,

VAXatation, MicroVAX, VAX

8000 , VAX 8000 \& VAX 8000

Sories of computers (under VMS 5.4

Verion 8.0

(BASE

1000720W1.11021)

Cyclone CVME 44, CVME 46 \& CVME 48; Force CPU 21, CPU 29, CPU 30, CPU 31, CPU32, CPU 37 \& Colden Triangle firepower, Heurlkon HKB8/N30 Sories, VZE Sorios a VZF Series; Integrated Solutions VMEQ8K2O, VME68K30, VME68225 \&. Uberator SBC; Matrix MS-CPU2ZO \& MS-CPU320; Mizar MZ7120, MZ7122, MZ7124, MZ7130, MZ8120 \& MZ8130; Sun Microsydems 3E Board Set; Motorola MNME147 Series \& MVME141 (MC88030), MMME133 Seriee, MVME134, MVME135 \& MVME138 (MC88020), MVME-165 \& MMME187; Tedpole TP32V \& TP33M (bare machines)

Intel ISBC 386/32 (bare machine)

\section{VAXMMS V5.2}

Corporation

VADS VAX/MMS $=>$ intel

388, VMS 5.2 ,

VAda-110-03315

Verion 6.0

(1000720W1.11022)

Walldatod by Ragiatration

Verdbx

DEC VAX-11, VAXserver,

Corporation

VADS

VAX MMS $=>$ Intel

386, VMS 5.3,

VAde-110-03315,

Version 8.0

(BASE

1000720W1.11022)
VAXetation, MicroVAX, VAX 8000 , VAX $8000 \&$ VAX 8000 Series of computers (under VMS 5.3) Intel ISEC 386/32 pare machino)
Verdbx

Corporation

VADS DEC-FISK $=>68 \mathrm{~K}$

Ultrix 3.1,

VAde-110-61125,

Veraion 6.0

(1000726W1.11024)

Walldated by Rogietration

Vordbx

Corporation

VADS DECAISC $=>$

C:K, Uitrix 4.0,

VAda-110-61125

Verion 6.0

(EASE

$1000720 W 1.11024$
DECutation 2100,3100, 5000 2.5200; and DECoystem 3100 , $5000,5100,5200,5400$, $5500,5810,5820,5830$ \& 5840 (under ULTRaX 4.0)
DECatation 3100 (under Utirtx 3.1)

Cyclone CVME 48; Force CPU 21, CPU 29, CPU 30, CPU 31, CPU 32, CPU 37; Hourlikon HKB8/NZFb Series, HKB8/N3O Sorles, \& HKB8/V3E Sorlos; Matrix MS-CPUZ2O, MS-CPU320, 2. MS-CPU330; Mizar MZ7122, $M Z 7124, M Z 7130, M Z 8120, \&$ MZ8130; Motorola MVME133 Sorles, MMME134, MMME135, 8 MVME147 Series; Radetone CPU-2A; SBE VCOM-24; Tedpole TP32V; and Tektronix MN Syztem, MN 68020 Support Syztem using TekDB Verion 5.0 .2 emulation colmare (bare machine simulation) (bare mechinea)

Motorola MMME147 (MCssoso) (bere machino)

Cycione CVME 44, CVME 46 \& CVME 48; Force CPU 21, CPU 29, CPU 30, CPU 31, CPU 32, CPU 37 \& Golden Triangle firepowor, Heurikon HKB8/NsO Sorber, VZE Sorlee \& VZF Sorles; Integrated Solutions VME68K20, VME68K30, VME68225 2 Lberator SBC; Matrix MS-CPU220 \& MS-CPU320; Mizar MZ7 120, MZ7122, MZ7124,

MZ7 130, MZ8120 \& MZ8130; Sun

Microsystems 3E Board Set; Motorole MMME147 Sorios (MC88030), MMME133 Sorles, MMME134 \& MMME135 (MC88020): Tedpole TP32V \& TP33M pare machinee) 


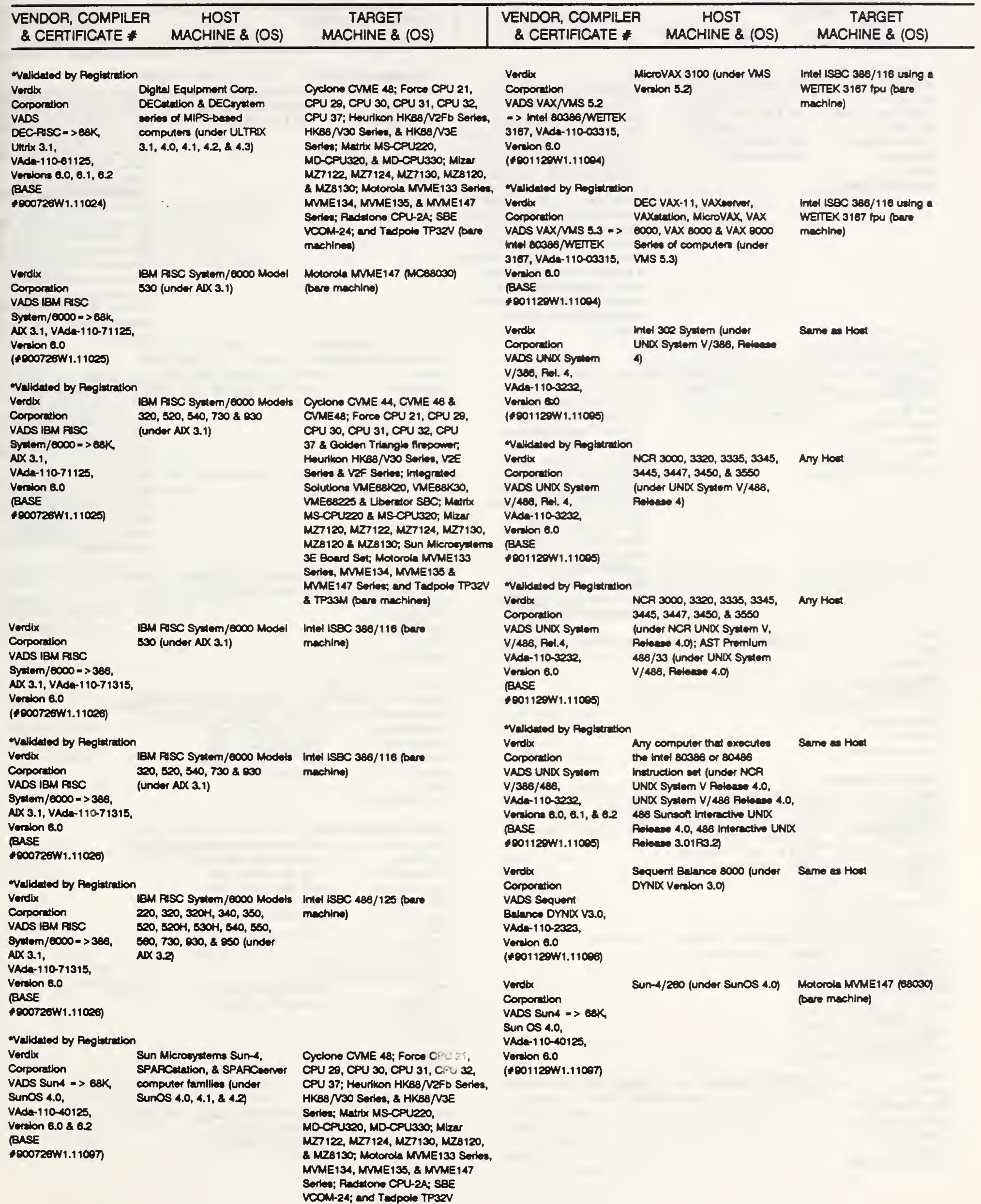




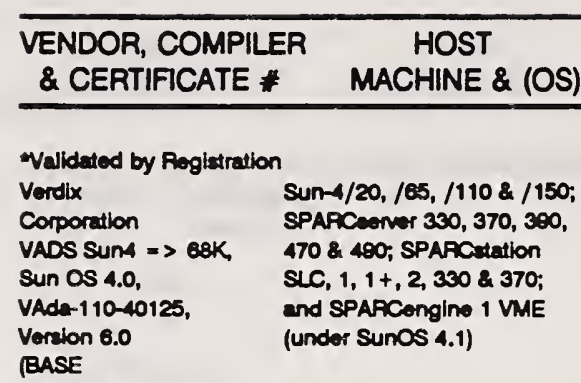

1801120W1.11097)

\begin{tabular}{|l|l}
\hline TARGET & VENDOR, COMPILER \\
\hline
\end{tabular}
MACHINE \& (OS) \& CERTIFICATE *

HOST MACHINE \& (OS)
TARGET

MACHINE \& (OS)

Welldated by Rogitration

Cyelone CVME 44, CVME 46 \& CVME 48; Fores CPU 21, CPU 29, CPU 30, CPU 31, CPU 32 , Hourikon HKB8/V3O Sorles, V2E Sorles a Vef Serles; Integrated Solutions VME68K20, VME68K 30 , VME68225 a Uberator SBC; Matrix MS-CPUE20 \& MS-CPU320; MI2ar MZ7120, MZ7122, MZ7 124, MZ7130, MZ8120 \& MZ8130; Sun Microoystems $3 E$ Board Set; Motorola MMME1 10 (MC88000), MMME133 Sorios, MMME134, MMME135 2. MMME138 (MC88020), MMME147 Sories \& MMME141 (MC88030), MMME-165 \& MMME-167 (MC88040); Tedpole TP32V \& TP33M (bare mechines)

Validated by Roglatration
Verdix
Corporation
VADS SUMA = $88 \mathrm{~K}$
SUn OS 4.1 ,
VAda-110-40125,
Verion 8.0
(BASE
1801120W1.11007

Sun Microsydems Sun-4, 2 SPARCengine computer families (under SunOS 4.1)

$\$ 1001120 W 1.11007$

Cyclone CVME 44, 46, \& 48; force CPU 21, CPU $29, \mathrm{CPU}$ 30, CPU 31, CPU 32, CPU 37. \& Golden Triangle Flrepower, Heurikon HKB8/N2E Sorles, N2F Serles, \& N30 Serles; integrated Solutions VMEE8K20, 68K30, 68225, \&

\author{
Verdibx \\ 4.0) \\ Corporation \\ VADS Sun-4 => \\ sum-3, sun os \\ 4.0, \\ VAda-1 10-4013, \\ Verion 8.0
}

(\$001120W1.11008)

- Valdated by Rogitration

Verdlx

Corporation

VADS SUn-4 =>

Sun-3, Sun OS

4.0,

VAda-110-4013,

Verion 6.0

(BASE

1001129W1.11008)

Verdix

Corporation

VADS ATET

382/600G UND

Syctem V, Roloase

3.22 ,

VAda-110-5151,

Verion 8.0

(4801129W1.11099)

Verdlx

Corporation

VADS HP- $6000 / 300$

= $>88 \mathrm{~K}, \mathrm{HP}-\mathrm{UX} 7.0$

VAda-1 10-15125,

Verion 6.0

(H.01120W1.11100) CPU 37 \& Golden Triangle firepower,
Verdix .

Corporation

VADS HP- $+000 / 300$

7.0,

YAde-110-15125,

Veraion 6.0

(BASE

$\$ 001120 W 1.11100$ )
$\Rightarrow$ 88K, HP-UX

HP 9000 Sories 300 Models $310,320,330,340,350$,

$360 \& 370$ (under HP-UX 7.0) SPARCerver, SPARCetation, Uberator SBC; Matrix MS-CPU220 \& MS-CPU320; Mizar MZ7120, MZ7122, MZ7124, MZ7130, MZ8120, MZ8130, 8 CPU330; Motorola MMME133 Soribs, MMME134, MMME135, 2 Sun-3/260 (under SunOs

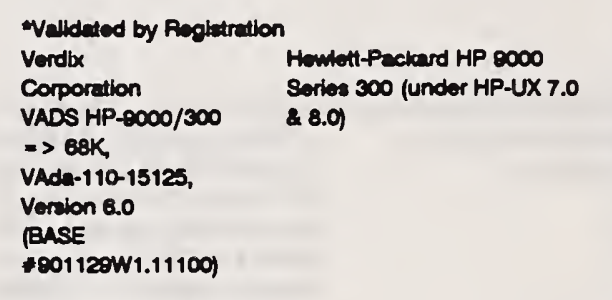

(1)
MMME147 Sories; Sun

Microystems 3E board set; and Tedpole Technology TP32V a TP33M (bare mechines)

Sun-4/260 (under SunOS 4.0)

Sun- $4 / 20, / 65, / 110, / 150$, /200 \& /280; SPARCoorver $330,370,390,470 \& 400$; SPARCatation SLC, $1,1+, 2$, $330 \& 370$; and SPARCengine 1 VME (under SunOs 4.1)

Sun $3 / 50, / 160, / 80, / 150$, $/ 180, / 280, / 280, / 470 \&$

/480 (under SunOS 4.1)

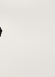

Verdix

Corporation

VAOS BCS/88K

AVilon DGUX 4.3,

VAde-110-8080,

Version 6.1

(\$001129W1.11101)

Validated by Rogituration

Sytem V, Rolase (under UNI

Same eas Hot

HP $\$ 000$ Modal 350 (under HP-UX 7.0)
Motorda MMME133A (88020) (bare mechino)
Data General AVION Model 5120 (under DG/UX 4.3)
VAda-110-8080,

Cyclone CVME 44, CVME 48 \& CVME 48; Fores CPU 21, CPU 29, CPU 30, CPU 31, CPU 32, CPU 37 \& Golden Triangle firepower, Hourikon HKB8/V3O Series, VZE Sories \& VZF Sories; Imtegrated Solution VME68K20, VME68K30, VME68225 2. Lberator SBC; Matrix MS-CPU220 \& MS-CPU320; Mizar MZ7120, MZ7122, MZ7124, MZ7130, MZ8120 \& MZ8130; Sun Microsyctems 3E Board Set; Motorola MMME147 Sories (MC88030), MMME133 Series, MMME134 \& MMME135 (MC88020); Tedpole TP32V \& TP33M pare machines)

Cyclone CVME 48; Force CPU 21, CPU 29, CPU 30, CPU 31, CPU 32, CPU 37; Hourikon HKB8/VZFb Sorles, HKB8/N30 Sorles, 2 HK68/V3E Sories; Matrix MS-CPUE20, MS-CPUizo, \& MS-CPU330; Mizar MZ7122, MZ7124, MZ7130, MZ8120, \& MZ8130; Motorda MMME133 Sories, MMME134, MMME135, \& MMME147 Sorles; Radatone CPU-2A; SBE VCOM-24; and Tedpole TP32V

Same es Hot
Verdbx

Corporation

VADS BCS/88K

AVilon DGUX 4.3,

VAda-110-8000,

Verion 6.1

(BASE

\$01120W1.11101)

-Validated by Rogiatration

Verdbx

Corporation

VADS BCS/88K

AVion DGUX 5.4,

VAda-110-8080,

Vertion 6.1

(BASE

\$901129W1.11101)

Velidated by Reglatration

Verdbx

Corporation

VADS BCS/88K

Veralon 6.1

(BASE

DG AVION Modets 4000 , $4000 \mathrm{GH}, 4020,4100,4120$, $5010,5200,5220,5240$, $5300,5310,5400,5402$, $5410,5412,6200 \& 6220$ (Under DG/UX 4.3)

+801120W1.11101)

Validated by Regiatration Verdix Corporation VADS ECS/88K VAde-110-8000, Vercion 6.1

(BASE

+001120W1.11101)
Data General AVION Modets $4000,4000 \mathrm{GH}, 4020,4100$, $4120,5010,5200,5220$, $5240,5300,5310,5400$, $5402,5410,5412,6200$ \& 6220; MODCOMP Rod Star family (under DG/UX 5.4)

MODCOMP Roal Star Family Any Hot (under REAL/OX C.0.2)
Any Hox
Motorda 8000 Della Series Amy Hot (MC88000), al modets (under Unix Syctem V/88, R32v3)
Any Hoet 


\begin{tabular}{ccc|ccc}
\hline $\begin{array}{c}\text { VENDOR, COMPILER } \\
\text { \& CERTIFICATE * }\end{array}$ & HOST & TARGET & VENDOR, COMPILER & HOST & TARGET \\
MACHINE \& (OS) & MACHINE \& (OS) & CERTIFICATE * & MACHINE \& (OS) & MACHINE \& (OS) \\
\hline
\end{tabular}

\section{Verdlx}

Corporation

VADS SUnA $=$ ?

SPARC, SUn OS 4.1

VAda-1 10-40440,

Veralon 6.0

(\$001120\%1.11102)

Velldatod by Rogiatration

Verdbx

Corporation

VADS SUnA $=>$ SPARC

Sun OS 4.1, 1200; SPARCom 330, 370

300,470 4 490; and

SPARCutation SLC, 1, $1+, 2$,

$330 \& 370$ (under SunOS 4.1)

Voriton 6.0

BASE

$\$ 801129 \% 1.11102$

Validated by Rogiatration

Vordix

Corporetion

Sun Microoyitom Sund

VADS SUnA = > SPARC

Sun OS4.1,

BPARCution, \& SPARCan

computer tamillos (under

sunos 4.0, 4.1, \& 4?

VAda-110-40140,

Veraton 6.086 .1

(BASE

$\$ 001129 W 1.11109$

Verdb

Corporation

Sun 3/260 (under Sunos

Potoeses 4.0)

Motorola MMME1es (88040)

(bare machino)

SPARCongine 1E \& renics

N-SPARC-334 bare mechinee)

68k, VAda-110-13140,

Verion 6.0

(\$810517W1.11149)

-Validated by Rogiatration

Verdix

Sun Mierooyutems Sun-3

computer femily (under

Corporation

SunOs 4.1)

68k, VAde-110-13140,

Veraton 6.0

BASE

1010517W1.11149

VADS DEC-FISC $\Rightarrow>$

Motorola AMME 165 (MC88040) (bere machine)
Verdix

Corporation

VADS Sun-4 SunOS =

68k, VAde-1 10-40140

Verion 6.0

(4010517W1.11152)

- Velldated by Rogietration

Verdbr

Sun Microsyotems Sun-4,

Corporation

VADS SunA SunOS

$\Rightarrow$ 68k

VAda-110-40140,

Veraton 6.0

BASE

$1010517 W 1.11152$

Velldated by Roglatretion

Vordbx

Sun Microoyztems Sun-4,

SPARCerver, \& SPARCatation

VADS sun-4 8unOS omputer femilies (under

$\Rightarrow 68040$,

Sunos 4.0,4.1, \& 4.2)

Verions $6.0 \& 6.2$

BASE

$010017 W 1.11152$

Verdbx SPARCoorver \& SPARCutation computer tanllies (under

sunos 4.1)

Corporation

VADS DEC-PISC $=>89 \mathrm{x}$

VAde-110-81680,

Veraion 8.1

(\$01051TW1.11153)

-Velidated by Rogletration

Verdbx

Corporation

VADS DEC-AISC $=>$

Rik VAde-110-81880

Verion 6.1

(EASE

$010017 W 1.11153$

Verdix

Corporation

VADSworto SunA $=$ >

68k, VAda-115-40800

Verion 20

(\$910517W1.11154)

Valldated by Rogletration Verdbx

Corporation

VADSurotos Sunt $=>$

88K,

VAde-115-40800,

Veraion 2.0

BASE

$010517 W 1.11154$

DEC DECatation \& DECrystem computer families (under ULTRIX 4.0

DECatation 2100 (under ULTRXX V4.0)

Sun $4 / 20$ (under SunOs

4.1.1 SPARCuenver \& SPARCestation computer families (under SunOs 4.1)
Motorola MMME165 (88040

(bare machino)

Motorole MMME185 (28090)

(bare machine)

DY 4 Sydern SVME-144; Force CPUnto Serlea/Eagle i

Motorda MMME1es, MMME167, MMME167A; PEP Moduler Computer VM40; and Tedpole TPA1V

Motorole MMME181 (bare mechine)

Motorola MMME181 (88000) (bere mechine)

Verdbx

Corporation

DECertition 5000-200 (under

MIPS R3000,

VAde-110-81620

Veraion 8.1

$(\$ 010517 W 1.11150)$

- Valideted by Rogistration

Verdbx

Corporation

MiPS R3000,

VAda-110-61620

Veralon 6.1

(BASE

$\$ \theta 10517 W 1.11150$

Vordix Corporation MicroVAX 3600 (under VMS VADS VIMS $\Rightarrow$ MIPS

Intograted Device Technology R3000, VAda-1 10-03620,

Verion 0.1

(*910517W1.11151)

- Valldated by Roglatration

Vordbx

DEC DECatation \& DECoytem computer families (under ULTRIX 4.0)

Loctheed Sanders STAR MMP (R3000) (bare machino)

Loctineed Sander STAR MMP (R3000) (bare machine)

Integraded Device Technology IDT7RS302 (bare mechine)

CADS VMS $\Rightarrow$ MiPS 6000 , VAX 8000 \& VAX 8000

R3000, VAda-110-03620, Sorles of computers (under

Veraion 8.1

VMS 5.3)

(910517W1.11151)

\section{IDTRSSOS (pere machino) V(2)}




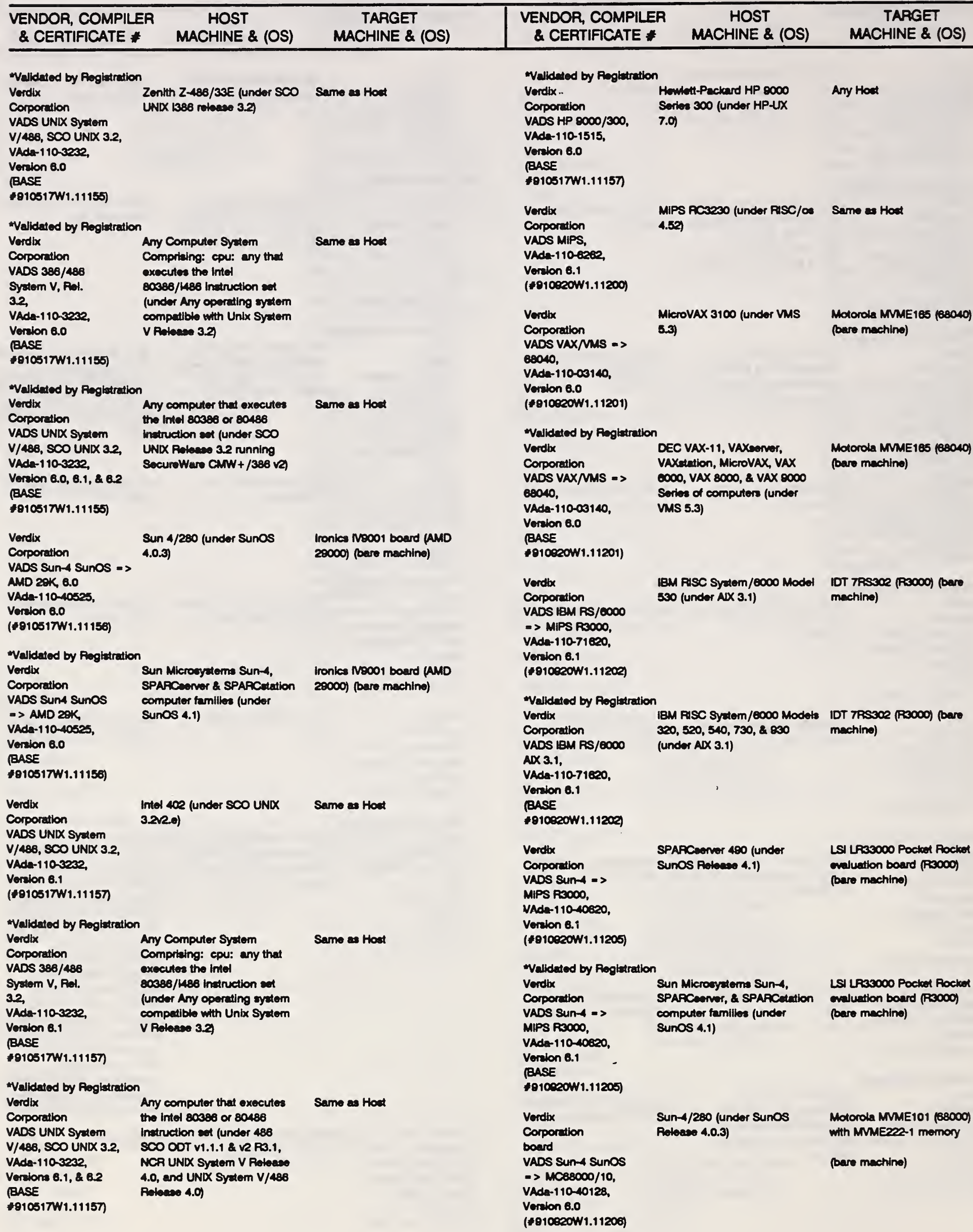


Valldeted by Aogiatration

Vordix Corporation

VADS SUnA $\Rightarrow$

Sun Mleroyyurms Sun-4, SPARCoonver, \& SPARCetation whth MVME222-1 mornory board MCs8000/10, VAda-110-40128, computer tamilies (under

Verelon 8.0 SunOs 4.1

(BASE

1010020W1.11208)

-Valdeted by Pogigatration

Vordix Corporation Sun Mlerooyntoms Sum-d,

VADS Sund $\Rightarrow \quad$ SPARCwerwer, SPARCitation,

MCB8000/10, SunOS \& SPARCongino computer

Voraton 8.0

tamlliee (under Sunos 4.1)

(BASE

(010020W1.11208)

Verdlu Corporetion Sund/280 (under SunOS

VADS SUn-4 SunOS $\Rightarrow>$ Reloeme 4.0.3)

CPL32, VAd -110-40150.

Veralon 8.0

(\$010820w1.11207)

Valldetod by Rogiatration

Vordic

Sun Microoyntoms Sun-d,

Corporation SPARCener, \& SPARCetention

VADS Sun-4 SunOS $\Rightarrow$ computer tamilles (under

CPU32, VAda-110-40150, SunOS 4.1)

Veraion 8.0

(BASE

\$010020W1.11207

Valideted by Roglatration

Vordle Sun Mlerooyntems Sun-4, Corporation SPARCoonver, SPARCutation,

VADS Sun-4 SunOS $=>$ \& SPARCongine computer CPU32, VAda-110-40150, tamillion (under SunOS 4.1) Veralon 8.0

(BASE

$\$ 010020$ W1.11207)

Vordbx

IBM PS/2 Model 80 (under

Corporation $A X 1.1)$

VADS IBM PS/2, ADX 1.1,

VAde-110-3535, Voration 6.1

(\$010020W1.11208)

Vordbx

Corporation

MIPS RC3230 (under RISC/0

VADS MIPS $=>$ MAPS

R3000, VAde-110-62620,

Verion 6.1

(\$010820W1.11200)

Verdid

Corporation

VADS Sum-3 SunOS

$\Rightarrow>68000 / 30$ ARTX

VAdil 110-13120,

Vorelon 8.0

(\$910020W1.11210)

Valldeted by Roghtstration

Verdlo

Corporation

VADS Sun3 SunOS

$\Rightarrow$ 68000/30 ARTX computer family (under

VAda-110-13120,

Vorelon 8.0

(BASE

\$010080W1.11210) \$8333, \& -68340 Evaluation Syderns (bere machinee)

Same as Hot

Locthoed Senders STAR MNP (R3000) (bare mechino) Ralaeve 4.0 (bare machins)

Gyelone CVME 44, 48, \& 48; torce CPU 21, CPU 29, CPU 30 . Triangle Flepower, Hourticon HKB8/NZE Sorles, NZF Sorie, SBC; Matrix MS-CPU220 \&
Motorole MMME101 188000 (bare machine)

Motorda 88302 ,

Phillipe-Signetica 68070, \&

Toehiba 68301 (bare

mechines)

Motorda CPU32 - Mes332EVS

Verdlix

Corporation

SPARCutation 2 (under SunOS

VADS SunA SunOS

$\Rightarrow$ 68020/30 ARTX.

VAde-110-40120,

Verion 6.0

(\$010020W1.11211)

- Velideted by Ragletration

Verdlox

Corportion

VADS Sund SunOS

- $88000 / 30$ ARTX

VAda-110-40120,

Vorion 6.0

(BASE

O10020W1.11211)

Motorole CPUS2 - M68332EVS ivchuation Syctem (88332) (baro mechino)

Velldated by Regletretion

Verdlex

Corportion

VADS Sund SunOS

$\Rightarrow$ 88020/30 ARTX,

VAda-110-40120,

Version 8.0 \&

6.2

BASE

1010020W1.11211)

Sun Microsyctems Sun-4, SPARCunner, \& SPARCutation computer familibe (under

SunOS 4.1)

Sun Microoymem Sun-d, SPARCutation, \& SPARCanver computer tamilibe (under sunos $4.0,4.1,24.24$ (bare mechine)
Motorola CPU32-68331

Sun-3/280 (under SunOs

Motorola MMME147 (28030) CPU 31, CPU 32, CPU 37, \& Golden a No Series; integrated Solutiona VME68K20, 88K30, 68225, \& Uberator MS-CPU320; Mizer MZ7122, MZ7124, MZ7130, MZ8120, \& MZ8130; Motorda MMME133 Sertee, MMME134, MMME135, MMME138, MMME141, MMME147 Sorles; Sun Microoystern: 3E board eot; and Tedpole Technology TP32V \& TP32M (baro machlnos)
Verdbx

Corporation

VADS IBM RISC

Sytem/6000 AX

$\Rightarrow 68020 / 30$ NRTX

VAd-110-71120.

Version 8.0

(\$910020W1.11212)

Vordbx

Corporation

VADS IEM RISC

Sytom $/ 8000$ AXX

- > 680020/30 NRTX.

VAda-110-71120,

Verion 2.0

BASE

1010020 W1.11212

Verdbx

Corporation

VADS SYSTEM V/800

REEASE 4,

VAde-110-0000

Verion 8.1

(\$010020W1.11213)

Verdibx

Corporation

VADS VMS $=$ >

AMDOS000,

VAde-110-0352s,

Verion 6.04

(\$010eeOW1.11214)

Welidated by Raglatration

Verdbx

Corporation

VADS VAX VMS $=$ >

AMD ZOK

VAda-110-00525,

Verion 6.04

BASE

(910020W1.11214)
Validated by Regintration

IBM RISC Sydem/6000 Models $320,520,540,730, \& 230$ (under $A \times 3.1$ )

IBM RISC Syctem/6000 Model 530 (under AX 3.1)

Oddeta 1800 Workatation (Under UNIX SYSTEM V/800 RELEASE $4 \mathrm{~V} 1.0$ )
MleroVAX 3000 (under VMS 5.4

DEC VAX-11, VAXeoner, VAXstation, MieroVAX, VAX 6000 , VAX 8000 , \& VAX 8000 Sorles of computers (under VMS 5.3)

Cycione CVME 48; Force CPU 21, CPU 29 , CPU 30, CPU 31 , CPU 32, CPU 37; Hourikon HKB8/VZFb Series, HKE8/N30 Series, HKB8/V3E Series; Matrix MS-CPU22O, MS-CPUSZO, 2 MS-CPU330; Mtzar MZ7122, MZ7124, MZ7130, MZ8120 \& MZ8130; Motorola MMME130 Serios, MMME134, MVME135, 2 MMME147 Series; Radetone CPU-2A; SBE VCOM-24; and Tedpole TP32N (bare machinea)

Motorda MMME147 (68030) (bare machine)

Motorola MMME147 (B8030) (bare mechine) Same as Hod

Ironice NeO01 board (AMD 20000) (Am2s000 bare VME machino)

Ironics MSO01 board (AMD 28000) (Am2e000 bare VME machlne) 


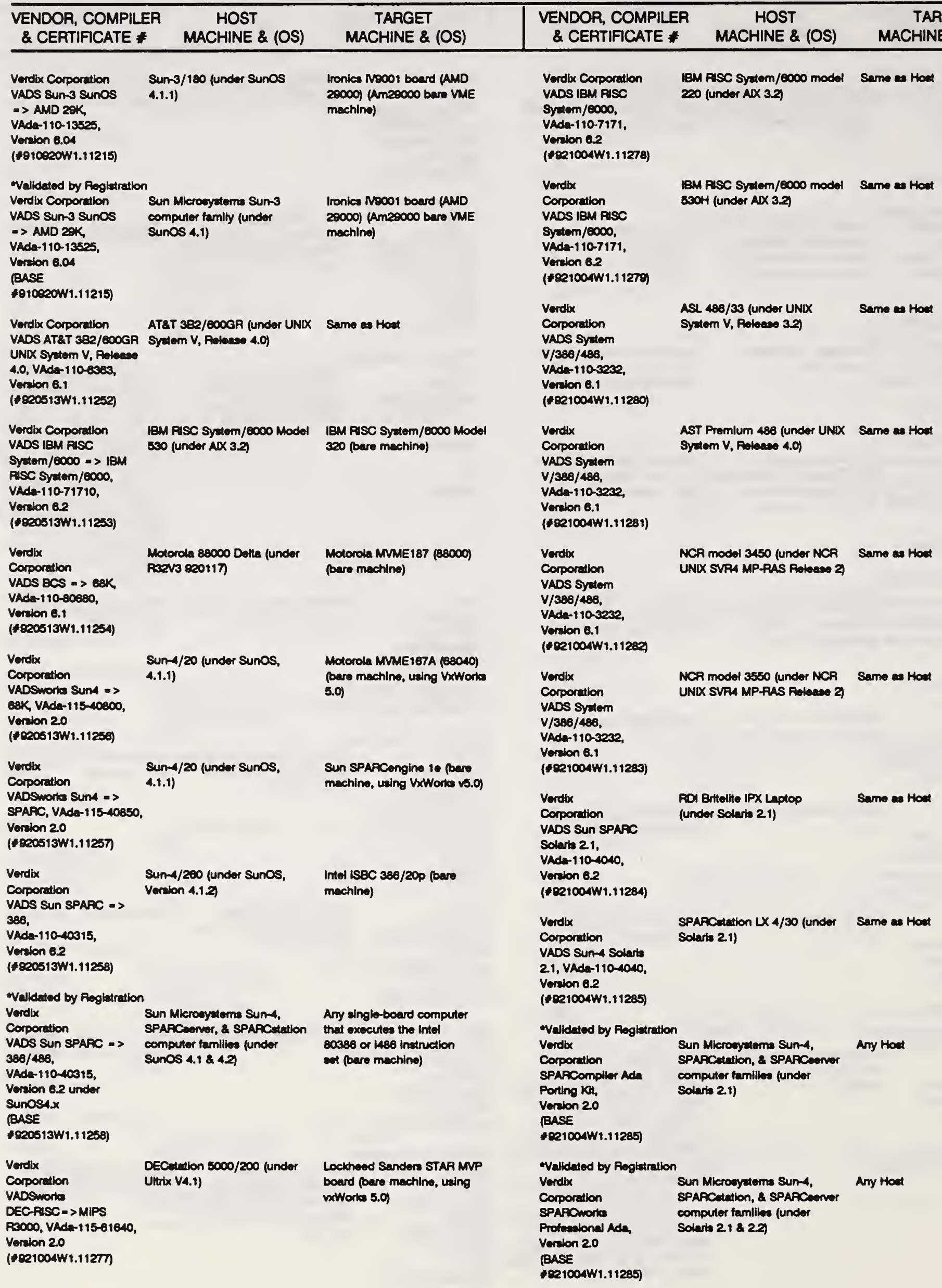




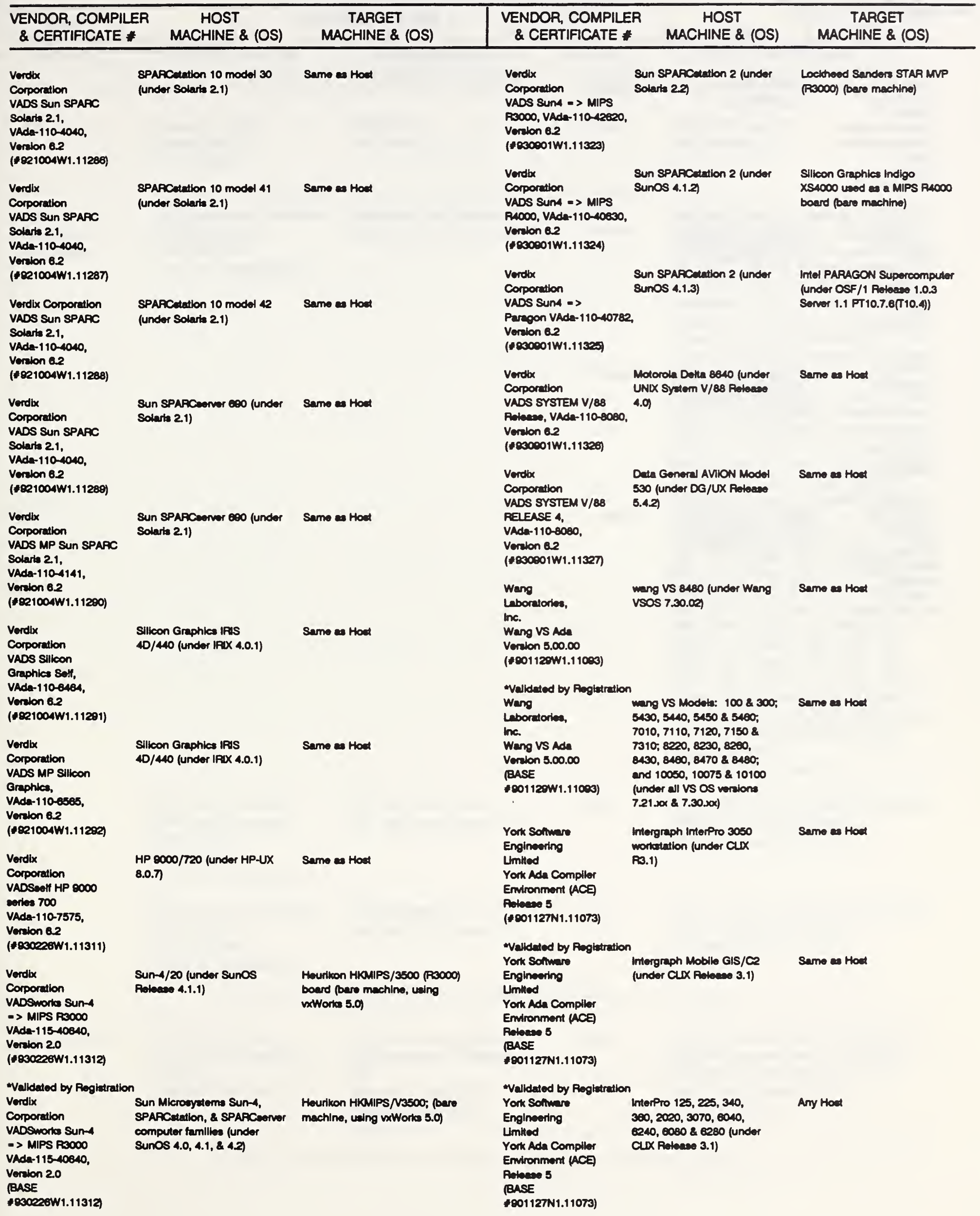




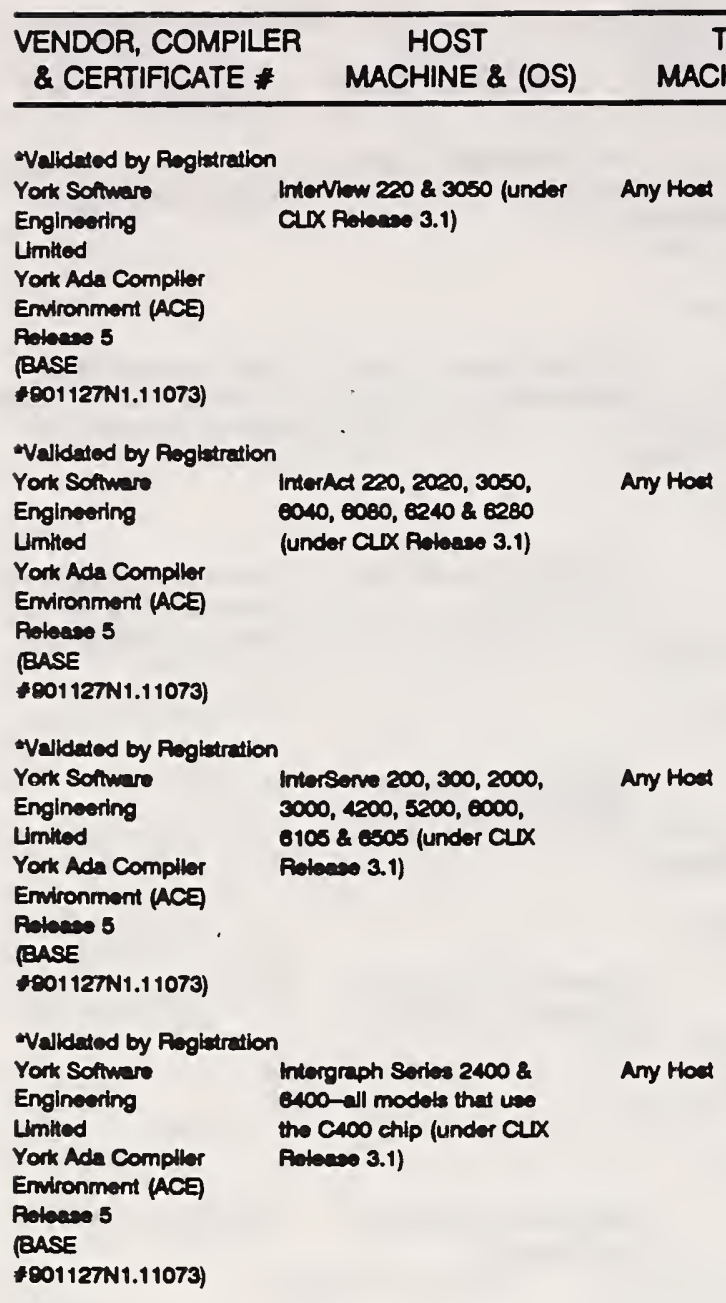




\section{VENDOR}

PROCESSOR ID

VSR \# \& LEVEL

HARDWARE \&
OPERATING SYSTEM

$D P X / 2250$

BOS, Version 2.1

\section{EXPIRY}

DATE

$4 / 1 / 94$

Version 01.01

PCVS/0005/F

\section{OTHER ENVIRONMENTS} HW/OS
Bull S.A.

Digital

Equipment

Corporation
DEC Pascal for OpenVMS

VAX Version 5.0

NIST-93/1762

Lovel 0/1 (Switchable)
VAXstation 3100 Model 70 OpenVMS VAX Version 5.5

DEC 3000 Model 500

DEC OSF/1 AXP Vession 1.2

OSF/1 AXP Systems

Version 5.0

NIST $-93 / 1763$

Level 0/1 (Switchable)

DEC Pascal for OpenVMS

AXP Version 5.0

NIST-93/1764

Lovel 0/1 (Switchable)
DEC 3000 Model 500

OpenVMS AXP Vension 1.5
$8 / 1 / 94$

$8 / 1 / 94$

VAXft 3000 Models $110,310,410,610$, 612; VAX-11 /730, /750, /780, /785; MicroVAX II; 2000; 3100 Models 10, 10E, $20,20 E, 30,40,80,90$; 3200; 3300; 3400; 3500; 3600; 3800; 3900; VAXstation II; II/GPX; II/QVSS; 2000; 2000/GPX, 2000/MFB; 3100 Models 30, $38,40,48,76 ; 3100 / \mathrm{GPX}$ Models 38, 48, 76; $3100 /$ SPX Models 38, 48, 76; 3200 ; 3500 ; 3520; 3540; 4000-VLC; 4000 Models 60, 90; VAXserver 3100 Models 10, 10E, 20, 20E; 3200; 3300; 3400; 3500; 3600; 3800; 3900; 4000 Models $200,300,400,500,600 ; 6000$ Models 210, 220, 310, 320, 410, 420, 510, 520; VAX 4000 Models 100, 200, 300, 500, 600 ; 6000 Models 210, 220, 230, 240, $310,320,330,340,360,410,420,430$, $440,450,460,510,520,530,540,550$, $560,610,620,630,640 ; 7000$ Models $610,620,630,640,650,660$; 8200; 8250; 8300; 8350; 8530; 8550; 8600; $8650 ; 8700 ; 8800 ; 8810 ; 8820 ; 8830$; 8840; 9000 Models 110, 110VP, 210 , 210VP, 310, 310VP, 320, 320VP, 330 , 330VP, 340, 340VP, 410, 410VP, 420, 420VP, 430, 430VP, 440, 440VP; 10000 Models 610, 620, 630, 640, 650, 660; VAXserver 8200, 8250, 8300, 8350, 8530, $8550,8600,8650,8700,8800,8810$, $8820,8830,8840$ OpenVMS VAX, Version 5.5

DEC 10000 , DEC 7000 , DEC 4000 , DEC 3000 , DEC 2000, DEC 1000 DEC OSF/1 AXP Version 1.2

$8 / 1 / 94$ DEC 10000, DEC 7000 , DEC 4000 , DEC 3000 , DEC 2000, DEC 1000 OpenVMS AXP Vession 1.5
IBM Canada LId. IBM AXX XL Pascal Compiler/6000 Version 1 Rolease 1 NIST-93/1462 Lovel 5.4
IBM RISC System/6000 POWERstation POWERserver 560 IBM AIX for IBM RISC System/6000, Version 3 Release 2
4/1/94 IBM RISC System/6000 POWERstation/ POWERserver Models 220, 22W, 22G, $340,350,550,560,580,970,980$ ADX for RISC System $/ 6000$ Version 3 Release 2 


\begin{tabular}{|c|c|c|c|c|}
\hline VENDOR & $\begin{array}{l}\text { PROCESSOR ID } \\
\text { VSR \# \& LEVEL } \\
\end{array}$ & $\begin{array}{c}\text { HARDWARE \& } \\
\text { OPERATING SYSTEM }\end{array}$ & $\begin{array}{r}\text { EXPIRY } \\
\text { DATE } \\
\end{array}$ & $\begin{array}{l}\text { OTHER ENVIRONMENTS } \\
\text { HW/OS } \\
\end{array}$ \\
\hline $\begin{array}{l}\text { Intergraph } \\
\text { Corporation }\end{array}$ & $\begin{array}{l}\text { Pascal-Clipper } \\
\text { Version 1.8.4B } \\
\text { NIST-94/1102 Lovel 0/1 }\end{array}$ & $\begin{array}{l}\text { Clipper Model } 2730 \\
\text { CLIX Version 7.1.3 }\end{array}$ & $12 / 1 / 94$ & $\begin{array}{l}\text { Clipper C300 and C400 } \\
\text { CLLX Version 7.1.3 }\end{array}$ \\
\hline \multirow[t]{3}{*}{$\begin{array}{l}\text { Siemens Nixdorf } \\
\text { Informations- } \\
\text { systeme AG }\end{array}$} & $\begin{array}{l}\text { Pascal-XT V2.2B } \\
\text { PCVS/0101/UK } \\
\text { Level I }\end{array}$ & $\begin{array}{l}7.582 \mathrm{TX}(\mathrm{H} 90) \\
\text { BS } 2000 \mathrm{~V} 11.0\end{array}$ & $9 / 1 / 94$ & $\begin{array}{l}7.582 \text { TX }(\mathrm{H}+9)) \\
\text { BS } 2000 \text { Version } 10.0\end{array}$ \\
\hline & $\begin{array}{l}\text { Pascal-XT V2.1C } \\
\text { PCVS/0102/UK } \\
\text { Lovell }\end{array}$ & $\begin{array}{l}M X 500(80386) \\
\text { SINLX-M VS.41 }\end{array}$ & $9 / 1 / 94$ & $\begin{array}{l}\text { SINIX-PC } \\
\text { SINIX-Z Version } 5.41\end{array}$ \\
\hline & $\begin{array}{l}\text { Pascal-XT V2.1C } \\
\text { PCVS/0103/UK } \\
\text { Lovel } 1\end{array}$ & $\begin{array}{l}\text { RM600 (MIPS R3000) } \\
\text { SINIX-P V5.41 }\end{array}$ & $9 / 1 / 94$ & $\begin{array}{l}\text { RM400 } \\
\text { SINIX-N Version } 5.41\end{array}$ \\
\hline
\end{tabular}




\section{VENDOR \\ Cray Research} Inc.

\section{Control Data}

Digital

Equipment

Corporation

\section{PROCESSOR ID VSR \# \& LEVEL}

\author{
Cray Standard C Compiler \\ Release 3.0.4 \\ NIST-92/2301
}

Cray Standard C Compiler

Release 3.0.4

NIST $-92 / 2302$

Cray Standard C Compiler

Release 3.0.4

NIST $-92 / 2303$

HARDWARE \&
OPERATING SYSTEM

Cray2/4-128

UNICOS Release 6.1.6

Cray Y.MP 81/8128

UNICOS Release 6.1.6

Cray Y-MP C9O

UNICOS Release 7C

\section{EXPIRY} DATE

\section{OTHER ENVIRONMENTS} HW/OS

12/1/93 Cray2

(pending) UNICOS Release 6.1.6

12/1/93 Cray Y.MP

(pending) UNICOS Release 6.1.6

\section{ANSI C Version 3.11 NIST-93/1103}

CN2 Version 2.5 NIST.93/1104
Control Data 4680

EP/LX Version 2.1 .1

CYBER $180-932$

NOS/VE Version 1.7.1 Level 803
$12 / 1 / 93$

(pending)
DEC OSF/ 1 for AXP C

Compiler Version 1

NIST-93/1313

DEC C Version 1.3 for OpenVMS AXP Systems NIST-93/1314 OpenVMS VAX Systems NIST-93/1315
DEC C Version 1.3 for

\section{DEC/3000 Model 400 AXP}

DEC OSF/1 AXP, Version 1.2

DEC/3000 Model 400 OpenVMS AXP, Version 1.5

VAXstation 4000, Model 60 OpenVMS VAX, Version 5.5

DEC 3000 Model 400 $D E C$ OSF $/ 1$ for AXP Version 2.0

NIST.93/2144

DEC C for RISC/Ultrix Version 1.2a Release 1 NIST $-93 / 2145$
DECstation 5000/200

Utixix Version 4.4

\section{2/1/94 Control Data 4000}

$E P / L X$ Version 2.1 .1

2/1/94 CYBER 180, 200

NOS/NE Version 1.7.1 Level 803
$3 / 1 / 94$
DEC/ $10000, / 7000, / 4000, / 3000, / 2000$, $/ 1000$
DEC OSF/1 AXP, Version 1.2

3/1/94 DEC/10000, DEC/7000, DEC/4000, DEC/3000, DEC/2000, DEC/1000 OpenVMS AXP, Version 1.5

3/1/94 VAX 4000 Models 200, 300; VAX 6000 Models 200,300, 400, 500; VAX 8200, $8250,8300,8350,85 \times x, 8600,8650$, $8700,8800,8810,8820,8830,8840$; VAX 9000 Models 210, 400; VAXt 3000 Model 310; VAX11/730, VAX11/750, VAX11/780, VAX11/785; MicroVAX's II, $2000,3100,3300,3400,3500,3600$, 3800,3900 ; VAXstation's II, 2000, 3100, $3200,3500,3520,3540$; VAXsenvers $3100,3300,3400,3500,3600,3602$, $3800,3900,4000$ Models 200, 300 , VAXserver 6000 Models 210/220, $310 / 320,410 / 420,510 / 520$ OpenVMS VAX, Version 5.5

12/1/94 DEC 3000400,500 ; DEC 4000610 ; DEC 7000 610; DEC 10000610 DEC OSF/1 for AXP Version 2.0

$12 / 1 / 94$

DECstation 2100, 3100; Personal DECstation 5000 Models 20/25 MX, HX, TX, PXG +, PXG Turbo +; DECstation 5000 Models 120/125/133 MX, CX, $H X$, PX, TX, PXG, PXG+, PXG Turbo, PXG Turbo +; Models $200 M X, C X, H X, P X$, TX, PXG, PXG +, PXG Turbo, PXG Turbo +; Models 240 MX, HX, TX, PXG +, PXG Turbo +; DECsystem 3100; 5000 Models 25, 200, 240; 5100; 5400; 5500; 5810; 5820; 5830; 5840; 5900 Ultrix Version 4.4 


\begin{tabular}{llcr}
\hline VENDOR & PROCESSOR ID & HARDWARE \& & EXPIRY \\
VSR \# \& LEVEL & OPERATING SYSTEM & DATE \\
\hline & $\begin{array}{lll}\text { DEC C for OpenVMS VAX } \\
\text { Version } 1.3\end{array}$ \\
NIST-93/1765 & $\begin{array}{c}\text { VAXstation } 3100 \text { Model } 48 \\
\text { OpenVMS VAX, Version } 6.0\end{array}$ & $8 / 1 / 94$ \\
& &
\end{tabular}

OTHER ENVIRONMENTS HW/OS

VAXft 3000 Models $110,310,410,610$, 612; VAX-11 /730, /750, /780, /785; MicroVAX II; 2000; 3100 Models 10, 10E, $20,20 E, 30,40,80,90$; 3200; 3300; 3400; 3500; 3600; 3800; 3500; VAXstation II; II/GPX; II/QVSS; 2000; 2000/GPX, 2000/MFB; 3100 Models 30, 38, 40, 48, 76; 3100/GPX Models 38, 48, 76; 3100/SPX Models 38, 48, 76; 3200; 3500; 3520; 3540; 4000-VLC; 4000 Models 60, S0; VAXserver 3100 Models 10, 10E, 20, 20E; 3200; 3300; 3400; 3500; 3600; 3800; 3500; 4000 Models 200, 300, 400, 500, 600; 6000 Models $210,220,310,320,410,420,510,520$; VAX 4000 Models $100,200,300,500$, 600 ; 6000 Models 210, 220, 230, 240, $310,320,330,340,360,410,420,430$, $440,450,460,510,520,530,540,550$, $560,610,620,630,640 ; 7000$ Models $610,620,630,640,650,660$; 8200; 8250; 8300; 8350; 8530; 8550; 8600; 8650; 8700; 8800; 8810; 8820; 8830; 8840; 9000 Models 110, 110VP, 210, 210VP, 310, 310VP, 320, 320VP, 330, 330VP, 340, 340VP, 410, 410VP, 420, 420VP, 430, 430VP, 440, 440VP; 10000 Models 610, 620, 630, 640, 650, 660; VAXserver $8200,8250,8300,8350,8530$, $8550,8600,8650,8700,8800,8810$, $8820,8830,8840$ OpenVMS VAX, Version 6.0

Encore Computer C Version 1.3.0 Corporation Company

Hewlett-Packard

$$
\text { NIST-93/2261 }
$$

Encore 91

UMAX V, Version 3.0.9
$11 / 1 / 94$

ENCORE INFINITY $90, \mathrm{R} / \mathrm{T}$

UMAX V, Version 3.0.9

ENCORE 93

UMAX V, Version 3.1.3

\begin{tabular}{|c|c|c|c|}
\hline $\begin{array}{l}\text { HP C/HP-UX Version } \\
\text { A.09.60 Release HP-UX } \\
9.0 \\
\text { NIST-94/1221 }\end{array}$ & $\begin{array}{c}\text { HP9O00 Model } 720 \\
\text { HP.UX Version } 9.0\end{array}$ & $1 / 1 / 95$ & $\begin{array}{l}\text { HP9000 Models } 8 \times x, 7 x x, 6 x x, \text { FxO } \\
\text { GxO, HxO, 1xO } \\
\text { HP-UX Vension } 9.0\end{array}$ \\
\hline $\begin{array}{l}\text { HP C/HP-UX Version } \\
\text { B.08.36 Release HP-UX } \\
\text { B.09.00 } \\
\text { NIST-94/1223 }\end{array}$ & $\begin{array}{l}\text { HP9000 Model 42S } \\
\text { HP-UX Version B.09.00 }\end{array}$ & $1 / 1 / 95$ & $\begin{array}{l}\text { HP9000 Models } 3 \times x, 4 \times x \\
\text { HP.UX Version B.09.00 }\end{array}$ \\
\hline $\begin{array}{l}\text { HP C/iX Version A.05.02 } \\
\text { Release MPE/iX } 5.0 \\
\text { NIST-94/1222 }\end{array}$ & $\begin{array}{l}\text { HP3000 Model } 967 \\
\text { MPE/iX Version } 5.0\end{array}$ & $1 / 1 / 95$ & $\begin{array}{l}\text { HP3000 Model 9xx } \\
\text { MPE/AX Version } 5.0\end{array}$ \\
\hline $\begin{array}{l}\text { C/370 Compiler Version } 2 \\
\text { Release } 1 \\
\text { NIST }-93 / 1051\end{array}$ & $\begin{array}{l}\text { ES/9000 } \\
\text { MVS/ESA SP Version } 4 \\
\text { Release } 2\end{array}$ & $1 / 1 / 94$ & $\begin{array}{l}3090,308 X, 43 \times \times, 937 X \\
\text { MVS/ESA SP Version } 4 \text { Release } 2\end{array}$ \\
\hline
\end{tabular}




\begin{tabular}{ccccc}
\hline VENDOR & PROCESSOR ID & HARDWARE \& & EXPIRY & OTHER ENVIRONMENTS \\
& VSR \# \& LEVEL & OPERATING SYSTEM & DATE & HW/OS \\
\hline
\end{tabular}

C/370 Compiler Version 2
Release 1
NIST-93/1052
C/370 Compiler Version 2
Release 1
NIST-93/1053
C/370 Compiler Version 2
Release 1
NIST-93/1054
SAA AD/CYCLE C/400
Version 1 Release 1.1
NIST-93/1055

$X \perp$ C Compiler Version 1 Release 2 NIST-93/1056

XL C Compiler Version 1 Release 2 NIST-93/1057

IBM C/C + + TOOLS, Version 2 Release 0 NIST-93/1461

\section{ES/9000}

VM/ESA Version 1 Release 1.1

$1 / 1 / 94$

ES/9000

VM/SP Version 1 Release 6

ES/9000

$V M / X A$ SP Version 1 Release 2

ES/9000

MVS/ESA SP Version 4

Release 2

IBM RISC 6000 Model 220 ALX for RISC 6000 Version 3 Release 2

IBM RISC 6000 Model $530 \mathrm{H}$ ALX for RISC 6000 Version 3 Release 2

IBM PS/2 486 Model 70 OS $/ 2$ Version 2 Release 0

$1 / 1 / 94$

$1 / 1 / 94$

$4 / 1 / 94$
$3090,308 X, 43 X X, 937 X$

VM/ESA Version 1 Release 1.1

1/1/94 3090, 308X, 43XX, 937X

VM/SP Version 1 Release 6

$3090,308 x, 43 \times x, 937 X$

VM/XA SP Version 1 Release 2

1/1/94 3909, $908 X, 43 \times X, 937 X$

MVS/ESA SP Version 4, Release 2

IBM RISC 6000 POWERstation/ POWERservers $320,320 \mathrm{H}, 340,350$, $520,52 \mathrm{OH}, 530,530 \mathrm{H}, 540,550,560$, 580; POWER-server(s) 730, 930, 950, 970,980

ALX for RISC 6000 Version 3

Release 2

1/1/94 IBM RISC 6000 POWERstation/ POWERservers $220,320,320 \mathrm{H}, 340$, $350,520,520 \mathrm{H}, 530,540,550,560,580$; POWER-server(s) $730,930,950,970$, 980

ALX for RISC 6000 Version 3 Release 2

IBM PS/ 12133 386SX/25, 486SX /20, 486DX/20, 486DX/40; $21553865 X / 25$, $486 \mathrm{SX} / 20,486 \mathrm{DX} / 20,486 \mathrm{DX} / 33$, 486DX/40; IBM PS/2 $8540386 S X / 20$, $8543386 \mathrm{SX} / 20,8555386 \mathrm{SX} / 16,8556$ $386 S X / 20,386 S L C / 20,85573865 X / 20$, $386 S L C / 20,8565386 S X / 16,8570$ $386 \mathrm{DX} / 16-25,486 \mathrm{DX} / 25,8573$ $386 \mathrm{DX} / 16-20,8575$ 486DX/33, IBM PS/2 $8580386 \mathrm{DX} / 20-25,8590486 \mathrm{SX} / 25$, $486 \mathrm{DX} / 25-33,486 \mathrm{D} \times 2 / 50-66,8595$ $486 \mathrm{SX} / 25,486 \mathrm{DX} / 25-33,486 \mathrm{DX} / 50-66$, 9556486 SLC2, 9557 486SLC2, 9576 486SX, 486DX2, 9577 486SX, 486DX2, 9585 486SX, 486DX2, 9595 486DX, 486DX2, 295 486DX; IBM PS/ThinkPad N45SL 386SL, N51SX 386SX, N51SLC 386SLC, CL57SX 386SX, 300 386SL, 700 486SLC, 700C 486SLC, IBM PS/ ValuePoint 325T, 425SX, 433DX, 466DX2 OS $/ 2$ Version 3 Release 0
$X \perp$ C Compiler Version 1.3 NIST-93/1961
RISC System/6000 Model 530 ADX Version 3.2
$10 / 1 / 94$ 


\begin{tabular}{|c|c|c|c|}
\hline VENDOR & $\begin{array}{l}\text { PROCESSOR ID } \\
\text { VSR \# \& LEVEL }\end{array}$ & $\begin{array}{c}\text { HARDWARE \& } \\
\text { OPERATING SYSTEM }\end{array}$ & $\begin{array}{r}\text { EXPIRY } \\
\text { DAT } \\
\end{array}$ \\
\hline & $\begin{array}{l}\text { C Set }++ \text { for AIX } \\
\text { Version } 2.1 \\
\text { NIST-93/1962 }\end{array}$ & $\begin{array}{l}\text { RISC System } / 6000 \text { Model } 530 \\
\text { ALX Version } 3.2\end{array}$ & $10 / 1 / 94$ \\
\hline & $\begin{array}{l}\text { IBM ILE C/400 Version } 2 \\
\text { Release } 3 \\
\text { NIST-93/1963 }\end{array}$ & $\begin{array}{l}\text { AS/400 Model 9406/ESO } \\
\text { OS/400 Version } 2 \text { Release } 3\end{array}$ & $10 / 1 / 94$ \\
\hline
\end{tabular}

Intel SSD

Intergraph

Corporation

CC Compiler Version R4.5 XP/5

$$
\text { NIST-93/1782 }
$$

OSF/LAD Release RI.1

Clipper Advanced

Optimizing C Version 1.57 NIST-94/1103

Clipper Advanced Optimizing C Version 2.0 NIST-94/1105
Clipper Model 2730

CLIX Version 7.1
OTHER ENVIRONMENTS HW/OS
9402 System Models DO2, E02, CO4, DO4, E04, CO6, DO6, E06, F02, F04, F06: 9404 System Models B10, C10, D10, E10, B2O, C2O, D2O, E2O, C25, D25, E25, F10, F2O, F25; 9406 System Models B30, B35, B40, B45, B50, B60, $B 70$, D35, E35, D45, E45, D50, E50, D60, E6O, F80, F90, F95, D70, E70, D80, E80, E9O, E95, F35, F45, F50, F6O, F70 OS/400 Version 2 Release 3

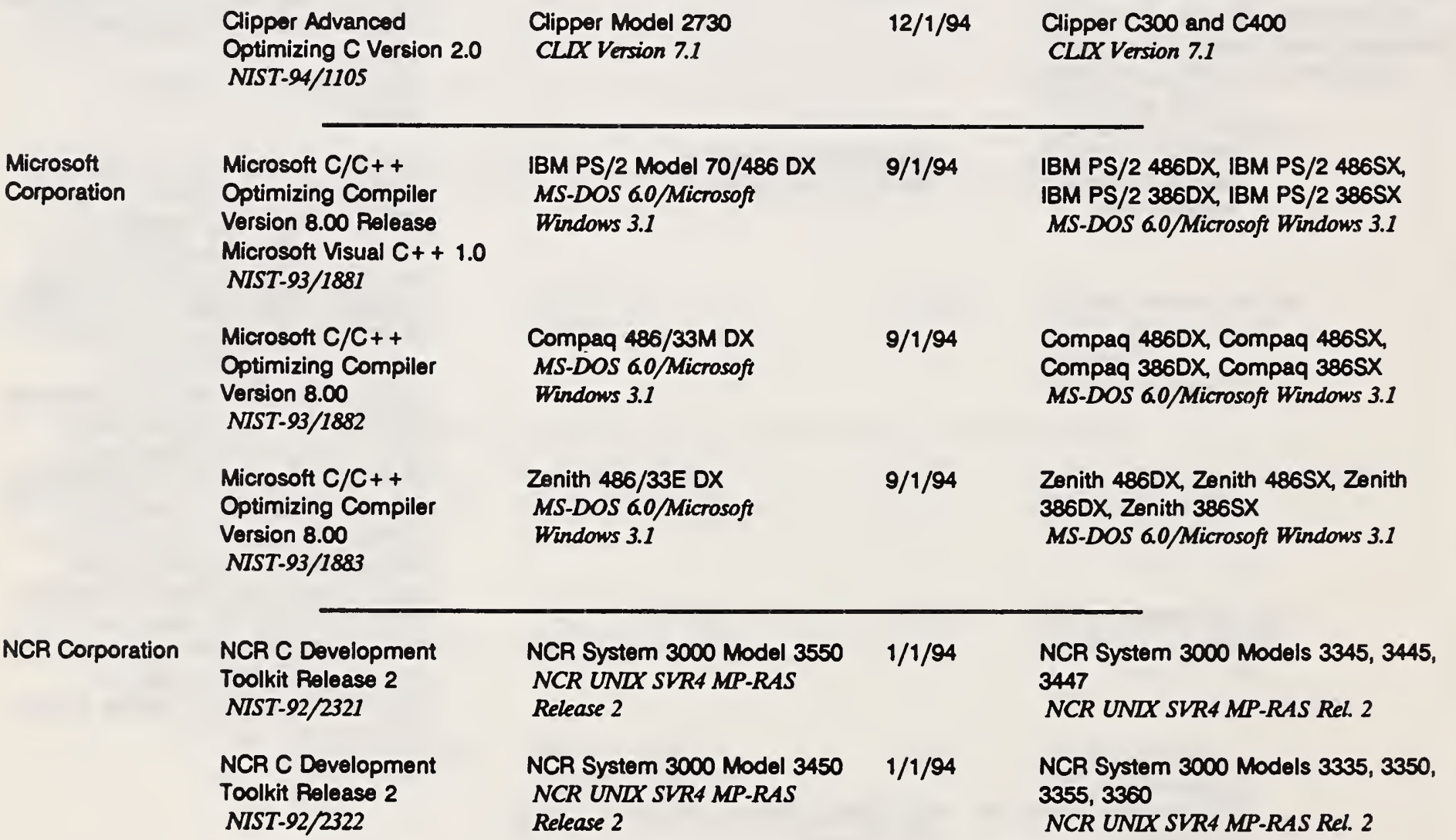




\begin{tabular}{|c|c|c|c|c|}
\hline VENDOR & $\begin{array}{l}\text { PROCESSOR ID } \\
\text { VSR \# \& LEVEL } \\
\end{array}$ & $\begin{array}{c}\text { HARDWARE \& } \\
\text { OPERATING SYSTEM }\end{array}$ & $\begin{array}{r}\text { EXPIRY } \\
\text { DATE }\end{array}$ & $\begin{array}{l}\text { OTHER ENVIRONMENTS } \\
\text { HW/OS }\end{array}$ \\
\hline & $\begin{array}{l}\text { NCR C Development } \\
\text { Toolkit Release } 2 \\
\text { NIST-94/1121 }\end{array}$ & $\begin{array}{l}\text { NCR System } 3000 \text { Model } 3550 \\
\text { NCR UNIX SVR4 MP-RAS } \\
\text { Release } 2 \text { Version SVR4 }\end{array}$ & $1 / 1 / 95$ & $\begin{array}{l}\text { NCR System } 3000 \text { Models } 3335,3340 \text {, } \\
3345,3350,3360,3410,3430,3445 \text {, } \\
3447,3450,3455,3470,3475,3520 \text {, } \\
3525,3555,3570,3575,3600 \\
\text { NCR UNLX SVR4 MP-RAS Rel. } 2\end{array}$ \\
\hline $\begin{array}{l}\text { Pyramid } \\
\text { Technology Corp. }\end{array}$ & $\begin{array}{l}\text { DC/OSx ANSI C, Version } \\
\text { 1.1 Release } 92006 x \\
\text { NIST-93/1341 }\end{array}$ & $\begin{array}{l}\text { MISserver-S } \\
\text { DC/OSx Version } 1.1 \\
\text { Release } 92 \text { c06x }\end{array}$ & $5 / 1 / 94$ & $\begin{array}{l}\text { MISserver-ES } \\
\text { DC/OSx Version } 1.1 \text { Release } 92 c 06 X\end{array}$ \\
\hline $\begin{array}{l}\text { Sequent } \\
\text { Computer } \\
\text { Systems, Inc. }\end{array}$ & $\begin{array}{l}\text { ptx/C Version } 2 \\
\text { Release } 1.0 \\
\text { NIST-93/2003 }\end{array}$ & $\begin{array}{l}\$ 2000 / 250 \\
\text { DYNLX/ptx Version } 2 \text { Release } \\
1.0\end{array}$ & 2/1/95 & $\begin{array}{l}S 2000 / 450, S 2000 / 750 \\
\text { DYNLX/pax Version } 2 \text { Release } 1.0\end{array}$ \\
\hline \multirow[t]{2}{*}{$\begin{array}{l}\text { Silicon Graphics } \\
\text { Computer } \\
\text { Systems }\end{array}$} & $\begin{array}{l}\text { ANSI C Version } 3.11 \\
\text { NIST-93/1161 }\end{array}$ & $\begin{array}{l}M / 120 \\
\text { RISC/OS Release } 5.01\end{array}$ & $4 / 1 / 94$ & $\begin{array}{l}\mathrm{M} / 800, \mathrm{M} / 1000, \mathrm{RS} 3260, \mathrm{RC} 3240 \text {, } \\
\mathrm{RC} 2030, \mathrm{RS} 2030, \mathrm{RC} 4230, \mathrm{RS} 4230 \text {, } \\
\mathrm{RC} 6280 \\
\text { RISC/OS Release } 5.01\end{array}$ \\
\hline & $\begin{array}{l}\text { C Rolease } 4.0 \\
\text { NIST-93/1162 }\end{array}$ & $\begin{array}{l}\text { IRIS 40/25 } \\
\text { IRLX Release } 5.0\end{array}$ & $4 / 1 / 94$ & $\begin{array}{l}\text { Personal IRIS, IRIS, IRIS 4D/50, 4D/70, } \\
4 D / 120,4 D / 220,4 D / 280 \\
\text { IRLX Release } 5.0\end{array}$ \\
\hline \multirow[t]{2}{*}{ SUNPRO } & $\begin{array}{l}\text { SPARCompiler C } \\
\text { Version } 3.0 \\
\text { NIST-93/2184 }\end{array}$ & $\begin{array}{l}\text { SPARCstation LX } \\
\text { SPARCserver } 1000 \\
\text { Solaris Version } 23\end{array}$ & $2 / 1 / 95$ & \\
\hline & $\begin{array}{l}\text { ProCompiler C Version } \\
2.0 .1 \\
\text { NIST-93/1902 }\end{array}$ & $\begin{array}{l}\text { DEUL Model 433DE } \\
\text { Solaris Version } 2.1 \text { Release X86 }\end{array}$ & $8 / 1 / 94$ & \\
\hline $\begin{array}{l}\text { Tandem } \\
\text { Computers } \\
\text { Incorporated }\end{array}$ & $\begin{array}{l}\text { C Version D20 Rolease } \\
\text { D20 } \\
\text { NIST-93/2041 }\end{array}$ & $\begin{array}{l}\text { NonStop Vx } \\
\text { NonStop Kernel Version } \\
\text { D20 Release D20 }\end{array}$ & $10 / 1 / 94$ & \\
\hline Unisys & $\begin{array}{l}\text { UCS C (UC) Version 4R1 } \\
\text { Rolease SB5R1 } \\
\text { NIST-93/2221 }\end{array}$ & $\begin{array}{l}2200 \text { Model } 900 \\
1100 \text { OS EXEC Version } \\
44 R I \text { Release SBSRI }\end{array}$ & $11 / 1 / 94$ & $\begin{array}{l}1100 / 90,2200 / 400,2200 / 600 \\
1100 \text { OS ECEC/44RI }\end{array}$ \\
\hline
\end{tabular}




\begin{tabular}{ccccc}
\hline VENDOR & PROCESSOR ID & HARDWARE \& & EXPIRY \\
\&VSR \# & OPERATING SYSTEM & LEVEL & DATE & OTHER ENVIRONMENTS \\
HW/OS
\end{tabular}

No entries at this time. 
2.8 LANGUAGE PROCESSORS

WITH REGISTERED REPORTS ONLY

\subsubsection{COBOL PROCESSORS WITH NONCONFORMITIES}

\begin{tabular}{|c|c|c|c|c|c|}
\hline VENDOR & $\begin{array}{c}\text { PROCESSOR ID } \\
\& V S R \# \\
\end{array}$ & $\begin{array}{c}\text { HARDWARE \& } \\
\text { OPERATING SYSTEM }\end{array}$ & $\begin{array}{r}\text { EXPIRY } \\
\text { DATE }\end{array}$ & SUBSET & $\begin{array}{c}\text { OTHER ENVIRONMENTS } \\
\text { HW/OS }\end{array}$ \\
\hline $\begin{array}{l}\text { Digital } \\
\text { Equipment } \\
\text { Corporation }\end{array}$ & $\begin{array}{l}\text { DEC COBOL Version } \\
\text { OpenVMS AXP Systen } \\
\text { NIST-93/1311 }\end{array}$ & $\begin{array}{l}\text { DEC } 3000 \\
\text { Model } 500 \text { AXP } \\
\text { OpenVMS AXP } \\
\text { Version } 1.0\end{array}$ & $3 / 1 / 94$ & High & $\begin{array}{l}\mathrm{DEC} / 10000, \mathrm{DEC} / 7000, \mathrm{DEC} / 4000 \text {, } \\
\mathrm{DEC} / 3000, \mathrm{DEC} / 2000, \mathrm{DEC} / 10000 \\
\text { OpenVMS AXP, Version } 1.0\end{array}$ \\
\hline
\end{tabular}




\section{2- 62}




\section{DATABASE LANGUAGE (SQL)}

\subsection{FIPS Database Language Standards}

As specified by the FIPS, FIRMR and the associated Federal ADP and Telecommunications Standards Index, Federal agencies, when acquiring SQL processors, are responsible for assuring that processors are in accordance with the applicable FIPS PUB 127, Database Language SQL. On December 3, 1993, FIPS PUB 127-2 superseded FIPS PUB 127-1.

\subsection{Organization of Database Language Processor Entries}

Each entry in the VPL is a very limited extract from the Validation Summary Report (VSR) available from the Software Standards Validation Group at NIST. See 3.4 and 3.5 below.

Products validated for conformance to FIPS PUB 127-2 are listed first, followed by products validated for FIPS PUB 127-1. Products that demonstrated one or more nonconformities, as assessed by the SQL Validation System, are listed separately at the end. (These products are considered "provisionally" validated, pending correction of nonconformities.) The entries in the VPL for database language processors are presented as follows:

- The VENDOR ID column contains the name of the Vendor of the processor.

- The PROCESSOR ID column contains the name of the processor, its version number, the VSR number, and the Expiry date of the Validation Certificate or the Registered VSR. The term "Pre-release" means that the vendor has designated the SQL processor as "not commercially available" at the time of validation. The product is listed to assist users in planning for future procurements.

- The INTERFACES \& COMPILERS column contains the names of associated interactive SQL or programming language interfaces, and identification of the programming language compilers that interface with the SQL processor. A listing in the COMPILERS column is not an indication that the compiler has been validated for the applicable programming language standard. See the preceding "Programming Languages" Section for a list of validated compilers.

- The HARDWARE \& OPERATING SYSTEM column presents the hardware and operating system environment used during the validation.

- The entries in the OTHER HW/SW ENVIRONMENTS column include other hardware and operating system environments in which the processor operates, and the programming language compilers that interface with the SQL processor. The listings of the compilers and operating systems may contain a range of versions that are supported. Rebadged or renamed software are also listed here. This column is restricted to binary-compatible hardware environments.

- The NONCONFORMITIES column lists the number and type of nonconformities for each programming language interface tested. "Schema" nonconformities are deficiencies in support for standard schema definition language constructs. "FIPS Flagger" in this column indicates that the mandatory FIPS Flagger requirement of FIPS 127 was not implemented. Refer to the VSR for details. The number of nonconformities is only one limited measure of the quality of an SQL interface. It is more important to analyze the nature of each individual nonconformity and its impact on meeting user requirements. 
Refer to Database Language SOL Validation Procedures. The requirements for validation of database language processors are the same as those for programming language processors, listed in section 2.3.1, with several exceptions. Expired VSRs are deleted from the VPL to motivate vendors to test new releases of their SQL processors and to demonstrate conformance to more comprehensive versions of the SQL Test Suite. Information about expired VSRs or vendor selftesting with the SQL Test Suite may be available from the vendor.

\subsection{Certificate of Validation}

A Certificate of Validation is issued for those SQL processors that have been tested and are considered to be in compliance with FIPS as specified by the FIPS, by the FIRMR, and the associated Federal ADP and Telecommunications Standards Index.

\subsection{Registered Report}

A Validation Summary Report (VSR) that indicates that the SQL processors did not meet the criteria for a Certificate of Validation may be registered by the Computer Systems Laboratory. A VSR is considered registered by CSL when it contains a signed notice that the VSR will be listed in the CSL Validated Products List (VPL).

\subsection{Validation Procedures}

SQL processors are tested in accordance with the procedures described in the NIST Database Language SQL Validation Procedures. To request a copy of the validation procedures and/or to request the validation of an SQL processor, contact:

National Institute of Standards and Technology

Computer Systems Laboratory

Software Standards Validation Group

Building 225, Room A266

Gaithersburg, Maryland 20899 (U.S.A.)

Telephone (301) 975-2490 (Voice)

(301) $975-3274$ (Voice)

(301) 948-6213 (FAX)

e-mail: dashiell@speckle.ncsl.nist.gov (INTERNET)

\subsection{SQL Validation System}

To request a copy of the SQL Validation System and/or to submit questions regarding the SQL Validation System, contact:

National Institute of Standards and Technology

Computer Systems Laboratory

Database Languages Group

Building 225, Room A266

Gaithersburg, Maryland 20899 (U.S.A.)

Telephone (301) 975-3258 (Voice)

(301) 975-3263 (Voice)

(301) 948-6213 (FAX)

e-mail: sullivan@ecf.ncsl.nist.gov (INTERNET) 


\begin{tabular}{lllll}
\hline VENDOR & PROCESSOR ID & INTERFACES & HARDWARE \& & OTHER HW/SW \\
& VSR\# \& EXP DATE & \& COMPILERS & OPERATING SYS. & ENVIRONMENTS \\
\hline
\end{tabular}

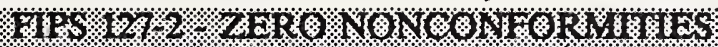

[Entry FIPS 127-2 exceeds requirements for FIPS 127-1 with Integrity Enhancement Option]

Informix
Software, Inc.
$\begin{aligned} & \text { AN6.0 PIX Pre-release } \\ & \text { NIST-94/7041 1/1/95 }\end{aligned}$
Features Tested:
Entry FIPS 127-2
FIPS Sizing Defaults

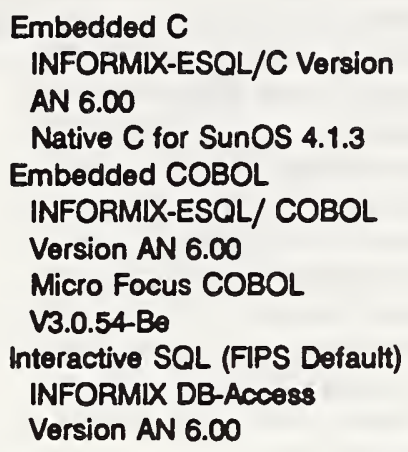

Sun SPARCstation 10

SunOS Version 4.1.3_DBE1.3

\section{Embedded C}

Pro*C, Version 1.6

Native C, bundled with

SunOS Version 4.1.3

\section{SUN SPARCstation 10} SunOS Version 4.1.3
(Beta) Pro-release NIST-93/7521 11/1/94

Features Tested:

Entry FIPS $127-2$

FIPS Sizing Defaults

ORACLE7, Rolease 7.1 (Beta)

Pro-release

NIST-93/7522 11/1/94

Features Tested:

Entry FIPS $127-2$

FIPS Sizing Defaults

ORACLE7, Release 7.1 (Bota)

Pre-release

NIST-93/7523 11/1/94

Features Tested:

Entry FIPS $127-2$

FIPS Sizing Defaults
Embedded C

Pro*C, Version 2.0

Native C, bundled with

SunOS Version 4.1.3
SUN SPARCstation 10

SunOS Version 4.1.3 $\begin{array}{lc}\text { Embedded Ada } & \text { SUN SPARCstation } 10 \\ \text { Pro*Ada, Version 1.6 } & \text { SunOS Version 4.1.3 }\end{array}$
Sybase, Inc. Sybase System 10 Pre-release

10.0.1

NIST-94/7061 $1 / 1 / 95$

Features Tested:

Entry FIPS 127-2

FIPS Sizing Defaults

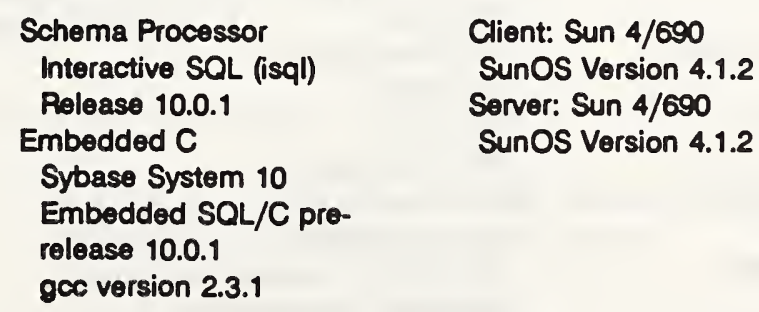

Client: Sun 4/690

SunOS Version 4.1.2

Server: Sun 4/690

SunOS Version 4.1.2 


\begin{tabular}{lllll}
\hline VENDOR & PROCESSOR ID & INTERFACES & HARDWARE \& & OTHER HW/SW \\
& VSR\# \& EXP DATE & \& COMPILERS & OPERATING SYS. & ENVIRONMENTS \\
\hline
\end{tabular}

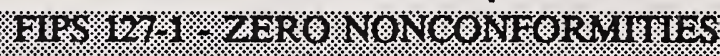

Cincom Systems, Inc.

SUPRA Server, Version 3.2

Pro-release

NIST-93/7481 12/1/94

Features Tested:

Lovel 2 ANSI SQL

Integrity Enhancement

FIPS Sizing Dofaults

FPS Flagger

SUPRA Server, Version 3.2

Pre-release

NIST-93/7482 12/1/94

Features Tested:

Lovel 2 ANSI SQL

Integrity Enhancement

FIPS Sizing Dofaults

FIPS Flagger
Embedded SQL C SUPRA C Precompiler Version 3.2 Pre-release $C$ bundled with SunOS

Release 4.1.1

Embedded SQL COBOL SUPRA COBOL Precompiler Version 3.2 Pre-releaso SUN COBOL Version 1.0 Interactive SQL SUPRA I/SQL Version 3.2

Pre-release

Embedded SQL Ada SUPRA Ada Precompiler, Version 3.2 Pre-release SUN Ada Version 1.1
SUN SPARCstation 2

SunOS Release 4.1.1

SUN SPARCstation 2

SunOS Release 4.1.1

$\begin{array}{ll}\text { Digital } & \text { VAX Rdb/NMS } \\ \text { Equipment } & \text { Version 4.1 } \\ \text { Corporation } & \text { NIST-92/7351 10/01/94 } \\ & \\ & \text { Features Tested: } \\ & \text { Level } 2 \text { ANSI SOL } \\ & \text { Integrity Enhancement } \\ & \text { Option } \\ & \text { FIPS Sizing Defaults } \\ & \text { FIPS Flagger }\end{array}$

VAX Rdb/NMS

Version 4.1

NIST-92/7352 10/01/94

Features Tested:

Lovel 2 ANSI SQL

Integrity Enhancement

Option

FIPS Sizing Defaults

FIPS Flagger

\author{
Embedded Ada \\ Module Ada \\ VAX Ada Version 2.2 \\ Embedded C \\ Module C \\ VAX C Version 3.2 \\ Embedded COBOL \\ Module COBOL \\ VAX COBOL Version 4.4 \\ Embedded FORTRAN \\ Module FORTRAN \\ VAX FORTRAN Version 5.7 \\ Embedded PASCAL \\ Module PASCAL \\ VAX Pascal Version 4.2 \\ Interactive SQL (FIPS Default) \\ Embedded Ada \\ Module Ada \\ VAX Ada Version 2.1 \\ Embedded C \\ Module C \\ VAX C Version 3.2 \\ Embedded COBOL \\ Module COBOL \\ VAX COBOL Version 4.4 \\ Embedded FORTRAN \\ Module FORTRAN \\ VAX FORTRAN Version 5.7 \\ Embedded PASCAL \\ Module PASCAL \\ VAX Pascal Version 4.2 \\ Interactive SQL (FIPS Default) \\ VAXstation 4000 Cluster \\ VAX/NMS Ver. 5.5-2
}

\author{
VAX, MicroVAX, and \\ VAXstation \\ VMS Versions 5.0 - 5.43 \\ VAX Ada V2.0 - 2.2 \\ VAX C V $3.0-3.2$ \\ VAX COBOL V4.2 4.4 \\ VAX Fortran V5.0 - 5.7 \\ VAX Pascal V3.9 - 4.2
}

\author{
VAX, MicroVAX, and \\ VAXstation \\ VMS Versions 5.0 - 5.5-2 \\ VAX Ada V2.0 - 2.1 \\ VAX C V $3.0-3.2$ \\ VAX COBOL V4.2 - 4.4 \\ VAX Fortran V5.0 - 5.7 \\ VAX Pascal V3.9 - 4.2
}




\begin{tabular}{ll}
\hline VENDOR & PROCESSOR ID \\
& VSR\# \& EXP DATE \\
\hline & VAX Rdb/MMS \\
& Version 4.2 \\
& NIST-92/7353 10/01/94 \\
& Features Tested: \\
& Lovel 2 ANSI SQL \\
& Integrity Enhancement \\
& Option \\
& FIPS Sizing Defaults \\
& FIPS Flagger
\end{tabular}

\section{INTERFACES \\ \& COMPILERS}

Embedded Ada
Module Ada

VAX Ada Version 2.2

Embedded C

Module C

VAX C Version 3.2

Embedded COBOL

Module COBOL

VAX COBOL Version 4.4

Embedded FORTRAN

Module FORTRAN

VAX FORTRAN Version 5.7

Embedded PASCAL

Module PASCAL

VAX Pascal Version 4.2

Interactive SOL (FIPS Default)

VAX Rdb/NMS
Version 4.2
NIST-92/7354 10/01/94
Features Tested:
Level 2 ANSI SQL
Integrity Enhancement
Option
FIPS Sizing Defaults
FIPS Flagger

VAX Rdb/NMS

Features Tested:

Lovel 2 ANSI SQL

Integrity Enhancement

FIPS Sizing Defaults

FIPS Flagger

$\begin{array}{ll}\text { Embedded Ada } & \text { VAXstation } 4000 \\ \text { Module Ada } & \text { Cluster } \\ \text { VAX Ada Version 2.1 } & \text { VAXNMS Ver. 5.5-2 }\end{array}$

Embedded C

Module C

VAX C Version 3.2

Embodded COBOL

Module COBOL

VAX COBOL Version 4.4

Embedded FORTRAN

Module FORTRAN

VAX FORTRAN Version 5.7

Embedded PASCAL

Module PASCAL

VAX Pascal Version 4.2

Interactive SQL (FIPS Default)

$\begin{array}{ll}\text { HARDWARE \& } & \text { OTHER HW/SW } \\ \text { OPERATING SYS. } & \text { ENVIRONMENTS }\end{array}$

VAXstation 3500 and VAX 8800

VAXNMS Ver. 5.4-3

VAX, MicroVAX, and

VAXstation

VMS Versions $5.0 \cdot 5.4-3$

VAX Ada V $2.0 \cdot 2.2$

VAX C V3.0 -3.2

VAX COBOL V4.2 - 4.4

VAX Fortran V5.0 - 5.7

VAX Pascal V $3.9 \cdot 4.2$

VAX, MicroVAX, and

VAXstation

VMS Versions $5.0 \cdot 5.5-2$

VAX Ada V2.0 - 2.1

VAX C V $3.0 \cdot 3.2$

VAX COBOL V4.2 $\cdot 4.4$

VAX Fortran V5.0 -5.7

VAX Pascal V3.9 - 4.2

$\begin{array}{ll}\text { IBM } & \text { Database } 2 \text { (DB2) Version } 3 \\ \text { Corporation } & \text { NIST-93/7441 8/1/94 } \\ & \text { Features Tested: } \\ & \text { Level } 2 \text { ANSI SQL } \\ & \text { FIPS Sizing Defaults } \\ & \text { FIPS Flagger }\end{array}$

Embedded C IBM C/370 Version 2 Release 1

Embedded COBOL

IBM SAA AD/CYCLE COBOL/370 Version 1

Release 1

Embedded Fortran

IBM VS FORTRAN Version 2

Release 5
IBM ES90009021-720

MVS/ESA SP Version 4

Release 1
Informix

Software Inc.
INFORMIX-OnLine/Secure

Version 5.00

NIST-93/7301 12/1/94

Features Tested:

Lovel 2 ANSI SQL

Integrity Enhancement

Option

FIPS Sizing Dofaults

FIPS Flagger

\author{
Module Ada \\ INFORMIX-ADA/SAME \\ Version 5.00 \\ SunAda Version 2.0 \\ Embedded C \\ INFORMDX-ESQL/C Version \\ 5.00 \\ Sun ANSI C Version 2.0.1 \\ Interactive SQL (FIPS Default) \\ INFORMIX DB-Access \\ Version 5.00 \\ RDI Britelite IPX Laptop \\ Solaris 2.1
}

Sun4c sparc

Solaris 2.1 


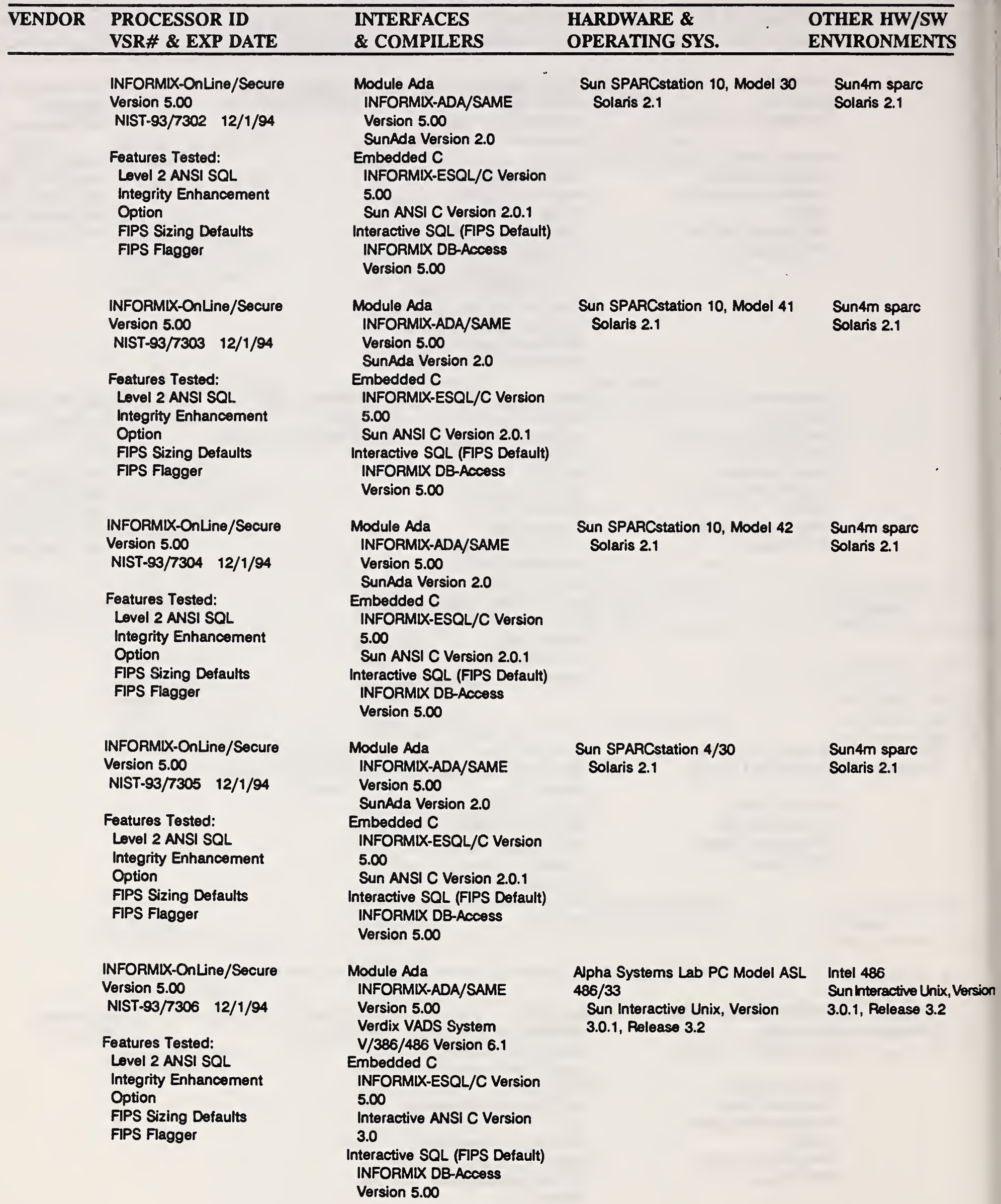




\section{INTERFACES}

\& COMPILERS

HARDWARE \&
OPERATING SYS.

Features Tested:

Lovel 2 ANSI SQL

Integrity Enhancement

Option

FIPS Sizing Defaults

FIPS Flagger

INFORMIX-OnLine/Secure

Version 5.00

NIST-93/7308 12/1/94

Features Tested:

Lovel 2 ANSI SQL Integrity Enhancement

Option

FIPS Sizing Defaults

FIPS Flagger

INFORMIX-OnLine/Secure

Version 5.00

NIST-93/7309 12/1/94

Features Tested:

Lovel 2 ANSI SQL

Integrity Enhancement

Option

FIPS Sizing Defaults

FIPS Flagger

INFORMIX-OnLine Version

5.02

NIST-94/7042 1/1/95

Features Tested:

Lovel 2 ANSI SOL

Integrity Enhancement

Option

FIPS Sizing Defaults

FIPS Flagger
Module Ada

INFORMIX-ADA/SAME

Version 5.00

Alsys Ada for HP9000

Series 800, Version A.05.35

Hewlett-Packard 9000 Series 800

Model 867

HP BLS A.08.08

Embedded C

INFORMIX-ESQL/C Version

5.00

HP C Version A.08.17

Interactive SQL (FIPS Default)

INFORMIX DB-Access

Version 5.00

Module Ada

INFORMIX-ADA/SAME

Version 5.00

Asys Ada for HP9000

Series 800, Version A.05.35

Embedded C

INFORMIX-ESQL/C Version

5.00

HP C Version A.08.17

Interactive SQL (FIPS Default)

INFORMIX DB-Access

Version $\mathbf{5 . 0 0}$

Module Ada

INFORMIX-ADA/SAME

Version 5.00

Asys Ada for HP9000

Series 800, Version A.05.35

Embedded C

INFORMIX-ESQL/C Version

5.00

HP C Version A.08.17

Interactive SQL (FIPS Default)

INFORMIX DB-Access

Version 5.00

Module Ada

INFORMIX-Ada/SAME

Version 5.02

Sun Ada Version 1.10

Embedded Ada

INFORMIX-Ada/SAME

Version 5.02

Sun Ada Version 1.10

Embedded C

INFORMIX-ESQL/C Version

5.02

Native C for SunOS 4.1.3

Interactive SQL (FIPS Default)

INFORMIX DB-ACcess

Version 5.02

\author{
Sun Sparc4 \\ SunOS 4.1.3_DBE1.3
}

Hewlett-Packard 9000 Series 800

Model 827

HP BLS A.08.08

Hewlett-Packard 9000 Series 800 Model 807

HP BLS A.08.08

HP9000 Series 800 , Series 700

HP BLS A.08.08-09
HP9000 Series 800 , Series 700

HP BLS A.08.08-09
HP9000 Series 800 , Series

HP BLS A.08.08-09 


\begin{tabular}{|c|c|c|c|}
\hline VENDOR & $\begin{array}{l}\text { PROCESSOR ID } \\
\text { VSR\# \& EXP DATE }\end{array}$ & $\begin{array}{l}\text { INTERFACES } \\
\text { \& COMPILERS } \\
\end{array}$ & $\begin{array}{l}\text { HARDWARE \& } \\
\text { OPERATING SYS. }\end{array}$ \\
\hline \multirow[t]{3}{*}{$\begin{array}{l}\text { Ingres, The } \\
\text { ASK Group }\end{array}$} & $\begin{array}{l}\text { ASK INGRES SOL, Rolease } \\
6.5 \text { Pre-release } \\
\text { NIST-93/7501 10/1/94 } \\
\text { Features Tested: } \\
\text { Level } 2 \text { ANSI SQL } \\
\text { Integrity Enhancement } \\
\text { Option } \\
\text { FIPS Sizing Defaults } \\
\text { FIPS Flagger }\end{array}$ & $\begin{array}{l}\text { Embedded Ada } \\
\text { Verdix Ada, Version 6.0.3 } \\
\text { Embedded C } \\
\text { SUN C Version } 1.0 \\
\text { Embedded FORTRAN } \\
\text { SUN Fortran Version } 1.4\end{array}$ & $\begin{array}{l}\text { SUN Sparcstation2 } \\
\text { SunOS Version 4.1.3 }\end{array}$ \\
\hline & $\begin{array}{l}\text { ASK INGRES SQL, Release } \\
6.5 \text { Pre-release } \\
\text { NIST-93/7502 10/1/94 } \\
\text { Features Tested: } \\
\text { Lovel } 2 \text { ANSI SQL } \\
\text { Integrity Enhancement } \\
\text { Option } \\
\text { FIPS Sizing Defaults } \\
\text { FIPS Flagger }\end{array}$ & $\begin{array}{l}\text { Embedded Ada } \\
\text { VAX Ada, Version 2.0-18 } \\
\text { Embedded C } \\
\text { VAX C, Version 3.1-051 } \\
\text { Embedded FORTRAN } \\
\text { VAX FORTRAN, Version 4.5- } \\
219\end{array}$ & $\begin{array}{l}\text { VAX } 6000-410 \\
\text { VAXNMS Version } 5.5\end{array}$ \\
\hline & $\begin{array}{l}\text { ASK INGRES SQL, Release } \\
6.5 \text { Pre-release } \\
\text { NIST-93/7503 10/1/94 } \\
\text { Features Tested: } \\
\text { Level } 2 \text { ANSI Enhancement } \\
\text { Integrity Enhancement } \\
\text { Option } \\
\text { FIPS Sizing Dofaults } \\
\text { FIPS Flagger }\end{array}$ & 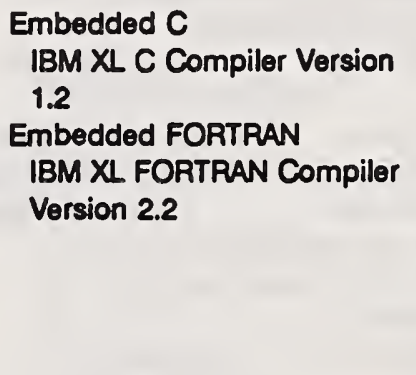 & $\begin{array}{l}\text { IBM RISC System 6000, Model } 530 \\
\text { IBM AIX for RISC System } 6000 \\
\text { Version 3.2.3 }\end{array}$ \\
\hline
\end{tabular}

ESQL/Nerdix Ada, Rel. 6.5 ESQL/C Rel. 6.5 ESOL/FORTRAN Rel. 6.5
ESOL/Ada, Rel 6.5

ESQL/C, Rel. 6.5 ESOL/FORTRAN, Rel. 6.5
ESQL/C Rol. 6.5 ESOL/FORTRAN Rol. 6.5

$\begin{array}{ll}\begin{array}{l}\text { Oracle } \\ \text { Corporation }\end{array} & \text { ORACLE7, Release } 7.0 \\ & \text { NIST-93/7101 11/1/94 } \\ & \text { Features Tested: } \\ & \text { Level } 2 \text { ANSI SQL } \\ \text { Integrity Enhancement } \\ \text { Option } \\ \text { FIPS Sizing Defaults } \\ \text { FIPS Flagger } \\ \text { ORACLE7, Release } 7.0 \\ \text { NIST-93/7102 11/1/94 } \\ \text { Features Tested: } \\ \text { Lovel } 2 \text { ANSI SOL } \\ \text { Integrity Enhancement } \\ \text { Option } \\ \text { FIPS Sizing Defaults } \\ \text { FIPS Flagger }\end{array}$

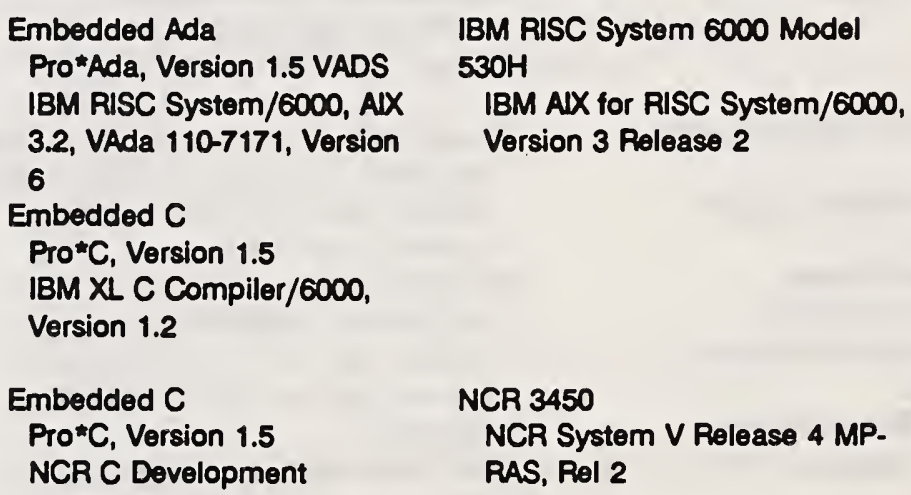

IBM RISC System 6000 Model $530 \mathrm{H}$

IBM AIX for RISC System/6000, Version 3 Release 2

NCR 3450

NCR System V Release 4 MP. RAS, Rel 2

IBM RISC System 6000 Models 220, 320, 320H, 340, $350,520,520 \mathrm{H}, 540,550$, $560,730,930,950,970$ AlXfor RISC System/6000, Version 3 Release 2
NCR Series 3000, to include $3335,3345,3447,3550,3600$ NCR Systom V Paleese 4 Mr RAS, Rel 2 
INTERFACES

\& COMPILERS

Embodded C

Pro*C, Version 1.5

NCR C Development

Toolkit, Rel 2

\section{HARDWARE \&} OPERATING SYS.

NCR 3550

NCR System V Release 4 MP. RAS, Rel 2

\section{Features Tested: \\ Lovel 2 ANSI SQL \\ Integrity Enhancement \\ Option \\ FIPS Sizing Dofaults \\ FIPS Flagger}

\section{ORACLE7, Release 7.0 \\ NIST-93/7104 11/1/94 \\ Features Tested: \\ Lovel 2 ANSI SQL \\ Integrity Enhancement \\ Option \\ FIPS Sizing Defaults \\ FIPS Flagger}

\author{
ORACLE7, Release 7.0 \\ NIST-93/7105 11/1/94 \\ Features Tested: \\ Lovel 2 ANSI SQL \\ Integrity Enhancement \\ Option \\ FIPS SizIng Dofaults \\ FIPS Flagger \\ Trusted ORACLE7, Release \\ 7.0 \\ NIST.93/7106 11/1/94 \\ Features Tested: \\ Lovel 2 ANSI SQL \\ Integrity Enhancement \\ Option \\ FIPS Sizing Dofaults \\ FIPS Flagger

Trusted ORACLE7, Release
7.0
NIST-93/7107 11/1/94
Features Tested:
Lovel 2 ANSI SQL
Integrity Enhancement
Option
FIPS Sizing Defaults
FIPS Flagger
Trusted ORACLE7, Release
7.0
NIST-93/7108 11/1/94
Features Tested:
Level 2 ANSI SOL
Integrity Enhancement
Option
FIPS Sizing Defaults
FIPS Flagger

\section{Embodded Ada \\ Pro*Ada, Version 1.5 Verdix Corp. VADS UNIX System

\author{
NCR 3450 \\ NCR System V Release 4 MP. \\ RAS, Rel 2
} $V / 386$, Release 4, Version 6.1

\section{Embedded Ada}

Pro"Ada, Version 1.5 Verdix Corp. VADS UNIX System $V / 386$, Release 4, Version 6.1

Embedded Ada Pro*Ada, Version 1.5 Assys Ada HP-B2425, Version A.05.35

Embedded C Pro*C, Version 1.5 HP C HP 92453-01, Version A.08.17

\section{Embedded Ada Pro*Ada, Version 1.5 Alsys Ada HP.B2425, Version A.05.35 \\ Embedded C \\ Pro ${ }^{\star} \mathrm{C}$, Version 1.5 HP C HP 92453-01, Version A.08.17}

\section{Embedded Ada \\ Pro*Ada, Version 1.5 \\ Alsys Ada HP.B2425, \\ Version A.05.35 \\ Embedded C \\ Pro*C, Version 1.5 HP C HP 92453-01, Version A.08.17}

\section{NCR 3550}

NCR System V Release 4 MP. RAS, Rel 2

Hewlett-Packard $9000 / 807$
HP-UX BLS, Version 8.08

Hewlett-Packard 9000/817 HP.UX BLS, Version 8.08
Hewlett-Packard $9000 / 847$
HP.UX BLS, Version 8.08
OTHER HW/SW

ENVIRONMENTS

NCR Series 3000 , to include 3335, 3345, 3447, 3550, 3500 NCR System V Rabese 4 MP RAS, Rel 2
NCR Series 3000, to include 3335, 3345, 3447, 3550, 3500 NCR Sstam V Rabese 4 MPRAS, Rel 2
NCR Series 3000, to include $3335,3345,3447,3500,3000$ NCR Sstam V Release 4 MP RAS, Rel 2
HP $9000 / 7 x x$ HP.UX BLS Release 8.09 HP $9000 / 8 x x$ HP-UX BLS Release 8.08
HP 9000/7xx HP.UX BLS Release 8.09 HP $9000 / 8 x x$ HP-UX BLS Release 8.08
HP $9000 / 7 \times x$

HP.UX BLS Release 8.09 HP $9000 / 8 x x$ HP-UX BLS Release 8.08 


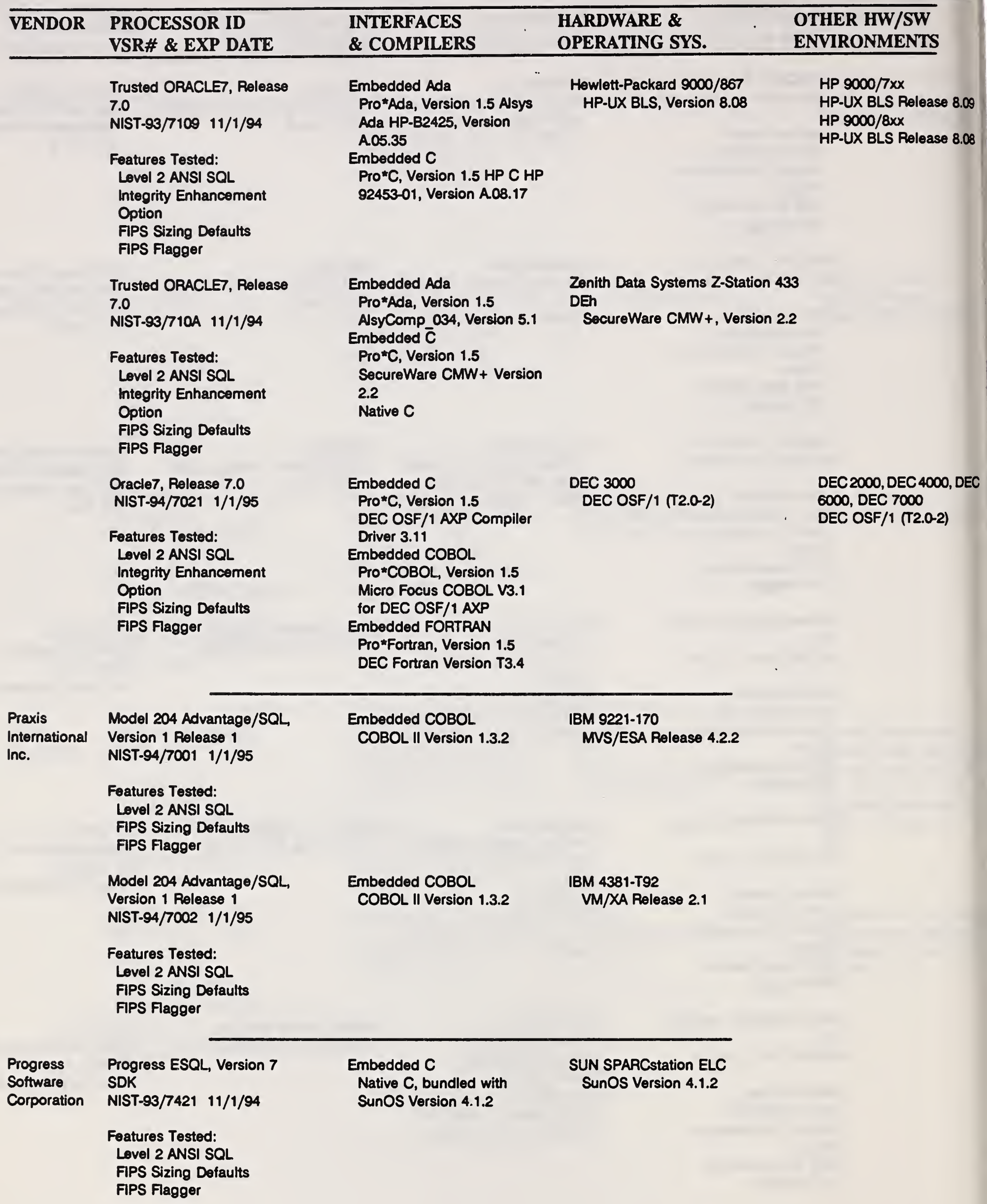




\begin{tabular}{lllll}
\hline VENDOR & PROCESSOR ID & INTERFACES & HARDWARE \& & OTHER HW/SW \\
& VSR\# \& EXP DATE & \& COMPILERS & OPERATING SYS. & ENVIRONMENTS \\
\hline
\end{tabular}

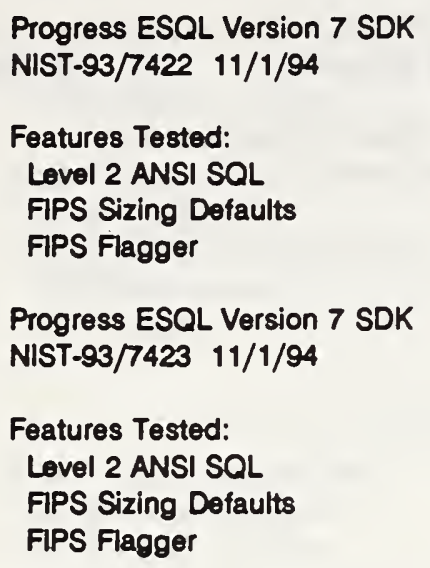

\section{Embedded C Microsoft C for UNIX Version 6}

\section{Embedded C} Microsoft $\mathrm{C} / \mathrm{C}++$ Version 7

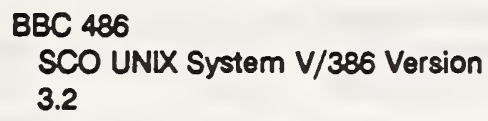

BBC 486

MS-DOS Version 5.0
Sybase, Inc. Sybase System 10/Beta

Version Prerelease NIST-93/7061 9/1/94

Features Tested:
Lovel 2 ANSI SQL
Integrity Enhncement Optiz Defaults
FIPS Sizing Diger
FIPS Flagger
Sybase System 10
Version 10.0
NIST-93/7062 10/1/94
Features Tested:
Lovel 2 ANSI SQL
Integrity Enhancement
Option
FIPS Sizing Defaults
FIPS Flagger

Sybase System 10

Version 10.0 NIST-93/7063 10/1/94

Features Tested: Lovel 2 ANSI SQL Integrity Enhancement Option FIPS Sizing Dofaults FIPS Flagger
Schema Processor

Sybase Interactive SQL

(isql) System 10 Version 10

Embedded C System 10 Beta Version $\propto$ bundled with HP.UX 9.0 Operating System

\section{Schema Processor Sybase Interactive SOL (isql) System 10 Version 10.0 \\ Embedded C Sybase ESQL/C System 10 Version 10.0 Metaware High C Release 2.3 \\ Embedded COBOL Sybase ESQL/COBOL System 10 Version 10.0 Microfocus COBOL Version 3.0 \\ Other Software Sybase SQL Server System 10 Version 10.0}

\section{Schema Processor Sybase Interactive SQL (isql) System 10 Ver 10.0 Embedded C Sybase ESQL/C System 10 Version 10.0 Sparc Compiler C Ver 2.0 Embedded COBOL Sybase ESQL/COBOL System 10 Version 10.0 Microfocus COBOL Compiler 3.1 Other Software} Sybase SQL Server System 10 Version 10.0

HP $9000 / 140$

HP.UX 9.0

HP $9000 / H 30$ HP-UX 9.0

NCR 3000 Series

Client: NCR 3550

NCR OS Version 2.00.02 NCR SVR $42 . x$

Server: NCR 3550

NCR OS Version 2.00.02

Client: Sun 4/65

Sun Solaris Version 2.2

Server: Sun 4/65

Sun Solaris Version 2.2 all Sun4, Sparcstation 10, Sparcserver 600 series Sun Solaris 2.2 


\begin{tabular}{ll}
\hline VENDOR & PROCESSOR ID \\
& VSR\# \& EXP DATE \\
\hline & Sybase System 10 \\
& Version 10.0 \\
& NIST-93/7064 $\quad 10 / 1 / 94$ \\
& Features Tested: \\
& Level 2 ANSI SQL \\
Integrity Enhancement \\
Option \\
FPS Sizing Defaults \\
FIPS Flagger
\end{tabular}

Sybase System 10

Version 10.0

NIST-93/7065 10/1/94

Features Tested:

Lovel 2 ANSI SQL

Integrity Enhancement Option

FIPS Sizing Dofaults

FIPS Flagger

\author{
Sybase System 10 \\ Version 10.0 \\ NIST-93/7066 10/1/94 \\ Features Tested: \\ Level 2 ANSI SQL \\ Integrity Enhancement \\ Option \\ FIPS Sizing Dofaults \\ FIPS Flagger
}

Sybase System 10

Version 10.0 NIST-93/7067 10/1/94

Features Tested: Lovel 2 ANSI SOL Integrity Enhancement Option FIPS Sizing Dofaults FPS Flagger

INTERFACES
\& COMPIIERS

Schema Processor Sybase Interactive SQL (isql) System 10 Ver 10.0

Embodded C Sybase ESQL/C System 10 Version 10.0 IBM ALX XLC Version 1.2 Embedded COBOL Sybase ESQL/COBOL System 10 Version 10.0 Microfocus COBOL

Compiler 3.1

Other Software

Sybase SQL Server System 10 Version 10.0

\section{Schema Processor}

Sybase Interactive SQL (isql) System 10 Ver 10.0

Embedded C Sybase ESQL/C System 10 Version 10.0

HP Native C, HP C A.09.30

Embodded COBOL Sybase ESQL/COBOL System 10 Version 10.0 HP COBOL Version 3.0

Other Software

Sybase SQL Server System 10 Version 10.0

Schema Processor Sybase Interactive SOL (isql) System 10 Ver 10.0

Embedded C Sybase ESQL/C System 10 Version 10.0

GCC Version 2.3.1

Embedded COBOL Sybase ESQL/COBOL System 10 Version 10.0 Microfocus COBOL

Compiler Version 3.0

Other Software Sybase SQL Server System 10 Version 10.0

\section{Schema Processor Sybase Interactive SQL (isql) System 10 Ver 10.0 \\ Client: DEC VAX 6000-450
Vax/MMS 5.4-1 A} Embedded C Sybase ESQL/C System 10 Version 10.0

Vax C Version 3.1

Embedded COBOL Sybase ESQL/COBOL System 10 Version 10.0 Vax COBOL Version 5.1 Other Software

Sybase SQL Server System 10 Version 10.0

\section{HARDWARE \&} OPERATING SYS.

Client: IBM RS/6000 Model 520

IBM ALX Version 3.2

OTHER HW/SW

ENVIRONMENTS

Server: IBM RS/6000 Model 520

IBM ALX Version 3.2

Client: HP 9000/827

HP-UX A.09.00

HP 700 Series, HP 800 Series HP.UX A.09.00

Server: HP 9000/827

HP.UX A.09.00

all DEC Vax ruming OS lovel
Client: Sun Sparcserver 690

\section{SunOS 4.1.3}

Server: Sun Sparcserver 690 SunOS 4.1.3 Vax/MMS 5.4

all Sun4, Sparcstation 10, Sparcserver 600 series SunOS 4.1.3

Server: DEC VAX $6000-450$ Vax/NMS 5.4-1 A 


\begin{tabular}{lllll}
\hline VENDOR & PROCESSOR ID & INTERFACES & HARDWARE \& & OTHER HW/SW \\
& VSR\# \& EXP DATE & \& COMPILERS & OPERATING SYS. & ENVIRONMENTS \\
\hline
\end{tabular}

\author{
Sybase System 10 \\ Version 10.0 \\ NIST-93/7068 10/1/94 \\ Features Tested: \\ Lovel 2 ANSI SQL \\ Integrity Enhncement Option \\ FIPS Sizing Defaults \\ FIPS Flagger
}

\section{Schema Processor \\ Sybase Interactive SQL \\ Client: DEC 3000 Model 500 \\ OSF/1 Version 1.2}

(isql) System 10 Ver 10.0

Embedded C

Sybase ESQL/C System 10

Version 10.0

DEC OSF/AXP C 3.11

Other Software

Sybase SQL Server System

10 Version 10.0
Server: DEC 3000 Model 500

OSF/1 Version 1.2 al DECAXP ruming OS level below Dec OSF/1 Version 12

\section{Unisys RDMS 2200, Release Level \\ Corporation 6R2 (SB5R2), Pre-release NIST-23/7461 12/1/94 \\ Features Tested: \\ Lovel 2 ANSI SQL Integrity Enhancement Option FIPS Sizing Defaults FIPS Flagger}

Embedded C

UCS C (UC) Version SB5P2

3.11

Embedded COBOL

UCS COBOL (UCOB)

Version SB5R2

Module Fortran

UCS Fortran (UFTN) Version

SB5R2
$2200 / 444$ System

OS 2200 Version SB5R2 (EXEC 44R2) IOS 1100 is renamed OS 2200]
$1100 / 90,2200 / 500$,

$2200 / 600,2200 / 900$

OS 2200 Version SB5R2

(EXEC 44R2)
White Cross WHITE CROSS 9000 Release Systems Ltd. $\quad 1.0 .0$

NIST-93/7251 1/1/94
Features Tested:
Lovel 2 ANSI SQL
FIPS Sizing Defaults
WHITE CROSS 9000 Release
1.0 .0
NIST-93/7252 1/1/94
Features Tested:
Lovel 2 ANSI SQL
FIPS Sizing Defaults

\author{
WHITE CROSS 9000 Release \\ 1.0 .0 \\ NIST-93/7253 1/1/94 \\ Features Tested: \\ Level 2 ANSI SQL \\ FIPS Sizing Defaults
}

WHITE CROSS 9000 Release 1.0.0

NIST-93/7254 1/1/94

Features Tested:

Lovel 2 ANSI SQL

FIPS Sizing Defaults

\section{Embedded C}

WHITE CROSS 9000 Client

Utilities Release 1.0.0

MICROSOFT C/C ++

Optimizing Compiler

Version 7.00

Communications

FTP PC/TCP Version 2.05

(over Ethemet)

Embedded C

WHITE CROSS 9000 Client

Utilities Rolease 1.0.0

NeXTSTEP Objective C

Release 3.0

Communications

TCP/IP software bundled

with OS (over Ethemet)

Embedded C

WHITE CROSS 9000 Client

Utilities Release 1.0.0

C Optimizing Compiler

Version $\mathbf{5 . 1 0}$

Communications

TCP/IP software bundled

with OS (over Ethemet)

Embedded C

WHITE CROSS 9000 Client

Utilities Rolease 1.0.0

SPARCompiler C Ver 2.0.1

Communications

TCP/IP software bundled

with OS (over Ethernet)
Client: Custom-built 80486-based PC

MICROSOFT MS-DOS Version 5.00

Server: WHITE CROSS 9000

Model WCS/9010

Client: NeXTstation

NeXTSTEP Rolease 3.0

Server: WHITE CROSS 9000

Model WCS/9010

Client: Custom-built 80486-based

PC

SCO UNIX SYSTEM V/386

Development System Release 3.2

Server: WHITE CROSS 9000

Model WCS/9010

Client: SPARCstation IPX

SunOS Release 4.1.2

Server: WHITE CROSS 9000

Model WCS/9010 


\begin{tabular}{llllll}
\hline VENDOR & PROCFSSOR ID & INTERPACES & HARDWARE \& & OTHER HW/SW & NONCON \\
& VSR \& EXP DATE & \& COMPILERS & OPERATING SYS & ENVIRONMIENIS & FORMTIIES \\
\hline
\end{tabular}

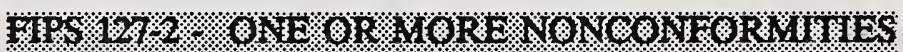

[Entry FIPS 127-2 exceeds requirements for FIPS 127-1 with Integrity Enhancement Option]

No entries for this quarter.

\section{HPS WY ONE OR WORE WOWCONORMMES}

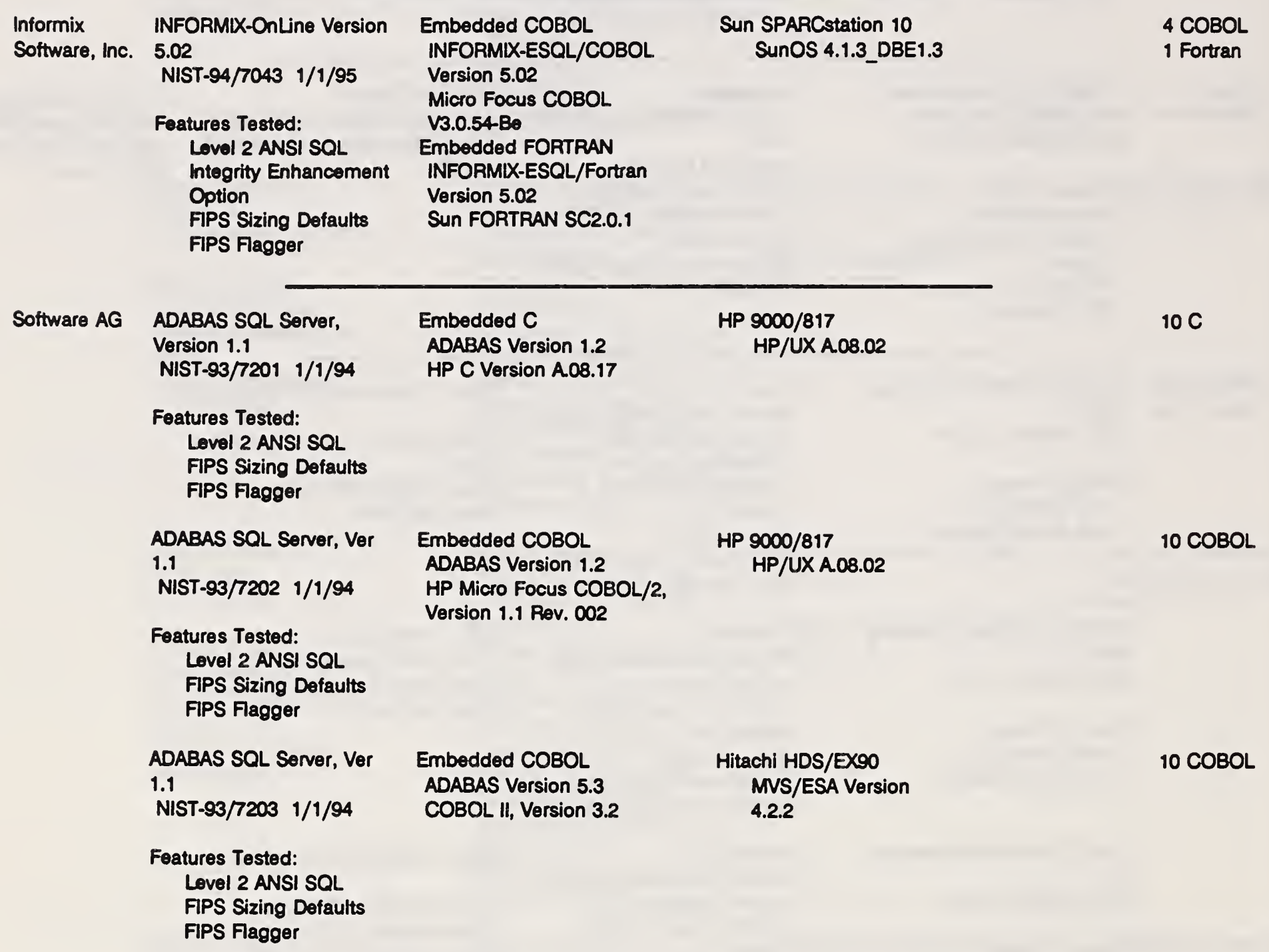




\section{GRAPHICS CONFORMANCE TESTING}

\subsection{FIPS GKS Standard}

The Graphical Kernel System (GKS) is a two-dimensional graphics tool box which provides for the display and manipulation of pictures and graphical input from the operator. The purpose of GKS is to promote portability of graphics applications for use on a variety of graphics workstations. It provides a functional interface between an application program and a configuration of graphical devices. The interface is at such a level of abstraction that hardware peculiarities are shielded from the application program.

FIPS PUB 120-1, GKS, is the first Federal Information Processing Standard Publication (FIPS PUB) registered for computer graphics systems. In accordance with FIPS PUB 120-1, twodimensional graphics toolbox packages acquired for Federal use after November 3, 1986 should implement FIPS GKS. Conformance testing of GKS implementations protects Federal investment by ensuring adherence to the graphics standard. FIPS PUB 120-1 requires that GKS implementations offered to Federal agencies be tested using the NIST Test Suite to ensure that a particular implementation meets the specifications of the FIPS. The GKS Validation Test Suite (Fortran) is available from:

Ms. Susan Sherrick

National Institute of Standards and Technology

Building 225, Room A266

Gaithersburg, MD 20899

(301) $975-3268$

\subsubsection{Organization of GKS Entries}

The entries in the VPL for GKS implementations are presented as follows:

- The VENDOR ID column contains the name of the Vendor of the implementation.

- The GKS NAME column contains the name of the implementation, its version number, the VSR number, and the Expiry date of the certificate of validation.

- The HARDWARE \& OP. SYSTEM column presents the hardware and operating system environment used during the validation.

- The GRAPHICS DEVICES column includes the graphics devices that were validated.

- The GKS LEVEL column indicates the level of GKS that was validated.

- The entries in the OTHER HW/OS column include other hardware and operating system environments in which the processor operates.

- The NONCONFORMITIES column indicates whether or not the GKS implementation conforms to the applicable FIPS in one or more cases as evidenced by the validation. The VSR should be reviewed for details of the nonconformities. 
PHIGS stands for Programmer's Hierarchical Interactive Graphics System. PHIGS is a system for interactive 3-dimensional (3D) graphics applications that provides programmers with a set of features enabling them to manipulate and display complex $3 \mathrm{D}$ objects. It is called hierarchical because the complex objects can be built up from simpler objects. PHIGS also provides a rich set of facilities for real-time interaction with the user. While it borrows many concepts from the Graphical Kernel System (GKS) standard, it also introduces many new features, such as a "graphics data base" (the centralized structure store), and support for modeling and viewing.

In accordance with FIPS PUB 153, (PHIGS), 3D graphics packages acquired for Federal use should implement FIPS PHIGS. Conformance testing of PHIGS implementations protects Federal investment by ensuring adherence to the graphics standard. FIPS PUB 153 requires that PHIGS implementations offered to Federal agencies be tested using the NIST PVT (PHIGS Validation Tests) test suite. The test suite ensures that a particular implementation meets the specifications set forth in the FIPS. The PHIGS PVT test suite is available from:

Project Leader, PHIGS Validation Tests

National Institute of Standards and Technology

Computer Systems Laboratory

Bldg. 225, Room A-266

Gaithersburg, MD 20899

phone: (301) 975-3265

e-mail: phigs@speckle.ncsl.nist.gov

\subsubsection{Organization of PHIGS Entries}

The entries in the VPL for PHIGS implementations are as follows:

- The VENDOR column contains the name of the vendor of the implementation.

- The PHIGS name column contains the name of the implementation, its version number, the Validation Summary Report (VSR) number, and the expiry date of the certification of validation.

- The HARDWARE \& OP.SYSTEM column presents the hardware and operating system environment used during the validation.

- The GRAPHICS DEVICES column includes the graphics devices that were validated.

- The entries in the REGISTERED ENVIRONMENTS HW/OS column includes registered hardware and operating systems for the implementation tested. The vendor of the implementation has certified that the identified processor, when operating under the environments included in this column, produces the same test results exhibited during the validation. Test results and other information from these environments may be required as evidence for entries to be included in this column.

- The NONCONFORMITIES column indicates whether or not the PHIGS implementation conforms to the FIPS in one or more cases as evidenced by the validation. The VSR should be reviewed for more details of the nonconformities. 
The FIPS 128-1, Computer Graphics Metafile (CGM) is a data interchange standard for the storage and retrieval of picture information in a device independent manner. The purpose of the CGM is to facilitate the transfer of graphical information among different computer systems, devices and/or applications.

The FIPS 128-1 requires the use of a CGM application profile. In particular, the Military Specification MIL-D-28003A, commonly known as the CALS CGM profile, is required for Federal government applications when the representation of graphical information in digital form is to be used in technical illustrations and publications, or when the use of a general-purpose, graphical interchange mechanism is required.

NIST offers two CGM Test Services: metafile testing and generator testing. The purpose of the Test Services is to determine the degree to which the metafile or CGM generator conforms to the FIPS 128-1 and the CALS profile. Presently, the Test Service addresses only CGM Version 1.

\subsubsection{CGM Test Labs and Test Suite}

CGM Validation Testing is available from the National Institute of Standards and Technology through its Computer Systems Laboratory (NIST/CSL).

National Institute of Standards and Technology

CGM Test Service

Building 225, Room A266

Gaithersburg, MD 20899

(301) 975-3265

The CGM Validation Test Software is based on CTS/Metacheck, version 2.10 and is available for purchase from

CGM Technology Software

1527 Route 12, Box 648

Gales Ferry, CT 06335

(203) 464-2623

\subsubsection{Certificate of Validation}

For metafile testings, a certificate of validation is issued for those CGM files that have been tested and are in compliance with the FIPS 128-1 and MIL-D-28003A. Conformance of a metafile does NOT necessarily imply conformance of the CGM generator, CGM interpreter, or other CGMs created on the same hardware and software platform.

For generator testing, a certificate of validation is issued for a CGM generator that has been tested and is in compliance with the FIPS 128-1 and MIL-D-28003A.

\subsubsection{Validated Metafiles}

The metafiles presented in Section 4.6 have been tested for conformity with FIPS PUB 128 or MIL-D-28003. Each entry in the VPL is a very limited extract from the Validation Summary Report (VSR) available from NIST/CSL. 


\subsection{Raster Graphics Standards}

FIPS PUB 150 adopts EIA-538 which defines the facsimile coding schemes and their control functions for Group 4 facsimile apparatus, i.e., ITU-T (formerly CCITT) Recommendation T.6. It defines a standard compression algorithm (T.6 - Group 4) suitable for the storage, retrieval, and interchange of raster graphics images.

Military Specification MIL-R-28002 specifies the structure and encoding of raster data files to be delivered to the government. It specifies the use of the standard compression algorithm defined in FIPS PUB 150. It also specifies the use of standard file headers which are defined in MILSTD-1840. MIL-STD-1840 standardizes the format and structure of digital technical data files for the purpose of interchange between organizations or systems.

\subsubsection{Certificate of Validation}

The Raster Graphics Validation Test Service tests an implementation's capablility of both receiving and generating raster graphics data conforming to the specifications in FIPS PUB 150 and MIL-R-28002.

A certificate of validation is issued for an implementation that passes the validation test and conforms to FIPS PUB 150 and MIL-R-28002.

\subsubsection{Information Pack}

Upon request, a Raster Graphics Validation Test Information Pack is available from:

National Institute of Standards and Technology (NIST)

Computer Systems Laboratory

Raster Graphics Validation Test Service

Technology Building, Room A266

Gaithersburg, MD 20899

Telephone (301) 975-3265 


\subsection{GKS IMPLEMENTATIONS}

\begin{tabular}{|c|c|c|c|c|c|c|}
\hline VENDOR & $\begin{array}{c}\text { GKS NAME } \\
\text { EXPIRY \& VSR \# }\end{array}$ & $\begin{array}{c}\text { HARDWARE \& } \\
\text { OP. SYSTEM }\end{array}$ & $\begin{array}{l}\text { GRAPHICS } \\
\text { DEVICES } \\
\end{array}$ & $\begin{array}{r}\text { GKS } \\
\text { LEVEL }\end{array}$ & $\begin{array}{c}\text { REGISTERED } \\
\text { ENVIRONMENTS }\end{array}$ & $\begin{array}{l}\text { NONCON. } \\
\text { FORMITIES }\end{array}$ \\
\hline $\begin{array}{l}\text { Advanced } \\
\text { Technology } \\
\text { Center }\end{array}$ & $\begin{array}{l}\text { GRAFPAK-GKS } \\
\text { Rolease } 4.0 \\
12 / 1 / 94 \\
\text { NIST/NCC-92/967 }\end{array}$ & $\begin{array}{l}\text { NCR } 3450 \\
\text { Unix System V } \\
\text { Release } 4\end{array}$ & $\begin{array}{l}\text { X Window System V11 } \\
\text { PostScript Portrait } \\
\text { Oriented Workstation } \\
\text { (using OMS PS } 810 \\
\text { Laser Printer) }\end{array}$ & Lovel $2 c$ & & No \\
\hline & $\begin{array}{l}\text { GRAFPAK-GKS } \\
\text { Rolease } 4.0 \\
12 / 1 / 94 \\
\text { NIST/NCC-92/968 }\end{array}$ & $\begin{array}{l}\text { NCR } 3550 \\
\text { Unix System V } \\
\text { Release } 4\end{array}$ & $\begin{array}{l}\text { X Window System V11 } \\
\text { PostScript Portrait } \\
\text { Oriented Workstation } \\
\text { (using OMS PS } 810 \\
\text { Laser Printer) }\end{array}$ & Lovel $2 c$ & & No \\
\hline & $\begin{array}{l}\text { GRAFPAK-GKS } \\
\text { Rolease } 4.0 \\
12 / 1 / 94 \\
\text { NIST/NCC-92/969 }\end{array}$ & $\begin{array}{l}\text { IBM RS6000 } \\
\text { Model } 220 \\
\text { AlX } 3.2\end{array}$ & $\begin{array}{l}\text { X Window System V11 } \\
\text { PostScript Portralt } \\
\text { Oriented Workstation } \\
\text { (using OMS PS } 810 \\
\text { Laser Printer) }\end{array}$ & Lovel $2 c$ & & No \\
\hline & $\begin{array}{l}\text { GRAFPAK-GKS } \\
\text { Release } 4.0 \\
12 / 1 / 94 \\
\text { NIST/NCC-92/970 }\end{array}$ & $\begin{array}{l}\text { IBM RS6000 } \\
\text { Model } 530 \mathrm{H} \\
\text { AIX } 3.2\end{array}$ & $\begin{array}{l}\text { X Window System V11 } \\
\text { PostScript Portrait } \\
\text { Oriented Workstation } \\
\text { (using OMS PS } 810 \\
\text { Laser Printer) }\end{array}$ & Level $2 c$ & & No \\
\hline & $\begin{array}{l}\text { GRAFPAK-GKS } \\
\text { Release } 4.0 \\
12 / 1 / 94 \\
\text { NIST/NCC-92/971 }\end{array}$ & $\begin{array}{l}\text { HP- } 9000 / 817 \\
\text { HP-UX } 8.08\end{array}$ & $\begin{array}{l}\text { X Window System V11 } \\
\text { PostScript Portrait } \\
\text { Oriented Workstation } \\
\text { (using OMS PS } 810 \\
\text { Laser Printer) }\end{array}$ & Lovel $2 c$ & & No \\
\hline & $\begin{array}{l}\text { GRAFPAK-GKS } \\
\text { Rolease } 4.0 \\
12 / 1 / 94 \\
\text { NIST/NCC-92/972 }\end{array}$ & $\begin{array}{l}\text { HP. } 9000 / 827 \\
\text { HP.UX } 8.02\end{array}$ & $\begin{array}{l}\text { X Window System V11 } \\
\text { PostScript Portrait } \\
\text { Oriented Workstation } \\
\text { (using OMS PS } 810 \\
\text { Laser Printer) }\end{array}$ & Level $2 c$ & & No \\
\hline & $\begin{array}{l}\text { GRAFPAK-GKS } \\
\text { Release } 4.0 \\
12 / 1 / 94 \\
\text { NIST/NCC-92/974 }\end{array}$ & $\begin{array}{l}\text { Sun Sparcstation } \\
1 \\
\text { Solaris } 2.1\end{array}$ & $\begin{array}{l}\text { X Window System V11 } \\
\text { PostScript Portralt } \\
\text { Oriented Workstation } \\
\text { (using OMS PS } 810 \\
\text { Laser Printer) }\end{array}$ & Lovel $2 c$ & & No \\
\hline
\end{tabular}


4.6 COMPUTER GRAPHICS METAFILES

\begin{tabular}{|c|c|c|c|c|c|}
\hline CLIENT & $\begin{array}{l}\text { VSR \# \& } \\
\text { DATE } \\
\end{array}$ & $\begin{array}{c}\text { \# CGM Submitted } \\
\text { /Conforming }\end{array}$ & $\begin{array}{l}\text { CGM/SIZE/ } \\
\text { DATE }\end{array}$ & GENERATOR & $\begin{array}{c}\text { PLATFORM } \\
\text { (As Reported by Ven }\end{array}$ \\
\hline $\begin{array}{l}\text { Interleaf, Inc } \\
\text { El Segundo, CA }\end{array}$ & $\begin{array}{c}\text { NIST-M-92/003-001 } \\
9 / 2 / 92\end{array}$ & $1 / 1$ & $\begin{array}{l}\text { asg.com } 8880 \\
8 / 31 / 92\end{array}$ & Interleaf Inc MDL/G & $\begin{array}{l}\text { Interleaf } 5 \text { v5.3, } \\
\text { HP9000/700, HP } \\
\text { UX v8.07 }\end{array}$ \\
\hline \multirow{5}{*}{$\begin{array}{l}\text { IBM Corporation } \\
\text { Federal Sector } \\
\text { Division } \\
\text { Oswego, NY }\end{array}$} & $\begin{array}{c}\text { NIST-M-92/005-002 } \\
10 / 28 / 92\end{array}$ & $5 / 5$ & $\begin{array}{l}\text { gcgm_1220.cgm } \\
5280-10 / 27 / 92\end{array}$ & GRAFPAK-CGM 1.1.2 & $\begin{array}{l}\text { IBM RS6000 Model } \\
\text { 220, AIX } 3.2\end{array}$ \\
\hline & & & $\begin{array}{l}\operatorname{gcgm} 1530 . c g m \\
5280-10 / 27 / 92\end{array}$ & GRAFPAK-CGM 1.1.2 & $\begin{array}{l}\text { IBM RS6000 Model } \\
530, \text { AIX } 3.2\end{array}$ \\
\hline & & & $\begin{array}{l}g c g m \_n 345 . c g m \\
5280-10 / 27 / 92\end{array}$ & GRAFPAK-CGM 1.1.2 & $\begin{array}{l}\text { NCR } 3450, \text { NCR } \\
\text { UNIX SVR4 }\end{array}$ \\
\hline & & & $\begin{array}{l}g c g m, n 355 . c g m \\
5280-10 / 27 / 92\end{array}$ & GRAFPAK-CGM 1.1.2 & $\begin{array}{l}\text { NCR } 3550, \text { NCR } \\
\text { UNIX SVR4 }\end{array}$ \\
\hline & & & $\begin{array}{l}\text { gks } 1530 . c g m \\
2368010 / 27 / 92\end{array}$ & GRAFPAK-GKS 4.0 & $\begin{array}{l}\text { IBM RS6000 Model } \\
530, \text { AIX } 3.2\end{array}$ \\
\hline \multirow[t]{5}{*}{$\begin{array}{l}\text { ESRI } \\
\text { Boulder Co }\end{array}$} & $\begin{array}{c}\text { NIST-M-93/006-003 } \\
1 / 26 / 93\end{array}$ & $5 / 5$ & $\begin{array}{l}\text { sun.cgm } 181680 \\
1 / 19 / 93\end{array}$ & ARC/INFO & $\begin{array}{l}\text { SUN SparcStation, } \\
\text { Sun OS 4.1.3 }\end{array}$ \\
\hline & & & $\begin{array}{l}\text { Ibm.cgm } 181680 \\
1 / 19 / 93\end{array}$ & ARC/INFO & $\begin{array}{l}\text { IBM RS6000, AIX } \\
3.2\end{array}$ \\
\hline & & & $\begin{array}{l}\text { dg.cgm } 181680 \\
\text { 1/19/93 }\end{array}$ & ARC/INFO & $\begin{array}{l}\text { Data General } \\
\text { AViON, DG/UX } \\
\text { 5.4.1 }\end{array}$ \\
\hline & & & $\begin{array}{l}\text { dec.cgm } 181680 \\
\text { 1/19/93 }\end{array}$ & ARC/INFO & $\begin{array}{l}\text { DecStation } 5000 \text {, } \\
\text { ULTRIX 4.2a }\end{array}$ \\
\hline & & & $\begin{array}{l}\text { sgi.cgm } 181680 \\
1 / 19 / 93\end{array}$ & ARC/INFO & $\begin{array}{l}\text { Silicon Graphics } \\
\text { Indigo, IRIX 4.0.2 }\end{array}$ \\
\hline \multirow[t]{3}{*}{$\begin{array}{l}\text { EDS } \\
\text { Herndon, VA }\end{array}$} & $\begin{array}{c}\text { NIST-M-93/007-004 } \\
1 / 29 / 93\end{array}$ & $3 / 3$ & $\begin{array}{l}\text { demo5.com } 13280 \\
1 / 28 / 93\end{array}$ & GRAFPAK-GKS 4.0 & $\begin{array}{l}\text { SPARCStation } 10 \\
\text { Model } 30 \text {, Solaris } \\
2.1\end{array}$ \\
\hline & & & $\begin{array}{l}\text { demo7.cgm } 5360 \\
1 / 28 / 93\end{array}$ & GRAFPAK-GKS 4.0 & $\begin{array}{l}\text { SPARCStation } 10 \\
\text { Model } 30 \text {, Solaris } \\
2.1\end{array}$ \\
\hline & & & $\begin{array}{l}\text { demo8.cgm } 3840 \\
1 / 28 / 93\end{array}$ & GRAFPAK-GKS 4.0 & $\begin{array}{l}\text { SPARCStation } 10 \\
\text { Model } 30 \text {, Solaris } \\
2.1\end{array}$ \\
\hline
\end{tabular}




\subsection{PHIGS APPLICATIONS}

VENDOR

PHIGS

NAME

HARDWARE \&
GRAPHICS

DEVICES

REGISTERED ENVIRONMENTS

NONCON-

OP. SYSTEM

No entries at this time. 
$4 \cdot 8$ 


\section{U.S. GOSIP TESTING PROGRAM REGISTER DATABASE SYSTEM (GRD)}

\subsection{Description}

The United States Government Open Systems Interconnection Profile (GOSIP) Testing Program was defined to assist Federal Agencies in assuring conformance to the GOSIP Standard. Testing for conformance to the Open Systems Interconnection (OSI) standards and for interoperability with other OSI implementations is available.

NISTIR 4594, "GOSIP Conformance and Interoperation Testing and Registration" establishes the framework for the establishment of registers for Test Suites, Test Systems (Means of Testing), Conformance Testing Laboratories, and Interoperability Testing Services.

\subsection{U.S. GOSIP Register Database (GRD)}

The U.S. GOSIP Register Database (GRD) is an online database facility developed by NIST. It provides up-to-date reference information for the following list of registers:

1. U.S. GOSIP Abstract Test Suites (ATS).

2. Assessed Means of Testing (MOT).

3. NVLAP Accredited Test Laboratories.

4. Conformance Tested GOSIP Products.

5. Interoperability Test Suites (ITS) for OSI Products.

6. Reference Entities for Means of Testing Assessment(s).

7. Interworking GOSIP Products.

8. Interoperability Test and Registration Services.

\subsection{How To Access the GOSIP Register Database (GRD)}

The GOSIP register files available for downloading are now located a different host. These files are available via anonymous ftp over the internet from:

Host Name: huachuca-jitcosi

IP Address: 138.27.7.2

Files are available in both ASCII (.ask) and WordPerfect 5.1 (.w51) formats. Just login with "anonymous" and use your internet address for the password. For .w51 files, binary transfer is required.

For any questions, problems or comments dealing with the GRD or the U.S. GOSIP Testing Program please contact:

Ken Thomas

Joint Interoperability Test Center - TCBB

Fort Huachuca, AZ 85613-7020

(602) $538-5170$

e-mail: C3A-TCB@huachuca-EMH2.army.mil 



\subsubsection{REGISTER OF CONFORMANCE TESTING LABORATORIES}

Conformance testing laboratories for the U.S. GOSIP Testing Program are listed here. All registered laboratories are deemed qualified to conduct conformance testing for U.S. GOSIP, for the test method identified.

Laboratory Code: 0354

Laboratory Name: Control Data Corporation, OSI Accredited Test Center 4201 North Lexington Avenue Aden Hills, MN 55126-6198

Contact and Phone: Ronald D Swan, Telephone: (612) $482-6257$ Fax: (612) $482-3616$

Scope of Registration: X400-1984 MHS: P2/P1/RTS/(Session), TP4,

TPO, TP2, CLNP, X25:PLP/HDLC LAP-B

Lab Type: Conformance Testing

Registration Type: FULL

Rogistered Until: 01JUL-94

Laboratory Code: 0355

Laboratory Name: Bull HN Conformance Test Center 13430 N Black Canyon Hwy, P.0. Box 8000 Phoenlx, AZ 85029

Contact and Phone: Oscar V Hefner Telephone: (602) 862-6001 Fax: $\quad(602) 862-6051$

Scope of Registration: FTAM/ACSE/Presentation/(Session), X400-1984 MHS: P2/P1/RTS/(Session), TP4, CLNP

Lab Type: Conformance Testing

Registration Type: FULL

Registered Until: 01 JUL-94

Laboratory Code: 0357

Laboratory Name: National Computing Centre Limited Oxford Road

Manchester

M17ED United Kingdom

Contact and Phone: A. E. J. Pink Telephone: +4461 2286333

Fax: $\quad+44612364715$

Scope of Registration: FTAM/ACSE/Presentation/(Session), X400-1984 MHS: P2/P1/RTS/(Session), Lab Type: Conformance Testing Session, TP4, TPO, TP2, CLNP

Registration Type: FULL

Registered Until: 01 JUL-94
Laboratory Codo: 0361

Laboratory: IBM Corportation - Networking Systems Protocol Center

Dept C70/Building 673, P.O. Box 12195

Research Triangle Park, NC $27709-2195$

Contact and Phone: Robert M Amy Telephone: (919) 254-4141 Fax: $\quad$ (919) 2545410

Scope of Reglstration: X.25:PLP/HDLC LAP.B

Lab Type: Conformance Testing

Registration Type: FULL

Registered Until: 01 JUL-94

Laboratory Code: 032

Laboratory Name: Digital Equipment Corp 550 King Street Littleton, MA 01460-1289

Contact and Phone: Richard A Duhamel Telephone: (508)486-5021 Fax: (508) 486-7417

Scope of Registration: FTAM/ACSE/Presentation/(Session), X400-1984 MHS: P2/P1/RTS/(Session), TP4, TP2, TPO, CLNP, X25:PLP/HDLC LAP.B

Lab Type: Conformance Testing

Registration Type: FULL

Registered Until: 01JUL-94

Laboratory Code: 0053

Laboratory Name: Corporation for Open Systems Intemational Test Center 8260 Willow Oaks Corp Drive, Suite 700 Fairfax, VA 22031

Contact and Phone: Andrea Reitzel Telephone: (703) 205-2809 Fax: (703) $846-8590$

Scope of Registration: FTAM/ACSE/Presentation/(Session), X400-1984 MHS:P2/P1/RTS/(Session), TP4, TPO, TP2, CLNP, X.25:PLP/HDLC LAP-B

Lab Type: Conformance Testing

Registration Type: FULL

Registered Until: 01JUL-94 
Laboratory Code: 0354

Laboratory Name: CDA, tncorporated Open Systems Development Group

8301 Greensboro Drive, Suite 610

McLean, VA 22102-3603

Contact and Phone: Kevin P Murray 821-1858 Fax: $\quad$ (703) $821-9859$

Scope of Registration: FTAM/ACSE/Pressentation/(Session), X400-1984 MHS: P2/P1/RTS/(Sossion), TPO, TP2, TP4, CLNP, X25: PLP/HDLC LAP-B

Lab Type: Conformance Testing

Registration Type: FULL

Registered Until: 01JUL-94

Laborationy Code: 0355

Laboratory Name: Hewlett-Packard Company, OSI Conformance Test Conter 19420 Homestead Road

Cupertino, CA $95014-9810$

Contact and Phone: Murali Subbarao Telephone: (408) 447-2822

Fax: $\quad$ (408) $447-3660$

Scope of Registration: FTAM/ACSE/Presentation(Session), X.400-1984 MHS: P2/P1/RTS(Session), Session, TP4, TP2, TPO, CLNP

Lab Type: Conformance Testing

Registration Type: FULL

Registered Until: $\quad$ 01JUL-94

Laboratory Codo: 0567

Laboratory Name: UNISYS - Open Systems Interconnection Laboratory

P.O. Box 203, 2450 Swedesford Road Paoli, PA 19301

Contact and Phone: Andrew Kalish Telephone: (215) 993-7044

Fax: (215) $993-7425$

Scope of Registration: FTAM/ACSE/Presentation/(Session), X.400-1984 MHS: P2/P1/RTS(Session), TP4, TP2, TPO, CLNP, X.25:PLP/HDLC LAP-B

Lab Typo: Conformance Testing

Rogistration Typo: FULL

Registered Until: 01-JUL-94
Laboratory Codo: 0370

Laboratory Name: Conformance Expert Center for OSI Bull CECOB

Rue Jean Jaures, B.P. 68

78430 Les Clayes/Bois

France

Contact and Phone: Gerard Vanderschooten Telephone: + 331

30806811

Fax: $\quad+33130807879$

Scope of Registration: Session, TP4, TPO, TP2, CLNP, 8802.2, 8802.3, X25:PLP/HDLC LAP-B

Lab Type: Conformance Testing

Registration Type: FULL

Registered Until: $\quad 01-0 C T-94$

Laboratory Code: 0371

Laboratory Name: Acatel TITN Incorporated Conformance, Accreditation and Test Conter 7011 Koll Conter Parkway, \# Suite 200 Pleasanton, CA 94566-3101

Contact and Phone: Sanjay P Lokare Telephone: (510) 4845764

Fax: $\quad(510) 484-4078$

Scope of Registration: FTAM/ACSE/Presentation/(Session), X400-1984 MHS: P2/P1/RTS/(Session), Session, TP4, TP2, TPO, CLNP

Lab Type: Conformance Testing

Registration Type: FULL

Registered Until: 01-OCT-94

Laboratory Code: 0385

Laboratory Name: Dept of Defense, Joint Interoperability Test Conter ATTN: TCDBA, Building 57305

Ft. Huachuca, AZ 85613-7020

Contact and Phone: Kenneth Thomas Telephone: (602) $538-5170$ Fax: $\quad(602) 538-4375$

Scope of Registration: FTAM/ACSE/Presentation/(Session) X.400-1984 MHS: P1/P2/RTS/(Session), Session, TP4, TP2, TPO, CLNP, 8802.2/8802.3, X.25:PLP/HDLC LAP-B

Lab Type: Conformance Testing

Rogistration Type: FULL

Registered Until: 01-APR-94 


\section{Laboratory Codo: 0391}

Laboratory Name: Data General Corporation, OSI Conformance Test Center

4400 Computer Drive, MS/D216

Westborough, MA 01580

$\begin{array}{lll}\text { Contact and Phone: } & \text { Charles Stakus } & \begin{array}{l}\text { Telephone: (508) } \\ 870-6392\end{array} \\ \text { Fax: } & \text { (508) } 898-4694 \\ \text { Scope of Registration: } & \text { FTAM/ACSE/Presentation/(Session), } \\ & \text { X400-1984 MHS: P2/P1/RTS/(Session), } \\ \text { Session, TP4, TP2, TPO, CLNP }\end{array}$

Lab Type: Conformance Testing

Rogistration Typo: FULL

Registered Until: $\quad$ 01-OCT-e3 (Did not renow)

Leboratory Code: 002

Laboratory Name: IBM Rome Networking Systems Laboratory Via Paolo DiDono, 44 00144 Rome taly

Contact and Phone: Aberto Sinibaldi Telephone: 396 5966-2281

Fax: $\quad 3965966-2467$

Scope of Registration: FTAM/ACSE/Presentation (Session), X.400-1984 MHS: P2/P1/RTS/(Session), Session, TP4, TP2, TPO, CLNP

Lab Type: Conformance Testing

Registration Type: FULL

Reglstered Until: 01-JUL-94

Laborationy Code: 0394

Laboratory Name: Telecommunications Laboratories Test Center P.O. Box 71

Chung-L, 320

Taiwan

Contact and Phone:

$$
\begin{gathered}
\text { Dr. Ching-Sung w } \begin{array}{c}
\text { Telephone: +886 } 3 \\
424-4377 \\
\text { Fax: }+8863 \\
490-4464
\end{array}
\end{gathered}
$$

Scope of Registration: X.400-1984 MHS: P2/P1/RTS/(Session), FTAM/ACSE/Presentation/(Session), Session, TP4, TP2, TPO, CLNP

Lab Type: Conformance Testing

Registration Typo: FULL

Registered Until: $01-0 C T-94$
Laboratory Code: 0397

Laboratory Name: OSI Conformance Testing Services, ICL Wenlock Way

West Gorton, Manchester

M12 5DR, United Kingdom

$\begin{array}{ll}\text { Contact and Phone: } \quad \text { R G Medley } & \text { Telephone: } 4461 \\ & \text { Fax: } \begin{array}{c}223-1301 \\ 4461223-0482\end{array}\end{array}$

Scope of Registration: FTAM/ACSE/Presentation(Session), X.400-1984 MHS: P2/P1/RTS/(Session), Session, TP4, TP2, TPO, CLNP

Lab Type: Conformance Testing

Registration Type: FULL

Registered Until: 01-APR-94

\subsubsection{REGISTER OF APPROVED US GOSIP MOT VALIDATION LABORATORIES}

MLAP Laboratory Cods: 0385

Laboratory Name: Department of Defense Joint Interoperability Test Center Building $\mathbf{5 7 3 0 5}$

Fort Huachuca, AZ 85613-7020

Contact: Kenneth Thomas Tel (602) 538-5170

MOT Validation Scope of Registration:

FTAM/ACSE/Presentation(Session), Session, MHS 84, MHS 88, TP4, TPO, TP2, CLNP, ES-IS 8802-2/8802-3, X.25 PLP/HDLC, LAB B, ISDN (Layer 2 and 3), X.500, CMIP, IS-IS

Type of Laboratory: MOT Qualification

Type of Reglstration (Full or Provisional): Full

Registered Until: 01-MAR-94

PWE Accreditation Number: $77.90 / 01$

Laboratory Name: ACERL

Domaine Technologique de Saclay

Batiment AZUR

4, rue Rene Razel, SACLAY 91982 ORSAY CEDEX

France

Contact: J-P Baconnet Tel +33160191310 Fax +33160191146

MOT Validation Scope of Registration:

FTAM, MMS, and $8804-4$

Type of Laboratory: MOT Qualification

Type of Registration (Full or Provisional): Full

Registered Until: 01-MAR-94 


\subsubsection{REGISTER OF CONFORMANCE TESTED GOSIP PRODUCTS}

\section{Product Code/Type: P-01 WAN}

Product DD. 174

Supplier: ALCATEL Data Networks, Inc. 12502 Sunrise Valley Drive Reston, VA 22096

Contact: K. Trumble Tol: (703) 689-6287

Fax:

GOSIP Product Name: TP4900/LPM Network Release 6 Version/Release: Release 6

Release Date: 08-JUN-93

Additional Info:

Registration:

Date: 19JUL-93 Basis: BASE

Type: FULL, GOSIP VER 2

Hardware and Operating System Platforms: HW: TP4900/LPM

Connectivity: V.35 OS: Network Rolease 6

Underlying Stack: None

Protocols and Profiles: $\quad$ X.25 PLP/(X.25 LAP-B) X.25 PLP [ISO 8208:1990] \{CCITT 1984\} X.25 LAP-B [ISO 7776:1986] \{CCITT 1984\}

ATS Used: ATS:2-2 AND ATS:2-1

Conformance Lab: NVAP* 0364

CDA, incorporated Open Systems Development Group

Product Code/Type: P-001 WAN

Product 1D: $\quad 175$

Supplier: ALCATEL Data Networks, Inc. 12502 Sunrise Valley Drive Reston, VA 22096

Contact: K. Trumble Tel: (703) 6896287 Fax:

GOSIP Product Name: TP4900/TCP/CF Network Release 6 Version/Release: Rolease 6

Release Date: 08-JUN-93

Additional Info:

Reglstration:

Date: 19JUL-93 Basis: BASE

Type: $\quad$ FULL, GOSIP Version 2

Hardware and Operating System Platforms:

HW: TP4900/TCP/CF

OS: Network Rolease 6

Connectivity: V.35

Underlying Stack: None

Protocols and Profiles: $\quad$ X.25 PLP/(X.25 LAP-B)

X.25 PLP [ISO 8208:1990] \{CCITT 1984\}

X.25 LAP-B [ISO T776:1986] \{CCIT 1984\}

\section{ATS Used: ATS:2-2 AND ATS:2-1}

Conformance Lab: NVAP* 0364

CDA, incorporated Open Systems Development Group
Product Code/Type: P-001 WAN

Product D: $\quad 173$

Supplier: ALCATEL Data Networks, Inc. 12502 Sunrise Valley Drive Reston, VA 22096

Contact: K. Trumble Tel: (703) 689-6287 Fax:

GOSIP Product Name:TP8000 Network Rolease 6

Version/Release: Release 6

Rolease Date: 08-JUN-93

Additional Info:

Registration:

Date: 19JUL-93 Basis: BASE

Type: FULL, GOSIP VER 2

Hardware and Operating System Platforms:

HW: TP8000

OS: Network Rolease 6

Connectivity: RS-232

Underlying Stack: None

Protocols and Profiles: X.25 PLP/( 225 LAP-B)

X.25 PLP [ISO 8208:1990] \{CCIT 1984\}

ATS Used: ATS:2-2 AND ATS:2-1

X.25 LAP-B [ISO T776:1986] \{CCITT 1984\}

Conformance Lab: NVLAP 0364

CDA, Incorporated Open Systems Development Group

Product Code/Typo: P-001 WAN

Product D:

Supplier: AT \& T Computer Systems 307 Middletown - Lncroft Road Lincroft, NW 07738

Contact: Reginald Lowis Tel: (908) 898-6005 Fax: (\$08) 8\$8-3717

GOSIP Product Name:A.T. \& T. X.25 Network Interface Product Version/Release: Release 2.0

Rolease Date: 01JAN-91

Additional Info:

Reglstration:

Date: CoAPR-91 Basis: BASE

Type: $\quad$ PROV, Gmdtthrd, V1-> V2

Hardware and Operating System Platforms:

AT \& T 6386 StarServer S (or StarServer E), UNIX System V, Rolease 4.0; GPSC-AT, or GPSC-AT/E Synchronous Card

Connectivity: RS-232C, V.35, X21bis

Underlying Stack: None

Protocols and Profiles: $\quad$ X.25 PLP/(X25 LAP-B) X.25 PLP [ISO 8208:1990] \{CCITT 1984\} X.25 LAP-B [ISO T776:1986] \{CCIT 1984\}

ATS Usod: ATS:1-1 and ATS:1-2

Conformance Lab: NVAP\# 0363

Corporation for Open Systems international Test Center 
Product Code/Type: P-001 WAN

Product D: $\quad 93$

Supplier: Bull Information Systems Incorporated 13430 North Black Canyon Highway Phoenix, AZ 85020

Contact: Bill George Tel: (602) 862-6008 Fax: (602) 862-6105

GOSIP Product Name: DATANET DCP 7500

Version/Release: DNS Ver 4 U1

Release Date: 01-JAN-92

Additional Info:

Registration:

Date: 13-NOV-92 Basis: BASE

Type: $\quad$ PROV, Gmdfthrd, V1-> V2

Hardware and Operating System Platforms:

DCP 7500

O/S DNS Ver 4 Update 1

Connectivity: RS-232C

Underlying Stack: None

Protocols and Profiles: $\quad$ X25 PLP/(X25 LAP-B) X.25 PLP [ISO 8208:1990] \{CCITT 1984\} X.25 LAP-B [ISO $7776: 1986$ ] \{CCITT 1984\}

ATS Used: ATS:1-1 and ATS:1-2

Conformance Lab: NVLAF 0370

Conformance Expert Conter for OSI Bull - CECOB

Product Code/Typo: P-001 WAN

Product 1D: $\quad 91$

Supplier: Bull Information Systems Incorporated 13430 North Black Canyon Highway Phoonix, AZ 85029

Contact: Bill George Tel: (602) 862-6008

Fax: (602) 862-6105

GOSIP Product Name: DPX/2 B.O.S. (Stack B)

Version/Release: Ver B.O.S. 2

Release Date: 01-OCT-92

Additional Info:

Rogistration:

$\begin{array}{lll}\text { Date: } & \text { 13-NOV-92 } & \text { Basis: BASE } \\ \text { Type: } & \text { PROV, Gmdfthrd, V1-> V2 }\end{array}$

Hardware and Operating System Platforms:

DPX/2 200 with MTB board, O/S B.O.S. 2

Connectivity: RS-232C

Underlying Stack: None

Protocols and Profiles: X.25 PLP/(X.25 LAP-B)

$X .25$ PLP [ISO 8208:1990] \{CCITT 1984\}

X.25 LAP-B [ISO T776:1986] \{CCITT 1984\}

ATS Used: ATS:1-1 and ATS:1-2

Conformance Lab: NVAP* 0370

Conformance Expert Center for OSI Bull - CECOB
Product Code/Type: P-001 WAN

Product D: 92

Supplier: Bull Information Systems Incorporated 13430 North Black Canyon Highway Phoenix, AZ 85029

Contact: Bill George Tel: (602) 862-6008 Fax: (602) $862-6105$

GOSIP Product Name: DPX/2 B.O.S. (Stack B) Version/Release: Ver B.O.S. 2

Release Date: 01-OCT-92

Additional Info:

Registration:

Date: 13-NOV-92 Basis: BASE

Type: $\quad$ PROV, Grndfthrd, V1.> V2

Hardware and Operating System Platforms:

DPX/2 200 with ECP Board, O/S B.O.S. 2

Connectivity: RS-232C

Underlying Stack: None

Protocols and Profiles: $\quad X .25$ PLP/(X.25 LAP.B)

X.25 PLP [ISO 8208:1990] \{CCITT 1984\}

X.25 LAP.B [ISO T776:1986] \{CCITT 1984\}

ATS Usod: ATS:1-1 and ATS:1-2

Conformance Lab: NVAP* 0370

Conformance Expert Center for OSI Bull - CECOB

Product Code/Type: P.01 WAN

Product 1D: 176

Supplier: Cisco Systems

1525 O'Brien Drive, P.O. Box 3075

Menlo Park, CA 94026-1435

Contact: William Miskovetz Tel: (415) 688-4682

Fax: (415) 688-4575

GOSIP Product Name: AGS+ $/ 3$ X.25 Version 2.0

Version/Release: Version 2.0

Release Date: 13UUL-93

Additional Info:

Registration:

Date: 02-AUG-93 Basis: BASE

Type: $\quad$ FULL, GOSIP Version 2

Hardware and Operating System Platforms:

$H W: A G S+/ 3$

OS: $g s 3-k 9.1(30)$

Connectivity: V.35

Underlying Stack: None

Protocols and Profiles: $\quad$ X.25 PLP/(X.25 LAP-B)

X.25 PLP [ISO 8208:1990] \{CCITT 1984\}

X.25 LAP.B [ISO T776:1986] \{CCITT 1984\}

ATS Used: ATS:2-2 AND ATS:2-1

Conformance Lab: NVLAP* 0364

CDA, Incorporated Open Systems Dovelopment Group 
Product Code/Type: P-D01 WAN

Product 1D: 177

Supplier: Cisco Systems 1525 O'Brien Drive, P.O. Box 3075 Menlo Park, CA 94026-1435

Contact: William Miskovetz Tel: (415) 688-4682 Fax: (415) 688-4575

GOSIP Product Name: AGS $+/ 4 \times 25$ Version 2.0

Version/Release: Version 2.0

Release Date: 13-JUL-93

Additional Info:

Rogistration:

Date: 02-AUG-93 Basis: BASE

Type: FULL, GOSIP Version 2

Hardware and Operating System Platforms:

HW: AGS $+/ 4$

OS: gs3-k 9.1(30)

Connectivity: V.35

Underlying Stack: None

Protocols and Profiles: $\quad$ X25 PLP/(X.25 LAP-B) X.25 PLP [ISO 8208:1990] \{CCITT 1984\} X.25 LAP-B [ISO $7776: 1986]$ \{CCITT 1984\}

ATS Used: ATS:2-2 AND ATS:2-1

Conformance Lab: MLAP\# 0364

CDA, Incorporated Open Systems Development Group

Product Code/Type: P-001 WAN

Product 1D: 178

Supplier: Cisco Systems

1525 O'Brien Drive, P.O. Box 3075

Menlo Park, CA 94026-1435

Contact: William Miskovetz Tel: (415) 688-4682

Fax: (415) 688-4575

GOSIP Product Name: CGS/24X.25 Version 2.0

Version/Release: Version 2.0

Release Date: 13-JUL-93

Additional Info:

Registration:

Date: 02-AUG-93 Basis: BASE

Type: $\quad$ FULL, GOSIP Version 2

Hardware and Operating System Platforms:

$H W:$ CGS $/ 3$

OS: gs3-k $9.1(30)$

Connectivity: V.35

Underlying Stack: None

Protocols and Profiles: $\quad$ X.25 PLP/(X.25 LAP-B)

X.25 PLP [ISO 8208:1990] \{CCITT 1984\}

X.25 LAP-B [ISO T776:1986] \{CCITT 1984\}

ATS Used: ATS:2-2 AND ATS:2-1

Conformance Lab: NVAP\# 0364

CDA, Incorporated Open Systems Development Group
Product Code/Type: P-001 WAN

Product D: 179

Supplier: Cisco Systems

1525 O'Brien Drive, P.O. Box 3075

Menlo Park, CA 94026-1435

Contact: William Miskovetz Tel: (415) 688-4682

Fax: (415) 688-4575

GOSIP Product Name: CGS/4 X.25 Version 2.0

Version/Release: Version 2.0

Release Date: 13-JUL-93

Additional Info:

Registration:

Date: 02-AUG-93 Basis: BASE

Type: $\quad$ FULL, GOSIP Version 2

Hardware and Operating System Platforms:

HW: CGS/4

OS: $g s 3-k 9.1(30)$

Connectivity: V.35

Underlying Stack: None

Protocols and Profiles: $\quad$ X25 PLP/(X25 LAP-B)

X25 PLP [ISO 8208:1990] \{CCITT 1984\}

X.25 LAP-B [ISO T776:1986] \{CCITT 1984\}

ATS Used: ATS:2-2 AND ATS:2-1

Conformance Lab: NVAP\# 0364

CDA, Incorporated Open Systems Development Group

Product Code/Type: P-C01 WAN

Product ID: 180

Supplier: Cisco Systems

1525 O'Brien Drive, P.O. Box 3075

Menlo Park, CA 94026-1435

Contact: William Miskovetz Tel: (415) 688-4682

Fax: (415) 688-4575

GOSIP Product Name: CGS/4 X.25 Version 2.0

Version/Release: Version 2.0

Release Date: 13JUL-93

Additional Info:

Registration:

Date: 02-AUG-93 Basis: BASE

Type: $\quad$ FULL, GOSIP Version 2

Hardware and Operating System Platforms:

HW: IGS/R

OS: lgs-kr 9.1(30)

Connectivity: V.35

Underlying Stack: None

Protocols and Profiles: $\quad$ X25 PLP/(X25 LAP-B) X25 PLP [ISO 8208:1990] \{CCITT 1984\}

ATS Used: ATS:2-2 AND ATS:2-1

X.25 LAP-B [ISO 7776:1986] \{CCITT 1984\}

Conformance Lab: NVAP* 0364

CDA, Incorporated Open Systems Development Group 
Product Code/Type: P001 WAN

Product D: 181

Supplier: Cisco Systems

1525 O'Brien Drive, P.O. Box 3075

Menlo Park, CA 94026-1435

Contact: William Miskovetz Tel: (415) 688-4682

Fax: (415) 688-4575

GOSIP Product Name: MGS/3 X.25 Version 2.0

Version/Release: Version 2.0

Release Date: 13-JUL-93

Additional Info:

Registration:

Date: 02-AUG-93 Basis: BASE

Type: $\quad$ FULL, GOSIP Version 2

Hardware and Operating System Platforms:

HM: MGS $/ 3$

OS: g83-k 9.1(30)

Connectivity: RS-232

Underlying Stack: None

Protocols and Profiles: $\quad$ X.25 PLP/ (X25 LAP-B)

X.25 PLP [ISO 8208:1990] \{CCITT 1984\}

X.25 LAP-B [ISO 7776:1986] \{CCITT 1984\}

ATS Usod: ATS:2-2 AND ATS:2-1

Conformance Lab: MVLAP 0364

CDA, Incorporated Open Systems Development Group

Product Code/Type: P-001 WAN

Product D: 182

Supplier: Cisco Systems

1525 O'Brien Drive, P.O. Box 3075

Menlo Park, CA 94026-1435

Contact: William Miskovetz Tel: (415) 688-4682

Fax: (415) 688-4575

GOSIP Product Name: MGS/4 X.25 Version 2.0

Version/Release: Version 2.0

Release Date: 13-JUL-93

Additional Info:

Registration:

$\begin{array}{lll}\text { Date: } & \text { 02-AUG-93 Basis: BASE } \\ \text { Type: } & \text { FULL, GOSIP Version } 2\end{array}$

Hardware and Operating System Platforms:

HW: MGS/4

OS: 9 s3-k $9.1(30)$

Connectivity: RS-232

Underlying Stack: None

Protocols and Profiles: $\quad$ X.25 PLP/(X25 LAP-B)

X.25 PLP [ISO 8208:1990] \{CCITT 1984\}

X.25 LAP-B [ISO $7776: 1986]$ \{CCITT 1984\}

ATS Usod: ATS:2-2 AND ATS:2-1

Conformance Lab: NVLAP 0364

CDA, Incorporated Open Systerns Development Group
Product Code/Type: P-001 WAN

Product D: 31

Supplier: Control Data Corporation 4201 North Lexington Avenue Arden Hills, MN 55126-6198

Contact: Ronald D Swan Tel: (612) $482-6527$ Fax: (612) 465-4996

GOSIP Product Name: CDCNET

Version/Release: Ver 1.6.1 L780A

Release Date: 01-MAR-92

Additional Info:

Rogistration:

Date: 30JAN-92 Basis: BASE

Type: $\quad$ PROV, Gmdthrd, V1-> V2

Hardware and Operating System Platforms:

CDCNET Dovice Interiaco

Connectivity: RS-232C, X.21

Underlying Stack: None

Protocols and Profiles: $\quad$ X.25 PLP/(X.25 LAP.B)

X.25 PLP [ISO 8208:1990] \{CCITT 1984\}

X.25 LAP-B [ISO $7776: 1986$ ] \{CCITT 1984\}

ATS Used: ATS:1-1 and ATS:1-2

Conformance Lab: NMAP\# 0354

Control Data Corporation, OSI Accredited Test Center

Product Code/Typo: P-001 WAN

Product D: $\quad 40$

Supplier: Data General Corporation

4400 Computer Drive, MS/D216

Westborough, MA 01580

Contact: Charles Stakus Tel: (508) 870-6392

Fax: (508) 898-4694

GOSIP Product Name: X.25 for AViiON Systems

Version/Release: Rel 2.20

Release Date: 01-FEB-92

Additional Info:

Registration:

Date: 18-FEB-92 Basis: BASE

Type: $\quad$ PROV, Gindfthrd, V1.> V2

Hardware and Operating System Platforms:

AViON 5000/6000 Series

DG/UX System for AViiON Systems Rov. 5.4.1

Connectivity: RS-232C

Underlying Stack: None

Protocols and Profiles: X.25 PLP/(X.25 LAP-B)

X.25 PLP [ISO 8208:1990] \{CCITT 1984\}

X.25 LAP-B [ISO $7776: 1986]$ \{CCITT 1984\}

ATS Usod: ATS:1-1 and ATS:1-2

Conformanco Lab: MLAP 0363

Corporation for Open Systems International Test Center 
Product Code/Typo: P-001 WAN

Product 1D: 204

Supplier: Data General Corporation 4400 Computer Drive Westboro, MA 01580

Contact: Peter Conway Tel: (508) 898-5000 Fax: $(508) 898-4212$

GOSIP Product Name: X.25 for AViiON Systems

Version/Release: Version 2.40

Rolease Date: 01-OCT-93

Additional Info: Vendor claims support of 1988 X.25.

Registration:

Date: 29NOV-93 Basis: BASE

Type: $\quad$ FULL, GOSIP Version 2

Hardware and Operating System Platforms:

HW: AViiON AV500, AV4000, AV5000, AV6000,

AV7000, AV8000, AV8500, AV 9000

OS: DG/UX System for AViiON Computers, Rovision 5.4R2.10

Connectivity: RS-232

Underlying Stack: None

Protocols and Profiles: $\quad$ X.25 PLP/(X.25 LAP-B)

X.25 PLP [ISO 8208:1990] \{CCITT 1984\}

X.25 LAP.B [ISO $7776: 1986]$ \{CCITT 1984\}

ATS Used: ATS $2-2$ and ATS 2-1

Conformance Lab: NMAP\# 0364

CDA, Incorporated Open Systems Development Group

\section{Product Code/Type: P-001 WNW}

Product D: 161

Supplier: Digital Equipment Corp

550 King Street

Littleton, MA 01460-1289

Contact: Richard A Duhamel

Tel: $(508)$ 486-5021

Fax: (508) $486-7417$

GOSIP Product Name: DEC Wide Area Device Drivers for ULTRIX

(Data Link Layer)

Version/Release: Ver 2.0

Release Date: 01-MAR-92

Additional Info: None

Registration:

Date: 19MAR-93 Basis: BASE

Type: $\quad$ PROV, Gmdthrd, V1-> V2

Hardware and Operating System Platforms:

MicroVAX/NAXstation 3000 Series

DEC ULTRIX Ver 4.2

Connectivity: X21bis

Underlying Stack: None

Protocols and Profiles: X.25 LAP-B [ISO 7776:1986] [CCITT 1984\}

ATS Used: ATS:1-1 and ATS:1-2

Conformance Lab: NVLAF 0362

Digital Equipment Corporation, OSI Conformance Interoperability

Test Center
Product Code/Typo: P-001 WAN

Product D: $\quad 140$

Supplier: Digital Equipment Corp

550 King Street

Littleton, MA 01460-1289

Contact: Richard A Duhamel

Tel: $(508)$ 486-5021

Fax: (508) 486-7417

GOSIP Product Name: DEC X.25 for ULTRIX

Version/Release: Ver 1.0

Rolease Date: 01-MAR-93

Additional Info: None

Registration:

Date: 19MAR93 Basis: BASE

Type: $\quad$ PROV, Grndfthrd, V1-> V2

Hardware and Operating System Platform

MicroVAX/NAXstation 3000 Series

DEC ULTRIX Ver 4.2

Connectivity: X25 LAP-B/X21bis

Underlying Stack: DEC Wide Area Device Drivers for ULTRIX Ver 2.0 01-MAR-92

Protocols and Profiles: X.25 PLP [ISO 8208:1990] \{CCITT 1984\}

ATS Used: ATS:1-1 and ATS:1-2

Conformance Lab: NVLAP* 0362

Digital Equipment Corporation, OSI Conformance Interoperability

Test Center

Product Code/Type: P-001 WAN

Product 1D: 100

Supplier: Digital Equipment Corp

550 King Street

Littleton, MA 01460-1289

Contact: Richard A Duhamel Tel: (508) 486-5021

Fax: (508) 486-7417

GOSIP Product Name: VAX Packetnet System Interface for DECnet-VAX (TM)

Version/Rolease: Ver 5.4 Extensions

Release Date: 01-SEP-91

Additional Info: None

Registration:

Date: 26JAN-93 Basis: BASE

Type: $\quad$ PROV, Gmdthrd, V1->V2

Hardware and Operating System Platforms:

MicroVAX 3800 with DSV11-SA Card VMS Ver 5.4

Connectivity: X.25 LAP.B/X.21bis

Underlying Stack: VAX Wide Area Notwork Device Drivers for DECnet-VAX(TM) Ver 5.4 Extensions

Protocols and Profiles: X.25 PLP [ISO 8208:1990] \{CCITT 1984\}

ATS Used: ATS:1-1 and ATS:1-2

Conformance Lab: NVAP\# 0362

Digital Equipment Corporation, OSI Conformance Interoperability Test Center 


\section{Product Code/Type: P-001 WAN}

Product tD: 147

Supplier: Digital Equipment Corp 550 King Street Littleton, MA 01460-1289

Contact: Richard A Duhamel Tel: $(508)$ 486-5021

Fax: (508) $486-7417$

GOSIP Product Name: VAX Wide Area Networks Device Driver for DECnet-VAX (TM) (Data Link Layer)

Version/Release: Ver 5.4 Extensions

Release Date: 01-SEP-91

Additional Info:

Registration:

$\begin{array}{lll}\text { Date: } & \text { 26-JAN-93 Basis: BASE } \\ \text { Type: } & \text { PROV, Grndfthrd, V1->V2 }\end{array}$

Hardware and Operating System Platforms:

MicroVAX 3800 with DSV11-SA Card

VMS Ver 5.4

Connectivity: $\mathbf{X} 21$ bis

Underlying Stack: None

Protocols and Profiles: X25 LAP-B [ISO 7776:1986] [CCIT 1984\}

ATS Usod: ATS:1-1 and ATS:1-2

Conformance Lab: NVLAP*0362

Digital Equipment Corporation, OSI Conformance Interoperability Test Conter

\section{Product Code/Type: P-001 WAN}

Product D: 172

Supplier: Digital Equipment Corporation Imperial Way

Reading, Berks RG20TE United Kingdom

Contact: Ken Chamberlain Tel:

Fax:

GOSIP Product Name: DEC Network Integration Server Software (DECNIS)

Version/Release: Ver 2.1

Release Date: 30-APR-93

Additional Info:

Rogistration:

Date: 29-JUL-93 Basis: BASE

Type: FULL, GOSIP Version 2

Hardware and Operating System Platforms:

HW: DECNIS 500, DECNIS 600

OS: DECNIS V2.1 with embedded OS

Connectivity: V.35

Underlying Stack: None

Protocols and Profiles: $\quad$ X.25 PLP/(X.25 LAP-B)

X.25 PLP [ISO 8208:1990] \{CCITT 1984\}

X.25 LAP-B [ISO $7776: 1986]$ \{CCITT 1984\}

ATS Usod: ATS:2-1 AND ATS:2-2

Conformance Lab: NVLAP* 0362

Digital Equipment Corporation, OSI Conformance Interoperability

Test Center
Product Code/Type: P-001 WAN

Product DD: 47

Supplier: Encore Computing Corporation 6901 West Sunrise Boulevard

Ft. Lauderdale, FL $33313-4499$

Contact: Augie Gonzales

Tel: (305) 587-2900

Fax: (305) 797-5807

GOSIP Product Name: Encore Infinity 90 Series GPIO I with EnComm X25 and PAD

Version/Rolease: Rov 3.0

Release Date: 01لJUL-92

Additional Info:

Registration:

Date: 24JUN-92 Basis: BASE

Type: $\quad$ PROV, Gmdthrd, V1->V2

Hardware and Operating System Platforms:

ENCORE Infinity 90 Series GPIO with VME Serial

Synchronous Controller (VSSC) Model 8523-443 UMAX 3.0.7

Connectivity: RS-232C

Underlying Stack: None

Protocols and Profiles: $\quad$ X25 PLP/(X25 LAP-B) X25 PLP [ISO 8208:1990] \{CCITT 1984\}

X25 LAP-B [ISO 7776:1986] \{CCITT 1984\}

ATS Usod: ATS: 1-1 and ATS:1-2

Conformance Lab: NVLAP 0364

CDA, Incorporated Open Systems Development Group

Product Code/Type: P-001 WAN

Product D: 18

Supplier: Harris Adacom Corporation

16001 Dallas Parkway

Dallas, TX 75248

Contact: Gregory Prynn Tel: (214) 386-2000

Fax: (214) 386-2524

GOSIP Product Name: Challenger ES/174-10

Version/Release: Rel 2.1

Release Date: 07-OCT-91

Additional Info:

Registration:

Date: 30-OCT-91 Basis: DERIVED

Type: $\quad$ PROV, Gmdfthrd, V1->V2

Hardware and Operating System Platforms:

Challenger ES/174-10 Ver 2.1

Connectivity: RS-232C

Underlying Stack: None

Protocols and Profiles: $\quad$ X.25 PLP/ $($ 225 LAP-B)

$X 25$ PLP [ISO 8208:1990] \{CCITT 1984\}

$X .25$ LAP-B [ISO $7776: 1986$ ] \{CCITT 1984\}

ATS Used: ATS:1-1 and ATS: $1-2$

Conformance Lab: NVLAP\# 0364

CDA, Incorporated Open Systems Development Group 
Product Code/Type: P-001 WAN

Product io:

Supplier: Harris Adacom Corporation 16001 Dallas Parkway

Dallas, TX 75248

Contact: Gregory Prynn Tel: (214) 386-2000

Fax: (214) 386-2524

GOSIP Product Name: Challenger ES/174-20

Version/Release: Rel 2.1

Release Date: 07-OCT-91

Additional Info:

Registration:

Date: 17-OCT-91 Basis: BASE

Type: PROV, Grndfthrd, V1-> V2

Hardware and Operating System Platforms:

Challenger ES/174-20 Rol 2.1

DTE/DCE Environment

Connectivity: RS-232C

Underlying Stack: None

Protocols and Profiles: $\quad$ X.25 PLP/ $(2.25$ LAP-B)

X.25 PLP [ISO 8208:1990] \{CCITT 1984\}

X.25 LAP.B [ISO T776:1986] \{CCITT 1984\}

ATS Used: ATS:1-1 and ATS:1-2

Conformance Lab: NVLAP\# 0364

CDA, Incorporated Open Systems Development Group

Product Code/Type: P-001 WAN

Product 10:

Supplier: Harris Adacom Corporation 16001 Dallas Parkway

Dallas, TX 75248

Contact: Gregory Prynn Tel: (214) 386-2000

Fax: (214) 386-2524

GOSIP Product Name: Challenger ES/174-60

Version/Release: Rel 2.1

Release Date: 07-OCT-91

Additional Info:

Registration:

Date: 30-0CT-91 Basis: DERIVED

Type: $\quad$ PROV, Gmdtthrd, V1->V2

Hardware and Operating System Platforms:

Challenger ES/174-60 Ver 2.1

Connectivity: RS-232C

Underlying Stack: None

Protocols and Profiles: $\quad$ X.25 PLP/(X.25 LAP-B)

X.25 PLP [ISO 8208:1990] \{CCITT 1984\}

X.25 LAP-B [ISO T776:1986] \{CCITT 1984\}

ATS Usod: ATS:1-1 and ATS:1-2

Conformance Lab: NVLAP\# 0364

CDA, Incorporated Open Systems Development Group
Product Code/Type: P-001 WAN

Product D.

45

Supplier: IBM Corporation

P.O. Box 12195

Research Triangle Park, NC 27709-2195

Contact: John P Streck Tel: (919) 254-4360

Fax: (919) 2545410

GOSIP Product Name: IBM 6611 Network Processor Model 140

Version/Release: Ver 1.0

Release Date: 26-JUN-92

Additional Info:

Registration:

Date: 25-MAR-92 Basis: DERIVED

Type: $\quad$ PROV, Gmdfthrd, V1-> V2

Hardware and Operating System Platforms: IBM 6611 Network Processor

Based on IBM AIX Ver 3.2 for RISC Sys/6000

IBM Multiprotocol Network Program

Connectivity: V.24 or RS-232C, V.35, X.21bis

Underlying Stack: None

Protocols and Profiles: $\quad$ X.25 PLP/(X.25 LAP-B) X.25 PLP [ISO 8208:1990] \{CCITT 1984\}

X.25 LAP-B [ISO 7776:1986] \{CCITT 1984\}

ATS Usod: ATS:1-1 and ATS:1-2

Conformance Lab: NVLAP\# 0361

IBM Corportation - Networking Systems Protocol Center

Product Code/Type: P-001 WAN

Product D:

46

Supplier: IBM Corporation

P.O. Box 12195

Research Triangle Park, NC 27709-2195

Contact: John P Streck

Tel: (919) 254-4360

Fax: (919) 2545410

GOSIP Product Name: IBM 6611 Network Processor Model 170

Version/Release: Ver 1.0

Release Date: 26-JUN-92

Additional Info:

Registration:

Date: 25-MAR-92 Basis: DERIVED

Type: $\quad$ PROV, Gmdthrd, V1->V2

Hardware and Operating System Platforms:

IBM 6611 Network Processor

Based on IBM AIX Ver 3.2 for RISC Sys $/ 6000$

IBM Multiprotocol Network Program

Connectivity: V.24 or RS-232C, V.35, X.21bis

Underlying Stack: None

Protocols and Profiles: $\quad$ X.25 PLP/(X.25 LAP-B)

X.25 PLP [ISO 8208:1990] \{CCITT 1984\}

X.25 LAP-B [ISO TT76:1986] \{CCITT 1984\}

ATS Used: ATS:1-1 and ATS:1-2

Conformance Lab: NVLAP\# 0361

IBM Corportation - Networking Systems Protocol Center 
Product Code/Typo: P-DO1 WAN

Product D: 16

Supplier: IBM Corporation

P.O. Box 12195

Research Triangle Park, NC $27709-2195$

Contact: John P Streck Tel: (919) 254-4360

Fax: (919) 254-5410

GOSIP Product Name: IBM AS/400 X25 Communication Support Program

Version/Release: Ver 2 Rel 1

Release Date: 24-MAY-91

Additional Info:

Rogistration:

Date: 25-SEP-91 Basis: BASE

Type: PROV, Grndfthrd, V1-> V2

Hardware and Operating System Platforms:

Processor IBM 9406

OS/400 Ver 2 Rel 1

Connectivity: V.24 or RS-232C, V.35, X.21bis

Underlying Stack: None

Protocols and Profiles: $\quad$ X25 PLP/(X.25 LAP-B)

X.25 PLP [ISO 8208:1990] \{CCITT 1984\}

X25 LAP.B [ISO T776:1986] \{CCIT 1984]

ATS Used: ATS:1-1 and ATS:1-2

Conformance Lab: NVLA * 0361

IBM Corportation - Networking Systems Protocol Center

\section{Product Code/Type: P-001 WAN}

Product D: 15

Supplier: IBM Corporation

P.O. Box 12195

Research Triangle Park, NC 27709-2195

Contact: John P Streck Tel: (919) 254-4360

Fax: (919) 254-5410

GOSIP Product Name: IBM AS/400 X25 Communication Support Program

Version/Roiease: Ver 2 Rol 1

Release Date: 24-MAY-91

Additional Info:

Registration:

Date: 25-SEP-91 Basis: DERIVED

Type: PROV, Gmdfthrd, V1->V2

Hardware and Operating System Platforms:

Processor IBM 9402, 9404

OS/400 Ver 2 Rel 1

Connectivity: V.24 or RS-232C, V.35, X21bis

Underlying Stack: None

Protocols and Profiles: $\quad$ X25 PLP/(X.25 LAP-B)

X.25 PLP [ISO 8208:1990] \{CCITT 1984\}

X25 LAP-B [ISO 7776:1986] \{CCITT 1984\}

ATS Used: ATS:1-1 and ATS:1-2

Conformance Lab: NMAP 0361

IBM Corportation - Networking Systems Protocol Center
Product Code/Typo: P-001 WAN

Product ID: 5

Supplier: IBM Corporation

P.O. Box 12195

Pesearch Triangle Park, NC $27709-2195$

Contact: John P Streck Tel: (919) 254-4360

Fax: (919) 254-5410

GOSIP Product Name: IBM X25 NCP Packet Switching Interface Version/Release: Ver 3 Rel 4

Release Date: 28-JUN-91

Additional Info:

Registration:

Date: 10-JUL-91 Basis: BASE

Type: $\quad$ PROV, Gmdfthrd, V1->V2

Hardware and Operating System Platforms:

Communications Controllers IBM 3745

MVS/XA Notwork Control Program (NCP) V5R4

System Support Program (SSP) V3R6

Virtual Telecommunications Accoss Method (NTAM) Ver 3

Connectivity: V.24 or RS-232C, V.35, X.21bis

Underlying Stack: None

Protocols and Profiles: X.25 PLP/(X.25 LAP-B)

X25 PLP [ISO 8208:1990] \{CCITT 1984\}

X.25 LAP-B [ISO T776:1986] \{CCIT 1984\}

ATS Used: ATS:1-1 and ATS:1-2

Conformance Lab: NVLA* 0361

IBM Corportation - Notworking Systems Protocol Center

Product Code/Typo: P-001 WAN

Product 1D: 2

Supplier: IBM Corporation

P.O. Box 12195

Research Triangle Park, NC 27709-2195

Contact: John P Streck Tol: (919) 254-4360

Fax: (919) 254-5410

GOSIP Product Name: IBM X25 NCP Packet Switching Interface Version/Release: Ver 3 Rol 4

Release Date: 28-JUN-91

Additional Info:

Registration:

Date: 10JUL-91 Basis: BASE

Type: $\quad$ PROV, Grndfthrd, V1-> V2

Hardware and Operating System Platforms:

Communications Controllers IBM 3745

MVS/SP Notwork Control Program (NCP) V5R4

System Support Program (SSP) V3R6

Virtual Telecommunications Access Method (NTAM) Ver 3

Connectivity: V.24 or RS-232C, V.35, X21bis

Underlying Stack: None

Protocols and Profiles: $\quad$ X25 PLP/(X.25 LAP-B)

X25 PLP [ISO 8208:1990] \{CCITT 1984\}

X.25 LAP-B [ISO $7776: 1986]$ \{CCIT 1984\}

ATS Usod: ATS:1-1 and ATS:1-2

Conformance Lab: NVAP\# 0361

IBM Corportation - Networking Systems Protocol Center 


\section{Product Code/Typo: P-001 WAN}

Product D:

8

Supplier: IBM Corporation

P.O. Box 12195

Research Triangle Park, NC $27709-2195$

Contact: John P Streck Tel: (919) 254-4360

Fax: (919) 254-5410

GOSIP Product Name: IBM X.25 NCP Packet Switching Interface Version/Release: Ver 3 Rel 4

Release Date: 28-JUN-91

Additional Info:

Registration:

Date: 10-JUL-91 Basis: DERIVD

Type: $\quad$ PROV, Grndfthrd, V1->V2

Hardware and Operating System Platforms:

Cornmunications Controllers IBM 3745

MVS/ESA Notwork Control Program (NCP) V5R4

System Support Program (SSP) V3R6

Virtual Telecommunications Access Mothod (MTAM) Version 3

Connectivity: V.24 or RS-232C, V.35, X.21bis

Underlying Stack: None

Protocols and Profiles: $\quad$ X.25 PLP/(X.25 LAP-B)

X.25 PLP [ISO 8208:1990] \{CCITT 1984\}

X.25 LAP-B [ISO T776:1986] \{CCITT 1984\}

ATS Usod: ATS:1-1 and ATS:1-2

Conformance Lab: NMAP* 0361

IBM Corportation - Networking Systems Protocol Center

\section{Product Code/Type: P-001 WAW}

Product D:

7

Supplier: IBM Corporation

P.O. Box 12195

Research Triangle Park, NC 27709-2195

Contact: John P Streck Tel: (919) 254-4360

Fax: (919) 254-5410

GOSIP Product Name: IBM X.25 NCP Packet Switching Interface Version/Rolease: Ver 3 Rel 4

Release Date: 28JUN-91

Additional Info:

Registration:

Date: 10-JUL-91 Basis: DERIVED

Type: $\quad$ PROV, Grndfthrd, V1->V2

Hardware and Operating System Platforms:

Comrnunications Controllers IBM 3745

VM/SP Network Control Program (NCP) V5R4

System Support Program (SSP) V3R6

Virtual Telecornmunications Access Method (NTAM) Version 3

Connectivity: V.24 or RS-232C, V.35, X.21bis

Underlying Stack: None

Protocols and Profiles: X.25 PLP/(X.25 LAP-B)

X.25 PLP [ISO 8208:1990] \{CCIT 1984\}

X.25 LAP.B [ISO T776:1986] \{CCIT 1984\}

ATS Used: ATS:1-1 and ATS:1-2

Conformance Lab: NVAP 0361

IBM Corportation - Networking Systems Protocol Center
Product Code/Typo: P-001 WAN

Product D:

Supplier: IBM Corporation

P.O. Box 12195

Research Trlangle Park, NC 27709-2195

Contact: John P Streck Tel: (919) 254-4360

Fax: (919) 254-5410

GOSIP Product Name: IBM X.25 NCP Packet Switching Interface

Version/Release: Ver 3 Rel 4

Release Date: 28JUN-91

Additional Info:

Registration:

Date: 10JUL-91 Basis: DERIVED

Type: $\quad$ PROV, Gmdthrd, V1->V2

Hardware and Operating Systern Platforms:

Communications Controllers IBM 3745

VM/XA Notwork Control Program (NCP) V5R4

System Support Program (SSP) V3R6

Virtual Telecommunications Access Method (NTAM) Version 3

Connectivity: V.24 or RS-232C, V.35, X.21bis

Underlying Stack: None

Protocols and Profiles: X.25 PLP/(X.25 LAP-B)

X.25 PLP [ISO 8208:1990] \{CCIT 1984\}

X.25 LAP-B [ISO T776:1986] \{CCITT 1984\}

ATS Used: ATS:1-1 and ATS:1-2

Conformance Lab: NVLAP* 0361

IBM Corportation - Networking Systems Protocol Center

Product Code/Type: P-001 WAN Product D: 12

Supplier: IBM Corporation

P.O. Box 12195

Research Triangle Park, NC 27709-2195

Contact: John P Strock Tol: (919) 254-4360

Fax: (919) 254-5410

GOSIP Product Name: IBM X.25 NCP Packet Switching Interface Version/Release: Ver 3 Rel 4

Rolease Date: 28JUN-91

Additional Info:

Registration:

Date: 10-JUL-91 Basis: DERIVED

Type: $\quad$ PROV, Grndthrd, V1->V2

Hardware and Operating System Platforms:

Communications Controllers IBM 3720

VM/XA Network Control Program (NCP) V5R4

System Support Program (SSP) V3R6

Virtual Telecommunications Access Method (NTAM) Version 3

Connectivity: V.24 or RS-232C, V.35, X.21bis

Underlying Stack: None

Protocols and Profiles: $\quad$ X.25 PLP/(X.25 LAP-B)

X.25 PLP [ISO 8208:1990] \{CCITT 1984\}

X.25 LAP-B [ISO T776:1986] \{CCITT 1984\}

ATS Used: ATS:1-1 and ATS:1-2

Conformance Lab: NMAP\# 0361

IBM Corportation - Networking Systems Protocol Center 


\section{Product Code/Type: P-001 WAN}

Supplier: IBM Corporation

P.O. Box 12195

Research Triangle Park, NC 27709-2195

Contact: John P Streck Tol: (919) 254-4360

Fax: (919) 254-5410

GOSIP Product Name: IBM X25 NCP Packet Switching Interface Version/Rolease: Ver 3 Rol 4

Release Date: 28-JUN-91

Additional Info:

Registration:

Date: 10JUL-91 Basis: DERIVED

Type: $\quad$ PROV, Gmdthrd, V1-> V2

Hardware and Operating System Platforms:

Communications Controllers IBM 3720

VM/SP Network Control Program (NCP) V5R4

System Support Program (SSP) V3R6

Virtual Telecommunications Access Method (NTAM) Version 3

Connectivity: V.24 or RS-232C, V.35, X.21bis

Underlying Stack: None

Protocols and Profiles: $\quad$ X25 PLP/(X.25 LAP-B) X.25 PLP [ISO 8208:1990] \{CCITT 1984\} X.25 LAP-B [ISO T776:1986] \{CCITT 1984\}

ATS Used: ATS:1-1 and ATS:1-2

Conformance Lab: NVLAP* 0361

IBM Corportation - Notworking Systems Protocol Center

\section{Product Code/Typo: P-001 WN}

Product D:

Supplier: IBM Corporation

P.O. Box 12195

Research Triangle Park, NC 27709-2195

Contact: John P Strock Tol: (919) 254-4360

Fax: (919) 2545410

GOSIP Product Name: IBM X.25 NCP Packet Switching Interface Version/Rolease: Vor 3 Rel 4

Release Date: 28JUN-91

Additional Info: None

Registration:

Date: 10JUL-91 Basis: DERNED

Type: $\quad$ PROV, Grndthrd, V1->V2

Hardware and Operating System Platforms:

Communications Controllers IBM 3720

MVS/XA Network Control Program (NCP) V5R4

System Support Program (SSP) V3R6

Virtual Telecommunications Access Method (NTAM) Version 3

Connectivity: V.24 or RS-232C, V.35, X.21bis

Underlying Stack: None

Protocols and Profiles: $\quad$ X.25 PLP/(X.25 LAP-B)

X.25 PLP [ISO 8208:1990] \{CCITT 1984\}

X25 LAP.B [ISO T776:1986] \{CCITT 1984\}

ATS Used: ATS:1-1 and ATS:1-2

Conformance Lab: NVAP 0361

IBM Corportation - Networking Systems Protocol Center
Product Code/Typo: P-001 WAN

Product 1D:

8

Supplier: IBM Corporation

P.O. Box 12195

Research Triangle Park, NC $27709-2195$

Contact: John P Streck Tel: (919) 254-4360

Fax: (919) 254-5410

GOSIP Product Name: IBM X25 NCP Packet Switching Interface Version/Release: Ver 3 Rel 4

Release Date: 28-JUN-91

Additional Info: None

Rogistration:

Date: 10JUL-91 Basis: DERIVED

Type: $\quad$ PROV, Gmdthrd, V1.>V2

Hardware and Operating System Platforms:

Communications Controllers IBM 3720 MVS/ESA Network Control Program (NCP) V5R4 System Support Program (SSP) V3R6 Virtual Telecommunications Access Method (NTAM) Version 3

Connectivity: V.24 or RS-232C, V.35, X.21bis

Underlying Stack: None

Protocols and Profiles: $\quad$ X.25 PLP/(X.25 LAP-B)

X.25 PLP [ISO 8208:1990] \{CCITT 1984\}

X.25 LAP-B [ISO T776:1986] [CCITT 1984\}

ATS Used: ATS: 1-1 and ATS:1-2

Conformance Lab: NYLAF 0361

IBM Corportation - Networking Systems Protocol Center

Product Code/Typo: P-001 WAN

Product D: 10

Supplier: IBM Corporation

P.O. Box 12195

Research Triangle Park, NC 27709-2195

Contact: John P Streck Tel: (919) 254-4360

Fax: (919) 2545410

GOSIP Product Name: IBM X25 NCP Packet Switching Interface

Version/Release: Ver 3 Rel 4

Release Date: 28-JUN-91

Additional Info: None

Registration:

Date: 10JUL-91 Basis: DERIVED

Type: $\quad$ PROV, Gmdfthrd, V1.> V2

Hardware and Operating System Platforms:

Communications Controllers IBM 3720

VM/XA Network Control Program (NCP) V5R4

System Support Program (SSP) V3R6

Virtual Telecommunications Access Method (NTAM) Version 3

Connectivity: V.24 or RS-232C, V.35, X.21bis

Underlying Stack: None

Protocols and Profiles: $\quad$ X.25 PLP/(X.25 LAP-B)

X.25 PLP [ISO 8208:1990] \{CCIT 1984\}

X25 LAP-B [ISO $7776: 1986]$ \{CCITT 1984\}

ATS Used: ATS:1-1 and ATS:1-2

Conformance Lab: NVLAP\# 0361

IBM Corportation - Networking Systems Protocol Center 
Product Code/Type: P-001 WAN

Product D: 183

Supplier: Intemational Business Machines Corporation 3605 Highway 52 North

Rochester, MN 55901-7820

Contact: Glenn Van Benschoten Tel: (507)253-5723 Fax:

GOSIP Product Name: AS/400 X.25 Communication Support Version/Release: Version 2 Release 3

Release Date: 05-NOV-93

Additional Info:

Registration:

Date: O3-AUG-93 Basis: BASE

Type: $\quad$ FULL, GOSIP Version 2

Hardware and Operating System Platforms:

HW: IBM 9402, 9404, 9406

OS: OS/400 Version 2 Release 3

Connectivity: RS-232C, V.35

Underlying Stack: None

Protocols and Profiles: X.25 PLP/(X.25 LAP-B)

X.25 PLP [ISO 8208:1990] \{ISO 8208\}

X.25 LAP-B [ISO $7776: 1986]$ \{ISO 7776$\}$

ATS Used: ATS:2-2 and ATS:2-1

Conformance Lab: NVLAP 0361

IBM Corportation - Notworking Systems Protocol Center

Product Code/Type: P-001 WAN

Product 1D: 43

Supplier: Intemational Business Machines Corporation 11400 Bumet Road

Austin, TX $78758-3493$

Contact: John P Streck Tol: (919) 254-4360

Fax: (919) 254-5410

GOSIP Product Name: IBM AIX FOR RISC SYSTEM/6000, X.25

WAN SPT, FOR IBM 7015, Al Models

Version/Rolease: Ver 3.2

Rolease Date: 28-FEB-92

Additional Info:

Registration:

$\begin{array}{lll}\text { Date: } & \text { 25-MAR-92 } & \text { Basis: BASE } \\ \text { Type: } & \text { PROV, Grndtthrd, V1->V2 }\end{array}$

Hardware and Operating System Platforms:

RISC System/6000 Products

Operating System IBM AXX Version 3.2 For RISC System $/ 6000$

Connectivity: V.24 or RS-232C, V.35, X.21bis

Underlying Stack: None

Protocols and Profiles: X.25 PLP/(X.25 LAP-B)

X.25 PLP [ISO 8208:1990] \{CCITT 1984\}

X25 LAP-B [ISO T776:1986] \{CCITT 1984\}

ATS Used: ATS:1-1 and ATS:1-2

Conformance Lab: NVAP 0361

IBM Corportation - Networking Systems Protocol Conter
Product Code/Type: P-001 WAN

Product DD:

41

Supplier: Intemational Business Machines Corporation 11400 Bumet Road

Austin, TX 78758-3493

Contact: John P Streck Tel: (919) 254-4360

Fax: (919) 2545410

GOSIP Product Name: IBM AIX for RISC System/6000, X.25 WAN Spt, for IBM 7012, Al Models

Version/Rolease: Ver 3.2

Rolease Date: 28-FEB-92

Additional Info:

Rogistration:

Date: 25-MAR-92 Basis: BASE

Type: $\quad$ PROV, Gmdfthrd, V1->V2

Hardware and Operating System Platforms:

RISC System/6000 Products

Operating System IBM AIX Version 3.2 For RISC System $/ 6000$

Connectivity: V.24 or RS-232C, V.35, X.21bis

Underlying Stack: None

Protocols and Profiles: $\quad$ X.25 PLP/(X.25 LAP-B)

X.25 PLP [ISO 8208:1990] \{CCIT 1984\}

ATS Usod: ATS:1-1 and ATS:1-2

X.25 LAP-B [ISO $7776: 1986$ ] \{CCITT 1984\}

Conformance Lab: NVLAP*0361

IBM Corportation - Networking Systems Protocol Center

Product Code/Type: P-001 WAN

Product D: 4

Supplier: Intemational Business Machines Corporation 11400 Bumet Road

Austin, TX $78758-3493$

Contact: John P Streck Tel: (919) 254-4360

Fax: (919) 2545410

GOSIP Product Name: IBM AX for RISC System/6000, X.25 WAN

Spt, for IBM 7016, Al Models

Version/Rolease: Ver 3.2

Release Date: 28-FEB-92

Additional Info:

Registration:

Date: 25-MAR-92 Basis: BASE

Type: PROV, Gmdfthrd, V1->V2

Hardware and Operating System Platforms:

RISC System/6000 Products

Operating System IBM AIX Version 3.2 for RISC System $/ 6000$

Connectivity: V.24 or RS-232C, V.35, X.21bis

Underlying Stack: None

Protocols and Profiles: $\quad$ X.25 PLP/(X.25 LAP-B) X.25 PLP [ISO 8208:1990] \{CCITT 1984\}

X.25 LAP-B [ISO T776:1986] \{CCITT 1984\}

ATS Used: ATS:1-1 and ATS:1-2

Conformance Lab: NVLAP 0361

IBM Corportation - Networking Systems Protocol Center 
Product Code/Typo: P-CO1 WAN

Product iD: 30

Supplier: International Business Machines Corporation 11400 Burnet Road

Austin, TX 78758-3493

Contact: John P Streck Tel: (919) 254-4360

Fax: (919) 2545410

GOSIP Product Name: IBM AIX for RISC System/6000, X.25 WAN Support, IBM 7011, All Models

Version/Rolease: Vor 3.2

Release Date: 28-FEB-92

Additional Info:

Registration:

Date: 25-MAR-92 Basis: BASE

Type: $\quad$ PROV, Gmdfthrd, V1-> V2

Hardware and Operating System Platforms:

RISC System/6000 Products

Operating System IBM AX Version 3.2 For RISC System $/ 6000$

Connectivity: V.24 or RS-232C, V.35, X.21bis

Underlying.Stack: None

Protocols and Profiles: $\quad$ X.25 PLP/(X.25 LAP-B) X.25 PLP [ISO 8208:1990] \{CCITT 1984\}

X.25 LAP-B [ISO $7776: 1986]$ \{CCITT 1984\}

ATS Usod: ATS:1-1 and ATS:1-2

Conformance Lab: NVLAP 0361

IBM Corportation - Networking Systems Protocol Center

\section{Product Code/Typo: P-001 WAN}

Product 1D: 42

Supplier: Intemational Business Machines Corporation 11400 Bumet Road

Austin, TX $78758-3493$

Contact: John P Streck Tel: (919) 254-4360

Fax: (919) 254-5410

GOSIP Product Name: IBM AVX for RISC System/6000, X.25 WAN

Support, for IBM 7013, Al Models

Version/Release: Ver 3.2

Release Date: 28-FEB-92

Additional Info:

Registration:

Date: 25-MAR-92 Basis: BASE

Type: $\quad$ PROV, Grndfthrd, V1->V2

Hardware and Operating System Platforms:

RISC System/6000 Products Operating System IBM ALX Version 3.2 For RISC System $/ 6000$

Connectivity: V.24 or RS-232C, V.35, X.21bis

Underlying Stack: None

Protocols and Profiles: X.25 PLP/(X.25 LAP-B)

X.25 PLP [ISO 8208:1990] \{CCITT 1984\}

X.25 LAP.B [ISO $7776: 1986]$ \{CCITT 1984\}

ATS Used: ATS:1-1 and ATS:1-2

Conformance Lab: MVAP\# 0361

IBM Corportation - Networking Systems Protocol Center
Product Code/Typo: P.001 WAN

Product 10: 102

Supplier: International Business Machines Corporation 3605 Highway 52 North

Rochester, MN 55901-7829

Contact: John P Streck Tel: (919) 254-4360

Fax: (919) 2545410

GOSIP Product Name: IBM AS/400 X.25

Version/Rolease: Ver 2 Rol 2

Release Date: 25-SEP-92

Additional Info:

Registration:

Date: 13-NOV-92 Basis: BASE

Type: $\quad$ PROV, Gmdfthrd, V1->V2

Hardware and Operating System Platforms:

AS/400 Model IBM 9404

OS/400 Ver 2 Rel 2

Connectivity: RS-232C, V.35

Underlying Stack: None

Protocols and Profiles: $\quad$ X.25 PLP/(X.25 LAP-B) X.25 PLP [ISO 8208:1990] \{CCITT 1984\}

$X .25$ LAP-B [ISO T776:1986] \{CCIT 1984\}

ATS Usod: ATS:1-1 and ATS:1-2

Conformance Lab: NVAP* 0361

IBM Corportation - Networking Systems Protocol Center

Product Code/Type: P-001 WAN

Product 10. 103

Supplier: Intemational Business Machines Corporation 3605 Highway 52 North

Rochester, MN 55901-7829

Contact: John P Strock Tol: (919) 254-4360

Fax: (919) 254-5410

GOSIP Product Name: IBM AS/400 X25 Communication Support Version/Release: Ver 2 Rel 2

Rolease Date: 26-JUN-92

Additional Info:

Registration:

Date: 13-NOV-92 Basis: DERIVE

Type: $\quad$ PROV, Grndfthrd, V1->V2

Hardware and Operating System Platforms:

AS/400 Model IBM 9402, 9406

OS/400 Ver 2 Rel 2

Connectivity: RS-232C, V.35

Underlying Stack: None

Protocols and Profiles: $\quad$ X25 PLP/(X.25 LAP-B)

X.25 PLP [ISO 8208:1990] \{CCITT 1984\}

X.25 LAP-B [ISO T776:1986] \{CCITT 1984\}

ATS Used: ATS:1-1 and ATS:1-2

Conformance Lab: NVLA* 0361

IBM Corportation - Networking Systems Protocol Center 
Supplier: Intemational Business Machines Corporation 3605 Highway 52 North Rochester, MN 55901-7820

Contact: John P Streck Tel: (919) 254-4360

Fax: (919) 254-5410

GOSIP Product Name: IBM AS/400 X25 Communlcations Support Version/Release: Ver 2, Rol 1.1

Release Date: 26-JUN-92

Additional Info:

Registration:

$\begin{array}{lll}\text { Date: } & \text { 13-NOV.92 } & \text { Basis: DERNED } \\ \text { Type: } & \text { PROV, Gmdfthrd, V1->V2 }\end{array}$

Hardware and Operating System Platforms:

IBM AS/400 Models 9402, 9404, 9406

OS/400 Ver 2 Rol 1.1

Connectivity: RS-232C, V.35

Underlying Stack: None

Protocols and Profiles: $\quad$ X.25 PLP/ $\times .25$ LAP-B) X.25 PLP [ISO 8208:1990] \{CCITT 1984\}

X.25 LAP-B [ISO T776:1986] \{CCIT 1984\}

ATS Usod: ATS:1-1 and ATS:1-2

Conformance Lab: NMAP 0361

IBM Corportation - Networking Systems Protocol Center

\section{Product Code/Type: P-001 WAN}

Product 1D: 104

Supplier: Intemational Business Machines Corporation 3605 Highway 52 North Rochester, MN 55901.7829

Contact: John P Streck Tel: (919) 254-4360

Fax: (919) 2545410

GOSIP Product Name: X25 Network Control Program Packet Switching Interface

Version/Rolease: Ver 3 Rel 5

Release Date: 25-SEP-92

Additional Info:

Registration:

Date: 13-NOV-92 Basis: BASE

Type: $\quad$ PROV, Grndfthrd, V1->V2

Hardware and Operating System Platforms:

IBM 3745 Communication Controller Network Control Program Ver 6

Connectivity: RS-232C, V.35

Underlying Stack: None

Protocols and Profiles: $\quad$ X.25 PLP/(X.25 LAP-B)

X.25 PLP [ISO 8208:1990] \{CCITT 1984\}

X.25 LAP-B [ISO TT76:1986] \{CCITT 1984\}

ATS Used: ATS:1-1 and ATS:1-2

Conformance Lab: NVAP\# 0361

IBM Corportation - Networking Systems Protocol Center
Product Code/Type: P-001 WAN

Product D: 162

Supplier: International Business Machines Corporation P.O. Box 12195

Research Triangle Park, NC 27709

Contact: Miriam Green Tel: (919) $254-6323$ Fax:

GOSIP Product Name: X25 Network Control Program Packet Switchlng Interface

Version/Release: Version 3 Release 6

Rolease Date: 30-APR-93

Additional Info:

Registration:

Date: 28-MAY-93 Basis: BASE

Typo: $\quad$ FULL, GOSIP VER 2

Hardware and Operating System Platforms:

IBM 3745

Network Control Program Ver 6 Rel 2

Connectivity: V.24 or RS-232C (X.21bis), V.35 X.21 switched and non-switched ISDN via X.21 (IBM 7820 Terminal Adaptor)

Underlying Stack: None

Protocols and Profiles: $\quad$ X.25 PLP/(X.25 LAP-B) X25 PLP [ISO 8208:1990] \{ISO 8208\} X25 LAP-B [ISO $7776: 1986]$ \{ISO 7776$\}$

ATS Used: ATS:2-1 and ATS:2-2

Conformance Lab: NMLA* 0361

IBM Corportation - Networking Systems Protocol Center

Product Code/Type: P-001 WAN

Product D: 22

Supplier: MEMOREX TELEX Corporation - Federal Systems 205 Van Buren Steet, Suite $\# 180$ Hemdon, VA 22070

Contact: Kevin Good Tel: (703) 318-5600 Fax: (703) 318-7575

GOSIP Product Name: 1174-10R

Version/Rolease: Ver B1.3

Release Date: 17-OCT-91

Additional Info:

Registration:

Date: $\quad 30-0 C T-91 \quad$ Basis: DERIVED

Type: $\quad$ PROV, Gmdfthrd, V1->V2

Hardware and Operating System Platforms: 1174-10R Ver B1.3

Connectivity: RS-232C

Underlying Stack: None

Protocols and Profiles: $\quad$ X.25 PLP/(X.25 LAP-B) X.25 PLP [ISO 8208:1990] \{CCITT 1984\} X.25 LAP-B [ISO T776:1986] \{CCITT 1984\}

ATS Used: ATS:1-1 and ATS:1-2

Conformance Lab: NMLAF 0364

CDA, Incorporated Open Systems Development Group 
Product Code/Typo: P-001 WAN

Product D: $\quad 20$

Supplier: MEMOREX TELEX Corporation - Foderal Systems 205 Van Buren Steet, Suite \#180 Herndon, VA 22070

Contact: Kevin Good Tel: (703) 318-5600

Fax: (703) 318-7575

GOSIP Product Name: 1174-60R

Version/Release: Ver B1.3

Release Date: 17-OCT-91

Additional Info:

Registration:

$\begin{array}{lll}\text { Date: } & 30-O C T-91 & \text { Basis: BASE } \\ \text { Type: } & \text { PROV, Grndfthrd, V1->V2 }\end{array}$

Hardware and Operating System Platforms:

1174-60R Ver B1.3

Connectivity: RS-232C

Underlying Stack: None

Protocols and Profiles: $\quad$ X.25 PLP/(X.25 LAP-B)

X.25 PLP [ISO 8208:1990] \{CCITT 1984\}

X.25 LAP.B [ISO TT76:1986] \{CCITT 1984\}

ATS Used: ATS:1-1 and ATS:1-2

Conformance Lab: NMAP 0364

CDA, Incorporated Open Systems Development Group

Product Code/Typo: P-001 WAN

Product D:

Supplier: MEMOREX TELEX Corporation - Foderal Systems 205 Van Buren Steet, Suite $\# 180$ Hemdon, VA 22070

Contact: Kevin Good Tel: (703) 318-5600

Fax: (703) 318-7575

GOSIP Product Name: 1174-9OR

Version/Release: Ver B1.3

Release Date: 17-OCT-91

Additional Info:

Registration:

Date:

30-OCT-91 Basis: DERIVED

Type:

PROV, Gmofthrd, V1-> V2

Hardware and Operating System Platforms:

1174-90R Ver B1.3

Connectivity: RS-232C

Underlying Stack: None

Protocols and Profiles: $\quad$ X25 PLP/(X.25 LAP-B) X.25 PLP [ISO 8208:1990] \{CCITT 1984\} X.25 LAP.B [ISO TT76:1986] \{CCITT 1984\}

ATS Usod: ATS:1-1 and ATS:1-2

Conformance Lab: NVAP\# 0364

CDA, Incorporated Open Systems Development Group
Product Code/Type: P-001 WAN

Product 1D: 29

Supplier: McData Corporation 310 Interlocken Parkway Broomfield, CO $80021-3464$

Contact: Steve Cartwright Tel: (303) 460-9200

Fax:

GOSIP Product Name: LinkMaster 7100 Model 10 Version/Release: Rel 3.0

Release Date: 11-NOV-91

Additional Info:

Registration:

Date: 20-JAN-92 Basis: DERIVED

Type: $\quad$ PROV, Grndfthrd, V1-> V2

Hardware and Operating System Platforms:

LinkMaster 7100 Model 10

Connectivity: RS-232C, V.35, X.21

Underlying Stack: None

Protocols and Profiles: X.25 PLP/(X.25 LAP.B) X.25 PLP [ISO 8208:1990] \{CCITT 1984\} X.25 LAP.B [ISO TT76:1986] \{CCITT 1984\}

ATS Used: ATS:1-1 and ATS:1-2

Conformance Lab: NVLAP\# 0364

CDA, Incorporated Open Systems Development Group

Product Code/Typo: P-001 WAN

Product D:

27

Supplier: McData Corporation

310 Interlocken Parkway

Broomfield, CO $80021-3464$

Contact: Steve Cartwright Tel: (303) $460-9200$

Fax:

GOSIP Product Name: LinkMaster 7100 Model 2OR

Version/Release: Rel 3.0

Release Date: 11-NOV-91

Additional Info:

Registration:

Date: 17-DEC-91 Basis: BASE

Type: PROV, Gmdfthrd, V1->V2

Hardware and Operating System Platforms:

LinkMaster 7100 Model 20R

Connectivity: RS-232C, V.35, X.21

Underlying Stack: None

Protocols and Profiles: $\quad$ X.25 PLP/(X.25 LAP-B)

X.25 PLP [ISO 8208:1990] \{CCITT 1984\}

X25 LAP.B [ISO T776:1986] \{CCITT 1984\}

ATS Used: ATS:1-1 and ATS:1-2

Conformance Lab: NVAP\# 0364

CDA, Incorporated Open Systems Development Group 
Product Code/Typo: R-001 WAN

Product D:

Supplier: McData Corporation

310 Interlocken Parkway

Broomfield, CO 80021-3464

Contact: Steve Cartwright Tel: (303) 460-9200

Fax:

GOSIP Product Name: LinkMaster 7100 Model 60

Version/Rolease: Rel 3.0

Release Date: 11-NOV-91

Additional Info:

Registration:

Date: 29JAN-92 Basis: DERNED

Type: $\quad$ PROV, Grndfthrd, V1->V2

Hardware and Operating System Platforms:

LinkMaster 7100 Model 60

Connectivity: RS-232C, V.35

Underlying Stack: None

Protocols and Profiles: $\quad$ X.25 PLP/(X.25 LAP-B)

X.25 PLP [ISO 8208:1990] \{CCIT 1984\}

X.25 LAP-B [ISO T776:1986] \{CCITT 1984\}

ATS Usod: ATS:1-1 and ATS:1-2

Conformance Lab: NVAP* 0364

CDA, Incorporated Open Systems Development Group

\section{Product Code/Typo: P-001 WAN}

Product 1D: 28

Supplier: McData Corporation

310 Interlocken Parkway

Broomfield, CO $80021-3464$

Contact: Steve Cartwright Tel: (303) $460-9200$

Fax:

GOSIP Product Name: LinkMaster 7100 Model 90

Version/Release: Rel 3.0

Release Date: 11-NOV-91

Additional Info:

Registration:

$\begin{array}{lll}\text { Date: } & \text { 29JAN-92 } \quad \text { Basis: DERIVED } \\ \text { Typo: } & \text { PROV, Grndfthrd, V1-> V2 }\end{array}$

Hardware and Operating System Platforms:

LinkMaster 7100 Model 90

Connectivity: RS-232C, V.35

Underlying Stack: None

Protocols and Profiles: $\quad$ X.25 PLP/(X.25 LAP-B)

X25 PLP [ISO 8208:1990] \{CCITT 1984\}

X.25 LAP-B [ISO T776:1986] \{CCITT 1984\}

ATS Used: ATS:1-1 and ATS:1-2

\section{Conformance Lab: NVLAP 0364}

CDA, Incorporated Open Systems Development Group
Product Code/Type: P-001 WAN

Product D. $\quad 180$

Supplier: NCR

9900 Old Grove Road

San Diego, CA 92131

Contact: Wendy Morrison Tel: (619) 693-5665

Fax: (619) 693-5705

GOSIP Product Name: NCR MUOE HDLC

Version/Release: Rolease 1.04

Rolease Date: 01-AUG-92

Additional Info: Requires NCR System 3000, X25 Network Services product for full $X .25$ capability.

Registration:

Date: $\quad$ OOSEP-92 Basis: BASE

Typo: $\quad$ PROV, Gmdfthrd, V1->V2

Hardware and Operating System Platforms:

HW: NCR System 3000 consisting of the following hardware models: $3320,3340,3345,3447,3450,3550$, and 3600

OS: NCR UNIX SVRA, (MP-RAS), Rol 2

Connectivity: RS-232

Underlying Stack: None

Protocols and Profiles: $\quad$ X.25 LAP-B [ISO 7776:1986] \{ISO T776\}

ATS Used: ATS:1-1

Conformance Lab: NVLA\# 0363

Corporation for Open Systems Intemational Test Center

Product Code/Type: P-001 WAN

Product D: $\quad 62$

$\begin{array}{ll}\text { Supplier: } & \text { NCR } \\ & \text { 9900 Old Grove Road } \\ & \text { San Diego, CA } 92131\end{array}$

Contact: Wendy Morrison Tel: (619) 693-5665

Fax: (619) $693-5705$

GOSIP Product Name: NCR System 3000, X.25 Network Services Version/Release: Release 1.04

Release Date: 01-AUG-92

Additional Info: Packet Layer Protocol Only.

Registration:

Date: 09 SEP-92 Basis: BASE

Type: $\quad$ PROV, Gmdfthrd, V1->V2

Hardware and Operating System Platforms:

HW: NCR System 3000 consisting of the following hardware models: $3320,3340,3345,3447$,

3450, 3550, and 3600 OS: NCR UNIX SVR4, (MP-RAS), Rol 2

Connectivity: X.25 LAP-B/RS-232

Underlying Stack: NCR MUOE HDLC, Release 1.04

Protocols and Profiles: X.25 PLP [ISO 8208:1990] $\{$ ISO 8208\}

ATS Used: ATS:1-2

Conformance Lab: NVLAP\# 0363

Corporation for Open Systems Intemational Test Center 


\section{Product Code/Typo: P-001 WNN}

Supplier: NETRIX Corporation 13595 Dulles Technology Drive Hemdon, VA 22071

Contact: Ted Ritter Tel: (703) $742-6000$

Fax: $(703) 742-4048$

GOSIP Product Name: NETRIX *1-ISS GOSIP X.25, GOSIP X.25 INTERFACE MODULE

Version/Release: Ver 1.0 Rel 1

Release Date: 01-SEP-92

Additional Info:

Registration:

Date: 06-0CT-92 Basis: BASE

Type: $\quad$ PROV, Gmdfthrd, V1-> V2

Hardware and Operating System Platforms:

Netrix *1-ISS Series 1.0, Notrix Operating System Rol 2.7

Connectivity: RS-232C

Underlying Stack: None

Protocols and Profiles: $\quad$ X.25 PLP/(X.25 LAP.B)

X.25 PLP [ISO 8208:1990] \{CCITT 1984\}

X.25 LAP.B [ISO TT76:1986] \{CCITT 1984\}

ATS Used: ATS:1.1 and ATS:1-2

Conformance Lab: NVLAP 0364

CDA, Incorporated Open Systems Development Group

\section{Product Code/Type: P-001 WAN}

Product D. 143

Supplier: NETRIX Corporation

13595 Dulles Technology Drive

Hemdon, VA 22071

Contact: Ted Ritter Tel: (703) 742-6000

Fax: $(703) 742-4048$

GOSIP Product Name: NETRIX 1-ISS GOSIP X.25, GOSIP X.25 INTERFACE MODULE

Version/Release: Ver 1.0 Rel 1

Release Date: 01-SEP-92

Additional Info:

Registration:

Date: 31-MAR-93 Basis: DERNED

Type: $\quad$ PROV, Gmdithrd, V1->V2

Hardware and Operating System Platforms:

Netrix 1-ISS Series 1000

Notrix OS Rol 1.1

Connectivity: RS-232C

Underlying Stack: None

Protocols and Profiles: $\quad$ X.25 PLP/ $\left.\chi_{225} L A P \cdot B\right)$ $X .25$ PLP [ISO 8208:1990] \{CCITT 1984\}

X.25 LAP.B [ISO T776:1986] \{CCIT 1984\}

ATS Usod: ATS:1-1 and ATS:1-2

\section{Conformance Lab: NVLAP 0364}

CDA, Incorporated Open Systems Development Group
Product Code/Typo: P-001 WAN

Product D: 165

Supplier: NETRIX Corporation 13595 Dulles Technology Drive Hemdon, VA 22071

Contact: Ted Ritter Tel: (703) 742-6000

Fax: $(703) 742-4048$

GOSIP Product Name: Netrix BRX GOSIP X.25 Interface Module 1.0 Version/Release: Version 1.0

Release Date: 01JUN-93

Additional Info:

Registration:

Date: 24JUN-93 Basis: BASE

Type: FULL, GOSIP Version 2

Hardware and Operating System Platforms:

H/W BRX Branch Router/Concentrator O/S BRX O/S Rel 3.3

Connectivity: V.35

Underlying Stack: None

Protocols and Profiles: X.25 PLP/(X25 LAP-B) X.25 PLP [ISO 8208:1990] \{CCIT 1984\}

ATS Usod: ATS:2-2 and ATS:2-1 X.25 LAP-B [ISO T776:1986] \{CCIT 1984\}

Conformance Lab: NVAP* 0364

CDA, Incorporated Open Systems Development Group

Product Code/Type: P-001 WAN

Product D: 164

Supplier: NETRIX Corporation 13595 Dulles Technology Drive Hemdon, VA 22071

Contact: Ted Ritter Tel: (703) 742-6000 Fax: $(703) 742-4048$

GOSIP Product Name: Netrix S100 GOSIP X.25 Interface Module 1.0 Version/Release: Version 1.0

Release Date: 01JUN-93

Additional Info:

Registration:

Date: 24JUN-93 Basis: BASE

Type: $\quad$ FULL, GOSIP Version 2

Hardware and Operating System Platforms:

HW: Netrix Series 100

OS: S100 O/S Rol 2.1

Connectivity: RS-232

Underiying Stack: None

Protocols and Profiles: X.25 PLP/(X.25 LAP.B) X25 PLP [ISO 8208:1990] \{CCITT 1984\}

X.25 LAP-B [ISO T776:1986] \{CCITT 1984\}

ATS Used: ATS:2-2 and ATS:2-1

Conformance Lab: NVLAP\# 0364

CDA, incorporated Open Systems Development Group 
Product Code/Typo: P-001 WN

Product D: 168

Supplier: Northern Telecom ATTN: NORTEL Federal Systems 2010 Corporate Ridge, Suite 800

McLean, VA 22102

Contact: Torre Abritton Tel: (703) 712-8764 Fax: (703) $712-8982$

GOSIP Product Name: Magellan DPN-100 Version/Release: G30S002

Release Date: 01-JAN-93

Additional Info:

Registration:

Date: 08JUL-93 Basis: BASE

Typo: $\quad$ FULL, GOSIP Version 2

Hardware and Operating System Platforms:

HW: Magellan DPN-100

OS: Magellan DPN-100 G30

Connectivity: RS-232

Underlying Stack: None

Protocols and Profiles: X.25 PLP/(X.25 LAP-B)

X25 PLP [ISO 8208:1990] \{ISO 8208\}

X.25 LAP-B [ISO $7776: 1986]$ \{ISO 7776$\}$

ATS Usod: ATS:2-2 and ATS:2-1

Conformance Lab: NVLAP 0363

Corporation for Open Systems Intemational Test Center

Product Code/Type: P-001 WAN

Product D: 152

Supplier: SUN MicroSystems, Inc. International Centre for Network Computing

32 Rue du Vieux Chene

F-38240 Moylan, France

Contact: Tom Hull Tel: +3376414218

Fax: +3376414241

GOSIP Product Name: SUNLink X.25 8.0 Rev B

Version/Release: 8.0 Rev B

Release Date: 01-JUN-93

Additional Info:

Registration:

Date: O6-MAY-93 Basis: BASE

Type: FULL, GOSIP VER 2

Hardware and Operating System Platforms:

HW: SPARCstation 10 Model 41 OS: Solaris 2.1

Connectivity: RS-232C

Underlying Stack: None

Protocols and Profiles: $\quad$ X.25 PLP/(X.25 LAP-B) X.25 PLP [ISO 8208:1990] \{CCITT 1984\}

X25 LAP-B [ISO T776:1986] \{CCITT 1984\}

ATS Used: ATS:2-1 and ATS:2-2

Conformance Lab: NMLAP\# 0364

CDA, Incorporated Open Systems Development Group
Product Code/Type: P-001 WAN

Product 1D: $\quad 65$

Supplier: SUN MicroSystems, Inc. Intemational Centre for Network Computing

32 Rue du Vieux Chene

F.38240 Meylan, France

Contact: Tom Hull Tel: +3376 414218

Fax: +3376414241

GOSIP Product Name: SUNNet X.25

Version/Release: Ver 7.0.1 Rel 1

Release Date: 01-OCT-92

Additional info:

Registration:

Date: 07-OCT-92 Basis: BASE

Type: $\quad$ PROV, Gmdthrd, V1->V2

Hardware and Operating System Platforms:

SUN 4/75

SUNNet O/S 4.1.2 (Solaris 1.0.1)

Connectivity: RS-232C

Underlying Stack: None

Protocols and Profiles: X.25 PLP/(X.25 LAP-B)

X.25 PLP [ISO 8208:1990] \{CCITT 1984\}

X.25 LAP-B [ISO T776:1986] \{CCIT 1984\}

ATS Usod: ATS:1-1 and ATS:1-2

Conformance Lab: NMAP* 0364

CDA, Incorporated Open Systems Dovelopment Group

Product Code/Typo: P-001 WAN

Product 1D: 151

Supplier: Sync Research, Incorporated

7 Studebaker

Invine, CA 92718

Contact: Sal Mungo Tel: (714) 588-2070

Fax: (714) $588-2080$

GOSIP Product Name: Network Access Concentrator (NAC) 4000 Version/Release: Ver 8.150

Release Date: 31-MAY-93

Additional Info:

Registration:

Date: 29-APR-93 Basis: BASE

Type: $\quad$ FULL, GOSIP VER 2

Hardware and Operating System Platforms:

Network Access Concentrator (NAC) $\mathbf{4 0 0 0}$ Proprietary

Connectivity: V.24, RS-232C, V.35

Underlying Stack: None

Protocols and Profiles: $\quad$ X.25 PLP/(X.25 LAP-B) X.25 PLP [ISO 8208:1990] \{CCIT 1984\}

X.25 LAP-B [ISO TT76:1986] \{CCITT 1984\}

ATS Used: ATS:2-1 and ATS:2-2

Conformance Lab: NVLAP* 0361

IBM Corportation - Networking Systems Protocol Center 
Product Code/Type: P-001 WAN

Product D. 153

Supplier: Sync Research, Incorporated 7 Studebaker

Irvine, CA 92718

Contact: Sal Mungo Tel: (714) 588-2070 Fax: (714) 588-2080

GOSIP Product Name: SNA Network Access Converter (SNAC) 6000 Version/Rolease: Ver 10.010

Release Date: 30-SEP-93

Additional Info:

Registration:

$\begin{array}{ll}\text { Date: } & \text { O6-MAY-93 Basis: DERVED } \\ \text { Type: } & \text { FULL, GOSIP VER } 2\end{array}$

Hardware and Operating System Platforms:

Notwork Access Concentrator (NAC) 4000 SNAC 6000 Proprietary

Connectivity: V.24, RS-232C, V.35

Underlying Stack: None

Protocols and Profiles: $\quad$ X.25 PLP/( 2.25 LAP-B) X.25 PLP [ISO 8208:1990] \{CCITT 1984\} X25 LAP-B [ISO $7776: 1986$ ] \{CCITT 1984\}

ATS Used: ATS:2-1 and ATS:2-2

Conformance Lab: NVLA 0361

IBM Corportation - Notworking Systems Protocol Center

Product Code/Typo: P-001 WAN

Product DD: 185

Supplier: Telematics Intemational 1201 Cypress Crook Road Fort Lauderdale, FL 33309

Contact: Terry Rihel Tel: (818) $880-4900$ Fax: (818) $880-4726$

GOSIP Product Name: ACP50 X25 Version 10.04 Version/Rolease: Version 10.04

Release Date: 30JUL-93

Additional Info:

Rogistration:

Date: 05-AUG-93 Basis: BASE

Type: $\quad$ FULL, GOSIP Version 2

Hardware and Operating System Platforms:

HW: ACP5O

OS: N/A

Connectivity: RS-232

Underlying Stack: None

Protocols and Profiles: $\quad$ X.25 PLP/(X.25 LAP-B) X.25 PLP [ISO 8208:1990] \{CCITT 1984\} X25 LAP-B [ISO $7776: 1986$ \} \{CCIT 1984\}

ATS Usod: ATS:2-1 and ATS:2-2

Conformance Lab: NMAP* 0364

CDA, Incorporated Open Systems Development Group
Product Code/Type: P-001 WAN

Product D: 131

Supplier: UNISYS Corporation 8008 West Park Drive McLean, VA 22102

Contact: Dale Pluta Tel: (703) 556-5682 Fax: (703) 556-5172

GOSIP Product Name: CP2000 X.25 Protocol Version/Release: Ver 30.00 .192

Rolease Date: 30-SEP-92

Additional Info:

Registration:

Date: 01-FEB-93 Basis: BASE

Type: $\quad$ PROV, Gmdfthrd, V1 $->$ V2

Hardware and Operating System Platforms:

CP2000 with LMH Card, O/S CP2000 Operating Software Ver 3.0

Connectivity: RS-232C

Underlying Stack: None

Protocols and Profiles: X.25 PLP/ $\left(\mathrm{C}_{25}\right.$ LAP-B)

X25 PLP [ISO 8208:1990] \{CCITT 1984\}

X25 LAP-B [ISO T776:1986] \{CCITT 1984\}

ATS Usod: ATS:1-1 and ATS:1-2

Conformance Lab: NVLAP 0367

UNISYS - Open Systems Interconnection Laboratory

Product Code/Type: P-001 WAN

Product D:

$\infty$

Supplier: UNISYS Corporation 8008 Westbrook Drive McLean, VA 22102

Contact: Keith Fretz Tel: (703) 556-5665 Fax: (703) 556-5172

GOSIP Product Name: $X 25$ PSCS

Version/Rolease: 51RA \& PCRs UP TO 1929 INCLUSIVE

Release Date: 01-OCT-92

Additional Info:

Registration:

Date: 19-0CT-92 Basis: BASE

Type: $\quad$ PROV, Gmdtthrd, V1->V2

Hardware and Operating System Platforms:

DCP.15, DCP/OS 5P2A, TELCON 9R1A

Connectivity: RS-232C, V.35

Underlying Stack: None

Protocols and Profiles: $\quad$ X.25 PLP/ ( 25 LAP-B)

X25 PLP [ISO 8208:1990] \{CCITT 1984\}

X.25 LAP.B [ISO T776:1986] \{CCITT 1984\}

ATS Usod: ATS:1-1 and ATS:1-2

Conformance Lab: NVLAP\# 0367

UNISYS - Open Systems Interconnection Laboratory 


\section{Product Code/Type: P-D01 WAN}

Product D.

201

Supplier: Wellfleet Communlcations, Inc. 8 Foderal Streot Billerlca, MA 01821

Contact: Kathy Huber Tel: (508) 436-3791 Fax: (508) $670-5747$

GOSIP Product Name: Access Feeder Node (AFN) S/W Version 5.81 Version/Release: Version 5.81

Release Date: 24SEP.93

Additional Info: The AFN System is a stand alone seli-contained router.

Registration:

Date: 13-OCT-93 Basis: BASE

Type: $\quad$ FULL, GOSIP Version 2

Hardware and Operating System Platforms:

H/W: Access Feeder Node (AFN)

O/S: Embedded In Registered Product

Connectivity: V.35

Underlying Stack: None

Protocols and Profiles: $\quad$ X.25 PLP/(X.25 LAP-B) X.25 PLP [ISO 8208:1990] \{CCIT 1984\}
X.25 LAP-B [ISO T776:1986] \{CCIT 1984\}

ATS Used: ATS:2-2 and ATS:2-1

Conformance Lab: NVLAP* 0364

CDA, Incorporated Open Systems Development Group

\section{Product Code/Type: P-001 WAN}

Product 1D: $\quad 200$

Supplier: Wellfleet Communlcations, Inc.

8 Federal Street

Billerica, MA 01821

Contact: Kathy Huber Tel: (508) 436-3791

Fax: (508) $670-5747$

GOSIP Product Name: Link Node (LN) S/W Version 5.81

Version/Rolease: Version $\mathbf{5 . 8 1}$

Release Date: 24-SEP.93

Additional Info: The LN System is a stand-alone self contained router.

Registration:

$\begin{array}{lll}\text { Date: } & \text { 13-OCT-93 Basis: BASE } \\ \text { Type: } & \text { FULL GOSIP Version 2 }\end{array}$

Hardware and Operating System Platforms:

HW: Link Node (LN) w/ ACE 20 Processor \& Dual Sync Dual Ethemet(DSDE) I/O Card

O/S: Embedded In GOSIP product

Connectivity: V.35

Underlying Stack: None

Protocols and Profiles: X.25 PLP/(X.25 LAP-B)

X.25 PLP [ISO 8208:1990] \{CCIT 1984\}

X25 LAP-B [ISO $7776: 1986$ ] \{CCITT 1984\}

ATS Usod: ATS:2-2 and ATS:2-1

Conformance Lab: NVAP* 0364

CDA, Incorporated Open Systems Development Group
Product Code/Type: P-00e LAN

Product D.

37

Suppller: 3 Com Corporation

5600 Bayfront Plaza, PO Box 58145

Santa Clara, CA $95052-8145$

Contact: Howard Chan Tel: (408) 764-5827 Fax:

GOSIP Product Name: Etherlink 16, 3C507

Version/Release: Rev A

Release Date: 01-AUG-90

Additional info:

Registration:

Date: 14FEB-92 Basis: BASE

Type: PROVSIONAL, GOSIP VER 1

Hardware and Operating System Platforms:

PC AT 80386, MS DOS 3.3

Connectivity: PLS $\{802.3\} / 10$ Base5

Underlying Stack: Information not available

Protocols and Profiles: MAC [ISO 8802-3:1989]

ATS Used: ATS:1-3

Conformance Lab: NVLAP* 0363

Corporation for Open Systems International Test Center

Product Code/Typa: P-00e LAN

Product D:

3

Supplier: Bull information Systems Incorporated Technology Park

Billerica, MA 01821-4199

Contact: Kenneth B Finkenauer Tel: $(508) 2942909$ Fax:

GOSIP Product Name: Local Area Controller Subsystem (LACS)

Version/Release:

Release Date:

Additional Info:

Registration:

Date: 01-APR91 Basis: BASE

Type: PROVSIONAL, GOSIP VER 1

Hardware and Operating System Platforms: DPS6000/HVS6 Release 2

Connectivity: 10Base5

Underlying Stack: None

Protocols and Profiles: $\quad \amalg C 1\{802.2\} / M A C \&$ PLS $\{802.3\}$

LLC1 [ISO 8802-2:1989]

MAC [ISO 8802-3:1989]

PLS [ISO 8802-3:1989]

ATS Used: ATS: 13 and ATS: $1-6$

Conformance Lab: NVLAP* 0363

Corporation for Open Systems Intemational Test Center 


\section{Product Code/Type: P-002 LN}

Product 1D: 79

Supplier: Control Data Corporation 4201 North Lexington Avenue Arden Hills, MN 55126-6198

Contact: Ronald D Swan Tel: (612) $482-6257$ Fax: (612) $482-3616$

GOSIP Product Name: CDCNET Ethernet Serial Channel Interface Version/Release: LLC/MAC 1.7.1, PLS 1.6.1

Release Date: 01-OCT-92

Additional Info:

Registration:

Date: 15-OCT-92 Basis: BASE

Type: PROVISIONAL, GOSIP VER 1

Hardware and Operating System Platforms:

Device Interface Model *GH120B, Equipment \#DY0227-B, Product *2608-6 (Stand-alone Machine) O/S None

Connectivity: 10Base5

Underlying Stack: None

Protocols and Profiles: $\quad \amalg C 1\{802.2\} / M A C \&$ PLS $\{802.3\}$

LLC1 [ISO 8802-2:1989]

MAC [ISO 8802-3:1989]

PLS [ISO 8802-3:1989]

ATS Usod: ATS: $1-3$ and ATS: $1-6$

Conformance Lab: NVLAP* 0363

Corporation for Open Systems Intemational Test Center

Product Code/Type: P-003 WIERMEDLATE SYSTEM Product ID: 167

Supplier: 3Com Corporation

5400 Bayfront Plaza

Santa Clara, CA $95052-8145$

Contact: Cyndi Jung Tol: (408) 7645173

Fax: (408) $764-5002$

GOSIP Product Name: NETBullder II Extended WAN 3C6242A-GOSIP

Version/Rolease: Version 6.1.5

Release Date: 04JUN-93

Additional Info:

Rogistration:

Date: 23JUN-93 Basis: BASE

Type: Provisional, GOSIP Ver. 2

Hardware and Operating System Platforms:

NETBuilder II (4 or 8 port chassis) with 2 Ethernet Modules SW/NB IIWX, V6.1.5

Connectivity: Port1: LLC1\{802.2\}/MAC $\{802.3\} / 10 B a s e 2$ PORT2: UC1 $\{802.2\} / M A C\{802.3\} / 10 B a s e 5$

Underlying Stack: NETBullder II Ethemet Module (Embedded in Product)

Protocols and Profiles: CLNP $\{$ IS $\}$ [ISO 8473:1988] \& ES-IS $\{$ IS $\}$ [ISO 9542:1988] \{Dynamic Routing Claimed\}

ATS Used: ATS:2-7.1

Conformance Lab: NVLAP* 0371

Acatel TITN Test Center
Product Code/Type: P-003 WTERMEDATE SYSTEM Product 1D: 192

Supplier: Cabletron Systems, Inc. 35 Industrial Way, P.O. Box 5005 Rochester, NH $03867-5005$

Contact: Ronald Fotino Tel: (603) $337-1769$ Fax: (603) $337-1697$

GOSIP Product Name: CRM-L Version/Release: Version 9.1 (3.5)

Release Date: 01-MAR-93

Additional info: The product is a stand-alone system with the O/S and $1 / O$ functions embedded in the product.

Registration:

Date: 24-SEP-93 Basis: BASE

Type: Provisional, GOSIP Ver. 2

Hardware and Operating System Platforms:

(1) HW: CRM-L

(1) OS: CRM-L Version 9.1 (3.5)

(2) HW: Cisco IGS Router

(2) OS: CRM-L Version 9.1 (3.5)

Connectivity: Port 1: LLC1 $\{802.2\} / M A C\{802.3\} / 10$ Base5 Port 2: $U \mathrm{C} 1\{802.2\} / \mathrm{MAC}\{802.3\} / 10 \mathrm{Base5}$

Underlying Stack: Embedded in Product

Protocols and Profiles: CLNP\{IS\} [ISO 8473:1988] \& ES-IS $\{$ IS $\}$ [ISO 9542:1988] \{Dynamic Routing

ATS Used: ATS:2-7.1 Claimed\}

Conformance Lab: NVLAP* 037

Acatel TITN Test Conter

Product Code/Type: P-003 WIERAEDLATE SYSTEM Product D: 197

Supplier: Cisco Systems

1525 O'Brien Drive, P.O. Box 3075

Menio Park, CA 94026-1435

Contact: Aex Tweodley Tel: (415) 688-8114

Fax: (415) 688-4575

GOSIP Product Name: AGS $+/ 3$ X.25 Version 2.0

Version/Release: Version 2.0

Release Date: 13JUL-93

Additional Info:

Registration:

Date: 15-OCT-93 Basis: BASE

Type: $\quad$ Provisional, GOSIP Ver. 2

Hardware and Operating System Platforms:

HW: AGS $+/ 3$

OS: $g s 3+k 9.1(30)$

Connectivity: Port 1: $\amalg C 1\{802.2\} / M A C\{802.3\} / 10 B a s e T$

Port 2: X.25 PLP/X.25 LAP-B/N.35

Underlying Stack: WAN: AGS $+/ 3 \times 25$ Version 2.0 LAN: gs3-k $9.1(30)(L L C)$ and Lance AMD(MAC)

Protocols and Profiles: CLNP\{IS\} [ISO 8473:1988] \& ES-IS $\{$ IS [ISO 9542:1988] [Dynamic Routing Claimod\}

ATS Usod: ATS:2-7.1

Conformance Lab: NVLA* 0364

CDA, Incorporated Open Systems Development Group 


\section{Product Code/Typo: P-003 WTERIEDLTE SYSTEM Product DD: 196}

Supplier: Cisco Systems

1525 O'Brien Drive, P.O. Box 3075

Menlo Park, CA 94026-1435

Contact: Aex Tweedley Tel: (415) 688-8114

Fax: (415) 688-4575

GOSIP Product Name: AGS $+/ 4$ X.25 Version 2.0

Version/Release: Version 2.0

Release Date: 13-JUL-93

Additional Info:

Registration:

Date: 15-OCT-93 Basis: BASE

Type: Provisional, GOSIP Ver. 2

Hardware and Operating System Platforms:

HW: $A G S+/ 4$

OS: $g s 3+9.1(30)$

Connectivity: Port 1: ULC1 $\{802.2\} / M A C\{802.3\} / 10 B a s e T$ Port 2: X.25 PLP/X.25 LAP-B/N.35

Underlying Stack: $\quad$ WAN: AGS + $/ 4 \times 25$ Version 2.0 LAN: 9 s3-K $9.1(30)(L C)$ and Lance AMD(MAC)

Protocols and Profiles: CLNP $\{$ IS $\}$ [ISO 8473:1988] \& ES-IS $\{$ IS $\}$ [ISO 9542:1988] \{Dynamic Routing Claimed\}

ATS Used: ATS:2-7.1

Conformance Lab: NVLAP* 0364

CDA, Incorporated Open Systems Development Group

Product Code/Type: P-003 WIERMEDATE SYSTEM Product D: 127

Supplier: Cisco Systems

1525 O'Brien Drive, P.O. Box 3075

Menlo Park, CA 94026-1435

Contact: Aex Twoedley Tel: (415) 688-8114

Fax: (415) 688-4575

GOSIP Product Name: CGS/3 X.25 Version 2.0

Version/Release: Version 2.0

Rolease Date: 13-JUL-93

Additional Info:

Registration:

Date: 15-OCT-93 Basis: BASE

Type: Provisional, GOSIP Ver. 2

Hardware and Operating System Platforms:

HW: CGS $/ 3$

OS: 9 sł $9.1(30)$

Connectivity: Port 1: LLC1 $\{802.2\} / M A C\{802.3\} / 10 B a s e T$ Port 2: X.25 PLP/X.25 LAP-BN.35

Underlying Stack: WAN: CGS/3 X.25 Version 2.0. LAN: 9s3-k 9.1(30) (LLC) and Lance AMD(MAC)

Protocols and Profiles: CLNP $\{$ IS $\}$ [ISO 8473:1988] \& ES-IS $\{$ IS $\}$ [ISO 9542:1988] \{Dynamic Routing Claimed\}

ATS Used: ATS:2-7.1

Conformance Lab: NVLAP* 0364

CDA, Incorporated Open Systems Development Group
Product Code/Type: P-003 WTERMEDATE SYSTEM Product ID: 198

Supplier: Cisco Systems

1525 O'Brien Drive, P.O. Box 3075

Menlo Park, CA 94026-1435

Contact: Alex Tweedley Tel: (415) 688-8114

Fax: (415) 688-4575

GOSIP Product Name: CGS/4 X.25 Version 2.0

Version/Release: Version 2.0

Release Date: 13-JUL-93

Additional Info:

Registration:

Date: $\quad$ 15-OCT-93 Basis: BASE

Type: $\quad$ Provisional, GOSIP Ver. 2

Hardware and Operating System Platforms:

HW: CGS/4

OS: $g s 3-k .1(30)$

Connectivity: Port 1: $\amalg C 1\{802.2\} / M A C\{802.3\} / 10 B a s e T$ Port 2: X.25 PLP/X.25 LAP-BN.35

Underlying Stack: WAN: CGS/4 X.25 Version 2.0 LAN: gs3-k 9.1(30)(LLC) and Lance AMD(MAC)

Protocols and Profiles: CLNP $\{$ IS $\}$ [ISO 8473:1988] \& ES-IS $\{$ IS $\}$ [ISO 9542:1988] \{Dynamic Routing Claimed\}

ATS Used: ATS:2-7.1

Conformance Lab: NVLAP\# 0364

CDA, Incorporated Open Systems Development Group

Product Code/Type: P-003 WTERUEDLTE SYSTEM Product ID: 199

Supplier: Cisco Systems 1525 O'Brien Drive, P.O. Box 3075 Menlo Park, CA 94026-1435

Contact: Alex Twoedley Tel: (415) 688-8114 Fax: (415) 688-4575

GOSIP Product Name: IGS/R X.25 Version 2.0 Version/Release: Version 2.0

Release Date: 13-JUL-93

Additional Info:

Registration:

Date: 15-OCT-93 Basis: BASE

Type: Provisional, GOSIP Ver. 2

Hardware and Operating System Platforms:

HW: IGS/R

OS: igs-kr 9.1(30)

Connectivity: Port 1: $L C 1\{802.2\} / M A C\{802.3\} / 10 B a s e T$ Port 2: X.25 PLP/X.25 LAP-B/N.35

Underlying Stack: WAN: IGS/R X25 Version 2.0 LAN: gs3-k 9.1(30)(LC) and Lanco AMD(MAC)

Protocols and Profiles: CLNP IIS $\}$ [ISO 8473:1988] \& ES-IS $\{$ IS [ISO 9542:1988] \{Dynamic Routing Claimed

ATS Used: ATS:2-7.1

Conformance Lab: NVLAP * 0364

CDA, incorporated Open Systems Development Group 
Product Codo/Typo: P-003 WTERMEDLTE SYSTEM Product lD: 105

Supplier: Cisco Systems 1525 O'Brien Drive, P.O. Box 3075

Menlo Park, CA 94026-1435

Contact: Alex Tweedley

Tel: (415) 688-8114

Fax: (415) 688-4575

GOSIP Product Name: MGS/3 X.25 Version 2.0

Version/Rolease: Version 2.0

Release Date: 13jUL-93

Additional Info:

Registration:

Date: 15-OCT-93 Basis: BASE

Type: $\quad$ Provisional, GOSIP Ver. 2

Hardware and Operating System Platforms:

HW: MGS/3

OS: 983 - $9.1(30)$

Connectivity: Port 1: $\amalg C 1\{802.2\} / M A C\{802.3\} / 10 B a s e T$ Port 2: X.25 PLP/X.25 LAP-B/RS-232

Underlying Stack: WAN: MGS/3 X.25 Version 2.0 LAN: gs3-k $9.1(30)(L L C)$ and Lanco AMD(MAC)

Protocols and Profiles: CLNP\{IS\} [ISO 8473:1988] \& ES-IS $\{$ IS $\}$ [ISO 9542:1988] [Dynamic Routing Claimed\}

ATS Used: ATS:2-7.1

Conformance Lab: NVLA * 0364

CDA, Incorporated Open Systems Development Group

\section{Product Code/Typo: P-003 WTERMEDATE SYSTEM Product ID: 190}

Supplier: Cisco Systems

1525 O'Brien Drive, P.O. Box 3075

Menlo Park, CA 94026-1435

Contact: Aex Tweedley

Tel: (415) 688-8114

Fax: (415) 688-4575

GOSIP Product Name: MGS/4 X.25 Version 2.0

Version/Release: Version 2.0

Release Date: 13NUL-93

Additional Info: The MGS/4 runs link and packet X.25 plus CLNP on the same board/CPU with the OS.

Registration:

Date: 17-SEP-93 Basis: BASE

Type: Provisional, GOSIP Ver. 2

Hardware and Operating System Platforms:

HW: MGS/4

OS: $953+k$ 9.1(30)

Connectivity: Port 1: LC1 $\{802.2\} / M A C\{802.3\} / 10 B a s e T$

Port 2: X.25 PLP/X.25 LAP.B/RS-232

Underlying Stack: WAN: MGS/4 X.25 Version 2.0 LAN: gs3-k $9.1(30)$ (LC) and Lance AMD(MAC)

Protocols and Profiles: CLNP $\{$ IS $\}$ [ISO 8473:1988] \& ES-IS $\{$ IS $\}$ [ISO 9542:1988] \{Dynamic Routing Claimed\}

ATS Used: ATS:2-7.1

\section{Conformance Lab: NMLAP* 0364}

CDA, Incorporated Open Systems Development Group
Product Code/Typo: P-003 INTERMEDIATE SYSTEM Product D: 148

Supplier: Cisco Systems, Incorporated 1525 O'Brien Drive

Menlo Park, CA 94025

Contact: Ms Susan Scheer Tel: (415) 688-8131

Fax: (415) $688-7666$

GOSIP Product Name: Cisco Systems Router

Version/Release: Ver 9 Rol 1

Release Date: 01-NOV-92

Additional Info:

Registration:

Date: 20-APR-93 Basis: BASE

Type: $\quad$ PROVSIONAL, GOSIP VER 2

Hardware and Operating System Platforms:

MGS, CGS, AGS +, IGS, cisco3000, cisco 4000 , cisco 7000

Cisco Systems Router (Stand-alone System)

Connectivity: Port 1: $\amalg C 1\{802.2\} / M A C\{802.3\} / 10 B a s e 5$

Port 2: LLC1 $\{802.2\} / M A C\{802.3\} / 10 B a s e 5$

Underlying Stack: Embedded in Cisco Router Product Using CSC-2E2T Interface Hardware

Protocols and Profiles: CLNP\{IS\} [ISO 8473:1988] \& ES-IS $\{$ IS $\}$ [ISO 9542:1988] \{Dynamic Routing Claimed\}

ATS Used: ATS:2-7.1

Conformance Lab: NVLA\# 0371

Acatel TITN Test Center

Product Code/Type: P-003 INTERUEDIATE SYSTEM Product ID: 184

Supplier: Digital Equipment Corporation Imperial Way

Reading, Berks RGzOTE United Kingdom

Contact: Ken Chamberlain Tel:

Fax:

GOSIP Product Name: DEC Network Integration Server Software (DECNIS)

Version/Release: Version 2.1

Release Date: 30-APR-93

Additional info:

Registration:

Date: 05-AUG-93 Basis: BASE

Type: Provisional, GOSIP Ver. 2

Hardware and Operating System Platforms:

HW: DECNIS 500, DECNIS 600

OS: DECNIS V.21 with embedded OS

Connectivity: Port 1: $L C_{1}\{802.2\} / M A C\{802.3\} / 10 B a s e 5$ Port 2: X.25 PLP/X.25 LAP-BN.35

Underlying Stack: LAN: DEC Network Integration Server S/W, DNSAE-AA, BNEA4D-02, and H4005. WAN: DEC Notwork Integration Server S/W and DNSAB-AA

Protocols and Profiles: CLNP\{IS\} [ISO 8473:1988] \& ES-IS $\{$ IS $\}$ [ISO 9542:1988] \{Dynamic Routing Claimed\}

ATS Used: ATS:2-7.1

Conformance Lab: NVLAP* 0362

Digital Equipment Corporation, OSI Conformance Interoperability Test Center 


\section{Product Code/Type: P-003 WTEPMEDUTE SYSTEM Product D: 70}

Supplier: UNISYS Corporation 8008 Westbrook Drive McLean, VA 22102

Contact: Keith Fretz Tel: (703) 556-5665

Fax: (703) 556-5172

GOSIP Product Name: DCP OSITS

Version/Release: 2R1A + 192-194,197,199,202,203,205,207

Release Date: O8-APR-92

Additional Info:

Registration:

Date: 16-0CT-92 Basis: BASE

TyP: $\quad$ PROVISIONAL, GOSIP VER 1

Hardware and Operating System Platforms:

DCP-15 through DCP-55 Front End Processors

DCP/OS Ver 5R2A and TELCON Ver 9R1A

Connectivity: Port 1: LLC1 $\{802.2\} / M A C\{802.3\}$

Port 2: X.25 PLP/X.25 LAP-B

Underlying Stack: LAN Platform 2R2A, DCP 802.3 Lan Line Module, Feature *F5137-00.

WAN: PSCS Ner 51RA \& PCRs), DCP-15.

Protocols and Profiles: CLNP\{IS\} [ISO 8473:1988] [Static

ATS Used: ATS:1-7.1

Routing\}

Conformance Lab: NVLAP 0367

UNISYS - Open Systems Interconnection Laboratory

Product Code/Type: P-003 WTERMEDATE SYSTEM Product ID: 202

Supplier: Wellfleet Communications, Inc.

8 Foderal Street

Billerica, MA 01821

Contact: Kathy Huber Tel: (508) 436-3791

Fax: (508) 670-5747

GOSIP Product Name: Access Feeder Node (AFN) S/W Version 5.81

Version/Release: Version 5.81

Rolease Date: 24SEP-93

Additional Info: The AFN is a sealed-standalone router with the CLNP, OS, and underlying stack contained within the product.

Registration:

Date: O4NOV-93 Basis: BASE

Type: Provisional, GOSIP Ver. 2

Hardware and Operating System Platforms:

H/W: Access Feeder Node (AFN)

O/S: Embedded in Registered Product

Connectivity: Port 1: LLC1 $\{802.2\} / M A C\{802.3\} / 10 B a s e T$

Port 2: X.25 PLP/X.25 LAP-B/N.35

Underlying Stack: WAN: Embedded In Product (Access Feoder Node (AFN) S/W Version 5.81) $L A N=>S M$ : Embedded In Product; I/O Module: Lance AMD(MAC)

Protocols and Profiles: CLNP $\{$ IS\} [ISO 8473:1988] \& ES-IS $\{$ IS $\}$ [ISO

ATS Used: ATS:2-7.1 9542:1988] \{Dynamic Routing Claimed\}

Conformance Lab: NMAP* 0364

CDA, Incorporated Open Systems Development Group
Product Code/Typo: P-003 INTERMEDUTE SYSTEM Product ID: 203

Supplier: Wellfleet Communications, Inc. 8 Foderal Stroet

Billerica, MA 01821

Contact: Kathy Huber Tel: (508) 436-3791

Fax: (508) $670-5747$

GOSIP Product Name: Link Node (LN) S/W Version 5.81

Version/Release: Version 5.81

Release Date: 24SEP-93

Additional Info: The LN is a standalone router with the CLNP, OS, and underlying stack contained within the product.

Registration:

Date: 04NOV-93 Basis: BASE

Type: $\quad$ Provisional, GOSIP Ver. 2

Hardware and Operating System Platforms:

H/W: Link Node (LN) w/ ACE 20 Processor

O/S: Embedded in Registered Product

Connectivity: Port 1: $\amalg C_{1}\{802.2\} / M A C\{802.3\} / 10 B a s e T$

Port 2: X.25 PLP/X25 LAP-BN.35

Underlying Stack: $\quad L A N=>$ SW: Embedded in Product; $1 / 0$ Module: DSDE (Dual Sync Dual Ethernet) $W A N=>$ Embedded in the Product (Link Node (LN) S/W Version 5.81)

Protocols and Profiles: CLNP\{IS\} [ISO 8473:1988] \& ES-IS\{IS\} [ISO 9542:1988] \{Dynamic Routing

ATS Used: ATS:2-7.1 Claimed\}

Conformance Lab: NVLAP* 0364

CDA, Incorporated Open Systems Development Group

Product Codo/Typo: P-003 WTIEREEDLATE SYSTEM Product ID: 186

Supplier: Wellfleet Communications, Inc.

8 Federal Street

Billerica, MA 01821

Contact: Dan Mulvey Tel: (703) 7396710

Fax: (703) $739-1394$

GOSIP Product Name: Wellfleet Communications Router

Version/Release: Version 5.81

Release Date: 24SEP-93

Additional Info:

Registration:

Date: 24AUG-93 Basis: BASE

Type: $\quad$ Provisional, GOSIP Ver. 2

Hardware and Operating System Platforms:

HW: Concentrator Node (CN) w/ACEO2O proc; OS: Embedded in product

HW: Concentrator Node (CN) w/ACE030 proc;

HW: Link Node (LN) w/ ACE020 processor;

HW: Link Node (LN) W/ ACEO30 processor;

HW: Feeder Node (FN) w/ ACEO20 processor;

HW: Feeder Node (FN) w/ ACEO30 processor;

OS: Embedded in product

Connectivity: Port 1: $\amalg C 1\{802.2\} / M A C\{802.3\} / 10 B a s e 5$

Port 2: $L \mathrm{LC1}\{802.2\} / \mathrm{MAC}\{802.3\} / 10$ Base5

Underlying Stack: SW: Embodded in Product; I/O Modules: DE (Dual Port Ethernet), DSDE (Dual Ethemet/Dual Port Sync), and/or QENET (Quad Port Ethernet)

Protocols and Profiles: CLNP\{IS\} [ISO 8473:1988] \& ES-IS $\{$ IS $\}$ [ISO 9542:1988] \{Dynamic Routing Claimed\}

ATS Usod: ATS:2-7.1

Conformance Lab: NVAP* 0371

Acatel TITN Test Center 
Product Code/Typo: P-OO4 TRANSPOPT

Product D: 88

Supplier: Bull Information Systems, Incorporated 13430 North Black Canyon Highway Phoenix, AZ 85029

Contact: Oscar V Hefner Tel: (602) 862-6001

Fax: (602) $862-6051$

GOSIP Product Name: DATANET DCP 7500

Version/Release: DNS Ver 4 Update 1

Rolease Date: 01JAN-92

Additional Info: None

Registration:

$\begin{array}{lll}\text { Date: } & \text { 13-NOV-92 } & \text { Basis: BASE } \\ \text { Type: } & \text { PROV, Gmdtthrd, V1-> V2 }\end{array}$

Hardware and Operating System Platforms: DCP 7500

O/S DNS Ver 4 Update 1

Connectivity: UC1 $\{802.2\} / M A C\{802.3\}$

Underlying Stack: Datanet DCP 7500 ISO 8802-2/3 (LAN)

Protocols and Profiles: TP4CLNS/CLNP $\{E S\}$ Transport Class 4-CLNS[ISO 8073:1988/Add2:

ATS Usod: ATS:1-7 AND ATS:1-9 1989] CLNP \{ES\} [ISO 8473:1988] \{Static Routing\}

Conformance Lab: NVLA \# 0370

Conformance Expert Center for OSI Bull - CECOB

Product Code/Typo: P-004 TRANSPORT

Product D: 168

Supplier: Bull Information Systems, Incorporated 13430 North Black Canyon Highway Phoenix, AZ 85029

Contact: Oscar V Hefner Tel: $(602)$ 862-6001

Fax: (602) 862-6051

GOSIP Product Name: DATANET DCP 7500

Version/Release: DNS Ver 4 Update 1

Release Date: 01-JAN-92

Additional Info: None

Registration:

Date: 13-NOV-92 Basis: DERIVED

Type: $\quad$ PROV, Gindtthrd, V1-> V2

Hardware and Operating System Platforms:

DCP 7500

O/S DNS Ver 4 Update 1

Connectivity: X.25 PLP/X.25 LAP-B

Underlying Stack: Datanet DCP 7500 X.25 Packet Layer; Datanet DCP 7500 X.25 Frame Layer.

Protocols and Profiles: TP4CLNS/CLNP $\{$ ES $\}$

Transport Class 4-CLNS[ISO 8073:1988/Add2:1989]

CLNP \{ES\} [ISO 8473:1988] \{Static Routing\}

ATS Used: ATS:1-7 AND ATS:1-9

Conformance Lab: NVAP 0370

Conformance Expert Center for OSI Bull - CECOB
Product Code/Typo: P-004 TRANSPORT

Product D: 89

Supplier: Bull Information Systems, Incorporated 13430 North Black Canyon Highway Phoenix, AZ 85029

Contact: Oscar V Hefner $\quad$ Tel: $(602) 862-6001$

Fax: (602) 862-6051

GOSIP Product Name: DATANET DCP 7500

Version/Release: DNS Ver 4 Update 1

Release Date: 01JAN-92

Additional Info: None

Registration:

Date: 13-NOV-92 Basis: DERIVED

Type: $\quad$ PROV, Gmdfthrd, V1-> V2

Hardware and Operating System Platforms:

DCP 7500

O/S DNS Ver 4 Update 1

Connectivity: X.25 PLP/X.25 LAP-B

Underlying Stack: Datanet DCP $7500 \times 25$ Packet Layer; Datanet DCP 7500 X.25 Frame Layer

Protocols and Profiles: Transport Class 4-CONS [ISO 8073:1988]

ATS Used: ATS:1-9

Conformance Lab: NVAP* 0370

Conformance Expert Conter for OSI Bull - CECOB

Product Code/Typo: P-004 TRANSPORT Product ID: 101

Supplier: Bull Information Systems, Incorporated 13430 North Black Canyon Highway Phoenix, AZ 85029

Contact: Oscar V Hefner Tel: (602) 862-6001 Fax: (602) 862-6051

GOSIP Product Name: DPX/2 B.O.S (Stack B)

Version/Release: 2

Release Date: 01-OCT-92

Additional Info: None

Rogistration:

Date: 13-NOV-92 Basis: DERIVED

Type: PROV, Grndtthrd, V1->V2

Hardware and Operating System Platforms:

DPX/2 200, O/S B.O.S. 2

Connectivity: X.25 PLP/X.25 LAP-B

Underlying Stack: DPX/2 X.25 Packet Layer; DPX/2 X.25 Frame Layer

Protocols and Profiles: Transport Class 4-CONS [ISO 8073:1988]

ATS Usod: ATS:1-9

Conformance Lab: NVLAP* 0370

Conformance Expert Center for OSI Bull - CECOB 
Product Code/Type: P-CO4 TRANSPORT

Product 1D: 84

Supplier: Bull Information Systems, Incorporated 13430 North Black Canyon Highway Phoonix, AZ 85029

Contact: Oscar V Hefner Tel: (602) 862-6001

Fax: (602) 862-6051

GOSIP Product Name: DPX/2 B.O.S. (Stack B)

Version/Release: Ver 2

Release Date: 01-OCT-91

Additional Info: None

Registration:

Date: 13-NOV-92 Basis: BASE

Type: $\quad$ PROV, Gmdfthrd, V1->V2

Hardware and Operating System Platforms:

DPX/2 200, O/S B.O.S. 2

Connectivity: X.25 PLP/X.25 LAP-B

Underlying Stack: DPX/2 X.25 Packet Layer; DPX/2 X.25 Frame Layer

Protocols and Profiles: Transport Class 0 [ISO 8073:1988]

ATS Used: ATS: $1-8$

Conformance Lab: NVLAP* 0370

Conformance Expert Center for OSI Bull - CECOB

\section{Product Code/Type: P-004 TRANSPORT}

Product 1D: 97

Supplier: Bull Information Systems, Incorporated 13430 North Black Canyon Highway Phoenix, AZ 85029

Contact: Oscar V Hefner Tel: (602) 862-6001 Fax: (602) 862-6051

GOSIP Product Name: DPX/2 B.O.S. (Stack B)

Version/Release: Ver/Rol 2

Release Date: 01-OCT-91

Additional Info: None

Registration:

Date: 13-NOV-92 Basis: BASE

Type: $\quad$ PROV, Grndfthrd, V1->V2

Hardware and Operating System Platforms:

DPX/2 200, O/S B.O.S. 2

Connectivity: $\amalg C_{1}\{802.2\} / M A C\{802.3\}$

Underlying Stack: DPX/2, BOS 2, ISO 8802-2/3 (LAN)

Protocols and Profiles: TP4-CLNS/CLNP $\{E S\}$

Transport Class 4CLNS[ISO 8073:1988/Add2:1989]

ATS Used: ATS:1-7 AND ATS:1.9

CLNP \{ES\} [ISO 8473:1988] \{Static Routing\}

Conformance Lab: NVLAP* 0370

Conformance Expert Center for OSI Bull - CECOB
Product Code/Type: P-004 TRANSPORT

Product D: 96

Supplier: Bull Information Systems, Incorporated 13430 North Black Canyon Highway Phoenix, AZ 85029

Contact: Oscar V Hefner Tel: (602) 862-6001

Fax: (602) $862-6051$

GOSIP Product Name: DPX/2 B.O.S. (Stack B)

Version/Release: Ver 2

Rolease Date: 01-OCT-91

Additional Info: None

Registration:

Date: 13-NOV-92 Basis: DERIVED

Type: $\quad$ PROV, Gmdfthrd, V1-> V2

Hardware and Operating System Platforms: DPX/2 200, O/S B.O.S. 2

Connectivity: X25 PLP/X.25 LAP.B

Underlying Stack: DPX/2 X.25 Packet Layer; DPX/2 X.25 Frame Layer

Protocols and Profiles: TP4-CLNS/CLNP $\{E S\}$

Transport Class 4-CLNS[ISO 8073:1988/Add2:1989]

CLNP\{ES\} [ISO 8473:1988] \{Static Routing\}

ATS Used: ATS:1-7 AND ATS:1-9

Conformance Lab: NVLAP* 0370

Conformance Expert Center for OSI Bull - CECOB

Product Code/Type: P-004 TRANSPORT

Product D: 85

Supplier: Bull Information Systems, Incorporated 13430 North Black Canyon Highway Phoenix, AZ 85020

Contact: Oscar V Hefner Tel: (602) 862-6001 Fax: (602) $862-6051$

GOSIP Product Name: Datanet (DCP 7500)

Version/Release: DNS Ver 4 Update 1

Release Date: 01JAN-92

Additional Info: None

Registration:

Date: 13-NOV-92 Basis: BASE

Type: $\quad$ PROV, Gmdtthrd, V1->V2

Hardware and Operating System Platforms:

DCP 7500

O/S DNS Ver 4 Update 1

Connectivity: X.25 PLP/X.25 LAP.B

Underlying Stack: DATANET DCP 7500 X25 Packet Layer DATANET DCP $7500 \times 25$ Frame Layer

Protocols and Profiles: Transport Class 0 [ISO 8073:1988]

ATS Usod: ATS: $1-8$

Conformance Lab: NVLAP* 0370

Conformance Expert Center for OSI Bull - CECOB 


\section{Product Code/Typo: P-004 TRANSPORT}

Product D: 170

Supplier: Control Data Corporation 4201 North Lexington Avenue

Arden Hills, MN 55126-6198

Contact: Ronald D Swan Tel: (612) $482-6257$

Fax: (612) $482-3616$

GOSIP Product Name: CDCNET

Version/Release: 1.7.1/BCU \#803AA

Release Date: 01-MAR-92

Additional Info: None

Registration:

$\begin{array}{lll}\text { Date: } & \text { OFFEB-93 } \quad \text { Basis: BASE } \\ \text { Type: } & \text { PROV, Gmdfthrd, V1-> V2 }\end{array}$

Hardware and Operating System Platforms:

CYBER 930 Host and Mainframe Device Interface (MDI), Mainframe Device Interface (TDI), or Integrated Communlcations Adapter (ICA) CYBER O/S, NOSNer 1.7.7, ICA Is stand-alono (Self Contained)

Connectivity: UC1 $\{802.2\} / M A C\{802.3\}$

Undertying Stack: CDCNET Ethernet Serial Channel Interface Product 46 (P-2 LAN) (Registered October 15, 1992)

Protocols and Profiles: TP4-CLNS/CLNP\{ES\}

Transport Class 4-CLNS[ISO 8073:1988/Add2:1989] CLNP\{ES\} [ISO 8473:1988] \{Static Routing\}

ATS Usod: ATS: 1.7 AND ATS:1.9

Conformance Lab: NMLAP 0354

Control Data Corporation, OSI Accredited Test Center

\section{Product Code/Type: P-CO4 TRWNPORT}

Product D: 88

Supplier: Control Data Corporation 4201 North Lexington Avenue Arden Hills, MN 55126-6198

Contact: Ronald D Swan Tel: (612) $482-6257$

Fax: (612) $482-3616$

GOSIP Product Name: CDCNET

Version/Release: Ver 1.6.1 / B720

Release Date: 01-MAR-92

Additional Info: None

Registration:

$\begin{array}{lll}\text { Date: } & \text { O9-NOV-92 } & \text { Basis: BASE } \\ \text { Type: } & \text { PROV, Gmdfthrd, V1->V2 }\end{array}$

Hardware and Operating System Platforms: CDCNET Device Interface DY-227-8, O/S NONE

Connectivity: X25 PLP/X.25 LAP-B

Undertying Stack: CDCNET, Ver 1.6.1, X.25

Protocols and Profiles: Transport Class 0 [ISO 8073:1988]

ATS Usod: ATS:1-8

Conformance Lab: NVAP 0354

Control Data Corporation, OSI Accredited Test Center
Product Code/Type: P-OO4 TRANSPORT

Product 1D: 38

Supplier: Control Data Corporation 4201 North Lexington Avenue Arden Hills, MN 55126-6198 Contact: J.F. Carey Tel: (612) $482-2567$
Fax: $(612) 482-2791$

GOSIP Product Name: Control mData EP/L Access \& Directory Version/Release: Ver 1.4.2

Release Date: 27-NOV-91

Additional Info: None

Registration:

Date: 25-FEB-92 Basis: BASE

Type: $\quad$ PROV, Grndthrd, V1-> V2

Hardware and Operating System Platforms: Control Data 4000

Control Data EP/ $/ X$ Version 1.4.2

Connectivity: PLS\{802.3\}/10Base5

Underlying Stack:

Protocols and Profiles: TP4-CLNS/CLNP\{ES\}

Transport Class 4-CLNS[ISO 8073:1988/Add2: 1989] CLNP \{ES\} [ISO 8473:1988] \{Static Routing\}

ATS Usod: ATS:1-7 and ATS:1-9

Conformance Lab: NVAP* 0354

Control Data Corporation, OSI Accroditod Test Center

Product Code/Typo: P-004 TRANSPORT

Product D. 76

Supplier: Data General Corporation

4400 Computer Drive, MS/D216

Westborough, MA 01580

Contact: Charies Stakus Tel: (508) $870-6392$

Fax: (508) 898-4694

GOSIP Product Name: OSI/Platform for AViiON Systems

Version/Release: Ver 3.0

Release Date: 01-JUN-92

Additional Info:

Registration:

Date: 23-OCT-92 Basis: BASE

Type: $\quad$ PROV, Gmdfthrd, V1->V2

Hardware and Operating System Platforms: AViON 5000/6000 Series

DG/UX System for AViiON Systems Rev. 5.4.1

Connectivity: $\amalg C 1\{802.2\} / M A C\{802.3\}$

Underlying Stack: Protocols and Profiles: 0003

TP4-CLNS/CLNP $\{$ ES $\}$

Transport Class 4CLNS[ISO 8073:1988/Add2:1989]

CLNP\{ES\} [ISO 8473:1988] \{Static Routing\}

ATS Used: ATS:1.7 and ATS:1-9

Conformance Lab: NMAP* 0371

Acatel TITN Test Conter 
Product Code/Type: P-OO4 TRANSPORT

Supplier: Data General Corporation

4400 Computer Drive, MS/D216

Westborough, MA 01580

Contact: Charles Stakus Tel: (508) $870-6392$

Fax: (508) 898-4694

GOSIP Product Name: OSI/Platform for AViiON Systems

Version/Release: Ver 3.0

Release Date: 01JUN-92

Additional Info:

Registration:

Date: 23-OCT-92 Basis: BASE

Type: $\quad$ PROV, Grndfthrd, V1->V2

Hardware and Operating System Platforms:

AViiON 5000/6000 Series

DG/UX System for AViON Systems Rev. 5.4.1

Connectivity: X.25 PLP/X.25 LAP-B

Underlying Stack:

Protocols and Profiles: Transport Class 0 [ISO 8073:1988]

ATS Used: ATS: $1-8$

Conformance Lab: NVLAP 0371

Acatel TITN Test Center

\section{Product Code/Typo: P-CO4 TRANSPORT}

Product 1D: 89

Supplier: Data General Corporation 4400 Computer Drive, MS/D216 Westborough, MA 01580

Contact: Charles Stakus

Tel: (508) $870-6392$

Fax: (508) 898-4694

GOSIP Product Name: OSI/Platform for AViOON Systems

Version/Release: Ver 3.0

Release Date: 01-JUN-92

Additional Info: None

Registration:

Date: 13-NOV-92 Basis: DERIVED

Type: $\quad$ PROV, Gmdfthrd, V1->V2

Hardware and Operating System Platforms:

AViiON 5000/6000 Series

DG/UX System for AViiON Systems Rev. 5.4.1

Connectivity: X.25 PLP/X.25 LAP.B

Underlying Stack: X.25 for AViON Systems, Ver. 2.20

Protocols and Profiles: TP4-CLNS/CLNP $\{E S\}$

Transport Class 4-CLNS[ISO 8073:1988/Add2:

1989] CLNP \{ES\} [ISO 8473:1988] \{Static Routing\}

ATS Used: ATS:1-7 AND ATS:1-9

Conformance Lab: NMAP* 0391

Data General Corporation, OSI Conformance Test Center
Product Code/Type: P-004 TRANSPORT

Product D. 193

Supplier: Data General Corporation

4400 Computer Drive

Westboro, MA 01580

Contact: Peter Conway Tel: (508) 898-7176

Fax: (508) $898-4212$

GOSIP Product Name: OSI/Platform for AViON Systems

Version/Release: Version 3.30

Release Date: 01-OCT-93

Additional Info: Product tested on Model AV8500 platform. Vendor claims support for other $H / W$ platforms as listed.

Registration:

Date: 30-SEP-93 Basis: BASE

Type: Provisional, GOSIP Ver. 2

Hardware and Operating System Platforms:

HW: AViiON AV500, AV4000, AV5000, AV6000,

AV7000, AV8000, AV8500, AV9000

OS: DG/UX System for AViON Computers, Revision 5.4R2.10

Connectivity: $\amalg C 1\{802.2\} / M A C\{802.3\} / 10 B a s e T$

Underlying Stack: LAN: ULC and MAC contained in DG/UX 5.4R2.10, embedded in product.

Protocols and Profiles: TP4-CLNS/CLNP $\{E S\}$ \& ES-IS $\{E S\}$ Transport Class 4-CLNS [ISO 8073:1988/Add2: 1989] CLNP\{ES\} [ISO 8473:1988] \& ES-IS $\{E S\}$ [ISO 9542:1988] \{Dyn Ring Claimed\}

ATS Used: ATS:2-7 and ATS:2-9.2

Conformance Lab: NVLAP* 0364

CDA, Incorporated Open Systems Development Group

Product Code/Type: P-004 TRANSPORT

Product D: 128

Supplier: Digital Equipment Corp 550 King Street Littleton, MA 01460-1289

Contact: Richard A Duhamel Tel: (508) 486-5021

Fax: (508) 486-7417

GOSIP Product Name: DECnet-VAX (TM) Ver 5.4 EXTENSIONS

Version/Release: Ver 5.4

Release Date: 01-SEP-91

Additional Info: None

Registration:

Date: 26-JAN-93 Basis: BASE

Type: $\quad$ PROV, Gmdfthrd, V1-> V2

Hardware and Operating System Platforms:

MicroVAX 3800

VMS Ver 5.4

Connectivity: X.25 PLP/X.25 LAP-B

Underlying Stack: VAX Packet System Interface for DECnet(TM) 5.4 Extensions, September 1991

Protocols and Profiles: Transport Class 0 [ISO 8073:1988]

ATS Used: ATS:1-8

Conformance Lab: NVLAP\# 0362

Digital Equipment Corporation, OSI Conformance Interoperability Test Center 


\section{Product Code/Type: P-004 TRANSPORT}

Product 1D: 129

Supplier: Digital Equipment Corp 550 King Street Littleton, MA 01460-1289

Contact: Richard A Duhamel Tel: (508) 486-5021

Fax: (508) 486-7417

GOSIP Product Name: DECnet/OSI for OpenVMS VAX V5.5/NOTS, V3.0A

Version/Release: Ver 3.0A

Release Date: 11-SEP-92

Additional Info: None

Registration:

\section{Date: 13JAN-93 Basis: DERIVED}

Type: $\quad$ PROV, Grndthrd, V1->V2

Hardware and Operating System Platforms: MicroVAX - II, 2000, $3100,3300 / 3400,3500,3600,3800,3900$; VAXstation - II, 2000, $3100,3200,3500,3520,3540,4000$; VAXserver - 3100, 3300/3400, $3500,3600,3602,3800$, 3900, 6000; VAX - 11/730, 11/750, 11/780, 11/785. $500,6000,8200,8250,8300,8350,85 x x, 8600,8650$, 8700 , 8350, 8810,8820, 8830, 8840, 9000; VAXt - M110/310, M410/610/612, WTH O/S OpenVMS V5.5

Connectivity: LC1 $\{802.2\} / M A C\{802.3\}$

Underlying Stack: Digital DESQA CSMA/CD LAN Controller; DEC (MAU) 44005; DEC (AUI) BNE4D-02; DEC (LLC) OpenVMS for VAX, Ver 5.5-2

Protocols and Profiles:

TP4-CLNS/CLNP $\{$ ES $\}$

Transport Class 4-CLNS[ISO 8073:1988/Add2:1989]

CLNP\{ES\} [ISO 8473:1988] \{Static Routing\}

ATS Used: ATS:1-7 AND ATS:1-9

Conformance Lab: NMAP* 0362

Digital Equipment Corporation, OSI Conformance Interoperability Test Conter

Product Code/Type: P-004 TRANSPORT

Product D: 144

Supplier: Digital Equipment Corp 550 King Street Littleton, MA 01460-1289

Contact: Richard A Duhamel Tel: (508) $486-5021$

Fax: (508) 486-7417

GOSIP Product Name: DECnet/OSI for ULTRIX

Version/Release: Ver 5.1-ECO0193

Release Date: 02JAN-93

Additional Info: None

Rogistration:

Date: 31-MAR-93 Basis: BASE

Type: $\quad$ PROV, Gmdthrd, V1-> V2

Hardware and Operating System Platforms:

1. DECstations 2100,3100s, 5000/20,5000/25, 5000/120, $5000 / 125,5000 / 200$, and $5800 / 240 w$ Integrated Ethemet Controller ULTRIX Worksystem Software, Ver 4.2A

2. DECsystems $5000 / 200,5000 / 240,5100,5400,5500,5810$, $5820,5830,5840,5900$ with Integrated Ethemet Controller ULTRIX Ver $4.2 A$

Connectivity: UC1 1802.2$\} / M A C\{802.3\}$

Underlying Stack: DECNet/OSI for ULTRIXVer 5.1-ECO0193 Layered product for ULTRIX; Chipset integrated with the CPU

Protocols and Profiles: TP4-CLNS/CLNP\{ES\} Transport Class 4-CLNS[ISO 8073:1988/Add2:1989] CLNP\{ES\} [ISO 8473:1988] \{Static Routing\}

ATS Used: ATS:1-7 and ATS: 1.9

Conformance Lab: NMLA* 0362

Digital Equipment Corporation, OSI Conformance Interoperability Test Conter
Product Code/Type: P-OOA TRANSPORT

Product D: 54

Supplier: Digital Equipment Corporation

Digital Park

Reading, RG2 OTE United Kingdom

Contact: Bill Daley Tel: Fax:

GOSIP Product Name: DECnet-VAX (TM) Extensions Ver 5.4A NOTS Ver $3.0 \mathrm{~A}$

Version/Release: 5.4A

Release Date: 01-APR-92

Additional Info: None

Rogistration:

Date: 16-AUG-92 Basis: BASE

Type: $\quad$ PROV, Grndfthrd, V1->V2

Hardware and Operating System Platforms:

Digital VAX Computer with VMS C5.4A + Operating System

Connectivity: UC1 $\{802.2\} / \operatorname{MAC}\{802.3\}$

Underlying Stack:

Protocols and Profiles: TP4-CLNS/CLNP $\{E S\}$

Transport Class 4-CLNS[ISO 8073:1988/Add2:1989]

CLNP\{ES\} [ISO 8473:1988] \{Static Routing\}

ATS Usod: ATS:1-7 and ATS:1-9

Conformance Lab: NVLAP\# 0362

Digital Equipment Corporation, OSI Conformance Interoperability Test Center

\section{Product Code/Type: P-004 TRANSPORT}

Product 1D: 57

Supplier: Encore Computing Corporation 601 West Sunrise Boulevard Ft. Lauderdale, FL 33313-4499

Contact: Augie Gonzales Tel: (305) 587-2900 Fax: (305) 797-5807

GOSIP Product Name: EnComm ISO Transport Services

Version/Release: Ver 3.0.0

Release Date: 01JUL-92

Additional Info: None

Registration:

Date: 31-AUG-92 Basis: BASE

Type: $\quad$ PROV, Gmdfthrd, V1->V2

Hardware and Operating System Platforms:

Encore Infinity 90 Series GPIO I, O/S UMAX Ver 3.0.7

Connectivity: X.25 PLP/X.25 LAP-B

Underlying Stack: Encore EnComm X.25 and PAD Rov 3.0.0, 15-JUN-92

Protocols and Profiles: Transport Class 0 [ISO 8073:1988]

ATS Used: ATS:1-8

Conformance Lab: NMAP\# 0364

CDA, Incorporated Open Systems Development Group 


\section{Product Code/Typo: P-OO4 TRANSPORT}

Supplier: Encore Computing Corporation 6901 West Sunrise Boulevard Ft. Lauderdale, FL 33313-4499

Contact: Augie Gonzales Tel: (305) 587-2900 Fax: $(305) 797-5807$

GOSIP Product Name: EnComm ISO Transport Services Version/Release: Ver 3.0 .0

Release Date: 01-AUG-92

Additional Info: None

Rogistration:

Date: 31-AUG-92 Basis: BASE

Type: $\quad$ PROV, Gindfthrd, V1->V2

Hardware and Operating System Platforms:

Encore Infinity 90 Series GPIO I, O/S UMAX Ver 3.0.7

Connectivity: $\operatorname{CLNP}\{E S\} / L C 1\{802.2\} / M A C\{802.3\}$ CLNP $\{E S\} / X .25$ PLP/X.25 LAP-B

Underlying Stack:

Encore/SynOptics LattisNet Mdl 3030 CLNP\{ES\}/LAN;

Encore/SynOptics Departmental Hub CLNP\{ES\}/LAN;

EnComm ISO CLNP $\{E S\} / E n C o m m ~ X 25$ and PAD Revision 3.0

Protocols and Profiles:

Transport Class 4-CLNS [ISO 8073:1988 / Add2:1989]

ATS Usod: ATS:1-9

Conformanco Lab: MLAP: 0364

CDA, Incorporated Open Systems Development Group

\section{Product Code/Type: P-CO4 TRANSPORT}

Product 10: 55

Supplier: Encore Computing Corporation

6901 West Sunrise Boulevard

Ft. Lauderdale, FL 33313-4499

Contact: Augie Gonzales Tel: (305) 587-2000

Fax: (305) 797-5807

GOSIP Product Name: Encore EnComm ISO Transport Services

Version/Release: Ver 3.0.0

Release Date: 01-AUG-92

Additional Info:

Registration:

Date: Basis: BASE

Type: PROV, Gmdfthrd, V1->V2

Hardware and Operating System Platforms:

Encore Infinity 90 Series GPIO I, O/S UMAX Ver 3.0.7

Connectivity: X.25 PLP/X.25 LAP-B

Underlying Stack:

EnComm VME Ethemet Drvr 2.1 over

Encore VME Ethemet Cntrilr (NSSC) *

8513-047 SynOptics Enterprise/Dpt Hub Configuration

Protocols and Profiles: CLNP\{ES\} [ISO 8473:1988] \{Static Routing\}

ATS Usod: ATS:1-7

Conformance Lab: NVLAP* 0364

CDA, Incorporated Open Systems Development Group

\section{Product Code/Type: P-004 TRANSPOPT}

Product D: 58

Supplier: Encore Computing Corporation 6901 West Sunrise Boulevard Ft. Lauderdale, FL 33313-4499

Contact: Augie Gonzales Tel: (305) 587-2900 Fax: (305) $797-5807$

GOSIP Product Name: Encore EnComm ISO Transport Services SynOptics LattisNet Mal 3030 Concentrator Version/Release: VER 3.0.0

Release Date: 01-AUG-92

Additional Info: None

Rogistration:

Date: 27-OCT-92 Basis: BASE

Type: $\quad$ PROV, Gmdfthrd, V1-> V2

Hardware and Operating System Platforms:

Encore Infinity 90 Series GPIO I, O/S UMAX Ver 3.0.7

Connectivity: $\amalg C 1\{802.2\} / M A C\{802.3\} / 10 B a s e T$

Underlying Stack: EnComm VME Ethemet Drvr 2.1 over Encore VME Ethemet Cntrllr (NSSC) \$8523-444 SynOptics LattisNet Model 3030 Concentrator, 3313 Ethernet MMM, and 3308 Host

Protocols and Profiles: CLNP\{ES\} [ISO 8473:1988] \{Static Routing\}

ATS Usod: ATS:1-7

Conformance Lab: NVLAP* 0364

CDA, Incorporated Open Systems Development Group

Product Code/Type: P-CO4 TRANSPORT

Product 1D. 78

Supplier: Encore Computing Corporation $\$ 01$ West Sunrise Boulevard

Ft. Lauderdale, FL $33313-4499$

Contact: Augie Gonzales Tel: (305) 587-2900

Fax: (305) $797-5807$

GOSIP Product Name: Encore EnComm ISO Transport Services over SynOptics Enterprise/Dpt Hub Configur.

Version/Release: Ver 3.0.0

Release Date: 01-AUG-92

Additional Info: Components of SynOptics Enterprise/Department Hub Configuration listed in underlying stack.

Registration:

Date: 27-OCT-92 Basis: BASE

Type: $\quad$ PROV, Gmdfthrd, V1-> V2

Hardware and Operating System Platforms:

Encore Infinity 90 Series GPIO I, O/S UMAX Ver 3.0.7

Connectivity: UC1 1802.2$\} / M A C\{802.3\} / 10 B a s e T$

Underlying Stack:

EnComm VME Ethnt Drvr 2.1, Encore VME Ethnt

Cntrl 8513-047, Hub (3000N, 33134-04, 3314A-04,

504A, 3595A-01, 3308A, 2813-04, 3304-ST, 3301,

33235, 3383-02, 508A)

Protocols and Profiles: CLNP\{ES\} [ISO 8473:1988] \{Static Routing\}

ATS Used: ATS:1-7

Conformance Lab: NVLAP* 0364

CDA, Incorporated Open Systems Development Group 
Product Code/Type: P-004 TRANSPORT

Supplier: Hewlett-Packard Company 19420 Homestead Road Cupertino, CA 95014-9810

Contact: Bruce Talley Tel: (408) 447-3599 Fax: (408) 447.3660

GOSIP Product Name: HP OSI Transport Services/9000, P/N 32070A

Version/Release: Ver C.02.00

Release Date: 10-JUN-91

Additional Info:

Registration:

Date: 28-MAY-91 Basis: BASE

Type: $\quad$ PROV, Gmdfthrd, V1->V2

Hardware and Operating System Platforms:

HP 9000 Series 800/HP-UX Operating System, Version 8.0

Connectivity: ШC1 $\{802.2\} / M A C\{802.3\}$

Underlying Stack:

Protocols and Profiles: TP4-CLNS/CLNP $\{$ ES\}

Transport Class 4-CLNS[ISO 8073:1988/Add2:1989]

CLNP\{ES\} [ISO 8473:1988] \{Static Routing\}

ATS Used: ATS:1.7 and ATS:1.9

Conformance Lab: NVLAP 0365

Hewlett-Packard Company, OSI Conformance Test Center

\section{Product Code/Type: P-004 TRANSPORT}

Product D: 117

Supplier: IBM Corporation, Rome Networking Systems Laboratory Via Paolo DiDona, 44 00144 Rome taly

Contact: Gerard Bonnes Tel: +33921141 22

Fax: + 3393247157

GOSIP Product Name: IBM AIX OSI Messaging and Filing/6000 Version/Release: Ver 1, Level 180

Release Date: 01-DEC-90

Additional Info:

Rogistration:

Date:

Type:

04JAN-93 Basis: BASE

PROV, Gmdfthrd, V1->V2

Hardware and Operating System Platforms:

IBM RISC System/6000 Al Models

$(7011,7012,7013,7015,6016$, OS AIX/6000

Connectivity: LLC1 $\{802.2\} / M A C\{802.3\}$

Underlying Stack: Software:

IBM AIX/6000 Ver 3.1.5; Hardware: Ethemet Adapter 7013-2890

Protocols and Profiles: TP4-CLNS/CLNP $\{$ ES $\}$

Transport Class 4-CLNS[ISO 8073:1988/Add2:1989]

CLNP\{ES\} [ISO 8473:1988] \{Static Routing\}

ATS Used: ATS:1-7 AND ATS:1-9

Conformance Lab: NVLAP 0369
Product Code/Typo: P-004 TRANSPORT

Product 1D: 23

Supplier: IBM Corporation, Rome Networking Systems Laboratory Via Paolo DiDona, 44

00144 Rome, Italy

Contact: Gerard Bonnes Tel: +3392114122 Fax: + +3393247157

GOSIP Product Name: OSI/Communications Subsystem Version/Release: Ver 1 Rel 1.1

Release Date: 01-DEC-90

Additional Info: None

Registration:

Date: 01-NOV-91 Basis: BASE

Type: $\quad$ PROV, Gmdthrd, V1-> V2

Hardware and Operating System Platforms:

(1) HW: IBM Enterprise System $/ 390$

(1) OS: IBM MVS/ESA Ver 3 Rel 1

(2) HW: IBM Enterprise System/370

(2) OS: IBM MVS/ESA Ver 3 Rel 1

Connectivity: X.25 PLP/X25 LAP.B

Underlying Stack: Provided by IBM NCP Packet Switching Interface.

Protocols and Profiles: Transport Class 0 [ISO 8073:1988]

ATS Usod: ATS: $1-8$

Conformance Lab: NVLAP\# 0369

Product Code/Typo: P-004 TRANSPORT

Product 1D: 26

Supplier: IBM Corporation, Rome Networking Systems Laboratory Via Paolo DiDona, 44 0144 Rome Italy

Contact: Gerard Bonnes

Tel: +3392114122

Fax: + 3393247157

GOSIP Product Name: OSI/Communications Subsystem

Version/Release: Ver 1 Rel 1.1

Release Date: 01-DEC-90

Additional Info:

Registration:

Date: 01-NOV-91 Basis: BASE

Type: $\quad$ PROV, Gmdfthrd, V1 $>$ V2

Hardware and Operating System Platforms:

IBM Enterprise System 370/390

IBM MVS/XA Ver 2 Rol 2

Connectivity: X.25 PLP/X.25 LAP-B

Underlying Stack: Provided by IBM NCP Packet Switching Interface.

Protocols and Profiles: Transport Class 0 [ISO 8073:1988]

ATS Usod: ATS: 1.8

Conformance Lab: NVLAP* 0369 
Supplier: IBM Corporation, Rome Notworking Systems Laboratory Via Paolo DiDona, 44 00144 Rome Italy

Contact: Gerard Bonnes

Tel: +3392114122

Fax: +3393247157

GOSIP Product Name: OSI/Communications Subsystem Version/Release: Ver 1 Rel 1.1

Release Date: 01-DEC-90

Additional Info:

Registration:

Date: 12-FEB-92 Basis: BASE

Type: $\quad$ PROV, Gmdfthrd, V1-> V2

Hardware and Operating System Platforms:

(1) HW: IBM Enterprise System/390

(1) OS: IBM MVS/ESA Ver 3 Rel 1

(2) HW: IBM Enterprise System/370

(2) OS: IBM MVS/ESA Ver 3 Rel 1

Connectivity: X.25 PLP/X.25 LAP-B

Underlying Stack: Software: IBM OSI/Comm Subsystem

V.1 R1.1 in IBM/S/390[802.2];

Interconnect Controller Program V1.0 on IBM

Interconnect Controller Program (802.3). Hardware: IB

Protocols and Profiles: TP4-CLNS/CLNP\{ES\}

Transport Class 4-CLNS[ISO 8073:1988/Add2:1989]

CLNP\{ES\} [ISO 8473:1988] \{Static Routing\}

ATS Used: ATS:1.7 and ATS: $1-9$

Conformance Lab: NVAP\# 0369

\section{Product Code/Type: P-004 TRANSPORT}

Product D: 33

Supplier: IBM Corporation, Rome Networking Systems Laboratory Via Paolo DiDona, 44 00144 Rome Italy

Contact: Gerard Bonnes

Tel: +3392114122

Fax: +3393247157

GOSIP Product Name: OSI/Communications Subsystem

Version/Release: Ver 1 Rel 1.1

Release Date: 01-DEC-S0

Additional Info: None

Registration:

Date: 12-FEB-92 Basis: BASE

Type: $\quad$ PROV, Gmdfthrd, V1->V2

Hardware and Operating System Platforms:

(1) HW: IBM Enterprise System $/ 390$

(1) OS: IBM MVS/ESA Ver 3 Rel 1

(2) HW: IBM Enterprise System $/ 370$

(2) OS: IBM MVS/ESA Ver 3 Rel 1

Connectivity: X.25 PLP/X.25 LAP-B

Underlying Stack: Provided by IBM NCP Packet Switching Interface.

Protocols and Profiles:

TP4-CLNS/CLNP $\{$ ES $\}$

Transport Class 4-CLNS[ISO 8073:1988/Add2:1989]

CLNP\{ES\} [ISO 8473:1988] \{Static Routing\}

ATS Used: ATS: 1-9

Conformance Lab: NVLAP\# 0369
Product Code/Type: P-004 TRANSPORT

Product D: 116

Supplier: IBM Corporation, Rome Networking Systems Laboratory Via Paolo DiDona, 44

Co144 Rome ttaly

Contact: Gorard Bonnes

Tel: + 3392114122

Fax: +3393247157

GOSIP Product Name: OSI/Communications Subsystem

Version/Release: Ver 1 Rel 1.1

Release Date: 01-DEC-S0

Additional Info: None

Registration:

Date: O4JAN-93 Basis: BASE

Type: $\quad$ PROV, Grndfthrd, V1-> V2

Hardware and Operating System Platforms:

IBM Enterprise System/390 Al Models (S/390, 43xx, 30xx),

IBM MVS/ESA, MVS/XA, IBM VM/SP, VM/ESA

Connectivity: $L L C 1\{802.2\} / M A C\{802.3\}$

Underlying Stack: Provided by IBM NCP Packet Switching Interface.

Protocols and Profiles: TP4-CLNS/CLNP $\{$ ES $\}$

Transport Class 4-CLNS[ISO 8073:1988/Add2:1989]

CLNP\{ES\} [ISO 8473:1988] \{Static Routing\}

ATS Used: ATS:1-7 AND ATS:1-9

Conformance Lab: NVAP* 0369

Product Code/Typo: P-004 TRANSPORT

Product 1D: 24

Supplier: IBM Corporation, Rome Networking Systems Laboratory Via Paolo DiDona, 44

00144 Rome traly

Contact: Gerard Bonnes Tel: +33921141 22

Fax: +3393247157

GOSIP Product Name: OSI/Communications Subsystem

Version/Release: Ver 1 Rel 1.1

Release Date: 01-DEC-90

Additional Info:

Registration:

Date: 01-NOV-91 Basis: DERIVED

Type: $\quad$ PROV, Grndfthrd, V1-> V2

Hardware and Operating System Platforms:

IBM Enterprise System /390

IBM VM/ESA Ver 1 Rel 1

IBM Enterprise System $/ 370$

IBM VM/ESA Ver 1 Rel 1

Connectivity: X.25 PLP/X.25 LAP-B

Underlying Stack:

Provided By IBM NCP Packet Switching Interface.

Protocols and Profiles: Transport Class 0 [ISO 8073:1988]

ATS Used: ATS:1-8

Conformance Lab: NVLAP\# 0369 


\section{Product Code/Type: P-004 TRANSPORT}

Product D. 25

Supplier: IBM Corporation, Rome Networking Systems Laboratory Via Paolo DiDona, 44 00144 Rome taly

Contact: Gerard Bonnes Tel: + $\quad 3392114122$ Fax: +3393247157

GOSIP Product Name: OSI/Communications Subsystem Version/Release: Ver 1 Rel 1.1

Release Date: 01-DEC-90

Additional Info:

Registration:

Date: 01-NOV-91 Basis: DERIVED

Type: $\quad$ PROV, Gindfthrd, V1-> V2

Hardware and Operating System Platforms:

IBM System 370/390

IBM VM/SP Rel 5

Connectivity: X.25 PLP/X.25 LAP-B

Underlying Stack:

Provided by IBM NCP Packet Switching Interface.

Protocols and Profiles: Transport Class 0 [ISO 8073:1988]

ATS Usod: ATS: $1-8$

Conformance Lab: NVLAP 0359

Product Code/Typo: P-004 TRANSPORT

Product D: 34

Supplier: IBM Corporation, Rome Networking Systems Laboratory Via Paolo DiDona, 44 00144 Rome italy

Contact: Gerard Bonnes Tel: + +3392114122

Fax: + 3393247157

GOSIP Product Name: OSI/Communications Subsystem

Version/Release: Ver 1 Rel 1.1

Rolease Date: 01-DEC-90

Additional Info:

Registration:

Date: 12-FEB-92 Basis: DERIVED

Type: $\quad$ PROV, Gmdfthrd, V1->V2

Hardware and Operating System Platforms:

IBM System 370/390

IBM MVS/XA Ver 2 Rel 2

IBM VM/SP Rol 5

IBM VM/ESA Ver 1 Rol 1

Connectivity: X.25 PLP/X.25 LAP-B

Underlying Stack:

Provided by IBM NCP Packet Switching Interface.

Protocols and Profiles: TP4-CLNS/CLNP $\{E S\}$

Transport Class 4-CLNS[ISO 8073:1988/Add2:1989]

CLNP\{ES\} [ISO 8473:1988] \{Static Routing\}

ATS Usod: ATS:1-9

Conformance Lab: NVLAP: 0359
Product Codo/Typo: P-COA TRANSPORT

Product 1D: 36

Supplier: IBM Corporation, Rome Networking Systems Laboratory Via Paolo DiDona, 44 00144 Rome traly

Contact: Gerard Bonnes Tel: +3392114122

Fax: + 3393247157

GOSIP Product Name: OSI/Communications Subsystem

Version/Rolease: Ver 1 Rel 1.1

Release Date: 01-DEC-S0

Additional Info:

Registration:

Date: 12-FEB-92 Basis: DERIED

Type: $\quad$ PROV, Gmdtthrd, V1->V2

Hardware and Operating System Platforms:

IBM System 370/390

IBM MVS/XA Ver 2 Rol 2

IBM VM/SP Rel 5

IBM VM/ESA Ver 1 Rol 1

Connectivity: X25 PLP/X25 LAP-B

Underlying Stack:

Provided by IBM NCP Packet Switching Interface.

Protocols and Profiles: Transport Class 4CONS [ISO 8073:1988]

ATS Usod: ATS:1-9

Conformance Lab: NVLAP 0369

Product Code/Type: P-004 TRANSPORT

Product D: 112

Supplier: IBM Corporation, Rome Networking Systems Laboratory Via Paolo DiDono, 44

00144 Rome traly

Contact: Michael Sullivan Tel: + 39651872517

Fax: + 39651872467

GOSIP Product Name: OSI/Communications Subsystem/400

Version/Release: Ver 2 Rol 1.1

Release Date: 01-MAR-92

Additional info:

Registration:

Date: 18-DEC-92 Basis: BASE

Type: $\quad$ PROV, Gmdfthrd, V1->V2

Hardware and Operating System Platforms:

AS/400 9404

OS/400 Ver 2 Rel 1.1

Connectivity: X25 PLP/X.25 LAP-B

Underlying Stack:

IBM AS/400 X.25 Communications Support Program, Ver 2, Rolease 1.1

Protocols and Profiles: Transport Class 0 [ISO 8073:1988]

ATS Used: ATS: $1-8$

Conformance Lab: NVAP, 0392

IBM Rome Networking Systems Laboratory 
Supplier: NCR

9900 Old Grove Road

San Diego, CA 92131

Contact: Wendy Morrison Tel: (619) $693-5665$

Fax: (619) $693-5705$

GOSIP Product Name: NCR UNIX OSI Network Services

Version/Release: Ver 2.00.02

Release Date: 17-APR-92

Additional Info: Same product also registered over $X .25$ (Product

ID 82) and for TPO over X.25 (Product ID 83)

Pegistration:

Date: 07-AUG-92 Basis: BASE

Type: PROV, Gmdfthrd, V1-> V2

Hardware and Operating System Platforms:

HW: NCR System 3000 consisting of the following hardware

models: $3320,3340,3345,3447,3450,3550$, and 3600

OS: NCR UNIX SVRA, (MP-RAS), Rel 2

Connectivity: $L C 1\{802.2\} / M A C\{802.3\}$

Underlying Stack:

NCR System 3000, Integrated LAN Driver Ver 2.00 Westem Digital WD8003

Protocols and Profiles: TP4CLNS/CLNP\{ES\}

Transport Class 4-CLNS[ISO 8073:1988/Add2:1989]

CLNP\{ES\} [ISO 8473:1988] \{Static Routing\}

ATS Used: ATS: 1-7 and ATS: $1-9$

Conformance Lab: NVLAP 0363

Corporation for Open Systems Intemational Test Center

\section{Product Code/Type: P-004 TRANSPORT}

Product D: 83

Supplier: NCR

9900 Old Grove Road

San Diego, CA 92131

Contact: Wendy Morrison Tel: (619) $693-5665$

Fax: (619) $693-5705$

GOSIP Product Name: NCR UNIX OSI Network Services

Version/Rolease: Ver 2.01

Rolease Date: 08-SEP-92

Additional Info: Same product also registered for TP4 (Product ID 51 and Product ID 82)

Registration:

Date: 27-OCT-92 Basis: BASE

Type: $\quad$ PROV, Gmdfthrd, V1-> V2

Hardware and Operating System Platforms:

HW: NCR System 3000 consisting of the following

hardware models:

$3320,3340,3345,3447,3450,3550$, and 3600

OS: NCR UNIX SVRA, (MP-RAS), Rol 2

Connectivity: X25 PLP/X.25 LAP-B

Underlying Stack:

NCR System 3000, X.25 Ntwrk Sves [Packet Layer]; NCR MUOE HDLC Ver 1.04 [Link Layer]; NCR Multi-Protocol Communications Adapter/ Firmware Ver 1.1A [Phys Layer]

Protocols and Profiles: Transport Class 0 [ISO 8073:1988]

ATS Used: ATS:1-8

Conformance Lab: NMAP* 0363

Corporation for Open Systems Intemational Test Center
Product Code/Type: P-004 TRANSPORT

Product D: 82

Supplier: NCR

9900 Old Grove Road

San Diego, CA 92131

Contact: Wendy Morrison Tel: (619) 693-5665

Fax: (619) 693-5705

GOSIP Product Name: NCR UNIX OSI Network Services

Version/Release: Ver 2.01

Release Date: 08-SEP-92

Additional Info: Same product also registered over LAN (Product

ID 51) and for TPO over X.25 (Product ID 83)

Registration:

Date: 27-OCT-92 Basis: DERIVE

Type: $\quad$ PROV, Gmdtthrd, V1->V2

Hardware and Operating System Platforms:

HW: NCR System 3000 consisting of the following hardware models: $3320,3340,3345,3447,3450,3550$, and 3600

OS: NCR UNIX SVRA, (MP-RAS), Rel 2

Connectivity: X.25 PLP/X.25 LAP-B

Underlying Stack:

NCR System 3000, X.25 Ntwrk Sves [Packet Layer]; NCR MUOE HDLC Ver 1.04 [Link Layer]; NCR Multi-Protocol Communications Adapter/ Firmware Ver 1.1A [Phys Layer]

Protocols and Profiles: TP4CLNS/CLNP\{ES\}

Transport Class 4-CLNS[ISO 8073:1988/Add2:1989]

CLNP\{ES\} [ISO 8473:1988] \{Static Routing\}

ATS Used: ATS:1-7 and ATS:1-9

Conformance Lab: NVLA* 0363

Corporation for Open Systems Intemational Test Center

Product Code/Type: P-COA TRANSPOPT

Product D: 49

Supplier: Novell Incorporated

2180 Fortune Drive

San Jose, CA 95131

Contact: Jan Provan

Tel: $(408)$ 473-8422

Fax: (408) 433-9827

GOSIP Product Name: NotWare FTAM Transport Component Version/Rolease: Ver 1.2 Rov $B$

Release Date: 20-APR-92

Additional Info: None

Registration:

Date: 24JUN-92 Basis: BASE

Type: $\quad$ PROV, Gmdfthrd, V1.>V2

Hardware and Operating System Platforms:

AST Premium 386/33 with 8 MB RAM

NOVELL 3.11 Operating System over NOVELL NE2000 Ethemet card (802.3)

Connectivity: $U \mathrm{C} 1\{802.2\} / \mathrm{MAC}\{802.3\}$

Underlying Stack:

NOVEUL 3.11 Operating System over NOVELL NE2000 Ethemet

Protocols and Profiles: TP4CLNS/CLNP\{ES $\}$

Transport Class 4-CLNS[ISO 8073:1988/Add2:1989]

CLNP\{ES\} [ISO 8473:1988] \{Static Routing\}

ATS Used: ATS:1-7 and ATS:1-9

Conformance Lab: NMAP\# 0357

National Computing Centre Limited 
Product Code/Typo: P-004 TRANSPORT

Product D: 50

Supplier: Novell Incorporated

2180 Fortune Drive

San Jose, CA $\$ 5131$

Contact: Jan Provan

Tel: (408) 473-8422

Fax: (408) 433-9827

GOSIP Product Name: NetWare FTAM Transport Component

Version/Release: Ver 1.2 Rev B

Rolease Date: 20-APR-92

Additional Info: None

Registration:

$\begin{array}{lll}\text { Date: } & \text { 24JUN-92 } & \text { Basis: DERNED } \\ \text { Type: } & \text { PROV, Gmdtthrd, V1-> V2 }\end{array}$

Hardware and Operating System Platforms:

AST Premium $386 / 33$ with 8 MB RAM

NOVELL 3.11 Operating System over NOVELL NE2000 Ethemet card (802.3)

Connectivity: LLC1\{802.2\}/MAC $\{802.3\}$

Underlying Stack:

NOVELL Ver 3.11 Operating System Over $L C_{1}\{802.2\} /$

MAC $\{802.4\}$ NE2000 and IBM 4MB Token Ring Card

Protocols and Profiles: TP4-CLNS/CLNP $\{$ ES $\}$

Transport Class 4CLNS[ISO 8073:1988/Add2:1989]

CLNP\{ES\} [ISO 8473:1988] \{Static Routing\}

ATS Used: ATS: $1-7$ and ATS: $1-9$

Conformance Lab: NVLAP 0357

National Computing Centre Limited

\section{Product Code/Type: P-004 TRANSPORT}

Product DD: 111

Supplier: Retix

2401 Colorado Avenue

Santa Monica, CA 90404

Contact: Jeff Stono Tel: (310) 828-3400

Fax: (310) $828-2255$

GOSIP Product Name: LT-610

Version/Release: Ver 2.3.0

Release Date: 01-OCT-92

Additional Info:

Registration:

Date: 18-DEC-92 Basis: BASE

Type: PROV, Gmdfthrd, V1-> V2

Hardware and Operating System Platforms:

Vendor claims all Intel 386 and 486 based platforms using

stated 0/8. Test platform was Intel 486DX (Alpha Systems

Laboratory, Incorporated ASL486/33 ASL433), O/S UNIX

System V Release 3.2 (SUN Soft Interactive Ver 3.0)

Connectivity: $U \mathrm{C} 1\{802.2\} / \mathrm{MAC}\{802.3\}$

Underlying Stack:

SM: Retix LT-610, Ver 2.3.0; H/W: Westem Digital Model WD8003.

Protocols and Profiles: TP4-CLNS/CLNP\{ES\}

Transport Class 4-CLNS[ISO 8073:1988/Add2:1989]

CLNP \{ES\} [ISO 8473:1988] \{Static Pouting\}

ATS Usod: ATS: 1.7 and ATS: 1.9

Conformance Lab: NMLAP 0357

National Computing Centro Limited
Product Code/Type: P-004 TRANSPORT

Product D: 132

Supplier: Rotix

2401 Colorado Avenue

Santa Monica, CA 90404

Contact: Jeff Stone

Tel: (310) $828-3400$

Fax: (310) 828-2255

GOSIP Product Name: LT-610

Version/Release: Ver 2.3.0

Release Date: 01-OCT-92

Additional Info: None

Registration:

Date: $\quad$ 05-FEB-93 Basis: BASE

Type: $\quad$ PROV, Gmofthrd, V1 -> V2

Hardware and Operating System Platforms:

intel 386 (Tatung) O/S UNIX System V Rel 3.2 (SCO UNLX 4.0)

Connectivity: $L C 1\{802.2\} / M A C\{802.3\}$

Underlying Stack:

Retix LT-610, Ver 2.3.0; Western Digital Model WD8003

Protocols and Profiles: TP4-CLNS/CLNP $\{$ ES $\}$

Transport Class 4-CLNS[ISO 8073:1988/Add2: 1989]

CLNP\{ES\} [ISO 8473:1988] \{Static Routing\}

ATS Usod: ATS: 1.7 and ATS:1-9

Conformance Lab: NVLAP 0357

National Computing Centre Limited

Product Code/Type: P-CO4 TRANSPORT

Product D: 187

Supplier: SUN MicroSystems, Inc.

32 Chemin du Vieux Chene

Meylan F-38240 France

Contact: Tom Hull

Tel: +3376414243

Fax: + 3376414241

GOSIP Product Name: SUNLink OSI

Version/Release: Version 8.0

Release Date: 08-AUG-93

Additional Info:

Registration:

Date: 26-AUG-93 Basis: BASE

Type: Provisional, GOSIP Ver, 2

Hardware and Operating System Platforms:

HW: AI SUN SPARC Hardware

OS: Solaris 2.1

Connectivity: X.25 PLP/X.25 LAP-B

Underlying Stack:

Sunlink X25 8.0

Protocols and Profiles:

Transport Class 0 [ISO 8073:1988]

ATS Usod: ATS:2-8

Conformance Lab: NVLAP\# 0357

National Computing Contre Limited 
Product Code/Typo: P-004 TRANSPORT

Product D: 188

Supplier: SUN MicroSystems, Inc. 32 Chemin du Vieux Chene Meylan F-38240 France

Contact: Tom Hull Tel: +3376414243

Fax: + 3376414241

GOSIP Product Name: Sunlink OSI

Version/Release: Version 8.0

Release Date: O8-AUG-93

Additional Info:

Registration:

Date: 09-SEP-93 Basis: BASE

Type: Provisional, GOSIP Ver. 2

Hardware and Operating System Platforms:

HW: SparcStation 10 Model 41

OS: Solaris 2.1

HW: Vendor clalms support for all SUN SPARC Hardware

Connectivity:

UC1 $\{802.2\} / M A C\{802.3\} / 10 B a s e T$

Underlying Stack:

Sunlink OSI 8.0 UC Module, implementing UC1, the Solaris 2.1

Lance Ethemet driver and Am7990 combination-implementing

ISO8802-3 Sun Motherboard LAN Interface

Protocols and Profiles:

TP4-CLNS/CLNP $\{E S\}$ \& ES-IS $\{$ ES $\}$

Transport Class 4-CLNS [ISO 8073:1988/Add2:1989]

CLNP\{ES\} [ISO 8473:1988] \& ES-IS $\{E S\}$ [ISO 9542:1988] \{Dyn

Rting Claimed

ATS Used: ATS:2-9.2 and ATS:2-7

Conformance Lab: NVLAP* 0357

National Computing Contre Limited

Product Code/Type: P-004 TRANSPORT

Product 1D: 66

Supplier: SUN MicroSystems, Inc. Intemational Centre for Network Computing

32 Rue du Vieux Chene

F-38240 Meylan France

Contact: Tom Hull Tol: +3376 414218

Fax: + 3376414241

GOSIP Product Name: SunNet OSI (Transport)

Version/Release: Ver 7.1

Release Date: 01-OCT-92

Additional Info:

Registration:

Date: $\quad$ 14-OCT-92 Basis: BASE

Type: $\quad$ PROV, Grndfthrd, V1-> V2

Hardware and Operating System Platforms:

SUN MicroSystems SPARCstation 2-4/75

SUN O/S 4.1.2 (Solaris 1.0.1)

Connectivity: LC 1 \{802.2\}/MAC $\{802.3\}$

Underlying Stack:

SUNNET OSI Ver 7.1 LC1 (HW) SUn CPU board LAN Interface (802.3)

Protocols and Profiles: TP4-CLNS/CLNP $\{E S\}$

Transport Class 4-CLNS[ISO 8073:1988/Add2:1989]

CLNP\{ES\} [ISO 8473:1988] \{Static Routing\}

ATS Usod: ATS:1-7 AND ATS: 1-9

Conformance Lab: NVLAP* 0357

National Computing Contre Limited
Product Code/Type: P-CO4 TRANSPORT

Product D: 87

Supplier: SUN MicroSystems, Inc. Intemational Centre for Network Computing

32 Rue du Vieux Chene

F-38240 Meylan France

Contact: Tom Hull Tol: +3376414218

Fax: +3376414241

GOSIP Product Name: SunNet OSI (Transport)

Version/Release: Ver 7.1

Release Date: 01-OCT-92

Additional Info: None

Reglstration:

Date: 25-NOV-92 Basis: BASE

Type: $\quad$ PROV, Grndfthrd, V1->V2

Hardware and Operating System Platforms:

SUN MicroSystems SPARCstation 2-4/75

SUN O/S 4.1.2 (Solaris 1.0.1)

Connectivity: X.25 PLP/X.25 LAP-B

Underlying Stack: SunNet X.25 Ver 7.0.1

Protocols and Profiles: Transport Class 0 [ISO 8073:1988]

ATS Usod: ATS:1-8

Conformance Lab: NVLAP* 0357

National Computing Centre Limited

Product Code/Type: P-004 TRANSPORT

Product 1D: 85

Supplier: SUN MicroSystems, Inc. Intemational Centre for Network Computing

32 Rue du Vieux Chene

F-38240 Meylan France

Contact: Tom Hull Tel: +3376414218

Fax: +3376414241

GOSIP Product Name: SunNet OSI (Transport)

Version/Release: Ver 7.1

Release Date: 01-OCT-92

Additional Info: None

Registration:

Date: 25-NOV-92 Basis: DERMED

Type: $\quad$ PROV, Gmdfthrd, V1->V2

Hardware and Operating System Platforms:

SUN MicroSystems SPARCstation 2-4/75

SUN O/S 4.1.2 (Solaris 1.0.1)

Connectivity: X.25 PLP/X.25 LAP-B

Underlying Stack: SunNet X.25 Ver 7.0.1

Protocols and Profiles: TP4CLNS/CLNP $\{E S\}$

Transport Class 4-CLNS[ISO 8073:1988/Add2:1989]

CLNP\{ES\} [ISO 8473:1988] \{Static Routing\}

ATS Usod: ATS:1-7 AND ATS:1-9

Conformance Lab: NVAP\# 0357

National Computing Contre Limited 
Supplier: SUN Microsystems Federal, Incorporated 2650 Park Tower Drive, Suite 500

Vienna, VA 22180-7306

Contact: Michael Barnes Tel: (703) 204-4100

Fax: (703) 204-4782

GOSIP Product Name: SUN SPARCstation 10 Model 30 w/SUNUnk OSI 8.0

Version/Release: Ver 8.0

Release Date: 04-AUG-92

Additional Info: None

Registration:

Date: 01-DEC-92 Basis: BASE

Type: $\quad$ PROV, Grndfthrd, V1->V2

Hardware and Operating Systern Platforms:

HW: SPARCstation 10 Model 30

OS: Solaris 2.1

Connectivity: $\amalg C 1\{802.2\} / M A C\{802.3\}$

Underlying Stack:

AMD, Lance 7990 Ethernet Controller SUN Solaris 2.1 Ethernet

Driver

Protocols and Profiles: TP4-CLNS/CLNP $\{$ ES $\}$

Transport Class 4-CLNS[ISO 8073:1988/Add2:1989]

CLNP\{ES\} [ISO 8473:1988] \{Static Routing\}

ATS Used: ATS:1-7 and ATS:1.9

Conformance Lab: NMAP* 0364

CDA, Incorporated Open Systerns Developrnent Group

Product Code/Type: P-004 TRANSPORT

Product D: 142

Supplier: SUN Microsystems Federal, Incorporated

2650 Park Tower Drive, Suite 500

Vienna, VA 22180-7306

Contact: Michael Barnes Tel: (703) 204-4100

Fax: (703) 204-4782

GOSIP Product Name: SUN SPARCstation 10 Model 41 w/SUNUink OSI 8.0

Version/Release: Ver 8.0

Release Date: 04AUG-92

Additional Info:

Registration:

Date: 01-DEC-92 Basis: BASE

Type: $\quad$ PROV, Grndthrd, V1-> V2

Hardware and Operating Systern Platforms:

HW: SPARCstation 10 Model 41

OS: Solaris 2.1

Connectivity: $L C 1\{802.2\} / M A C\{802.3\}$

Underlying Stack:

AMD, Lance 7990 Ethernet Controller SUN Solaris 2.1 Ethernet

Driver

Protocols and Profiles: TP4-CLNS/CLNP $\{E S\}$

Transport Class 4-CLNS[ISO 8073:1988/Add2:1989]

CLNP\{ES\} [ISO 8473:1988] \{Static Routing\}

ATS Usod: ATS:1.7 and ATS:1.9

Conformance Lab: NMLAP 0364

CDA, Incorporated Open Systerns Developrnent Group
Product Code/Typo: P-CO4 TRANSPORT

Product D: 106

Supplier: SUN Microsysterns Federal, Incorporated 2650 Park Tower Drive, Suite 500

Vienna, VA $22180-7306$

Contact: Michael Barnes Tel: (703) 204-4100

Fax: (703) 204-4782

GOSIP Product Name: SUN SPARCstation 10 Model 42 w/SUNLink OSI 8.0

Version/Release: Ver $\mathbf{8 . 0}$

Release Date: O4AUG-92

Additional Info:

Registration:

Date: 01-DEC-92 Basis: BASE

Type: $\quad$ PROV, Grndfthrd, V1-> V2

Hardware and Operating Systern Platforms:

HW: SPARCstation 10 Model 42

OS: Solaris 2.1

Connectivity: $L C 1\{802.2\} / M A C\{802.3\}$

Underlying Stack:

AMD, Lance 7990 Ethernet Controller. SUN Solaris 2.1 Ethernet

Driver

Protocols and Profiles: TP4-CLNS/CLNP $\{$ ES $\}$

Transport Class 4-CLNS[ISO 8073:1988/Add2:1989]

CLNP\{ES\} [ISO 8473:1988] \{Static Routing\}

ATS Usod: ATS:1-7 and ATS:1-9

Conformance Lab: NVLAP\# 0364

CDA, Incorporated Open Systerns Development Group

Product Code/Type: P-004 TRANSPORT Product ID: 107

Supplier: SUN Microsysterns Federal, Incorporated

2650 Park Tower Drive, Suite 500

Vienna, VA 22180-7306

Contact: Michael Barnes Tel: (703) 204-4100

Fax: (703) 204-4782

GOSIP Product Name: SUN SPARCstation 4/30 w/SUNLink OSI 8.0 Version/Release: Ver $\mathbf{8 . 0}$

Release Date: 04-AUG-92

Additional Info:

Registration:

Date: 01-DEC-92 Basis: BASE

Type: PROV, Grndfthrd, V1-> V2

Hardware and Operating Systern Platforms:

HW: SPARCstation 4/30

OS: Solaris 2.1

Connectivity: LLC1 $\{802.2\} / M A C\{802.3\}$

Underlying Stack:

AMD, Lance 7990 Ethernet Controller. SUN Solaris 2.1 Ethernet

Driver

Protocols and Profiles:

TP4-CLNS/CLNP $\{E S\}$

Transport Class 4-CLNS[ISO 8073:1988/Add2:1989]

CLNP\{ES\} [ISO 8473:1988] \{Static Routing\}

ATS Usod: ATS:1-7 and ATS:1-9

Conformance Lab: NMLAP* 0364

CDA, incorporated Open Systerns Developrnent Group 
Product Code/Type: P-004 TRANSPORT

Product D: 109

Supplier: SUN Microsystems Federal, Incorporated 2650 Park Tower Drive, Suite 500

Vienna, VA 22180-7306

Contact: Michael Barnes Tel: (703) 204-4100 Fax: (703) 204-4782

GOSIP Product Name: SUN, RDI BrightLite Model IPX Color Laptop Workstation w/ SUNLink OSI 8.0

Version/Release: Ver 8.0

Release Date: O4-AUG-92

Additional Info: None

Registration:

Date: 01-DEC-92 Basis: BASE

Type: $\quad$ PROV, Grndthrd, V1-> V2

Hardware and Operating System Platforms:

RDI BrightLite IPX Color Laptop Workstation Solaris 2.1

Connectivity: LC1 $\{802.2\} / M A C\{802.3\}$

Underlying Stack:

AMD, Lance 7990 Ethemet Controller. SUN Solaris 2.1 Ethemet Driver

Protocols and Profiles: TP4-CLNS/CLNP\{ES\}

Transport Class 4-CLNS[ISO 8073:1988/Add2: 1989]

CLNP\{ES\} [ISO 8473:1988] \{Static Routing\}

ATS Usod: ATS:1-7 and ATS:1-9

Conformance Lab: NVAP* 0364

CDA, Incorporated Open Systems Development Group

Product Code/Typo: P-004 TRANSPORT

Product D: 138

Supplier: UNISYS Corporation

8008 West Park Drive

McLean, VA 22102

Contact: Dale Pluta

Tel: (703) 556-5682

Fax: (703) 556-5172

GOSIP Product Name: A-Series CP2000 OSHIPC Sottware

Version/Rolease: Ver 30.00 .199

Release Date: 11-DEC-92

Additional info:

Registration:

Date: 05-FEB-93 Basis: BASE

Type: $\quad$ PROV, Gmdthrd, V1-> V2

Hardware and Operating System Platforms:

Mainframe: A6 (TP4), FEP: CP2000 (CLNP)

O/S Mainframe: A-Series System Software, Ver 4.0 (TP4)

FEP: CP2000 Operating Software, V3.0 (CLNP)

Connectivity: $\amalg C_{1}\{802.2\} / M A C\{802.3\}$

Underlying Stack:

S/W: CP2000 O/S V3.0; H/W: CP2000 with LMH Card

Protocols and Profiles: TP4-CLNS/CLNP $\{E S\}$

Transport Class 4-CLNS[ISO 8073:1988/Add2:1989]

CLNP\{ES\} [ISO 8473:1988] \{Static Routing\}

ATS Used: ATS:1-7 and ATS:1-9

Conformance Lab: NMAP 0367

UNISYS - Open Systems Interconnection Laboratory
Product Code/Typo: P-004 TRANSPORT

Product 1D: 137

Supplier: UNISYS Corporation

8008 West Park Drive

MicLean, VA 22102

Contact: Dale Pluta

Tel: $(703)$ 556-5682

Fax: (703) 556-5172

GOSIP Product Name: A-Series/CP2000 OSHIPC Software Version/Release: Ver $\mathbf{3 0 . 0 0 . 2 0 0}$

Release Date: 05-JAN-93

Additional Info:

Registration:

Date: 16-FEB-93 Basis: BASE

Type: $\quad$ PROV, Gmdtthrd, V1->V2

Hardware and Operating System Platforms:

A-Series Processor

A-Series System Software, Version 4.0

Connectivity: X.25 PLP/X.25 LAP-B

Underlying Stack: CP2000 X.25, Ver 30.00.192, 30-SEP-92

Protocols and Profiles: Transport Class 4-CONS [ISO 8073:1988]

ATS Usod: ATS:1-9

Conformance Lab: NVLAP 0367

UNISYS - Open Systems Interconnection Laboratory

Product Code/Type: P-004 TRANSPORT

Product D: 145

\author{
Supplier: UNISYS Corporation \\ 8008 West Park Drive \\ McLean, VA 22102 \\ Contact: Dale Pluta Tel: (703) 556-5682 \\ Fax: (703) 556-5172
}

GOSIP Product Name: A-Series/CP2000 OSHIPC Software

Version/Release: Ver 30.00.199

Rolease Date: 11-DEC-92

Additional Info:

Registration:

Date: 16-FEB-93 Basis: DERMED

Type: $\quad$ PROV, Gmdthrd, V1->V2

Hardware and Operating System Platforms:

H/W: Mainframe: A6 (TP4), FEP: CP2000 (CLNP)

O/S: Mainframe: A-Series System Software, Ver 4.0

(TP4), FEP: CP2000 Operating Software, Ver 3.0 (CLNP)

Connectivity: X.25 PLP/X.25 LAP-B

Underlying Stack: CP2000 X.25 Ver 30.00.192, 30-SEP-92

Protocols and Profiles: TP4-CLNS/CLNP\{ES\}

Transport Class 4-CLNS[ISO 8073:1988/Add2:1989]

CLNP\{ES\} [ISO 8473:1988] \{Static Routing\}

ATS Usod: ATS: $1-7$ and ATS: $1-9$

Conformance Lab: NVLAP: 0367

UNISYS - Open Systems Interconnection Laboratory 
Product Code/Typo: P-004 TRANSPORT

Product D: 136

Supplier: UNISYS Corporation 8008 West Park Drive

McLoan, VA 22102

Contact: Dale Pluta

Tel: (703) 556-5682

Fax: $(703)$ 556-5172

GOSIP Product Name: ASeries/CP2000 OSHIPC Software, Unisys Product 113/P4.3

Version/Release: Ver 30.00 .200

Release Date: 05-JAN-93

Additional Info:

Registration:

Date: 26-FEB-93 Basis: BASE

Type: $\quad$ PROV, Gindtthrd, V1->V2

Hardware and Operating System Platforms:

A-Series Processor

A-Series System Software, Version 4.0

Connectivity: X.25 PLP/X.25 LAP.B

Underlying Stack: CP2000 X25, Ver 30.00.192, 30-SEP-92

Protocols and Profiles: Transport Class 0 [ISO 8073:1988]

ATS Usod: ATS: 18

Conformance Lab: NMLAP 0367

UNISYS - Open Systems Interconnection Laboratory

Product Code/Typo: P-OO4 TRANSPORT

Product 1D: 50

Supplior: UNISYS Corporation

8008 Westbrook Drive

McLean, VA 22102

Contact: Keith Fretz

Tol: (703) 556-5665

Fax: (703) 556-5172

GOSIP Product Name: CMS 1100/OSITS

Version/Release: 7R2B + PCR15312/2R1A+ PCRs192,193,194,197

Release Date: 01-MAR-92

Additional Info:

Registration:

Date: 01-SEP-92 Basis: BASE

Type: $\quad$ PROV, Gmdtthrd, V1->V2

Hardware and Operating System Platforms:

2200 System and 1100/90 Processors, DCP-15 through DCP-55

Front End Processors. Operating System OS 1100 Exec on processor DCP/OS 5R2A, TELCON 9R1A on Front End Processors Connectivity: LC1 $\{802.2\} / \mathrm{MAC}\{802.3\}$

Underlying Stack:

LAN Platform 2R2A, DCP 802.3; LAN Line Module Feature * F5137-00

Protocols and Profiles: TP4-CLNS/CLNP \{ES\}

Transport Class 4-CLNS[ISO 8073:1988/Add2:1989]

CLNP\{ES\} [ISO 8473:1988] \{Static Routing\}

ATS Used: ATS:1-7 AND ATS:1-9

Conformance Lab: NVAP 0367

UNISYS - Open Systems Interconnection Laboratory
Product Code/Typo: P-CO4 TRANSPORT

Product D: 81

Supplier: UNISYS Corporation

8008 Westbrook Drive

MicLan, VA 22102

Contact: Koith Fretz Tel: (703) 556-5665

Fax: (703) 556-5172

GOSIP Product Name: CMS 1100/OSITS Ver 7R2B + PCR 15312, 1 92-194, 197, 199, 202, 203, 205, 207

Version/Rolease: Rol 2R1A

Rolease Date: 01-MAR-92

Additional info:

Registration:

Date: 16-OCT-92 Basis: DERMED

Type: $\quad$ PROV, Gmdfthrd, V1->V2

Hardware and Operating System Platforms:

OS1100 Exec. Ver 43R2 running on 2200 systems and $1190 / 90$ Processors DCP.15 through DCP-55 Front End Processors, O/S OS1100 Exec. on Processors DCP/OS 5R2A, Telcon 9R1A on Front End Processors

Connectivity: X.25 PLP/X.25 LAP-B

Underlying Stack:

X25 PSCS Ver 51RA plus PCRs 1891-1899, 1902, 1911, 1923, 1929.

Protocols and Profiles: TP4-CLNS/CLNP $\{$ ES $\}$

Transport Class 4-CLNS[ISO 8073:1988/Add2:1989]

CLNP\{ES\} [ISO 8473:1988] \{Static Routing\}

ATS Usod: ATS:1-7 AND ATS:1-9

Conformance Lab: NVLAP 0367

UNISYS - Open Systems Interconnection Laboratory

Product Code/Typo: P-DO4 TRANSPORT

Product 1D: 73

Supplier: UNISYS Corporation

8008 Westbrook Dive

McLean, VA 22102

Contact: Koith Fretz Tol: (703) 556-5665

Fax: (703) 556-5172

GOSIP Product Name: DCP OSITS

Version/Release: 2R1A + 192-194,197,199,202,203,205,207

Release Date: 01-MAR-92

Additional Info:

Registration:

Date: $\quad$ 19OCT-92 Basis: BASE

Type: $\quad$ PROV, Gmdthrd, V1.>V2

Hardware and Operating System Platforms:

DCP.15 through DCP.55 Front End Processors

DCP/OS Ver 5R2A and TELCON Ver 9R1A

Connectivity: UC1\{802.2\}/MAC $\{802.3\}$

Underlying Stack:

LAN Platform 2R2A, DCP 802.3 LAN Line Module, Feature \# F5137-00

Protocols and Profiles: TP4-CLNS/CLNP $\{E S\}$

Transport Class 4-CLNS[ISO 8073:1988/Add2:1989]

CLNP\{ES\} [ISO 8473:1988] \{Static Routing\}

ATS Used: ATS:1-7 AND ATS:1.9

Conformance Lab: NVLA 0367

UNISYS - Open Systems Interconnection Laboratory 


\section{Product Code/Typo: P-004 TRANSPORT}

Supplier: UNISYS Corporation

8008 Westbrook Drive

McLoan, VA 22102

Contact: Keith Fretz

Tel: (703) 556-5665

Fax: (703) 556-5172

GOSIP Product Name: DCP OSITS

Version/Release: 2R1A + 192-194,197,199,202,203,205,207

Release Date: 01-MAR-92

Additional Info:

Registration:

Date: $\quad$ 19-OCT-92 Basis: BASE

Type: $\quad$ PROV, Grndtthrd, V1->V2

Harcware and Operating System Platforms:

DCP-15 through DCP-55 Front End Processors

DCP/OS Ver 5R2A and TELCON Ver 9R1A

Connectivity: X.25 PLP/X.25 LAP-B

Underlying Stack:

Protocols and Profiles: Transport Class 0 [ISO 8073:1988]

ATS Usod: ATS: 18

Conformance Lab: NVLAP\# 0367

UNISYS - Open Systems Interconnection Laboratory

Product Code/Type: P-004 TRANSPORT

Product D: 71

Supplier: UNISYS Corporation

8008 Westbrook Drive

McLean, VA 22102

Contact: Koith Fretz Tel: (703) 556-5665

Fax: (703) 556-5172

GOSIP Product Name: DCP OSITS

Version/Release: 2R1A + 192-194,197,199,202,203,205,207

Release Date: 01-MAR-92

Additional Info:

Registration:

Date: 19-OCT-92 Basis: DERIVED

Type: $\quad$ PROV, Gindthrd, V1-> V2

Hardware and Operating System Platforms:

DCP.15 through DCP.55 Front End Processors

DCP/OS Ver 5R2A and TELCON Ver 9R1A

Connectivity: X.25 PLP/X.25 LAP.B

Underlying Stack:

Protocols and Profiles: TP4-CLNS/CLNP $\{$ ES $\}$

Transport Class 4-CLNS[ISO 8073:1988/Add2:1989]

CLNP\{ES\} [ISO 8473:1988] \{Static Routing\}

ATS Used: ATS:1-7 AND ATS:1-9

Conformance Lab: NVAP* 0367

UNISYS - Open Systems Interconnection Laboratory
Product Code/Typo: P-004 TRANSPORT

Product D: 17

Supplier: UNISYS Corporation

8008 Westbrook Drive

MicLean, VA 22102

Contact: Koith Fretz Tel: (703) 556-5665

Fax: (703) $556-5172$

GOSIP Product Name: DCP OSITS

Version/Rolease: 2R1A, + 192-194,197,199,202,203,205,207

Rolease Date: 01-MAR-92

Additional Info: None

Registration:

Date: 19-OCT-92 Basis: DERIVED

Type: $\quad$ PROV, Grndfthrd, V1 $>$ V2

Hardware and Operating System Platforms:

DCP-15 through DCP-55 Front End Processors

DCP/OS Ver 5R2A and TELCON Ver 9R1A

Connectivity: X.25 PLP/X.25 LAP-B

Underlying Stack:

Protocols and Profiles: Transport Class 4-CONS [ISO 8073:1988]

ATS Used: ATS:1.9

Conformance Lab: MVAP* 0367

UNISYS - Open Systems Interconnection Laboratory

Product Code/Type: P-006 MHS

Product D: 154

Supplier: Control Data Systems, Incorporated

4201 North Lexington Avenue

Arden Hills, MN $55126-6198$

Contact: B. Sokhon Tol: (612) $482-3868$

Fax: (612) $482-2791$

GOSIP Product Name: MAIL *HUB MHS/4000

Version/Release: Ver 2.0.1

Release Date: 29JAN-93

Additional Info: This product does not support MTA Relaying

Registration:

Date: O4-MAY.93 Basis: BASE

Type: PROVISIONAL, GOSIP VER 1

Hardware and Operating System Platforms:

Control Data CD 4000 Series

EP/X 2.0.1

Connectivity:

TP4-CLNS/CLNP/LAN

Underlying Stack:

Control Data EP/X Access and Directory Ver 1.4.2

Protocols and Profiles:

0045

MHS/(Sossion)

MHS [X400:1984] \{P2, P1, RTS\}

MHS Sossion [X.410:1984]

ATS Used:

ATS:1-13, ATS: $1-14$ and ATS:1-15

Conformance Lab: NVLAP* 0354

Control Data Corporation, OSI Accredited Test Center 
Product Code/Typo: P-006 MHS

Product D: 138

Supplier: Encore Computing Corporation $œ 01$ West Sunrise Boulevard

Ft. Lauderdale, FL $33313-4499$

Contact: Augie Gonzales Tel: (305) 587-2900

Fax: (305) 797-5807

GOSIP Product Name: EnComm $\times 400$

Version/Release: Ver 2.0.1

Release Date: 01-JAN-93

Additional Info: None

Registration:

Date: 11-MAR-93 Basis: BASE

TyPe: $\quad$ PROVSIONAL, GOSIP VER 1

Hardware and Operating System Platforms:

Encore Infinity 90 Series GPIO I, O/S UMAX Ver 3.0.7

Connectivity: TPO/WAN

Underlying Stack:

EnComm ISO Transport Services, Rev 3.0.0 [TPO]. EnComm X25

Rev 3.0 (x.25)

Protocols and Profiles: MHS/(Session)

MHS [X.400:1984] \{P2, P1, RTS\}

MHS Session [X,410:1984]

ATS Used: ATS:1-13, ATS:1-14 and ATS:1-15

Conformance Lab: NVLA * 0364

CDA, Incorporated Open Systems Development Group

\section{Product Code/Type: P-006 NHS}

Product D: 191

Supplier: Enterprise Solutions Limited

32603 Bowman Knoll Drive

Westlake Village, CA 91361

Contact: Barry Sandever Tel: (818) 597-8943

Fax: (818) 595-9621

GOSIP Product Name: Enterprise Mail User Agent

Version/Release: Version 2.11

Release Date: 01-MAY-93

Additional Info: Must be used w NCR StarPRO Message Central 400, Ver 2.0, 01-MAR-93. This combination of products provides set of MHS: P2, P1, RTS, \& embedded

Registration: Session protocols

Date: 17-SEP-93 Basis: BASE

Type: $\quad$ Provisional, GOSIP Ver. 1

Hardware and Operating System Platforms:

$H W$ : NCR System 3000 consisting of the following

hardware models:

$3320,3340,3345,3447,3450,3550$, and 3600

OS: NCR UNIX SVRA, (MP-RAS), Rel 2

Connectivity:

TPS-CLNS/CLNP/LAN

Underlying Stack:

NCR UNIX OSI Network Services, Version 2.00.02

Protocols and Profiles: MHS/(Session)

MHS [X.400:1984] \{P2, P1, RTS\}

MHS Session [X 410:1984]

ATS Used: ATS:2-13.4

\section{Conformance Lab: NMAP 0353}

Corporation for Open Systems Intemational Test Conter
Product Code/Type: P.006 MHS

Product D: 13

Supplier: Hewlett-Packard Company, OSI Conformance Test Center 19420 Homestead Road, MS 43UW

Cupertino, CA 95014-9810

Contact: Murali Subbarao

Tel: $(408)$ 447-2822

Fax: (408) 447-2697

GOSIP Product Name: HP X400/9000 P/N HP32032A;

(X.400 Interface) HP OpenMail, P/N B1600A

Version/Release: Ver C.02.00; Ver A.00.02.03

Release Date: 10-JUN-91

Additional Info: None

Roglstration:

Date: 19AUG-91 Basis: BASE

TyPe: $\quad$ PROVSIONAL, GOSIP VER 1

Hardware and Operating System Platforms:

HP 9000 Series 800/HP.UX Operating System, Ver 8.0

Connectivity: TP4-CLNS/CLNP/LAN

Underlying Stack:

HP OSI Transport Services/9000 Series 800

Protocols and Profiles: MHS/(Session)

MHS [X.400:1984] \{P2, P1, RTS\}

MHS Session [X410:1984]

ATS Used: ATS:1-13, ATS:1-14 AND ATS:1-15

Conformance Lab: NVLAP\# 0365

Howlett-Packard Company, OSI Conformance Test Center

Product Code/Type: P-006 MHS

Product D: 141

Supplier: IBM Corporation, Rome Networking Systems Laboratory Via Paolo DiDono, 44

Do144 Rome Italy

Contact: Michael Sullivan Tel: +39651872517

Fax: + 39651872467

GOSIP Product Name:X400 PROFS Connection

Version/Release: V1R3

Release Date: 01JUN-91

Additional Info: Must be used with Open Network Distribution Services, V1R1, 01-OCT-90, \& OSI/Communications Subsystem, V1R1.1, 01-DEC-90

Registration:

Date: 19MAR-93 Basis: BASE

Type: PROVSIONAL, GOSIP VER 1

Hardware and Operating System Platforms:

IBM Enterprise System $/ 390$

IBM VM/ESA Ver 1 Rel 1

IBM Enterprise System/370

IBM VM/ESA Ver 1 Rel 1

Connectivity: TPO/WAN

Undertying Stack:

OSI/Communications Subsystem Ver 1 Rel 1.1 IBM NCP Packet

Switching Interface

Protocols and Profiles: MHS/(Session)

MHS [X400:1984] \{P2, P1, RTS\}

MHS Session [X.410:1984]

ATS Used: ATS:1-13, ATS:1-14 AND ATS:1-15

Conformance Lab: NVLAP 0392

IBM Rome Networking Systems Laboratory 
Product Code/Type: P-006 MHS

Product D: 194

Supplier: IBM Rome Networking Systems Laboratory

Via Paolo DiDono, 44

00143 Rome Haly

Contact: Aberto Sinibaldi Tel: 396 5966-2281

Fax: $3965966-2467$

GOSIP Product Name: Open Network Distrlbution Services

Version/Release: Vèrsion 1 Release 1

Release Date: 01-OCT-90

Additional Info: Must be used with OSI/Communications

Subsystem, V1R1.1, 01-DEC-90, to provide the P1/RTS and

Embedded Session protocols

Registration:

Date: 15-OCT-93 Basis: DERIVED

Type: $\quad$ Provisional, GOSIP Ver. 1

Hardware and Operating System Platforms:

(1) HW: IBM Enterprise System/390

(1) OS: IBM MVS/ESA Ver 3 Rel 1

(2) HW: IBM Enterprise System $/ 370$

(2) OS: IBM MVS/ESA Ver 3 Rel 1

Connectivity: TPO/WAN

Underlying Stack:

OSI/Communications Subsystem, V1R1.1, 01-DEC-90, and IBM NCP Packet Switching Interface

Protocols and Profiles: MHS/(Session)

MHS [X400:1984] \{P1,RTS\}

MHS Session [X 400:1984]

ATS Used: ATS: $1-13$ and ATS:1-14

Conformance Lab: NVLAP\# 0392

IBM Rome Networking Systems Laboratory

Product Code/Type: P-006 MHS

Product D. 195

Supplier: IBM Rome Networking Systems Laboratory

Via Paolo DiDono, 44

00143 Rome Haly

Contact: Aberto Sinibaldi Tel: $3965966-2281$

Fax: $3965966-2467$

GOSIP Product Name: $\times 400$ DISOSS Connection

Version/Release: Version 1 Release 2

Release Date: 01-MAR-91

Additional Info: Must be used with Open Network Distribution Services, V1R1, 01-OCT-90, and OSI/Communications Subsystem, V1R1.1, 01-DEC-SO

Registration:

Date: 13-OCT-93 Basis: BASE

Type: $\quad$ Provisional, GOSIP Ver. 1

Hardware and Operating System Platform:

(1) HW: IBM Enterprise System/390

(1) OS: IBM MVS/ESA Ver 3 Rel 1

(2) HW: IBM Enterprise System $/ 370$

(2) OS: IBM MVS/ESA Ver 3 Rel 1

Connectivity: MHS \{P1, RTS\}/Session/TPO/WAN

Underlying Stack:

Open Network Distribution Services, V1R1, 01-OCT-90, OSI/

Communications Subsystem V1R1.1, 01-DEC-90, and IBM NCP

Packet Switching Interface

Protocols and Profiles: MHS [X.400:1984] \{P2\}

ATS Usod: ATS:1-15

Conformance Lab: NVLAP* 0392

IBM Rome Networking Systems Laboratory
Product Code/Type: P-006 MHS

Product D: $\quad 155$

Supplier: International Business Machines Corporation 3605 Highway 52 North

Rochester

MN 55901 USA

Contact: Michael Sullivan

Tel: $(39-06) 51872517$

Fax: $(39-06) 51872467$

GOSIP Product Name: IBM OSI Message Services/400

Version/Release: Ver 2 Rel 1.1

Release Date: 01-MAR-92

Additional Info: Must be used with OSI Communications

Subsystem/400 Ver 2 Rel 1.1 01-MAR-92

Registration:

Date: 04-MAY-93 Basis: BASE

TyPe: PROVSIONAL, GOSIP VER 1

Hardware and Operating System Platforms:

AS $/ 400$

IBM OS/400 Vor 2 Rel 1.1

Connectivity: TPO/WAN

Underlying Stack:

TPO: IBM OSI Communication Subsystem/400 V2 R1.1 WAN: IBM AS/400 X.25 Communication Support Program V2 R1.1

Protocols and Profiles:

MHS/(Session)

MHS [X.400:1984] \{P2, P1, RTS\}

MHS Session [X.410:1984]

ATS Used: ATS:1-13, ATS:1-14 and ATS:1-15

Conformance Lab: NVAP* 0392

IBM Rome Networking Systems Laboratory

Product Code/Typo: P-006 MHS

Product ID:

124

Supplier: Retix

2401 Colorado Avenue

Santa Monica, CA $\mathbf{9 0 4 0 4}$

Contact: Jeff Stone Tel: (310) $828-3400$

Fax: (310) 828-2255

GOSIP Product Name: Retix User Agent Model MH-423,

Retix OpenServer Model MH-4420

Version/Release: Ver 1.41

Release Date: 01-OCT-92

Additional Info: None

Registration:

Date: O6JAN-93 Basis: BASE

Type: PROVSIONAL, GOSIP VER 1

Hardware and Operating System Platforms:

Test platform was Apha Systems Lab (Intel 486), with Westem Digital 8003 for 802.3

O/S Sunsoft's Interactive UNIX Ner 3.2), Version 3.0 Vendor claims all Intel 80386 and 80486 based platforms using stated O/S

Connectivity: TP4-CLNS/CLNP/LAN

Underlying Stack: Retix LT-610, Ver 2.3.0 (TP4/CLNP/LAN)

Protocols and Profiles: MHS/(Session)

MHS [X.400:1984] \{P2, P1, RTS $\}$

MHS Session [X.410:1984]

ATS Usod: ATS: 1-13, ATS:1-14 AND ATS:1-15

Conformance Lab: NVAP* 0357

National Computing Contre Limited 
Product Code/Typo: P-006 MHS

Product D: 123

Supplier: Retix

2401 Colorado Avenue

Santa Monica, CA 90404

Contact: Jeff Stone Tel: (310) 828-3400

Fax: (310) 828-2255

GOSIP Product Name: Retix User Agent Model MH-423,

Retix OpenServer Model MH-4430

Version/Release: Ver 1.41

Release Date: 01-OCT-92

Additional Info: None

Registration:

$\begin{array}{lll}\text { Date: } & \text { O6JAN-93 Basis: BASE } \\ \text { Type: } & \text { PROMSIONAL, GOSIP VER } 1\end{array}$

Hardware and Operating System Platforms:

Test platform was a Tatung (Intel 386), with a Westem Digital 8003 for 802.3

O/S SCO Ver 4.0 (UNIX V Rel 3.2) Vendor claims all Intel 386 and 486 based platforms using stated O/S

Connectivity: TP4-CLNS/CLNP/LAN

Underlying Stack: Retix LT-610, Ver 2.3.0 (TP4/CLNP/LAN)

Protocols and Profiles: MHS/(Session)

MHS [X.400:1984] \{P2, P1, RTS\}

MHS Session [X 410:1984]

ATS Usod: ATS:1-13, ATS:1-14 AND ATS:1-15

Conformance Lab: NVAP 0357

National Computing Contre Limited

\section{Product Code/Typo: P-006 MHS}

Product D: $\quad 149$

Supplier: UNISYS Corporation

8008 West Park Drive

McLoan, VA 22102

Contact: Dale Pluta

Tel: (703) 556-5682

Fax: (703) 556-5172

GOSIP Product Name: A-Series OSHMHS

Version/Release: 2.0 .048

Release Date: 17-DEC-92

Additional Info: Used In conjunction with A-Series/CP2000 OSHIPC Software, Version 30.00.199, 11-Dec-92.

Rogistration:

Date: 04-MAY-93 Basis: BASE

TyPe: PROVSIONAL, GOSIP VER 1

Hardware and Operating System Platforms:

A-Series/CP2000

- A-Series System Software Ver 4.0

- CP2000 Operating Software Ver 3.0

Connectivity: TP4-CLNS/CLNP/LAN

Undertying Stack:

A-Series/CP2000 OSHIPC (TP4-CLNS/CLNP). CP2000 Operating

Software (LAN)

Protocols and Profiles: MHS/(Session)

MHS [X.400:1984] \{P2, P1, RTS\}

MHS Session [X.410:1984]

ATS Used: ATS:1-13, ATS:1-14, ATS:1-15

Conformance Lab: NVAP * 0357

UNISYS - Open Systems Interconnection Laboratory
Product Code/Typo: P-006 NHS

Product D: $\quad 163$

Supplier: UNISYS Corporation

8008 West Park Drive

McLean, VA 22102

Contact: Dale Pluta

Tel: (703) 556-5682

Fax: (703) 556-5172

GOSIP Product Name: A-Series OSHMHS

Version/Release: Ver 2.0.048

Release Date: 17-DEC-92

Additional Info: In conjunction with A-Series CP/2000 OSHIPC Software Ver 30.00 .200 , Rel 05-JAN-93

Registration:

Date: 18JUN-93 Basis: DERIVED

Type: $\quad$ PROVSIONAL, GOSIP VER 1

Hardware and Operating System Platforms:

ASeries/CP2000

- ASeries System Software Ver 4.0

- CP2000 Operating Software Ver 3.0

Connectivity: TP4-CONS/WAN

TPO/WAN

Underlying Stack:

A-Series/CP2000OSHIPCSoftware/CP2000X.25;A-Series/CP2000

OSHIPC Unisys Product 113/P4.3

Protocols and Profiles: MHS/(Session)

MHS [X400:1984] \{P2, P1, RTS $\}$

MHS Session [X 410:1984]

ATS Used: ATS:1-13, ATS:1-14 and ATS:1-15

Conformance Lab: MVAP* 0367

UNISYS - Open Systems Interconnection Laboratory

Product Code/Type: P-006 MHS

Product 1D: 74

Supplier: UNISYS Corporation

8008 Westbrook Drive

McLean, VA 22102

Contact: Koith Fretz

Tel: (703) 556-5665

Fax: (703) 556-5172

GOSIP Product Name: DDP.PPC \& OS 1100 OSHMHS

Version/Release: Ver DDP.PPC 5RLA + PCR 987

Release Date: 30-MAR-92

Additional Info: None

Registration:

Date: 16-OCT-92 Basis: DERIVED

Type: $\quad$ PROVISIONAL, GOSIP VER 1

Hardware and Operating System Platforms:

Model $1100 / 90$ or any 2200 System using OS 1100 EXEC Ver 43R2

and DCP-15 through DCP-55 using DCP/OS Ver 5R2A and

TELCON Ver 9R1A

Connectivity:

TP4-CLNS/CLNP/WAN or TP4-CONS/WAN or TPOMAN

Underlying Stack: ASeries/CP2000 OSHIPC Ver 30.00.199

(TP4/CLNP). [ISO 8208] X25

DCP OSITS, Ver 2RIA(TP4). [ISO 8208]

X25 CMS 1100/OSITS Ver 7R2B(TPO).

[ISO 8208] X.25 (X.25)

Protocols and Profiles: MHS/(Session)

MHS [X.400:1984] \{P2, P1, RTS\}

MHS Session [X410:1984]

ATS Used: ATS:1-13, ATS:1-14 AND ATS:1-15

Conformance Lab: NVLAP* 0367

UNISYS - Open Systems Interconnection Laboratory 


\section{Product Code/Type: P-006 MHS}

Product D

Supplier: UNISYS Corporation

8008 Westbrook Drive

McLoan, VA 22102

Contact: Keith Fretz

Tel: $(703)$ 556-5665

Fax: (703) 556-5172

GOSIP Product Name: OS1100 OSHMHS DDP-PPC: 5RIA \& PCR 987

Version/Release: Ver OSHHS 2R1B

Release Date: 06-MAY-92

Additional Info: None

Registration:

Date: 10-SEP-92 Basis: BASE

Typo: $\quad$ PROVISIONAL, GOSIP VER 1

Hardware and Operating System Platiorms:

$1100 / 90$ and 2200 Series Processors, OS1100 Exec. Ver 43R2

Connectivity: TP4-CLNS/CLNP/LAN

Underlying Stack: CMS $11007 R 2 B$ + PCR 15312(TP4).

OSITS 2RIA + PCR 192, 193, 194, 197 CMS 1100 7R2B + PCR 15312 (CLNP).

DCP 802.3 LAN Line Module (LAN)

Protocols and Profiles:

MHS/(Session)

MHS [X.400:1984] \{P2, P1, RTS\}

MHS Session [X.410:1984]

ATS Used: ATS:1-13, ATS:1-14 AND ATS:1-15

Conformance Lab: NVLA* 0367

UNISYS - Open Systems Interconnection Laboratory

Product Code/Type: P.006 MHS Rolay

Product 1D: 110

Supplier: Data General Corporation

4400 Computer Drive, MS/D216

Westborough, MA 01580

Contact: Charles Stakus Tel: (508) 870-6392

Fax: (508) $898-4694$

GOSIP Product Name: $\times \mathbf{4 0 0}$ for AViiON Sys

(X.400 for AViiON Sys/3.0) (OSI for AViON)

Version/Release: Ver 3.10

Release Date: 01-AUG-92

Additional info: None

Registration:

$\begin{array}{lll}\text { Date: } & \text { 01-DEC-92 } \quad \text { Basis: BASE } \\ \text { Type: } & \text { PROVIONAL, GOSIP VER } 1\end{array}$

Hardware and Operating System Platforms:

AViiON Series 5000, VSC Synchronous Comms Controller,

O/S, DG/UX Rel. 5.4.1, AV/X.25 Rel. 2.2.0

Connectivity: TPO/WAN

Underlying Stack:

OSI/Platform for AViON System Ver 3.0 [TPO]. X.25 for AViiON

System Ver 2.20

Protocols and Profiles: MHS Relay/(Session)

MHS Relay [X.400:1984] \{P1, RTS

MHS Session [X.410:1984]

ATS Used: ATS:1-13 and ATS:1-14

Conformance Lab: NVLAP 0391

Data General Corporation, OSI Conformance Test Center
Product Code/Type: P-006 MHS Rolay

Product D. 130

Supplier: Digital Equipment Corporation

P.O. Box 121

Worton Grange, Reading, Berks RG2 OTE United Kingdom

Contact: Richard A Duhamel Tel: (508) 486-5021

Fax: (508) 486-7417

GOSIP Product Name: VAX Message Router X.400 Gateway

Version/Release: Ver 2.2G

Rolease Date: 18-DEC-92

Additional Info: None

Registration:

Date: 11-MAR-93 Basis: BASE

Type: PROVISIONAL, GOSIP VER 1

Hardware and Operating System Platforms:

MicroVAX 3400

VAXNMS Ver $5.4 \& 5.5$

Connectivity: TPO/WAN

Underlying Stack:

DECNet-VAX (TM) Extensions Ver5.4A (TD1111)

Protocols and Profiles:

MHS Rolay/(Session)

MHS Rolay [X.400:1984] \{P1, RTS\}

MHS Session [X410:1984]

ATS Used: ATS:1-13 AND ATS:1-14

Conformance Lab: NVLAP* 0357

National Computing Contre Limited

Product Code/Type: P-006 MHS Polay

Product D: 114

Supplier: NCR Network Products Division

9900 Old Grove Road

San Diego, CA 92131

Contact: Rolf Krause Tel: (619) $693-5788$

Fax: (619) 693-5705

GOSIP Product Name: NCR StarPRO Message Central 400

Version/Rolease: Ver 2.0

Rolease Date: 01-MAR-93

Additional Info: This Product also registered for TP4 over LAN (Product ID 115)

Registration:

Date: 29-DEC-92 Basis: BASE

Typo: PROVISIONAL, GOSIP VER 1

Hardware and Operating System Platforms:

HW: NCR System 3000 consisting of the following hardware models: $3320,3340,3345,3447,3450,3550$, and 3600

OS: NCR UNIX SVR4, (MP-RAS), Rel 2

Connectivity: TPO/WAN

Underlying Stack:

NCR UNIX OSI Network Services, Ver 2.01 (Product ID 83)

Protocols and Profiles: MHS Relay/(Session)

MHS Relay [X 400:1984] \{P1, RTS\}

MHS Session [X.410:1984]

ATS Used: ATS:1-13 AND ATS:1-14

Conformance Lab: NVLAP* 0363

Corporation for Open Systems Intemational Test Center 


\section{Product Codo/Type: P-006 MHS Ralay}

Product 1D: 115

Supplier: NCR Notwork Products Division 9900 Old Grove Road

San Diego, CA 92131

Contact: Rolf Krause

Tel: (619) 693-5788

Fax: (619) $693-5705$

GOSIP Product Name: NCR StarPRO Message Central 400 Version/Release: Ver 2.0

Release Date: 01-MAR-93

Additional Info: This Product also registered for TPO over WAN (Product ID 114)

Registration:

Date: 29-DEC-92 Basis: DERIVD

Type: PROVISIONAL, GOSIP VER 1

Hardware and Operating System Platforms:

HW: NCR System 3000 consisting of the following

hardware models:

$3320,3340,3345,3447,3450,3550$, and 3600

OS: NCR UNIX SVR4, (MP-RAS), Rel 2

Connectivity: TP4-CLNS/CLNP/LAN

Underlying Stack:

NCR UNIX OSI Network Services,

Ver 2.00.02 (TP4/CLNP, Product ID 51)

Protocols and Profiles: MHS Relay/(Session)

MHS Relay [X.400:1984] \{P1, RTS\}

MHS Session [X.410:1984]

ATS Usod: ATS:1-13 AND ATS:1-14

Conformanco Lab: NVAP* 0363

Corporation for Open Systems Intemational Test Conter

Product Code/Typo: P006 MHS Ralay

Product D:

Suppller: SUN MIcroSystems, Inc. Intemational

Contre for Network Computing

32 Rue du Vieux Chene

F-38240 Meylan France

Contact: Tom Hull Tol: +337641 4218

Fax: +3376414241

GOSIP Product Name: SUNNET MHS

Version/Release: Ver 7.1

Release Date: 01-OCT-92

Additional Info: None

Rogistration:

Date: 25-NOV-92 Basis: DERMED

Type: $\quad$ PROVSIONAL, GOSIP VER 1

Hardware and Operating System Platforms:

SUN 4, Station 2-4/75

SUN O/S 4.0.3, 4.1, 4.1.1

Connectivity: TPO/WAN

Underlying Stack: SunNet X.25, Ver 7.0.1 Rel 1

Protocols and Profiles: MHS Relay/(Session)

MHS Relay [X.400:1984] \{P1, RTS\}

MHS Session [X.410:1984]

ATS Used: ATS:1-13 AND ATS:1-14

Conformance Lab: NVAP* 0357

National Computing Centre Limited
Product Code/Typo: P006 MHS Ralay

Product 1D: 67

Supplier: SUN MicroSystems, Inc.

International Contre for Network Computing

32 Rue du Vieux Chene

F-38240 Meylan France

Contact: Tom Hull Tol: +33764142 18

Fax: +3376414241

GOSIP Product Name: SUNNET MHS Gateway

Version/Release: Ver 7.1

Rolease Date: 01-OCT-92

Additional Info: None

Registration:

Date: 14-OCT-92 Basis: BASE

Type: PROVISIONAL, GOSIP VER 1

Hardware and Operating System Platforms:

SUN 4, Station $24 / 75$

SUN O/S 4.0.3, 4.1, 4.1.1

Connectivity: TP4-CLNS/CLNP/LAN

Underlying Stack: SunNet OSI Ver 7.1 Rel 1

Protocols and Profiles: MHS Relay/(Session)

MHS Rolay [X.400:1984] \{P1, RTS\}

MHS Session [X.410:1984]

ATS Usod: ATS:1-13 and ATS:1-14

Conformance Lab: NVLAP* 0357

National Computing Centre Limited

Product Code/Type: P-006 MHS Ralay

Product D: 118

Supplier: SUN Microsystems Federal, Incorporated 2650 Park Tower Drive, Suite 500

Vienna, VA $22180-7306$

Contact: Michael Barnes Tel: (703) 204-4100

Fax: (703) 204-4782

GOSIP Product Name: SunLink OSI

Version/Release: Ver 8.0

Release Date: 04-AUG-92

Additional Info: None

Registration:

Date: $\quad 04 J A N-93 \quad$ Basis: BASE

Type: PROVISIONAL, GOSIP VER 1

Hardware and Operating System Platforms:

SUN, SPARCstation 10 model 30

- MAC HMW - AMD, Lance 7990 Ethemet Controller

- MAC S/W - SUN, Solaris 2.1 Ethernet Driver, O/S Solaris 2.1

Connectivity: TP4-CLNS/CLNP/LAN

Underlying Stack: SunLink OSI 8.0 (TP4/CLNP)

Protocols and Profiles: MHS Relay/(Session)

MHS Relay [X 400:1984] \{P1, RTS\}

MHS Session [X.410:1984]

ATS Used: ATS:1-13 AND ATS:1-14

Conformance Lab: NMAP* 0364

CDA, Incorporated Open Systems Development Group 
Product Code/Type: P-006 MHS Palay

Product D: 119

Supplier: SUN Microsystems Federal, Incorporated 2650 Park Tower Drive, Suite 500

Vienna, VA $22180-7306$

Contact: Michael Barnes Tel: (703) 204-4100 Fax: (703) 204-4782

GOSIP Product Name: SunLink OSI

Version/Release: Ver 8.0

Release Date: O4-AUG-92

Additional Info: None

Registration:

Date:

04JAN-93 Basis: BASE

Type:

PROVISIONAL, GOSIP VER 1

Hardware and Operating System Platforms:

SUN, SPARCstation 10 Model 42

- MAC HM - AMD, Lance 7990 Ethernet Controller

- MAC S/W - SUN, Solaris 2.1 Ethernet Drlver, O/S Solaris 2.1

Connectivity: TP4-CLNS/CLNP/LAN

Underlying Stack: SunLink OSI 8.0 (TP4/CLNP)

Protocols and Profiles: MHS Relay/(Sesslon)

MHS Relay [X.400:1984] \{P1, RTS\}

MHS Session [X410:1984]

ATS Used: ATS:1-13 AND ATS:1-14

Conformance Lab: NVLAP* 0364

CDA, Incorporated Open Systems Development Group

Product Code/Type: P-006 MHS Relay

Product D: 120

Supplier: SUN Microsysterns Federal, Incorporated

2650 Park Tower Drive, Suite 500

Vienna, VA 22180-7306

Contact: Michael Barnes Tel: (703) 204-4100

Fax: (703) 204-4782

GOSIP Product Name: SunLink OSI

Version/Release: Ver $\mathbf{8 . 0}$

Release Date: O4-AUG-92

Additional Info: None

Registration:

Date: O4JAN-93 Basis: BASE

Type: $\quad$ PROVSIONAL, GOSIP VER 1

Hardware and Operating System Platforms:

SUN, SPARCstation 10 Model 41

- MAC H/W - AMD, Lance 7990 Ethemet Controller

- MAC SN - SUN, Solaris 2.1 Ethernet Driver, O/S Solaris 2.1

Connectivity: TP4-CLNS/CLNP/LAN

Underlying Stack: SunLink OSI 8.0 (TP4/CLNP)

Protocols and Profiles: MHS Relay/(Session)

MHS Relay [X.400:1984] \{P1, RTS\}

MHS Session [X410:1984]

ATS Used: ATS:1-13 AND ATS:1-14

Conformance Lab: NVLAP 0364

CDA, Incorporated Open Systerns Developrnent Group
Product Code/Type: P-006 MHS Raley

Product D: 121

Supplier: SUN Microsystems Federal, Incorporated 2650 Park Tower Drive, Suite 500

Vienna, VA 22180-7306

Contact: Michael Barnes Tel: (703) 204-4100

Fax: (703) 204-4782

GOSIP Product Name: SunLink OS

Version/Release: Ver $\mathbf{8 . 0}$

Release Date: O4-AUG-92

Additional Info: None

Peglstration:

Date: O4JAN-93 Basis: BASE

Type: $\quad$ PROVIONAL, GOSIP VER 1

Hardware and Operating System Platforms:

SUN, SPARCstation 4/30

- MAC HM - AMD, Lance 7990 Ethernet Controller

- MAC SM - SUN, Solaris 2.1 Ethernet Driver, O/S Solaris 2.1

Connectivity: TP4-CLNS/CLNP/LAN

Underlying Stack: SunLink OSI 8.0 (TP4/CLNP)

Protocols and Profiles: MHS Relay/(Session)

MHS Rolay [X 400:1984] \{P1, RTS \}

MHS Session [X.410:1984]

ATS Used: ATS:1-13 AND ATS:1-14

Conformance Lab: NVAP; 0364

CDA, Incorporated Open Systerns Development Group

Product Code/Type: P-006 MHS Rolay

Product D: 122

Supplier: SUN Microsysterns Federal, Incorporated 2650 Park Tower Drive, Suite $\mathbf{5 0 0}$

Vienna, VA $22180-7306$

Contact: Michael Barnes

Tel: (703) 204-4100

Fax: (703) 204-4782

GOSIP Product Name: SunLink OSI

Version/Release: Ver $\mathbf{8 . 0}$

Release Date: O4AUG-92

Additional Info: None

Registration:

Date: O4JAN-93 Basis: BASE

Type: PROVSIONAL, GOSIP VER 1

Hardware and Operating System Platforms:

RDI BRIGHTUTE Model IPX Color Laptop Workstation

- MAC HM - AMD, Lance 7990 Ethernet Controller

- MAC S/W - SUN, Solaris 2.1 Ethernet Driver, O/S Solaris 2.1

Connectivity: TP4-CLNS/CLNP/LAN

Underlying Stack: SunLink OSI 8.0 (TP4/CLNP)

Protocols and Profiles: MHS Relay/(Session)

MHS Relay [X.400:1984] \{P1, RTS\}

MHS Session [X.410:1984]

ATS Used: ATS:1-13 AND ATS:1-14

Conformarice Lab: NVLAP\# 0364

CDA, Incorporated Open Systerns Development Group 
Product Code/Type: P-007 FTAM

Product 1D:

113

Supplier: Bull information Systems, Incorporated 13430 North Black Canyon Highway

Phoenix, AZ 85029

Contact: Oscar V Hefner Tel: (602) 862-6001

Fax: (602) 862-6051

GOSIP Product Name: FTAMX

Version/Release: Ver 02.01.06

Release Date: 01-AUG-92

Additional Info: None

Registration:

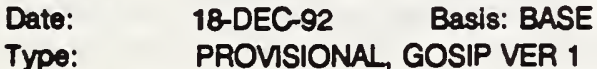

Hardware and Operating System Platforms:

DPX/2 200, 0/S B.O.S. 2

Connectivity: Session/TP4-CLNS/CLNP/LAN

Underlying Stack: LT-610, Bull Information Systems

Protocols and Profiles: FTAM [ISO 8571:1988] \{T1, M1\}

ATS Used: ATS:1-16

Conformance Lab: NMAP: 0370

Conformance Expert Center for OSI Bull - CECOB

\section{Product Code/Typo: P-007 FTAM}

Product D: $\quad 80$

Supplier: Data General Corporation

4400 Computer Drive, MS/D216

Westborough, MA 01580

Contact: Charles Stakus Tel: (508) $870-6392$

Fax: (508) 898-4694

GOSIP Product Name: FTAM For AViON Systems

Version/Release: Vor 3.10

Release Date: 01-AUG-92

Additional Info: None

Registration:

Date: 13-NOV-92 Basis: BASE

Type: $\quad$ PROVSIONAL, GOSIP VER 1

Hardware and Operating System Platforms: AViiON 5000/6000 Series

DG/UX System for AViON Systems Rov. 5.4.1

Connectivity: Session/TP4-CLNS/CLNP/LAN

Underlying Stack: OSI/Platform for AViiON Systems

Protocols and Profiles: FTAM [ISO 8571:1988] \{T1, M1\}

ATS Usod: ATS:1-16

Conformance Lab: NVAP 0391

Data General Corporation, OSI Conformance Test Center
Product Code/Typo: P-OOT FTAM

Product D: 130

Supplier: Digital Equipment Corp

550 King Street

Uittleton, MA 01460-1289

Contact: Richard A Duhamel Tel: (508) 486-5021

Fax: (508) 486-7417

GOSIP Product Name: DECnet/OSI for OpenVMS

Version/Release: Ver V5.5

Release Date: OANOV-92

Additional info: None

Rogistration:

Date: 13-JAN-93 Basis: DERIVED

Type: PROVISIONAL, GOSIP VER 1

Hardware and Operating System Platforms:

Digital VAX

VMS Ver 5.5

Connectivity: Session/TP4-CLNS/CLNP/LAN

Underlying Stack:

DECNet - OSI for Open VMS VAX Ver 5.5/NOTS Ver 3.0A

Protocols and Profiles: FTAM [ISO 8571:1988] \{T1\}

ATS Usod: ATS:1-16

Conformance Lab: MLAP* 0362

Digital Equipment Corporation, OSI Conformance Interoperability Test Center

Product Code/Type: P-oor FTAM

Product D: $\quad 53$

Supplier: Digital Equipment Corporation

550 King Street

Littleton, MA 01460-1289

Contact: Ladan Porooshani Tel: (508) $486-7123$

Fax:

GOSIP Product Name: DECNET-VAX (TM) Extensions

Ver 5.4ANAX FTAM Ver 2.0A

Version/Rolease: Ver 2.0A

Release Date: 01-MAR-92

Additional Info: None

Rogistration:

Date: 16-AUG-92 Basis: BASE

TYPe: $\quad$ PROVISIONAL, GOSIP VER 1

Hardware and Operating System Platforms:

Digital VAX with VMS V5.4 and DECNET-VAX V5.4 Extensions

Connectivity: TP4-CLNS/CLNP/LAN

Underlying Stack: DECNet - OSI for OpenVMS VAX

Ver 5.5NOTS Ver 3.0A

Protocols and Profiles: FTAM/(ACSE)/(Presentation)/(Session)

FTAM [ISO 8571:1988] \{T1\}

FTAM ACSE [ISO 8650:1988]

FTAM Presentation [ISO 8823:1988]

FTAM Session [ISO 8327:1987]

ATS Used: ATS:1-16

Conformance Lab: NMAP\# 0362

Digital Equipment Corporation, OSI Conformance Interoperability Test Center 


\section{Product Code/Typo: P-007 FTAM}

Product D:

63

Supplier: Encore Computing Corporation

\$01 West Sunrise Boulevard

Ft. Lauderdale, FL 33313-4499

Contact: Augie Gonzales Tel: (305) 587-2900

Fax: (305) 797-5807

GOSIP Product Name: EnComm FTAM

Version/Release: Ver 2.0.1

Release Date: 01-SEP.92

Additional Info: None

Rogistration:

Date: 23-SEP-92 Basis: BASE

TyPo: $\quad$ PROVISIONAL, GOSIP VER 1

Hardware and Operating System Platiorms:

Encore Infinity 90 Series GPIO I, O/S UMAX Ver 3.0.7

Connectivity: TP4-CLNS/CLNP/LAN

Underlying Stack: Encore Infininty 90 Series GPIO I

Protocols and Profiles: FTAM/(ACSE)/(Presentation)/(Session)

FTAM [ISO 8571:1988] \{T1\}

FTAM ACSE [ISO 8650:1988]

FTAM Presentation [ISO 8823:1988]

FTAM Session [ISO 8327:1987]

ATS Used: ATS:1-16

Conformance Lab: NVLAP* 0364

CDA, Incorporated Open Systems Development Group

Product Code/Type: P-0OT FTAM

Product D: 32

Supplier: Hewlett-Packard Company

19420 Homestead Road

Cupertino, CA 950149810

Contact: Kelly Emo Tel: (408) 447-2822

Fax: (408) 447-3660

GOSIP Product Name: HP FTAM/9000 Series 800

Version/Release: Ver C.02.03

Release Date: 10JUN-91

Additional Info: None

Rogistration:

Date: 30-JAN-92 Basis: BASE

Typo: PROVSIONAL, GOSIP VER 1

Hardware and Operating System Platforms:

HP9000 Series 800 computers which support LAN/9000 link product;

HP-UX Operating System, Version 8.0

Connectivity: TP4-CLNS/CLNP/LAN

Underlying Stack: Unknown. Documentation was not retained.

Protocols and Profiles: FTAM/(ACSE)/(Presentation)/(Session)

FTAM [1SO 8571:1988] \{T1\}

FTAM ACSE [ISO 8650:1988]

FTAM Presentation [ISO 8823:1988]

FTAM Session [1SO 8327:1987]

ATS Used: ATS:1-16

\section{Conformance Lab: NMAP* 0365}

Hewlett-Packard Company, OSI Conformance Test Center
Product Code/Typo: P-oor FTNM

Product D: 135

Supplier: IBM Corporation, Rome Notworking Systems Laboratory Via Paolo DiDono, 44

Co144 Rome, Haly

Contact: Michael Sullivan Tel: +3965187 2517

Fax: + 39651872467

GOSIP Product Name: IBM AIX OSI Messaging and Filing / 6000 Version/Release: Ver 1

Release Date: 01-DEC-90

Additional Info: None

Pegistration:

Date: 25-FEB-93 Basis: BASE

Type: PROVSIONAL, GOSIP VER 1

Hardware and Operating System Platforms:

IBM AIX/6000, Ver 3.1.5, O/S RISC System 6000

Connectivity: TP4-CLNS/CLNP/LAN

Underlying Stack: None

Protocols and Profiles: FTAM/(ACSE)/(Presentation)/(Session)

FTAM [ISO 8571:1988] \{T1\}

FTAM ACSE [ISO 8650:1988]

FTAM Presentation [ISO 8823:1988]

FTAM Session [ISO 8327:1987]

ATS Usod: ATS:1-16

Conformance Lab: NVAP* 0392

IBM Rome Notworking Systems Laboratory

Product Codo/Typo: P-007 FTAM

Product D: 166

Supplier: IBM Corporation, Rome Networking Systems Laboratory Via Paolo DiDono, 44

00144 Rome ttaly

Contact: Michael Sullivan Tel: + 39651872517

Fax: + 39651872467

GOSIP Product Name: IBM OSI/File Services

Version/Release: Version 1 Release 1

Release Date: 01JUN-90

Additional Info: Must be used with OSI/Communications

Subsystem Version 1 Rolease 1.1, 01-DEC-90

Registration:

Date: 24JUN-93 Basis: BASE

Type: $\quad$ Provisional, GOSIP Ver. 1

Hardware and Operating System Platforms:

(1) HW: IBM Enterprise System/390

(1) OS: IBM MVS/ESA Vor 3 Rol 1

(2) HW: IBM Enterprise System $/ 370$

(2) OS: IBM MVS/ESA Ver 3 Rol 1

Connectivity: TP4-CONS/WAN

Underlying Stack:

IBM OSI/Communications Subsystem V1R1.1 IBM X.25 NCP

Packet Switching Interface V3R4

Protocols and Profiles: FTAM/(ACSE)/(Presentation)/(Session)

FTAM [ISO 8571:1988] \{T1\}

FTAM ACSE [ISO 8650:1988]

FTAM Presentation [ISO 8823:1988]

FTAM Session [ISO 8327:1987]

ATS Used: ATS:1-16

Conformance Lab: NVLAP* 0392

IBM Rome Notworking Systems Laboratory 
Product Code/Type: P-007 FTAM

Product D: 52

Supplier: NCR

9900 Old Grove Road

San Diego, CA 92131

Contact: Wendy Morrison

Tel: (619) 693-5665

Fax: (619) $693-5705$

GOSIP Product Name: NCR OSI STAR PRO FTAM

Version/Rolease: Ver 2.00.00

Release Date: 01-JUL-92

Additional Info: None

Registration:

Date: 07-AUG-92 Basis: BASE

Typo: PROVSIONAL, GOSIP VER 1

Hardware and Operating System Platforms:

HW: NCR System 3000 consisting of the following

hardware models:

$3320,3340,3345,3447,3450,3550$, and 3600

OS: NCR UNIX SVR4, (MP-RAS), Rol 2

Connectivity: TP4-CLNS/CLNP/LAN

Underlying Stack:

Product ID* 51: NCR UNIX OSI Network Services, Ver 2.00 .02

Protocols and Profiles: FTAM/(ACSE)/(Presentation)/(Session)

FTAM [ISO 8571:1988] \{T1\}

FTAM ACSE [ISO 8650:1988]

FTAM Presentation [ISO 8823:1988]

FTAM Sossion [ISO 8327:1987]

ATS Used: ATS:1-16

Conformance Lab: NVAP* 0363

Corporation for Open Systems International Test Center

\section{Product Code/Typo: P-007 FTAM}

Product 1D: 48

Supplier: Novell Incorporated

2180 Fortune Drive

San Jose, CA 95131

Contact: Jan Provan

Tel: (408) 473-8422

Fax: (408) 433-9827

GOSIP Product Name: NETWARE FTAM

Version/Rolease: Ver 1.2 Rov B

Release Date: 20-APR-92

Additional Info: None

Registration:

$\begin{array}{ll}\text { Date: } & \text { 24JUN-92 } \\ \text { Type: } & \text { PROVSIONAL, GOSIP VASE } \\ & \end{array}$

Hardware and Operating System Platforms:

FTAM Initiator Hub and Responder AST Premium 386/33; Netware 3.11; Novell Ethemet Card FTAM Initiator Executable AST Premium 386/33; DOS 3.3; Novell NE2000 Ethemet Card

Connectivity: TP4-CLNS/CLNP/LAN

Underlying Stack:

Unknown. Documentation was not retained.

Protocols and Profiles: FTAM/(ACSE)/(Presentation)/(Session)

FTAM [ISO 8571:1988] \{T1\}

FTAM ACSE [ISO 8650:1988]

FTAM Presentation [ISO 8823:1988]

FTAM Sossion [ISO 8327:1987]

ATS Used: ATS:1-16

Conformance Lab: NVAP 0357

National Computing Centre Limited
Product Code/Typo: P-007 FTAM

Product 1D: 134
Supplier: Retix

2401 Colorado Avenue

Santa Monica, CA 90404

Contact: Jeff Stone Tel: (310) 828-3400

Fax: (310) 828-2255

GOSIP Product Name: Retix FTAM FT-820

Version/Rolease: Ver 1.80

Release Date: 01-OCT-92

Additional Info: None

Registration:

Date:

01-FEB-92 Basis: BASE

Typo:

PROVSIONAL, GOSIP VER 1

Hardware and Operating System Platforms:

Tatung (Intel 386), O/S UNIX System V Rel 3.2 (SCO Ver 4.0)

Connectivity: TP4-CLNS/CLNP/WAN

Underlying Stack: Retix LT-610

Protocols and Profiles: FTAM/(ACSE)/(Presentation)/(Session)

FTAM [ISO 8571:1988] \{T1\}

FTAM ACSE [ISO 8650:1988]

FTAM Presentation [ISO 8823:1988]

FTAM Session [ISO 8327:1987]

ATS Usod: ATS:1-16

Conformance Lab: NMAP* 0357

National Computing Centre Limited

Product Code/Type: P-COT FTAM

Product D: 126

Supplier: Retix

2401 Colorado Avenue

Santa Monica, CA 90404

Contact: Jeff Stone Tel: (310) $828-3400$

Fax: (310) $828-2255$

GOSIP Product Name: Retix FTAM Model FT-820

Version/Release: Ver 1.80

Rolease Date: 01-OCT-92

Additional Info: None

Rogistration:

Date: 21 JAN-93 Basis: BASE

Typo: PROVSIONAL, GOSIP VER 1

Hardware and Operating System Piatforms:

Intel 486 Apha Systems Lab

UNIX System V Rol 3.2

Connectivity: Session/TP4-CLNS/CLNP/LAN

Underlying Stack: Retix LT-610

Protocols and Profiles: FTAM [ISO 8571:1988] \{T1\}

ATS Used: ATS:1-16

Conformance Lab: NVLAP\# 0357

National Computing Centre Limited 
Supplier: SUN MicroSystems, inc. Intemational Contre for Network Computing

32 Rue du Vieux Chene

F.38240 Meylan France

Contact: Tom Hull Tol: +3376 414218

Fax: +3376414241

GOSIP Product Name: SUNNET OSI FTAM

Version/Release: Ver 7.1

Release Date: 01-OCT-92

Additional Info: None

Registration:

$\begin{array}{lll}\text { Date: } & \text { 14OCT-92 } & \text { Basis: BASE } \\ \text { Type: } & \text { PROVSIONAL, GOSIP VER } 1\end{array}$

Hardware and Operating System Platforms:

SUN 4

SUN O/S 4.0.3, 4.1, 4.1.1, 4.1.2

Connectivity: TP4-CLNS/CLNP/LAN

Underlying Stack: SUNNet OSI 7.0

Protocols and Profiles: FTAM/(ACSE)/(Presentation)/(Session)

FTAM [ISO 8571:1988] \{T1\}

FTAM ACSE [ISO 8650:1988]

FTAM Presentation [ISO 8823:1988]

FTAM Session [ISO 8327:1987]

ATS Used: ATS:1-16

Conformance Lab: NVAP* 0357

National Computing Centre Limited

Product Code/Type: P-oot FTAM

Product iD:

Supplier: SUN MicroSystems, Inc.

Intemational Centre for Network Computing

32 Rue du Vieux Chene

F.38240 Meylan France

Contact: Tom Hull Tel: +3376414218

Fax: +3376414241

GOSIP Product Name: SUNNET OSI FTAM

Version/Release: Ver 7.1

Release Date: 01-OCT-92

Additional Info: None

Registration:

Date: 25-NOV-92 Basis: DERIVED

Type: $\quad$ PROVISIONAL, GOSIP VER 1

Hardware and Operating System Platforms:

SUN 4

SUN O/S 4.0.3, 4.1, 4.1.1, 4.1.2

Connectivity: TPO/WAN

Underlying Stack: SUNNet X25

Protocols and Profiles: FTAM [ISO 8571:1988] \{T1\}

ATS Used: ATS:1-16

Conformance Lab: NVLAP* 0357

National Computing Centre Limited
Product Code/Type: P-007 FTNM

Product D: 156

Supplier: SUN Microsystems Federal, Incorporated 2650 Park Tower Drive, Suite 500

Vienna, VA 22180-7306

Contact: Michael Barnes Tel: (703) $204-4100$

Fax: (703) 204-4782

GOSIP Product Name: SUNLink OSI FTAM

Version/Release: Ver $\mathbf{8 . 0}$

Release Date: O4-AUG-92

Additional Info: None

Reglstration:

Date: O4JAN-93 Basis: BASE

Type: PROVISIONAL, GOSIP VER 1

Hardware and Operating System Platforms:

HW: SPARCstation $4 / 30$

OS: Solaris 2.1

Connectivity: TP4CLNS/CLNP/LAN

Underlying Stack: SUNLink OSI 8.0

Protocols and Profiles: FTAM/(ACSE)/(Presentation)/(Session)

FTAM [ISO 8571:1988] \{T1\}

FTAM ACSE [ISO 8650:1988]

FTAM Presentation [ISO 8823:1988]

FTAM Session [ISO 8327:1987]

ATS Used: ATS:1-16

Conformance Lab: NVLAP* 0364

CDA, Incorporated Open Systems Development Group

Product Code/Type: P-007 FTAM

Product 1D: 150

Supplier: SUN Microsystems Federal, Incorporated

2650 Park Tower Drive, Suite 500

Vienna, VA 22180-7306

Contact: Michael Barnes

Tel: (703) 204-4100

Fax: (703) 204-4782

GOSIP Product Name: SUNLink OSI FTAM

Version/Release: Ver 8.0

Rolease Date: O4-AUG-93

Additional Info: None

Registration:

Date: O4JAN-93 Basis: BASE

Type: $\quad$ PROVISIONAL, GOSIP VER 1

Hardware and Operating System Platforms: HW: SPARCstation 10 Model 42

OS: Solaris 2.1

Connectivity: TP4-CLNS/CLNP/LAN

Underlying Stack: SUNLink OSI 8.0

Protocols and Profiles: FTAM/(ACSE)/(Presentation)/(Session)

FTAM [ISO 8571:1988] \{T1\}

FTAM ACSE [ISO 8650:1988]

FTAM Presentation [ISO 8823:1988]

FTAM Session [ISO 8327:1987]

ATS Used: ATS:1-16

Conformance Lab: NVLAP* 0364

CDA, Incorporated Open Systems Development Group 


\section{Product Code/Type: P-007 FTAM}

Product D: 158

Supplier: SUN Microsystems Federal, Incorporated

2650 Park Tower Dive, Suite 500

Vienna, VA 22180-7306

Contact: Michael Barnes Tel: (703) 204-4100

Fax: (703) 204-4782

GOSIP Product Name: SUNLink OSI FTAM

Version/Release: Ver 8.0

Release Date: O4-AUG-83

Additional Info: None

Registration:

$\begin{array}{ll}\text { Date: } & \text { O4JAN-93 } \\ \text { Type: } & \text { PROVISIONAL, BASE }\end{array}$

Hardware and Operating System Platforms:

HW: SPARCstation 10 Model 41

OS: Solaris 2.1

Connectivity: TP4-CLNS/CLNP/LAN

Underlying Stack: SUNUink OSI 8.0

Protocols and Profiles: FTAM/(ACSE)/(Presentation)/(Session)

FTAM [ISO 8571:1888] \{T1\}

FTAM ACSE [ISO 8650:1988]

FTAM Presentation [ISO 8823:1988]

FTAM Session [ISO 8327:1987]

ATS Used: ATS:1-16

Conformance Lab: NVLAP * 0364

CDA, Incorporated Open Systems Development Group

\section{Product Code/Typo: P-007 FTNM}

Product D: 157

Supplier: SUN Microsystems Federal, Incorporated

2650 Park Tower Drive, Suite 500

Vienna, VA 22180-7306

Contact: Michael Barnes Tel: (703) 204-4100

Fax: (703) 204-4782

GOSIP Product Name: SUNLink OSI FTAM

Version/Release: Ver 8.0

Pelease Date: O4AUG-93

Additional info: None

Registration:

Date: O4JAN-93 Basis: BASE

Type: PROVSIONAL, GOSIP VER 1

Hardware and Operating System Platforms:

HW: SPARCstation 10 Model 30

OS: Solaris 2.1

Connectivity: TP4-CLNS/CLNP/LAN

Underlying Stack: SUNLink 8.0

Protocols and Profiles: FTAM/(ACSE)/(Presentation)/(Session)

FTAM [ISO 8571:1988] \{T1\}

FTAM ACSE [ISO 8650:1988]

FTAM Presentation [ISO 8823:1988]

FTAM Session [ISO 8327:1987]

ATS Used: ATS:1-16

Conformance Lab: NMAP 0364

CDA, Incorporated Open Systems Development Group
Product Code/Type: P-007 FTAM

Product D: 160

Supplier: SUN Microsystems Federal, incorporated 2650 Park Tower Drive, Suite 500

Vienna, VA $22180-7306$

Contact: Michael Barnes Tel: (703) 204-4100

Fax: (703) 204-4782

GOSIP Product Name: SUNLink OSI FTAM

Version/Rolease: Ver 8.0

Pelease Date: O4-AUG-93

Additional info: None

Registration:

Date: O4JAN-93 Basis: BASE

Type: PROVIIONAL, GOSIP VER 1

Hardware and Operating System Platforms:

RDI BrightLite IPX Color Laptop Workstation

Solaris 2.1

Connectivity: TP4-CLNS/CLNP/LAN

Undertying Stack: SUNLink OSI 8.0

Protocols and Profiles: FTAM/(ACSE)/(Presentation)/(Session)

FTAM [ISO 8571:1988] \{T1\}

FTAM ACSE [ISO 8550:1988]

FTAM Presentation [ISO 8823:1988]

FTAM Session [ISO 8327:1987]

ATS Usod: ATS:1-16

Conformance Lab: NVLAP* 0364

CDA, incorporated Open Systems Development Group

Product Code/Type: P-OO7 FTAM

Product D: 146

Supplier: UNISYS Corporation

8008 West Park Drive

McLean, VA 22102

Contact: Dale Pluta Tel: (703) 556-5682

Fax: (703) 556-5172

GOSIP Product Name: A-Series OSI FTAM

Version/Release: Ver $\mathbf{4 0 . 4 0 0 . 0 0 0 8}$

Release Date: 16-NOV-92

Additional info: In conjunction with A-Series/CP2000 OSHIPC Ver 30.00.199, Rel 11-Dec-92

Registration:

Date: 13-APR-93 Basis: BASE

TyPe: $\quad$ PROVISIONAL, GOSIP VER 1

Hardware and Operating System Platforms:

A6

A-Series System Ver 4.0

Connectivity: TP4-CLNS/CLNP/LAN

Undertying Stack: A-Series/CP2000 OSHIPC Ver 30.00 .199 11-DEC-92

Protocols and Profiles: FTAM/(ACSE)/(Presentation)/(Session)

FTAM [ISO 8571:1988] \{T1\}

FTAM ACSE [ISO 8650:1988]

FTAM Presentation [ISO 8823:1988]

FTAM Session [ISO 8327:1987]

ATS Used: ATS:1-16

Conformance Lab: NYLAP* 0367

UNISYS - Open Systems Interconnection Laboratory 
Supplier: UNISYS Corporation

8008 West Park Drive

McLean, VA 22102

Contact: Dale Piuta Tel: (703) 556-5682

Fax: (703) 556-5172

GOSIP Product Name: A-Series OSHFTAM Product 115/P7.1a

Version/Release: Ver $\mathbf{4 0 . 4 0 0 . 0 0 0 8}$

Release Date: 16-NOV-92

Additional Info: Session Layer Is not registered at the request of the vendor UNISYS

Registration:

$\begin{array}{ll}\text { Date: } & \text { O6-MAY-93 } \quad \text { Basis: DERNED } \\ \text { Type: } & \text { PROVSIONAL, GOSIP VER } 1\end{array}$

Hardware and Operating System Platforms:

A6

A-Series System Vor 4.0

Connectivity: TP4-CONS/WAN

Underlying Stack: Unisys Product 113/P4.2

Protocols and Profiles: FTAM/(ACSE)/(Presentation)

FTAM [ISO 8571:1988] \{T1\}

FTAM ACSE [ISO 8650:1988]

FTAM Presentation [ISO 8823:1988]

ATS Used: ATS-16

Conformance Lab: MVAP* 0367

UNISYS - Open Systems Interconnection Laboratory

Product Code/Type: P-0OT FTAM

Product 1D: 75

Supplier: UNISYS Corporation

8008 Westbrook Drive

MoLean, VA 22102

Contact: Keith Fretz Tel: (703) 556-5665

Fax: (703) 556-5172

GOSIP Product Name: OSLFTAM

Version/Release: Rel 2R1A

Rolease Date: O3-MAY-92

Additional Info: None

Registration:

Date: 16-OCT-92 Basis: DERIVED

Typo: PROVISIONAL, GOSIP VER 1

Hardware and Operating System Platforms:

$1100 / 90$ and all 2000 Series Systems, OS1100, Release 43R1

Connectivity: TP4-CLNS/CLNP/WAN

Underlying Stack: CP $2000 \times 25$

Protocols and Profiles: FTAM/(ACSE)/(Presentation)/(Session)

FTAM [ISO 8571:1988] \{T1\}

FTAM ACSE [ISO 8650:1988]

FTAM Presentation [ISO 8823:1988]

FTAM Session [ISO 8327:1987]

ATS Used: ATS:1-16

Conformance Lab: NVAP* 0367

UNISYS - Open Systems Interconnection Laboratory
Product Code/Type: P-007 FTAM

Product D: 80

Supplier: UNISYS Corporation

2450 Swedesford Road

Paoli, PA 19301

Contact: Ed Kelly

Tel: (215) 993-7208

Fax:

GOSIP Product Name: OSLFTAM

Version/Rolease: Rel 2R1A

Rolease Date: O3-MAY-92

Additional Info: None

Registration:

Date:

01-SEP-92 Basis: BASE

Type:

PROVISIONAL, GOSIP VER 1

Hardware and Operating System Platforms:

1100/90 and all 2000 Series Systems OS1100 Release 43R1

DCP-15 through DCP-55, DCP/OS Ver 5R2A/TELCON Ver 9R1A

Connectivity: TP4-CLNS/CLNP/LAN

Underiying Stack: DCP OSITS

Protocols and Profiles: FTAM/(ACSE)/(Presentation)/(Session)

FTAM [ISO 8571:1988] \{T1\}

FTAM ACSE [ISO 8650:1988]

FTAM Presentation [ISO 8823:1988]

FTAM Session [ISO 8327:1987]

ATS Used: ATS:1-16

Conformance Lab: NVLAP 0367

UNISYS - Open Systems Interconnection Laboratory

Product Code/Typo: P-007 FTAM

Product 1D: $\quad 60$

Supplier: UNISYS Corporation

2450 Swedesford Road

Paoli, PA 19301

Contact: Ed Kelly

Tel: (215) 993-7208

Fax:

GOSIP Product Name: OSLFTAM

Version/Rolease: Rol 2R1A

Release Date: 03-MAY-92

Additional Info: None

Registration:

Date: 10-SEP-92 Basis: BASE

Type: PROVISIONAL, GOSIP VER 1

Hardware and Operating System Platforms:

1100/90 and all 2000 Series Systems OS1100 Release 43R1

DCP-15 through DCP-55, DCP/OS Ver 5R2A/TELCON Ver 9R1A

Connectivity: TP4-CLNS/CLNP/LAN

Underlying Stack: CMS 1000 7R2B PLS PCR 15312

Protocols and Profiles: FTAM/(ACSE)/(Presentation)/(Session)

FTAM [ISO 8571:1988] \{T1\}

FTAM ACSE [ISO 8650:1988]

FTAM Presentation [ISO 8823:1988]

FTAM Session [ISO 8327:1987]

ATS Used: ATS:1-16

Conformance Lab: NVAP* 0367

UNISYS - Open Systems Interconnection Laboratory 
Test Suites for the GOSIP Interoperabllity Testing provisions are llsted here. Entries on this reglster are Provisional, valid until December 31, 1994.

\section{$\pi S_{-1} \times 400$}

OSINET, Message Handling Systems Interoperability Tests, Version 1, Edition 2, September 1990, avallable from: OSINET Corporation, 1750 Od Meadow Road Sulte 400, McLean, VA 22102, Tol. (703) 883-2797

\section{TS-2 FTAM}

OSINET, File Transfer, Access and Management Interoperabllity Tests, Version 1, Edition 2, June 1990, available from: OSINET Corporation, 1750 Old Meadow Road Sulte 400, McLean, VA 22102, Tol. (703) 883-2797

\subsubsection{REGISTER OF GOSIP INTEROPERABILITY TEST AND REGISTRATION SERMCES}

Interoperabllity Test and Registration Services which meet the GOSIP Interoperability Testing provisions are listed here. Entries on this register are Provisional.

\section{ITRS-1 OSINET}

c/o Corporation for Open Systems Intemational 1750 Old Meadow Road Suite 400, McLean, VA 22102 Tol: (703) 883-2797

ITRS-2 Process to Support Interoperability (PSI) SPAG, PSI Operator, SPAGsa Avenue Louise 165, Box 6,B-1050 Brussels, Belgium Tol. 3226457811

Fax. 3226450879
Means of Testing (MOT) for the GOSIP program of conformance testing are listed here. These MOTs relate to the protocols Identified in FIPS 146 GOSIP Version 1 and FIPS 146-1 GOSIP Version 2. For further details of each MOT listed, please contact the named supplier.

Version 1 MOT entries are prefixed by "1.".

Version 2 MOT entries are prefixed by "2.".

\section{MEANS OF TESTING REGISTER: WIDE AREA NETWORK}

MOT 1-1 WAN TEST SYSTEMS

NOTE: Wide Area Test Systems reglstered under GOSIP Version 1 were deleted from the Register as of March 14, 1903.

MEANS OF TESTING REGISTER: LOCAL AREA NETWORK

NOTE: Local Area Tost Systems registered under GOSIP Version 1 and not related to FTAM or MHS were deleted from the Register as of March 14, 1993.

\section{MOT 1.2 LAN TEST SYSTEMS}

Supplier. Corporation for Open System: 1750 Old Meadow Road McLoan, VA 22102 USA

Contact: Andrea Reitzel

Tel (703) 205-2809

Fax (703) $848-4572$

Test System Name, Release and Date:

COS 802.3 Test System, Ver 1.0, May 1989.

Hardware and Operating System Platform(s):

HP 16500 A Logic Analyzer System Mainframe, Ver 2.0

HP 16531A 400 Mhz Scope Card, Ver 2.01

HP 16530 A Timebase Card, Ver 2.0

HP 86568 Signal Generator, options $001 \& 002$ HP $8770 A$ Arbitrary Waveform Synthesizer HP 4972A LAN Protocol Analyzer with RS-232C Option, Ver B.04.01

Base/Dorived: Base

Connectivity: 8802-3, Physical

Underlying Stack:

Protocols and Profiles: MAC[ISO 8802-3] (Physical Layer Signalling)

Abstract Test Suite used: ATS:1-3

Date Registered: October 30, 1990

Type of Registration and Expiration Date: Provisional, until October 01, 1992 GOSIP Version 1 
Supplier: Corporation for Open Syctam 1750 Old Meadow Road McLean, VA 22102 USA

Contact: Andrea Reltzel

Tol (703) 205-2809

Fax (703) 848-4572

Test System Name, Release and Date: COS 802.2/802.3 10Base5 Layer 2 Test System, Ver 1.1. May 1989.

Hardware and Operating System Platform(s): MS-DOS 3.1, IBM PC/AT Compatible HP 4972A LAN Protocol Analyzer with RS-232C Option, Ver B.04.01

HP 8568B Signal Generator

HP 8770A Arbitrany Waveform Synthesizer

Base/Derived: Base HP 16500A Loglc Analyzer

Connectivity: MAC[802.3], Physical

Underlying Stack:

Protocols and Profiles:

LC1[8802-2]/MAC[8802-3]

Abstract Test Suite used: ATS:1-3 and ATS:1-6

Date Registered: October 30, 1990

Type of Reglstration and Explration Date:

Provisional, until October 01, 1992 GOSIP Version 1

Supplier: Corporation for Open Systems

1750 Old Meadow Poad

McLean, VA 22102

USA

Contact: Andrea Roltzel

Tel (703) 205-2809

Fax (703) 848-4572

Test System Name, Release and Date:

COS 802.2/802.3 10Base5 Layer 2 Test System, Ver 1.4 December 01, 1990

Hardware and Operating System Platform(s): MS-DOS 3.1, IBM PC/AT Compatible HP 4972A LAN Protocol Analyzer with RS-232C Option, Ver B.04.01

HP 8568 B Signal Generator

HP $8770 A$ Arbitrary Waveform Synthesizer

HP 16500 A Logic Analyzer

Base/Derived: Derived

Connectivity: MAC[802.3], Physical

Underlying Stack:

Protocols and Profiles:

\section{LCI[ISO 8802-2]/MAC[ISO 8802-3]}

Abstract Test Suite used: ATS:1-3 and ATS:1-6

Date Reglstered: July 21, 1992

Type of Reglstration and Explration Date:

Provisional, until October 01, 1992 GOSIP Version 1
MEANS OF TESTING REGISTER: CLNP

\section{MOT 13 CLNP TEST SYSTEMS}

NOTE: CLNP Test Systems registered under GOSIP Version 1 were deleted from the Register as of March 14, 1993.

\section{MEANS OF TESTING REGISTER: TRANSPORT}

\section{MOT 1-4 TRANSPORT TEST SYSTEMS}

NOTE: Transport Test Systems reglstered under GOSIP Version 1 were deleted from the Register as of March 14, 1993. 
MEANS OF TESTING REGISTER: SESSION

\section{MOT 1.5 SESSION TEST SYSTEMS}

Supplier: Acatel TITN hoorporatod 7011 Koll Conter Parkway

Suite 200

Pleasanton, CA 94566-3101

Contact: Scott M. Schmitz Tel (703) 715-0800

Fax (703) 715-0804

Test System Name, Release and Date:

XRTLE, Sossion

Ver 1.1

January 30, 1992

Hardware and Operating System Platform(s):

80386 PC, Interactive 386/1X UNIX Ver 2.2

Twice source code modified Acatel TITN Answare for $\times 25$

Base/Derived: Derived

Connectivity: TP4-CLNS/CLNP\{ES\}/LAN

TPONAN

TPO/X.25/PLP/HDLC/RS-232C

Protocols and Profiles:

[ISO 8327] SESSION/FULL SESSION

Abstract Test Suite used: ATS:1-10

Date Reglstered: August 03, 1992

Type of Reglstration and Expiration Date:

Provisional, until October 01, 1992 GOSIP Version 1

Supplier. The National Computing Centre Limited

Oxford House, Oxford Road

Manchester, M1 7ED

United Kingdom

U.S. Contact: Andrea Reitzel Tol (703) 205-2809

U.K. Contact: Peter Bird Tel (44) 61 228-6333

Test System Name, Release and Date:

NCC:COS Session Tester Ver 1.0

August 01, 1991

Hardware and Operating System Platform(s):

SUN 3 Series, SUN OS 4.1.1 or later

SUN 4 Series, SUN OS 4.1.1 or later

Base/Derived: Derived

Connectivity: TPO/X.25 PLP/X.25 LAP-B/RS-232C

TP2 $X .25$ PLP/X.25 LAP.B/N.35

TP4/X.25 PLP/X.25 LAP-B

TP4-CLNS/CLNP $\{E S\} / L L C 1\{802.2\} / M A C\{802.3\}$

TP4-CLNS/CLNP $\{E S\} / X .25$ PLP/X.25 LAP-B

Protocols and Profiles:

[ISO 8327] Session/Full Session

Abstract Test Sulte used: ATS:1-10

Date Reglstered: July 14, 1992

Type of Reglstration and Expiration Date:

Provisional, until October 01, 1992 GOSIP Version 1

\section{MEANS OF TESTING REGISTER: MHS}

MOT 1-6 MHS TEST SYSTEMS

Supplier: GSHDanet incorporated 1380 Old Froeport Road Pittsburgh, PA 15238

Contact: Hans-Ludwig Heil Tel (412) 967-0834

Test System Name, Release and Date: OSITEST $/ 400$,

Ver 3.3

July 01,1990

Hardware and Operating System Platform(s):

SUN 3 Series, SUN OS 3.5

SUN 4 Series, SUN OS 4.1

DEC MicroVAX, ULTRIX 2.2

Base/Derived: Base

Connectivity: Session/TPO/X.25 PLP/X.25 LAP-B/RS-232C Sossion/TPO/X.25 PLP/X.25 LAP.B/N.35

Sossion/TP4/CLNP $\{E S\} / L C 1\{8802.2\} / M A C\{8802.3\}$

Underlying Stack:

Protocols and Profiles: MHS- $84[X, 400]\{P 2, P 1, R T S\}$

MHS-84 Relay[X.400]\{P1,RTS $\}$

MHS Session [X.410:1984]

Abstract Test Suite used: ATS:1-13, ATS:1-14, ATS:1-15

Date Reglstered: September 30, 1990

Type of Registration and Explration Date: Provisional, until October 01, 1992 GOSIP Version 1

Supplier. The National Computing Centre Limited Oxtord House, Oxford Road

Manchester, M1 7ED

Unlted Kingdom

U.S. Contact: Andrea Reitzel

Tel (703) 205-2809

Tel (44) $61228-6333$

Test System Name, Release and Date:

NCC:COS MHS Tester,

Ver 2.2.2

July 01,1991

Hardware and Operating System Platform(s): SUN 4 Series, SUN OS 4.1

Base/Derived: Derived

Connectivity: Session/TPO/X.25 PLP/X.25 LAP-B/RS-232C Sossion/TPO/X.25 PLP/X.25 LAP-B/N.35 Session/TP4/CLNP/X.25 PLP/X.25 LAP.B/RS-232C Session/TP4/CLNP/X.25 PLP/X.25 LAP-B/N.35 Session/TP4/CLNP/LLC1[8802-2]/MAC[8802-3] Session/TP4/CLNP/LLC1[8802-2]/MAC[8802-4]

Underlying Stack:

Protocols and Profiles: MHS-84[X,400] \{P2,P1,RTS $\}$ MHS-84 Relay $[X .400]\{P 1, R T S\}$ MHS Session[X.410:1984]

Abstract Test Suite used: ATS:1-13, ATS:1-14, ATS:1-15

Date Registered: August 19, 1991

Type of Registration and Expiration Date: Provisional, until October 01, 1992 GOSIP Version 1 
MEANS OF TESTING REGISTER: FTAM

\section{MOT 1.7 FTAM TEST SYSTEMS}

Supplier: Ncatal TITN Incorporated 12030 Sunrise Valley Drive Reston, VA 22091

Contact: Scott M. Schmitz Tel (703) 715-0800 Fax (703) 715-0804

Test System Name, Release and Date: XRTLE, FTAM Ver 1.0 April 10, 1991

Hardware and Operating System Platform(s): SUN 3 Series, SUN O.S. 4.0 .3

SUN 4 Series, SUN 0.S. 4.1

Base/Derived: Derived

Connectivity:

SESSION/TPO/X.25 PLP/X.25 LAP.B/RS-232C SESSION/TPO/X.25 PLP/X.25 LAP-B.V.35 SESSION/TP4/X.25 PLP/X25 LAP-BN.35 SESSION/TP4/X.25 PLP/X.25 LAP-B/RS-232C Underlying Stack: SESSION/TP4/CLNP/LC1[802.2]/MAC[802.3]

Protocols and Profiles: FTAM[ISO 8571]

Abstract Test Suite used: ATS:1-16

Date Registered: June 04, 1991

Type of Registration and Expiration Date: Provisional, until October 01, 1992 GOSIP Version 1

Supplier: Acatel TITN incorporated

7011 Koll Center Parkway, Suite 200

Pleasanton, CA 94566-3101

Contact: Scott M. Schmitz Tel (703) 715-0800

Fax (703) $715-0804$

Test System Name, Release and Date: XRTLE, FTAM Ver 1.2 January 30, 1992

Hardware and Operating System Platform(s): 80386 PC, Interactive 386/iX UNIX Ver 2.2

Base/Derived: Derived

Connectivity:

Session/TP4/CLNP/LLC1[802.2]/MAC[802.3]

Session/TPO/X25 PLP/HDLC/RS-232C

Session/TP4/X.25 PLP/X.25 LAP.B/RS-232C and V.35

Underlying Stack:

Protocols and Profiles: FTAM[ISO 8571]

Abstract Test Suite used: ATS:1-16

Date Rogistered: August 03, 1992

Type of Registration and Expiration Date:

Provisional, until October 01, 1992 GOSIP Version 1
Supplier: GSHDanet incoporated 1380 Old Freeport Road Pittsburgh, PA 15238

Contact: Hans-Ludwig Heil Tol (412) 967-0834

Test System Name, Release and Date: OSITEST/FTAM,

Ver 2.4

July 01, 1990

Hardware and Operating System Platform(s):

SUN 3 Series, SUN OS 3.5

SUN 4 Series, SUN OS 4.1

DEC MicroVAX, ULTRIX 2.2

Base/Derived: Base

Connectivity: Session/TPO/WAN Session/TP4/CLNP/LAN

Protocols and Profiles: ISO 8571 FTAM; ISO 8823 Presentation; ISO 8650 ACSE FTAM Session[ISO 10607-1:1990]

Abstract Test Suite used: ATS:1-16

Date Registered: September 30, 1990

Type of Registration and Expiration Date: Provisional, until October 01, 1992 GOSIP Version 1

Supplier: The National Computing Centre Limited Oxford House, Oxford Road Manchester, M1 7ED United Kingdom

U.S. Contact: Andrea Reitzel Tel (703) 205-2809

U.K. Contact: Peter Bird Fax (703) 846-8590 Tel (44) $61228-6333$

Test System Name, Release and Date: NCC:COS FTAM Tester, Ver 2.2 .2 July 01, 1991

Hardware and Operating System Platform(s): SUN 4 Series, SUN OS 4.1

Base/Derived: Derived

Connectivity: Session/TPO/X.25 PLP/HDLC LAP-B/RS-232C Session/TPO/X.25 PLP/HDLC LAP-B/N.35 Session/TP4/CLNP/X25 PLP/HDLC LAP-B/RS-232C Session/TP4/CLNP/X.25 PLP/HDLC LAP-B/N.35 Session/TP4/CLNP/MAC[802.3]/UC1[802.2] Session/TP4/CLNP/MAC[8802-4]/LC1[8802-2]

Protocols and Profiles: FTAM[ISO 8571]; Presentation[ISO 8823]; ACSE[ISO 8650] FTAM Session[ISO 8327: 1987]

Abstract Test Suite used: ATS:1-16

Date Registered: August 01, 1991

Type of Registration and Expiration Date: Provisional, until October 01, 1992 GOSIP Version 1 
Supplier: The National Computing Centre Limited Oxford House, Oxford Road Manchester, M1 7ED United Kingdom

U.S. Contact: Andrea Reitzel

U.K. Contact: Peter Bird
Tel (703) 205-2809

Fax (703) 846-8590

Tel (44) $61228-6333$
Test System Name, Release and Date: NCC:COS FTAM Tester,

Ver 2.3.1

March 18, 1992

Hardware and Operating System Platform(s):

SUN 3 Series, SUN OS 4.1 or later

SUN 4 Series, SUN OS 4.1 or later

Base/Derived: Derived

Connectivity: ACSE/Presentation/Session/TPO/X.25 PLP/X25 LAP.B ACSE/Presentation/Session/TP2/X25 PLP/X.25 LAP-B ACSE/Presentation/Session/TP4/X25 PLP/X.25 LAP-B ACSE/Presentation/Session/TP4/CLNP

Protocols and Profiles: FTAM[ISO 8571]; Presentation[ISO 8823]; ACSE[ISO 8650] FTAM Session Platform [ISO 8327: 1987]

Abstract Test Suite used: ATS:1-16

Date Registered: April 23, 1992

Type of Registration and Expiration Date: Provisional, until October 01, 1992 GOSIP Version 1

Supplier. The National Computing Centro Limited Oxford House, Oxford Road Manchester, M1 7ED United Kingdom

U.S. Contact: Andrea Reitzel Tel (703) 205-2809

U.K. Contact: Peter Bird Tel (44) $61228-6333$

Test System Name, Release and Date: NCC:COS FTAM Tester,

Ver 2.4

March 05, 1992

Hardware and Operating System Platform(s): SUN 3 Series, SUN OS 4.1 or later SUN 4 Series, SUN OS 4.1 or later

Base/Derived: Derived

Connectivity: Session/TPO/X.25 PLP/X.25 LAP.B

Session/TP2/X.25 PLP/X.25 LAP-B

Session/TP4/X.25 PLP/X.25 LAP-B

Session/TP4/CLNP

Protocols and Profiles:

FTAM[ISO 8571]; ACSE[ISO 8650]; Presentation[ISO 8823]; FTAM Session[ISO 10607-1]

Abstract Test Sulte used: ATS:1-16

Date Registered: July 14, 1992

Type of Registration and Expiration Date:

Provisional, until October 01, 1992 GOSIP Version 1

\section{PEGISTER OF GOSIP MEANS OF TESTING GOSIP VERSION 2}

Means of Testing (MOT) for the GOSIP program of conformance testing are listed here. These MOTs relate to the protocols identified in FIPS 146-1 GOSIP Version 2. The type of registration and expiration date are listed with each MOT. For further details of each MOT listed, please contact the named supplier.

\section{MEANS OF TESTING REGISTER: WIDE AREA NETWORKS}

MOT 2-1 WIDE AREA NETWORK

Supplier. Intemational Business Machines P.O. Box 12195

Research Triangle Park, NC 27709 USA

Contact: John G. Conner Tel (919) 254-2679 Fax

Test System Name, Release and Date: Automated Protocol Test System/2 (APTS/2), Ver 1.1 August 03, 1992

Hardware and Operating System Platform(s): IBM PS/2 Model 70, 80, or 90 w 8MB memory, OS/2 Ver 1.3 Base/Derived: Base

Connectivity: RS-232C, V.3

Protocols and Profiles:

X.25 PLP[8208] X.25 LAP.B[7T76]) \{CCIT 1980, 1984, ISO 8208\}

Abstract Test Suite used: ATS:2-1 (99.7\%) and ATS:2-2 (100\%) FULL $X 25$ WTH THE INCLUSION OF ANSI DEFECT REPORTS.

Date Registered: December 14, 1992

Type of Registration and Expiration Date: FULL, until October 01, 1994 GOSIP Version 2

Supplier: Howlett-Packard Company 42115 Street Edmonton, Aberta, T6E5R5 Canada

Contact: Bill Mortimer Tel (403) $462-4545$ Fax

Test System Name, Release and Date: ISO 8882 Test Suite

Ver 3.0

September 01, 1992

Hardware and Operating System Platform(s): PT300, РT302, PT500, PT502, and PT540, O/S Ver 2.0

Base/Derived: Base

Connectivity: RS-232C, V.35

Protocols and Profiles: X25 PLP[8208] (X.25 LAP-B[7776]) \{CCITT 1980, 1984, ISO 8208\}

Abstract Test Suite used: ATS:2-1 (99.7\%) and ATS:2-2 (100\%) FULL $X .25$ WITH THE INCLUSION OF ANSI DEFECT REPORTS.

Date Registered: January 29, 1993

Type of Registration and Expiration Date: FULL, until October 01, 1994 GOSIP Version 2 


\section{Supplier. Howlett-Pacteard Company 42115 Street Edmonton, Aberta, T6E5R5 Canada}

Contact: Bill Mortimer Tel (403) $462-4545$ Fax

Test System Name, Release and Date: ISO 8882 Test Suite

Ver 3.1

February 01, 1993

Hardware and Operating System Platform(s): PT300, PT302, PT500, PT502, and PT540, O/S Ver 2.0 (PT500/300), 2.7 (PT502/302) and 2.8 (PT540)

Base/Derived: Derived

Connectivity: RS-232C, V.35

Protocols and Profiles: X.25 PLP[8208] (X.25 LAP.B[7776]) \{CCITT 1980, 1984, ISO 8208\}

Abstract Test Suite used: ATS:2-1 (99.7\%) and ATS:2-2 (100\%) FULI X25 WITH THE INCLUSION OF ANSI DEFECT REPORTS.

Date Registered: February 17, 1993

Type of Registration and Expiration Date: FULL, until October 01, 1994 GOSIP Version 2

Supplier: TEKEEC

26580 W. Agoura Road

Calabasas, CA 91302

Contact: Siamak Pousabahian Tel (818) 880-7952 Fax (818) 880-6993

Test System Name, Release and Date:

Chameleon 32 Plus, ISO 8882 Test Suite

Ver 1.0

July 14, 1992

Hardware and Operating System Platform(s):

Chameleon 32 Plus

MTOS-UX

Base/Derived: Base

Connectivity: RS-232C, V.35

Protocols and Profiles:

X.25 PLP/X.25 LAP-B

X.25 PLP[ISO 8208:1990] \{CCITT 1984\}

X.25 LAP-B[ISO T776:1986] \{CCITT 1984\}

Abstract Test Suite used: ATS:2-1 (100\%) and ATS:2-2 (100\%) FULL $X .25$ WTTH THE INCLUSION OF ANSI DEFECT REPORTS.

Date Registered: February 08, 1993

Type of Pegistration and Expiration Date:

FULL, until October 01, 1994 GOSIP Version 2
Supplier: TEKEEC

26580 W. Agoura Road

Calabasas, CA 91302

Contact: Pierre Prescott Tel (880) $880-7780$

Fax (818) $880-6993$

Test System Name, Release and Date:

Chameleon 32 Plus, ISO 8882 Test Suite

Vor 2.0

December 17, 1992

Hardware and Operating System Platform(s):

Chameleon 32 Plus

MTOS-UX

Base/Derived: Derived

Connectivity: RS-232C, V.35

Protocols and Profiles:

X.25 PLP/X.25 LAP.B

X.25 PLP[ISO 8208:1990] \{CCITT 1984\}

X.25 LAP-B[ISO 7776:1986] \{CCITT 1984\}

Abstract Test Suite used: ATS:2-1 (100\%) and ATS:2-2 (100\%)

Date Registered: May 12, 1993

Type of Registration and Expiration Date:

FULL, until October 01, 1994 GOSIP Version 2

\section{MEANS OF TESTING REGISTER: CLNP}

\section{MOT 2.3 CLNP TEST SYSTEMS}

Supplier: Acatal TITN incorporated

7011 Koll Center Parkway, Suite 200

Pleasanton, CA 94566-3101

Contact: Scott M. Schmitz

Tel (703) $715-0800$

Fax (703) 715-0804

Test System Name, Release and Date:

XRTLE Ver 4.21

CLNP Ver 1.1

January 29, 1992

Hardware and Operating System Platform(s):

80386 PC, Interactive 386/iX UNIX Version 2.2, 3.0, and 3.0.1 over (LAN) CMC 640 A.T. Type CMC

Base/Derived: Base

Connectivity: $\operatorname{LC} 1\{802.2\} / \mathrm{MAC}\{802.3\}$

Protocols and Profiles: CLNP $\{E S\}$ [ISO 8473]

Abstract Test Suite used: ATS:2-7 (90.9\% Wuse of manual assessment)

Date Registered: January 11, 1993

Type of Registration and Expiration Date:

Provisional until October 01, 1994 GOSIP Version 2 
Supplier: The National Computing Centre Limited

Oxford House, Oxford Road

Manchester M1 7ED

United Kingdom

Contact: Peter Bird

Tel (44) 612286333

Fax

Test System Name, Release and Date: NCC IP (CLNP) Test System,

Ver 2.2.1

March 01, 1991

ITS Ver 2.3

Hardware and Operating System Platform(s):

SUN OS 4.1.2, SUN SPARCstation 330, SUNNET OSI Ver 7.0

Base/Derived: Base

Connectivity: $\operatorname{LLC1}\{802.2\} / \mathrm{MAC}\{802.3\}$

Protocols and Profiles:

CLNP\{ES\}[ISO 8473: 1988]

Abstract Test Suite used: ATS:2-7 (97.0\% With use of manual assessment)

Date Registered: March 03, 1993

Type of Registration and Expiration Date:

Provisional until October 01, 1994 GOSIP Version 2 (Lovel 1)

\section{MEANS OF TESTING REGISTER: ES-IS(ES)}

\section{MOT 2-3 ES-IS TEST SYSTEMS}

Supplier: Acatel TIN, hc.

7011 Koll Center Parkway, Suite 200

Pleasanton, California 94566-3101

Contact: Mr. Scott Schmitz

National Technical Manager (703) 715-0800

Test System Name, Release and Date:

Acatel TITN, Inc. XRTLE V5.20

ES-IS(ES) V1.1 June 1993.

Hardware and Operating System Platform:

PC 386/486

Interactive UNIX V2.2, V2.2.1, V3.0, V3.0.1

Base/Derived: Base

Connectivity: LLC1 1802.2$\} / M A C\{802.3\} / 10 B a s e 5$

Underlying Stack:

TWCE sottware modified by Acatel TITN ANSWARE.

Protocols and Profiles: SIA VER 3:ES-IS $\{E S\}$ [ISO 9542:1988]

Abstract Test Suite used: $\quad$ ATS:2-18

\section{Percentage of ATCs Passed:}

Literal Passes: $\quad 87 \%$

Passes Based on Manual Assessment: $8 \%$

Total Percentage Passed: $\quad 95 \%$

Date Registered: 01-OCT-93

Type of Registration: Provisional GOSIP V2

Expiration Date:

\section{MEANS OF TESTING REGISTER: TRANSPORT}

MOT 2-4 TRANSPORT TEST SYSTEMS

Supplier. Alcatal TITN incorporated

7011 Koll Center Parkway, Suite 200

Pleasanton, CA 94566-3101

Contact: Scott M. Schmitz Tel (703) 715-0800

Fax (703) 715-0804

Test System Name, Release and Date:

XRTLE Ver 4.21

TRO2(TPO) Ver 1.1

(Transport Class 0)

January 29, 1992

Hardware and Operating System Platform(s): 80386 based PC, Interactive 386/iX UNX Ver 2.2 TWCE Source code modified by Acatel TITN ANSWARE

Base/Derived: Base

Connectivity: X.25 PLP/X.25 LAP-B/RS-232C

Underlying Stack:

Protocols and Profiles: ISO 8073:1988, Transport Class 0

Abstract Test Suite used: ATS:2-8 (87\%)

Date Registered: February 01, 1993

Type of Registration and Expiration Date:

Provisional until October 01, 1994 GOSIP Version 2

Supplier: Ncatt TITN hcorporated

7011 Koll Center Parkway, Suite 200

Pleasanton, CA 94566-3101

Contact: Scott M. Schmitz Tol (703) 715-0800

Fax (703) 715-0804

Test System Name, Release and Date:

XRTLE Ver 4.21

TRO2(TP2) Ver 1.1

(Transport Class 2)

January 29, 1992

Hardware and Operating System Platform(s):

80386 based PC

Interactive 386/1X UNIX Ver 2.2

TWCE Source code modified by Alcatel TITN ANSWARE for X.25

Base/Derived: Base

Connectivlty: X.25 PLP/X.25 LAP-B/RS-232C

Protocols and Profiles:

Transport Class 2 [ISO 8073:1988]

Abstract Test Suite used: ATS:2-20 (92.7\%) (93.7\% With use of manual assessment)

Date Registered: March 05, 1993

Type of Registration and Expiration Date:

Provisional until October 01, 1994 GOSIP Version 2 
Supplier. Ncatel TITN incorporated

7011 Koll Conter Parkway, Suite 200

Pleasanton, CA 94566-3101

Contact: Scott M. Schmitz

$$
\begin{aligned}
& \text { Tel }(703) 715-0800 \\
& \text { Fax }(703) 715-0804
\end{aligned}
$$

Test System Name, Rolease and Date:

XRTLE Ver 4.21

TP4-CONS Ver 2.3

January 29,1992

Hardware and Operating System Platform(s):

80386 based PC

Interactive 386/1X UNIX Ver 2.2

TWCE Source code modified by Acatel TITN ANSWARE for $\times .25$

Base/Derived: Base

Connectivity: X.25 PLP/HDLC LAP-B/RS-232C

Protocols and Profiles: TP4-CONS[ISO 8073:1988]

Abstract Test Suite used: ATS:2-9.2 (85.5\%) (89.1\% With use of manual assessment)

Date Registered: March 02, 1993

Type of Registration and Expiration Date:

Provisional until October 01, 1994 GOSIP Version 2

Supplier: Ncattel TITN Incorporated

7011 Koll Center Parkway, Suite 200

Pleasanton, CA 94566-3101

Contact: Scott M. Schmitz Tel (703) $715-0800$

Fax (703) 715-0804

Test System Name, Release and Date:

XRTLE Ver 4.21

TP4-CLNS Ver 2.41

January 29, 1992

Hardware and Operating System Platform(s):

80386 based PC, Interactive 386/1X UNIX Ver 2.2, TWCE

Source code modified by Acatel TITN ANSWARE for CLNP

Base/Derived: Base

Connectivity: CLNP $\{$ ES $\} / L C 1\{802.2\} / M A C\{802.3\}$

Protocols and Profiles:

TP2[ISO 8073:1988]

Abstract Test Suite used: ATS:2-20 (94.7\%) (95.3\% With use of manual assessment)

Date Registered: March 05, 1993

Type of Registration and Expiration Date:

Provisional until October 01, 1994 GOSIP Version 2
Supplier. The Nations Computing Centre Limited Oxford House, Oxford Road . Manchester M1 7ED United Kingdom

Contact: Peter Bird Tel 011-44-612-286-333 Fax 011-44-612-369-877 Internet peter@ncc.co.uk

USA: Robert Clark Technical Support Executive

Tel (510) $687-3002$

Fax (510) 685-2864

Intemetrob@premenos.sf.ca.us

Test System Name, Relesse and Date: NCC ITS Transport Test System, Class 0 Release 3.0

January 04, 1992

ITS Protocol Engine Ver 2.3

Hardware and Operating System Platform(s):

SUN 3 or SUN 4 Series operating SUN OS 4.1.1 or later SUNNot $7.0 \times 25+$ OSI

Base/Derived: Base

Connectivity: X.25 PLP/X.25 LAP-B/RS-232C

Protocols and Profiles: TPO[ISO 8073:1988]

Abstract Test Suite used: ATS:2-8 (85.2\%) (88.9\% With use of

Date Registered: March 31, 1993 manual assessment)

Type of Registration and Expiration Date:

Provisional until October 01, 1994 GOSIP Version 2

Supplier. The National Computing Centre Limited

Oxford House, Oxford Road

Manchester M1 7ED

United Kingdom

Contact: Peter Bird Tel 011-44-612-286-333

Fax 011-44-612-369-877

Internet peter@ncc.co.uk

USA: Robert Clark, Technical Support Executive

Tel (510) $687-3002$

Fax (510) 685-2864

Intemetrob@premenos.sf.ca.us

Test System Name, Release and Date:

NCC ITS Transport Test System, Class 2

Release 3.0

January 04, 1992

ITS Protocol Engine Ver 2.3

Hardware and Operating System Platform(s):

SUN 3 or SUN 4 Series operating SUN OS 4.1 .1 or later SUNNet $7.0 \times .25+$ OSI

Base/Derived: Base

Connectivity: X.25 PLP/X.25 LAP-B/RS-232C

Protocols and Profiles:

TP2[ISO 8073:1988]

Abstract Test Suite used: ATS:2-20 (66.5\%) (71.8\% With use of manual assessment)

Date Registered: March 31, 1993

Type of Registration and Expiration Date:

Provisional until October 01, 1994 GOSIP Version 2 
Supplier: The Nations Computing Centre Limited

Oxford House, Oxford Road

Manchester M1 7ED

United Kingdom

$\begin{array}{lll}\text { Contact: Peter Bird } & \text { Tel } & 011-44-612-286-333 \\ & \text { Fax } & 011-44-612-369-877\end{array}$

Intemet peter@ncc.co.uk

USA:

Robert Clark, Technical Support Executive

Tei (510) 687-3002

Fax (510) 685-2864

Internet rob@premenos.sf.ca.us

Test System Name, Release and Date:

NCC ITS Transport Test System, Class 4/CONS

Release 3.0

January 04, 1992

ITS Protocol Engine Ver 2.3

Hardware and Operating System Platform(s):

SUN 3 or SUN 4 Series operating SUN OS 4.1.1 or later SUNNet X.25 7.0

Base/Derived: Base

Connectivity: X.25 PLP/X.25 LAP-B/RS-232C

Protocois and Profiles: TP4-CONS[ISO 8073:1988]

Abstract Test Sulte used: ATS:2-9.2 (77.8\%) (81.6\% With use of

Date Registered: March 31, 1993 manual assessment)

Type of Registration and Expiration Date:

Provisional untii October 01, 1994 GOSiP Version 2

Supplier: The Nationd Computing Centre Limited

Oxford House, Oxford Road

Manchester M1 7ED

United Kingdom

Contact: Peter Bird Toi 011-44-612-286-333

Fax 011-44-612-369-877

Internet peter@ncc.co.uk

USA: Robert Clark, Technical Support Executive

Tol (510) 687-3002

Fax (510) 685-2864

Intemetrob@premenos.sf.ca.us

Test System Name, Reiease and Date:

NCC ITS Transport Test System, Class 4/CLNS

Reiease 3.0

May 01, 1992

ITS Protocol Engine Ver 2.3

Hardware and Operating System Platform(s):

SUN 3 or SUN 4 Series operating SUN OS 4.1 .1 or later SUNNet OSI 7.0 or later

Base/Derived: Base

Connectivity: $\amalg C_{1}\{802.2\} / M A C\{802.3\}$

Protocois and Profilies:

TP4-CLNS[ISO 8073:1988/Add.2:1989]

Abstract Test Suite used: ATS:2-9.2 (86.5\%) (88.9\% With use of

Date Registered: March 31, 1993

manual assessment)

Type of Registration and Expiration Date:

Provisional until October 01, 1994 GOSIP Version 2
MEANS OF TESTING REGISTER: SESSION

MOT 2-5 SESSION TEST SYSTEMS

Supplier. Acatal TITN Incorporated

7011 Koil Center Parkway, Suite 200

Pleasanton, CA 94566-3101

Contact: Scott M. Schmitz

Tei (703) $715-0800$

Fax (703) 715-0804

Test System Name, Release and Date:

XRTLE Ver 4.21

SESSION Ver 1.1

January 29, 1992

Hardware and Operating System Platform(s):

80386 PC, Interactive 386/iX UNIX (tested with Version 3.0, vendor clalms support for 2.2 and higher) over TWCE Source code modified by Acatei TITN ANSWARE for Transport, CLNP, LC1

Base/Derived: Base

Connectivity: TP4/CLNP $\{E S\} / \amalg C 1\{802.2\} / M A C\{802.3\}$

Protocois and Profiies: Session[iSO 8327]

Abstract Test Suite used:

ATS:2-10.1 (98.4\%) (98.6\% With use of manual assessment)

Date Registered: January 14, 1993

Type of Registration and Expiration Date:

Provisional untii October 01, 1994 GOSIP Version 2

\section{MEANS OF TESTING REGISTER: MHS}

\section{MOT 2-6 MHS TEST SYSTEMS}

\section{Supplier: Alcatel TITN Inconporated}

7011 Koll Center Parkway, Suite 200

Pleasanton, CA 94566-3101

Contact: Scott M. Schmitz Tei (703) 715-0800

Fax (703) 715-0804

Test System Name, Reiease and Date:

GENEPX400 Ver 2.6

June 28, 1992

Hardware and Operating System Platform(s): 80386 PC, Interactive 386/iX UNIX Ver 2.2 over SEMA group software for Session, TPO, OST PCXNet Card running OST X.25 SM

Base/Derived: Base

Connectivity: TPO/X.25 PLP/X.25 LAP-B/RS-232C

Protocois and Profiles: MHS-84[x.400] \{P2, P1, RTS $\}$

Abstract Test Suite used: ATS:2-13.2 (67.16\%) (82.09\% With use of manual assessment) ATS:2-13.3 (90.53\%) ATS:2-13.4 (94.18\%) (96.83\% With use of manual assessment)

Date Registered: February 04, 1993

Type of Registration and Expiration Date:

Provisional until October 01, 1994 GOSiP Version 2 
Supplier. The Nations Computing Centre Limited

Oxford House, Oxford Road

Manchester M1 7ED

United Kingdom

Contact: Poter Bird Tol 011-44-61-228-6333

Fax 011-44-61-236877

internet peter@ncc.co.uk

Test System Name, Release and Date:

$84 \mathrm{mhs}$

Ver 1.0

Fobruary 01, 1993

Additional Information: ITS Protocol Engine Release 24

Hardware and Operating System Platform(s):

SUN SPARCstation $4 / 330$ with SUnOS 4.1.2

SUNNET $7.0 \times .25$ + SUNNET OSI 7.0 (TPO)

OpenWindows 2.0 (not recommended) OpenWindows 3.0

SunLink 8.0 X.25 + SunLink OSI 8.0 (TPO)

Base/Derived: Base

Connectivity: TPO/WAN

Protocols and Profiles: MHS[X 400:1984] \{P2, P1, RTS\}

MHS Session [X.410:1984]

Abstract Test Suite used: $\quad$ Session 2-13.1 (65.4\%)

RTS 2-13.2 (97.1\%)

P1 2-13.3 (85.21\%, 1992.3\%)

Date Registered: May 24, 1993 P2 2-13.4 (82.01\%)

Type of Registration and Expiration Date:

Provisional until October 01, 1994 GOSIP Version 2

\section{MEANS OF TESTING REGISTER: FTAM}

\section{MOT 2.7 FTAM TEST SYSTEMS}

Supplier: Ncattel TITN incorporated

7011 Koll Center Parkway, Suite 200

Pleasanton, CA 94566-3101

Contact: Scott M. Schmitz

Tel (703) 715-0800

Fax (703) 715-0804

Test System Name, Release and Date:

XRTLE Ver $\mathbf{4 . 2 1}$

FTAM Ver 1.31

January 20,1992

Hardware and Operating System Platform(s):

80386 PC, Interactive 386/iX UNIX Ver 2.2 over TWICE Source code modified by Acatel TITN ANSWARE for Session, Transport, CLNP, UC1, X.25

Base/Derived: Base

Connoctivity: Session/TP4/CLNP $\{$ ES $\} / L L C 1\{802.2\} / M A C\{802.3\}$

Session/TP4/X.25 PLP/X.25/RS-232C

Session/TPO/X.25 PLP/X.25/RS-232C

Protocols and Profiles:

FTAM[ISO 8571: 1988], \{T1\}Simple File Transfer Protocol

Abstract Test Suite used: ATS:2-16.1 (78\%) (84\% With use of

Date Registered: January 25, 1993 manual assessment)

Type of Registration and Expiration Date:

Provisional until October 01, 1994 GOSIP Version 2
Supplier: GSHDanet, incorporated

1380 Old Freoport Road

Pittsburgh, PA 15237

Contact: Hans-Ludwig Heil Tel (412) $957-0834$

Fax

Test System Name, Version, Release and Date: OSITEST/FTAM

Version 2.6.3.2

01-MAR-93

Additional Information: OSITEST/TCM Version 2.1.4

Hardware and Operating System Platform(s): Sun4 330 , SunOS 4.1.1

Base/Derived: Base

Connectivity: TP4-CLNS/CLNP $\{$ ES $\} / L A N$ TPONAN

Underlying Stack: SunNet OSI 7.0

SunNet X25 7.0

Protocols and Profiles:

FTAM/(ACSE)/(Presentation)/(Session)

FTAM[ISO 8571:1988] \{T1, M1, T2, A1, R\&R\}

FTAM ACSE[ISO 8650:1988]

FTAM Presentation [ISO 8823:1987]\{ASN.1\}

FTAM Session [ISO 8327:1987]\{R\&R\}

Abstract Test Suite used:

ATS:2-16.2(91\%), ATS:2-16.4(83\%), AT S:2-16.6(90\%), ATS:2-16.7(87\%), ATS:2-16.8(93\%), ATS:2-16.9(82\%),

ATS:2-16.10(81\%), ATS:2-16.11(95\%), ATS:2-16.12(90\%),

ATS:2-16.13(73\%)

Date Registered: 28-JUN-93

Type of Registration and Expiration Date:

Provisional, GOSIP VER 2, until 01-OCT-94

Supplier: National Computing Centre, Ld Oxford Road

Manchester M17ED

United Kingdom

Contact: Robert Clark Tel (510) 687-3002

Test System Name, Version, Release and Date: FTAM Version 3.0

Additional Information:

FTAM Test System includes the PICStool and the Integrated Tool

Set includes the NCC Test Engine Release 2.4, PIXIT Tool, PCTR

Tool, OSllook, OSHool, X-BROWSE, OsmOSis analysis tools

Hardware and Operating System Platform(s):

Sun4 330 , SunOS 4.1.2

Base/Derived: Base

Connectivity: TP4-CLNS/CLNP\{ES\}/LAN

TP4-CLNS/CLNP\{ES\}/WAN

TPO/WAN

Underlying Stack: SunNet OSI 7.0

SunNet X25 7.0

Protocols and Profiles:

FTAM/(ACSE)/(Presentation)/(Session)

FTAM[ISO $8571: 1988]\{T 1, M 1, T 2, A 1, R \& R\}$

FTAM ACSE[ISO 8650:1988]

FTAM Presentation [ISO 8823:1987] \{ASN.1\}

FTAM Session [ISO 8327:1987]\{R\&R\}

Abstract Test Suite used:

ATS:2-16.2(94\%), ATS:2-16.3(75\%), AT S:2-16.6(90\%),

ATS:2-16.7(96\%), ATS:2-16.8(88\%), ATS:2-16.9(100\%),

ATS:2-16.10(95\%), ATS:2-16.11(95\%), A T S:2-16.12(94\%), ATS:2-26.13(97\%)

Date Rogistered: June 28, 1993

Type of Registration and Expiration Date:

Provisional, GOSIP VER 2, until October 01, 1994 


\subsubsection{US GOSIP PICS PROFORMA}

PICS Proforma 1D: 1

Protocol/Profile: X.25 HDLC PLP

GOSIP Version: 2

Reference ATS: $\quad 2-2$

Organization: NIST/JITC

Title: US GOSIP Protocol Implementation Conformance Statement Proforma for Packet Layer (ISO 8208)

lssue/Number: None

Date: March 1992

Addl Document: None

Organization:

Title:

tssue/Number:

Date:

Additional Info: None

PICS Proforma D: 2

Protocol/Profile: X.25 HDLC LAP-B

GOSIP Version: 2

Reference ATS: $\quad 2.1$

\section{Organization: NIST/JITC}

Title: US GOSIP Protocol Implementation Conformance Statement Proforma for Data Link Layer (ISO 7776)

lssue/Number: None

Date: March 1992

\section{Addl Document: None}

Organization:

Title:

issue/Number:

Date:

Additional Info: None

PICS Proforma 1D: 3

Protocol/Profile: CLNP

GOSIP Version: 2

Reference ATS: $\quad 2.7$

\section{Organization: NIST/JITC}

Title: US GOSIP Protocol Implementation Conformance Statement Proforma for Connectionless Network Layer Protocol (ISO 8473)

\section{ksue/Number: None}

Date:

March 1992

\section{Addl Document: None}

Organization:

Titte:

kssue/Number:

Date:
PICS Proforma 1D: 4

Protocol/Profile: ES-IS

GOSIP Version: 2

Reference ATS: TBD

Organization: ISO

Title: Annex A, PICS Proformas [ISO 9542:1988(E)]

lssue/Number: $\quad$ ISO 9542

Date: 15 August 1988

Addl Document: Interim Profile Specific ICS

Organization: JITC

Title: $\quad$ Interim ISO 9542 (1988) Profile Specific ICS Proforma

lssue/Number: None

Date: July 1992

Additional Info: ICS to be submitted to OW for consideration

PICS Proforma 1D: 5

Protocol/Profile: TPO, TP2, TP4

GOSIP Version: 2

Reference ATS: $\quad 2-8,2-9.1,2-9.2,2-9.3,2-20$

Organization: NIST/JITC

Title: US GOSIP Protocol Implementation Conformance Statement Proforma for TRANSPORT Class 0, 2, and 4 Protocols (ISO 8073:1988)

lssue/Number: None

Date: June 1993

Addl Document: None

Organization:

Title:

issue/Number:

Date:

Additional Info: None

PICS Proforma 1D: 6

Protocol/Profile: TPO, TP4

GOSIP Version: 2

Reference ATS: $\quad 1-8,1-9,2-8,2-9.1,2-9.2,2-9.3$

Organization: NIST/JITC

Title: US GOSIP Protocol Implementation Conformance Statement Proforma for Transport Class 0 and 4 Protocols (ISO 8073)

Issue/Number: None

Date: March 1992

Addl Document: None

Organization:

Title:

issue/Number:

Date:

Additional Info: None 
PICS Proforma 1D: 7

Protocol/Profile: CLTP

GOSIP Version: 2

Reference ATS:

$\begin{array}{ll}\text { Organization: } & \text { ISO } \\ \text { Title: } & \text { Draft Amendment ISO 8602:1987/DAM 1:PICS } \\ \text { Proforma } \\ \text { lssue/Number: None } \\ \text { Date: } \quad \text { April } 1993\end{array}$

Addl Document: None

Organization:

Title:

kssue/Number:

Date:

Additional Info: Voting Terminates on 29 Oct 1993 for ISO 8602:1987/DAM 1

PICS Proforma 1D: 8

Protocol/Profile: MHS-84 \{RTS, P1, P2\}

GOSIP Version: 1

Reference ATS: $1-13,1-14,1-15$

Organization: Corporation for Open Systems (COS)

Title: COS Stack Specification COS/AMH113

lssue/Number: Version 1.2

Date: 15 April 1992

Addl Document: None

Organization:

Title:

issue/Number:

Date:

Additional Info: None

PICS Proforma 1D: 9

Protocol/Profile: MHS-84 \{RTS, P1, P2\}

GOSIP Version: 2

Reference ATS: $\quad 2-13.2,2-13.3,2-13.4$

Organization:

Title:

Corporation for Open Systems (COS)

ksue/Number:

COS Stack Specification COS/AMH113

Date:

Version 1.2

15 April 1992

Addl Document: None

Organization:

Title:

lssue/Number:

Date:

Additional Info: None
PICS Proforma D: 10

Protocol/Profile: Session

GOSIP Version: 2

Peference ATS: $\quad 2-10.1,2-10.2$

Organization: ISO

Title: Basic connection oriented session Protocol Implementation Conformance Statement (PICS) Proforma

lssue/Number: $\quad 8327-2$

Date: 22 Oct 1992

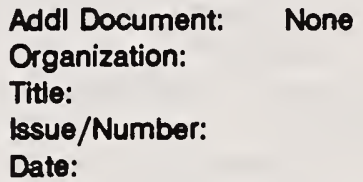

Title:

lssue/Number:

Date:

Additional Info: JITC is producing an addendum - several changes to align this PICS with US GOSIP

Pics Proforma 1D: 11

Protocol/Profile: MHS-84 \{Session\}

GOSIP Version: 2

Roference ATS: $2-13.1$

Organization: Corporation for Open Systems (COS)

Title: $\quad$ COS Stack Specification COS/AMH113

lssue/Number: Version 1.2

Date: 15 April 1992

Addl Document: None

Organization:

Title:

lssue/Number:

Date:

Additional Info: None

PICS Proforma 1D: 12

Protocol/Profile: FTAM ACSE

GOSIP Version: 2

Reference ATS: $\quad 2-16.9$

Organization: ISO

Title: $\quad$ ACSE Protocol Implementation Conformance Statement (PICS) Proforma

ksue/Number: $\quad 8650-2$

Date: $\quad 22$ October 1992

Addl Document: None

Organization:

Title:

issue/Number:

Date:

Additional Info: Adjust requirements to conform to FTAM 
PICS Proforma ID: 13

Protocol/Profile: FTAM

GOSIP Version: 2

Reference ATS: $\quad 2-16$ Series

Organization: ISO/IEC

Title: Protocol Implementation Conformance Statement ISO 8571, Part 5

ksue/Number: First Edition

Date: 15 December 1990

Addl Document: Profile Requirements List for NIST OW FTAM Phase 3

Organization: NIST/JITC

Title: $\quad$ Stable Implementation Agreements for OSI Protocols: Part 10 - FTAM Phase 3

lssue/Number: Version 5, Edition 1

Date: December 1991

Additional Info: None

PICS Proforma 1D: 14

Protocol/Profile: FTAM Presentation

GOSIP Version: 2

Reference ATS: $\quad 2-16.10$

Organization: $\quad$ ISO

Title: Presentation Protocol Implementation

issue/Number:

Presentation Protocol Implementation

Date: $\quad 22$ Oct 1992

Addl Document: None

Organization:

Title:

Issue/Number:

Date:

Additional Info: Adjust requirements to conform to FTAM

PICS Proforma 1D: 15

Protocol/Profile: FTAM Session

GOSIP Version: 2

Reference ATS: $\quad 2-16.12$

Organization: ISO

Title: Basic connection oriented session Protocol Implementation Conformance Statement (PICS) Proforma

Issue/Number: $\quad$ 8327-2

Date: $\quad 22$ October 1992

Addl Document: None

Organization:

Title:

lssue/Number:

Date:

Additional Info: Adjust requirements to conform to FTAM
5.4.8 GOSIP Register Database: Abstract Test Suites (ATS)

ATS Code: ATS:1-1

Protocol:

HDLC LAP.B

00000000001

High Lovel Data Link Control, Logical Access Procedure

B

GOSIP Version: 1

Expire Date:

Comments:

Title: X.25-DTE Conformance Testing: Text for Data Link Layer Test Suite

Organization: ISO/IEC JTC1/SC2

lssue Date: 30-JUNSO

lssue: ISO/IEC DP $8882-2$

Comments:

ATS Code: ATS:1-10

Protocol:

00000000011

Session

GOSIP Version: 1

Expire Date:

Comments:

Title: $\quad$ Session Abstract Test Suite; Volumes 1, 2 and 3

Organization: CTS-WAN

000068

Issue Date: 01-SEP-88

Issue: CTS-WAN/T\&S/ABS/SES/CS/1.0

Comments:

ATS Code: ATS:1-13

Protocol: MHS-84\{RTS\}

Message Handling System - 1984, Reliable Transfer Service Profile

GOSIP Version: 1

Expire Date:

Comments: Also issued under NIST cover, August 1990.

Title: $\quad$ MHS RTS Tests and Testing Guide

Organization: National Computing Centre Limited Oxford Road

Manchester

M17ED United Kingdom

lssue Date: 01-APR-90

lssue: lssue 2

Comments: 
ATS Code: ATS:1-14

Protocol: MHS-84\{P1\}

Message Handling System - 1984, P1 Profile

GOSIP Version: 1

Expire Date:

Comments: Also issued under NIST cover, August 1990.

Title: $\quad$ MHS P1 Tests and Testing Guide

Organization: National Computing Centre Limited Oxford Road

Manchester M17ED United Kingdom

ksue Date: 01-APR-90

kssue: lssue 3

Comments:

ATS Code: ATS:1-15

Protocol: MHS-84\{P2\}

Message Handling System - 1984, P2 Profile

GOSIP Version: 1

Expire Date:

Comments: Also issued under NIST cover, August 1990.

Title: $\quad$ MHS P2 Tests and Testing Guide

Organization: National Computing Centre Limited Oxford Road

Manchester M17ED United Kingdom

lssue Date: 01-APR-90

Issue: ksue 3

Comments:

ATS Code: ATS:1-16

Protocol: FTAM $\{$ T1,T2\}/ACSE/Presentation

File Transfer, Access, and Management: T1 and T2 Profiles, Association Control Service Element, and Presentation

GOSIP Version: 1

Expire Date:

Comments:

T1 and T2 profiles from NIST; ACSE and Presentation from NCC.

Title: $\quad$ FTAM Abstract Test Suite for GOSIP Version 1

Organization: National Institute of Standards and Technology (NIST)

Building 225, Room B151

Gaithersburg MD 20899 USA

ksue Date: 01-AUG-90

Issue:

Comments:

Title: $\quad$ FTAM Tests and Testing Guide

Organization: National Computing Centre Limited Oxford Road

Manchester M17ED United Kingdom

ksue Date: 03-AUG-90

issue: NCC/TPD - 89/016

Comments:
ATS Code: ATS:1-2

Protocol: $\quad$ X.25 PLP

$X .25$ Packet Layer Protocol

GOSIP Version: 1

Expire Date:

Comments:

Title: $\quad$ DIS $8882-3$ London Output.

Organization: ISO/IEC JTC1/SC2

Issue Date: 20JUN-90

Issue: ISO/IEC JTC1/SC6/N5608 Revised

Comments:

\section{ATS Code: ATS:13}

Protocol: $\quad$ MAC \& PLS [ISO 8802/3]

Medium Accoss Control and Physical Layer Signaling [ISO 8802/3]

GOSIP Version: 1

Expire Date:

Comments: Incorporates four test suites submitted by Corporation for Open Systems (COS) International, October 1989.

Title: $\quad$ 802.3 Draft Abstract Test Suite for GOSIP Version 1.

Organization: National Institute of Standards and Technology (NIST)

Building 225, Room B151

Gaithersburg MD 20899 USA

ksue Date: 01-AUG-90

Issue:

Comments:

ATS Code: ATS: $1-4$

Protocol: $\quad$ MAC \& PLS [ISO 8802/4]

Medium Access Control and Physical Layer Signaling [ISO $8802 / 4$ ]

GOSIP Version: 1

Expire Date:

Comments:

Title: $\quad$ 802.4 MAC Sublayer Conformance Test System Test Case Reference Guide.

Organization: Corporation for Open Systems Intemational Test Center 8260 Willow Oaks Corp Drive, Suite 700
Fairfax
VA 22031
USA

Issue Date: 01-MAR-88

lssue: COS/TPD-88/008, issue 1

Comments:

ATS Code: ATS: 15

Protocol: $\quad$ MAC \& PLS [ISO 8802/5]

Medium Access Control and Physical Layer Signaling

GOSIP Version: 1 [ISO 8802/5]

Expire Date:

Comments: No registered ATS 
ATS Code: ATS:1-6

Protocol: LLC-1

Logical Unk Control Type 1

GOSIP Version: 1

Expire Date:

Comments:

Title: $\quad 802.2$ UC (Type 1) Abstract Test Suite Submission (extract from 802.3 10BASE5 Layer 2 Test System Results Analysis Guide - LC Sublayer).

Organization: Corporation for Open Systems International Test Center 8260 Willow Oaks Corp Drive, Suite 700 Fairfax VA 22031 USA

ksue Date: 01-AUG-90

Issue: lssue 1

Comments:

\section{ATS Code: ATS:1-7}

Protocol: CLNP $\{E S\}$

Connectionless Network Protocol, End System

GOSIP Version: 1

Expire Date:

Comments:

Title: Internet Protocol Tests and Testing Guide for ISO 8473 Connectionless Network Protocol Implementations.

Organlzation: National Computing Contre Limited Oxford Road Manchester M17ED United Kingdom

lssue Date: 01JUL-90

lssue: NCC/TPD-90/003, issue 2

Comments:

\section{ATS Code: ATS:1-7.1}

Protocol: CLNP $\{$ IS $\}$

Connectionless Network Protocol, Intermediate System GOSIP Version: 1

Expire Date:

Comments:

Title: Interim Abstract Test Suite for CLNP Intermediate Systems Testing.

Organization: National Institute of Standards and Technology (NIST)

Bullding 225, Room B151

Gaithersburg MD 20899

USA

Issue Date: 30-OCT-90

Issue:

Comments:

\section{ATS Code: ATS: $1-8$}

Protocol: TPO

GOSIP Version: 1

Transport Protocol Class 0

Explre Date:

Comments:

Title: ISO/OSI Transport Class O CTS-WAN Abstract Test Suite

Organization: National Computing Centre Limited Oxford Road

Manchester M17ED United Kingdom

lssue Date: 13-OCT-88

lssue: CTS-WAN/T\&S/ABS/TRO/CS/1.0, Rov 2.1

Comments:
ATS Code: ATS:1-9

Protocol: TP4

Transport Protocol Class 4

GOSIP Version: 1

Expire Date:

Comments:

Title: Transport Class 4 Tests and Testing Guide for ISO 8073 Transport Class 4 Implementations.

Organization: National Computing Centre Limited Oxford Road Manchester M17ED United Kingdom

lssue Date: 01JUL-S0

lssue: NCC/TPD - $90 / 002$, Issue 2

Comments:

\section{ATS Codo: ATS:2-1}

Protocol: HDLC LAP.B

High Level Data Link Control, Logical Access Procedure B

GOSIP Version: 2

Expire Date:

Comments:

Title:

Information Technology-Telecommunications and Information Exchange Between Systems- X.25 DTE Conformance Testing. Part 2: Data Link Layer Conformance Test Suite

Organization: ISO/IEC JTC1/SC6

Issue Date: 20-JAN-92

Issue: ISO/IEC 8882-2:1992(E), First Edition

Comments:

Title: Defect Report to ISO/IEC 8882-2: 1992

Organization: ISO/IEC JTC1/SC6

ksue Date: 30-MAR-92

Issue: $\quad X 353.4 / 92-54$

Comments:

ATS Code: ATS:2-10.1

Protocol: Session

GOSIP Version: 2

Expire Date:

Comments: Should be superseded by ATS:2-10.2

Title: $\quad$ Session Abstract Test Suite, Volumes 1, 2, and 3

Organization: CTS-WAN

Issue Date: 01-SEP-88

lssue: CTS/WAN/T\&S/ABS/SES/CS/1.0

Comments:

Title: Session CTS-WAN Abstract Test Suite, CTS2 Extension

Organization: Acatel TITN Incorporated 7011 Koll Conter Parkway, Suite 200 Pleasanton CA 94566-3101 USA

lssue Date: 19-MAR-91

Issue: Version 2.0

Comments: 
ATS Cods: ATS:2-10.2

Protocol: Session

GOSIP Version: 2

Expire Date:

Comments:

Title: Session Abstract Test Suite

Organization: Open Systems Testing Consortium Secretariat Rue du Trone, 12

B-1050 Brussels Belgium

Issue Date: 01-JAN-92

Issue: OSTC/T\&S/ABS/SES/CS/2.1

Comments:

ATS Code: ATS:2-13.1

Protocol: MHS-84 Session

GOSIP Version: 2

Message Handling System - 1984, Session Protocol

Expire Date:

Comments:

Title: Abstract Session Test Suite DSE MHS

Organization: Open Systems Testing Consortium Secretariat Rue du Trone, 12

lssue Date: 18JAN-89

B-1050 Brussels Bolgium

lssue: OSTC/MHS/ABS/SES/DSE/3.0, Version 3.0

Comments:

ATS Code: ATS:2-132

Protocol: MHS-84\{RTS\}

Message Handling System - 1984, Reliable Transfer Service Profile

GOSIP Version: 2

Expire Date:

Comments:

Title: $\quad$ MHS RTS Abstract Test Suite

Organization: Open Systems Testing Consortium Secretariat Rue du Trone, 12

B-1050 Brussels

Belgium

ksue Date: 01-JUL-91

ksue: OSTC/MHS/ABS/RTS/2.0

Comments:

ATS Code: ATS:2-13.3

Protocol: MHS-84\{P1\}

Message Handling System - 1984, P1 Profile

GOSIP Version: 2

Expire Date:

Comments:

Title: MHS Abstract Test Suite, P1 Protocol A/311 and A/3211

Organization: Open Systems Testing Consortium Secretariat Rue du Trone, 12

B-1050 Brussels Bolgium

ksue Date: 28-SEP-90

lssue: OSTC/MHS/ABS/P1/2.0

Comments:
ATS Code: ATS:2-13.4

Protocol: MHS-84\{P2 $\}$

GOSIP Version: 2

Message Handling System - 1984, P2 Profile

Expire Date:

Comments:

Title: $\quad$ MHS Abstract Test Suite, P2

Organization: Open Systems Testing Consortium Secretariat Rue du Trone, 12

B-1050 Brussels Belgium

lssue Date: 01-SEP-92.

kssue: OSTC/MHS/ABS/P2/2.0

Comments:

ATS Code: ATS:2-16.1

Protocol: FTAM $\{$ T1 $\}$

File Transfer, Access, and Management; Simple File Transfer Profile

GOSIP Version: 2

Expire Date:

Comments:

Title: T1-A/111, Volume 1 (Responder Tests) and Volume 2 (Initiator Tests)

Organization: Open Systems Testing Consortium Secretariat Rue du Trone, 12

B-1050 Brussels Belgium

ksue Date: 01-JAN-88

lssue: CTS-WAN/FTAM/ABS/FTAM-A111/1.0

Comments:

ATS Code: ATS:2-16.10

Protocol: FTAM Presentation

File Transfer, Access, and Management; Presentation Protocol

GOSIP Version: 2

Expire Date:

Comments:

Title: $\quad$ FTAM Presentation Abstract Test Suite

Organization: Open Systems Testing Consortium Secretariat

Rue du Trone, 12

B-1050 Brussels Belgium

Issue Date: 01JAN-92

lssue: OSTC/FTAM/ABS/FTAM/PRES

Comments:

ATS Code: ATS:2-16.11

Protocol: FTAM Presentation ASN.1

File Transfer, Access, and Management; Presentation Abstract Syntax Notation One

GOSIP Version: 2

Expire Date:

Comments:

Title: FTAM Presentation ASN.1 Abstract Test Suite

Organization: Open Systems Testing Consortium Secretariat

Rue du Trone, 12

B-1050 Brussels Belgium

lssue Date: 01-JAN-92

lssue: OSTC/FTAM/ABS/FTAM/PRES/ASN.1/BER

Comments: 
ATS Code: ATS:2-16.12

Protocol: FTAM Session

File Transfer, Accoss, and Management; Session Protocol GOSIP Version: 2

Expire Date:

Comments:

Thtle: $\quad$ FTAM Session Abstract Test Suite

Organization: Open Systems Testing Consortium Secretariat Rue du Trone, 12
B-1050 Brussels
Belgium

ksue Date: 17-SEP-91

lssue: OSTC/FTAM/ABS/FTAM/SES

Comments:

\section{ATS Code: ATS:2-16.13}

Protocol: FTAM Session R\&R

File Transfer, Access, and Management; Session Restart and Recovery

GOSIP Version: 2

\section{Expire Date:}

Comments:

Titte: FTAM Session Restart and Recovery Abstract Test Suite Organization: Open Systems Testing Consortium Secretariat Rue du Trone, 12
B-1050 Brussels
Belgium

ksue Date: 01-JAN-92

lssue: OSTC/FTAM/ABS/FTAM/SES/R\&R

Comments:

\section{ATS Codo: ATS:2-16.2}

Protocol: FTAM $\{$ T1\}

File Transfer, Accoss, and Management; Simple File Transfer Profile

GOSIP Version: 2

Expire Date:

Comments:

Title: FTAM A/111 (T1) Responder ATS and FTAM A/111 (T1) Initiator ATS

Organization: Open Systems Testing Consortium Secretariat Rue du Trone, 12

B-1050 Brussels

Belgium

ksue Date: 01-APR-91

Issue: CTS-WAN/FTAM/ABS/FTAM-A111/2.0, Version 2.0, Volumes. 1

Comments:

ATS Codo: ATS:2-163

Protocol: FTAM $\{R \& R\}$

File Transfer, Access, and Management; Restart and Recovery Profile

GOSIP Version: 2

Expire Date:

Comments:

Title: FTAM A/111 Restart and Rocovery, Abstract Test Suite

Organization: Open Systems Testing Consortium Secretariat Rue du Trone, 12

B-1050 Brussels Belgium

Lsue Date:

lssue: OSTC/FTAM/ABS/FTAM-A111 R\&R/1.0

Comments:
ATS Code: ATS:2-16.4

Protocol: FTAM $\{R \& R\}$

File Transfer, Access, and Management; Restart and Recovery Profile

GOSIP Version: 2

Expire Date:

Comments:

Title: FTAM A/111 Restart and Recovery, Abstract Test Suite Organization: Open Systems Testing Consortium Secretariat Rue du Trone, 12
B-1050 Brussels
Belgium

ksue Date: 29-OCT-91

lssue: OSTC/FTAM/ABS/FTAM-A111 R\&R $\backslash 2.0$

Comments:

ATS Code: ATS:2-16.5

Protocol: FTAM $\{M 1\}$

File Transfer, Access, and Management; Management Profile

GOSIP Version: 2

Expire Date:

Comments:

Title: $\quad$ FTAM A/13 ATS

Organization: Open Systems Testing Consortium Secretariat Rue du Trone, 12

B-1050 Brussels Belgium

ksue Date: 01-JAN-90

lssue: FTAM/ABS/FTAM-A13/1.0

Comments:

ATS Code: ATS:2-16.6

Protocol: FTAM $\{M 1\}$ File Transfer, Access, and GOSIP Version: 2

Management; Management Profile

Expire Date:

Comments:

Title: $\quad$ FTAM A/13 ATS, Version 2.0

Organization: Open Systems Testing Consortium Secretariat Rue du Trone, 12
B-1050 Brussels
Bolgium

Issue Date: 01-AUG-91

kssue: FTAM/ABS/FTAM/A13/2.0

Comments:

ATS Code: ATS:2-16.7

Protocol: FTAM $\{$ T2 $\}$

File Transfer, Access, and Management; Positional File Transfer Profile

GOSIP Version: 2

Expire Date:

Comments:

Title: $\quad$ FTAM A/112 Abstract Test Suite, Volume 1 (A/112 Responder) and Volume 2 (A/112 Initiator)

Organization: Open Systems Testing Consortium Secretariat Rue du Trone, 12

B-1050 Brussels Belgium

issue Date: 01JAN-92

lssue: OSTC/FTAM/ABS/FTAM-A/112

Comments: 
ATS Code: ATS:2-168

Protocol: FTAM $\{A 1\}$

File Transfer, Access, and Management; Simple File Access Profile

GOSIP Version: 2

Expire Date:

Comments:

Title: FTAM A/122 Responder Abstact Test Suite Nolume 1) and FTAM A/122 Initator Abstract Test Suite Nolume 2)

Organization: Open Systems Testing Consortium Secretariat Rue du Trone, 12

B-1050 Brussels Belgium

lssue Date: 01JAN-92

Issue: OSTC/FTAM/ABS/FTAM-A/122

\section{ATS Code: ATS:2-16.9}

Protocol: FTAM ACSE

File Transfer, Access, and Management; Association Control Service Element

GOSIP Version: 2

Expire Date:

Comments: ATS Consists of a common part and a specific annex.

Title: MHS '88 ACSE Abstract Test Suite (Common Part)

Organlzation: Open Systems Testing Consortium Secretariat

Rue du Trone, 12

B-1050 Brussels Belgium

Issue Date: 05-SEP-91

lssue: OSTC/MHS-88/ABS/ACSE/ALL/1.0

Comments: Without OSTC cover, document is identified as CTS2-ACSE V2.02

Title: $\quad$ FTAM ACSE Abstract Test Suite

Organization: Open Systems Testing Consortium Secretariat Rue du Trone, 12

B-1050 Brussels Belgium

Issue Date: 01-JAN-92

lssue: OSTC/FTAM/ABS/FTAM/ACSE

Comments: This document is Annex D of Document 1. Without OSTC cover, document is identified as ACSE_CTS2 FTAM.

ATS Code: ATS:2-17

Protocol: $\quad$ T Virtual Terminal

GOSIP Version: 2

Expire Date:

Comments: No registered ATS.

\section{ATS Code: ATS:2-18}

Protocol: ES-IS $\{$ ES $\}$

End System to Intermediate System in End System

GOSIP Version: 2

Expire Date:

Comments:

Title: $\quad$ ES/IS in ES Abstract Test Suite

Organization: Alcatel TITN Answare

$$
\text { Aix-en-Provence France }
$$

lssue Date: 01-MAY-93

ksue: Version 1.1
ATS Code: ATS:2-19

Protocol: CONS

Connection Oriented Network Service

GOSIP Version: 2

Expire Date:

Comments: No registered ATS.

ATS Codo: ATS:2-1A

Protocol: HDLC LAP-B

High Lovel Data Link Control, Logical Access Procedure B

GOSIP Version: 2

Expire Date:

Comments: This ATS consists of ATS:2-1 as modified by the documents below.

Title: Instructions to the Editor for the corrections of reported defects in ISO/IEC $8882-2$ and $8882-3$

Organization: ISO/IEC SC6

lssue Date: 12-FEB-93

Issue: SC6 N 7988

Comments: To be used with Defect Reports referenced within.

Title: Instructions to the Editor for the preparation of the 2nd Edition of ISO/IEC $8882-2$ and $8882-3$

Organization: ISO/IEC SC6

Issue Date: 23-JUL-92

lssue: SC6 N7565

Comments: To be used with Defect Reports referenced within.

ATS Code: ATS:2-2

Protocol: $\quad$ X.25 PLP

X.25 Packet Layer Protocol

GOSIP Version: 2

Expire Date:

Comments:

Title: Information Technology-Telecommunications and Information Exchange Between Systems-X.25-DTE Conformance Testing-Part 3: Packet Layer Conformance Test Suite

Organization: ISO/IEC JTC1/SC2/WG2

Issue Date: 16-JAN-91

lssue: ISO/IEC 8882-3:1991(E), First Edition

Title: Defect Report to ISO/IEC 8882-3:1991

Organization: ISO/IEC JTC1/SC2/WG2

Issue Date: 01-MAR-92

ksue: $\quad$ X353.7/92-49

\section{ATS Code: ATS:2-20}

Protocol: TP2

Transport Protocol Class 2

GOSIP Version: 2

Expire Date:

Comments: A revised version of this ATS is registered as ATS:2-20A

Title: $\quad$ OSTC Abstract Test Suite, Transport Class 2

Organization: Open Systems Testing Consontium Secretariat Pue du Trone, 12

B-1050 Brussels

Belgium

Issue Date: 31-JAN-91

lssue: $\quad$ OSTC/T\&S/ABS/TR2/CS/2.0 


\section{ATS Codo: ATS:2-20A}

Protocol: TP2

Transport Protocol Class 2

GOSIP Version: 2

Expire Date:

Comments: Minor revisions of ATS:2-20

Title: $\quad$ OSTC Abstract Test Suite, Transport Class 2

Organization: Open Systems Testing Consortium Secretariat Rue du Trone, 12

B-1050 Brussels Belgium

Issue Date:

lssue: $\quad$ OSTC/T\&S/ABS/TR2/CS/2.1

\section{ATS Code: ATS:2-21}

Protocol: ISDN

GOSIP Version: 2

Integrated Services Digital Network

Expire Date:

Comments: No registered ATS.

\section{ATS Code: ATS:2-2A}

Protocol: $\quad \times 25$ PLP

GOSIP Version: 2

X.25 Packet Layer Protocol

Expire Date:

Comments: This ATS consists of ATS:2-2 as modified by the documents below.

Title: Instructions to be Editor for the corrections of reported defects in ISO/IEC $8882-2$ and $8882-3$

Organization: ISO/IEC SC6

Issue Date: 12-FEB-93

ksue: $\quad$ SC6 N7988

Comments: To be used with Defect Reports referenced within.

Title: Instructions to the Editor for the preparation of the 2nd Edition of ISO/IEC $8882-2$ and $8882-3$

Organization: ISO/IEC SC6

lssue Date: 23 JUL-92

lssue: SC6 N7565

Comments: To be used with Defect Reports referenced within.

\section{ATS Code: ATS:23}

Protocol: $\quad$ MAC \& PLS [ISO 8802/3]

Medium Access Control and Physical Layer

GOSIP Version: 2

Signaling [ISO 8802/3]

Expire Date:

Comments: Incorporates four test suites submitted by Corporation for Open Systems (COS) Intemational in October 1989

Title: $\quad$ 802.3 Draft Abstract Test Suite for GOSIP Version 1

Organization: National Institute of Standards and Technology (NIST)

Building 225, Room B151

Gaithersburg MD 20899

USA

ksue Date: 01-AUG-90

lssue:
ATS Cods: ATS:24

Protocol: $\quad$ MAC \& PLS [ISO 8802/4]

Medium Access Control and Physical Layer

GOSIP Version: 2

Signaling [ISO 8802/4]

Expire Date:

Comments:

Title: 802.4 MAC Sublayer Conformance Test System Test Case Reference Guide

Organization: Corporation for Open Systems International Test Conter 8260 Willow Oaks Corp Drive, Suite 700 Fairfax VA 22031 USA

lssue Date: 01-MAR-88

lssue: COS/TPD-88/008, issue 1

ATS Codo: ATS:2-5

Protocol: $\quad$ MAC \& PLS [ISO 8802/5]

Medium Access Control and Physical Layer Signaling

GOSIP Version: 2 [ISO $8802 / 5$ ]

Expire Date:

Comments: No Rogistered ATS

ATS Code: ATS:2-6

Protocol: $\quad$ LC-1

GOSIP Version: 2 Logical Link Control Type 1

Expire Date:

Comments:

Title: $\quad 802.2$ UC (Type 1) Abstract Test Suite Submission (extract from 802.3 10BASE5 Layer 2 Test System Results Analysis Guide - UC Sublayer)

Organization: Corporation for Open Systems Intemational Test Conter 8260 Willow Oaks Corp Drive, Suite 700 Fairfax VA 22031 USA

lssue Date: 01-AUG-90

ksue: ksue 1

Comments:

\section{ATS Code: ATS:2-7}

Protocol: CLNP $\{E S\}$

Connectionless Network Protocol, End System

GOSIP Version: 2

Expire Date:

Comments:

Title: Intemet Protocol Tests and Testing Guide for ISO 8473 Connectionless Network Protocol Implementations

Organization: National Computing Centre Limited Oxford Road Manchester M17ED United Kingdom

ksue Date: 01-JUL-90

Issue: NCC/TPD-90/003, lssue 2

Comments: 
ATS Code: ATS-2-7.1

Protocol: CLNP\{IS\}

Connectionless Network Protocol, Intermediate System

GOSIP Version: 2

Expire Date:

Comments:

Title: Interim Abstract Test Suite for CLNP Intermediate Systems Testing

Organization: National Institute of Standards and Technology (NIST)

Building 225, Room B151

Gaithersburg MD 20899 USA

issue Date: 30-OCT-90

issue:

\section{ATS Cods: ATS:2-8}

Protocol: TPO Transport Protocol Class 0

GOSIP Version: 2

Expire Date:

Comments: A revised version of this ATS registered as ATS:2-8A

Title: $\quad$ OSTC Abstract Test Suite, Transport Class 0

Organization: Open Systems Testing Consortium Secretariat Rue du Trone, 12 B-1050 Brussels Belgium

ksue Date: 31-JAN-91

lssue: OSTC/T\&S/ABS/TRO/CS/2.0

\section{ATS Code: ATS:2-8A}

Protocol: TPO Transport Protocol Class 0

GOSIP Version: 2

Expire Date:

Comments: $\quad$ Minor revision of ATS $2-8$

Titie: $\quad$ OSTC Abstract Test Suite, Transport Class 0

Organization: Open Systems Testing Consortium Secretariat Rue du Trone, 12

B-1050 Brussels Belgium

Hsue Date:

lssue: $\quad$ OSTC/T\&S/ABS/TRO/CS/2.1

\section{ATS Code: ATS:2-9.1}

Protocol: TP4-CLNS

Transport Protocol Class 4 Over Connectionless Network Service

GOSIP Version: 2

Expire Date:

Comments: New ATS under development by EWOS.

Title: $\quad$ Transport Class 4 over CLNS Abstract Test Suite

Organization: Acatel TITN Answare

Aix-en-Provence France

Issue Date: 07-MAY-92

Issue: Version 2.2
ATS Code: ATS:2-9.2

Protocol: TP4

$$
\text { Transport Protocol Clașs } 4
$$

GOSIP Version: 2

Expire Date:

Comments: New ATS under development by EWOS.

Title: Transport Class 4 Tests and Testing Guide for ISO 8073 Transport Class 4 Implementations

Organization: National Computing Centre Limited Oxford Road

Manchester

M17ED

United Kingdom

Issue Date: 01-MAY-92

lssue: ISG-DEV-008-10-004, lssue 5

\section{ATS Code: ATS: $2-9.3$}

Protocol: TP4-CONS Transport Protocol Class 4 Over GOSIP Version: 2

\section{Connection Oriented Network Service}

Expire Date:

Comments: New ATS under development by EWOS

Title: $\quad$ Transport Class 4 over CONS Abstract Test Suite

Organization: Acatel TITN Answare

$$
\text { Aix-en-Provence France }
$$

Issue Date: 30-SEP-91

Issue: Version 2.0

ATS Codo: ATS:3-221

Protocol: MHS-88 Session

Message Handling System - 1988, Session

GOSIP Version: 3

Expire Date:

Comments:

Title: MHS-88 DSE Session ATS

Organization: Open Systems Testing Consortium Secretariat Rue du Trone, 12

$$
\text { B-1050 Brussels Belgium }
$$

lssue Date:

ksue: MHS-88/ABS/SES/DSE/1.0

ATS Code: ATS:3-2221

Protocol: MHS-88 \{PRES-MH\}

GOSIP Version: 3

Message Handling System - 1988, Presentation - MH

Expire Date:

Comments: ATS consists of a specific annex and a common part.

Title: $\quad$ MHS '88 Presentation Abstract Test Suite MH (88) Part

Organization: Open Systems Testing Consortium Secretariat Rue du Trone, 12

B-1050 Brussels

lssue Date: 20-DEC-91

Belgium

lssue: OSTC/MHS-88/ABS/PRES/MH/1.0

Comments: This document is Annex A of document 2. Without OSTC cover, document is identified as CTS2/XMHS/MH/PRES/ATS/ V3.1

Title: $\quad$ MHS '88 Presentation - Common Abstract Test Suite Organization: Open Systems Testing Consortium Secretariat Rue du Trone, 12

B-1050 Brussels

ksue Date: 20-DEC-91

Belgium

lesue: OSTC/MHS-88/ABS/PRES/ALL/1.0

Comments: Without OSTC cover, document is identified as CTS2/XMHS/AU/PRES/ATS/N4.0 CTS2_X_PRES 
ATS Codo: ATS:3223.1

Protocol: MHS-88 (ACSE-MH\} Message Handling System -1988 , Association Control Service Element

GOSIP Version: 3

Expire Date:

Comments: ATS consists of a specific annex and a common part.

Title: MHS '88 ACSE Abstract Test Suite MH

Organization: Open Systems Testing Consortium Secretariat Rue du Trone, 12

B-1050 Brussels Belgium

ksue Date: O4DEC-91

tssue: OSTC/MHS-88/ABS/ACSE/MH/1.0

Comments: Thls is Annex $C$ of Document 2. Without OSTC cover, document is Identified as ACSE_ATS_MHS_SPECIFIC_PART V2.03.

Title: $\quad$ MHS '88 ACSE Abstract Test Suite (Common Part)

Organization: Open Systems Testing Consortium Secretariat Rue du Trone, 12

B-1050 Brussels Bolgium

ksue Date: 05-SEP-91

lssue: OSTC/MHS-88/ABS/ACSE/ALL/1.0

Comments: Without OSTC cover, document is identified as CTS2-ACSE V2.02

\section{ATS Code: ATS:32232}

Protocol: MHS-88 \{ACSE-P3-UA\}

Message Handling System - 1988, Association Control Service Element-PZ-UA

GOSIP Version: 3

Expire Date:

Comments: ATS consists of a specific annex and a common part.

Title: $\quad$ MHS '88 ACSE Abstract Test Suite P3-UA

Organization: Open Systems Testing Consortium Secretariat Rue du Trone, 12

B-1050 Brussels Belgium

Issue Date: 01-DEC-91

issue: OSTC/MHS-88/ABS/ACSE/RA/1.0

Comments: This is Annex B4 of Document 2. Without OSTC cover, document is Identlfied as ACSE_ATS_RA_SPECIFIC_PART P3-UA V2.03

Titte: MHS '88 ACSE Abstract Test Suite (Common Part)

Organization: Open Systems Testing Consortium Secretariat Rue du Trone, 12

B-1050 Brussols Bolgium

Issue Date: 05-SEP-91

issue: OSTC/MHS-88/ABS/ACSE/ALL/1.0

Comments: Without OSTC cover, document is Identified as CTS2-ACSE V2.02
ATS Code: ATS:3-223.3

Protocol: MHS-88 \{ACSE-P3-MTA\}

Message Handling Sysțem - 1988, Association Control Service Element-P3-MTA

GOSIP Version: 3

Expire Date:

Comments: ATS consists of a specific annex and a common part.

Title: $\quad$ MHS '88 ACSE Abstract Test Suite P3-MTA

Organization: Open Systems Testing Consortium Secretariat Rue du Trone, 12

B-1050 Brussels Bolgium

lssue Date: 01-DEC-91

lssue: OSTC/MHS-88/ABS/ACSE/RA/1.0

Comments: This is Annex B5 of Document 2. Without OSTC cover, document is Identified as ACSE_ATS_RA_SPECIFIC_PART P3-MTA V2.03

Title: $\quad$ MHS '88 ACSE Abstract Test Suite (Common Part)

Organization: Open Systems Testing Consortium Secretariat Rue du Trone, 12 B-1050 Brussels Bolgium

lssue Date: 05-SEP-91

Issue: OSTC/MHS-88/ABS/ACSE/ALL/1.0

Comments: Without OSTC cover, document is identified as CTS2-ACSE V2.02

ATS Code: ATS:3-223.4

Protocol: MHS-88 \{ACSE-P3-MS\}

Message Handling System - 1988, Association Control Service Element-P3-MS

GOSIP Version: 3

Expire Date:

Comments: ATS consists of a specific annex and a common part.

Title: $\quad$ MHS '88 ACSE Abstract Test Suite P3-MS

Organization: Open Systems Testing Consortium Secretariat Rue du Trone, 12

B-1050 Brussels

Bolgium

lssue Date: 01-DEC-91

lssue: OSTC/MHS-88/ABS/ACSE/RA/1.0

Comments: This is Annex B3 of Document 2. Without OSTC cover, document is identified as ACSE_ATS_RA_SPECIFIC PART P3-MS V2.03

Title: MHS '88 ACSE Abstract Test Suite (Common Part)

Organization: Open Systems Testing Consortium Secretariat Rue du Trone, 12

B.1050 Brussels

Bolgium

Issue Date: 05-SEP-91

Hsue: OSTC/MHS-88/ABS/ACSE/ALL/1.0

Comments: Without OSTC cover, document is identified as CTS2-ACSE V2.02 
ATS Code: ATS:3-2235

Protocol: MHS-88 \{ACSE-P7-UA\} Message Handling System - 1988, Association Control Service Element-P7-UA

GOSIP Version: 3

Expire Date:

Comments: ATS consists of a specific annex and a common part.

Title: $\quad$ MHS '88 ACSE Abstract Test Suite P7-UA

Organization: Open Systems Testing Consortium Secretariat

Rue du Trone, 12

B-1050 Brussels Belgium

lssue Date: 01-DEC-91

lssue: OSTC/MHS-88/ABS/ACSE/RA/1.0

Comments: This is Annex B2 of Document 2. Without OSTC cover, document is identified as ACSE_ATS_RA_SPECIFIC_PART P7-UA V2.03

Title: MHS '88 ACSE Abstract Test Suite (Common Part)

Organization: Open Systems Testing Consortium Secretarlat Rue du Trone, 12

B-1050 Brussels

Bolgium

Issue Date: 05-SEP-91

lssue: OSTC/MHS-88/ABS/ACSE/ALL/1.0

Comments: Without OSTC cover, document is Identified as CTS2-ACSE V2.02

\section{ATS Cods: ATS:3-2236}

Protocol: MHS-88 \{ACSE-P7-MS\}

Message Handling System - 1988, Association Control Service Element-P7-MS

GOSIP Version: 3

Expire Date:

Comments: ATS consists of a specific annex and a common part.

Title: $\quad$ MHS '88 ACSE Abstract Test Suite P7-MS

Organization: Open Systems Testing Consortium Secretariat

Rue du Trone, 12

B-1050 Brussels Bolgium

issue Date: 01-DEC-91

ksue: OSTC/MHS-88/ABS/ACSE/RA/1.0

Comments: Thls is Annex B4 of Document 2. Without OSTC cover, document is identified as ACSE_ATS_RA_SPECIFIC_PART P7-MS V2.03

Title: MHS '88 ACSE Abstract Test Suite (Common Part)

Organization: Open Systems Testing Consortium Secretariat

Rue du Trone, 12

B-1050 Brussels Belgium

ksue Date: 05-SEP-91

$$
\text { Belgium }
$$

issue: OSTC/MHS-88/ABS/ACSE/ALL/1.0

Comments: Without OSTC cover, document is identified as CTS2-ACSE V2.02
ATS Code: ATS:3-22,4

Protocol: MHS-88 \{RTSE\}

Message Handling System - 1988, Reliable Transfer Service Element

GOSIP Version: 3

Expire Date:

Comments:

Title: $\quad$ MHS '88 RTSE Abstract Test Suite

Organization: Open Systems Testing Consortium Secretariat Rue du Trone, 12

B-1050 Brussels Bolgium

lesue Date: 15-OCT-91

lssue: OSTC/MHS-88/ABS/RTSE/1.0

Comments: Without OSTC cover, document is identified as CTS2/ XMHS/MH/RTSE/ATS/N6.0

ATS Code: ATS:3-225

Protocol: $\quad$ MHS-88 $\{$ P1 $\}$

Message Handling System - 1988, P1 Profile

GOSIP Version: 3

Expire Date:

Comments:

Title: $\quad$ MHS '88 P1 Abstract Test Suite

Organization: Open Systems Testing Consortium Secretariat Rue du Trone, 12

B-1050 Brussels

Bolgium

Issue Date: O8JAN-92

ksue: OSTC/MHS-88/ABS/P1/1.0

Comments: Without OSTC cover, document is identified as CTS2/ XMHS/MH/P1/ATS/N2.5.

ATS Code: ATS:3-226

Protocol: MHS-88 \{P2\}

Message Handling System - 1988, P2 Profile

GOSIP Version: 3

Expire Date:

Comments:

Title: $\quad$ MHS '88 P2 Abstract Test Suite

Organization: Open Systems Testing Consortium Secretariat Rue du Trone, 12

B-1050 Brussels Belgium

Issue Date: 26-MAY-91

lssue: OSTC/MHS-88/ABS/P2/1.0

Comments: Without OSTC cover, this document Is identified as CTS2/XMHS/MH/P2/ATS/N3.2 


\section{ATS Codo: ATS:3227.1}

Protocol: MHS-88 \{PZ-UA\} Message Handling System - 1988, P3-UA Profile

GOSIP Version: 3

Expire Date:

Comments:

Title: $\quad$ MHS '88 P3-UA Abstract Test Suite

Organization: Open Systems Testing Consortium Secretariat Pue du Trone, 12

B-1050 Brussels

Belgium

Issue Date: 01-SEP-91

issue: OSTC/MHS-88/ABS/P3-UA/1.0

Comments: Without OSTC cover, document is identified as CTS2/ XMHS/RA/P3-UA/ATSN4.0

\section{ATS Code: ATS:3-2272}

Protocol: MHS-88 \{P3-MTA\}

Message Handling System - 1988, P3-MTA Profile

GOSIP Version: 3

Expire Date:

Comments:

Title: $\quad$ MHS '88 P3-MTA Abstract Test Suite

Organlzation: Open Systems Testing Consortium Secretariat Rue du Trone, 12

B-1050 Brussels Belgium

Issue Date: 18-SEP-91

OSTC/MHS-88/ABS/P3-MTA/1.0

Comments: Without OSTC cover, document is identified as CTS2/ XMHS/RA/P3-MTA/ATS N4.0

\section{ATS Codo: ATS:3-228.1}

Protocol: MHS-88 \{P7-UA\}

Message Handling System - 1988, P7-UA Profile

GOSIP Version: 3

Expire Date:

Comments:

Title: $\quad$ MHS '88 P7-UA Abstract Test Suite

Organization: Open Systems Testing Consortium Secretariat Rue du Trone, 12

B-1050 Brussels Belgium

lssue Date: 18-SEP-91

issue: OSTC/MHS-88/ABS/P7-UA/1.0

Comments: Without OSTC cover, document is identified as CTS2/ XMHS/RA/P7-UA/ATS/N4.0
ATS Code: ATS:3-228.2

Protocol: MHS-88 \{P7-MS\}

Message Handling System - 1988, P7-MS Profile

GOSIP Version: 3

Expire Date:

Comments:

Title: $\quad$ MHS '88 P7-MS Abstract Test Suite

Organlzation: Open Systems Testing Consortium Secretariat Rue du Trone, 12

B-1050 Brussels Belgium

ksue Date: 19-SEP-91

ksue: OSTC/MHS-88/ABS/P7-MS/1.0

Comments: Without OSTC cover, document is identified as CTS2/XMHS/RA/P7-MS/ATS/N4.0

\section{ATS Code: ATS:3-23}

Protocol: $\quad \times 500$

Directory Services

GOSIP Version: 3

Expire Date:

Comments: No Registered ATS. NIST plans to establish a conformance testing Infrastructure for Directory Services by the end of 1993 



\section{NIST POSIX CONFORMANCE TESTING}

The National Institute of Standards and Technology through its Computer Systems Laboratory (NIST/CSL) has established a conformance testing program for the Federal Information Standard for POSIX (FIPS 151-1 and FIPS 151-2). FIPS 151-2 will replace FIPS 151-1 in its entirely on October 15, 1992. These standards are based on the IEEE POSIX Std. 1003.1-1988 (FIPS 151-1) and ISO/IEC 9945-1:1990 (FIPS 151-2). The testing model includes a Certification Authority, NVLAP Accredited Testing Laboratories, Clients and the official NIST POSIX Conformance Test Suites. The Certification Authority is the Director of NIST/CSL. The National Voluntary Laboratory Accreditation Program (NVLAP), part of NIST, accredits the testing laboratories. The test suites are NIST-PCTS:151-1 and NIST-PCTS:151-2 were developed by NIST/CSL and are based on the test assertions specified by the IEEE Standard for Information Technology - Test Methods for Measuring Conformance to POSIX, IEEE Std. 1003.3-1991 (NIST-PCTS:151-1) and the IEEE Standard for Information Technology - Test Methods for Measuring conformance to POSIX.1, IEEE Std 2003.1-1992 (NIST-PCTS:151-2).

\subsection{POSIX Test Procedures}

There are Accredited POSIX Testing Laboratories (APTLs) accredited by NVLAP for using one or both test suites. NVLAP accreditation is renewable after one year, and identifies the specific testing procedures which the lab is authorized to run. The labs provide testing and analysis services to their clients and may forward the final test results to NIST/CSL for evaluation and subsequent issuance of a Certificate of Validation by NIST/CSL.

Testing policy documents and registers of validated products and accredited laboratories and available on an electronic mail (email) file server system. For most email systems, send an email message to posix@nist.gov (mail posix@nist.gov). The first line of the message should contain a command to send index (send index). After issuing the send command and a carriage return, end the email message. A listing of all of the available files will be returned via email to the requesting email address.

\subsection{POSIX Test Suite}

The NIST-PCTS:151-2 is available from NIST/CSL, POSIX Certification Authority, Building 225 Room B266, National Institute of Standards and Technology, Gaithersburg, MD 20899. The NIST-PCTS: $151-1$ is available from the National Technical Information Services (NTIS), 5825 Port Royal Road, Springfield, VA 22161, (703) 487-4650. For ordering information call (301) 975-3290.

\subsection{Validation Requirements}

An acc edited lab may submit a "clean" test report to NIST/CSL for evaluation in anticipation of a Certification of Validation being issued. "Clean" implies no test assertion failures. The Certificate of Validation will confirm that the stated product has been tested using the official NIST-PCTS and that the test results have been validated by NIST/CSL. The Certificate of Validation and the Test Results Summary contain information on the product tested, the implementation that was tested, the suppliers, conditional features that were tested, configuration details and the identification of the testing laboratory. These certificates are issued by NIST/CSL through the testing lab. Fees for services by the testing labs are established by the labs. 


\subsection{TESTING LABORATORIES for NIST POSIX (FIPS 151-1)}

The National Voluntary Laboratory Accreditation Program (NVLAP) has accredited the following laboratories to test computer operating system interfaces for conformance with the Federal Information Processing Standard 151-1 (FIPS 151-1) using the NIST POSIX Conformance Test Suite (NIST. PCTS:151-1). Only accredited laboratories may submit test reports to NIST/CSL for validation.

\section{ACCREDTED NIST POSX TESTING LABORATORIES}

The National Voluntary Laboratory Accreditation Program (NMLP) has accredited the following laboratories to test computer operating system interfaces for conformance with the Federal Information Processing Standard 151-1 (FIPS 151-1) using the NIST POSIX Conformance Test Suite (NIST. PCTS:151-1). Only accredited laboratories may submit test reports to NIST/CSL for validation.

BUL SA / Laboratoire POSX

1 rue de Provence / BP208

38432 ECHIROLLES CEDEX (France)

DataFocus incorporated

12450 Fair Lakes Circle, Suite 400

Fairfax, VA 22033-3831

\section{Minderaft, the.}

410 Cambridge Avenue

Palo Alto, CA 94306

\section{PERENNIAL}

4699 Old Ironsides Drive, Suite 210

Santa Clara, CA 95054

UniSoft Comporation

6121 Hollis Street

Emeryville, CA 94608-2092
Contact: Mr. Georges Chardon

Phone: (33) 76397593

Contact: Mr. Glen McPherson

Phone: 703-631-6770

Contact: Mr. Bruce Weiner

Phone: 415-323-9000

Contact: Mr. Barry E. Hedquist

Phone: 408-748-2900

Contact: Ms. Audrey Ruelas

Phone: 510-420-6400 


\subsection{VALIDATED PRODUCTS \\ for NIST POSIX (FIPS 151-1)}

\section{NIST POSXX VALDATED PRODUCTS}

The following products have been tested by an Accredited POSIX Testing Laborafory (APTL) using the official National Institute of Standards and Technology POSIXConformance Test Suite (NIST-PCTS:151-1) for the Federal Information Processing Standards Publication 151-1 (FIPS PUB 151-1). A Certificate of Validation has been Issued by NIST/CSL. Additional information Is avallable from NIST/CSL on Conditional features supported, configuration details, and resolved test codes (if appropriate).

\section{PRODUCT SUPPLIERS REFERENCE FILE \#}

Amdahl Corporation Apple Computer Inc.

AT\&T

BULL S.A.

Control Data Corporation

CONVEX Computer Corporation

Cray Research, Inc.

Data General Corporation

Digital Equipment Corp.

Encore Computer Corporation ENC6897

\section{AMD5598 \\ APP2482, APP3355, APP7204, APP7224,} APP7235, APP8616, APP9125, APP9165 ATT1566

BUL2387, BUL6051

CDC1101, CDC5574, CDC5750

\section{CRA2641}

DGC2542, DGC4767, DGC8016, DGC8703, DGC9391, DGC9574

DEC0319, DEC0638, DEC4670, DEC5794, DEC7386, DEC7833, DEC7917, DEC8003, DEC9418, DEC 9672

ESIX/Everex Systems, Inc.

Harris Corporation

Howlett-Packard Company

EVROS01, EVR9749

HAR5240

HPCO115, HPCO303, HPCO535, HPCO603, HPC1581, HPC1992, HPC2540, HPC2698, HPC2952, HPC3574, HPC3760, HPC3897, HPC4246, HPC6304, HPC6391, HPC6637, HPC6906, HPC7051, HPC7716, HPC8098, HPC9185

Interactive Systems Corp. INT5154 Intergraph Corporation INT4675 Intemational Business Machines, Inc.

IBM0320, IBM0458, IBM1344, IBM2592, IBM3697

Lynx Real-Time Systems, Inc. LNX3076

Modular Computer Systems, Inc. MOD4817

Motorola Computer Group MOT1086, MOT5618

NCR Corporation

Next Computer, Inc.

NCR0554, NCR1448, NCR2047, NCR2805 NCR3061, NCR3331, NCR4518, NCR5533, NCR7380, NCR7549 NXT0623

Pyramid Technology Corporation PYR1271, PYR3067, PYR3233, PYR4970, PYR9863

Santa Cruz Operation Inc. SCO3664, SCO3832, SCO4102, SCO5199, SC06748, SCO8054, SCOS875

Sequent Computer Systems Inc. SEC8754

Silicon Graphics, Inc. SGI5507, SG19297

Sun Microsystems Computer Corp. SUN1065, SUN1442, SUN2031, SUN2727, SUN2930, SUN3272, SUN3402, SUN5684, SUN5782, SUN5970, SUN6602, SUN7188, SUN7793

SunSoft, Inc. SUNO617, SUN2241, SUN3129, SUN3403, SUN4529, SUN5382, SUN6635, SUN6859, SUN8720, SUN9763

Unisys Corporation

Univel

UNI0505, UNI1798, UNI3690, UNI5711, UNIS063, UNIS080

UNV0528, UNV2014, UNV3055, UNV3978, UNV9180

UNIX System Laboratories
USL2115, USL3610, USL6259

\section{SYSTEM SUPPUERS}

\section{REFERENCE FILE :}

AGI Computer, Inc.

Apha Systems Lab

Amdahl Corporation

Apple Computer Inc.

AST Research, Inc.

AT\&T

BUL S.A.

Compaq Computer Corporation INT5154, LNX3076, SUN6859

Control Data Corporation CONVEX Computer Corp.

Cray Research, Inc.

Data General Corporation

Dell Computer Corporation

Diamond Flower Incorporated SCO3664, SCOS054

Digital Equipment Corp. DEC0319, DEC0638, DEC4670, DEC5794, DEC7386, DEC7833, DEC7917, DEC8003, DEC9418, DEC 9572

Encore Computer Corporation ENC6897

ESIX/Everex Systems, Inc.

Harris Corporation

Howlett-Packard Company

EVR9749

HAR5240

HPCO115, HPC0303, HPCO535, HPC1581, HPC1992, HPC2540, HPC2698, HPC2952, HPC3574, HPC3760, HPC3897, HPC4246, HPC0603, HPC6304, HPC6391, HPC6637, HPC6906, HPC7051, HPCT716, HPC8098, HPC9185

Intergraph Corporation INT4675

Intemational Business Machines IBM0320, IBM0458, IBM1344, IBM2592, IBM3697

Modular Computer Systems, Inc. MOD4817

Motorola Computer Group MOT1086, MOT5618

NCR Corporation

NCR0554, NCR1448, NCR2047, NCR2805, NCR3061, NCR3331, NCR4518, NCR5533, NCR7380, NCR7549

NeXT Compputer, Inc. NXT0623

Pyramid Technology Corp. PYR1271, PYR3067, PYR3233, PYR4970,

RDI PYR9863

SUN3402

Sequent Computer Systems Inc. SEC8754

Silicon Graphics, Inc. SGI5507, SG19297

Sun Microsystems Corp.

SUN0617, SUN1442, SUN2031, SUN2241, SUN2727, SUN2930, SUN3129, SUN3272, SUN4529, SUN5382, SUN5684, SUN5782, SUN5970, SUN6602, SUN6635, SUN7188, SUN7793, SUN8720, SUN9763

Unisys Corporation SCO9875, UN10505, UNI1798, UNI3690, UNI5711, UNISO63, UNI9080, UNV0528, UNV2014, UNV3978

Zenith Data Systems 
Reference File \#: AMD5598

Product Supplier: Amdahl Corporation

Product Tested: UTS System Version: 4 Release: 1

System Supplier: Amdahl Corporation

System Hardware: 5995M Model: 4550

C Compiler: Amdahl C Version: 1.5 Release: June, 1993

PCTS: $151-1$ Version: $1.1 \cdot 05 / 21 / 92$

APTL: 0342 Mindcraft, Inc. Date lssued: 07/23/93

Reference File \#: APP2482

Product Supplier: Apple Computer Inc.

Product Tested: A/UX Version: 2.0.1 Release: 01/30/1991

System Supplier: Apple Computer Inc.

System Hardware: Macintosh Model: Ilfx

C Compiler: A/UX native C compiler (cc) Ver: 1.21 Rel: 1/13/1991

PCTS: 151-1 Version: 1.1 - 04/26/91

APTL: 0342 Mindcraft, Inc. Date Issued: 05/24/91

Reference File \#: APP3355

Product Supplier: Apple Computer Inc.

Product Tested: A/UX Version: 3.0 Release: March 9, 1992

System Supplier: Apple Computer Inc.

System Hardware: Macintosh Model: Quadra 700

C Compiler: A/UX native C compiler (cc) Ver: 1.23 Rel: Feb 9, 1992

PCTS: 151-1 Version: 1.1 - 01/22/92

APTL: 0342 Mindcraft, Inc. Date Issued: 04/16/92

Reference File *: APP7204

Product Supplier: Apple Computer Inc.

Product Tested: A/UX Version: 3.0.1 Release: April 23, 1993

System Supplier: Apple Computer Inc.

System Hardware: Workgroup Server Model: 80

C Compiler: A/UX Developer's Tools (c89) Ver: 1.1 Rel: Apr 1, 1992

PCTS: 151-1 Version: 1.1 - 05/21/92

APTL: 0342 Mindcraft, Inc. Date lssued: 06/24/93

Reference File *: APP7224

Product Supplier: Apple Computer Inc.

Product Tested: A/UX Version: 3.0 Release: March 9, 1992

System Supplier: Apple Computer Inc.

System Hardware: Macintosh Model: Quadra 950

C Compiler: A/UX native C compiler (cc) Ver: 1.23 Rel: Feb 9, 1992

PCTS: 151-1 Version: 1.1 - 01/22/92

APTL: 0342 Mindcraft, Inc. Date ksued: 05/14/92

Reference File *: APP7235

Product Supplier: Apple Computer Inc.

Product Tested: A/UX Version: 2.0.1 Release: 01/30/1991

Supplier: Apple Computer Inc. Hardware: Macintosh Model: Ilci C Compiler: A/UX native C compiler (c) Ver: 1.21 Rel: 01/13/1991

PCTS: 151-1 Version: $1.1 \cdot 04 / 26 / 91$

APTL: 0342 Mindcraft, Inc. Date lssued: 05/24/91

Reference File \#: APP8616

Product Supplier: Apple Computer Inc.

Product Tested: A/UX Version: 2.0.1 Release: 01/30/1991

Supplier: Apple Computer Inc. Hardware: Macintosh Model: Ilsi

C Compiler: A/UX native C compiler (cc) Ver: 1.21 Rel: 01/13/1991

PCTS: 151-1 Version: $1.1 \cdot 04 / 26 / 91$

APTL: 0342 Mindcraft, Inc. Date lssued: 05/24/91

Reference File \#: APP9125

Product Supplier: Apple Computer Inc.

Product Tested: A/UX Version: 3.0 Release: March 9, 1992

System Supplier: Apple Computer Inc.

System Hardware: Macintosh Model: Quadra 700

C Compiler: A/UX Developer's Tools (c89) Ver: 1.1 Rel: April 1, 1992

PCTS: $151-1$ Version: 1.1 - 05/21/92

APTL: 0342 Mindcraft, Inc. Date lssued: 08/11/92
Reference File \#: APP9165

Product Supplier: Apple Computer Inc.

Product Tested: A/UX Version: 3.0 Release: March 9, 1992

System Supplier: Apple Computer Inc.

System Hardware: Macintosh Model: Quadra 950

C Compiler: A/UX Developer's Tools (c89) Ver: 1.1 Rel: Apr 1, 1992

PCTS: 151-1 Version: 1.1 - 05/21/92

APTL: 0342 Mindcraft, Inc. Date lssued: 08/11/92

Reference File \#: ATT1566

Product Supplier: AT\&T

Product Tested: AT\&T UNIX System V Ver: Rolease 4 Rel: 4.0.3

System Supplier: AT\&T

System Hardware: AT\&T 382 R3 Series Model: 3B2/600 GR

C Compiler: AT\&T 3B2/RISC C Development System Version: 1.0

PCTS: $151-1$ Version: $1.1 \cdot 09 / 11 / 91$

APTL: 0343 DataFocus Incorporated Date lssued: 11/06/91

Reference File *: BUL2387

Product Supplier: BULL S.A.

Product Tested: BOS Version: 2 Release: 1

System Supplier: BULL S.A

System Hardware: DPX/2 Model: 200

C Compiler: C Compiler Version: 72 Release: 1

PCTS: 151-1 Version: 1.1 - 05/21/92

APTL: 0373 BULL S.A./Laboratoire POSIX Date issued: 2/24/93

Reference File \#: BUL6051

Product Supplier: BULL S.A.

Product Tested: BOS/X Version: 3 Release: 2

System Supplier: BULL S.A.

System Hardware: DPX/20 Model: 620

C Compiler: BOS/X XLC C Compiler Version: 1 Release: 02

PCTS: 151-1 Version: $1.1-05 / 21 / 92$

APTL: 0373 BULL S.A.Laboratoire POSIX Date lssued: 1/22/93

Reference File \#: CDC1101

Product Supplier: Control Data Corporation

Product Tested: EP/IX Version: 1.4.2 Release: November 27, 1991

System Supplier: Control Data Corporation

System Hardware: Control Data 4000 Model: 4680MP

C Compiler: EP/XX C Language RISCompiler V: C 2.11 Rel: July 1990

PCTS: 151-1 Version: 1.1-09/11/91

APTL: 0356 Applications Software Incorporated Date Issued: 1/29/92

Reference File *: CDC5574

Product Supplier: Control Data Corporation

Product Tested: EP/IX Version: 1.3.1 Release: 03/21/1991

System Supplier: Control Data Corporation

System Hardware: Control Data 4000 Model: $4330-250$

C Compiler: EP/XX C Language RISCompiler Version: 2.11 Release:

July 1990

PCTS: 151-1 Version: $1.1 \cdot 04 / 26 / 91$

APTL: 0356 Applications Software Incorporated Date lssued: $05 / 24 / 91$

Reference File \#: CDC5750

Product Supplier: Control Data Corporation

Product Tested: EP/IX Version: 1.3.1 Release: 03/21/1991

System Supplier: Control Data Corporation

System Hardware: Control Data $\mathbf{4 0 0 0}$ Model: $\mathbf{4 6 8 0}$

C Compiler: EP/IX C Language RISCompiler Version: 2.11 Release:

07/16/1990

PCTS: 151-1 Version: 1.1 - 04/26/91

APTL: 0356 Applications Software Incorporated Date Issued:

$05 / 24 / 91$ 
Reference File \#: $\mathrm{CONO2O2}$

Product Supplier: CONVEX Computer Corporation

Product Tested: ConvexOS Version: 10.1 Release: C200 Series

System Supplier: CONVEX Computer Corporation

System Hardware: C2 Model: C220

C Compiler: CONVEX C Version: 4.3.2

PCTS: 151-1 Version: 1.1 - 01/22/92

APTL: 0343 DataFocus Incorporated Date Issued: 05/11/92

Reference File *: CON2551

Product Supplier: CONVEX Computer Corporation

Product Tested: ConvexOS Version: 10.1 Release: $\mathrm{C} 3800$ Series

System Supplier: CONVEX Computer Corporation

System Hardware: C38 Model: C3810

C Compiler: CONVEX C Version: 4.3.2

PCTS: 151-1 Version: 1.1 - 01/22/92

APTL: 0343 DataFocus Incorporated Date Issued: 05/11/92

Reference File : CON6027

Product Supplier: CONVEX Computer Corporation

Product Tested: ConvexOS Version: 10.1 Release: C3400 Series

System Supplier: CONVEX Computer Corporation

System Hardware: C34 Model: C3440

C Compiler: CONVEX C Version: 4.3.2

PCTS: 151-1 Version: 1.1 - 01/22/92

APTL: 0343 DataFocus incorporated Date issued: 05/11/92

Reference File *: CRA2641

Product Supplier: Cray Research, Inc.

Product Tested: UNICOS Version: 7.0.5.bu Release: 7.0

System Supplier: Cray Research, Inc.

System Hardware: Cray Y-MP Model: YMP2E/232-4

C Compiler: Cray Standard C Compiler Release: 3.0 .5 (5/20/93)

PCTS: 151-1 Version: 1.1 - 05/21/92

APTL: 0343 DataFocus Incorporated Date Issued: 10/14/93

Roference File *: DEC0319

Product Supplier: Digital Equipment Corporation

Product Tested: DEC OSF/1 Version: 1.2 Release: March 1993

System Supplier: Digital Equipment Corporation

System Hardware: DEC/3000 Model: $\mathbf{5 0 0}$

C Compiler: DEC OSF/1 for AXP C Compiler Version: 1 Release:

March 1993

PCTS: 151-1 Version: $1.1-05 / 21 / 92$

APTL: 0342 Minderaft, Inc. Date lssued: 03/10/93

Reference File *: DEC0638

Product Supplier: Digital Equipment Corporation

Product Tested: VMS Version: 5 Release: 5 (with VMS POSIX, version 1.0)

System Supplier: Digital Equipment Corporation

System Hardware: VAXstation Model: 3100 M76

C Compiler: VAX C Version: 3 Release: 2

PCTS: 151-1 Version: 1.1 $00 / 11 / 91$

APTL: 0343 DataFocus incorporated Date issued: 01/29/92

Reference File *: DEC4670

Product Supplier: Digital Equipment Corporation

Product Tested: The ULTRIX Operating System Version: 4.3A

Release: July 1993

System Supplier: Digital Equipment Corporation

System Hardware: DECstation Model: $5000 / 150$

C Compiler: Mips C Compiler Version: 3.0

PCTS: 151-1 Version: 1.1 - 05/21/92

APTL: 0342 Mindcraft, the. Date lssued: 06/24/93
Reference File \#: DEC5794

Product Supplier: Digital Equipment Corporation

Product Tested: ULTRIX Version: 4.2 Release: May 31, 1991

System Supplier: Digital Equipment Corporation

System Hardware: VAXstation II Model: GPX

C Compiler: poc Version: 4.2

PCTS: 151-1 Version: 1.1 - 04/26/91

APTL: 0342 Mindcraft, Inc. Date lssued: 06/17/91

Reference File *: DEC7386

Product Supplier: Digital Equipment Corporation

Product Tested: The ULTRIX Operating System Version: 4.3 Release: August 1992

System Supplier: Digital Equipment Corporation

System Hardware: DECstation Model: 5000/200

C Compiler: Mips C Compiler Version: 2.10

PCTS: 151-1 Version: 1.1 - 05/21/92

APTL: 0342 Mindcraft, Inc. Date lssued: $09 / 18 / 92$

Reference File *: DEC7833

Product Supplier: Digital Equipment Corporation

Product Tested: OpenVMS VAX Version: 6 Release: 0 (with

OpenVMS VAX POSIX, Version X1.2-35E)

System Supplier: Digital Equipment Corporation

System Hardware: VAXstation Model: 3100 M76

C Compiler: VAX C Version: 3 Release: 2

PCTS: $151-1$ Version: $1.1-05 / 21 / 92$

APTL: 0343 DataFocus Incorporated Date issued: 10/14/93

Reference File \#: DEC7917

Product Supplier: Digital Equipment Corporation

Product Tested: the ULTRIX Operating System Version: 4.2A

Release: November 18, 1991

System Supplier: Digital Equipment Corporation

System Hardware: DECstation Model: 3100

C Compiler: MIPS C Compiler Version: 2.10

PCTS: $151-1$ Version: 1.1 - $09 / 11 / 91$

APTL: 0342 Mindcraft, Inc. Date Issued: 12/06/91

Reference File *: DEC8003

Product Supplier: Digital Equipment Corporation

Product Tested: The ULTRLX Operating System Version: 4.3A

Release: July 1993

System Supplier: Digital Equipment Corporation

System Hardware: DECstation Model: 5000/260

C Compiler: Mips C Compiler Version: 3.0

PCTS: 151-1 Version: 1.1 - 05/21/92

APTL: 0342 Mindcraft, Inc. Date lssued: 06/24/93

Reference File *: DEC9418

Product Supplier: Digital Equipment Corporation

Product Tested: ULTRIX Version: 4.2 Release: May 31, 1991

System Supplier: Digital Equipment Corporation

System Hardware: DECstation Model: 3100

C Compiler: MIPS C Compiler Version: 2.10

PCTS: 151-1 Version: 1.1 - 04/26/91

APTL: 0342 Mindcraft, Inc. Date issued: 06/17/91

Reference File \#: DEC9672

Product Supplier: Digital Equipment Corporation

Product Tested: The ULTRLX Operating System Version: 4.2A

Release: December 1991

System Supplier: Digital Equipment Corporation

System Hardware: DECstation Model: 5000/200

C Compiler: MIPS C Compiler Version: 2.10

PCTS: $151-1$ Version: $1.1 \cdot 09 / 11 / 91$

APTL: 0342 Mindcraft, Inc. Date issued: 02/12/92 
Reference File : DGC2542

Product Supplier: Data General Corporation

Product Tested: DG/UX Version: 5.4

System Supplier: Data General Corporation

System Hardware: AViion 5000 Model: AV/5240

C Compiler: GNU C Compiler for AViON Systems Version: 1.37.23

PCTS: 151-1 Version: 1.1 - 07/01/91

APTL: 0342 Mindcraft, Inc. Date lssued: $09 / 10 / 91$

Reference File \#: DGC4767

Product Supplier: Data General Corporation

Product Tested: DG/UX Version: 5.4.2 Release: August 1992

System Supplier: Data General Corporation

System Hardware: AVion AV/530/4600 Model: AV/532

C Compiler: GNU C Compiler for AViiON Systems Version: DG-2.2.3

Release: August 1992

PCTS: $151-1$ Version: 1.1 - 05/21/92

APTL: 0342 Minderaft, Inc. Date issued: 09/09/92

Reference File \#: DGC8016

Product Supplier: Data General Corporation

Product Tested: DG/UX Version: 5.4

System Supplier: Data General Corporation

System Hardware: AViion 400/4000 Model: AV/4100

C Compiler: GNU C Compiler for AViiON Systems Version: 1.37.23

PCTS: 151-1 Version: 1.1 - 07/01/91

APTL: 0342 Mindcraft, Inc. Date ksued: $\infty / 10 / 91$

Reference File \#: DGC8703

Product Supplier: Data General Corporation

Product Tested: DG/UX Version: 5.4

System Supplier: Data General Corporation

System Hardware: AViion 400/4000 Model: AV/412

C Compiler: GNU C Compiler for AViiON Systems Version: 1.37.23

PCTS: 151-1 Version: 1.1 - 07/01/91

APTL: 0342 Mindcraft, Inc. Date lssued: $\infty 9 / 10 / 91$

Reference File *: DGC9391

Product Supplier: Data General Corporation

Product Tested: DG/UX Version: 4.32

System Supplier: Data General Corporation

System Hardware: AViion AV/400/4000 Model: AV/410

C Compiler: GNU C Compiler for AViion Sys Version: 1.37.23

PCTS: 151-1 Version: 1.1 - 04/26/91

APTL: 0342 Mindcraft, Inc. Date lssued: 05/24/91

Reference File *: DGC9574

Product Supplier: Data General Corporation

Product Tested: DG/UX Version: 5.4.2 Release: August 1992

System Supplier: Data General Corporation

System Hardware: AViion AV/8000 Model: AV/6240

C Compiler: GNU C Compiler for AViON Systems Version: DG-2.2.3

Release: August 1992

PCTS: 151-1 Version: 1.1 - 05/21/92

APTL: 0342 Mindcraft, Inc. Date lssued: 11/03/92

Reference File *: ENC6897

Product Supplier: Encore Computer Corporation

Product Tested: UMAX V Release: 3.0.6

System Supplier: Encore Computer Corporation

System Hardware: 91 Series Model: 91-02427

C Compiler: Green Hills Software, Inc. C Release: 1.1

PCTS: 151-1 Version: 1.1 - 01/22/92

APTL: 0345 UniSoft Corporation Date kssued: 3/12/92
Reference File *: EVROg01

Product Supplier: ESIX/Everex Systems, Inc.

Product Tested: ESIX System V Release 4 Version: 4 Release: 4.0

System Supplier: AGI Computer, Inc.

System Hardware: AGI Model: $486 / 33$

C Compiler: ESIX ANSI C Compiler Version: 5.0

PCTS: 151-1 Version: 1.1 - 01/22/92

APTL: 0343 DataFocus Incorporated Date lssued: 05/28/92

Reference File \#: EVR9749

Product Supplier: ESIX/Everex Systems, Inc.

Product Tested: ESIX System V Release 4 Version: 4 Release: 4.0 System Supplier: ESIX/Everex Systems, Inc.

System Hardware: Everex Model: 3000 S 386/33

C Compiler: ESIX ANSI C Compiler Version: 5.0

PCTS: 151-1 Version: 1.1 - 01/22/92

APTL: 0343 DataFocus Incorporated Date Issued: 05/28/92

Reference File : HAR5240

Product Supplier: Harris Corporation

Product Tested: CX/UX Release: 5.3

System Supplier: Harris Corporation, Computer Systems Division

System Hardware: Night Hawk Model: HN4802

C Compiler: Harris C Compiler Release: 5.3

PCTS: 151-1 Version: $1.1-09 / 11 / 91$

APTL: 0342 Mindcraft, Inc. Date lssued: 12/16/91

Reference File \#: HPC0115

Product Supplier: Hewlett-Packard Company

Product Tested: HP.UX Version: 8.02 Release: 10/06/91

System Supplier: Hewlett-Packard Company

System Hardware: HP9000 Series 800 Model: $867 S$

C Compiler: HP C Compiler Version: A 08.71 Release: 10/06/91

PCTS: 151-1 Version: 1.1 - 05/21/92

APTL: 0342 Mindcraft, Inc. Date Issued: 12/08/92

Reference File *: HPCO303

Product Supplier: Howlett-Packard Company

Product Tested: HP-UX Version: 8.02 Release: 10/06/91

System Supplier: Hewlett-Packard Company

System Hardware: HP9000 Series 800 Model: $867 \mathrm{~s}$

C Compiler: HP C Compiler Version: A 08.17 Release: 10/06/91

PCTS: 151-1 Version: 1.1 - 05/21/92

APTL: 0346 Hewlett-Packard POSIX Conformance Test Conter Dete

Issued: $09 / 09 / 92$

Reference File *: HPC0535

Product Supplier: Howlett-Packard Company

Product Tested: Domain/OS Version: 10.4 Release: April 1992

System Supplier: Hewlett-Packard Company

System Hardware: Domain Series 4000 Model: DN4500

C Compiler: Domain/C Version: 6.9.M/MPX Release: May 1992

PCTS: $151-1$ Version: 1.1 - 05/21/92

APTL: 0346 Hewlett-Packard POSIX Conformance Test Conter Date tssued: $09 / 2 / 92$

Reference File *: HPCO603

Product Supplier: Howlett-Packard Company

Product Tested: HP.UX Version: 9.01 Release: January 4, 1993

System Supplier: Hewlett-Packard Company

System Hardware: HP9000 Series 700 Model: 735

C Compiler: HP C Compiler Version: HP92453-01 A.09.19 Release:

December, 1992

PCTS: 151-1 Version: 1.1 - 05/21/92

APTL: 0342 Mindcraft, Inc. Date lssued: 2/19/93 
Reference File *: HPC1581

Product Supplier: Hewlett-Packard Company

Product Tested: HP-UX Version: 8.02 Release: 10/06/91

System Supplier: Hewlett-Packard Company

System Hardware: HP9000 Series 800 Model: 827 S

C Compiler: HP C Compiler Version: A 08.71 Release: 10/06/91

PCTS: 151.1 Version: 1.1 - 05/21/92

APTL: 0342 Mindcraft, Inc. Date lssued: 12/08/92

Reference File *: HPC1992

Product Supplier: Howlett-Packard Company

Product Tested: HP-UX Version: 8.08 Release: 11/23/92

System Supplier: Hewlett-Packard Company

System Hardware: HP9000 Series 800 Model: $827 S$

C Compiler: HP C Compiler Version: A 08.71 Release: 10/06/91

PCTS: $151-1$ Version: 1.1 - 05/21/92

APTL: 0342 Mindcraft, Inc. Date Issued: 12/08/92

Reference File *: HPC2540

Product Supplier: Hewlett-Packard Company

Product Tested: HP-UX Version: 8.07 Release: December 1991

System Supplier: Hewlett-Packard Company

System Hardware: HP9000 Series 700 Model: 720

C Compiler: HP C Compiler Version: A 08.71 Release: Dec 1991

PCTS: 151-1 Version: 1.1 - 09/11/91

APTL: 0346 Hewlett-Packard POSLX Conformance Test Center Date ssued: 01/20/92

Reference File \#: HPC2698

Product Supplier: Hewlett-Packard Company

Product Tested: HP-UX Version: 8.02 Release: 10/06/91

System Supplier: Hewlett-Packard Company

System Hardware: HP9000 Series 800 Model: $817 \mathrm{~S}$

C Compiler: HP C Compiler Version: A 08.71 Release: 10/06/91

PCTS: 151-1 Version: 1.1 - 05/21/92

APTL: 0342 Mindcraft, Inc. Date lssued: 12/08/92

Reference File *: HPC2952

Product Supplier: Howlett-Packard Company

Product Tested: Domain/OS Version: 10.4 Release: April 1992

System Supplier: Hewlett-Packard Company

System Hardware: Domain Series 400 Model: $433 \mathrm{~s}$

C Compiler: Domain/C Version: 6.9.M/MPX Release: May 1992

PCTS: $151-1$ Version: 1.1 - 05/21/92

APTL: 0346 Hewlett-Packard POSIX Conformance Test Center Date

lssued: $09 / 2 / 92$

Reference File *: HPC3574

Product Supplier: Hewlett-Packard Company

Product Tested: HP.UX Version: 9.0 Release: October 7, 1992

System Supplier: Hewlett-Packard Company

System Hardware: HPS000 Series 400 Model: 4335

C Compiler: HP C Compiler Version: B23718.08.00 Intemal Revision

70.2 Release: October 7, 1992

PCTS: 151-1 Version: 1.1 - 05/21/92

APTL: 0342 Mindcraft, inc. Date lssued: 2/19/93

Reference File *: HPC3760

Product Supplier: Hewlett-Packard Company

Product Tested: HP.UX Version: 8.02 Release: 10/06/91

System Supplier: Hewlett-Packard Company

System Hardware: HP9000 Series 800 Model: 8475

C Compiler: HP C Compiler Version: A 08.71 Release: 10/06/91

PCTS: 151-1 Version: 1.1 - 05/21/92

APTL: 0342 Mindcraft, inc. Date lssued: 12/08/92
Reference File *: HPC3897

Product Supplier: Hewlett-Packard Company

Product Tested: HP.UX Version: 9.0 Release: October 7, 1992

System Supplier: Howlett-Packard Company

System Hardware: HP9000 Series 800 Model: $847 \mathrm{~S}$

C Compiler: HP C Compiler Version: A 09.19 Release: Oct 7, 1992

PCTS: 151-1 Version: 1.1 - 05/21/92

APTL: 0342 Mindcraft, Inc. Date lssued: 1/07/93

Reference File \#: HPC4246

Product Supplier: Howlett-Packard Company

Product Tested: HP-UX Version: 8.08 Release: 11/23/92

System Supplier: Hewlett-Packard Company

System Hardware: HP9000 Series 800 Model: 807 S

C Compiler: HP C Compiler Version: A 08.71 Release: 10/06/91

PCTS: 151.1 Version: $1.1 \cdot 05 / 21 / 92$

APTL: 0342 Mindcratt, Inc. Date lssued: 12/08/92

Reference File \#: HPC6304

Product Supplier: Hewlett-Packard Company

Product Tested: HP-UX Version: 9.01 Release: January 4, 1993

System Supplier: Hewlett-Packard Company

System Hardware: HPSO00 Series 700 Model: 720

C Compiler: HP C Compiler Ver: HP92453-01 A.09.19 Rel: Dec, 1992

PCTS: 151-1 Version: 1.1 - 05/21/92

APTL: 0342 Mindcraft, Inc. Date lssued: 2/19/93

Reference File \#: HPC6391

Product Supplier: Hewlett-Packard Company

Product Tested: HP.UX Version: 8.00 with PHCO_0800 (Patch)

Release: January 1991, January 1992 (Patch)

System Supplier: Hewlett-Packard Company

System Hardware: HPS000 Series 400 Model: 400 S

C Compiler: HP C Compiler Version: B 08.00 Release: Dec. 1991

PCTS: $151-1$ Version: 1.1 - 01/22/92

APTL: 0346 Hewlett-Packard POSIX Conformance Test Center Date

lssued: 04/17/92

Reference File \#: HPC6637

Product Supplier: Hewlett-Packard Company

Product Tested: HP.UX Version: 8.08 Release: 11/23/92

System Supplier: Hewlett-Packard Company

System Hardware: HP9000 Series 800 Model: $817 \mathrm{~S}$

C Compiler: HP C Compiler Version: A 08.71 Release: 10/06/91

PCTS: 151.1 Version: 1.1 - 05/21/92

APTL: 0342 Mindcraft, Inc. Date lssued: 12/08/92

Reference File \#: HPC6906

Product Supplier: Howlett-Packard Company

Product Tested: HP.UX Version: 9.01 Release: January 4, 1993

System Supplier: Hewlett-Packard Company

System Hardware: HP9000 Series 700 Model: 715

C Compiler: HP C Compiler Ver: HP92453-01 A.09.19 Rel: Dec. 1992

PCTS: 151-1 Version: 1.1 - 05/21/92

APTL: 0342 Mindcraft, Inc. Date lssued: 2/19/93

Reference File *: HPC7051

Product Supplier: Hewlett-Packard Company

Product Tested: HP-UX Version: 8.08 Release: 11/23/92

System Supplier: Hewlett-Packard Company

System Hardware: HP9000 Series 800 Model: 8675

C Compiler: HP C Compiler Version: A 08.71 Release: 10/06/91

PCTS: 151-1 Version: 1.1 - 05/21/92

APTL: 0342 Mindcraft, Inc. Date lssued: 12/08/92 
Roference File *: HPC7716

Product Supplier: Howlett-Packard Company

Product Tested: HP-UX Version: 8.08 Release: 11/23/92

System Supplier: Howlett-Packard Company

System Hardware: HP9000 Series 800 Model: 8475

C Compiler: HP C Compiler Version: A 08.71 Release: 10/06/91

PCTS: 151-1 Version: 1.1 - 05/21/92

APTL: 0342 Mindcraft, Inc. Date lssued: 12/08/92

Reference File *: HPC8098

Product Supplier: Howlett-Packard Company

Product Tested: HP-UX Version: 8.02 Release: 10/06/91

System Supplier: Hewlett-Packard Company

System Hardware: HP9000 Series 800 Model: 807 S

C Compiler: HP C Compiler Version: A 08.71 Release: 10/06/91

PCTS: 151-1 Version: 1.1 - 05/21/92

APTL: 0342 Mindcraft, Inc. Date Issued: 12/08/92

Reference File \#: HPC9185

Product Supplier: Howlett-Packard Company

Product Tested: HP-UX Version: 8 Release: 5/6/91

System Supplier: Howlett-Packard Company

System Hardware: HP9000 Series 800 Model: 835

C Compiler: HP C Compiler Version: A 08.17 Release: 5/6/91

PCTS: $151-1$ Version: $1.1-09 / 11 / 91$

APTL: 0346 Hewlett-Packard POSIX Conformance Test Center Date lssued: $12 / 18 / 91$

Reference File *: IBM0320

Product Supplier: Intemational Business Machines Inc.

Product Tested: AIX Version 3 for RISC System/6000 Version: 3

Release: 2

System Supplier: International Business Machines Inc.

System Hardware: RISC System/6000 Model: 220

C Compiler: xlc Version: 1 Release: 2

PCTS: 151-1 Version: 1.1 - 01/22/92

APTL: 0342 Mindcraft, Inc. Date lssued: 02/25/92

Reference File \#: IBM0458

Product Supplier: Intemational Business Machines Inc.

Product Tested: AIX Version 3 for RISC System/6000 Version: 3

Release: 2

System Supplier: International Business Machines Inc.

System Hardware: RISC System/6000 Model: $530 \mathrm{H}$

C Compiler: xlc Version: 1 Release: 2

PCTS: 151-1 Version: 1.1 - 01/22/92

APTL: 0342 Mindcraft, Inc. Date Issued: 02/25/92

Reference File \#: IBM1344

Product Supplier: International Business Machines Inc.

Product Tested: AIX Version: 3 Release: 1

System Supplier: International Business Machines Inc.

System Hardware: RISC System/6000 Model: 320

C Compiler: xlc Version: 3 Release: 1

PCTS: 151-1 Version: 1.1 - 04/26/91

APTL: 0342 Mindcraft, Inc. Date issued: 05/24/91

Reference File \#: 1BM2592

Product Supplier: International Business Machines Inc. Product Tested: AIX Version: 3 Release: 1

System Supplier: International Business Machines Inc.

System Hardware: RISC System/6000 Model: 530

C Compiler: xlc Version: 3 Release: 1

PCTS: 151-1 Version: 1.1 - 04/26/91

APTL: 0342 Minderaft, Inc. Date Issued: 05/24/91
Reference File \#: IBM3697

Product Supplier: International Business Machines Inc. Product Tested: AIX Version 3 for RiSC System/6000 Version: 3

Rolease: 2

System Supplier: International Business Machines Inc. System Hardware: RISC System/6000 Model: 320

C Compiler: xlc Version: 1 Release: 2

PCTS: 151-1 Version: 1.1 - 01/22/92

APTL: 0342 Mindcraft, Inc. Date lssued: 02/25/92

Reference File *: INT4675

Product Supplier: Intergraph Corporation

Product Tested: CLX Version: 06.02.01 Release: 3.1

System Supplier: Intergraph Corporation

System Hardware: Intergraph 6400 Series Workstation Model: 6450

C Compiler: CLIPPER Advanced Optimizing C Compiler Version:

06.00.01.43 Release: 28JAN-1992

PCTS: $151-1$ Version: 1.1 - 01/22/92

APTL: 0343 DataFocus Incorporated Date ksued: 05/28/92

Reference File \#: INT5154

Product Supplier: Interactive Systems Corp.

Product Tested: Interactive UNIX Operating System Version: $\mathbf{3 . 0}$

Rolease: 3.2

System Supplier: Compaq Computer Corporation

System Hardware: Compaq Model: System Pro

C Compiler: Interactive UNIX Software Development System Ver: 3.0

PCTS: $151-1$ Version: 1.1 - 09/11/91

APTL: 0345 UniSoft Corporation Date lssued: 10/16/91

Reference File *: LNX3076

Product Supplier: Lynx Real-Time Systems, Inc.

Product Tested: LynxOS Version: 2 Release: 2.2.0

System Supplier: Compaq Computer Corporation

System Hardware: ProLinea Model: 4/33

C Compiler: gcc Version: 1.42 Release: September 19, 1992

PCTS: $151-1$ Version: $1.1 \cdot 05 / 21 / 92$

APTL: 0342 Mindcraft, Inc. Date issued: 10/14/93

Reference File *: MOD4817

Product Supplier: Modular Computer Systems, Inc.

Product Tested: REAL/IX Version: V.3 Rolease: D.0

System Supplier: Modular Computer Systems, Inc.

System Hardware: REAL/STAR Model: 1000

C Compiler: GNU C Compiler for REAL/IX Systems Version: 1.37

PCTS: $151-1$ Version: 1.1 - 01/22/92

APTL: 0342 Mindcraft, Inc. Date lssued: 05/05/92

Reference File *: MOT1086

Product Supplier: Motorola Computer Group

Product Tested: UNIX System V/88 Release 4.0 Version: 3

Release: 4.0

System Supplier: Motorola Computer Group

System Hardware: Motorola Series 8000 Model: $8 \times 40$

C Compiler: Software Development System Version: T302.0 Release:

12/2/92

PCTS: 151-1 Version: $1.1 \cdot 05 / 21 / 92$

APTL: 0343 DataFocus, Inc. Date issued: $2 / 19 / 93$

Reference File \#: MOT5618

Product Supplier: Motorola Computer Group

Product Tested: UNIX System V/88 Release 4.0 Version: 3

Release: 4.0

System Supplier: Motorola Computer Group

System Hardware: Motorola Series 8000 Model: $8 \times 20$

C Compiler: Software Development System Ver: T302.0 Rel: 12/2/92

PCTS: 151-1 Version: 1.1 - 05/21/92

APTL: 0343 DataFocus, Inc. Date lssued: 2/19/93 
Reference File *: NCRO554

Product Supplier: NCR Corporation

Product Tested: NCR UNIX System V Ver: Release 4 Rel: 4.0.4

System Supplier: NCR Corporation

System Hardware: NCR 3B2 R3 Series Model: 3B2/1000 R3

(Military ID: 3B2/600 GR)

C Compiler: 3B2/RISC C Development System Release: 1.1

PCTS: $151-1$ Version: $1.1 \cdot 05 / 21 / 92$

APTL: 0343 DataFocus, Inc. Date lssued: 12/09/92

Reference File *: NCR1448

Product Supplier: NCR Corporation

Product Tested: NCR UNIX System V Release 4 MP-RAS, Rel 2

Version: SVR4 Release: 2

System Supplier: NCR Corporation

System Hardware: System 3000 Model: 3455

C Compiler: NCR C Development Toolkit Release: 2

PCTS: 151-1 Version: 1.1 - 05/21/92

APTL: 0343 DataFocus, Inc. Date ksued: 10/08/93

Reference File *: NCR2O47

Product Supplier: NCR Corporation

Product Tested: NCR System V Release 4 MP.RAS, Rel 2 Version:

SVR4 Release: 2

System Supplier: NCR Corporation

System Hardware: System 3000 Model: 3447

C Compiler: NCR C Development Toolkit Release: 2

PCTS: 151.1 Version: $1.1 \cdot 01 / 22 / 92$

APTL: 0343 DataFocus, inc. Date ksued: 06/26/92

Reference File \#: NCR2805

Product Supplier: NCR Corporation

Product Tested: NCR System V Release 4 MP-RAS, Rel 2 Version: SVR4 Release: 2

System Supplier: NCR Corporation

System Hardware: System 3000 Model: 3450

C Compiler: NCR C Development Toolkit Release: 2

PCTS: 151.1 Version: $1.1 \cdot 01 / 22 / 92$

APTL: 0343 DataFocus, inc. Date lssued: 06/26/92

Reference File *: NCR3061

Product Supplier: NCR Corporation

Product Tested: NCR UNIX System V Release 4 MP-RAS, Rel 2

Version: SVR4 Release: 2

System Supplier: NCR Corporation

System Hardware: System 3000 Model: 3555

C Compiler: NCR C Development Toolkit Release: 2

PCTS: $151 \cdot 1$ Version: $1.1 \cdot 05 / 21 / 92$

APTL: 0343 DataFocus, inc. Date issued: 10/08/93

Reference File *: NCR3331

Product Supplier: NCR Corporation

Product Tested: NCR System V Release 4 MP.RAS, Rel 2 Version: SVR4 Rolease: 2

System Supplier: NCR Corporation

System Hardware: System 3000 Model: 3345

C Compiler: NCR C Development Toolkit Release: 2

PCTS: 151-1 Version: 1.1 01/22/92

APTL: 0343 DataFocus, Inc. Date issued: 06/26/92

Reference File *: NCR4518

Product Supplier: NCR Corporation

Product Tested: NCR System V Release 4 MP-RAS, Rel 2 Version: SVR4 Release: 2

System Supplier: NCR Corporation

System Hardware: System 3000 Model: 3550

C Compiler: NCR C Development Toolkit Release: 2

PCTS: 151-1 Version: 1.1 - 01/22/92

APTL: 0343 DataFocus, Inc. Date lssued: 06/26/92
Reference File \#: NCR5533

Product Supplier: NCR Corporation

Product Tested: NCR UNIX System V Release 4 MP-RAS, Rel 2

Version: SVR4 Release: 2

System Supplier: NCR Corporation

System Hardware: System 3000 Model: $\mathbf{3 5 2 0}$

C Compiler: NCR C Development Toolkit Release: 2

PCTS: 151.1 Version: $1.1 \cdot 05 / 21 / 92$

APTL: 0343 DataFocus, Inc. Date Issued: 10/08/93

Reference File *: NCR7380

Product Supplier: NCR Corporation

Product Tested: UNIX System V Release 4.0 Version 3.1

Version: 3.1 Release: 4.0

System Supplier: NCR Corporation

System Hardware: StarServer E Model: Release 3

C Compiler: Optimized C Compiler Version: 5.0

PCTS: 151.1 Version: $1.1 \cdot 05 / 21 / 92$

APTL: 0343 DataFocus, Inc. Date issued: 03/10/93

Reference File *: NCR7549

Product Supplier: NCR Corporation

Product Tested: NCR UNIX System V Release 4 MP.RAS, Rel 2

Version: SVR4 Release: 2

System Supplier: NCR Corporation

System Hardware: System 3000 Model: 3525

C Compiler: NCR C Development Toolkit Release: 2

PCTS: $151-1$ Version: $1.1-05 / 21 / 92$

APTL: 0343 DataFocus, Inc. Date issued: 10/08/93

Reference File \#: NXT0623

Product Supplier: NeXT Computer, Inc.

Product Tested: NEXTSTEP Version: 3.2 Release: November 5, 1993 (with POSIX for NEXTSTEP version 1.0)

System Supplier: NeXT Computer, Inc.

System Hardware: NeXTstation Model: Color Turbo

C Compiler: NEXTSTEP DEVELOPER Version: 3.2 Release:

November 5, 1993

PCTS: 151.1 Version: $1.1 \cdot 05 / 21 / 92$

APTL: 0342 Mindcraft, Inc. Date issued: 10/08/93

Reference File *: PYR1271

Product Supplier: Pyramid Technology Corporation

Product Tested: OSx Version: 5.1a-92a023 Release: 0422s

System Supplier: Pyramid Technology Corporation

System Hardware: MIServer Model: MIS-2T

C Compiler: att $\propto$ Version: 5.1

PCTS: 151.1 Version: $1.1 \cdot 01 / 22 / 92$

APTL: 0343 DataFocus Incorporated Date lssued: 05/28/92

Reference File *: PYR3057

Product Supplier: Pyramid Technology Corporation

Product Tested: DataCenter/OSx Version: dcosx Release: 1.1. 920027

System Supplier: Pyramid Technology Corporation

System Hardware: MIServer Model: 2S

C Compiler: DataCenter/OSx C Compiler Release: 1.1-92C027

PCTS: $151-1$ Version: $1.1 \cdot 05 / 21 / 92$

APTL: 0343 DataFocus Incorporated Date issued: 09/09/92

Reference File *: PYR3233

Product Supplier: Pyramid Technology Corporation

Product Tested: DataCenter/OSx Version: dcosx Release: 1.1920027

System Supplier: Pyramid Technology Corporation

System Hardware: MIServer Model: 12S

C Compiler: DataCenter/OSx C Compiler Release: 1.1-920027

PCTS: $151-1$ Version: $1.1 \cdot 05 / 21 / 92$

APTL: 0343 DataFocus Incorporated Date issued: 10/05/92 
Reference Flie *: PYR4970

Product Supplier: Pyramid Tochnology Corporation Product Tested: DataCenter/OSx Version: dcosx Rel: 1.1-92c027

System Supplier: Pyramid Technology Corporation System Hardware: MIServer Model: 4S

C Compiler: DataCenter/OSx C Compiler Release: 1.1-92C027

PCTS: 151-1 Version: 1.1 - 05/21/92

APTL: 0343 DataFocus incorporated Date Issued: $09 / 09 / 92$

Reference File *: PYR9863

Product Supplier: Pyramid Technology Corporation

Product Tested: OSx Version: 5.1a Release: 0318t

System Supplier: Pyramid Technology Corporation

System Hardware: MIServer Model: MIS-4T

C Compiler: att $\propto$ Version: $\mathbf{5 . 1}$

PCTS: $151-1$ Version: $1.1 \cdot 01 / 22 / 92$

APTL: 0343 DataFocus Incorporated Date issued: 05/28/92

Reference File *: SCO3664

Product Supplier: Santa Cruz Operation Inc.

Product Tested: SCO Open Desktop Version: 2.0

System Supplier: Diamond Flower Incorporated

System Hardware: DFI Model: 486SX/25

C Compiler: Microsoft C Version: $\mathbf{5 . 1}$

PCTS: $151-1$ Version: $1.1 \cdot 05 / 21 / 92$

APTL: 0343 DataFocus Incorporated Date lssued: 11/02/92

Roference File *: SCO3832

Product Supplier: Santa Cruz Operation Inc.

Product Tested: SCO UNIX System V/386 Version: Release 3.2

System Supplier: Zenith Data Systems

System Hardware: Z Station Model: 433DEh

C Compiler: Microsoft C Version: 5.1

PCTS: 151-1 Version: 1.1 - 05/21/92

APTL: 0343 DataFocus Incorporated Date Issued: 09/28/92

Reference File : SCO4102

Product Supplier: Santa Cruz Operation, Inc.

Product Tested: SCO UNIX System V/386 Version: Release 3.2

System Supplier: AST Research, Inc.

System Hardware: Premium Series Model: 486/33

C Compiler: Microsoft C Version: $\mathbf{5 . 1}$

PCTS: 151-1 Version: 1.1 - 05/21/92

APTL: 0343 DataFocus, Inc. Date lssued: 07/01/92

Reference File : SCO5199

Product Supplier: Santa Cruz Operation Inc.

Product Tested: SCO UNIX System V/386 Version: 3.2

System Supplier: Zenith Data Systems

System Hardware: Zenith Data Systems Supersport Laptop Model:

Supersport SX

C Compiler: Microsoft C Version: $\mathbf{5 . 1}$

PCTS: 151-1 Version: 1.1 - 07/01/91

APTL: 0343 DataFocus incorporated Date lssued: $09 / 17 / 91$

Reference File *: SCOS748

Product Supplier: Santa Cruz Operation Inc.

Product Tested: SCO UNIX System V/386 Version: 3.2 Release: 2

System Supplier: Data General Corporation

System Hardware: Walkabout/SX Model: G2763

C Compiler: Microsoft C Optimizing Compiler Version: 5.1

PCTS: $151-1$ Version: 1.1 - 07/01/91

APTL: 0342 Minderaft, Inc. Date Issued: 09/10/91
Reference File *: SCO8054

Product Supplier: Santa Cruz Operation Inc.

Product Tested: SCO Open Desktop Version: 2.0

System Supplier: Diamond Flower Incorporated

System Hardware: DFI Model: 486/33

C Compiler: Microsoft C Version: 5.1

PCTS: $151-1$ Version: 1.1 - 05/21/92

APTL: 0343 DataFocus Incorporated Date Issued: 11/02/92

Reference File *: SCO9875

Product Supplier: Santa Cruz Operation Inc.

Product Tested: SCO UNIX System V/386 Version: 3.2

System Supplier: UNISYS Corporation

System Hardware: PW² Advantage 3000 Series Model: 3256

C Compiler: Microsoft C Version: 5.1

PCTS: $151-1$ Version: 1.1 - 09/11/91

APTL: 0343 DataFocus Incorporated Date lssued: 11/01/91

Reference File *: SEC8754

Product Supplier: Sequent Computer Systems Inc.

Product Tested: DYNIX/ptx Operating System Version: 1.3.0

System Supplier: Sequent Computer Systems inc.

System Hardware: Symmetry Series II Model: S27

C Compiler: C Tools Version: 1.12p

PCTS: $151-1$ Version: $1.1 \cdot 09 / 11 / 91$

APTL: 0345 UniSoft Corporation Date lssued: 12/09/91

Reference File *: SG15507

Product Supplier: Silicon Graphics, Inc.

Product Tested: IRIX Version: 4.0.5

System Supplier: Silicon Graphics, Inc.

System Hardware: IRIS Model: Crimson

C Compiler: IRIS Development Option Version: 2.20

PCTS: 151-1 Version: 1.1 - 01/22/92

APTL: 0342 Mindcraft, Inc. Date Issued: 06/15/92

Reference File *: SG19297

Product Supplier: Silicon Graphics, Inc.

Product Tested: IRIX Version: 4.0.5

System Supplier: Silicon Graphics, Inc.

System Hardware: IRIS Model: Indigo

C Compiler: IRIS Development Option Version: 2.20

PCTS: 151-1 Version: 1.1 - 01/22/92

APTL: 0342 Mindcraft, Inc. Date lssued: 06/15/92

Reference File *: SUN0617

Product Supplier: SunSoft, Inc.

Product Tested: Solaris Version: 1.0.1 Release: PC

System Supplier: Sun Microsystems Computer Corporation, Inc. System Hardware: SPARCstation IPC Model: GX

C Compiler: Solaris C Compiler Version: 1.0.1 Release: Dec 4, 1991

PCTS: $151-1$ Version: $1.1 \cdot 05 / 21 / 92$

APTL: 0343 DataFocus Incorporated Date Issued: 08/27/92

Reference File \#: SUN1065

Product Supplier: Sun Microsystems Computer Corporation, Inc. Product Tested: Solaris 2.1 for x86 Version: 2.1 Release: May 1993

System Supplier: Dell Computer Corporation System Hardware: 450 Model: DE

C Compiler: ProCompiler C Version: 2.0 .1 for x86 Rel: May 1993

PCTS: 151.1 Version: 1.1 - 05/21/92

APTL: 0342 Mindcraft, Inc. Date lssued: 05/20/93 
Reference File *: SUN1442

Product Supplier: Sun Microsystems Computer Corporation, Inc. Product Tested: Solaris Version: 2.2 Release: May 28, 1993

System Supplier: Sun Microsystems Computer Corporation, Inc. System Hardware: SPARCstation LX Model: 4/30

C Compiler: Sun C Compiler Version: 2.0.1 Release: Oct. 3, 1992

PCTS: 151-1 Version: $1.1-05 / 21 / 92$

APTL: 0342 Mindcraft, Inc. Date issued: 05/28/93

Reference File *: SUN2031

Product Supplier: Sun Microsystems Computer Corporation, Inc. Product Tested: Solaris Version: 2.1 Release: August 4, 1992 System Supplier: Sun Microsystems Computer Corporation, Inc. System Hardware: SunWorkstation 4/30 Model: 4/30

C Compiler: Sun C Compiler Version: 2.0 Release: June 30, 1992

PCTS: 151-1 Version: $1.1-05 / 21 / 92$

APTL: 0342 Mindcraft, Inc. Date lssued: 10/08/92

Reference File *: SUN2241

Product Supplier: SunSoft, inc.

Product Tested: Solaris Version: 2.0 Release: June 1992

System Supplier: Sun Microsystems Computer Corporation, Inc. System Hardware: SPARCstation 2 Model: 4/75

C Compiler: Sun C Compiler Version: 2.0 Release: 20 May 1992

PCTS: 151-1 Version: 1.1 - 01/22/92

APTL: 0342 Mindcraft, lnc. Date lssued: 07/02/92

Reference File *: SUN2727

Product Supplier: Sun Microsystems Computer Corporation, Inc. Product Tested: Solaris Version: 2.1 Release: December 7, 1992

System Supplier: Sun Microsystems Computer Corporation, Inc.

System Hardware: SPARCserver 10 Model: 42

C Compiler: Sun C Compiler Version: 2.0.1 Release: Oct. 3, 1992

PCTS: 151.1 Version: 1.1 - 05/21/92

APTL: 0342 Minderaft, Inc. Date lssued: 1/07/93

Reference File *: SUN2930

Product Supplier: Sun Microsystems Computer Corporation, Inc. Product Tested: Solaris Version: 2.2 Release: May 28, 1993

System Supplier: Sun Microsystems Computer Corporation, Inc. System Hardware: SPARCstation 2 Model: 4/75

C Compiler: Sun C Compiler Version: 2.0.1 Rolease: Oct. 3, 1992 PCTS: 151-1 Version: 1.1 - 05/21/92

APTL: 0342 Mindcraft, Inc. Date lssued: 05/28/93

Roference File \#: SUN3129

Product Supplier: Sunsoft, Inc.

Product Tested: Interactive Unix Operating System V/386 Version: 3.0.1 Rolease: 3.2

System Supplier: Compaq Computer Corporation System Hardware: Desk Pro Model: 386/20E

C Compiler: Interactive Unix Software Development System Version: 3.0 Release: December 4, 1991

PCTS: 151-1 Version: $1.1-05 / 21 / 92$

APTL: 0345 UniSoft Corporation Date ksued: $9 / 18 / 92$

Roference File \#: SUN3272

Product Supplier: Sun Microsystems Computer Corporation, Inc. Product Tested: Solaris Version: 2.2 Release: May 28, 1993

System Supplier: Sun Microsystems Computer Corporation, Inc. System Hardware: SPARCenter 10 Model: 54

C Compiler: Sun C Compiler Version: 2.0.1 Release: Oct. 3, 1992 PCTS: $151-1$ Version: 1.1 - 05/21/92

APTL: 0342 Mindcraft, Inc. Date lssued: 05/28/93
Reference File \#: SUN3402

Product Supplier: Sun Microsystems Computer Corporation, Inc. Product Tested: Solaris Version: 2.1 Rolease: August 4, 1992 System Supplier: RDI

System Hardware: BriteLite Model: IPX Color Laptop Workstation C Compiler: Sun C Compiler Version: 2.0 Release: June 30, 1992 PCTS: 151-1 Version: 1.1 - 05/21/92

APTL: 0342 Mindcraft, Inc. Date issued: 10/16/92

Reference File *: SUN3403

Product Supplier: SunSoft, Inc.

Product Tested: Interactive Unix Operating System V/386 Version: 3.0.1 Rolease: 3.2

System Supplier: Apha Systems Lab

System Hardware: ASL486/33 Model: ASL433

C Compiler: Interactive Unix Software Development System Version: 3.0

PCTS: 151.1 Version: 1.1 - 05/21/92

APTL: 0345 UniSoft Corporation Date lssued: 10/05/92

Reference File *: SUN4529

Product Supplier: SunSoft, Inc.

Product Tested: Solaris Version: 1.1 Version C Release: August 13, 1993

System Supplier: Sun Microsystems Computer Corporation, Inc. System Hardware: SPARCclassic Model: 4/15

C Compiler: Solaris C Compiler Version: 1.1 Rolease: August 13, 1993

PCTS: $151-1$ Version: 1.1 - 05/21/92

APTL: 0342 Mindcraft, Inc. Date ksued: 10/14/93

Reference File *: SUN5382

Product Supplier: SunSoft, Inc.

Product Tested: Solaris Version: 1.0.1 Release: PC

System Supplier: Sun Microsystems Computer Corporation, Inc.

System Hardware: SPARCstation IPX Model: GX

C Compiler: Solaris C Compiler Version: 1.0.1 Release: December 4, 1991

PCTS: $151-1$ Version: $1.1 \cdot 05 / 21 / 92$

APTL: 0343 DataFocus incorporated Date issued: 09/02/92

Reference File *: SUN5684

Product Supplier: Sun Microsystems Computer Corporation, Inc.

Product Tested: Solaris Version: 2.1 Release: December 7, 1992

System Supplier: Sun Microsystems Computer Corporation, Inc. System Hardware: SPARCclassic Model: 4/15

C Compiler: Sun C Compiler Version: 2.0.1 Release: October 3, 1992

PCTS: 151-1 Version: $1.1 \cdot 05 / 21 / 92$

APTL: 0342 Minderaft, lnc. Date kssued: 1/07/93

Reference File \#: SUN5782

Product Supplier: Sun Microsystems Computer Corporation, inc. Product Tested: Solaris Version: 2.1 Release: August 4, 1992

System Supplier: Sun Microsystems Computer Corporation, Inc. System Hardware: SPARCserver 10 Model: 30

C Compiler: Sun C Compiler Version: 2.0 Release: June 30, 1992

PCTS: 151-1 Version: 1.1 - 05/21/92

APTL: 0342 Mindcraft, Inc. Date Issued: 10/08/92

Reference File *: SUUN970

Product Supplier: Sun Microsystems Computer Corporation, Inc. Product Tested: Solaris Version: 2.1 Release: August 4, 1992

System Supplier: Sun Microsystems Computer Corporation, Inc. System Hardware: SPARCserver 10 Model: 41

C Compiler: Sun C Compiler Version: 2.0 Release: June 30, 1992

PCTS: 151.1 Version: $1.1 \cdot 05 / 21 / 92$

APTL: 0342 Mindcraft, Inc. Date lssued: 10/08/92 
Reference File *: SUN6602

Product Supplier: Sun Microsystems Computer Corporation, Inc. Product Tested: Solaris Version: 2.2 Release: May 28, 1993

System Supplier: Sun Microsystems Computer Corporation, Inc. System Hardware: SPARCenter 2000 Model: 01

C Compiler: Sun C Compiler Version: 2.0.1 Release: October 3, 1992

PCTS: 151-1 Version: 1.1 - 05/21/92

APTL: 0342 Mindcraft, Inc. Date Issued: 05/28/93

Reference File \#: SUN6635

Product Supplier: SunSoft, Inc.

Product Tested: Solaris Version: 1.0.1 Release: PC

System Supplier: Sun Microsystems Computer Corporation, Inc.

System Hardware: SPARCserver 690 Model: 140

C Compiler: Solaris C Compiler Version: 1.0.1 Release: December 4, 1991

PCTS: 151-1 Version: 1.1 - 01/22/92

APTL: 0342 Mindcraft, tnc. Date lssued: 02/19/92

Reference File *: SUN6859

Product Supplier: SunSoft, Inc.

Product Tested: INTERACTIE UNIX Operating System V/386

Version: 4.0 Release: 3.2

System Supplier: Compaq Computer Corporation

System Hardware: DeskPro Model: 66M

C Compiler: INTERACTIVE Software Development System Version: 4.0 Rolease: May 1993

PCTS: 151-1 Version: 1.1 - 05/21/92

APTL: 0342 Mindcraft, inc. Date lssued: $07 / 15 / 93$

Reference File *: SUN7188

Product Supplier: Sun Microsystems Computer Corporation, inc.

Product Tested: Solaris Version: 1.1 Release: August 24, 1992

System Supplier: Sun Microsystems Computer Corporation, Inc.

System Hardware: SPARCstation 10 Model: GX-30

C Compiler: Solaris C Compiler Version: 1.1 Release: August 24, 1992

PCTS: 151-1 Version: 1.1 - 05/21/92

APTL: 0342 Mindcraft, inc. Date issued: 08/27/92

Reference File *: SUN7793

Product Supplier: Sun Microsystems Computer Corporation, Inc. Product Tested: Solaris Version: 2.1 Release: August 4, 1992

System Supplier: Sun Microsystems Computer Corporation, Inc. System Hardware: SPARCserver 10 Model: 42

C Compiler: Sun C Compiler Version: 2.0 Release: June 30, 1992

PCTS: 151-1 Version: 1.1 - 05/21/92

APTL: 0342 Mindcraft, Inc. Date lssued: 10/08/92

Reference File *: SUN8720

Product Supplier: SunSoft, Inc.

Product Tested: Solaris Version: 1.1 Version C Release: August 13, 1993

System Supplier: Sun Microsystems Computer Corporation, Inc. System Hardware: SPARCstation Model: 4/30

C Compiler: Solaris C Compiler Version: 1.1 Release: Aug 13, 1993

PCTS: 151-1 Version: 1.1 - 05/21/92

APTL: 0342 Mindcraft, Inc. Date Issued: 10/14/93

Reference File \#: SUN9763

Product Supplier: SunSoft, Inc.

Product Tested: Solaris Version: 1.0.1 Release: PC

System Supplier: Sun Microsystems Computer Corporation, Inc. System Hardware: SPARCstation 2 Model: GX

C Compiler: Solaris C Compiler Version: 1.0.1 Release: Dec 4, 1991

PCTS: 151-1 Version: 1.1 - 01/22/92

APTL: 0342 Mindcraft, inc. Date kssued: 02/19/92
Reference File \#: UN10505

Product Supplier: Unisys Corporation

Product Tested: UNIX System V Rolease 4 Version: Revision 1.0.2

System Supplier: Unisys Corporation

System Hardware: Unisys U 6000 Series Model: U 6000/15

C Compiler: UNIX System V Release 4 Standard C Development

Environment Version: 1.0.2

PCTS: 151-1 Version: 1.1 - 01/22/92

APTL: 0342 Mindcraft, Inc. Date lssued: 04/30/92

Reference File *: UNI1798

Product Supplier: Unisys Corporation

Product Tested: UNIX System V Release 4 Version: Revision 1.0.2

System Supplier: Unisys Corporation

System Hardware: Unisys U 6000 Series Model: U 6000/65

C Compiler: UNIX System V Release 4 Standard C Development

Environment Version: 1.0.2

PCTS: 151-1 Version: 1.1 - 01/22/92

APTL: 0342 Mindcraft, Inc. Date Issued: 05/12/92

Reference File *: UNI3690

Product Supplier: Unisys Corporation

Product Tested: UNIX System V Release 4 Version: 1.1 Release: October 30, 1992

System Supplier: Unisys Corporation

System Hardware: Unisys U 6000 Series Model: U6000/65

C Compiler: UNIX System V Release 4 Standard C Development

Environment Version: 1.1

PCTS: 151-1 Version: $1.1-05 / 21 / 92$

APTL: 0342 Mindcraft, Inc. Date lssued: 09/28/92

Reference File *: UN15711

Product Supplier: Unisys Corporation

Product Tested: UNIX System V Release 4 Version: Revision 1.0.2

System Supplier: Unisys Corporation

System Hardware: Unisys U 6000 Series Model: U 6000/60

C Compiler: UNIX System V Release 4 Standard C Development Environment Version: 1.0.2

PCTS: 151-1 Version: 1.1 - 01/22/92

APTL: 0342 Mindcraft, Inc. Date lssued: 05/12/92

Reference File *: UNI9063

Product Supplier: Unisys Corporation

Product Tested: UNIX System V Release 4 Version: Rovision 1.0.2

System Supplier: Unisys Corporation

System Hardware: Unisys U 6000 Series Model: U 6000/35

C Compiler: UNIX System V Release 4 Standard C Development

Environment Version: 1.0.2

PCTS: 151-1 Version: 1.1 - 01/22/92

APTL: 0342 Mindcraft, Inc. Date Issued: 05/12/92

Reference File *: UNI9080

Product Supplier: Unisys Corporation

Product Tested: CTOS II Version: 3 Release: 3

System Supplier: Unisys Corporation

System Hardware: Unisys B-Series Model: NGEN

C Compiler: Microsoft C Version: 6.0

PCTS: 151-1 Version: 1.1 - 07/01/91

APTL: 0343 DataFocus Incorporated Date Issued: $09 / 17 / 91$ 
Reference File \#: UNVO528

Product Supplier: Univel Product Tested: UnixWare Version: 1.0 Release: June 1993

System Supplier: Unisys Corporation

System Hardware: Unisys U 6000/DT Series/PW 2 Advantage Plus Series Model: U6000/DT1 (MPE 4332)

C Compiler: Optimizing C Compilation Sys Ver: 2.0 Rel: Nov 2, 1992 PCTS: 151-1 Version: 1.1 - 05/21/92

APTL: 0342 Mindcraft, Inc. Date lssued: 05/18/93

Reference File \#: UNV2014

Product Supplier: Univel

Product Tested: UnixWare Version: 1.0 Release: June 1993

System Supplier: Unisys Corporation

System Hardware: Unisys U 6000/DT Series/PW 2 Advantage Plus Series Model: U6000/DT2 (MPE 4663)

C Compiler: Optimizing C Compilation System Version: 2.0 Release: Nov. 2, 1992

PCTS: $151-1$ Version: $1.1 \cdot 05 / 21 / 92$

APTL: 0342 Mindcraft, Inc. Date issued: 05/18/93

Reference File *: UNV3055

Product Supplier: Univel

Product Tested: UnixWare Application Server Version: 1.0

Release: October 1992

System Supplier: AST Research, Inc.

System Hardware: Premium 486/33 Model: 3V

C Compiler: UnixWare Software Development Kit Version: 1.0

Release: October 1992

PCTS: $151-1$ Version: $1.1 \cdot 05 / 21 / 92$

APTL: 0342 Mindcraft, Inc. Date Issued: 10/08/93

Reference File *: UNV3978

Product Supplier: Univel

Product Tested: UnixWare Version: 1.0 Release: June 1993

System Supplier: Unisys Corporation

System Hardware: Unisys PW2 Advantage Series Model: MPI 4336)

C Compiler: Optimizing C Compilation System Version: 2.0 Release: Nov. 2, 1992

PCTS: $151-1$ Version: $1.1 \cdot 05 / 21 / 92$

APTL: 0342 Mindcraft, Inc. Date issued: 05/18/93

Reference File \#: UNV9180

Product Supplier: Univel

Product Tested: UnixWare Personal Edition Version: 1.0 Release: October 1992

System Supplier: AST Research, Inc.

System Hardware: Premium 486/33 Model: $3 V$

C Compiler: UnixWare Software Development Kit Version: 1.0

Release: October 1992

PCTS: 151.1 Version: $1.1 \cdot 05 / 21 / 92$

APTL: 0342 Mindcraft, Inc. Date lssued: 10/08/93

Reference File *: USL2115

Product Supplier: UNIX System Laboratories, Inc.

Product Tested: UNIX System V Release 4 Version: 4 Rolease: 4.0

System Supplier: AST Research, Inc.

System Hardware: Premium Series Model: 486/33

C Compiler: Standard C Development Environment Version: 5.0

PCTS: $151-1$ Version: $1.1 \cdot 05 / 21 / 92$

APTL: 0343 DataFocus, Inc. Date Issued: 07/01/92
Reference File *: USL3610

Product Supplier: UNIX System Laboratories, Inc.

Product Tested: UNIX System V Release 4 for the Intel386"

Architecture Version: 4

Rolease: July 1991

System Supplier: AT\&T

System Hardware: AT\&T 6386/25 WGS Model: CPU 311 PC3B

C Compiler: Standard C Development Environment Version: issue 5

PCTS: $151-1$ Version: $1.1-09 / 11 / 91$

APTL: 0342 Mindcraft, inc. Date issued: 12/12/91

Reference File *: USL6259

Product Supplier: UNIX System Laboratories, Inc.

Product Tested: UNIX System V/386 Rolease 4 Version: 4.0T

Rolease: August 1992, with PATCH \#1 (Package Date: 11/20/92)

System Supplier: AST Research, Inc.

System Hardware: Premium 486/33 Model: 3V

C Compiler: UNIX System Laboratories Standard C Development

Environment Version: tssue 5

PCTS: 151-1 Version: 1.1 - 05/21/92

APTL: 0342 Minderaft, Inc. Date lssued: 2/12/93 


\subsection{TESTING LABORATORIES AND VALIDATED PRODUCTS for NIST POSIX (FIPS 151-2)}

\section{ACCREDIED NIST POSX TESTING LABORATORIES}

The National Voluntary Laboratory Accreditation Program (NVAP) has accredited the following laboratories to test computer operating system interfaces for conformance with the Federal information Processing Standard 151-2 (FIPS 151-2) using the NIST POSIXConformance Test Suite (NIST. PCTS:151-2). Only accredited laboratories may submit test reports to NIST/CSL for validation.

\section{Datafocus hcorporated}

12450 Fair Lakes Circle, Suite 400

Fairfax, VA 22033-3831

Nindcraft, he.

410 Cambridge Avenue

Palo Alto, CA 94306

\section{NIST POSX VALIDATED PRODUCTS}

The following products have been tested by an Accredited POSIX Testing Laboratory (APTL) using the official National Institute of Standards and Technology POSIX Conformance Test Suite (NIST-PCTS:151-2) for the Federal Information Processing Standards 151-2 (FIPS PUB 151-2). A Certificate of Validation has been issued by NIST/CSL. Additional Information Is available from NIST/CSL on conditional features supported, configuration details, and resolved test codes (if appropriate).

Information In this listing Includes product information on the Implementation and system tested and information on the type of implementation. FIPS 151-2 supports three types of implementations, native, hosted, and cooperating. A native implementation "refers to an Implementation of POSIX 1 that interfaces directly to an operating system kernel." A Cooperating implementation "refers to an implementation of POSIX.1 that interfaces directly to an operating system kemel but the load modules are not produceable on thls implementation." A hosted implementation "referes to an Implementation of POSIX.1 that is accomplished through interfaces from the POSIX.1 services to some altemate form of operating system kemel services."

Information Is also provided on the following primary conditional features:

General Terminal Interface devices (GTI),

Mountable File System (MFS),

Modem Control (MC), and

Apppropriate Privileges (AP).

\section{PROODUCT SUPPUERS}

Digital Equipment Corporation

Unisys Corporation

\section{SYSTEM SUPPUERS}

Digital Equipment Corporation

Unisys Corporation

\author{
PEFERENCE FIL : \\ 151-2DECO01 \\ 151-2UN1001, 151-2UN1002 \\ REFERENCE FIE : \\ 151-2DECO01 \\ 151-2UN1001, 151-2UN1002
}




\section{PRODUCTS}

151-2DECOO1 ksued: 08/12/93 Type: Hosted

Product Supplier: Digital Equipment Corporation

Product: POSX for Open VMS NXP Version X1.0-041

PCD: POSIX 1003.1-1990 Conformance Document for Open VMS AXP (July 1993)

GTI - NOT Provided by Product MC - NOT Provided by Product

MFS - Supported by Product AP - Supported by Product

Computer Hardware Supplier: Digital Equipment Corporation

Computer Hardware Product: DECsystem, Model 4000/610

Host Operating System Supplier: Digital Equipment Corporation

Host Operating System: OpenVMS AXP Version 1.5

C Compiler: DEC C Version 1, Release 3

APTL: 0343 DataFocus Incorporated

151-2UN1001 lssued: 12/02/93 Type: Native

Product Supplier: Unisys Corporation

Product: Unix System V Rolease 4 Rovision 12

PCD: UNIX System V Release 4.0 POSIX Conformance Programmer's Guide

GTI - Supportod by Product

MFS - Supported by Product

MC - Supported by Product

Computer Hardware Supplier: Unisys Corporation

AP - Supported by Product

Computer Hardware Product: Unisys U6000 Series U6000/65

C Compller: Unix System V Release 4 Standard C Development Environment Rev. 1.2

APTL: 0342 Mindcraft, Inc.

151-2UN1002 Issued: 12/02/93 Type: Native

Product Supplier: Unisys Corporation

Product: Unix System V Rolease 4 Rovision 12

PCD: UNIX System V Release 4.0 POSIX Conformance Programmer's Guide

GTI - Supported by Product

MFS - Supported by Product

MC - Supported by Product

Computer Hardware Supplier: Unisys Corporation

AP - Supported by Product

Computer Hardware Product: Unisys U6000 Series U6000/300

C Compiler: Unix System V Release 4 Standard C Development Environment Rov. 1.2

APTL: 0342 Mindcraft, Inc.

For further information on the NIST/CSL POSIX validation program contact Martha M. Gray, Computer Systems Laboratory, B266 Technology Bldg., NIST, Gaithersburg, MD 20899. Telephone: 301-975-3276, fax: 301-590-0932, e-mail: gray@swe.ncsl.nist.gov.

This register is also available on an electronic mail (email) file server system. To use the service, you must be able to send and receive email via the Internet. For most email systems, send an email message (mail posix@nist.gov) with the first line of the message containing a command to send 151.1reg and a carriage return. The next line should simply end your email message (on some systems a period and a carriage return). This register will be returned via email to your email address. There is also a register for FIPS 151-2 accredited laboratories and validated products. For this register use the command send 151-2reg. 



\section{COMPUTER SECURITY TESTING}

\subsection{Cryptographic Standards}

The lists in Sections 8.6, 8.7 and 8.8 provide technical information about products that have been validated as conforming to the following computer security FIPS:
a. Data Encryption Standard (DES), FIPS PUB 46-1,
b. Message Authentication Code (MAC), FIPS PUB 113, and
c. Key Management Using ANSI X9.17, FIPS PUB 171.

\subsection{Data Encryption Validation Tests}

FIPS PUB 46-1 specifies a cryptographic algorithm that converts plaintext to ciphertext using a 56bit key. Testing procedures for the validation of devices as conforming to FIPS PUB 46-1 are described in the NBS Special Publication 500-20, Validating the Correctness of Hardware Implementations of the NBS Data Encryption Standard. The validation of a device is performed by running the Monte Carlo test described in the publication. The Monte-Carlo test consists of eight million encryptions and four million decryptions, with two encryptions and one decryption making up a single test. The test is designed to use the Electronic Codebook Mode (ECB) of DES. Although the actual test described in NBS Special Publication 500-20 is the same test used to validate devices today, the procedures for administering the test have changed. Currently, the test is performed by the vendor using initial values supplied by NIST. The vendor uses the supplied information to run the Monte-Carlo test and sends the results to NIST.

\subsection{Message Authentication Code (MAC) Validation System}

FIPS PUB 113, Computer Data Authentication, specifies a Data Encryption Algorithm which may be used to detect unauthorized intentional and accidental modifications to data. This process is known as data authentication. The algorithm is based on DES and is used to authenticate an entire binary message. FIPS PUB 113 is compatible with ANSI X9.9 which provides methods for authenticating an entire binary message as well as all or parts of a message which are in a coded character format. Procedures for the validation of products which implement FIPS PUB 113 and ANSI X9.9 are described in NBS Special Publication 500-156, Message Authentication Code (MAC) Validation System: Requirements and Procedures.

\subsection{Key Management Validation System (KMVS)}

FIPS PUB 171 adopts ANSI X9.17 for Federal Government use. ANSI X9.17, Financial Institution Key Management (Wholesale), provides procedures and protocols for the secure generation, distribution, storage, entry, use and destruction of symmetric cryptographic keying material (e.g., DES). It provides key management solutions for a variety of operational environments, and as such, ANSI X9.17 contains a number of options. FIPS PUB 171 specifies a particular set of options whenever keying material is distributed using the protocols of ANSI X9.17. Procedures for the validation of products which conform to a subset of the options selected in FIPS PUB 171 are described in the Key Management Validation System: Point-to-Point Validation System document which is available from the Manager of the Security Group (see Section 8.5). 


\subsection{General}

\subsubsection{Request for Validation}

To validate a product, a vendor should send a formal request for validation which includes a clear indication of the product to be tested. The request must also include the name, address, and telephone number of the person within the vendor's organization who will be responsible for the validation testing. The request should be sent to:

Manager, Security Technology Group

Computer Security Division

Computer Systems Laboratory

Building 225, Room A216

National Institute of Standards and Technology

Gaithersburg, MD 20899

Telephone (301) 975-2920

\subsubsection{Information about Validated Products}

It should be noted that the purpose of the following lists (see Sections 7.6, 7.7 and 7.8) is to provide technical information about products that have been validated as conforming to the FIPS Standards listed in Section 7.1. NIST has made every attempt to provide complete and accurate information about the products described in the following lists. However, due to the possibility of changes made within individual companies, NIST cannot guarantee that this document reflects the current status of each product.

\subsubsection{Validation Documentation}

Copies of the above FIPS and Special Publications are for sale by the National Technical Information Service, U.S. Department of Commerce, Springfield, VA 22161. The KMVS validation requirements document discussed in Section 7.4 can be obtained by contacting the Manager of the Security Technology Group at the above address. 


\subsection{DES Validated Devices}

NOTE: The purpose of this document is to provide technical information about devices that have been validated as conforming to Federal Information Processing Standard Publication 46-1, Data Encryption Standard. The National Institute of Standards and Technology (NIST) has made every attempt to provide complete and accurate information about the devices described in this document. However, due to the possibility of changes made within individual companies, NIST cannot guarantee that this document reflects the current status of each product.

\begin{tabular}{ccccc}
\hline $\begin{array}{c}\text { MANUFACTURER } \\
\text { ADDRESS }\end{array}$ & $\begin{array}{c}\text { DEVICE } \\
\text { ID }\end{array}$ & PRODUCT & $\begin{array}{c}\text { VALIDATION } \\
\text { DATE }\end{array}$ & DESCRIPTION \\
\hline
\end{tabular}

\section{ADT Security Systems} 2560 Huntington Avenue

Fourth Floor

Alexandria, VA 22303

Hal Marriott

(703) $960-8548$

Advanced Micro Devices, inc. 4115 Freiderich Lane

Mail Stop 135

Austin, TX 78744

Patrick Soheili
(408) $749-2161$

ADT Universal Communicator

$7187-009$ Universal

$10 / 17 / 90$

Subsystem and

2995 Universal

Subsystem

\section{AmZ8068}

8068DC; 8068DCB; 8068JC; 8068PC;

$1 / 28 / 81$

\section{AM 9568}

9568DC; 9568DCB; $2 / 28 / 84$ 95681; 9568DMB; 9568JC; 9568PC;

AT\&T Smart Card Version 2.11/DES

Computer Security $5 / 3 / 91$ System Tolegraph Company (AT\&T) 6612 E. 75th Street

P.O. Box 1008 Indianapolis, IN 46206

Ken Zompol

(908) 658-6870
AT\&T Smart Card Version 3.0/DES (5E1)
Computer Security $7 / 19 / 91$ System
Chip is an on board component for Communicator products in the High Security Intrusion Detection System. System has integrated key management capabilities.
One 40-pin DIP package; n-channel Si-gate tochnology; ECB, CBC and 8-bit CFB modes; separate ports for key input, clear data and enciphered data; concurrent input, output and ciphering activities; extemal DMA control; interfaces with AmZ8000 CPU bus directly, and with the 2000 , 8080,8085 and 8048 families with minimum throughput greater than 1 Mbytes per second; greater than 1 Mbytes per second.

N-channel silicon gate LSI product containing the circuitry necessary to encrypt and decrypt data; can be used in terminals dedicated controllers, communication concentrators, and peripheral task processors in general processor systems; can be used in CF, ECB, or CBC operating modes; separate ports for key input, clear data, and enciphered data enhanced security; interface directly to the LAPX86, 88 bus; interfaces with 2900 and 8051 families with minimal extemal logic.

Card is part of a smart card based Computer Security System (CSS). The card is carried by an authorized user and permits the user to gain access to host computer systems that are protected by the CSS.

This version of the AT\&T Smart Card is designed to closely follow developments in the intemational standards arena in areas of card communication protocols, commands and file structures. It is a general purpose smart card that supports multiple applications and uses the DES as a basic part of its operating system.

Provides data encryption for messages sent and received on-line between and ATM/EFT Network switch processor and an IBM host participant in that network. DES key management is automatic and under system control.

Manufactured using CMOS technology; 40-pin DIP; oncryption modes include ECB, CBC, CFB, and OFB; throughput 1.882 Mbytes/second on-chip RAM and ROM program memory. 


\section{DES Validated Devices, Continued}

\begin{tabular}{ccccc}
\hline $\begin{array}{c}\text { MANUFACTURER } \\
\text { ADDRESS }\end{array}$ & DEVICE & PRODUCT & $\begin{array}{c}\text { VALIDATION } \\
\text { DATE }\end{array}$ & DESCRIPTION \\
\hline
\end{tabular}

AT\&T Bell Laboratories

25 Lindsley Drive

Room 2B-309

Morristown, N.J. 07960

William Oeschger

(201) 898-1198

\section{American Telephone}

and Telegraph Company

AT\&T Guilford Conter

L85 and Mt Hope Church Road

McLeansville, NC 27420

Mr. B. F. Bailey

(919) $279-3779$

American Telephone

and Telegraph Company

AT\&T Guilford Center

L-85 and Mt Hope Church Road

McLoansville, NC 27420

Mr. M. Zugay

(919) 279-3779

Chase Manhattan Bank, N.A. 199 Water Street 12th Floor

New York, NY 10081

Robert Stevenson

(212) $797-4153$

Collins Telecommunications

Collins Defense Communications

350 Collins Road, NE

Mail Stop 120-105

Codar Rapids, lowa 52498

Jim Perkins

(319) 395-5773

Computer Elektronik Infosys

of America, Inc.

512-A Herndon Parkway

Hemdon, VA 22070

A. Mark Brown

(703) $435-3800$

Cylink Corporation

110 South Wolfe Road

Sunnyvale, CA 94086

Les Nightingill

(408) $735-5800$

Datakey inc.

407 West Travelers Trail

Bumsville, MN 55337-9990

Michael Carenzo

(612) $890-6850$

$\begin{array}{ll}\text { DEP229ER } & \text { Not Commercially } \\ \text { (ME220ER) } & \text { Available }\end{array}$

(WE220ER) Available

AT\&T Mark E DES Not Available

Key Generator, PN

ON493049-1X

\section{AT\&T Mark ET \\ DES Key \\ Generator, Part}

No. AN10014-1

Not Available

$6 / 3 / 92$

3.5 micron NMOS tochnology; 40-pin DIP; encryption modes - ECB, CBC, OFB, CFB1, CFB8, CFB64; Throughput rate of $117 \mathrm{~K}$ ciphering operation/second.

$6 / 3 / 92$

Not available

Chase Encryption Not Available Device 1

$7 / 24 / 84$

Not Available

pMOS chip with 40 usec algorithm execution time; chip has approximately a 50 nsec state change; can perform I/O functions while the chip is in operation: part of network stand-alone encryptor.

Voice Privacy Device Only 10/6/81

Device VP430

Chip and Design $\quad 7 / 24 / 91$

SuperCrypt

Kit

Imbedded encryption device for commercial hand held communications devices.

Chip designed for high speed (12 Megabytes/sec data rates) encryption and decryption. ECB, CBC, CFB and OFB modes of DES supported as well as MAC generation. Available as a 120 Pin Flat Pack.

CryptCard CryptCard $\quad 1 / 12 / 93$

$\begin{array}{lll}\text { CY1045 Chip Only } & \text { 1/28/87 }\end{array}$

CryptCard is an access control and DES encryption adapter for notebook PCs that have a PCMCLA slot.

Not Available

Cylink Faxdes

Device Only

$7 / 1 / 87$

Not Available

12035-001,

Device Only

$6 / 3 / 92$

Not Available

12422-001, DES2M1CFB

Device Only

$8 / 27 / 92$

Not Available

HB-310 ASACS

HB-310 ASACS

$7 / 2 / 92$

Smart Card

The ASACS hardware consists of a credit-card sized smart card with an embedded Hitachi H8/310 microprocessor and a reader/writer interface which provides an RS-232 serial connection to a host computer. The smart card functions are implemented in firmware which is stored in the memory of the card's microprocessor. 
DES Validated Devices, Continued

\begin{tabular}{|c|c|c|c|c|}
\hline $\begin{array}{c}\text { MANUFACTURER } \\
\text { ADDRESS }\end{array}$ & $\begin{array}{c}\text { DEVICE } \\
\text { ID }\end{array}$ & PRODUCT & $\begin{array}{c}\text { VALIDATION } \\
\text { DATE }\end{array}$ & DESCRIPTION \\
\hline
\end{tabular}

Docutel/Olivetti Corporation 106 Decker Court Suite 300

inving, TX 75062

Division Intemational Marketing

(214) $550-5400$

\section{The Exchange \\ 15395 SE 30th Place \\ Bellevue, WA 98007 \\ Patricia Lenti-Crane \\ (206)644-7000}

Front Line Software

P.O. Box 217

Lowell, MA 01853

William Graham

(617) $452-3352$

GEMPLUS CARD INTERNATIONAL

6290 Montrose Road

Rockville, MD 20852

Gilles Lisimaque

(301) $770-1558$

General Electric Company

Mountain View Road

Lynchburg, VA 24502

Jim Eder

(804) 948-6187

Glenco Engineering, Inc.

270 LexIngton Drive

Buffalo Grove, IL 600896930

D. Wade Clark

(708) 808-0300

IBM Corporation

Foderal Systems Division

P.O. Box 100

Kingston, NY 12401

Robert Elander

(914) 385-6692

IBM Corporation

1001 W.T. Harris Blvd. West

Charlotte, NC 28257

William Rohland

(704) $594-8250$

$\begin{array}{ll}\text { Docutel Nordisk } & \text { Total Teller } \\ \text { Spardata Cash } & 2380 ; \text {;TS 5100; } \\ \text { Dispensing } & \\ \text { Terminal } & \end{array}$

$6 / 20 / 82$

Spardata Cash

Torminal

EXCRYPT
DEB-64-KM
(originally
EXCLUDE DEB-64
KM)

\section{6-8064 PROM Chip Only 12/1/86}

Device

Device Only $\quad 1 / 26 / 89$

$12 / 1 / 80$

\section{MCOS16K Card Only}

$3 / 18 / 91$

EEPROM/DES

Part Number $19 B 801375$

Not Available

$6 / 28 / 85$

Glen-DES PN

GL306051

Glen-DES PN
GL306051

$5 / 8 / 92$

4402182 WK4/988 Chip Only

$11 / 1 / 77$

P/N 8270094

using DES Chip

P/N 5898057

(originally

8269206)

Two TTL cards . 8632242 and 8679176

4745 Security Not Available

$8 / 25 / 78$

Interface Unit and

the Personal

Security Card
3846-Link

Encryption;3848-

Cyptographic Unit;

IBM 4753 Notwork

Security Processor

$10 / 10 / 90$

$9 / 21 / 79$

Firmware implementation of DES in ROM for PIN/communications security.

Encrypts and decrypts data; generates random keys; supports up to six security processor boards that can be run in parallel to enhance throughput; has storage capacity for up to $\mathbf{4 0 0 0}$ DES keys; developed for secure financial transactions.

4 K EPROM to be used with Intel IPAX family of microprocessors including all models of the IBM PC family; all modes of DES supported.

A multi-application smart card which complies with the ISO standard 7816 (parts 1, 2, and 3) for Integrated Circuit cards with contacts.

The GE DES IC is a microprocessor controlled, low speed asynchronous CMOS IC using DES. Intended to provide secure voice in commercial grade mobile radio applications.

The Glen-DES is a compact 20 pin design, using low power CMOS technology, operating at 3us using a $16 \mathrm{MHz}$ clock. The DES chip features nonvolatile intemal memory, an extemal key and a combined key. It is available with a simple CPU interface and it supports a DOS printer port implementation.

This card used in terminal equipment; the chip uses technology with PLA control to implement CBC;

This card is used in 3845 and 3846 equlpment for 8bit CFB.

Will operate at least at the 1.5 Mbytes 360 channel rate; card set is used in the 3848 cryptographic unit; uses "Emerald-5" technology.

Devices are used in a transaction security system to protect the privacy and integrity of data using a common cryptographic interface. The security interface unit communicates with the Personal Security Card and the cryptographic adaptor, if present. The Personal Security Card is an integrated-circuit chip card that contains a single chip security processor. 


\section{DES Validated Devices, Continued}

\begin{tabular}{|c|c|c|c|c|}
\hline $\begin{array}{l}\text { MANUFACTURER } \\
\text { ADDRESS } \\
\end{array}$ & $\begin{array}{l}\text { DEVICE } \\
\text { ID }\end{array}$ & PRODUCT & $\begin{array}{c}\text { VALIDATION } \\
\text { DATE } \\
\end{array}$ & DESCRIPTIOYN \\
\hline $\begin{array}{l}\text { Intel } \\
1900 \text { Prairie City Road } \\
\text { Folsom, CA } 95630 \\
\text { Joe Dragony } \\
\text { (916) } 351-5250\end{array}$ & $8294 A$ & $\begin{array}{l}8294 \text { chip; } 8294 A \\
\text { chip; }\end{array}$ & $6 / 20 / 82$ & $\begin{array}{l}\text { Agorithm is microcode which is bumed into a } 1 \\
\text { Kbyte ROM on a } 5 \text { volt, } 40 \text {-pin chip driven by a } 8042 \\
\text { microprocessor. } \\
\text { Same as the } 8294 \text { except for a maximum data } \\
\text { transfer rate of } 400 \text { bytes per second. }\end{array}$ \\
\hline $\begin{array}{l}\text { John E. Holt \& Associates } \\
2714 \text { Key Boulevard } \\
\text { Arlington, VA } 22201 \\
\text { John Holt } \\
\text { (703) } 524-2923\end{array}$ & Krypton Firmware & $\begin{array}{l}\text { Expansion board } \\
\text { available in two } \\
\text { models; }\end{array}$ & $2 / 12 / 86$ & $\begin{array}{l}\text { ROM chips for the standard IBM PC family include } \\
\text { eight } 3722 \text { chips, four } 2764 \text { chips and one } 27256 \\
\text { chip; 1024-bit CBC chaining; encryption speed } \\
\text { dependent on clock of PC; ROM can plug directly } \\
\text { into ROM slot. }\end{array}$ \\
\hline $\begin{array}{l}\text { Lexicon } \\
\text { ICOT Corporation } \\
3801 \text { Zanker Road } \\
\text { P.O. Box } 5143 \\
\text { San Jose, CA } 95150-5143\end{array}$ & $\begin{array}{l}\text { LEX-POS (Model } \\
600 \text { ) }\end{array}$ & Device Only & $11 / 28 / 84$ & $\begin{array}{l}\text { A Personal Identification Number (PIN) entry device; } \\
\text { used in conjunction with financial transaction } \\
\text { devices, } 16 \text { key keyboard, } 20 \text { character display, RS- } \\
232 \text { compatible, Lexicon sold LEX-POS to ICOT } \\
\text { Corporation. }\end{array}$ \\
\hline
\end{tabular}

Bob Lynch

(408) $433-3300$

\author{
LSI Logic/Dataco AS \\ Smedeholm 12-14 \\ DK-2730 Herlev \\ Denmark \\ Jens Kjelsbak \\ 4544530100
}

Matsushita Electronic Components High Frequency Products Division

One Pansonic Way

Socaucus, NJ 07094

Dursun Sakarya

(201) 348-7767

Micro Card Technologies, Inc.

14070 Proton Road

Dallas, TX 75244

Jeff Lang

(214) $788-4055$

Morse Security Group, inc.

12960 Bradiey Avenue

Sylmar, CA 91342-0128

Nalin Chheda

(800) 423-5669 (818) 367-5951

\section{Motorola}

Microprocessor Products Division

6501 William Cannon Drive West

Austin, TX 78735-8598

Don Ponder

(512) 440-2956

Nowbridge Microsystems

603 March Road

Kanata, Ontario

Canada K2K 2 M5

Tony Rosati

(613) 592-0714

\section{Dataco L5A4043 2030025402}

EBC 1642 IC Card Card Only

ScaNET PC TCP/IP 1/12/90

package; Gateway;

IBM 3270 Gateway;

Terminal Server;

IBM S/3X Terminal

Server; Buffered

Repeater;

$3 / 13 / 91$

Micro Card TB100 TB100 Integrated Integrated Circuit Circuit Card Family Card

\section{TRAP 5200 System}

MC6859 (originally MC6859 Chip MGD68NE)
Touch Response

Aarm; Touch

Response Transponder;
$9 / 19 / 90$
Custom DES IC was manufacturer by LSI Logic for Dataco. The DES chip is designed for optional use in ScaNet local area network products.

Card is designed to be a high security extemal storage media housing an 8 bit CPU and $64 \mathrm{Kbit}$. EEPROM.
A multi-application integrated circuit card which can simultaneously support several application data files. Ciphering and deciphering functions may be used to encrypt or decrypt extemal messages using DES.

Touch response alarm processor system, including a receiver processor located in a data gathering center and a series of transponders located at remote locations, contains DES to produce encrypted data that flows along a communication path.

Si-gate depletion mode, nMOS 24-pin DIP using CFB. single 5 volt power supply; implements ECB and
CA20C03A
Chip Only
$4 / 10 / 91$

A high performance WD20C03A compatible DES Data encryption processor with data transfer rates up to 4 Mbytes per second. Supports ECB and CBC; PLCC and PDIP packaging available. 


\begin{tabular}{|c|c|c|c|c|}
\hline $\begin{array}{l}\text { MANUFACTURER } \\
\text { ADDRESS }\end{array}$ & $\begin{array}{l}\text { DEVICE } \\
\text { ID }\end{array}$ & PRODUCT & $\begin{array}{c}\text { VALIDATION } \\
\text { DATE }\end{array}$ & DESCRIPTION \\
\hline
\end{tabular}

\author{
Nownet S.A. \\ Alsina $\mathbf{4 3 0}$ \\ Buenos Aires 1087 \\ Argentina \\ Daniel Ramos \\ 5413349732
}

Nixdorf Computer Corporation

168 Middlesex Tumpike

Burlington, MA 01803

Kevin Madden

(617) $890-3600$

Racal-Milgo

P.O. Box 407044

Ft. Lauderdale, FL 33340-7044

Richard Abbruscato

(305) $476-6800$

Rothenbuhler Engineering

P.O. Box 708

2191 Rhodes Road

Sedro Wolley, WA $98284-0708$

Andrew Benson

(206) $856-0836$

Socur-Data Systems, Inc.

Omega Conter

7340 Executive Way, Suite R

Frederick, MD 21701

Ronald Baum

(301) 68-9955

Texas instruments, inc.

P.O. Box 1443, M/S 736

Houston, TX 77001

Mike Polen

(713) 2743635

UNNAC

P.O. Box 3942

St. Paul, MN 55165

Jim Nolson

(612) $631-6728$

VSI Technology, Inc.

8375 S. River Parkway

Tompe, AZ 85284

R. Slusarczyk

(602) $752-8574$
Data Security Chip Only $7 / 2 / 91$

Device (DSD 9612)

VEM Module

Not Available

$1 / 7 / 80$

Datacryptor

Datacryptor II; $\quad 1 / 7 / 80$

Datacryptor III; Dial-

up Datacryptor II;

Datacryptor II

Model 1027

Standard 1027

CLS Series 5200 CLS Serles 5200

Encryption Module Polling System

$3 / 19 / 91$

DESPLEX

TM5; MP5

$2 / 2 / 89$

TMS 99541

TMS 7500 Chip;

Now name for the

$2 / 28 / 82$

TMS 99521 Chip:

End-End/Mass Not Available

Storage Encryptor

$1 / 29 / 80$

Prototype device for testing purposes only

This device is based on an eight bit INTEL data at speeds of 1200 to 9600 bps and ports. in the financial community; uses $T L$

Stand alone equipment with public key a contral monitoring facility.

Preprogrammed TMS7020 8-bit single chip microprocessor with 8 Kbytes of EPROM. Transfer communicates with other devices via EIA RS-232-C

The plug-in module is used with the Nixdorf 8864 CPU for encrypting data transmission blocks and file protection; may be used in terminal applications management remote distribution of master keys.

The CLS Series 5200 Encryption Module is used in a system which communicates 8 channels of electronic security information between a client and

Used in a CF configuration as part of a firmware operating system for processing and transmission of alarm sensor data as well as receiving and annuciating dat at an alarm monitoring facility. microprocessor; 40-pin DIP plastic package I/O pins are TL compatible; master and active key registers.

\begin{tabular}{|c|c|}
\hline $\begin{array}{l}\text { VMoo7 - Data } \\
\text { Encryption } \\
\text { Processor }\end{array}$ & VMoO7 Chip \\
\hline $\begin{array}{l}\text { VMoog Data } \\
\text { Encryption } \\
\text { Processor }\end{array}$ & $\begin{array}{l}\text { VMOOOB; } \\
\text { VMOOSABO; } \\
\text { VMOOOAB6; }\end{array}$ \\
\hline
\end{tabular}

The VM007 Data Encryption Processor is a programmable integrated circuit that provides a complete cryptographic system on a single chip. It contains a hardware implementation of the DES, RISC-based sequencer, data storage registers, and ROM-based microprogram. It is designed to provide very high data and key processing rates (up to 190 Megabits per second), flexible I/O interfacing, advanced security features and supports all DES modes of operation.

WP PN 5286/WP MP40/AS- 40; $\quad 5 / 26 / 89$ PN $5287 \quad M P 44 / A S-44$;
The monitor panels are intended for use in a monitoring station of a proprietary intrusion detection alarm system.
A Unit of Baker Protective Services 1010 North Glebe Road, Suite 680 Arlington, VA 22201

William Martin

(703) $247-4250$ 
DES Validated Devices, Continued

\section{MANUFACTURER ADDRESS \\ Western Digital Corporation 2445 McCabe Way \\ Irvine, CA 92714 \\ Product Marketing Manager \\ for Security Devices \\ (714) $474-2033 \times 7853$}

DEVICE

ID

PRODUCT VALIDATION

DATE

WD-2001/WD2002 WD-2001 Chip; WD-2002 Chip;

$8 / 9 / 79$

WD20C03 DES

Device

$2 / 19 / 87$

WD-20CO3 Chip

(2)

DESCRIPTION

Uses si-gate nMOS, TL compatible; ECB speods of up to 40 Kbytes/second, 161 Kbytes/second and 242 Kbytes/second.
Uses si-gate CMOS, TL compatible; ECB and CBC, speeds of up to $403 \mathrm{Kbytes} /$ second, 645 Kbytes/second and $807 \mathrm{Kbytes/second} \mathrm{in} \mathrm{ECB.}$ 


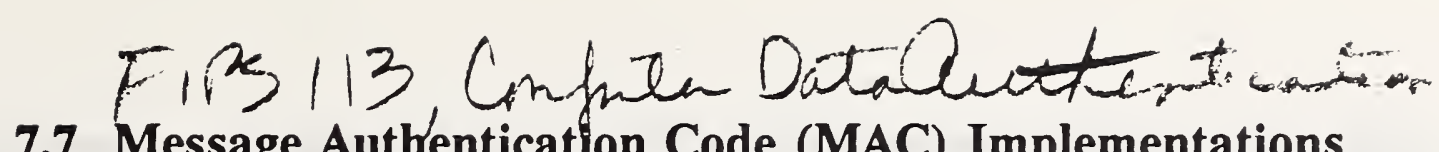 7.7 Message Authentication Code (MAC) Implementations}

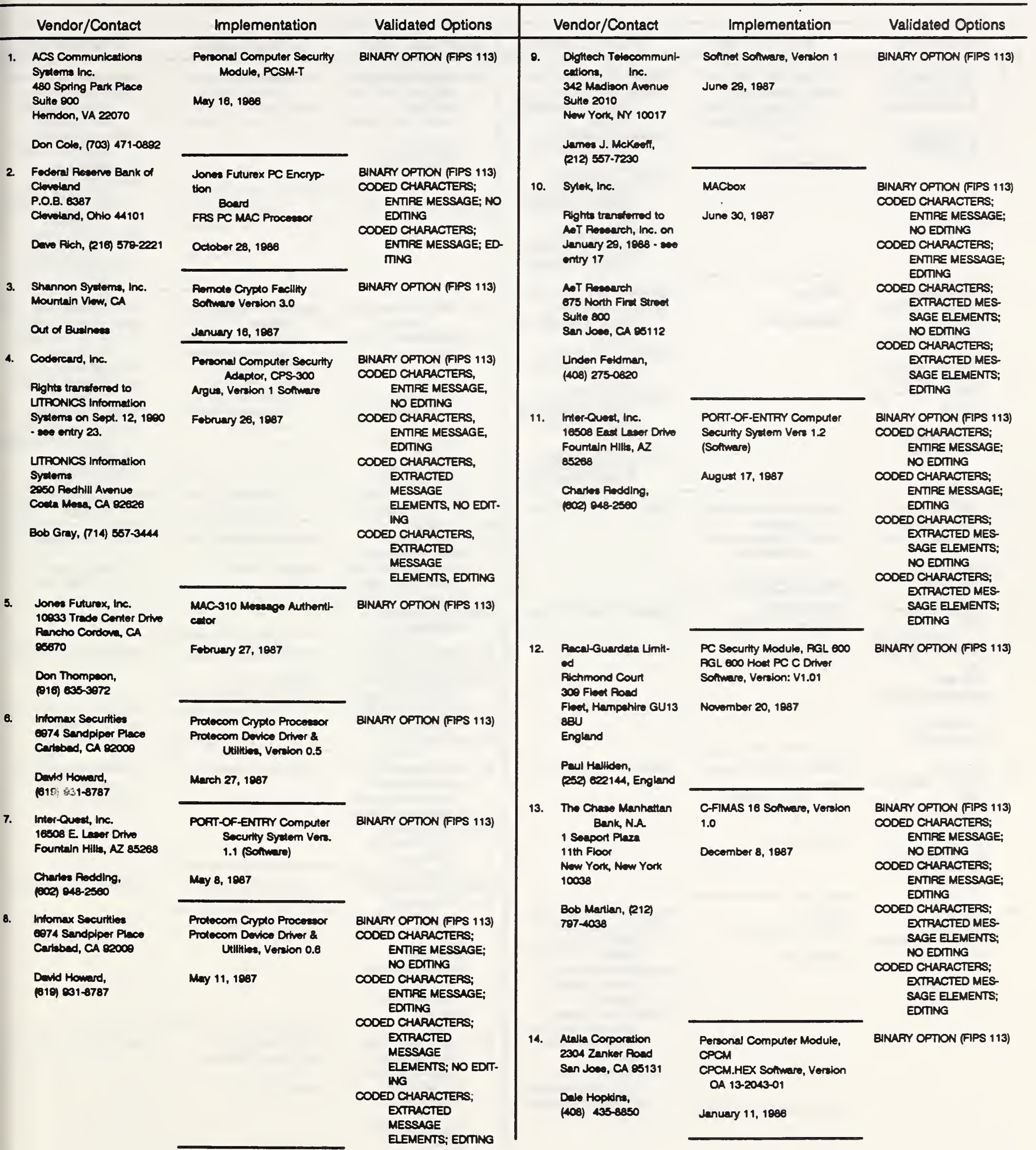




\section{Message Authentication Code (MAC) Implementations, Continued}

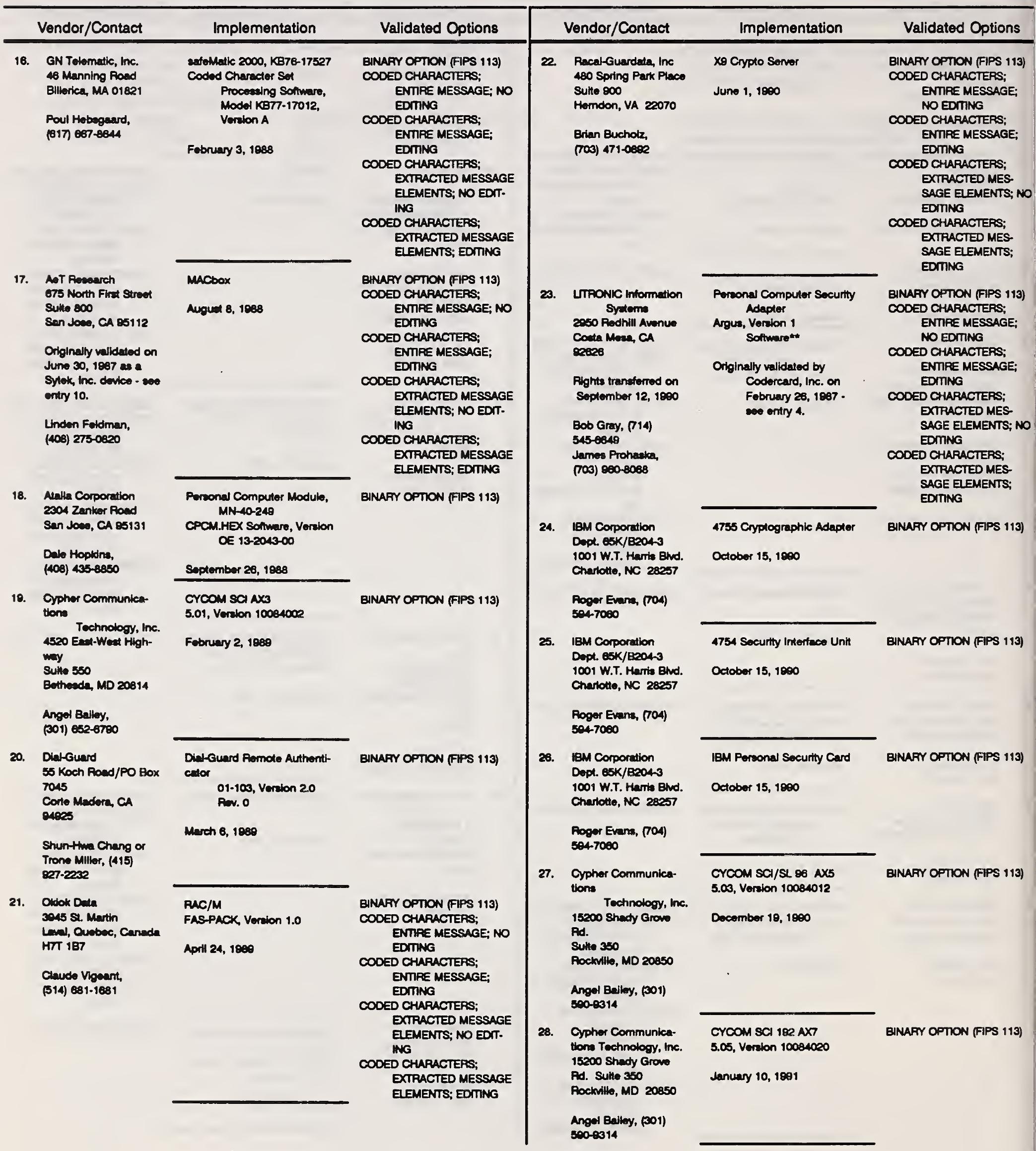




\section{Message Authentication Code (MAC) Implementations, Continued}

\begin{tabular}{|c|c|c|}
\hline Vendor/Contact & Implementation & Validated Options \\
\hline $\begin{array}{l}\text { Digital Equipmont } \\
\text { Corporation } \\
\text { Digital Ditwo- } \\
\text { MKO1-2/806 } \\
\text { Merrimeck, NH } 03054\end{array}$ & $\begin{array}{l}\text { PIN Pad } 201 \text { SMD } \\
\text { Model: P003-120-XX } \\
\text { March 25, } 1601\end{array}$ & BINAPY OPTION (FIPS 113) \\
\hline $\begin{array}{l}\text { Stove Lewrence, } \\
\text { (003) 884-3445 }\end{array}$ & & \\
\hline $\begin{array}{l}\text { Intormation Securitly } \\
\text { Corporation } \\
1141 \text { Late Cook Rond } \\
\text { Suthe D } \\
\text { Deerfield, IL } 60015\end{array}$ & $\begin{array}{l}\text { DES Module used in } \\
\text { SpyProofl } \\
\text { July 10, } 1001\end{array}$ & BINAFY OPTION (FIPS 113) \\
\hline $\begin{array}{l}\text { Mikheal Markoultz, } \\
(706) \text { 405-0500 }\end{array}$ & & \\
\hline $\begin{array}{l}\text { Dightw Signeture } \\
\text { Validatiod by } \\
\text { Intormation Securtly } \\
\text { Corporation } \\
1115 \text { N. Eant Avenue } \\
\text { Oak Park, IL } 60308\end{array}$ & 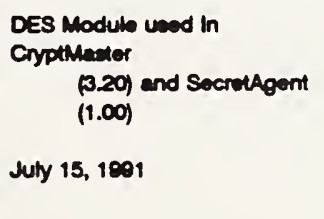 & BINARY OPTON (FIPS 113) \\
\hline $\begin{array}{l}\text { Micheol Markonitz, } \\
\text { (706) } 405-0500\end{array}$ & & \\
\hline $\begin{array}{l}\text { The Exchange Systems } \\
\text { 15ses SE 3oth Pleco } \\
\text { Bellown, WA sec07. } \\
\text { Csod }\end{array}$ & $\begin{array}{l}\text { PCE-3000 (BBM PS/2 } \\
\text { Microchannol) } \\
\text { January 8, } 1002\end{array}$ & $\begin{array}{l}\text { BINAFY OPTION (FIPS 113) } \\
\text { CODED CHWARACTERS; } \\
\text { ENTRE MESSAGE; NO } \\
\text { EDTMNG }\end{array}$ \\
\hline $\begin{array}{l}\text { Robert Adamson, } \\
\text { (206) 644-7000 X255 }\end{array}$ & & $\begin{array}{l}\text { COOED CHARACTERS; } \\
\text { ENTRE MESSAGE; } \\
\text { EDTHNG } \\
\text { COOED CHARACTERS; } \\
\text { EXTRACTED MESSAGE } \\
\text { ELEMENTS; NO } \\
\text { EDTMNG } \\
\text { COOED CHARACTERS; } \\
\text { EXTRACTED MESSAGE } \\
\text { ELEMENTS; EDTMNG }\end{array}$ \\
\hline $\begin{array}{l}\text { The Exchange Syetems } \\
15305 \text { SE 3oth Plece }\end{array}$ & PCE-1000 ISA Adeplor & $\begin{array}{l}\text { BINAFY OPTION (FIPS 113) } \\
\text { CODED CTHRACTERS; }\end{array}$ \\
\hline $\begin{array}{l}\text { Bellowns, WA } 80007 \text { - } \\
\text { eser }\end{array}$ & Jenuary 9,1802 & $\begin{array}{l}\text { ENTRE MESSAGE; NO } \\
\text { EDITING } \\
\text { COOED CHARACTERS, }\end{array}$ \\
\hline $\begin{array}{l}\text { Robort Adamson, } \\
\text { (200) } 7000 \text { X255 }\end{array}$ & & $\begin{array}{l}\text { ENTRE MESSAGE; } \\
\text { EDTING } \\
\text { COOED CHARACTERS; } \\
\text { EXTRACTED MESSAGE } \\
\text { ELMENTS; NO } \\
\text { EDTING } \\
\text { COOED CHARACTERS; } \\
\text { EXTRACTED MESSAGE } \\
\text { EIEMENTS; EDTHG }\end{array}$ \\
\hline
\end{tabular}




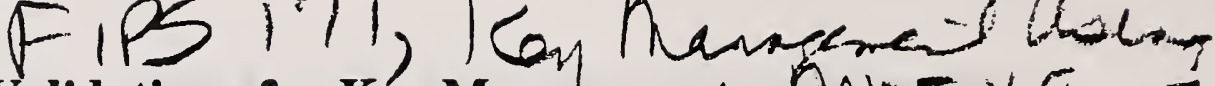 7.8 Validations for Key Management $A N \leq I \times 9,7$}

\begin{tabular}{|c|c|c|c|c|c|c|c|}
\hline & Vendor/Contect & Implementation & Validated Option & & Vendor/Contect & Implementation & Validated Option \\
\hline 1. & 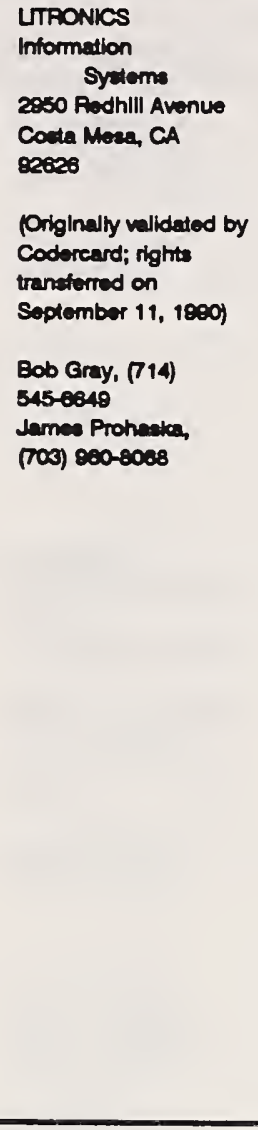 & $\begin{array}{l}\text { Hardware: Argus-PC } \\
\text { Model: CMS-100 } \\
\text { Sormare: Argus/MACE } \\
\text { Sothware Version: } \\
1.0 \\
\text { Soptember 23, } 1988\end{array}$ & 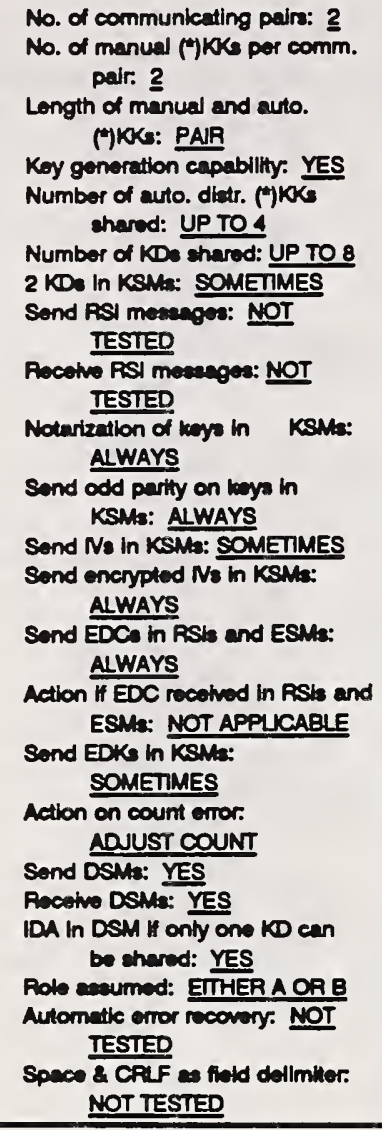 & 3. & $\begin{array}{l}\text { TECHNICAL } \\
\text { COMMUNICATIONS } \\
\text { COAPORATION } \\
100 \text { DOmIno Ditve } \\
\text { CONCORD, } \\
\text { Masaschusetts } \\
01742 \\
\text { John GIII, } \\
\text { (B17) } 862-0035\end{array}$ & $\begin{array}{l}\text { Hardware: } \text { CX5000 } \\
\text { Software: Version: } 2.0\end{array}$ & 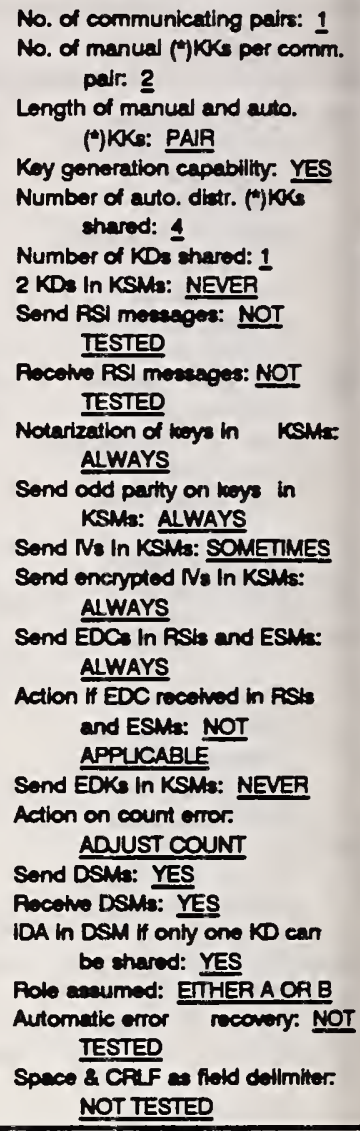 \\
\hline $\begin{array}{l}2 . \\
\text { Max }\end{array}$ & $\begin{array}{l}\text { TECHNICAL } \\
\text { COMMUNICATONS } \\
\text { CORPORATION } \\
100 \text { DOMInO Ditve } \\
\text { CONCORD, } \\
\text { achuastts } 01742 \\
\text { John GH, (B17) } 862 \text {. } \\
\text { Coss }\end{array}$ & $\begin{array}{l}\text { Harduare: } \frac{\text { Cx5000A }}{\text { Solmure: Version: } 1.0} \\
\text { May 6, } 1891\end{array}$ & 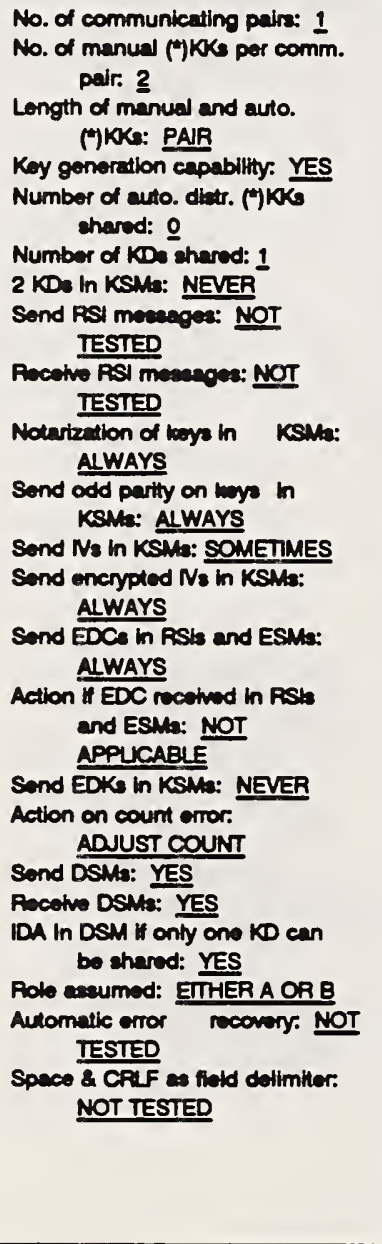 & 4. & $\begin{array}{l}\text { COMMUNKCATION } \\
\text { DEVCES, INC. } \\
1 \text { Fondmann Court } \\
\text { Clinon, NU OTO11 } \\
\text { Gone Harteell, } \\
\text { (201) Tr2-6097 }\end{array}$ & $\begin{array}{l}\text { Herctware: RSD/E } \\
\text { Software: Version } 7.2\end{array}$ & 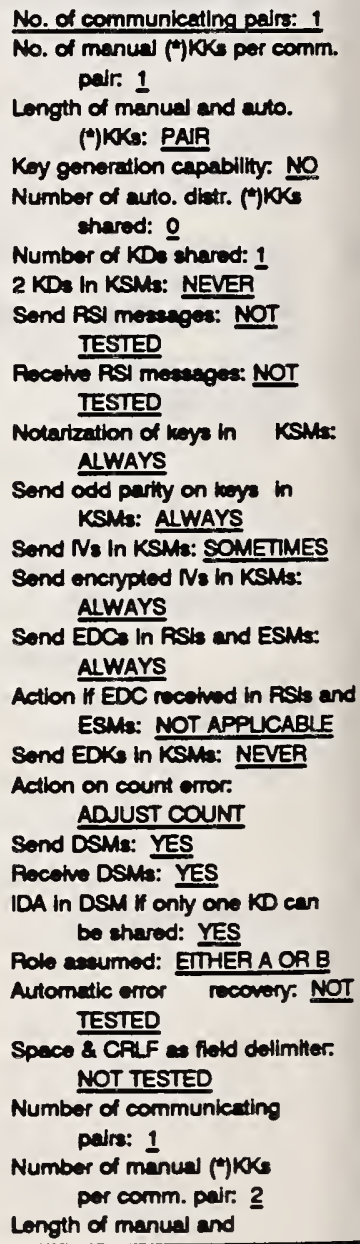 \\
\hline
\end{tabular}




\section{APPENDIX A}

\section{FIPS CONFORMANCE TESTING}

PRODUCTS AND SERVICES 



\section{APPENDIX A}

\section{FIPS CONFORMANCE TESTING PRODUCTS AND SERVICES}

The purpose of this appendix is to provide information about products and services that are available to Federal Agencies for assessing products for conformance to FIPS.

The entries in this list identify the topic, the standard tested, the NIST contact, and the product or service offered. The letters T, S, or C in the Product/Service column indicate a test method, testing service, or certificate/registered report respectively.

TOPIC

COBOL

Fortran

Pascal

C

Ada

MUMPS

SQL
STANDARD

FIPS PUB 21-3

FIPS PUB 69-1

FIPS PUB 109

FIPS PUB 160

FIPS PUB 119

FIPS PUB 125

FIPS PUB 127-1
CONTACT

Judy Kailey

NIST, Bldg. 225, Rm. A266

Gaithersburg, MD 20899

(301) 975-3259

Judy Kailey

NIST, Bldg. 225, Rm. A266

Gaithersburg, MD 20899

(301) $975-3259$

Carmelo Montanez

NIST, Bldg. 225, Rm. A266

Gaithersburg, MD 20899

(301) $975-2398$

Carmelo Montanez

NIST, Bldg. 225, Rm. A266

Gaithersburg, MD 20899

(301) 975-2398

William Dashiell

NIST, Bldg. 225, Rm. A266

Gaithersburg, MD 20899

(301) $975-2490$

William Dashiell

NIST, Bldg. 225, Rm. A266

Gaithersburg, MD 20899

(301) $975-2490$

Joan Sullivan

NIST, Bldg. 225, Rm. A266

Gaithersburg, MD 20899

(301) 975-3258
PRODUCT/SERVICE

T, S, C

T, S, C

T, S, C

T, S, C

T, S, C

T, S, C

T, S, C 


\begin{tabular}{|c|c|c|c|}
\hline TOPIC & STANDARD & CONTACT & PRODUCT \\
\hline GKS & FIPS PUB 120 & $\begin{array}{l}\text { Susan (Quinn) Sherrick } \\
\text { NIST, Bldg. 225, Rm. A266 } \\
\text { Gaithersburg, MD } 20899 \\
\text { (301) 975-3268 }\end{array}$ & $\mathrm{T}, \mathrm{S}, \mathrm{C}$ \\
\hline CGM & $\begin{array}{l}\text { FIPS PUB } 128 \\
\text { MIL-D-28003 }\end{array}$ & $\begin{array}{l}\text { Lynne Rosenthal } \\
\text { NIST, Bldg. 225, Rm. A266 } \\
\text { Gaithersburg, MD } 20899 \\
\text { (301) 975-3353 }\end{array}$ & $\mathrm{T}, \mathrm{S}, \mathrm{C}$ \\
\hline PHIGS & $\begin{array}{l}\text { FIPS PUB } 153 \\
\text { ANSI/ISO } 9592.1-1989\end{array}$ & $\begin{array}{l}\text { Kevin Brady } \\
\text { NIST, Bldg. 225, Rm. A266 } \\
\text { Gaithersburg, MD } 20899 \\
\text { (301) 975-3644 }\end{array}$ & $\mathrm{T}, \mathrm{S}, \mathrm{C}$ \\
\hline Raster & $\begin{array}{l}\text { FIPS PUB } 150 \\
\text { MIL-R-28002 }\end{array}$ & $\begin{array}{l}\text { Frank Spielman } \\
\text { NIST, Bldg. 225, Rm. A266 } \\
\text { Gaithersburg, MD } 20899 \\
\text { (301) 975-3257 }\end{array}$ & $\mathrm{T}$ \\
\hline IRDS & FIPS PUB 156 & $\begin{array}{l}\text { Alan Goldfine } \\
\text { NIST, Bldg. 225, Rm. A266 } \\
\text { Gaithersburg, MD } 20899 \\
\text { (301) 975-3252 }\end{array}$ & $\mathrm{T}, \mathrm{S}, \mathrm{C}$ \\
\hline POSIX & FIPS PUB $151-1$ & $\begin{array}{l}\text { Martha Gray } \\
\text { NIST, Bldg. } 225, \text { Rm. B266 } \\
\text { Gaithersburg, MD } 20899 \\
\text { (301) 975-3276 }\end{array}$ & $\mathrm{T}, \mathrm{S}, \mathrm{C}$ \\
\hline $\begin{array}{l}\text { Message } \\
\text { Authentication }\end{array}$ & FIPS PUB 113 & $\begin{array}{l}\text { Miles Smid } \\
\text { NIST, Bldg. 225, Rm. A216 } \\
\text { Gaithersburg, MD } 20899 \\
\text { (301) 975-2938 }\end{array}$ & $\mathrm{T}, \mathrm{S}, \mathrm{C}$ \\
\hline $\begin{array}{l}\text { Key Management } \\
\text { Validation }\end{array}$ & $\begin{array}{l}\text { FIPS PUB } 171 \\
\text { ANSI X } 9.17\end{array}$ & $\begin{array}{l}\text { Miles Smid } \\
\text { NIST, Bldg. 225, Rm. A216 } \\
\text { Gaithersburg, MD } 20899 \\
\text { (301) 975-2938 }\end{array}$ & $\mathrm{T}, \mathrm{S}, \mathrm{C}$ \\
\hline $\begin{array}{l}\text { Data Encryption } \\
\text { Standard }\end{array}$ & FIPS PUB 46-1 & $\begin{array}{l}\text { Miles Smid } \\
\text { NIST, Bldg. 225, Rm. A216 } \\
\text { Gaithersburg, MD } 20899 \\
\text { (301) 975-2938 }\end{array}$ & $\mathrm{T}, \mathrm{S}, \mathrm{C}$ \\
\hline GOSIP & FIPS PUB 146 & $\begin{array}{l}\text { Stephen Nightingale } \\
\text { NIST, Bldg. 225, Rm } 141 \\
\text { Gaithersburg, MD } 20899 \\
\text { (301) 975-3616 }\end{array}$ & $\mathrm{T}, \mathrm{S}$ \\
\hline
\end{tabular}


TOPIC

$1984 \times 25$

\section{ISDN Data Link}

Layer

Q921.LAPD

ANSI T1.602
CONTACT

David Su

NIST, BIdg. 223, Rm. B364

Gaithersburg, MD 20899

(301) $975-6194$

David Su

NIST, Bldg. 223, Rm. B364

Gaithersburg, MD 20899

(301) $975-6194$

David Su

ISDN Physical Layer S/T Interface

ANSI T1.605 (S/T Interface) NIST, Bldg. 223, Rm. B364

ANSI T1.601 (U Interface)

Gaithersburg, MD 20899

(301) 975-6194

ISDN Network Layer Q931

ANSI T1.607

ANSI T1.608

FIPS PUB (planned)

FDDI
David Su

NIST, Bldg. 223, Rm. B364

Gaithersburg, MD 20899

(301) 975-6194

David Su

NIST, Bldg. 223, Rm. B364

Gaithersburg, MD 20899

(301) 975-6194
PRODUCT/SERVICE

$\mathrm{T}$

$\mathrm{T}$

$\mathrm{T}$ (abstract)

$\mathrm{T}$

$\mathrm{T}$ 


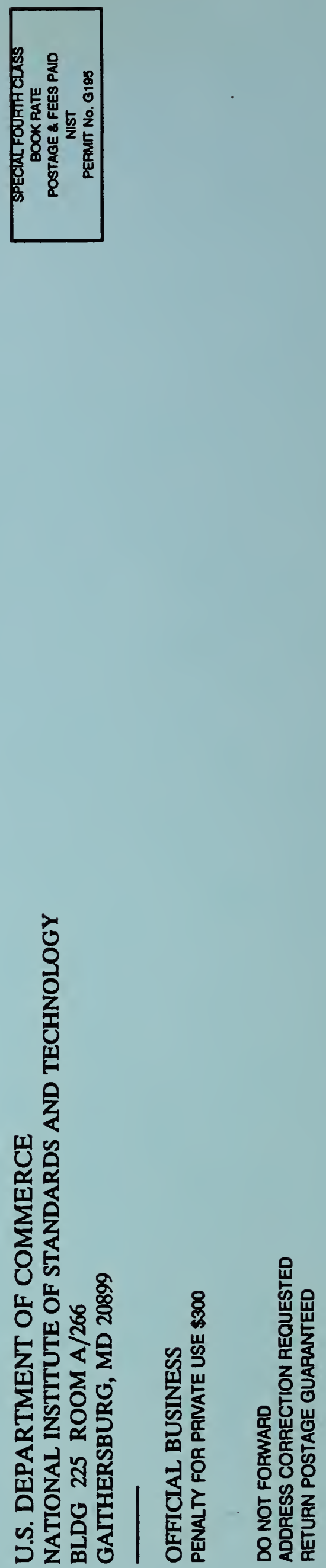